\title{
INVESTIGATIONS OF RING-OPENING REACTIONS OF CYCLOPROPANATED CARBOHYDRATES: TOWARDS THE SYNTHESIS OF THE NATURAL PRODUCT (-)-TAN-2483B
}

\author{
by \\ Russell James Hewitt
}

\section{Victoria \\ UNIVERSITY OF WELLINGTON \\ Te Whare Wānanga
te Ūpoko o te Ika a Māui}

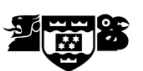

\author{
A thesis \\ submitted to Victoria University of Wellington \\ in fulfilment of the \\ requirements for the degree of \\ Doctor of Philosophy \\ in Chemistry
}

Victoria University of Wellington

2010 


\section{Abstract}

Cyclopropanes and carbohydrates are materials of great interest to chemists. Ring opening reactions of cyclopropanated carbohydrates have excellent potential for synthesis, due to the many diverse structures that may be obtained. The work described in this thesis explores the scope of such ring opening reactions, and extends to the synthesis and reactions of several novel cyclopropanated carbohydrates, in which synthesis of a natural product was also investigated.

Several bicyclic gem-dihalocyclopropanes, including 97, were synthesised. Basemediated cyclopropane ring opening of $\mathbf{9 7}$ in the presence of nucleophiles afforded a series of 2- $C$-branched glycosides 389 and 390 (Chapter 2), whereas silver-promoted ring expansion provided access to seven-membered rings (255 and 256) (Chapter 3). Studies on the mechanisms of the ring opening processes were also carried out.<smiles>CCCO[C@@H]1O[C@H](COBr)[C@@H](O)[C@H](OBr)/C1=C/Br</smiles>

389,390<smiles>BrC[C@H]1O[C@H](Br)[C@H](Br)[C@H](OBr)[C@H]1Br</smiles>

97<smiles>O[C@H]1O[C@H](COCc2ccccc2)[C@@H](Br)C=C(Br)[C@H]1Br</smiles>

255,256

Ring-opening reactions of carbohydrate-derived gem-dihalocyclopropanes were also applied to the exploration of possible routes to the natural product (-)-TAN-2483B (154). Attempts to convert D-galactose and D-xylose into the dihydropyran 193 are the subject of Chapter 4, while the transformation of D-mannose into 193 and subsequent efforts to prepare the natural product 154 are discussed in Chapter 5 .<smiles>[R]C1O[C@H](O)C(O)C(O)C1O</smiles>

$\mathrm{R}=\mathrm{H}, \mathrm{CH}_{2} \mathrm{OH}$

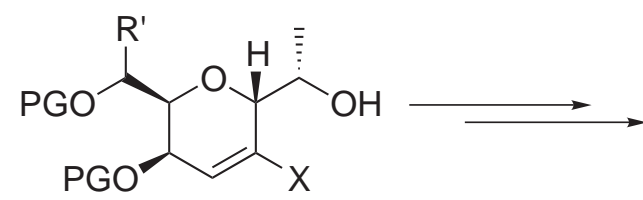

193

$\mathrm{R}^{\prime}=\mathrm{H}, \mathrm{CH}_{2} \mathrm{OPG}$

$\mathrm{PG}=$ protecting group<smiles>C/C=C/[C@H]1O[C@H]2C(=CC1O)C(=O)O[C@H]2C</smiles>

154

(-)-TAN-2483B 
To my Grandmother, Rev. Joan M. Pascoe 


\section{Acknowledgements}

Gandalf : "I am looking for someone to share in an adventure that I am arranging. .." Bilbo : "...I have no use for adventures. Nasty disturbing uncomfortable things! Make you late for dinner!"

J. R. R. Tolkien The Hobbit

First and foremost, thanks to Dr Joanne Harvey, it has been an absolute pleasure working for you. Your enthusiasm (for cyclopropanes especially) and knowledge of chemistry has been so beneficial. Whenever I hit a brick wall, you were always able to provide me with alternative ways around problems. No matter how small my research updates may have been, I was guaranteed of your interest, especially if I had another unusual set of spectra to show you.

Thanks also to the other organic synthesis academics, Dr Brendan Burkett and Dr Paul Teesdale-Spittle. You both had so many useful questions or comments at group meeting, which often helped me think about my chemistry from a different point of view.

I'm very grateful to my thesis editors, Joanne, Brendan, Lynton and Mark. Not only was your hard work of immense value, your kind comments helped my confidence in my storytelling immensely.

I have had a great bunch of labmates over the years. From the earlier days, thanks to Lynton (whose calm efficiency I could never emulate), Emma T, Rhys, Shivali, Emma D, and my labmates in 208 - Robert, Catherine, Ashna, Shi and Jim. For those more recent labmates who put up with a slightly odd "senior lab guy", thanks to Anita, Febly, Sam (best person to share a fumehood with), Thomas, Hemi, Jack, Mark, Peter M (my protégé), Peter C and Xuyu. 
Thanks to Assoc. Prof. Peter Northcote, Dr Mattie Timmer and Prof. John Spencer, and of course their students. Helpful advice, in addition to great communication between the labs was of great benefit.

The general staff at SCPS make postgraduate study so much easier. A big thanks to Lisa and Sally, who saw a lot more of me than most, and always had an answer for my numerous queries. Darren, Grant, Helen, Alan, Manu and John Ryan have all been very helpful in solving all sorts of problems that arose over the years. Thanks also to the lab technicians Rhys, Teresa, Jackie and Jaime-Anne, who frequently let me use or borrow their equipment.

And of course, the gradroom camaraderie has been another bonus, thanks to Anna, Azeem, Benjamin, Carla, Ramprasad and Srikanth. A special thanks to Ashna, who has been a great friend, and it was great to be able to share the latest lab results with Jacqui, one of the few people whose enthusiasm for research could match mine.

Thanks to Mina and John Ryan for providing very helpful $\mathrm{AT}_{\mathrm{E}} \mathrm{X}$ templates, and for all the others who recommended I change from Word!

Finally, I must thank those outside of SCPS. Thanks to my friends and numerous flatmates, especially to Chao and Jo, and Nancy (a wonderful neighbour) who could always help me take my mind off chemistry.

A final thanks to my family. Mum and Terry, Dad and Helen, and my sister Rebecca, you have always been there for me, and have made study so much easier with your support, despite the distances that separate us. 


\section{Table of Contents}

Abstract

Acknowledgments

Table of Contents vi

List of Figures $\quad$ ix

List of Schemes $\quad$ X

List of Tables $\quad$ xiv

Glossary $\quad$ Xv

Compound Numbering $\quad$ xvii

$\begin{array}{ll}\text { Publication } & \text { xviii }\end{array}$

1 Introduction $\quad 1$

1.1 Cyclopropanes . . . . . . . . . . . . . . . . . . 1

1.1.1 Synthesis of Cyclopropanes . . . . . . . . . . . . . 1

1.1.2 Ring Expansion of gem-Dihalocyclopropanes . . . . . . . . . . 2

1.2 Cyclopropanated Carbohydrates . . . . . . . . . . . . . . 3

1.2.1 Synthesis of Cyclopropanated Carbohydrates . . . . . . . . . . 4

1.2.2 Reactions of Cyclopropanated Carbohydrates . . . . . . . . 10

1.3 Oxepanes, Oxepines and Septanoses . . . . . . . . . . . . . . . . 19

1.4 Furo[3,4-b]pyran-5-ones . . . . . . . . . . . . . . . . . . . . 21

1.4.1 Syntheses of Furo[3,4-b]pyran-5-ones . . . . . . . . . . . 21

1.4.2 Furo[3,4-b]pyran-based Natural Products . . . . . . . . . . . . 23

1.4.3 Syntheses of Furo[3,4-b]pyran-Based Natural Products . . . . 26

1.5 Research Objectives . . . . . . . . . . . . . . . . . . . . . 30

1.5.1 Ring-Expansion Studies . . . . . . . . . . . . . . 30

1.5.2 Synthesis of $(-)$-TAN-2483B . . . . . . . . . . . . . 32 
2 Base-Mediated Ring-Opening Reactions 35

2.1 Introduction . . . . . . . . . . . . . . . . . . . . . . 35

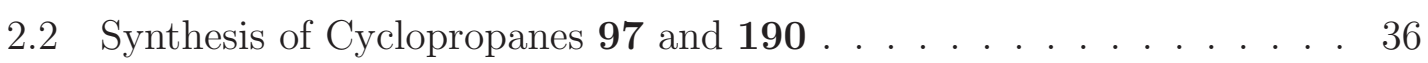

2.3 Base-Mediated Methanolysis of Cyclopropanes 97 and 190 . . . . . 39

2.4 Mechanistic Studies . . . . . . . . . . . . . . . . . . . . . . . 43

2.5 Base-Mediated Ring Opening with Alternative Nucleophiles . . . . . 48

2.6 Palladium-Catalysed Coupling Reactions . . . . . . . . . . . . . . . . 49

2.7 Reactions of Galactose and Xylose Derivatives . . . . . . . . . . . . . 50

2.8 Summary of Chapter $2 \ldots \ldots \ldots$. . . . . . . . . . . . 53

3 Silver-Mediated and Thermal Ring-Opening Reactions $\quad 54$

3.1 Introduction . . . . . . . . . . . . . . . . . . . . . . 54

3.2 Silver-Mediated Ring-Expansion Reactions in Acetic Acid . . . . . . 55

3.3 Ring-Expansions of Cyclopropane 97 with Alternative Nucleophiles . 57

3.4 Scope of Oxepine Synthesis . . . . . . . . . . . . . . . . . . . 60

3.5 Oxepines Derived from Galactose and Xylose . . . . . . . . . . . . . 62

3.6 Oxepine Derivatisation Reactions . . . . . . . . . . . . . . 63

3.7 Synthesis of Furanosyl Sugars . . . . . . . . . . . . . . . . . . . . . 64

3.8 Summary of Chapter $3 \ldots \ldots$. . . . . . . . . . . . . . . . . . 69

4 Galactose and Xylose-Based Approaches to (-)-TAN-2483B $\quad 70$

4.1 Introduction . . . . . . . . . . . . . . . . . . . . . 70

4.2 Synthesis and Cyclopropanations of 2,3-Unsaturated Sugar 290 . . . 72

4.3 Synthesis of Alternative 2,3-Unsaturated Carbohydrates . . . . . . . . 74

4.4 -Methoxybenzyl-Protected Xylal Route to (-)-TAN-2483B . . . . . 76

4.5 Benzyl-Protected Xylal Route to $(-)$-TAN-2483B . . . . . . . . . . . 79

4.6 Summary of Chapter $4 \ldots \ldots$. . . . . . . . . . . . . . . . . 81

5 Approaches to (-)-TAN-2483B from Mannose 82

5.1 Introduction . . . . . . . . . . . . . . . . . . . . . 82

5.2 Synthesis of Mannose-Based Glycal 209 . . . . . . . . . . . . . . . 84

5.3 Synthesis of the Pyran Ring of $(-)$ TAN-2483B . . . . . . . . . . 85

5.3 .1 Cyclopropanation of Glycal 209 . . . . . . . . . . . . 86

5.3.2 Ring Expansion of Mannose-Derived Cyclopropane 340 . . . . 87

5.4 Functionalisation of the Pyran Ring of (-)-TAN-2483B . . . . . . . . 89

5.4.1 Ethylidene Wittig-Based Route . . . . . . . . . . . . . . . 89

5.4 .2 Methylidene Wittig-Derived Route . . . . . . . . . . . . . . . 91

5.4.3 Stabilised Wittig-Based Route to (-)-TAN-2483B . . . . . . . 93

5.4.4 HWE/Ramberg-Bäcklund Route to (-)-TAN-2483B . . . . . 94

5.5 Approaches to the furo[3,4-b]pyran-5-one skeleton of (-)-TAN-2483B 99

5.6 Summary of Chapter 5 . . . . . . . . . . . . . . . . . 102 
6.1 Ring Opening Studies . . . . . . . . . . . . . . . . . . . 103

6.2 Natural Product Synthesis . . . . . . . . . . . . . . . . . . . . . 104

7 Experimental $\quad 106$

7.1 General Experimental Details . . . . . . . . . . . . . . 106

7.2 Chapter Two Experimental . . . . . . . . . . . . . . . 107

7.3 Chapter Three Experimental . . . . . . . . . . . . . . . . . . . 127

7.4 Chapter Four Experimental . . . . . . . . . . . . . . . 145

7.5 Chapter Five Experimental . . . . . . . . . . . . . 155

$\begin{array}{ll}\text { Spectra } & 180\end{array}$

$\begin{array}{ll}\text { References } & 193\end{array}$ 


\section{List of Figures}

1.1 Simmons-Smith derived cyclopropanes . . . . . . . . . . . . . 6

1.2 Formation of septanoside-based trisaccharides via cyclopropanes . . . 18

1.3 Seven-membered ring natural products . . . . . . . . . . . . . . . . 19

1.4 Seven-membered oxacycles . . . . . . . . . . . . . . . . . . . . 19

1.5 Examples of septanose carbohydrates . . . . . . . . . . . . . 20

1.6 Furo[3,4-b]pyran-5-one . . . . . . . . . . . . . . . . . . . 21

1.7 Selected examples of furo[3,4-b]pyran-based natural products . . . . . 23

1.8 Fusidilactones A-E . . . . . . . . . . . . . . . . . . 24

1.9 Massarilactones A-D . . . . . . . . . . . . . . . . 25

1.10 TAN-2483 natural products . . . . . . . . . . . . . . 25

1.11 Initial and revised structures of waol A and B . . . . . . . . . 26

$2.1{ }^{1} \mathrm{H}-\mathrm{NMR}$ spectra of 213 and $214 \ldots \ldots$. . . . . . . . . . . . . . . 40

2.2 Selected NMR correlations of 213 and 214 . . . . . . . . . . . . . . 40

2.3 Selected methylene and halomethylene compounds . . . . . . . . . . . 41

$4.1(R, R)$-Jacobsen's catalyst . . . . . . . . . . . 78

5.1 Cyclopropanes 290 and $243 \ldots \ldots$. . . . . . . . . . 82 


\section{List of Schemes}

1.1 Common cyclopropanation methodologies . . . . . . . . . . 2

$1.2 \mathrm{gem}$-Dihalocyclopropane ring opening . . . . . . . . . . . . . 2

1.3 Conversion of gem-dihalocyclopropane 7 into an allylic acetate . . . . 2

1.4 Ring expansion of cyclopropanated pyran 10 . . . . . . . . . . . 3

1.5 Exemplification of DePuy's "cis-in, trans-out" rule . . . . . . . . 3

1.6 Exocyclic carbanion-mediated cyclopropanation . . . . . . . . . 5

1.7 Silver-mediated synthesis of 4,5-cyclopropanated carbohydrate 20 . . 5

1.8 Ring-contraction cyclopropanation . . . . . . . . . . 5 5

1.9 Simmons-Smith cyclopropanation of 2,3-unsaturated sugar 25 . . . 6

1.10 Ethyl diazoacetate-mediated cyclopropanation . . . . . . . . . . . . 7

1.11 Preparation of a cyclopropyl-linked fullerene-sugar conjugate . . . . 7

1.12 HWE-type cyclopropanation . . . . . . . . . . . . . 8

1.13 A cyclopropane synthesised from an $S$-ylide . . . . . . . . . . 8

1.14 The first reported cyclopropanated carbohydrate $(39) \ldots$. . . . . . . 9

1.15 Formation of spirocyclopropane sugars . . . . . . . . . . . . . . 9

1.16 Cyclopropanations reported by Nagarajan . . . . . . . . . . . . . . . 9

1.17 Stereoselective cyclopropanations of 4,5-unsaturated sugars . . . . . . 10

1.18 Ring opening and ring expansion of cyclopropanes . . . . . . . . . . . 10

1.19 Cascade radical cyclisation of cyclopropane 53 . . . . . . . . . . . . 11

1.20 Ring-opened and ring-expanded cyclopropane derivatives . . . . . . . 11

1.21 Synthesis of epothilones via cyclopropane 59 . . . . . . . . . . . . . . 12

1.22 Synthesis of various cyclopropane-derived disaccharides . . . . . . . . 13

1.23 Synthesis of glyco-amino acid $72 \ldots \ldots$. . . . . . . . . . . 13

1.24 Platinum-catalysed ring opening of cyclopropane 73 . . . . . . . . 14

1.25 Ring opening reactions of cyclopropanated sugar 77 . . . . . . . . . 14

1.26 Synthesis of $(+)$-calystegine B2 . . . . . . . . . . . . . 15

1.27 Glucal and galactal-derived oxepines . . . . . . . . . . . . . . 16

1.28 Ring expansion of cyclopropanated sugar 90 . . . . . . . . . . . 16

1.29 Oxazepine formation and Suzuki coupling . . . . . . . . . . . . 16

1.30 Reported ring expansion of glycal-derived cyclopropane 97 . . . . . 17

1.31 Formation of septanosides via cyclopropane 101 . . . . . . . . . . . 17

1.32 Formation of septanosides via cyclopropane 105 . . . . . . . . . . . 18 
1.33 RCM-based synthesis of septanoses . . . . . . . . . . . . . . . . 20

1.34 Synthesis of a fragment of lasonalide A . . . . . . . . . . . . . 21

1.35 Synthesis of furo[3,4-b]pyran-5-ones via a multi-component reaction . 22

1.36 Vitamin C-derived furo[3,4-b]pyran-5-ones . . . . . . . . . . . . 22

1.37 Synthesis of furo[3,4-b]pyran-5-ones from 2,3-dihydropyran . . . . . . 23

1.38 Syntheses of (-)-TAN-2483A (153) and (-)-waol A (157) . . . . . 27

1.39 Synthesis of $(-)$-massarilactone B (150) and diol 169 . . . . . . . 27

1.40 Attempted synthesis of $(-)$-TAN-2483B . . . . . . . . . . . . 28

1.41 Attempted epoxide-mediated synthesis of (-)-TAN-2483B . . . . . . 29

1.42 Synthesis of hexose-derived cyclopropanes . . . . . . . . . . . . 30

1.43 Synthesis of acetyl and benzyl glycals . . . . . . . . . . . . . . . . 31

1.44 Synthesis of glucose-derived cyclopropanes 97 and 190 . . . . . . . 31

1.45 Cyclopropane ring-expansion . . . . . . . . . . . . . . 32

1.46 Cyclopropyl carbohydrate-based retrosynthesis of (-)-TAN-2483B . . 32

1.47 Retrosynthesis of galactose-based (-)-TAN-2483B route . . . . . . . 33

1.48 Retrosynthesis of (-)-TAN-2483B using a xylose-based route . . . . . 34

1.49 Retrosynthesis of mannose-based $(-)$-TAN-2483B route . . . . . . . 34

2.1 Nagarajan's reported oxepine synthesis . . . . . . . . . . . . . . 36

2.2 Synthesis of acetyl glucal (179) and benzyl glucal (188) . . . . . . 36

2.3 Cyclopropanation of tri- $O$-acetyl-D-glucal (179) . . . . . . . . . 37

2.4 Base-mediated ring opening of cyclopropane 97 . . . . . . . . . . . . 40

2.5 Lithium-halogen exchange of bromoalkene 214 . . . . . . . . . . . 41

2.6 Synthesis of tetradeutero pyranoside sugars 219 and 220 . . . . . . 44

2.7 Mechanism of base-mediated ring opening of cyclopropane $\mathbf{9 7}$. . . . 45

2.8 Reaction of cyclopropane $\mathbf{9 7}$ with $n$-butyllithium . . . . . . . . . . 47

2.9 Synthesis of glycosylamines 240 and $241 \ldots \ldots$. . . . . . . . . . 49

2.10 Attempted Suzuki coupling of 2- $C$-branched sugar 214 . . . . . . . 50

2.11 Stille coupling of 2 - $C$-branched sugar $214 \ldots \ldots$. . . . . . . 50

2.12 Nagarajan's reported synthesis of oxepines 244, 245, 246 and $\mathbf{2 4 7}$. 51

2.13 Synthesis of galactose- and xylose-based glycals . . . . . . . . . . . 51

2.14 Synthesis of galactose- and xylose-based cyclopropanes . . . . . . . . 52

2.15 Ring opening of galactose-based cyclopropane 45 . . . . . . . . . . . 53

2.16 Ring opening of xylose-based cyclopropane $243 \ldots \ldots$. . . . . . . . 53

3.1 Ring expansion of cyclopropane $97 \ldots \ldots$. . . . . . . . . . 54

3.2 Synthesis of acetoxy oxepines 257,258 and $259 \ldots \ldots$. . . . . . 56

3.3 Mechanism of the ring-expansion reaction of cyclopropane $\mathbf{9 7}$. . . 59

3.4 Synthesis of bicyclic acetal 267 . . . . . . . . . . . . . 60

3.5 Synthesis of 2-C-branched sugars and oxepines . . . . . . . . . . . . 61

3.6 Formation of galactose-derived oxepine 269 . . . . . . . . . . . . 63 
3.7 Formation of xylose-derived oxepines 271 and $272 \ldots$. . . . . . 63

3.8 Lithium-halogen exchange of allyl oxepine 261 . . . . . . . . . . . 64

3.9 Heck cyclisation of allyl oxepine 261 . . . . . . . . . . . . . . . . 64

3.10 Formation of methylated $C$-furanosyl sugars 275, 276 and 277 . . . 65

3.11 Formation of allylated $C$-furanosyl sugars 278, 279 and 280 . . . . 66

3.12 Lithium-halogen exchange of bromoalkene 280 . . . . . . . . . . . 66

3.13 Mechanism of conversion from oxepines into furanosyl products . . . 68

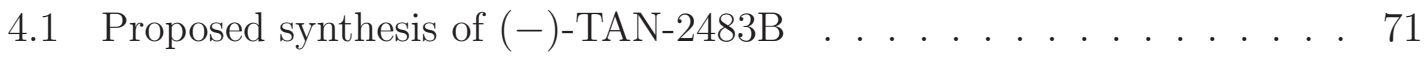

4.2 Synthesis of thioglycoside $289 \ldots \ldots$. . . . . . . . . . . . 73

4.3 Synthesis of galactose-based substrate 290 . . . . . . . . . . . . 73

4.4 Unsuccessful epimerisation of 290 . . . . . . . . . . . . . . . 74

4.5 Synthesis of carbonate $304 \ldots \ldots$. . . . . . . . . . . . . 75

4.6 Isomerisation of $180 \ldots \ldots$. . . . . . . . . . . . . . 75

4.7 Hydrolysis of glycal $\mathbf{1 8 0} \ldots \ldots \ldots$. . . . . . . . . . . . . 76

4.8 Formation of trichloroacetimidate $309 \ldots \ldots$. . . . . . . 76

4.9 Proposed xylose-based synthesis of $($-)-TAN-2483B . . . . . . . . 77

4.10 Synthesis and cyclopropanation of xylose-based glycal 314 . . . . . 78

4.11 Unsuccessful ring-expansion of cyclopropane 315 . . . . . . . . . . . 79

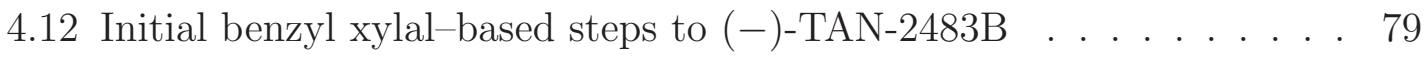

4.13 Formation of furanosyl aldehyde $322 \ldots$. . . . . . . . . . . . . 80

4.14 Intramolecular 1,4 -addition of xylose derivative 323 . . . . . . . . . 81

4.15 Attempted deprotection/olefination of oxepines 271 and 272 . . . . 81

5.1 Intramolecular conjugate addition of 323 vs. $326 \ldots$. . . . . . 83

5.2 Proposed furanoglycal-based route to (-)-TAN-2483B . . . . . . . 83

5.3 Proposed mannose-based synthesis of $(-)$-TAN-2483B . . . . . . . . 84

5.4 Synthesis of mannose-based glycal 209 . . . . . . . . . . . . . . . 85

5.5 Key cyclopropanation/expansion sequence . . . . . . . . . . . . 85

5.6 Attempted gem-dibromocyclopropanation of glycal 209 . . . . . . . 86

5.7 Synthesis of mannose-based cyclopropane 340 . . . . . . . . . . 86

5.8 Ring-expansion of mannose-derived cyclopropane 340 . . . . . . . . 87

5.9 Cyclopropanation and ring-expansion of mannose-derived glycal 20988

5.10 Synthesis of key hemiacetal 345 . . . . . . . . . . . . . . . . . 89

5.11 Ethylidene Wittig/epoxide route . . . . . . . . . . . . . . . . . . . 90

5.12 Ethylidene Wittig/iodoetherification route . . . . . . . . . . . . . . 91

5.13 Proposed methylidene Wittig-derived route to (-)-TAN-2483B . . . 91

5.14 Methylidene Wittig/epoxide route . . . . . . . . . . . . . . . . . . . . 92

5.15 Methylidene Wittig iodoetherification . . . . . . . . . . . . . . . . . 92

5.16 Ethoxycarbonylmethylene Wittig/epoxide route . . . . . . . . . . . 93

5.17 Mechanism of base-mediated ring opening of ester 361 . . . . . . . 94 
5.18 The Ramberg-Bäcklund reaction . . . . . . . . . . . . . . . . . 94

5.19 Proposed sulfone-based Ramberg-Bäcklund route . . . . . . . . . . . 95

5.20 Synthesis of sulfonated sugar 367 . . . . . . . . . . . . . . 96

5.21 Attempted synthesis of alkene 368 under Meyers conditions . . . . . 96

5.22 Attempted synthesis of vinyl sugar 368 under Chan conditions . . . . 97

5.23 Synthesis of $\alpha, \beta$-unsaturated sulfone $372 \ldots \ldots$. . . . . . . . . . . . 99

5.24 Attempted lactone formation of alcohols 357 and 358 . . . . . . . . 99

5.25 Ring-opening of iodosugar 359 . . . . . . . . . . . . . . . . 100

5.26 Carbonylation of diene 353 . . . . . . . . . . . . . . 100

5.27 Iodoetherification of ester $380 \ldots \ldots 101$

5.28 Proposed lactonisation of iodoesters 381 and $382 \ldots . . . . . .101$

6.1 Palladium-catalysed cross-coupling of 2-C-bromomethylene sugars . 103

6.2 Reactions of cyclopropane $\mathbf{9 7}$ with carbon and nitrogen nucleophiles . 104

6.3 Ring-opening reactions of cyclopropane 235 . . . . . . . . . . . . . . 104

6.4 Synthesis of $\alpha$-hydroxy ester 395 . . . . . . . . . . . . . 105

6.5 Synthesis of phenyl-TAN-2483B (397) . . . . . . . . . . . 105 


\section{List of Tables}

2.1 Cyclopropanation of tri- $O$-benzyl-D-glucal (188) . . . . . . . . 38

2.2 Base-mediated ring-opening of cyclopropanes $\mathbf{9 7}$ and $\mathbf{1 9 0}$. . . . . . 42

2.3 Comparison of the reactivity between cyclopropanes $\mathbf{9 7}$ and $\mathbf{1 9 0}$. . . 43

2.4 Ring opening of cyclopropane $\mathbf{9 7}$ with various nucleophiles . . . . . . 48

3.1 Acetic acid-mediated ring-expansions of cyclopropane 97 . . . . . . 56

3.2 Ring-expansion of cyclopropane $\mathbf{9 7}$ with various nucleophiles . . . . . 58

3.3 Miscellaneous ring-expansions of cyclopropane $97 \ldots$. . . . . . . 61

5.1 Attempts at $\alpha$-halogenating sulfone $367 \ldots \ldots$. . . . . . . . . 98 


\section{Glossary}

\begin{tabular}{|c|c|}
\hline AIBN & 2,2 '-azo-bis-isobutyronitrile \\
\hline Allyl & $\mathrm{CH}_{2} \mathrm{CHCH}_{2^{-}}$ \\
\hline BAIB & [bis-(acetoxy)iodo]benzene \\
\hline brs & broad singlet \\
\hline conc. & concentration \\
\hline COSY & correlation spectroscopy \\
\hline CSA & $(1 S)$-(+)-10-camphorsulfonic acid \\
\hline d & doublet \\
\hline DBU & 1,8-diazabicyclo[5.4.0]undec-7-ene \\
\hline $\mathrm{DCC}$ & $N, N^{\prime}$-dicyclohexylcarbodiimide \\
\hline DDQ & 2,3-dichloro-5,6-dicyanobenzoquinone \\
\hline DIBAL-H & diisobutylaluminium hydride \\
\hline DMAP & $N, N$-dimethyl-4-aminopyridine \\
\hline DMDC & $2^{\prime}$-deoxy-2'-methylidenecytidine \\
\hline DME & 1,2-dimethoxyethane (a.k.a. ethylene glycol dimethyl ether) \\
\hline $\mathrm{DMF}$ & $N, N$-dimethylformamide \\
\hline DMSO & dimethylsulfoxide \\
\hline dppf & 1,1'-bis(diphenylphosphino)ferrocene \\
\hline eq. & equivalents \\
\hline HMBC & heteronuclear multi bond correlation \\
\hline HMPA & hexamethylphosphoramide \\
\hline HRMS & high resolution mass spectrometry \\
\hline HWE & Horner-Wadsworth-Emmons \\
\hline IR & infrared \\
\hline KHMDS & potassium hexamethyldisilazide \\
\hline LDA & lithium diisopropylamide \\
\hline LiHMDS & lithium hexamethyldisilazide \\
\hline m.p. & melting point \\
\hline$m-\mathrm{CPBA}$ & $m$-chloroperbenzoic acid \\
\hline Ms & methanesulfonyl (a.k.a. mesyl) \\
\hline NBS & $N$-bromosuccinimide \\
\hline NIS & $N$-iodosuccinimide \\
\hline
\end{tabular}




$\begin{array}{ll}\text { NMR } & \text { nuclear magnetic resonance } \\ \text { nOe } & \text { nuclear Overhauser effect } \\ \text { NOESY } & \text { nuclear Overhauser effect spectroscopy } \\ \text { Nuc } & \text { nucleophile } \\ \text { PG } & \text { protecting group } \\ \text { PMB } & \text { p-methoxybenzyl } \\ \text { PMP } & \text { p-methoxyphenyl } \\ \text { PPTS } & \text { pyridinium p-toluenesulfonate } \\ \text { q } & \text { quartet } \\ \text { r.t. } & \text { room temperature } \\ \text { RCM } & \text { ring closing metathesis } \\ \text { Rf } & \text { retention factor } \\ \text { s } & \text { singlet } \\ \text { sat. } & \text { saturated } \\ \text { t } & \text { triplet } \\ \text { TBAI } & \text { tetrabutylammonium iodide } \\ \text { TBS } & t \text {-butyldimethylsilyl } \\ \text { TEBAC } & \text { benzyltriethylammonium chloride } \\ \text { TEMPO } & 2,2,6,6 \text {-tetramethylpiperidine-1-oxyl } \\ \text { Tf } & \text { trifluoromethanesulfonyl (a.k.a. triflyl) } \\ \text { THF } & \text { tetrahydrofuran } \\ \text { TLC } & \text { thin layer chromatography } \\ \text { TMS } & \text { trimethylsilyl } \\ \text { Ts } & p \text {-toluenesulfonyl (a.k.a. tosyl) } \\ & \end{array}$




\section{Compound Numbering}

The naming and numbering of the majority of compounds in this thesis is based on the IUPAC recommendations for carbohydrates. ${ }^{1}$ Thus, the main carbon backbone is numbered from either the anomeric carbon, or in the case of $C$-glycosyl derivatives, numbered from the terminal end of the aglycone. For the purposes of numbering when presenting experimental data, all well-known groups are listed using common abbreviations, others (e.g. allyl), are numbered from their point of connection with the main carbon chain; priority is given to carbon-carbon bonded sidechains. Compounds incorporating the furo[3,4-b]pyran-5-one ring system are named and numbered according to IUPAC recommendations for heterocyclic compounds (see below).

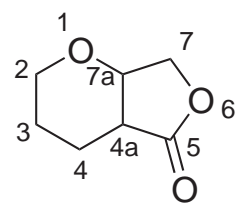




\section{Publication}

The following publication was based upon work carried out during the period of $\mathrm{PhD}$ candidature, discussed in chapters 2 and 3 of this thesis.

"Synthesis of Oxepines and 2-Branched Pyranosides from a D-Glucal-Derived gemDibromo-1,2-cyclopropanated Sugar"

Hewitt, R. J.; Harvey, J. E. J. Org. Chem. 2010, 75, 955-958. 


\section{Chapter 1}

\section{Introduction}

\subsection{Cyclopropanes}

Cyclopropanes are of interest in organic synthesis as targets themselves, or as substrates for further reactions, ${ }^{2}$ which can provide access to complex and synthetically challenging structures. These highly strained three-membered rings have been known for over a century, since W. H. Perkin described the first synthesis of a cyclopropane derivative in 1884, through attack of diethyl malonate dianion upon 1,2dibromoethane. ${ }^{3,4}$ Subsequently, a large volume of research involving cyclopropanes has evolved, and discussions of their synthesis and reactions have been the subject of several books ${ }^{5,6}$ and reviews. ${ }^{7-10}$

\subsubsection{Synthesis of Cyclopropanes}

The generation of cyclopropanes has been thoroughly investigated, with many welldocumented procedures providing cyclopropanes of varying functionality. Two of the most commonly employed procedures are the Simmons-Smith and Mąkosza cyclopropanations (Scheme 1.1). The Simmons-Smith reaction forms a cyclopropane (1) through formal addition of carbene $\left(: \mathrm{CH}_{2}\right)$ to an olefin $(2)$, which installs a methylene bridge. ${ }^{11}$ gem-Dihalocyclopropanes (3) are predominantly formed using the biphasic Mąkosza cyclopropanation. ${ }^{12}$ This reaction involves base-mediated deprotonation of a haloform, which then forms the corresponding dihalocarbene $\left(: \mathrm{CX}_{2}\right)$, 
which may be trapped by an olefin substrate. The research presented in this thesis involves the synthesis and reactions of ring-fused gem-dihalocyclopropanes.

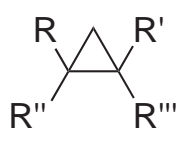

1

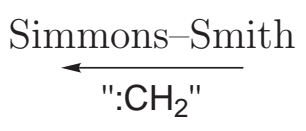

$: \mathrm{CH}_{2}$

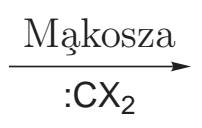

2

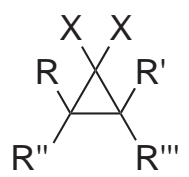

3

Scheme 1.1 Common cyclopropanation methodologies ${ }^{11,12}$

\subsubsection{Ring Expansion of gem-Dihalocyclopropanes}

Ring-fused cyclopropanes undergo ring-expansion reactions, in which the carbon provided by the cyclopropane is used to create a larger ring, as exemplified in Scheme 1.2. gem-Dihalocyclopropanes such as 4 undergo ring opening through either a thermal or halophile-mediated process. The use of silver salts is an excellent way of mediating this reaction, as the silver ion abstracts a halogen atom, to provide an intermediate such as $5 .^{8}$ The departed halogen will be trapped as a silver halide salt, therefore it cannot attack intermediate $\mathbf{5}$. Instead, in the presence of a chosen nucleophile, an allyl-functionalised product (6) is formed, which may be exploited using cross-coupling reactions or other processes to form a variety of complex targets, including natural products. ${ }^{13-15}$

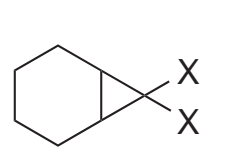

4
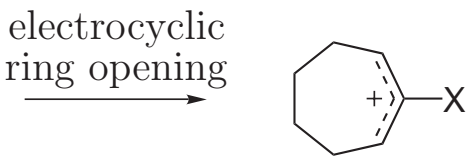

5
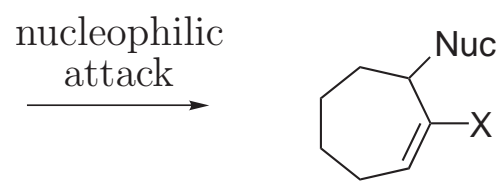

6

Scheme 1.2 gem-Dihalocyclopropane ring opening

Ring expansions of bicyclo[n.1.0] species offer elegant ways of synthesising rings that may be less readily available by other means. In 1967, Sandler demonstrated that cyclopropanes such as 7 can undergo ring-expansion reactions to generate allylic acetates (8) (Scheme 1.3). ${ }^{16}$ This sequence highlights the synthetic utility of these cyclopropanes, enabling the conversion of olefins $(\mathbf{9})$ into products such as $\mathbf{8}$.

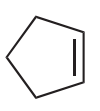

9
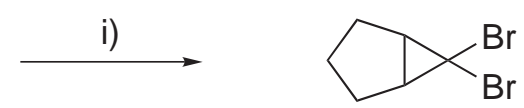

7
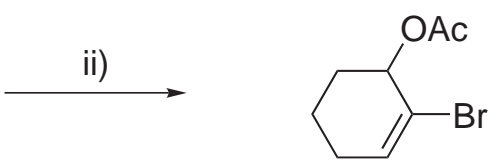

8

Reagents and Conditions: i) $\mathrm{CHBr}_{3}, \mathrm{KO}^{t} \mathrm{Bu}$, pentane, $0{ }^{\circ} \mathrm{C}$ to r.t.; ii) $\mathrm{AgOAc}, \mathrm{AcOH}$, reflux.

Scheme 1.3 Conversion of gem-dihalocyclopropane 7 into an allylic acetate ${ }^{16}$

Skattebøl reported one of the earliest examples of cyclopropane ring expansion, using pyran-based gem-dichlorocyclopropane 10. ${ }^{17}$ Upon treatment with potassium tert- 
butoxide, functionalised oxepine $\mathbf{1 1}$ was isolated in $79 \%$ yield (Scheme 1.4). The nucleophile selectively attacked the oxygenated carbon, as participation of the pyran oxygen(see 12), provided oxonium ion 13, which led to selective formation of acetal 11.<smiles>ClC1(Cl)C2CCCOC21</smiles>

10

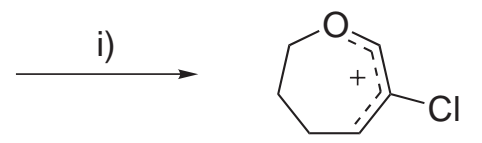

12

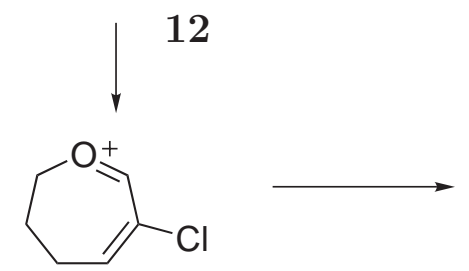

13

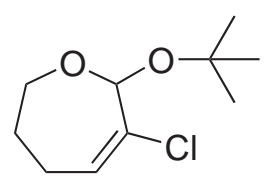

11

Reagents and Conditions: i) $\mathrm{KO}^{t} \mathrm{Bu},{ }^{t} \mathrm{BuOH}$, reflux.

Scheme 1.4 Ring expansion of cyclopropanated pyran $\mathbf{1 0}^{17}$

Several decades ago, the likely mechanism for electrocyclic ring-opening reactions was proposed by Woodward and Hoffmann, ${ }^{18}$ then later DePuy et al. established the "cis-in trans-out" rule for disrotatory gem-dihalocyclopropane electrocyclic ring opening. ${ }^{19,20}$ The diagram below (Scheme 1.5) illustrates the two possible modes for bicyclic gem-dihalocyclopropanated substrates, in which substituents cis- to the departing halogen rotate inwards, and trans-substituents rotate outwards. Cyclopropyl bicycles such as 4 , upon loss of halogen $\mathrm{X}_{2}$ would create highly strained transoid rings as exemplified by intermediate 14. A seven-membered ring such as 14 is incapable of incorporating a trans-double bond, which limits ring opening to the cis-in mode in which the endo-halogen departs, creating cisoid intermediates such as $\mathbf{1 5 .}$

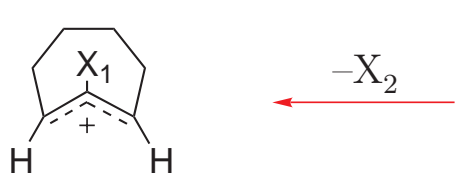

14

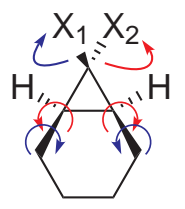

4
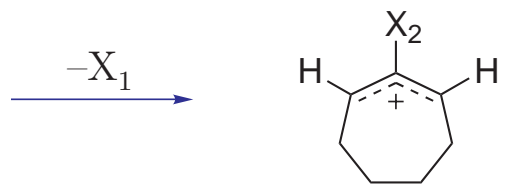

15

Scheme 1.5 Exemplification of DePuy's "cis-in, trans-out" rule 19,20

\subsection{Cyclopropanated Carbohydrates}

Carbohydrate chemistry is a well-established area of research that has experienced significant growth in recent years, with renewed interest in oligo- and polysaccharide synthesis, as well as increasing complexity in monosaccharide derivatisation. ${ }^{21}$ Carbohydrates are naturally occurring, readily available and inexpensive chiral pool reagents. They are comprised of polyhydroxylated scaffolds, containing many stere- 
ocentres which may be exploited in syntheses of chiral compounds. Established procedures for a variety of chemical transformations, such as protecting group strategies, are well grounded. Allowances for chemical sensitivity, in addition to strategies providing high selectivity and efficiency are often well precedented.

Synthesis and subsequent derivatisation of cyclopropyl carbohydrates remains a relatively small area of research and, of this work, only a small fraction is devoted to reactions of the cyclopropyl moiety. ${ }^{10,22}$ Research on cyclopropanated carbohydrates is almost equally divided between pyranose- and furanose-based sugars. The majority of furanoside cyclopropanes have been synthesised by Simmons-Smith cyclopropanation of unsaturated sugars, often yielding conformationally constrained nucleosides. ${ }^{23}$ Conversely, pyranoside chemistry within this field is more varied, as demonstrated below. The following section will provide selected literature examples of cyclopropanation of carbohydrates, via both intra- and intermolecular processes.

\subsubsection{Synthesis of Cyclopropanated Carbohydrates}

Cyclopropanes may be synthesised using a wide range of methods, usually through addition of a carbene source to an olefin (see subsection 1.1.1). The synthesis of cyclopropanated carbohydrates almost always involves construction of a cyclopropyl ring onto a pre-existing carbohydrate scaffold. A selection of examples detailing the synthesis of cyclopropanated sugars is shown below.

\section{Intramolecular Cyclopropanations}

While a great number of cyclopropanated carbohydrates are synthesised via intermolecular reactions, there have been many intramolecular syntheses, often using quite clever methodology. A notable example of cyclopropanation via an exocyclic carbanion was reported by Voelter et al., forming a 3,4-fused cyclopropyl ring (Scheme 1.6). Treatment of triflate $\mathbf{1 6}$ with acetonitrile and LDA, followed by treatment with additional base provided intermediate $\mathbf{1 7}$. This anion opened the neighbouring epoxide to afford cyclopropyl product $18 .{ }^{24}$

Spanevello and Testero found $\gamma$-bromoketone $\mathbf{1 9}$ provided access to cyclopropane $\mathbf{2 0}$ through treatment with silver fluoride in pyridine (Scheme 1.7). ${ }^{25}$ This presumably arose through formation of an enolate, due to deprotonation at C-4 and subsequent silver-assisted attack upon the bromomethyl moiety. An increase in yield was observed if the iodo-analogue was employed. 


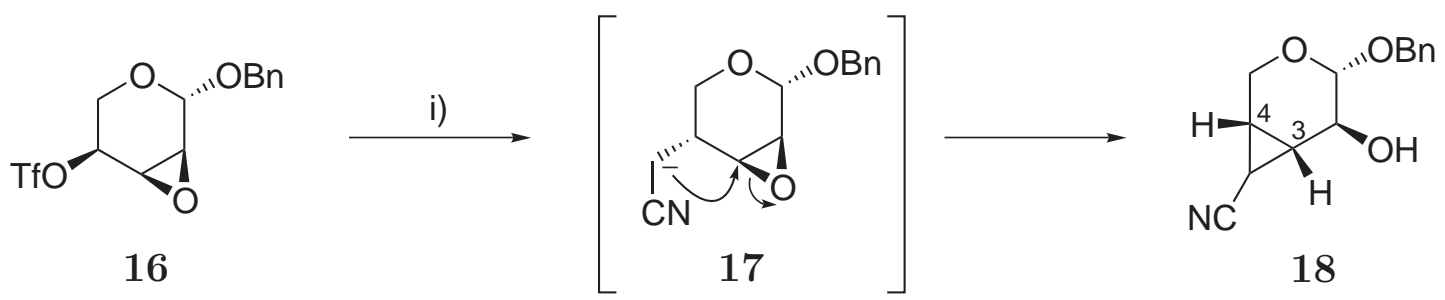

Reagents and Conditions: i) MeCN, LDA, HMPA, THF, $-78^{\circ} \mathrm{C}$.

Scheme 1.6 Exocyclic carbanion-mediated cyclopropanation ${ }^{24}$<smiles>[R6]O[C@@H]1C(=O)C[C@H](OC)O[C@@H]1CBr</smiles>

19

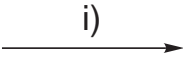

Reagents and Conditions: i) $\mathrm{AgF}$, pyridine, r.t.

Scheme 1.7 Silver-mediated synthesis of 4,5-cyclopropanated carbohydrate $\mathbf{2 0}^{25}$

An excellent example of cyclopropane formation through concomitant ring contraction has been reported by Okabe and Sun. ${ }^{26}$ Through the use of a homo-Ferrier rearrangement, ${ }^{27}$ dihydropyran derivative $\mathbf{2 1}$ was converted into a furanocyclopropane, through reaction with a protected cytidine base (22) and ethyl aluminium chloride (Scheme 1.8). As the mesylate leaving group was at the homoallylic position, the Ferrier-type rearrangement created a bond between C-2 and C-4. The cyclopropyl ring was formed stereoselectively, affording nucleosides 23 and 24 as a 1:1 anomeric mixture.

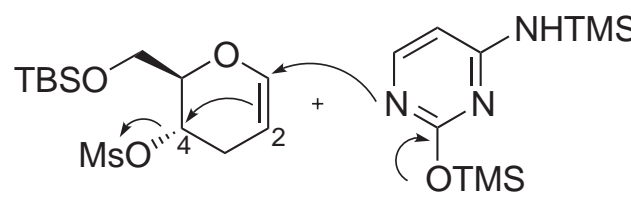

21

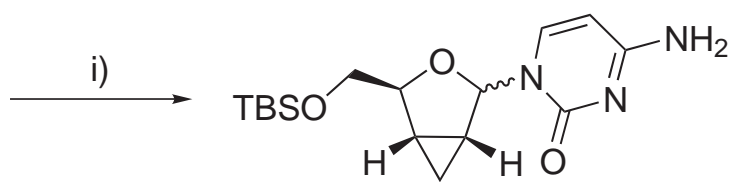

23,24

Reagents and Conditions: i) $\mathrm{EtAlCl}_{2}, \mathrm{MeCN}$, reflux.

Scheme 1.8 Ring-contraction cyclopropanation ${ }^{26}$

The above examples demonstrate that intramolecular cyclopropanation provides a range of intriguing structures. However, a large number of cyclopropyl sugars have also been synthesised through intermolecular cyclopropane formation. SimmonsSmith cyclopropanation (and variations) involves the addition of carbene, whilst other cyclopropanation methods usually append cyclopropanes with additional functionality. 


\section{Simmons-Smith Cyclopropanations}

The Simmons-Smith method of cyclopropanation ${ }^{11}$ has been used extensively, and is the most commonly employed procedure for the synthesis of cyclopropyl targets. An early application of this procedure with unsaturated sugars was published in 1969, involving protected 2,3-unsaturated pyranose sugar 25. ${ }^{28} \mathrm{~A}$ zinc-copper couple, prepared from zinc metal and copper diacetate, was used in the presence of diiodomethane to generate a carbenoid intermediate, providing cyclopropane 26 (Scheme 1.9). Due to zinc coordination with the oxygens adjacent to the olefin, carbene addition occurred exclusively at the $\alpha$-face.

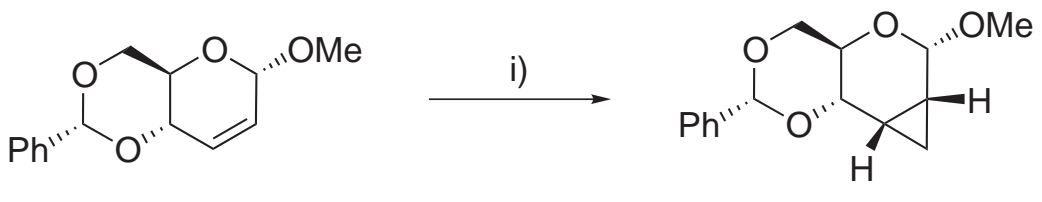

25

26

Reagents and Conditions: i) $\mathrm{CH}_{2} \mathrm{I}_{2}, \mathrm{Zn} / \mathrm{Cu}, \mathrm{Et}_{2} \mathrm{O}, 20{ }^{\circ} \mathrm{C}$.

Scheme 1.9 Simmons-Smith cyclopropanation of 2,3-unsaturated sugar $\mathbf{2 5}^{28}$

More recent publications report the cyclopropanation of sugars using the Furukawa modification of the Simmons-Smith methodology, in which the zinc-copper couple is replaced by diethyl zinc. ${ }^{29,30}$ Two products obtained in this way are shown below (Figure 1.1). Simmons-Smith cyclopropanation of an unsaturated D-xylose derivative provided cyclopropane $\mathbf{2 7}$ in a 60:1 diastereomeric ratio with the stereoisomeric cyclopropane. ${ }^{31}$ This cyclopropanated derivative was used in the synthesis of conformationally restricted nucleosides, a field which has led to a huge amount of research concerning cyclopropanated sugars. ${ }^{23}$ The Hoberg group synthesised glucal-derived cyclopropane $\mathbf{2 8}$ in $87 \%$ yield, as a single diastereoisomer. ${ }^{32}$

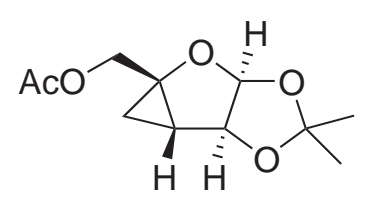

27

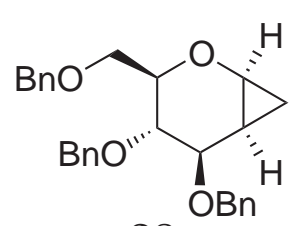

28

Figure 1.1 Simmons-Smith derived cyclopropanes ${ }^{31,32}$

\section{Cyclopropanations with Diazo Reagents}

Diazo-based carbenoid sources have often been used for cyclopropane synthesis. The most common method involves the catalysed addition of ethyl diazoacetate to olefins, thereby affording a cyclopropane with ethoxycarbonyl functionality. Furthermore, it is complementary to the Simmons-Smith reaction, as the direction of attack of the diazo reagent is controlled by steric factors. Several reports of 
diazoacetate-mediated cyclopropanations of glycals have been published, ${ }^{33,34}$ as exemplified below (Scheme 1.10). Alkene 29 in the presence of $\mathrm{Rh}_{2}(\mathrm{OAc})_{4}$ catalyst was treated with ethyl diazoacetate to form substituted cyclopropane $30 .{ }^{35}$ Carbenoid addition occurred exclusively at the less sterically hindered $\alpha$-face, with the ethoxycarbonyl moiety in the exo-configuration, whereas Simmons-Smith cyclopropanation of alkene $\mathbf{2 9}$ would likely provide carbene addition to the $\beta$-face, due to coordination of the zinc species with the C-3 silyloxy moiety.

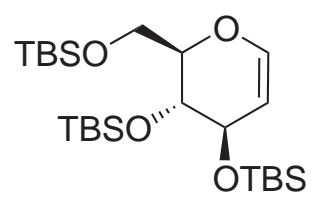

29

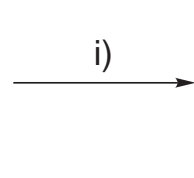

30

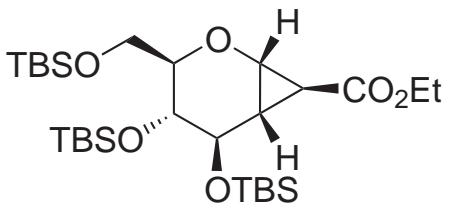

Reagents and Conditions: i) $\mathrm{CH}\left(\mathrm{N}_{2}\right) \mathrm{CO}_{2} \mathrm{Et}, \mathrm{Rh}_{2}(\mathrm{OAc})_{4}, \mathrm{CH}_{2} \mathrm{Cl}_{2}$, r.t.

Scheme 1.10 Ethyl diazoacetate-mediated cyclopropanation ${ }^{35}$

Diazomethane may undergo cycloaddition with unsaturated sugars, then subsequent photolysis may eliminate molecular nitrogen to provide a cyclopropane derivative. This provides methylene-functionalised cyclopropanes, analogous to SimmonsSmith cyclopropanation. Robins and Samano investigated the use of diazomethane to form spirocyclopropanes from furanose sugars with unsubstituted exocyclic double bonds. ${ }^{36}$

Vasella and co-workers have published numerous papers on reactions with glycosylidene diazirine carbenes, such as D-glucose derivative 31. This substrate has been used as a carbene source for the synthesis of several anomeric spirocyclic cyclopropanes, using olefins such as dihydrofuran and dihydropyran. ${ }^{37}$ Another report involved addition of fullerene $\mathrm{C}_{60}$ providing fullerene-cyclopropane-carbohydrate hybrid 32 (Scheme 1.11). ${ }^{38}$<smiles>BrC(Br)(Br)c1ccccc1</smiles>

31

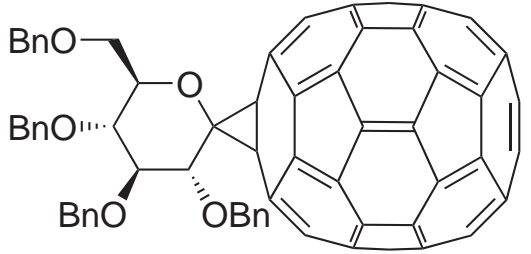

32 Reagents and Conditions: i) $\mathrm{C}_{60}$, toluene, $23{ }^{\circ} \mathrm{C}$.

Scheme 1.11 Preparation of a cyclopropyl-linked fullerene-sugar conjugate ${ }^{38}$

\section{Ylide-Based Cyclopropanations}

It has been shown that cyclopropane rings may be formed, or undergo reactions in a similar manner to olefins. An early synthesis of a cyclopropanated sugar used a phos- 
phonate ylide to convert an epoxide into a cyclopropane, analogous to the HornerWadsworth-Emmons (HWE) conversion of aldehydes into alkenes (Scheme 1.12). ${ }^{39}$ Thus, treatment of epoxide 33 with triethyl phosphonoacetate and sodium hydride allowed replacement of the bridging oxygen with an ethyl acetate group (34).<smiles>CO[C@H]1O[C@@H]2COC(c3ccccc3)O[C@H]2[C@H]2O[C@H]12</smiles>

33

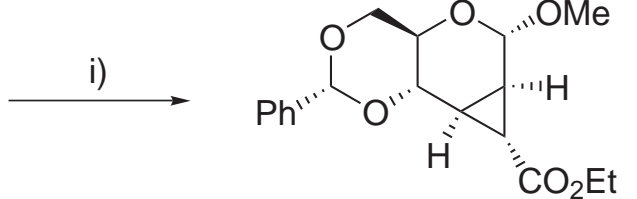

34

Reagents and Conditions: i) (EtO $)_{2} \mathrm{P}(\mathrm{O}) \mathrm{CH}_{2} \mathrm{CO}_{2} \mathrm{Et}, \mathrm{NaH}$, dioxane, $80{ }^{\circ} \mathrm{C}$.

Scheme 1.12 HWE-type cyclopropanation ${ }^{39}$

Sakakibara et al. have investigated the reaction of sulfur ylides with 3-nitro-2,3unsaturated carbohydrates. Reaction of $\beta$-anomer 35 led to the expected cyclopropane 36 in $75 \%$ yield, with no evidence of attack at the $\beta$-face (Scheme 1.13). ${ }^{40}$ Unlike most examples mentioned within this section, the authors believe cyclopropanation occurs in a stepwise manner, through ylide attack at $\mathrm{C}-2$ to generate intermediate 37, followed by attack of the anionic C-3 carbon, and ejection of DMSO.

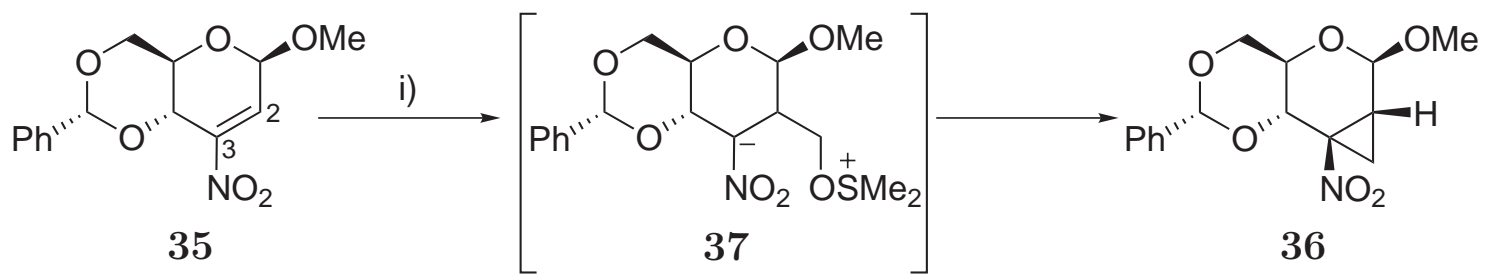

Reagents and Conditions: i) $\mathrm{Me}_{2} \mathrm{~S}(\mathrm{OMe}) \mathrm{I}, \mathrm{NaH}, \mathrm{DMSO}$, r.t.

Scheme 1.13 A cyclopropane synthesised from an $S$-ylide ${ }^{40}$

\section{Dihalocarbene-Based Cyclopropanations}

The first synthesis of a cyclopropanated sugar was published by the Brimacombe group four decades ago. ${ }^{41}$ The authors utilised dichlorocarbene, synthesised in situ from sodium methoxide and ethyl trichloroacetate, ${ }^{42}$ for the cyclopropanation of tri$O$-methyl-D-glucal (38). The authors assumed that carbene addition occurred exclusively at the $\alpha$-face due to steric constraints, providing gem-dichlorocyclopropane 39 in $82 \%$ yield (Scheme 1.14). This method of gem-dihalocyclopropanation has in more recent times been superseded by Mąkosza cyclopropanation, which provides similar reactivity and efficiency, while using more readily available reagents.

Petter and Powers synthesised spirocycles 40 and 41 through Mąkosza cyclopropanation of L-arabinose-derived sugar 42 (Scheme 1.15). Partial steric hindrance of both faces resulted in a diastereomeric mixture of cyclopropanes, slightly favouring 40 as the major product. ${ }^{43}$ 
<smiles>COC[C@H]1OC=C[C@@H](OC)[C@H]1OC</smiles>

38

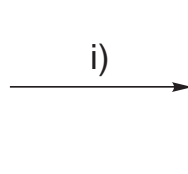

Reagents and Conditions: i) EtOCOCCl $3, \mathrm{NaOMe}$, hexane, r.t.

Scheme 1.14 The first reported cyclopropanated carbohydrate $(39)^{41}$<smiles>C=C1C(OCc2ccccc2)OCC2OC(C)(C)OC12</smiles>

42

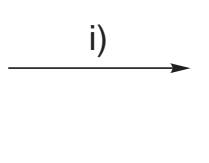

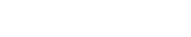

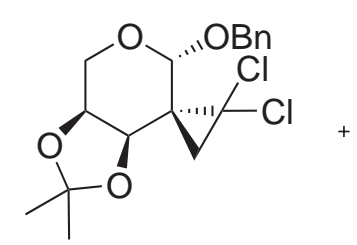

40

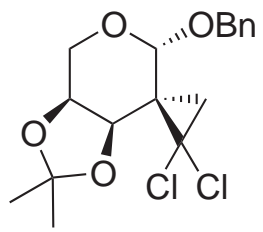

41

Reagents and Conditions: i) $\mathrm{CHCl}_{3}$, TEBAC, benzene, $\mathrm{NaOH}_{(\mathrm{aq})}$.

Scheme 1.15 Formation of spirocyclopropane sugars ${ }^{43}$

Recently, two fascinating reports of dihalocarbene addition onto unsaturated sugars have been published, both including comparisons with Simmons-Smith carbene addition. In 1995, Nagarajan et al. reported the cyclopropanation of several glycals, including tri- $O$-benzyl-D-galactal (43) and di-O-benzyl-L-rhamnal (44) (Scheme 1.16). ${ }^{44}$ As expected, Simmons-Smith cyclopropanation exclusively gave the oxygen-directed product stereoisomers. Cyclopropanes with opposite stereochemistry across the 1,2-bond were prepared using Mạkosza cyclopropanation. ${ }^{12}$ Thus, $\alpha$-cyclopropyl galactal (45), and rhamnal (46) derivatives were obtained stereoselectively in $92 \%$ and $95 \%$ yield respectively. Through the use of bromoform, the gem-dibromo- analogues were isolated in $64-84 \%$ yield. ${ }^{45}$<smiles>BrC(Br)(Br)c1ccccc1</smiles>

43<smiles>CC(C)C</smiles>

44<smiles>O[C@H]1[C@@H](COBr)O[C@@H](Cl)C(Cl)[C@H](Br)[C@@H]1Br</smiles>

45<smiles>C[C@@H]1O[C@H]2[C@@H]([C@H](O)[C@H](Br)[C@H]1Br)C2(Cl)Cl</smiles>

46

Reagents and Conditions: i) $\mathrm{CHCl}_{3}$, TEBAC, $\mathrm{NaOH}_{(\mathrm{aq})}$, r.t.

Scheme 1.16 Cyclopropanations reported by Nagarajan ${ }^{44,45}$

Experimentation with 4,5-unsaturated sugars by the Corsaro group has further extended the scope of this chemistry. Cyclopropanation of carbohydrates such as 47 was achieved stereoselectively to provide cyclopropane adducts including $\mathbf{4 8}$ (Scheme 1.17). Cyclopropanation of substrates with less-bulky protecting groups 
on the top face led to a mixture of cyclopropanes, due to the carbene species attacking both faces. ${ }^{46}$<smiles>COC1OC(COc2ccccc2)=C[C@H](OCc2ccccc2)[C@H]1Cc1ccccc1</smiles>

47

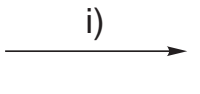

Reagents and Conditions:<smiles>COC1O[C@]2(COCc3ccccc3)C(Cl)[C@H](O)[C@H](Oc3ccccc3)[C@H]2C1(Cl)Cl</smiles>

48

Scheme 1.17 Stereoselective cyclopropanations of 4,5-unsaturated sugars ${ }^{46}$

As detailed above, a great number of cyclopropanated sugars have been synthesised over the last four decades, covering the majority of the most common methods of cyclopropanation. The following section presents and discusses the various reactions of cyclopropanated sugars that have so far been investigated.

\subsubsection{Reactions of Cyclopropanated Carbohydrates}

While many cyclopropanated carbohydrates have been reported, only a fraction have been derivatised through reaction of the cyclopropane moiety. There are several procedures commonly employed that retain the cyclopropyl ring, such as reductive dehalogenation of a $\mathrm{gem}$-dihalocyclopropane. Of particular relevance to this research are syntheses involving cleavage of the cyclopropane. For a bicyclic system such as generic cyclopropyl sugar 49, two types of cleavage are possible (Scheme 1.18). The
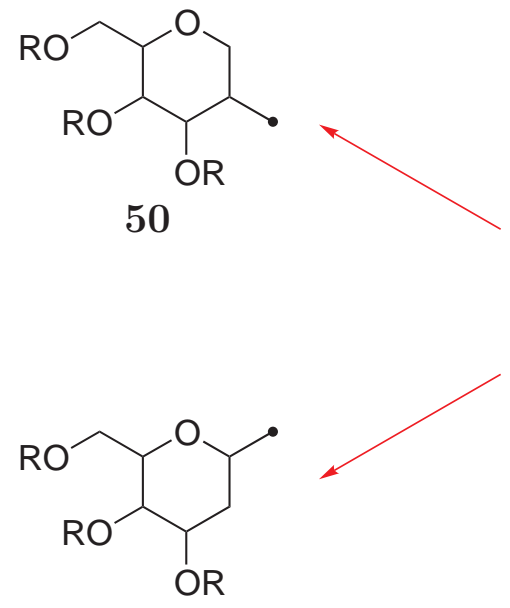<smiles>[R9]CC1OC2CC2C([R9])C1O</smiles>

49

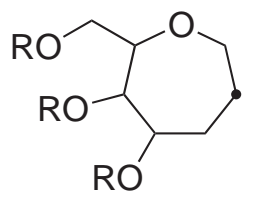

52

51

Scheme 1.18 Ring opening and ring expansion of cyclopropanes majority of ring cleavages occur through the red paths, in which either the 1,7- or the 2,7-bond is broken, to provide branched products 50 and/or 51. Such reactions can be regarded as cyclopropane ring-opening reactions, as the cyclopropane ring is opened, whereas the other ring remains unchanged. Alternatively, cleavage of the 
1,2-bond provides products such as 52 , via the blue path. This provides a single, unbranched ring one carbon greater in size than the larger ring of the starting material. This transformation will be referred to as a ring expansion.

\section{Ring-Opening Reactions of Cyclopropanated Carbohydrates}

\section{Radical-Initiated Reactions}

Some of the most commonly observed reactions of cyclopropanated carbohydrates are radical-mediated ring-opening processes. Within this field, the Gurjar group have published many fascinating results, including those involving spirocyclopropanated sugars. Functionalisation of a furanose sugar afforded alkyne $\mathbf{5 3}$ (Scheme 1.19), which upon reaction with tributyltin hydride and AIBN led to an elegant radical cascade reaction. Mechanistically, bromine abstraction led to cyclopropane ring opening, providing a C-3 allyl-substituted radical intermediate. Two sequential radical cyclisations took place involving the alkyne and the allylic $\pi$-bond to provide epimeric tetracycles $\mathbf{5 4}$ and $\mathbf{5 5 .}{ }^{47}$ Similar substrates lacking an alkyne tether have been shown to provide gem-diallyl functionality at C-3, in the presence of allyltributylstannane. ${ }^{48,49}$

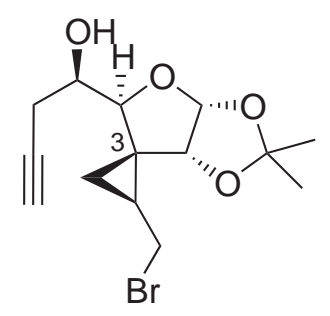

53

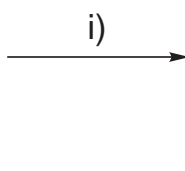

Reagents and Conditions: i) $\mathrm{Bu}_{3} \mathrm{SnH}, \mathrm{AIBN}$, toluene, $100{ }^{\circ} \mathrm{C}$.

Scheme 1.19 Cascade radical cyclisation of cyclopropane $53^{47}$

Glycal-derived cyclopropane $\mathbf{5 6}$ was prepared with a xanthate tether, to allow radical initiation at C-8 (Scheme 1.20). Ring-opened product 57 was obtained, in addition to a similar amount of ring-expanded sugar 58. ${ }^{50}$ This represents one of the few ring expansion reactions of non-halogenated cyclopropanated sugars.<smiles>CSC(=S)OC[C@H]1C[C@@H]1O[C@H](COCc1ccccc1)[C@H](Br)C(C)C</smiles>

56

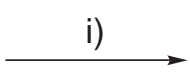

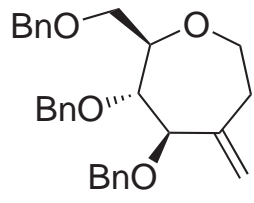

58

Reagents and Conditions: i) $\mathrm{Bu}_{3} \mathrm{SnH}$, AIBN, toluene, reflux.

Scheme 1.20 Ring-opened and ring-expanded cyclopropane derivatives ${ }^{50}$ 


\section{Electrophilic Halogen-Mediated Reactions}

1,2-cyclopropanated sugars can be opened using electrophilic halogenation, analogous to haloetherification. Danishefsky et al. utilised this methodology, involving pseudo-sugar 59 (Scheme 1.21). Treatment with $N$-iodosuccinimide and methanol provided ring-opened substrate $\mathbf{6 0}$, which was used to synthesise the highlighted portion of epothilone A (61) and B (62). ${ }^{51}$ Ley and co-workers utilised similar methodology in their work towards the total synthesis of altohyrtin A (spongistatin 1). ${ }^{52}$ These examples demonstrate the excellent utility of cyclopropanes in forming quaternary carbons, which otherwise may be inaccessible.

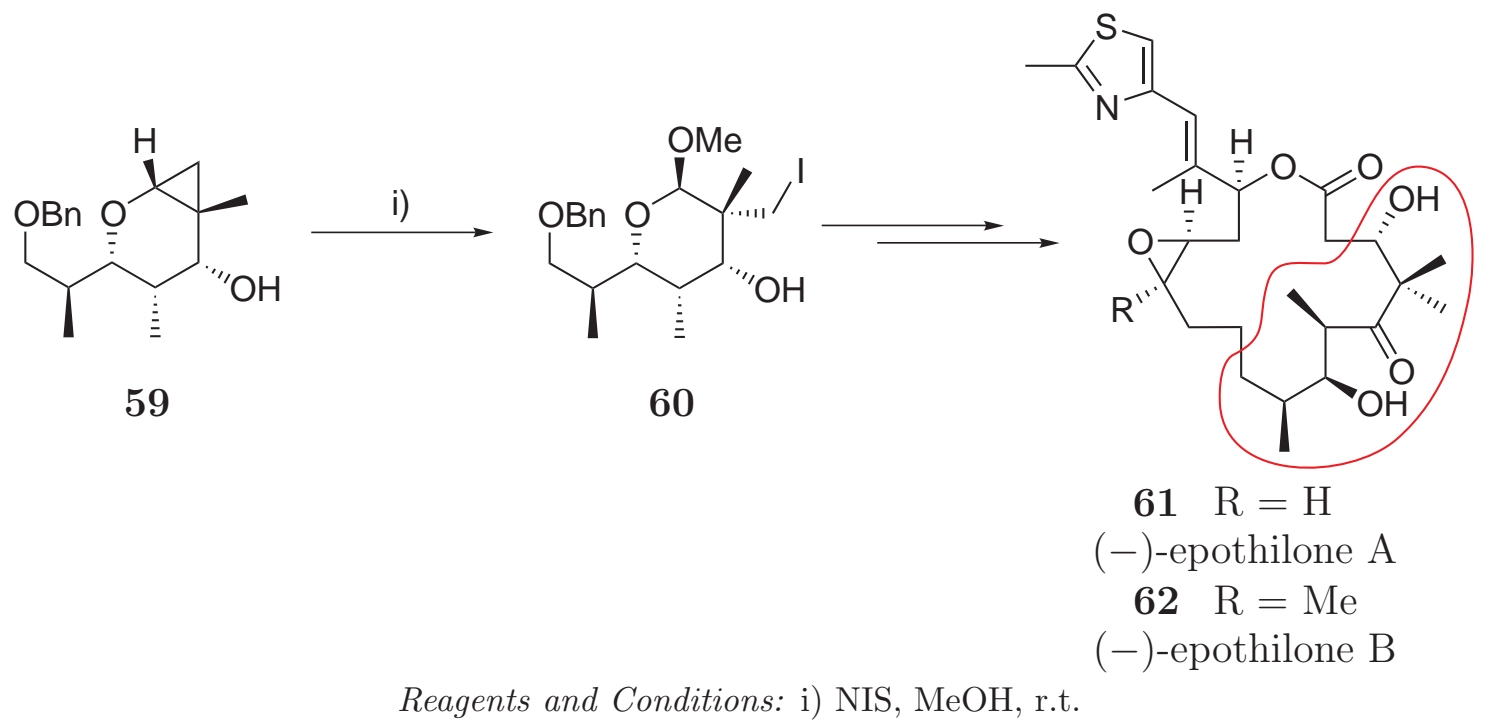

Scheme 1.21 Synthesis of epothilones via cyclopropane $\mathbf{5 9} \mathbf{9}^{51}$

Nagarajan used a haloetherification strategy with isomeric cyclopropanes 63 and 64, obtained through Simmons-Smith cyclopropanation and a Mąkosza cyclopropanation/reduction sequence, respectively. Cyclopropane 63 has the methylene bridge on the $\beta$-face due to zinc-coordination with the neighbouring benzyloxy substituent. Conversely, $\alpha$-face cyclopropane $\mathbf{6 4}$ was obtained due to sterically directed dihalocarbene addition. NBS-mediated ring opening provided a variety of glycosides in good yields, through solvolysis (Scheme 1.22). ${ }^{45}$ Simmons-Smith-derived cyclopropane 63 formed $\alpha$-glycosides exclusively; cyclopropane 64 provided anomeric mixtures of glycosides (65 and 66). A later report demonstrated similar reactivity with sugarderived nucleophiles. In this case, only three equivalents of nucleophile were used (in acetonitrile), rather than a solvolytic quantity. Thus, longer reaction times at room temperature were required to provide disaccharides in $60-66 \%$ yield. ${ }^{53}$

Sridhar and co-workers exploited the functionality of a methyl diazoacetate-derived cyclopropyl sugar to synthesise glyco-amino acids. ${ }^{33}$ Cyclopropane 69 was subjected to a haloetherification-type reaction in the presence of NIS and diacetone D-galactose (70), through promotion with catalytic TMSOTf (Scheme 1.23). The 


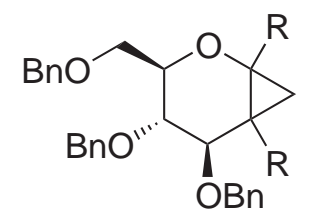

$63 \mathrm{R}=\cdots \cdot \mathrm{H}$

$64 \mathrm{R}=-\mathrm{H}$

$\mathrm{Nuc}=-\xi-\mathrm{OMe}$

$-\xi-O B n$

$-\xi-\mathrm{OH}$

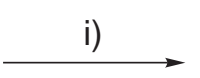

(Nuc

$67,68 \mathrm{R}=\cdots \cdot \mathrm{H}$

$65,66 \mathrm{R}=-\mathrm{H}$

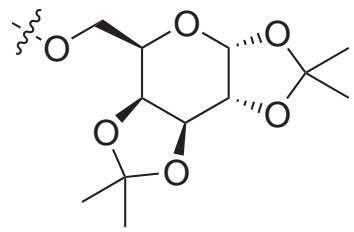

Reagents and Conditions: i) NBS, NucH, r.t.

Scheme 1.22 Synthesis of various cyclopropane-derived disaccharides ${ }^{45,53}$

obtained $\alpha$-iodoester 71 was then treated with sodium azide and subjected to a Staudinger reaction to provide glyco-amino acid 72. The authors extended this methodology to involve several different glycal-derived cyclopropanes, and numerous glycosyl acceptors.

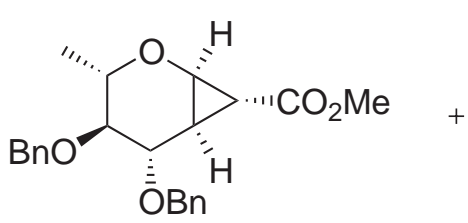

69

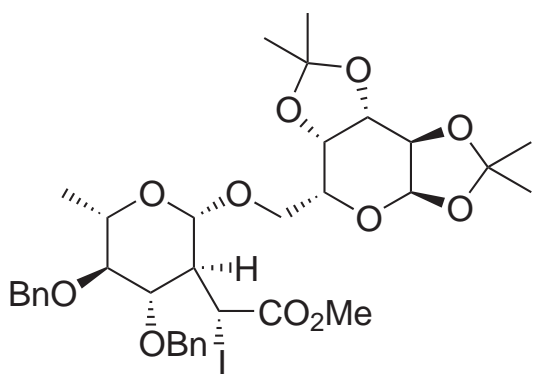

71<smiles>CC1(C)O[C@@H]2OC(C)(C)[C@@H]3OC(C)(C)O[C@@H]3[C@H]2O1</smiles>

70

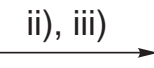

72

Reagents and Conditions: i) NIS, TMSOTf, $\mathrm{CH}_{2} \mathrm{Cl}_{2}, 0{ }^{\circ} \mathrm{C}$ to r.t.; ii) $\mathrm{NaN}_{3}$, DMF, r.t.; iii) $\mathrm{PPh}_{3}$, THF, r.t. then $\mathrm{H}_{2} \mathrm{O}$, reflux.

Scheme 1.23 Synthesis of glyco-amino acid $\mathbf{7 2}^{33}$

\section{Miscellaneous Ring Opening Reactions}

Madsen et al. have developed a synthesis of 2- $C$-branched carbohydrates through platinum-catalysed ring opening of cyclopropane 73 (Scheme 1.24). Treatment with Zeise's dimer led to insertion into the 1,7-bond. Nucleophilic attack by a range of alcohols, including monosaccharides, afforded 2-C-branched glycosides such as 74. ${ }^{54,55}$ Yields were consistently high, with predominant or selective formation of the $\alpha$-anomer. Work with the electrophilic catalyst $\mathrm{PdCl}_{2}(\mathrm{PhCN})_{2}$ and $\mathrm{BnOH}$, led to a rearrangement of cyclopropane 73 to provide olefin $75 .{ }^{55}$ 
<smiles>Brc1ccccc1</smiles>

73<smiles>BrC(Br)(Br)c1ccccc1</smiles>

73

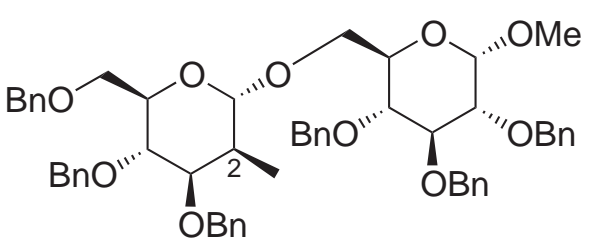

74<smiles>CC1=C[C@@H](Br)[C@H](COBr)O[C@H]1Br</smiles>

75

Reagents and Conditions: i) methyl 2,3,4-tri- $O$-benzyl- $\alpha$-D-glucoside, $\left[\mathrm{Pt}\left(\mathrm{C}_{2} \mathrm{H}_{4}\right) \mathrm{Cl}_{2}\right]_{2}, \mathrm{CH}_{2} \mathrm{Cl}_{2}$, r.t.; ii) $\mathrm{BnOH}, \mathrm{PdCl}_{2}(\mathrm{PhCN})_{2}, \mathrm{CH}_{2} \mathrm{Cl}_{2}$, r.t.

Scheme 1.24 Platinum-catalysed ring opening of cyclopropane $\mathbf{7 3}{ }^{54,55}$

The Theodorakis group used an acid-mediated cyclopropane ring-opening reaction in their studies towards the synthesis of the natural product $(+)$-norrisolide (76). Initial reaction of the ethyl diazoacetate-derived cyclopropane 77 with sulfuric acid in ethanol led to the expected solvolytic opening, with concomitant loss of the isopropylidene group, liberating epimeric diols $\mathbf{7 8}$ and $\mathbf{7 9}$ (Scheme 1.25). ${ }^{34} \mathrm{~A}$ later method used methanesulfonic acid to achieve the desired ring opening. In the absence of an external nucleophile, intramolecular lactonisation ensued, providing lactone $\mathbf{8 0}$, which retained the acetonide under the milder conditions. ${ }^{56,57}$ Gharpure et al. have used similar methodology to synthesise a range of products derived from either ring expansion or ring-opening processes. ${ }^{58}$ Branched furanone and pyranone products, in addition to several furanofuranone bicycles too numerous to discuss here, were all obtained from a single cyclopropane.<smiles>CCOC(C)[C@@H]1[C@H](C)O[C@@H]([C@H]2COC(C)(C)O2)[C@H]1O</smiles>

77<smiles>CCOC(=O)[C@H]1[C@H]2O[C@H]([C@H]3COC(C)(C)O3)[C@H](OBr)[C@@H]21</smiles>

77

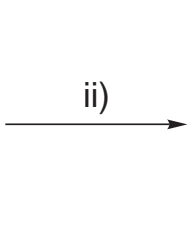<smiles>CCOC(=O)C[C@H]1O[C@H]([C@@H](O)CO)[C@@H](OBr)[C@@H]1OCC</smiles>

78,79

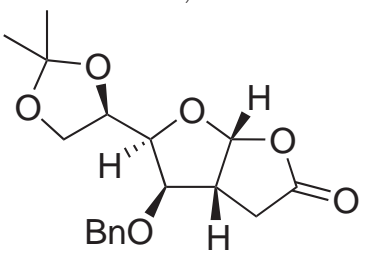

80

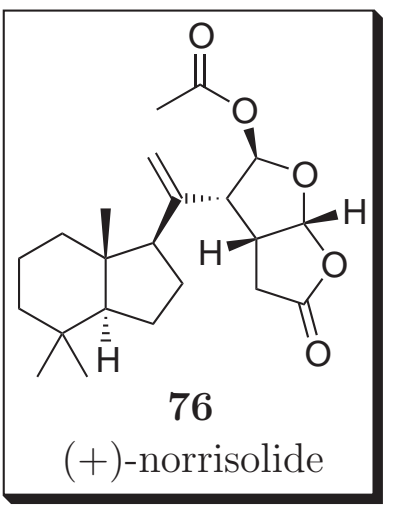

$(+)$-norrisolide

Reagents and Conditions: i) $\mathrm{H}_{2} \mathrm{SO}_{4}$, EtOH, $25{ }^{\circ} \mathrm{C}$; ii) $\mathrm{MeSO}_{3} \mathrm{H}$, Acetone, $25{ }^{\circ} \mathrm{C}$.

Scheme 1.25 Ring opening reactions of cyclopropanated sugar $77^{34,56,57}$ 


\section{Ring-Expansion Reactions of Cyclopropanated Carbohydrates}

While the above examples demonstrate ring opening of cyclopropyl sugars provides great scope for synthesis of branched chain sugars, there have been several reports of ring opening of cyclopropanated carbohydrates via cleavage of the fused bond. Such cleavage leads to ring expansion; for example, a bicyclo[4.1.0]heptane skeleton expands to form a seven-membered ring.

\section{Ring Expansions of Simmons-Smith Derived Cyclopropanes}

Boyer and Lallemand utilised a ring expansion as a crucial step in the total synthesis of (+)-calystegine B2 (81, Scheme 1.26). Cyclopropane $\mathbf{8 2}$ was treated with iron trichloride in DMF to provide chlorinated ketone $\mathbf{8 3} .{ }^{59}$

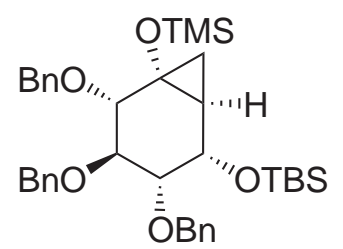

82

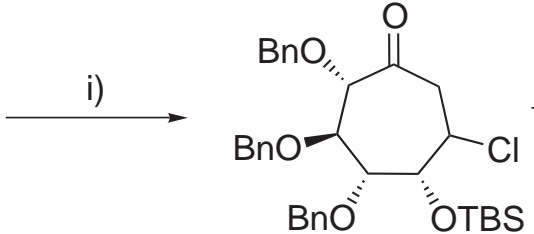

83

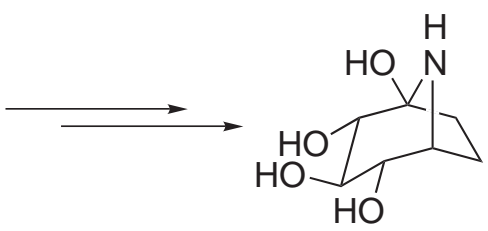

81

Reagents and Conditions: i) $\mathrm{FeCl}_{3}$, DMF, $70{ }^{\circ} \mathrm{C}$.

$(+)$-calystegine B2

Scheme 1.26 Synthesis of (+)-calystegine B2 ${ }^{59}$

Hoberg and co-workers have published several papers on the ring expansion of Simmons-Smith derived cyclopropanated glycals. Of particular interest are their studies on cyclopropanes such as $\mathbf{8 4}$, which have been used to provide sevenmembered oxacycles. Lewis acid-mediated reaction of cyclopropane $\mathbf{8 4}$ with several silylated nucleophiles afforded ring-expanded products 85 and 86 in yields of 67$93 \%$, along with small quantities of diene 87 (Scheme 1.27). ${ }^{60}$ While the anomeric stereoselectivity was poor, the galactal series provided much higher anomeric selectivity, using cyclopropane $\mathbf{8 8} .{ }^{61}$ This substrate incorporates a higher degree of steric hindrance upon the $\beta$-face, which leads to preference for nucleophilic attack at the $\alpha$-face. Diastereoselectivites for oxepines such as $\mathbf{8 9}$ were obtained in ratios of up to 80:1 in favour of the $\alpha$-anomer.

\section{Ring Expansions of Dihalocarbene-Derived Cyclopropanes}

gem-Dihalocyclopropyl carbohydrates undergo ring expansion reactions ${ }^{10}$ in the presence of halophilic silver salts. This leads to a monohalogenated $\pi$-allyl cationic species (see subsection 1.1.2), which then may be attacked by various nucleophiles. 
<smiles>CC(=O)OC1[C@H]2C[C@@H]2O[C@@H]2CO[Si](C(C)(C)C)(C(C)(C)C)OC[C@H]12</smiles>

84

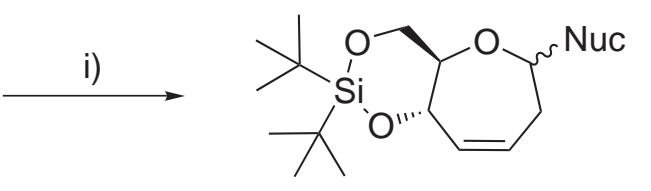

85,86<smiles>CC(C)(C)[Si]1(C(C)(C)C)OC[C@H]2OC=CC=C[C@H]2O1</smiles>

87<smiles>CC(C)(C)O[C@H]1[C@@H]2C[C@@H]2O[C@@H]2CO[Si](C(C)(C)C)(C(C)(C)C)O[C@H]12</smiles>

88

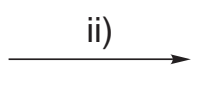<smiles>C=CC[C@H]1CC=C[C@@H]2O[Si](C(C)(C)C)(C(C)(C)C)OCC2O1</smiles>

89

Reagents and Conditions: i) TMSNuc, TMSOTf, MeCN, $-40{ }^{\circ} \mathrm{C}$ to r.t.; ii) TMSAllyl, TMSOTf, $\mathrm{MeCN},-40{ }^{\circ} \mathrm{C}$ to r.t.

Scheme 1.27 Glucal and galactal-derived oxepines ${ }^{60,61}$

The first reported ring expansion of a cyclopropyl sugar was published by Gross et al. in 1979. ${ }^{62}$ 3,4-Cyclopropyl sugar 90 was treated with silver perchlorate in methanol (Scheme 1.28), leading to an epimeric mixture of olefins 91 and 92 in $61 \%$ yield. The chlorinated analogue also underwent a similar ring-expansion reaction.<smiles>CC1(C)O[C@H]2OC(Br)([C@H]3COC(C)(C)[C@H]3Br)[C@@H]2O1</smiles>

90
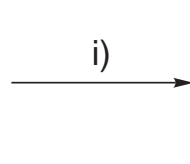<smiles>CO[C@@]1([C@@H]2COC(C)(C)O2)O[C@@H]2OC(C)(C)O[C@@H]2C=C1Br</smiles>

91,92

Reagents and Conditions: i) $\mathrm{AgClO}_{4}, \mathrm{~K}_{2} \mathrm{CO}_{3}, \mathrm{MeOH}, 60^{\circ} \mathrm{C}$.

Scheme 1.28 Ring expansion of cyclopropanated sugar $\mathbf{9 0}^{62}$

gem-Dibromocyclopropyl oxazines 93 and 94, accessible from D-glyceraldehyde as a diastereomeric mixture (Scheme 1.29), were reacted with excess potassium carbonate in refluxing methanol to provide oxazepine 95. The utility of the product structure was demonstrated through the use of palladium-catalysed cross-coupling reactions. Suzuki reaction of $\mathbf{9 5}$ with phenylboronic acid provided coupled product 96; similar products were obtained through Sonogashira, Stille and Heck methodologies. ${ }^{63}$<smiles>CC1(C)OC[C@H](C2N(Cc3ccccc3)OCC3C(Br)(Br)C23Br)O1</smiles>

93, 94
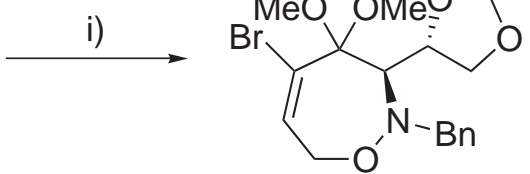

95

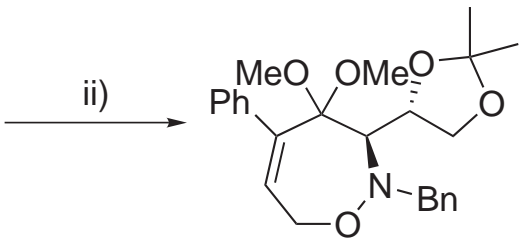

96

Reagents and Conditions: i) $\mathrm{K}_{2} \mathrm{CO}_{3}, \mathrm{MeOH}$, reflux; ii) $\mathrm{PhB}(\mathrm{OH})_{2}, \mathrm{Pd}(\mathrm{OAc})_{2}, \mathrm{PPh}_{3}, \mathrm{~K}_{2} \mathrm{CO}_{3}$, DMF, $70{ }^{\circ} \mathrm{C}$.

Scheme 1.29 Oxazepine formation and Suzuki coupling ${ }^{63}$

Ring expansion of gem-dihalocyclopropanated carbohydrates has also been reported by the Nagarajan group. Ring opening of cyclopropane $\mathbf{9 7}$ failed to occur in the 
presence of silver ion and Lewis acid catalysis in acetic acid; at room temperature, no reaction was observed, while under reflux the sugar was destroyed. However, the reaction proceeded well in a solution of excess potassium carbonate in refluxing methanol, and was reported to lead to the ring-expanded oxepine anomers $\mathbf{9 8}$ and 99. These products were isolated in a combined yield of $67 \%$ (1.3:1 ratio), though the stereochemistry of each anomer was not determined (Scheme 1.30). ${ }^{45}$ Similar results were obtained through using the corresponding D-galactose- and D-xylosebased cyclopropanes, providing mixtures of oxepines in yields of 55-70\%.<smiles>Br[C@H]1[C@H](Br)[C@H](Br)[C@@H](Br)[C@H]1Br</smiles>

97

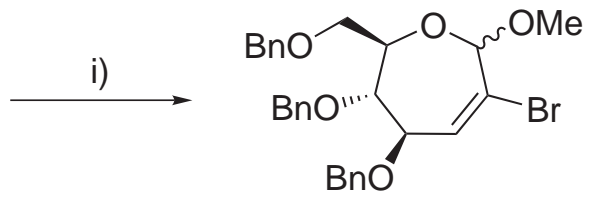

98,99

Reagents and Conditions: i) $\mathrm{K}_{2} \mathrm{CO}_{3}, \mathrm{MeOH}$, reflux.

Scheme 1.30 Reported ring expansion of glycal-derived cyclopropane $\mathbf{9 7} 7^{45}$

Jayaraman has recently extended this methodology to the synthesis of septanosides, the seven-membered ring glycosides. As shown in Scheme 1.31, 2-benzyloxyglucal 100 was cyclopropanated at the $\alpha$-face to provide cyclopropane 101, which was ring-expanded to provide oxepine derivative 102 in excellent yield. ${ }^{64}$ An oxidation, reduction and hydrogenolysis sequence ultimately provided methyl septanoside 103. Similar methodology using galactose-based precursors led to septanoside 104.<smiles>OC[C@H]1OC=C(Oc2ccccc2)[C@@H](OBr)[C@@H]1Br</smiles>

100<smiles>CC#CC</smiles>

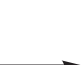

ii)

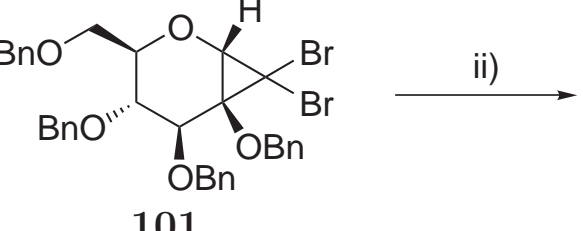<smiles>CO[C@H]1O[C@H](COBr)[C@@H](O)[C@H](OCc2ccccc2)C1Br</smiles>

102

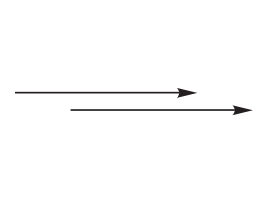

$\sqrt{20}$<smiles>CO[C@H]1O[C@H](CO)[C@@H](O)[C@H](O)[C@H]1O</smiles>

103

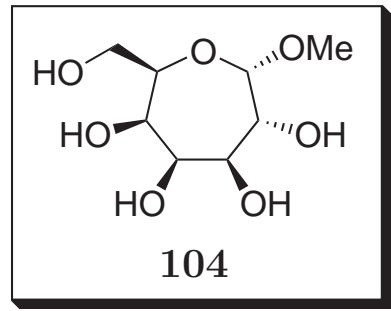

ii) $\mathrm{NaOMe}$, toluene, reflux, $94 \%$.

Scheme $\mathbf{1 . 3 1}$ Formation of septanosides via cyclopropane $\mathbf{1 0 1}^{64}$

Further extension of the above methodology to include a wide range of glycosyl acceptors as nucleophiles was recently reported. ${ }^{65} \mathrm{gem}$-Dichlorocyclopropane 105 was subjected to ring expansion in the presence of potassium carbonate in refluxing toluene. A variety of phenols and two sugars were used as nucleophiles, generating numerous oxepines as shown generically by structure 106 (Scheme 1.32). Yields were excellent when phenols were employed (76-85\%), while the sugar-derived nucle- 
ophiles provided good yields of $58 \%$ and $63 \%$. Subsequent oxidation, reduction and hydrogenation provided a variety of septanosides (107). A glycosyl amine analogue was obtained through use of sodium azide as a nucleophile in the ring expansion of cyclopropane $\mathbf{1 0 5} .^{65}$<smiles>CC(C)C</smiles>

105<smiles>CC#CCC#CC</smiles>

106<smiles>C[C@@H]1O[C@H](CO)[C@@H](O)[C@H](O)[C@H]1O</smiles>

107<smiles>[R]c1ccc(OC)c(OCCCC#N)c1</smiles><smiles>CO[C@H]1O[C@H](OC)[C@H](OC)[C@@H]1OCc1ccccc1</smiles><smiles>CO[C@H]1O[C@H](OC)[C@H](OCc2ccccc2)[C@@H](O)[C@@H]1OCc1ccccc1</smiles>

Reagents and Conditions: i) NucH, $\mathrm{K}_{2} \mathrm{CO}_{3}$, toluene, reflux; ii) $\mathrm{RuCl}_{3}, \mathrm{NaIO}_{4}$, EtOAc, $\mathrm{MeCN}$, r.t.; iii) $\mathrm{NaBH}_{4}, \mathrm{MeOH}, 0{ }^{\circ} \mathrm{C}$; iv) $\mathrm{Pd} / \mathrm{C}, \mathrm{H}_{2}$, EtOAc, $\mathrm{MeOH}$, r.t.

Scheme $\mathbf{1 . 3 2}$ Formation of septanosides via cyclopropane $\mathbf{1 0 5}^{65}$

A very recent paper by Jayaraman describes the synthesis of disaccharides 108 and 109 and trisaccharides 110 and 111 through similar methodology. ${ }^{66}$ Using glycals derived from D-lactose and D-maltose, the multi-step sequence discussed above (Scheme 1.32) provided disaccharides 108 and 109, through cyclopropane ring expansion with methanol. The D-lactose-derived cyclopropane was also ring expanded with the previously employed sugar nucleophiles (methyl 2,3,4-tri- $O$-benzyl- $\alpha$-Dglucoside and methyl 2,3-di- $O$-benzyl- $\alpha$-D-arabinoside) to provide trisaccharides $\mathbf{1 1 0}$ and 111 respectively (Figure 1.2), incorporating the seven-membered oxepine ring.<smiles>CO[C@H]1O[C@H](CO)[C@@H](O[C@H]2O[C@H](CO)[C@@H](O)[C@H](O)[C@H]2O)[C@H](O)[C@H]1O</smiles>

108<smiles>CO[C@H]1O[C@H](CO)[C@@H](O[C@H]2O[C@H](CO)[C@@H](O)[C@H](O)[C@H]2O)[C@H](O)[C@H]1O</smiles>

109<smiles>CO[C@H]1O[C@H](CO[C@H]2O[C@H](CO)[C@@H](O[C@H]3O[C@H](CO)[C@@H](O)[C@H](O)[C@H]3O)[C@H](O)[C@H]2O)[C@@H](O)[C@H](O)[C@H]1O</smiles>

110<smiles>CO[C@H]1O[C@H](CO[C@@H]2O[C@H](CO)[C@@H](O[C@H]3O[C@H](CO)[C@@H](O)[C@H](O)[C@H]3O)[C@H](O)[C@H]2O)[C@@H](O)[C@H]1O</smiles>

111

Figure 1.2 Formation of septanoside-based trisaccharides via cyclopropanes ${ }^{66}$ 


\subsection{Oxepanes, Oxepines and Septanoses}

The most common ring sizes encountered in organic compounds are undoubtedly the five- and six-membered rings. While seven-membered rings are much less common, they nevertheless occupy an important area of organic chemistry. These rings are present in the structures of several well-known natural products such as (-)colchicine (112) and ciguatoxin (113) (Figure 1.3).<smiles>COc1cc2c(c(OC)c1OC)-c1ccc(OC)c(=O)cc1[C@@H](N)CC[C@@H]2N[14CH3]</smiles>

Figure 1.3 Seven-membered ring natural products

Seven-membered oxacycles are often encountered in structures of synthetic targets. The parent structures are oxepane (114), the fully saturated seven-membered ring, and oxepine (115), the fully unsaturated analogue (Figure 1.4). Partially saturated derivatives are also commonly termed oxepines (or oxepenes). Thus, oxacycle 116 is named 2,5,6,7-tetrahydrooxepine, though for convenience may be referred to as an oxepine (or oxepene). The synthesis of oxepines and oxepanes has been achieved through numerous means, ${ }^{67,68}$ several of which involved application of cyclopropane chemistry. ${ }^{45,64-66}$

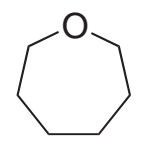

114 oxepane<smiles>C1=CC=COC=C1</smiles>

115<smiles>C1=CCCOCC1</smiles>

116

Figure 1.4 Seven-membered oxacycles

Sugars containing a seven-membered ring are termed septanoses (as compared to the pyranoses and furanoses). Relative to their smaller counterparts, little research has been published on these carbohydrates. ${ }^{69}$ D-Glucose exists in both pyranose and furanose forms when in aqueous solution, however a tiny portion is thought to be present as D-glucoseptanose (117) (Figure 1.5). While this structure contains an oxepane ring, it still is a six-carbon sugar (hexose). The seven carbon homo- 
logues of the naturally occurring sugars are known as the heptoses, and those that incorporate an oxepane ring (118) are termed heptoseptanoses. A few examples of heptoseptanosides have been investigated, leading to the synthesis of compounds such as 119, an oxepane-based nucleoside. ${ }^{70}$<smiles>O[C@H]1CO[C@@H](O)[C@@H](O)[C@H]1O</smiles>

117<smiles>OCC1O[C@H](O)C(O)C(O)C1O</smiles>

118<smiles>Cc1cn(C2CCC[C@@H](O)[C@H](CO)O2)c(=O)[nH]c1=O</smiles>

119

Figure 1.5 Examples of septanose carbohydrates ${ }^{70}$

There are many reports of interesting and varied syntheses of septanoses within the chemical literature, several of which utilise cyclopropanes (see section 1.2.2). Several cyclisation strategies ${ }^{68}$ such as ring-closing metathesis have been used, as exemplified by the work of Peczuh and co--workers. ${ }^{71,72}$ Diene 120 was reacted with Schrock catalyst $\mathbf{1 2 1}$ to provide a septanose glycal, which was later epoxidised to provide 122 (Scheme 1.33). Treatment of epoxide 122 with different nucleophiles under various conditions provided several D-idoseptanoside derivatives (123 and 124), with predominant or exclusive formation of the $\alpha$-anomers.<smiles>C=COC[C@H](OCc1ccccc1)[C@@H](OCc1ccccc1)C(Br)Br</smiles>

120

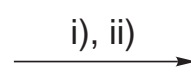

122<smiles>Br[C@H]1CO[C@@H]2O[C@H]2[C@H](Br)[C@H](Br)[C@H]1Br</smiles><smiles>CC#CC#CC</smiles>

123,124

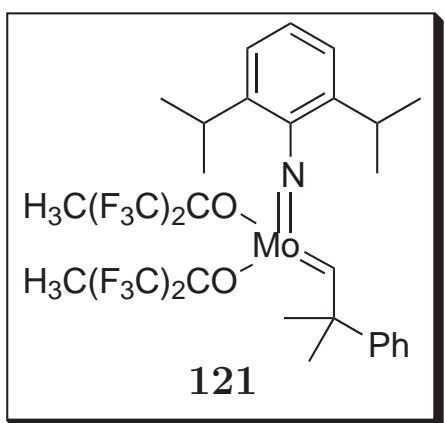

Reagents and Conditions: i) 121, $\mathrm{CH}_{2} \mathrm{Cl}_{2}$, reflux; ii) dimethyldioxirane, $\mathrm{CH}_{2} \mathrm{Cl}_{2}, 0{ }^{\circ} \mathrm{C}$ iii) Nuc, various conditions.

Scheme 1.33 RCM-based synthesis of septanoses ${ }^{71,72}$ 


\subsection{Furo[3,4-b]pyran-5-ones}

The furo[3,4-b]pyran-5-one bicycle (Figure 1.6) is an interesting heterocycle, though little research on this structure has been published. It is present in certain natural product families, and has featured as a synthetic target in several publications.

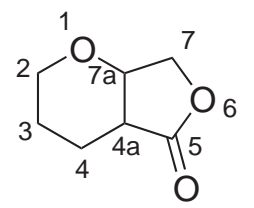

Figure 1.6 Furo[3,4-b]pyran-5-one

\subsubsection{Syntheses of Furo[3,4- $b]$ pyran-5-ones}

Hoffmann and Nowakowski synthesised a furo[3,4-b]pyran-5-one derivative in their approach to the synthesis of the macrolide lasonalide A (125) (Scheme 1.34). ${ }^{73}$ Starting from ketone 126 (obtained from furan and 1,1,3,3-tetrabromoacetone), functionalisation at the $\alpha$-centre provided quaternary compound $\mathbf{1 2 7}$. Ketone reduction and subsequent protection, ozonolysis of the alkene bridge, then reduction provided diol 128. Finally, to differentiate between the two hydroxyls, basemediated lactonisation provided furo[3,4-b]pyran-5-one derivative 129. This provided a masked form of the highlighted portion of lasonalide A (125).<smiles>O=C1CC2C=CC(C2)O1</smiles>

126

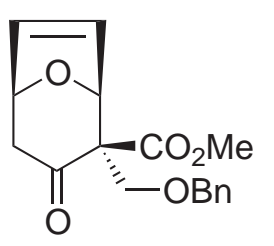

127

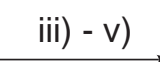

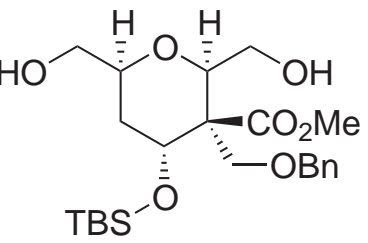

128

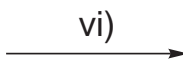<smiles>CC(C)(C)O[C@H]1CC(CO)O[C@H]2COC(=O)[C@]21O</smiles>

129

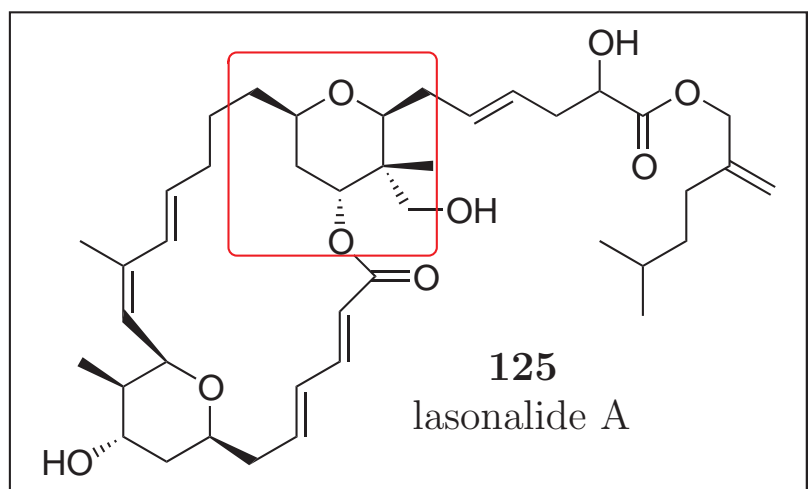

Reagents and Conditions: i) (-)-bis[(S)-1-phenylethyl]amidolithium, LiCl, $\mathrm{NCCO}_{2} \mathrm{Me}$, THF, $-94{ }^{\circ} \mathrm{C}$; ii) $\mathrm{NaH}, \mathrm{BnOCH}_{2} \mathrm{Cl}$, dioxane, $0{ }^{\circ} \mathrm{C}$ to r.t.; iii) $\mathrm{NaBH}_{4}, \mathrm{CeCl}_{3}, \mathrm{MeOH}, \mathrm{EtOH},-78{ }^{\circ} \mathrm{C}$; iv) TBSOTf, lutidine, $\mathrm{CH}_{2} \mathrm{Cl}_{2}, 0{ }^{\circ} \mathrm{C}$ to r.t.; v) $\mathrm{O}_{3}, \mathrm{MeOH}, \mathrm{CH}_{2} \mathrm{Cl}_{2},-78{ }^{\circ} \mathrm{C}$ then $\mathrm{NaBH}_{4}, \mathrm{EtOH}$, $-30{ }^{\circ} \mathrm{C}$; vi) imidazole, $\mathrm{MeCN}, 100{ }^{\circ} \mathrm{C}$.

Scheme 1.34 Synthesis of a fragment of lasonalide $\mathrm{A}^{73}$ 
Another synthesis of furo[3,4- $b]$ pyran-5-ones proceeds through a multi-component reaction. Shaabani and co-workers report that reaction of an isocyanide (130), a functionalised acetylene (131), and tetronic acid (132, 2,4(3H,5H)-furandione) provides complex furo[3,4-b]pyran-5-one products (133) (Scheme 1.35). ${ }^{74}$ Several different isocyanides and acetylenes were chosen, and yields ranged from 68-80\%, requiring very mild conditions to achieve formation of the bicycle.

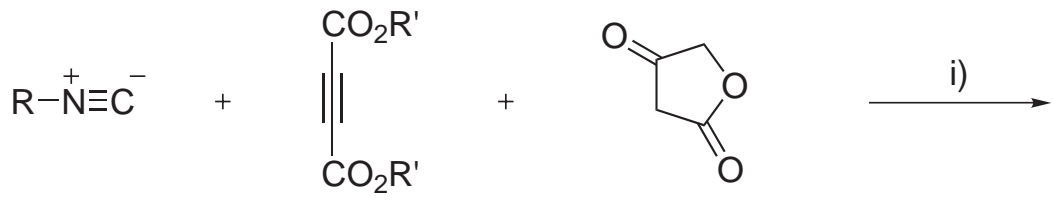

130<smiles>[R]NC1=C(C(=O)O[2H])C(C(=O)O)C2=C(COC2=O)O1</smiles>

133

$\mathrm{R}=$ cyclohexyl, ${ }^{t} \mathrm{Bu}$ $\mathrm{R}^{\prime}=\mathrm{Me}, \mathrm{Et}$

Reagents and Conditions: i) $\mathrm{CH}_{2} \mathrm{Cl}_{2}$, r.t.

Scheme 1.35 Synthesis of furo[3,4-b]pyran-5-ones via a multi-component reaction $^{74}$

A series of natural product-derived furo[3,4- $b]$ pyran-5-ones have been obtained from reaction of 6-O-palmitoyl-L-ascorbic acid (134) with acrolein (135), to provide intermediate 136 (Scheme 1.36). ${ }^{75}$ The unmasked ketone of intermediate 136 reacts with the alcoholic solvent, promoting cyclisation to provide furo[3,4-b]pyran-5-ones 137.<smiles>CC(C)C(C)(C)C(=O)OC[C@H](O)[C@H]1OC(=O)C(O)=C1O</smiles><smiles>CC(C)C(=O)OC[C@H](O)[C@H]1OC(=O)[C@](O)(CCC=O)C1=O</smiles>

136<smiles>CC(C)(C)C(=O)OC[C@H](O)[C@H]1OC(=O)[C@]2(O)CCC(O)O[C@]12O</smiles>

137

Reagents and Conditions: i) $\mathrm{ROH}, 70^{\circ} \mathrm{C}$.

$$
\mathrm{R}=\mathrm{Me}, \mathrm{Et}, n-\mathrm{Pr}
$$

Scheme 1.36 Vitamin C-derived furo[3,4-b]pyran-5-ones ${ }^{75}$

Paquette and Sivik reported the synthesis of simple furo[3,4- $b]$ pyran-5-ones derived from 2,3-dihydropyran (138). ${ }^{76}$ Thus, $\alpha$-chloropropionyl chloride (139) in the presence of triethylamine generated chloromethylketene, which provided bicycle 140, upon reaction with 138 (Scheme 1.37). Dechlorination with a zinc- 
copper couple, followed by Baeyer-Villiger oxidation provided furo[3,4- $b]$ pyran-5one 141. Phenylselenation at $\mathrm{C}-4 \mathrm{a}$, followed by oxidative elimination provided $\alpha, \beta$-unsaturated lactone 142. Isomerisation afforded bicycle 143, in which the dihydrofuran ring of this derivative was then converted into the fully unsaturated form, for use in Diels-Alder reactions.

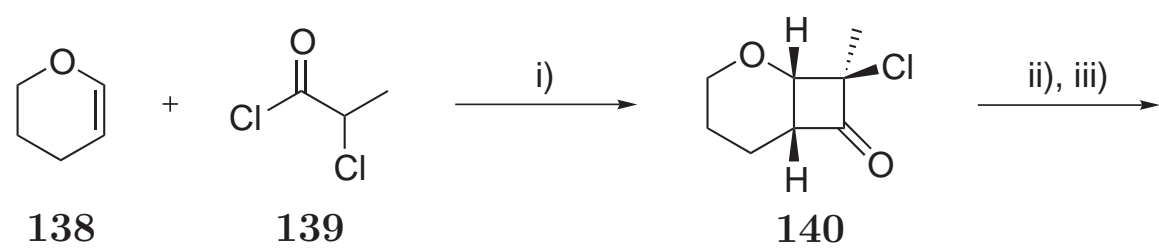<smiles>[CH][C@@H]1OC(=O)[C@H]2CCCO[C@H]12</smiles>

141<smiles>C[C@@H]1C[C@@H]2OCCC=C2C(=O)O1</smiles>

142<smiles>CC#CCCCC</smiles>

143

Reagents and Conditions: i) $\mathrm{NEt}_{3}$, hexane, $0{ }^{\circ} \mathrm{C}$; ii) $\mathrm{Zn}, \mathrm{Cu}(\mathrm{OAc})_{2}, \mathrm{NEt}_{3}, \mathrm{NH}_{4} \mathrm{Cl}, \mathrm{MeOH}, 0{ }^{\circ} \mathrm{C}$; iii) $m$-CPBA, $\mathrm{CH}_{2} \mathrm{Cl}_{2},-25{ }^{\circ} \mathrm{C}$; iv) $\mathrm{KHMDS}, \mathrm{PhSeCl}$, THF, $-78^{\circ} \mathrm{C}$ to r.t.; v) $m$-CPBA, $\mathrm{NaHCO}_{3}, \mathrm{CH}_{2} \mathrm{Cl}_{2}, 0{ }^{\circ} \mathrm{C}$ to r.t.; vi) $\mathrm{RhCl}_{3} \cdot 3 \mathrm{H}_{2} \mathrm{O}, \mathrm{NEt}_{3}$, EtOH, $90{ }^{\circ} \mathrm{C}$.

Scheme 1.37 Synthesis of furo[3,4-b] pyran-5-ones from 2,3-dihydropyran ${ }^{76}$

\subsubsection{Furo[3,4-b]pyran-based Natural Products}

Relatively few natural products are known to incorporate the furo[3,4- $b]$ pyran-5-one ring system; only the fusidilactones, ${ }^{77,78}$ massarilactones, ${ }^{79-81}$ (-)-TAN-2483 A and $\mathrm{B},{ }^{82}(-)$-waol $\mathrm{A}^{83}$ and waol $\mathrm{B}^{84}$ compounds appear to exhibit this ring structure. These compounds have all been isolated from various fungi, and exhibit numerous structural similarities and bioactivities. Shown below are representative examples from each of these natural product families (Figure 1.7).<smiles>CCC(C)CC(C)/C=C\[C@H]1OC2=C(C(=O)O[C@H]2C)[C@@H](O)[C@@H]1O</smiles>

(+)-fusidilactone A (-)-massarilactone D<smiles>C/C=C/[C@H](O)[C@H]1O[C@H](/C=C/C)[C@@H](O)C=C1C(=O)O</smiles>

waol B<smiles>C/C=C/[C@H]1O[C@@H]2C(=C[C@H]1O)C(=O)O[C@H]2C</smiles>

$(-)-\mathrm{TAN}-2483 \mathrm{~A}$

Figure 1.7 Selected examples of furo[3,4-b]pyran-based natural products 


\section{Fusidilactones}

Krohn and co-workers isolated fusidilactones A (144), B (145) and C (146) from cultures of the fungal endophyte Fusidium sp. (Figure 1.8). ${ }^{77}$ The crude extracts from which these products were isolated displayed antifungal activity against Eurotium repens and Fusarium oxysporum, and moderate antibacterial activity against E. coli and Bacillus megaterium. More recently, fusidilactones D (147) and E (148) were obtained, which differ from fusidilactones A and B only in the degree of hydroxylation in the sidechain. ${ }^{78}$ Hsung et al. have published initial results in their approach to the synthesis of the complex hexacycle $(+)$-fusidilactone $\mathrm{C}(\mathbf{1 4 6}) .{ }^{85}$

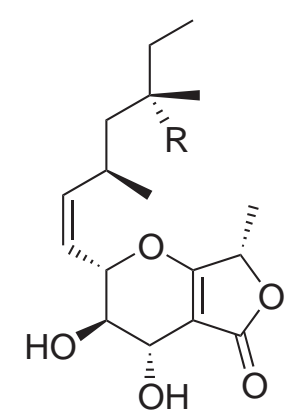

144,147

$$
\begin{gathered}
\mathrm{R}=\mathrm{H} \\
(+) \text {-fusidilactone A } \\
\mathrm{R}=\mathrm{OH} \\
\text { (+)-fusidilactone D }
\end{gathered}
$$

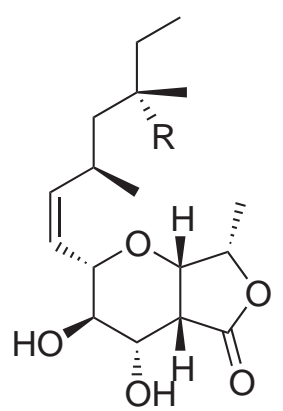

145,148

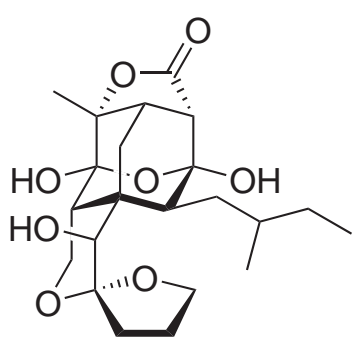

146

$$
\mathrm{R}=\mathrm{OH}
$$

(+)-fusidilactone C

$(+)$-fusidilactone B

$\mathrm{R}=\mathrm{H}$

(+)-fusidilactone E

Figure 1.8 Fusidilactones A-E $\mathrm{E}^{77,78}$

\section{Massarilactones}

The Gloer group isolated massarilactones A (149) and B (150) from the aquatic fungus Massarina tunicata (Figure 1.9). ${ }^{79}$ Later work by the Krohn group led to the discovery of additional furo[3,4- $b]$ pyran-based secondary metabolites, namely massarilactone C (151) and D (152), both isolated from the endophytic fungus Coniothyrium sp. ${ }^{80}$ Further work led to the isolation of massarilactones E-G, structurally similar to 149 and $\mathbf{1 5 1} .^{81}$ Massarilactones A and B exhibit antibacterial activity against Bacillus subtilis, with (-)-massarilactone B also able to inhibit Staphylococcus aureus. ${ }^{79}$ Despite promising activity in crude extracts, massarilactones C and D appeared to be biologically inactive against Bacillus megaterium, in addition to the Microbotryum violaceum fungus, and the Chlorella fusca green alga. ${ }^{80}$ 


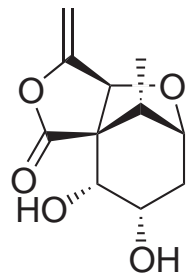

149<smiles>C/C=C/[C@H]1OC2=C(C(=O)O[C@H]2C)[C@@H](O)[C@@H]1O</smiles>

150<smiles>CC(=O)O[C@H]1C[C@@H]2C[C@@H](O)[C@@H](O)[C@@]1(OC(C)=O)O2</smiles>

151<smiles>C/C=C/[C@H]1OC2=C(C(=O)O[C@@]2(C)O)[C@@H](O)[C@@H]1O</smiles>

152

(+)-massarilactone A

(+)-massarilactone $\mathrm{C}$

(-)-massarilactone B

(-)-massarilactone D

Figure 1.9 Massarilactones A-D ${ }^{79,80}$

\section{(-)-TAN-2483 A and (-)-TAN-2483 B}

In 1998 Hayashi and co--workers published a patent regarding compounds (-)-TAN2483A (153) and (-)-TAN-2483B (154), isolated from a filamentous fungus culture (Figure 1.10). ${ }^{82}$ Initial biological tests upon TAN-2483A showed good inhibition of the c-src tyrosine kinase enzyme, responsible for cell growth, in addition to inhibition of bone resorption that is initiated by the parathyroid hormone. ${ }^{82}$<smiles>C/C=C/C1O[C@@H]2C(=C[C@H]1O)C(=O)O[C@H]2C</smiles>

153<smiles>C/C=C/C1O[C@H]2C(=CC1O)C(=O)O[C@H]2C</smiles>

154

(-)-TAN-2483A

(-)-TAN-2483B

Figure 1.10 TAN-2483 natural products ${ }^{82}$

\section{Waol Compounds}

Mizoue and co-workers reported the isolation of $\delta$-lactone FD-211 (155), which was obtained from the fungus Myceliophthora lutea (Figure 1.11). ${ }^{83}$ This lactone demonstrated activity against a broad spectrum of tumour cell lines. FD-211 was later termed waol A, when a close relative, waol B (156) was later isolated from the same fungal species. ${ }^{84}$ Later, a preliminary paper reported the partial synthesis of structure 155. ${ }^{86}$ More recently, the Snider group have published work indicating that waol A and B do not have structures 155 and 156, and the correct structures of waol A and B were confirmed by synthesis to be 157 and 158 respectively. ${ }^{87}$ 
<smiles>C/C=C/C1O[C@@H](/C=C/C)C2=CC(O)C(=O)OC21</smiles>

155<smiles>C/C=C/[C@@H]1OC(=O)C2=C[C@@H](O)[C@@H](/C=C/C)O[C@H]21</smiles>

157<smiles>C/C=C/[C@H]1O[C@H](/C=C/C)[C@H](O)/C1=C\C(O)C(=O)OC</smiles>

156

(-)-waol A<smiles>C/C=C/[C@H](O)[C@H]1O[C@H](/C=C/C)[C@@H](O)C=C1C(C)=O</smiles>

158

Figure 1.11 Initial and revised structures of waol $\mathrm{A}$ and $\mathrm{B}^{83,84,87}$

\subsubsection{Syntheses of Furo[3,4-b]pyran-Based Natural Prod- ucts}

The Snider group has published the only known reports on the total synthesis of these furo[3,4- $b$ ] pyran-5-one natural products. ${ }^{87,88}$ After proposing alternative structures for waol A and B, an elegant synthetic route was developed that provided access to representatives of the fusidilactone, massarilactone, waol and TAN-2483 natural products incorporating the furo[3,4- $b]$ pyran-5-one bicycle.

(-)-TAN-2483A and (-)-waol A were both synthesised using the route shown below (Scheme 1.38). Aldol reaction of lactone $\mathbf{1 5 9}$ and aldehyde $\mathbf{1 6 0}$ led to a mixture of $\beta$ hydroxy lactone 161 (38\%) and the corresponding C-4 epimer (14\%). ${ }^{87,88}$ Compound 161 was subjected to iodoetherification to provide 162 (88\%), with the required configuration at C-2. To complete the synthesis, treatment of iodide 162 with triethylamine led to the formation of an intermediate epoxide, which underwent elimination to afford (-)-TAN-2483A (153) in 79\% yield. Use of an alternative lactone (163), led to the synthesis of waol A (157), which was subjected to lactone hydrolysis and reaction with diazomethane, to generate waol B (158). ${ }^{87,88}$

Snider applied similar methodology to the synthesis of (-)-massarilactone B and an approach to (+)-fusidilactone B. ${ }^{88}$ Beginning from lactone 166 (Scheme 1.39), repetition of the above aldol coupling provided 167 and the corresponding C-4 epimer. Iodoetherification of $\mathbf{1 6 7}$ provided bicycle $\mathbf{1 6 8}$ in $33 \%$ yield over two steps. This intermediate was used to synthesise (-)-massarilactone B (150) and diol 169, a truncated form of (+)-fusidilactone B (145). 
<smiles>[R][C]1OC(=O)C[C@@H]1O</smiles>

$159 \mathrm{R}=\mathrm{Me}$

$163 \mathrm{R}=(1 E)$-propenyl

160

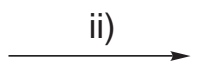<smiles>[R][C@H]1OC(=O)[C@@H]2[C@@H]1O[C@H](/C=C/C)[C@H](I)[C@@H]2O</smiles>

$162 \mathrm{R}=\mathrm{Me}$

$165 \mathrm{R}=(1 E)$-propenyl

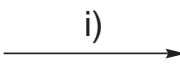

$161 \mathrm{R}=\mathrm{Me}$

$164 \mathrm{R}=(1 E)$-propenyl

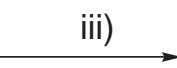

$153 \mathrm{R}=\mathrm{Me}$

(-)-TAN-2483A

$157 \mathrm{R}=(1 E)$-propenyl

(-)-waol A

Reagents and Conditions: i) LDA, THF, $-42{ }^{\circ} \mathrm{C}$; ii) bis-(sym-collidine) $\mathrm{AgPF}_{6}, \mathrm{I}_{2}, \mathrm{CH}_{2} \mathrm{Cl}_{2}$, $25{ }^{\circ} \mathrm{C}$; iii) $\mathrm{NEt}_{3}, \mathrm{CH}_{2} \mathrm{Cl}_{2}, 25^{\circ} \mathrm{C}$.

Scheme 1.38 Syntheses of (-)-TAN-2483A (153) and (-)-waol A $(\mathbf{1 5 7})^{87,88}$

$\mathrm{HO}$,<smiles>C[C@H]1CCC(=O)O1</smiles><smiles>CC1CCCCC1C</smiles>

166

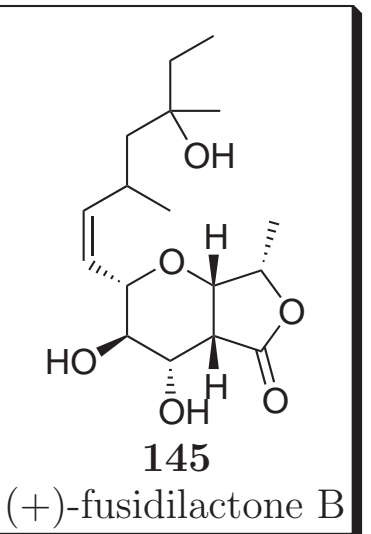<smiles>C/C=C/C=C/[C@H](O)[C@H]1C(=O)O[C@H](C)C1O</smiles>

167<smiles>CC(C)/C=C\[C@H]1O[C@H]2[C@@H](C)OC(=O)[C@@H]2[C@H](O)[C@H]1O</smiles>

169
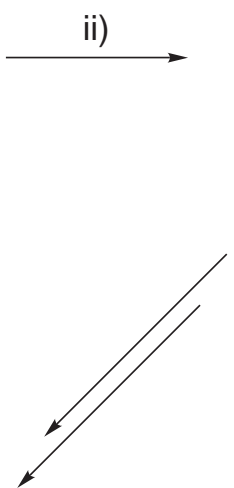

Reagents and Conditions: i) $160, \mathrm{LDA}, \mathrm{THF},-42{ }^{\circ} \mathrm{C}$; ii) bis-(sym-collidine) $\mathrm{AgPF}_{6}, \mathrm{I}_{2}$, $\mathrm{CH}_{2} \mathrm{Cl}_{2}, 25^{\circ} \mathrm{C}$.

Scheme 1.39 Synthesis of (-)-massarilactone B (150) and diol $169^{88}$ 
Finally, Snider attempted to synthesise (-)-TAN-2483B. As this compound is the C-7a epimer of (-)-TAN-2483A, the general synthetic scheme used in the previous syntheses needed to be elaborated. Initial attempts began with bicyclic iodide 168, a synthetic intermediate in the above syntheses (Scheme 1.39). ${ }^{88}$ Treatment of intermediate 168 with base provided compound 170 (Scheme 1.40), a stereochemical isomer of (-)-TAN-2483B in which the configurations at C-2 and C-3 are different. Epimerisation of the C-3 alcohol was achieved through oxidation to ketone $\mathbf{1 7 1}$, followed by reduction to alcohol $\mathbf{1 7 2}$, the C-2 epimer of (-)-TAN-2483B. Unfortunately, silica-based chromatographic purification of ketone $\mathbf{1 7 1}$ resulted in the rapid isomerisation to enol 173. It was proposed that this occurs through tautomerisation of the C-3 ketone to an enol across the 2,3-bond, followed by a Claisen rearrangement. Due to this observation, attempts to epimerise C-2 were abandoned.

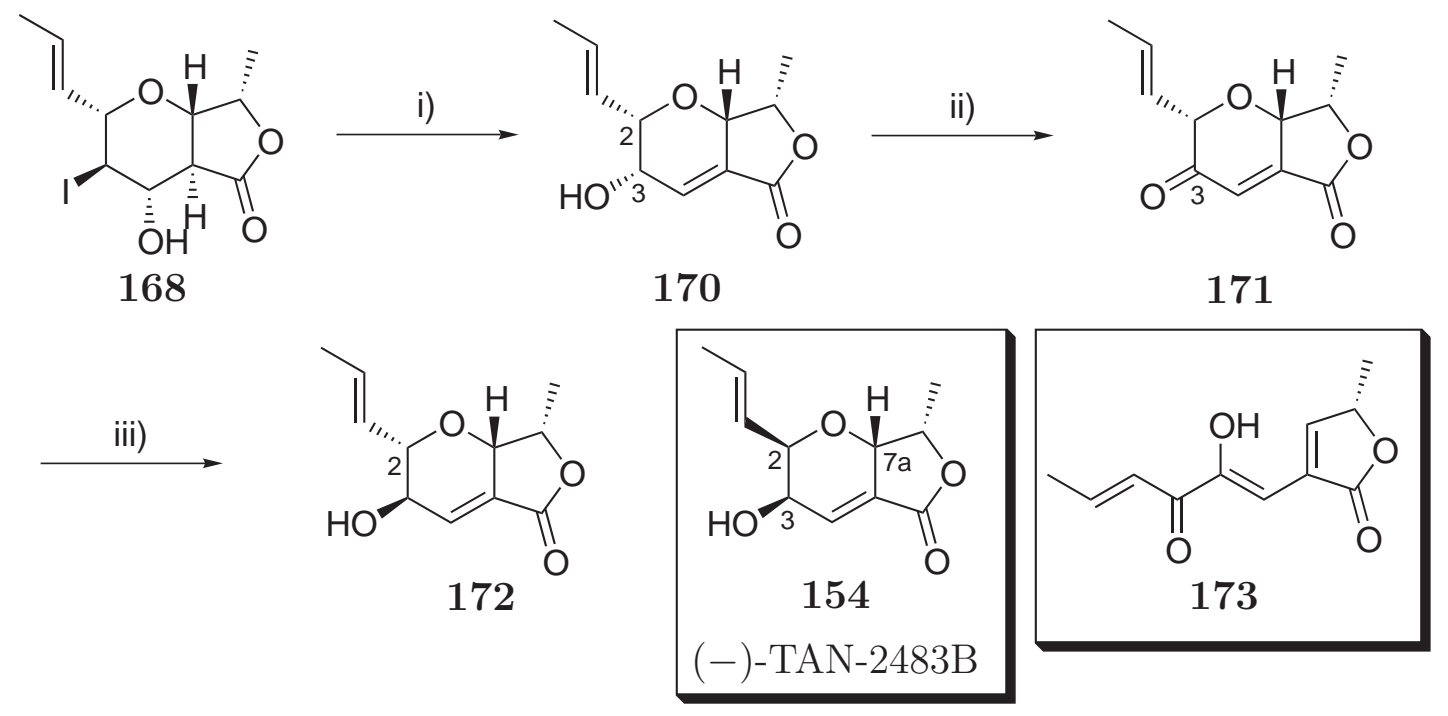

Reagents and Conditions: i) DBU, THF, $25{ }^{\circ} \mathrm{C}$; ii) Dess-Martin periodinane, $\mathrm{CH}_{2} \mathrm{Cl}_{2}, 2{ }^{\circ} \mathrm{C}$; iii) $\mathrm{NaBH}_{4}, \mathrm{CeCl}_{3} \cdot 7 \mathrm{H}_{2} \mathrm{O}, \mathrm{MeOH}, 25^{\circ} \mathrm{C}$.

Scheme 1.40 Attempted synthesis of (-)-TAN-2483B ${ }^{88}$

A final attempt at synthesising (-)-TAN-2483B involved an alternative method of pyran formation, using the previously undesired minor aldol product 174 (4-epi167) from the synthesis of (-)-massarilactone B (Scheme 1.39). ${ }^{88}$ It was expected that epoxidation of the 2,3-bond and subsequent attack by the C-7a alcohol would provide bicycles $\mathbf{1 7 5}$ and $\mathbf{1 7 6}$, of which the former could provide access to (-)TAN-2483B. However, epoxidation occurred at both olefins, and the 2,3-epoxide products underwent cyclisation to exclusively provide bicycle 176. It is noteworthy that the bicyclic configuration of (-)-TAN-2483B has the propenyl sidechain and the lactone moiety in a trans-configuration; in contrast, the methodology employed by Snider invariably produces a cis-configuration. Snider's several unsuccessful attempts to synthesise (-)-TAN-2483B indicate alternative methodology is required for the synthesis of this natural product. While many of the syntheses discussed in subsection 1.4.1 demonstrate the furo[3,4- $b]$ pyran-5-one skeleton is accessible using efficient sequences, none of the methods detailed provide functionality similar to that 


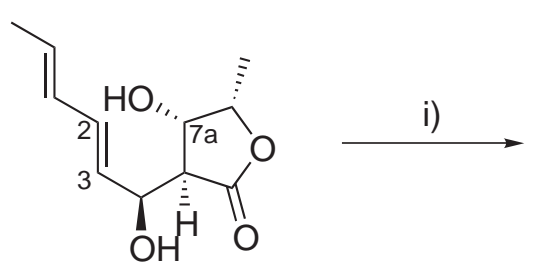

174

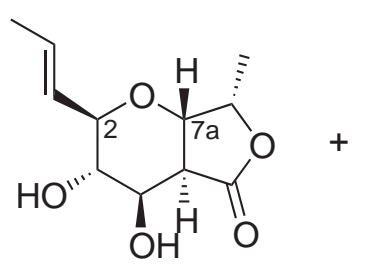

175

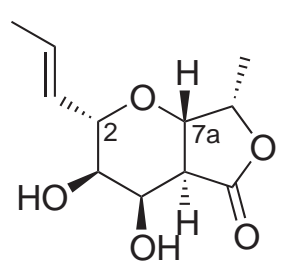

176

Reagents and Conditions: i) m-CPBA, $\mathrm{NaHCO}_{3}, \mathrm{CH}_{2} \mathrm{Cl}_{2}, 0{ }^{\circ} \mathrm{C}$.

Scheme 1.41 Attempted epoxide-mediated synthesis of (-)-TAN-2483B ${ }^{88}$

of the furo[3,4-b]pyran-5-one-based natural products. This therefore shows multistep sequences such as those used by Snider ${ }^{88}$ are valid, unless the products from multicomponent couplings such as that reported by Shaabani ${ }^{74}$ could be elaborated further to provide access to biologically relevant targets. 


\subsection{Research Objectives}

The research contained within this thesis is comprised of two main parts. Ring expansion studies of selected cyclopropanated carbohydrates will be explored, with the intention of extending the limited range of available reactions so far reported in the scientific literature. Secondly, through application of this methodology, or variations thereof, a synthesis of furo[3,4- $b]$ pyran-5-one-based natural products will be investigated.

\subsubsection{Ring-Expansion Studies}

In the interests of synthesising various oxepines of structure 177 (Scheme 1.42), methodology that extends the work of Nagarajan ${ }^{45}$ incorporating a variety of nucleophiles will be explored. Oxepines 177 might be obtained through ring-expansion reactions of cyclopropanated carbohydrates 178 .<smiles>[R9]CC1OC([NH2+])C(Br)=CC1O</smiles>

177<smiles>[R9]CC1OC2C(C(O)C1O[R])C2(Br)Br</smiles>

178

Scheme 1.42 Synthesis of hexose-derived cyclopropanes

This area of research will involve synthesis of cyclopropanated carbohydrates, via cyclopropanation of glycals. Peracetylated glycals 179, 180 and 181 will be formed from the respective carbohydrate parents D-glucose (182), D-galactose (183) and D-xylose (184), through the well-established Fischer glycal synthesis, via peracetylation, anomeric bromination and zinc reduction (Scheme 1.43). ${ }^{89}$ Deprotection to afford free glycals 185, 186 and 187, followed by benzylation will furnish the protected glycals $(\mathbf{1 8 8}, \mathbf{4 3}, \mathbf{1 8 9})$, used as cyclopropanation precursors.

The glycals will then be cyclopropanated using the established Mạkosza method of cyclopropane formation. ${ }^{12}$ Literature precedence indicates a mild alternative to the standard Mąkosza cyclopropanation procedure is probably required to avoid starting material degradation. ${ }^{45}$ The majority of work will focus on the cyclopropanation of D-glucose derivative 188 (Scheme 1.44), in which it is possible that both diastereomers 97 and 190 will be obtained, due to cyclopropanation from both the top and bottom face of glucal derivative 188. The reaction should favour cyclopropane $\mathbf{9 7}$, with carbene addition occurring from the opposite face to the neighbouring C-3 benzyloxy substituent. 


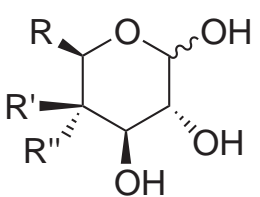

$182 \mathrm{R}=\mathrm{CH}_{2} \mathrm{OH}, \mathrm{R}^{\prime}=\mathrm{H}, \mathrm{R}^{\prime \prime}=\mathrm{OH}$

$183 \mathrm{R}=\mathrm{CH}_{2} \mathrm{OH}, \mathrm{R}^{\prime}=\mathrm{OH}, \mathrm{R}^{\prime \prime}=\mathrm{H}$

$184 \mathrm{R}=\mathrm{H}, \mathrm{R}^{\prime}=\mathrm{H}, \mathrm{R}^{\prime \prime}=\mathrm{OH}$ iv) or v)

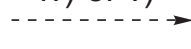

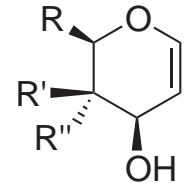

$185 \mathrm{R}=\mathrm{CH}_{2} \mathrm{OH}, \mathrm{R}^{\prime}=\mathrm{H}, \mathrm{R}^{\prime \prime}=\mathrm{OH}$ i), ii), iii)<smiles>[2H]C1OC=C[C@@H](OC(C)C)[C@@H]1P</smiles>

$179 \mathrm{R}=\mathrm{CH}_{2} \mathrm{OAc}, \mathrm{R}^{\prime}=\mathrm{H}, \mathrm{R}^{\prime \prime}=\mathrm{OAc}$

$180 \mathrm{R}=\mathrm{CH}_{2} \mathrm{OAc}, \mathrm{R}^{\prime}=\mathrm{OAc}, \mathrm{R}^{\prime \prime}=\mathrm{H}$

$181 \mathrm{R}=\mathrm{H}, \mathrm{R}^{\prime}=\mathrm{H}, \mathrm{R}^{\prime \prime}=\mathrm{OAc}$
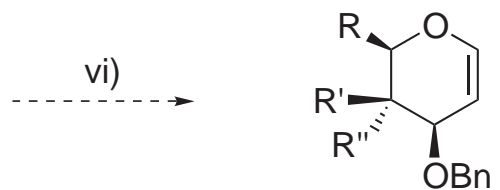

$188 \mathrm{R}=\mathrm{CH}_{2} \mathrm{OBn}, \mathrm{R}^{\prime}=\mathrm{H}, \mathrm{R}^{\prime \prime}=\mathrm{OBn}$

$43 \mathrm{R}=\mathrm{CH}_{2} \mathrm{OBn}, \mathrm{R}^{\prime}=\mathrm{OBn}, \mathrm{R}^{\prime \prime}=\mathrm{H}$

$189 \mathrm{R}=\mathrm{H}, \mathrm{R}^{\prime}=\mathrm{H}, \mathrm{R}^{\prime \prime}=\mathrm{OBn}$

Scheme 1.43 Synthesis of acetyl and benzyl glycals

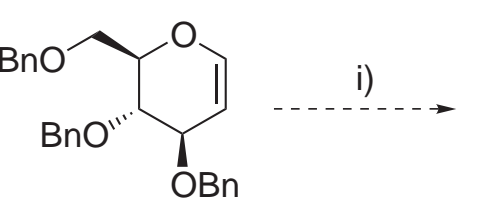

188

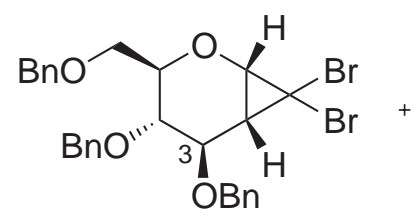

97

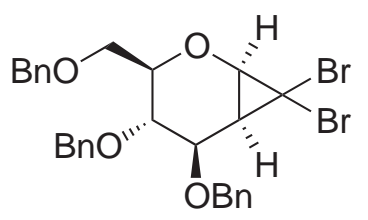

190

Reagents and Conditions: i) $\mathrm{CHBr}_{3}, \mathrm{TEBAC}, \mathrm{NaOH} / \mathrm{KF}_{(\mathrm{aq})}$, r.t.

Scheme 1.44 Synthesis of glucose-derived cyclopropanes 97 and 190

Upon generation of cyclopropanes such as 97, ring expansion studies will be carried out primarily by extending the methodology employed by Nagarajan. ${ }^{45}$ The base-mediated ring expansions will be attempted using a variety of nucleophiles, as discussed in chapter 2 (Scheme 1.45). While Nagarajan used potassium carbonate in methanol, it is likely that use of a slight excess of a stronger, nucleophilic base will also provide efficient reaction. This might permit the use of a non-nucleophilic solvent, hence more complicated nucleophiles could be used. As discussed earlier (page 3), participation of the pyran ring oxygen (forming a glycosyl cation) indicates nucleophilic attack should occur predominantly at the anomeric centre, to provide oxepines 191 and 192. Reaction rates and anomeric stereoselectivity will be of interest, particularly when this methodology is applied to D-galactose and D-xylose derivatives.

Nagarajan was unable to synthesise ring-expanded products when using silver salts. It is proposed that through careful analysis and modification of the reaction conditions, oxepines might be obtained without significant degradation of the starting material. This research will be covered in chapter 3 . 
<smiles>BrC(Br)(Br)c1ccccc1</smiles>

97

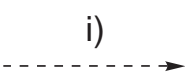

Reagents and Conditions: i) NaNuc, THF, reflux or $\mathrm{Ag}^{+}, \mathrm{NucH}$

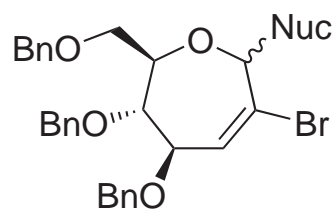

191,192

Scheme 1.45 Cyclopropane ring-expansion

\subsubsection{Synthesis of (-)-TAN-2483B}

Snider was able to synthesise many furo[3,4-b]pyran-5-one-based natural products, however the attempted synthesis of (-)-TAN-2483B was unsuccessful. ${ }^{88}$ Access to (-)-TAN-2483B (154) appears to be viable through use of a cyclopropanated carbohydrate, as indicated below (Scheme 1.46). (-)-TAN-2483B might be accessed from advanced intermediate 193, which comprises most of the carbon skeleton of (-)-TAN-2483B (Scheme 1.46); only sidechain elaboration and lactone formation are required. Intermediate 193 could be accessed from a simple naturally occurring hexose or pentose (194), through a cyclopropanation/ring expansion sequence, followed by further functional group interconversions.<smiles>C/C=C/[C@H]1O[C@H]2C(=CC1O)C(=O)O[C@H]2C</smiles>

154

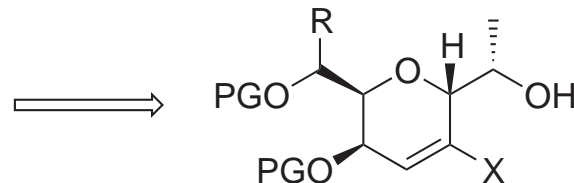

193

(-)-TAN-2483B<smiles>[R]C1O[C@H](O)C(O)C(O)C1O</smiles>

194

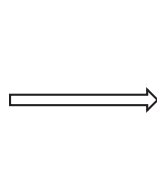

$\mathrm{R}^{\prime}=\mathrm{H}, \mathrm{CH}_{2} \mathrm{OH}$

Scheme 1.46 Cyclopropyl carbohydrate-based retrosynthesis of (-)-TAN-2483B

The stereogenic centres at positions C-2 and C-3 of (-)-TAN-2483B closely resemble those of D-galactose. Therefore, it was initially proposed that (-)-TAN-2483B might be obtained as shown in the retrosynthetic scheme below (Scheme 1.47). (-)-TAN2483B (154) could be formed via advanced intermediate 195, through regioselective deprotection of the $p$-methoxybenzylidene acetal, ${ }^{90}$ then an oxidation, olefination and deprotection sequence. It was thought alcohol 196 would provide lactone 195 through carbonylation or carboxylation of the alkenyl halide, then lactonisation. Alcohol 196 might be synthesised from cyclopropane 197 through an intramolecular cyclopropane-opening reaction, followed by chelation-controlled addition of a methyl group to the aldehyde. Cyclopropane 197 would be in equilibrium with the corresponding ring-closed sugar 198. This cyclopropane could be accessed from 2,3-unsaturated sugar 199, through cyclopropanation and protection/deprotection steps. Finally, 2,3-unsaturated sugar 199 would be easily obtained through a Ferrier rearrangement ${ }^{27}$ of tri- $O$-acetyl-D-galactal. 


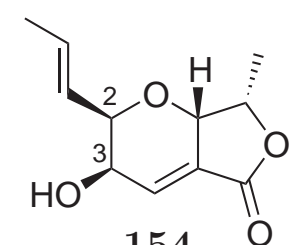

154

(-)-TAN-2483B

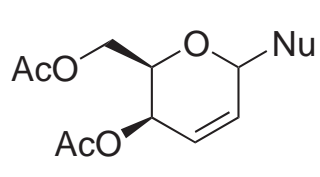

199

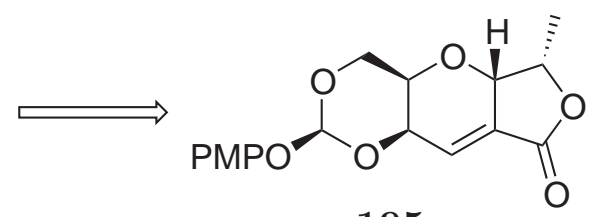

195

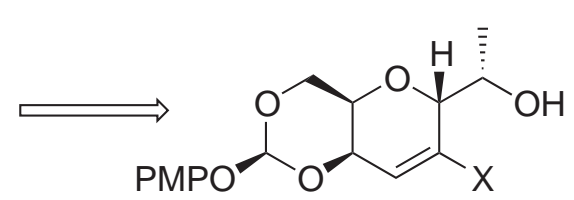

196

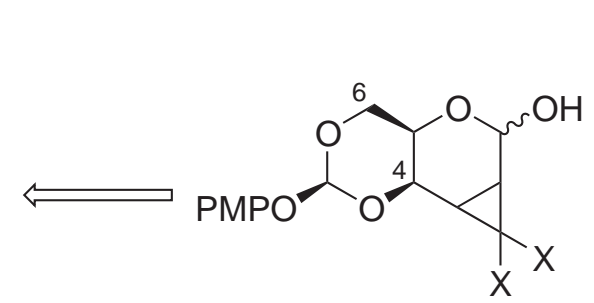

198

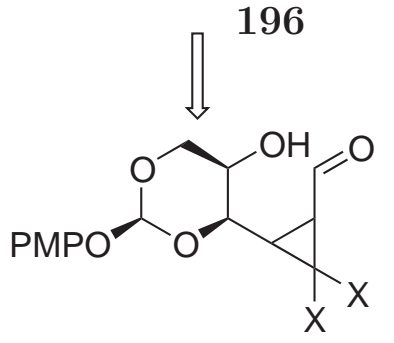

197

Scheme 1.47 Retrosynthesis of galactose-based (-)-TAN-2483B route

Alternatively, (-)-TAN-2483B could be accessed from a glycal-derived oxepine, such as the pentose sugar D-xylose. This could prove advantageous, due to the ease of synthesis of glycals. Furthermore, the above D-galactose route calls for intramolecular nucleophilic attack following cyclopropane ring-opening reaction. Obtaining the desired product, with the correct stereochemistry might be challenging. D-Xylose also contains the stereochemical configuration analogous to (-)-TAN-2483B, therefore the following retrosynthetic analysis (Scheme 1.48) could be effective for this total synthesis. It was proposed that (-)-TAN-2483B might be accessed from advanced intermediate 200, through a carbonylation/lactonisation sequence. Alcohol 200 could be accessed from triene 201 through cis-selective Jacobsen epoxidation; ${ }^{91}$ removal of protecting groups and intramolecular epoxide ring opening would likely provide 200. This would incorporate the only stereocentres not derived from the sugar starting material via asymmetric epoxidation. In turn, triene 201 could be synthesised from 202 through an oxidation/olefination sequence. Alcohol 202 could be accessed from 203 through deacetylation, followed by a $(Z)$-selective Wittig olefination. The required oxepine 203 could be synthesised from cyclopropane 204, through a ring-expansion reaction. Cyclopropane 204 could be accessed from di$O$-acetyl-D-xylal, through deprotection, then reprotection with an appropriate protecting group, followed by cyclopropanation. The $p$-methoxybenzyl protecting group was initially chosen, as it was expected to provide sufficient stability, while enabling an orthogonal deprotection strategy (using $\mathrm{DDQ}^{90}$ ).

Finally, a bicyclo[3.1.0] hexane-derived cyclopropanated carbohydrate could also provide access to (-)-TAN-2483B. D-Mannose could be used in this type of synthesis, as illustrated in Scheme 1.49. Using a deprotection/diol cleavage sequence, then olefination and deprotection, (-)-TAN-2483B (154) might be accessed from advanced intermediate 205. This lactone might in turn be generated from intermediate 206, through carbonylation and lactonisation. Alcohol 206 could arise from reducing 


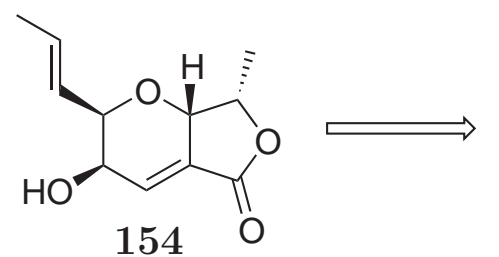

(-)-TAN-2483B

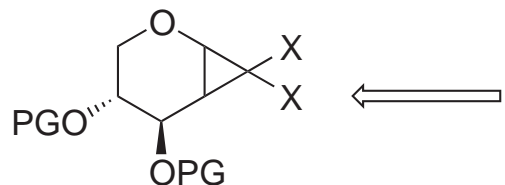

204<smiles>[X]C1=C[C@H](O)[C@@H](/C=C/C)O[C@H]1CO</smiles><smiles>[C]=C</smiles>
200<smiles>[R6]OC1OCC(O[R6])C(O[R6])C=C1[X]</smiles>

203

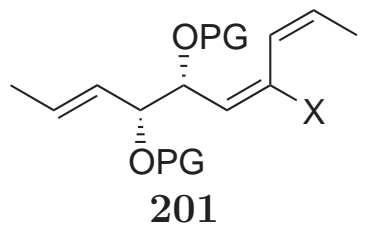<smiles>C=CC=C</smiles>

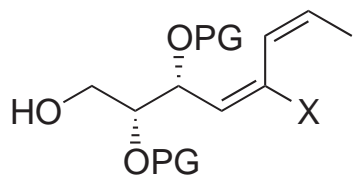

202

Scheme 1.48 Retrosynthesis of (-)-TAN-2483B using a xylose-based route

sugar 207 through an olefination/epoxidation route, followed by an epoxide ring opening to generate the pyran ring of 206. Substrate 207 could be synthesised from cyclopropane 208 through the application of a ring-expansion/deprotection sequence. Finally, cyclopropane might be obtained from known glycal 209, which is in turn derived from D-mannose. ${ }^{34}$<smiles>C/C=C/[C@H]1O[C@H](C)[C@H](O)C=C2C(=O)O[C@H](O)[C@@H]21</smiles>

154

(-)-TAN-2483B<smiles>CC1(C)OCC(C2OC=CC2O)O1</smiles>

209<smiles>C=CC</smiles>
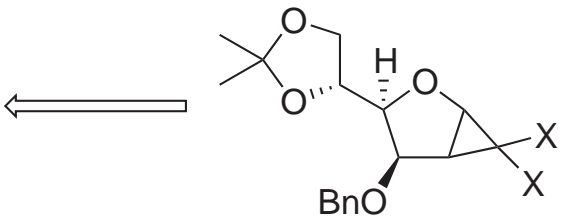

208<smiles>[X]C1=C[C@@H](O)[C@H]([C@H](C)O)O[C@H]1[C@H]1COC(C)(C)O1</smiles>

206<smiles>[C]=C=C</smiles><smiles>[X]C1=C[C@@H](O)[C@H]([C@@H]2COC(C)(C)O2)O[C@H]1O</smiles>

207

Scheme 1.49 Retrosynthesis of mannose-based (-)-TAN-2483B route

Efforts towards the synthesis of (-)-TAN-2483B through use of D-galactose and Dxylose are discussed in chapter 4 , and the successful synthesis of the carbon skeleton of the furo[3,4-b]pyran-5-one bicycle is discussed in chapter 5 . 


\section{Chapter 2}

\section{Base-Mediated Ring-Opening Reactions}

\section{$2.1 \quad$ Introduction}

Ring-opening reactions of cyclopropanated carbohydrates are of significant interest as a means of synthesising unusual carbohydrates and carbohydrate mimics. Studies of cyclopropyl sugars have been reported often, ${ }^{9,22}$ including a few examples of gem-dihalocyclopropanated carbohydrates used in ring-opening reactions. ${ }^{62,63}$ The Nagarajan group ${ }^{45}$ has described the synthesis of several carbohydrate derivatives incorporating an oxepine ring. Cyclopropane starting materials such as $\mathbf{9 7}$ (Scheme 2.1), easily accessible from protected glycals, were reported to undergo regioselective ring opening. Although several silver-promoted reaction conditions were investigated by Nagarajan, none proved fruitful. However, employing several equivalents of potassium carbonate in methanol ${ }^{92}$ under reflux temperatures, allowed conversion of cyclopropane $\mathbf{9 7}$ into oxepines $\mathbf{9 8}$ and $\mathbf{9 9}$. This base-mediated methodology would have great synthetic value if the nucleophile could be varied and used in only moderate excess. Repetition and extension of this methodology for preparing oxepines, through exploration of different cyclopropane precursors, nucleophiles and reaction conditions was a major focus of this research. 
<smiles>Cc1ccccc1Br</smiles>

97

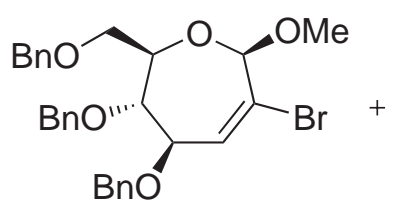

98

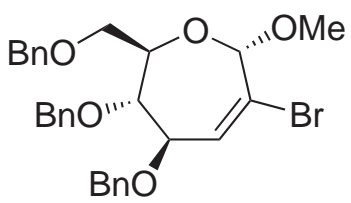

99

Reagents and Conditions: i) $\mathrm{K}_{2} \mathrm{CO}_{3}, \mathrm{MeOH}$, reflux.

Scheme 2.1 Nagarajan's reported oxepine synthesis ${ }^{45}$

\subsection{Synthesis of Cyclopropanes 97 and 190}

Studies began with D-glucose (182), a readily available sugar with good precedence as a cyclopropane substrate, as demonstrated by Nagarajan. ${ }^{45}$ Conversion of D-glucose into D-glucal involved the use of methodology pioneered by Fischer ${ }^{93-95}$ and refined by others. ${ }^{89,96}$ Synthesis began with peracetylation of D-glucose (182) using acetic anhydride and catalytic perchloric acid (Scheme 2.2); $\alpha$-bromoglycoside 210 was obtained through addition of hydrobromic acid. Upon isolation of crude glycosyl halide 210, reaction in the presence of zinc dust provided tri- $O$-acetyl-D-glucal (179), the first potential substrate for cyclopropanation. In the interests of incorporating more stable protecting groups, 179 was deprotected using triethylamine in aqueous methanol. The resulting D-glucal (185) was purified in good yield (66\% from D-glucose), by precipitation of impurities from a methanol/acetone/diethyl ether solvent mixture. D-Glucal (185) was then perbenzylated upon treatment with an excess of sodium hydride, followed by addition of benzyl bromide. Thus, triO-benzyl-D-glucal (188) was obtained in very good yield (84\%). Both protected glycals 179 and 188 were used as substrates for cyclopropanation.<smiles>OC[C@H]1O[C@H](O)[C@@H](O)[C@H](O)[C@H]1O</smiles>

182<smiles>CC#CC</smiles>

210<smiles>CC(=O)OC[C@H]1OC=C[C@@H](OC(C)=O)[C@H]1OC(C)=O</smiles>

179

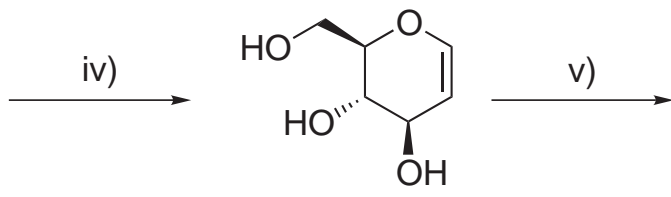

185<smiles>BrC(Br)(Br)c1ccccc1</smiles>

188

Reagents and Conditions: i) $\mathrm{Ac}_{2} \mathrm{O}, \mathrm{HClO}_{4}, 40-50{ }^{\circ} \mathrm{C}, 30 \mathrm{~min}$; ii) $\mathrm{HBr}, \mathrm{AcOH}$, r.t., 90 min; iii) $\mathrm{Zn}, \mathrm{AcOH}_{(\mathrm{aq})}, 0{ }^{\circ} \mathrm{C}$ to r.t., overnight; iv) $\mathrm{NEt}_{3}, \mathrm{MeOH}_{(\mathrm{aq})}$, r.t., $90 \mathrm{~min}, 66 \%$ from D-glucose; v) $\mathrm{BnBr}, \mathrm{NaH}, \mathrm{DMF}, 0{ }^{\circ} \mathrm{C}$ to r.t., overnight, $84 \%$.

Scheme 2.2 Synthesis of acetyl glucal (179) and benzyl glucal (188)

The original procedure for Mạkosza cyclopropanation involves a biphasic system, in which the aqueous phase is comprised of a caustic solution of $50 \%$ (w/w) sodium hydroxide in water. ${ }^{12}$ Nagarajan's synthesis of cyclopropane $\mathbf{9 7}$ from glycal 188 
utilised a subsequent modification by Mąkosza, wherein the aqueous phase employs a comparatively low concentration of sodium hydroxide. A high concentration of potassium fluoride is employed, to ensure the water activity of the solution is comparable to $50 \%(\mathrm{w} / \mathrm{w})$ sodium hydroxide in water. ${ }^{97}$ This modification allows for carbene addition to substrates that would normally not survive the highly basic conditions of standard Mạkosza cyclopropanation. With this in mind, attempts to synthesise $\mathrm{gem}$-dihalocyclopropanated carbohydrates began with tri- $O$-acetyl-Dglucal (179) (Scheme 2.3). This material was subjected to Mąkosza cyclopropanation, using the modified Mąkosza conditions employed by Nagarajan for the cyclopropanation of glycal 188 [6\% (w/w) sodium hydroxide, 47\% (w/w) potassium fluoride, $47 \%$ (w/w) water]. ${ }^{45}$ Unfortunately, glycal 179 was not stable under these conditions, and only degraded material was recovered after extraction with diethyl ether. This, together with TLC and NMR evidence, indicated significant loss of the acetate protecting groups. The use of ultrasound has been reported to improve cyclopropanations, ${ }^{9,98}$ providing much faster reaction rates. It was thought that ultrasonicating the reaction of $\mathbf{1 7 9}$ with bromoform and $50 \%(\mathrm{w} / \mathrm{w})$ aqueous sodium hydroxide might promote carbene addition before significant deprotection occurs. These conditions led to consumption of starting material in approximately 2 hours, but isolated yields of cyclopropanes 211 and 212 were poor, ranging from 5-10\%, presumably again due to loss of the acetate groups and subsequent retention of the resulting polar material in the aqueous phase upon workup.<smiles>CC(=O)OC[C@H]1OC=C[C@@H](OC(C)=O)[C@H]1OC(C)=O</smiles>

179

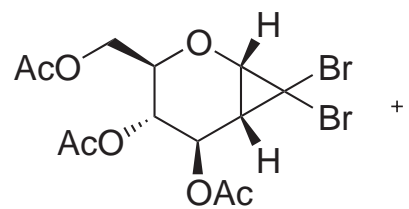

211<smiles>CC(=O)OC[C@H]1O[C@H]2[C@H]([C@H](OC(C)=O)[C@@H]1OC(C)=O)C2(Br)Br</smiles>

212

Reagents and Conditions: i) $\mathrm{CHBr}_{3}, \mathrm{TEBAC}, \mathrm{NaOH} / \mathrm{KF}_{(\mathrm{aq})}$, r.t.

Scheme 2.3 Cyclopropanation of tri- $O$-acetyl-D-glucal (179)

The use of chloroform to generate the corresponding gem-dichlorocyclopropanes encountered similar difficulties. Incorporation of a variety of cosolvents (dichloromethane, diethyl ether or toluene) was also explored, but failed to do more than aid stirring of the viscous reaction mixtures. These observations show that the acetate groups are too labile under Mąkosza conditions. Therefore, the more robust substrate tri-O-benzyl-D-glucal (188) was subsequently employed for cyclopropanations.

Nagarajan previously reported that the original Mąkosza cyclopropanation of tri- $O$ benzyl-D-glucal (188) was initially unsuccessful, presumably due to the use of $50 \%$ $(\mathrm{w} / \mathrm{w})$ sodium hydroxide in water. ${ }^{45}$ When this reaction was repeated under ultrasonication conditions (to promote efficient carbene addition), a complicated mixture 
of inseparable products was obtained. Therefore, the modified potassium fluoride method of Ma̧kosza was attempted. This reaction proceeded efficiently, using either ultrasonication or magnetic stirring. Thus, cyclopropane $\mathbf{9 7}$ was obtained in $66 \%$ yield, along with a minor amount (10\%) of isomer 190 (formed due to carbene addition from the top face), previously unreported by Nagarajan (entry 1, Table 2.1). Cyclopropane 97 was obtained as a white solid, which was stable at room temperature under air for several months. Cyclopropane 190 was isolated as an unstable yellow oil. It was stored under argon in the freezer, but even under these conditions it degraded into an unidentifiable mixture within a few months.

Table 2.1 Cyclopropanation of tri-O-benzyl-D-glucal $(\mathbf{1 8 8})^{a}$<smiles>BrC(Br)(Br)c1ccccc1</smiles>

188

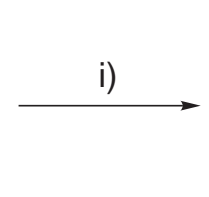

Consitions: i)

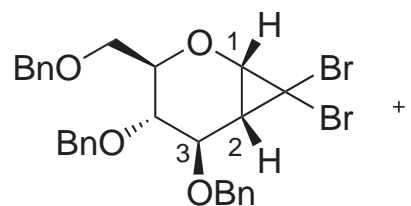

97<smiles>BrC[C@H]1O[C@H](Br)[C@@H](OCc2ccccc2)[C@@H]2[C@@H]1C2(Br)Br</smiles>

190

Reagents and Conditions: i) $\mathrm{CHBr}_{3}$, TEBAC, $\mathrm{NaOH} / \mathrm{KF}_{(\mathrm{aq})}$, r.t. or $20-30{ }^{\circ} \mathrm{C}$.

\begin{tabular}{|c|c|c|c|c|c|}
\hline Entry & Reaction method & Reaction time & Recovered 188 & Yield (97) & Yield (190) \\
\hline \hline 1 & magnetic stirring & $2 \mathrm{~d}$ & $1 \%$ & $66 \%$ & $10 \%$ \\
\hline 2 & magnetic stirring & $2 \mathrm{~d}$ & $16 \%$ & $63 \%$ & $7 \%$ \\
\hline 3 & ultrasonication & $10 \times 10 \mathrm{~min}$ & $42 \%$ & $35 \%$ & $5 \%$ \\
\hline 4 & ultrasonication & $8 \times 30 \mathrm{~min}$ & $30 \%$ & $48 \%$ & $6 \%$ \\
\hline 5 & ultrasonication & $12 \times 30 \mathrm{~min}$ & $28 \%$ & $43 \%$ & $7 \%$ \\
\hline 6 & ultrasonication & $12 \times 30 \mathrm{~min}$ & $30 \%$ & $48 \%$ & $8 \%$ \\
\hline
\end{tabular}

${ }^{a}$ all yields are of isolated products

The major isomer $\mathbf{9 7}$ was easily identified by NMR spectroscopy, with a characteristic doublet of doublets at $1.87 \mathrm{ppm}$ in the proton spectrum, corresponding to the H-2 methine at the ring junction. This proton coupled to $\mathrm{H}-1(J=7.8 \mathrm{~Hz})$, which was significantly further downfield at $3.94 \mathrm{ppm}$, due to the attached pyran ring oxygen. A coupling constant of $4.9 \mathrm{~Hz}$ was observed between H-2 and H-3. All characterisation data agreed very well with that reported by Nagarajan.

The minor isomer $\mathbf{1 9 0}$ had a similar proton NMR spectrum to that of $\mathbf{9 7}$. The H-2 methine appeared as a triplet $(J=8.1 \mathrm{~Hz})$ at $2.30 \mathrm{ppm}$, due to coupling with $\mathrm{H}-1$ (at $4.07 \mathrm{ppm}$ ), and H-3. This indicated all three protons were on the $\alpha$-face, therefore this product was the $\beta$-cyclopropyl product. Conversely, major isomer 97 was determined to be the $\alpha$-cyclopropyl product, due to the observed coupling constant $(4.9 \mathrm{~Hz})$ between $\mathrm{H}-2$ and $\mathrm{H}-3$.

The use of ultrasonication was explored to optimise this reaction (Table 2.1, entries 3-6). Extended periods of sonication caused the sonication bath to heat up, which could have allowed cyclopropane ring-opening reactions to occur, and reduce the yield of cyclopropanes. Therefore, the temperature was maintained between $20-$ 
$30{ }^{\circ} \mathrm{C}$ through replacement of the water in the sonication bath at regular intervals. Sonication led to improved conversion, as cyclopropanes were generated in good yield after several hours sonication. However, as starting material was usually recovered upon purification, reversion to the conventionally stirred reaction described by Nagarajan was deemed prudent, requiring a less complicated experimental procedure.

This cyclopropanation was now a very effective means of providing major cyclopropane 97, though it was occasionally quite temperamental (compare entries 1 and 2, Table 2.1). Typically once all reagents were added, a clear biphasic mixture was observed, which steadily turned dark-brown throughout the course of the reaction. However, several times when this reaction was performed, the mixture would only turn a pale-yellow colour, which usually indicated a lack of any significant reactivity. Presumably, this variable nature between successive reactions is responsible for several discrepancies in yield as shown in Table 2.1.

\subsection{Base-Mediated Methanolysis of Cyclopropanes 97 and 190}

Initial attempts to form oxepines began with repetition of Nagarajan's methodology, in which cyclopropane $\mathbf{9 7}$ was dissolved in methanol, treated with an excess of potassium carbonate, then refluxed. ${ }^{45}$ This provided a $1: 1.1(\beta: \alpha)$ mixture of anomeric sugars, with yield, anomeric ratio and analytical data all in excellent agreement with Nagarajan's report. The proton NMR spectra of the two separable anomers showed acetal resonances at 5.19 and $5.05 \mathrm{ppm}$, in addition to alkene peaks at 6.81 and $6.78 \mathrm{ppm}$ (Figure 2.1). However, the lack of significant coupling between the olefinic proton (presumed to be H-3) and the allylic proton (H-4) warranted closer analysis. Upon performing nOe experiments, an unexpected correlation between the acetal and olefinic protons was observed, in both directions, with both anomers. Subsequent investigation of the full series of both 1D and 2D NMR spectra confirmed that this reaction actually provided the 2- $C$-branched pyranose sugars $\mathbf{2 1 3}$ and $\mathbf{2 1 4}$, and not the oxepine structures previously assigned by Nagarajan ${ }^{45}$ (Scheme 2.4).

The anomeric configurations were individually assigned based on nOe correlations across the pyran ring oxygen (Figure 2.2). The less polar (minor) anomer was subjected to irradiation of the anomeric proton, which provided an enhancement of $\mathrm{H}-5$, indicative of the $\beta$-anomer, 213. The more polar (major) anomer was identified when, on irradiation of the anomeric proton, an enhancement of the H-6 

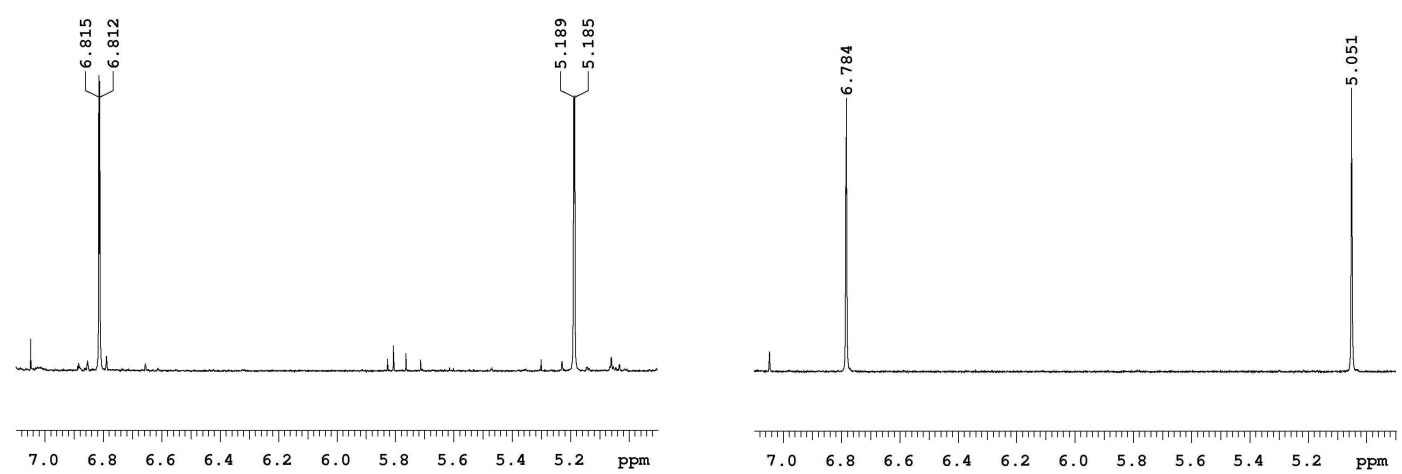

Figure $2.1{ }^{1} \mathrm{H}-\mathrm{NMR}$ spectra of 213 and 214

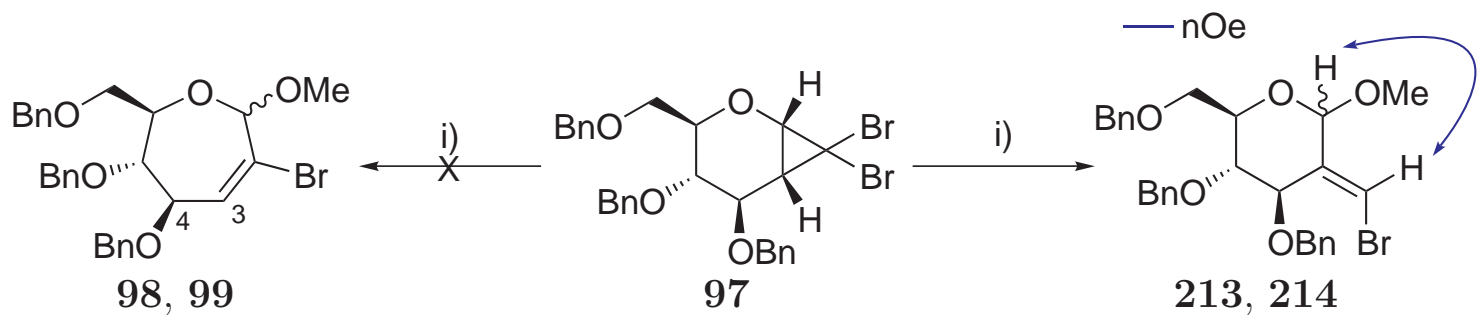

Reagents and Conditions: i) $\mathrm{K}_{2} \mathrm{CO}_{3}$, $\mathrm{MeOH}$, reflux, 8 h, $68 \%$.

Scheme 2.4 Base-mediated ring opening of cyclopropane $\mathbf{9 7}$

protons was observed, indicative of the $\alpha$-anomer, 214. In the proton NMR spectra (Figure 2.1), the olefinic proton of $\mathbf{2 1 3}\left(\delta_{\mathrm{H}} 6.81 \mathrm{ppm}\right)$ appeared as a narrow doublet $(J=1.7 \mathrm{~Hz}$ ), which was confirmed to be due to an allylic coupling with $\mathrm{H}-1$ upon examination of the COSY spectrum. The olefinic proton of $214\left(\delta_{\mathrm{H}} 6.78 \mathrm{ppm}\right)$ appeared as a singlet. The carbon NMR spectrum indicated the two carbon shifts of each product were quite different, with olefinic signals occurring at 137.5 (C-2) and 112.6 (C-7) ppm for 213, and 137.0 (C-2) and 114.7 (C-7) ppm for 214.
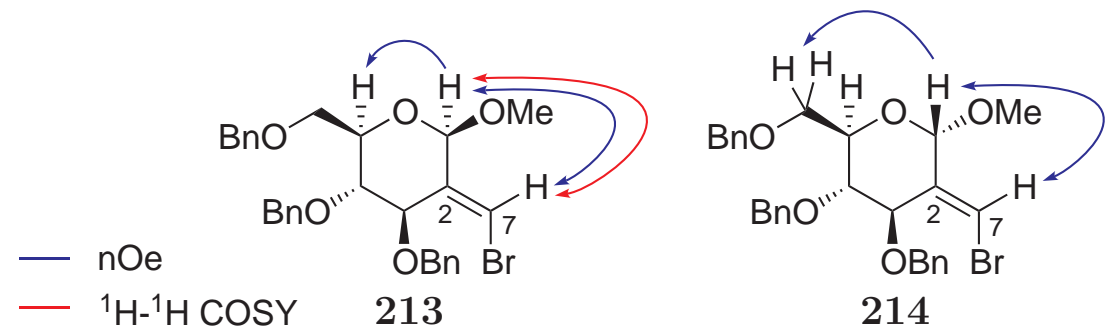

Figure 2.2 Selected NMR correlations of 213 and 214

Product 214 was then derivatised, which provided more conclusive proof of the unusual 2-C-branched structure. A portion of anomer 214 was subjected to lithiumhalogen exchange, through treatment with $n$-butyllithium. Upon generation of the corresponding lithiated alkene, addition of water led to the exocyclic methylene derivative 215 (Scheme 2.5). This structure was easily confirmed by proton NMR, with three singlets observed near $5 \mathrm{ppm}$, attributed to the acetal proton (5.06 ppm), and the two protons of the exocyclic methylene group (5.16, 5.30 ppm). Further- 
more, sugar $\mathbf{2 1 5}$ has been reported previously, ${ }^{99}$ obtained through a VilsmeierHaack reaction of tri- $O$-benzyl-D-glucal (188) and, after several functional group transformations, Ferrier rearrangement. Gratifyingly, the reported NMR data was in excellent agreement with that of product $\mathbf{2 1 5}$ obtained from the above lithiumhalogen exchange.<smiles>CO[C@H]1O[C@H](COc2ccccc2)[C@@H](OCc2ccccc2)[C@H](OBr)/C1=C\Br</smiles>

214

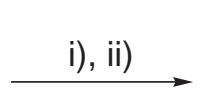<smiles>C=C1[C@H](OC)O[C@H](COc2ccccc2)[C@H](O)[C@@H]1OCc1ccccc1</smiles>

215

Reagents and Conditions: i) $n$-BuLi, THF, $-78{ }^{\circ} \mathrm{C}, 1 \mathrm{~h}$; ii) $\mathrm{H}_{2} \mathrm{O},-78{ }^{\circ} \mathrm{C}$ to r.t., 30 min, $29 \%$ over 2 steps.

Scheme 2.5 Lithium-halogen exchange of bromoalkene 214

2-C-Methylene sugars have received some attention in the literature, with many reports involving the synthesis of carbohydrates similar to $\mathbf{2 1 5}$, albeit derived from non-cyclopropyl precursors. ${ }^{100-102}$ 2- $C$-Halomethylene sugar derivatives have previously been obtained using Wittig chemistry. ${ }^{103}$ The halomethylene branch has also been observed in nature, as seen within malyngamides such as 216 (Figure 2.3). ${ }^{104}$ Even more exciting is the presence of these moieties within 2- $C$-substituted nucleoside derivatives. DMDC (217) and tezacitabine (218) both inhibit the ribonucleotide reductase enzyme, and have been evaluated in Phase I clinical trials. ${ }^{105}$<smiles>C=C(CC(=CCl)CN([14CH3])C(=O)CCC=CCC(=O)OC)OC</smiles>

216

Malyngamide $\mathrm{O}$<smiles></smiles>

217

DMDC<smiles>Nc1ccn(C2OC(CO)C(O)C2=CF)c(=O)n1</smiles>

218

Figure 2.3 Selected methylene and halomethylene compounds ${ }^{104,105}$

With the structures of pyranoside sugars 213 and 214 correctly identified, optimisation of this reaction was required prior to use with alternative substrates and nucleophiles. It was hypothesised that the use of sodium methoxide might lead to more efficient reactivity, due to the greater concentration of the methoxide anion, when compared to methanolic potassium carbonate. Therefore, cyclopropane $\mathbf{9 7}$ was initially reacted with an excess of commercially available sodium methoxide and the non-nucleophilic solvent THF. Unfortunately, only a fraction of starting material was consumed after reaction overnight, even though reflux conditions were used, providing a very similar temperature to that of refluxing methanol (methanol b.p. $64.7^{\circ} \mathrm{C}$, c.f. THF b.p. $65-67^{\circ} \mathrm{C}$ ). Preparation of fresh sodium methoxide was carried out by dissolving a known amount of sodium in methanol, then complete 
removal of methanol. However, this was even less successful, with no consumption of starting material observed at all after three hours reaction at THF reflux. It was intriguing to find that the addition of a small excess of methanol provided significantly greater reactivity, leading to isolation of the pyranoside sugars 213 and $\mathbf{2 1 4}$ in very good yield (up to 71\%). This reaction was performed using a freshly prepared solution of sodium methoxide (approximately 1.5 eq.) in a minimal volume of methanol. This solution was treated with the cosolvent THF, followed by the cyclopropane.

A comparison of various potassium carbonate and sodium methoxide-based reactions was instructive in optimising the reaction (Table 2.2). Nagarajan's method, ${ }^{45}$ with methanol as the solvent, provides isomers 213 and 214 in good yield (entry 1). Use of sodium methoxide enabled a faster reaction (entry 2); a relatively small excess of methanol is required, while still achieving very good yields (entry 3 ). While this reaction proceeds at room temperature, the reaction was more sluggish, and unreacted starting material $(27 \%)$ was recovered from the reaction after 1.5 hours (entry 4). Finally, the isomeric cyclopropane 190 was also subjected to these conditions (entry 5), but reacted much more slowly than 97. After 3 hours at reflux the reaction was terminated and a portion of starting material (16\%) was later isolated.

Table 2.2 Base-mediated ring-opening of cyclopropanes $\mathbf{9 7}$ and $\mathbf{1 9 0}^{a}$

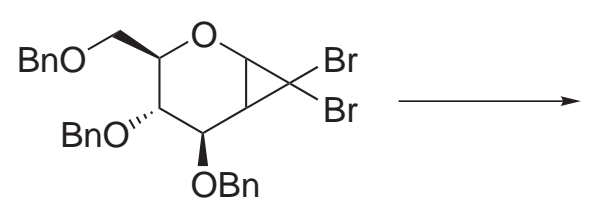

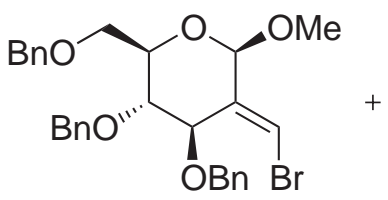

213

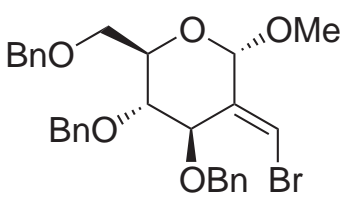

214

\begin{tabular}{|c|c|c|c|c|c|}
\hline Entry & Substrate & Base & Nucleophile, Solvent & Conditions & $\begin{array}{c}\text { Yield, Ratio } \\
(\mathbf{2 1 3 : 2 1 4})\end{array}$ \\
\hline \hline 1 & $\mathbf{9 7}$ & 6 eq. $\mathrm{K}_{2} \mathrm{CO}_{3}$ & 250 eq. MeOH & reflux, $8 \mathrm{~h}$ & $68 \%, 1.2: 1$ \\
\hline 2 & $\mathbf{9 7}$ & 6 eq. $\mathrm{NaOMe}$ & 34 eq. $\mathrm{MeOH}, \mathrm{THF}$ & reflux, $3 \mathrm{~h}$ & $64 \%, 1.5: 1$ \\
\hline 3 & $\mathbf{9 7}$ & 1.5 eq. $\mathrm{NaOMe}$ & 7.5 eq. $\mathrm{MeOH}, \mathrm{THF}$ & reflux, $1.25 \mathrm{~h}$ & $71 \%, 1: 2.2$ \\
\hline 4 & $\mathbf{9 7}$ & 1.0 eq. $\mathrm{NaOMe}$ & 14 eq. $\mathrm{MeOH}, \mathrm{THF}$ & r.t., $1.5 \mathrm{~h}$ & $60 \%, 1: 1$ \\
\hline 5 & $\mathbf{1 9 0}$ & 1.5 eq. $\mathrm{NaOMe}$ & 12 eq. $\mathrm{MeOH}, \mathrm{THF}$ & reflux, $3 \mathrm{~h}$ & $48 \%, 1: 2.3$ \\
\hline
\end{tabular}

${ }^{a}$ all yields and ratios are of isolated products

Discrepancies exist between different reactions, possibly due to the varying quality of the solvents, which could have been slightly wet. THF was distilled from the sodium benzophenone ketyl radical before use, whereas methanol was distilled over sodium, but then stored until required. It is likely the methanol could have become slightly wet before certain reactions were performed. For a reaction involving 1.5 equivalents of base (concentration of $\left.0.15 \mathrm{molL}^{-1}\right)$, only $0.3 \%(\mathrm{v} / \mathrm{v})$ of water is required to convert the base into sodium hydroxide. Therefore, it is likely that the differences in reaction rate, and also in anomeric ratios, could be due to varying amounts of sodium methoxide and sodium hydroxide in the reaction. 
The comparative reactivity of cyclopropanes $\mathbf{9 7}$ and $\mathbf{1 9 0}$ appeared quite different, therefore it was deemed necessary to perform a comparative reaction, that could be monitored by NMR experiment to prove the cyclopropanes were indeed reacting at different rates. A ca. 5:4 ratio mixture of cyclopropanes $\mathbf{9 7}$ and $\mathbf{1 9 0}$ was dissolved in THF- $d_{8}$, then treated with a solution of sodium methoxide in methanol. Proton NMR spectra were obtained at various times, with integrations of the relevant peaks ( $\mathrm{H}-2$ of 97 and 190, $\mathrm{H}-1$ and $\mathrm{H}-7$ of 213 and 214) used to determine approximate amounts of the components of the reaction mixture. It became evident that cyclopropane $\mathbf{9 7}$ is very much more reactive than isomeric cyclopropane $\mathbf{1 9 0}$ (Table 2.3). Unfortunately, an excessive amount of starting material was employed, which consequently provided poorly resolved NMR spectra. Therefore, the ratios of reaction components are not very accurate, except for the percentages obtained after isolation (entry $120 \mathrm{~min}$ ).

Table 2.3 Comparison of the reactivity between cyclopropanes $\mathbf{9 7}$ and $\mathbf{1 9 0}^{a b}$

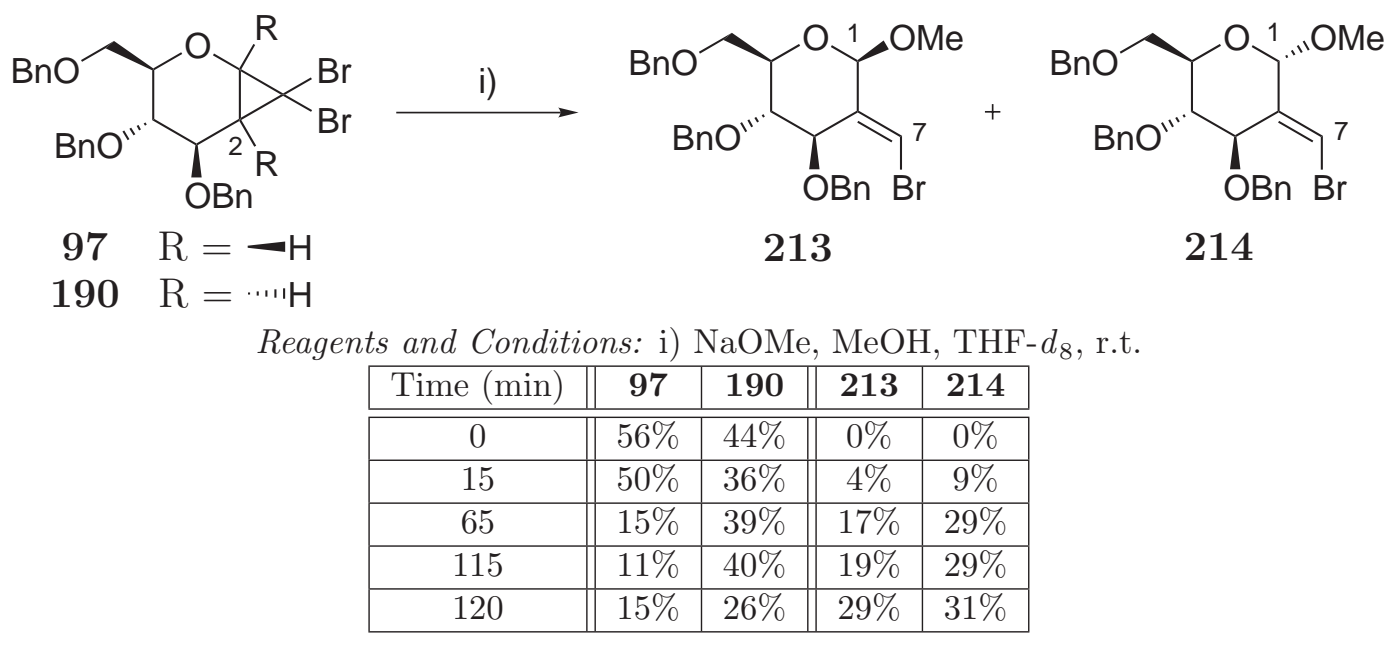

${ }^{a}$ all values were determined through ${ }^{1} \mathrm{H}-\mathrm{NMR}$ integrations

${ }^{b}$ approximate error $5-10 \%$ in all measurements

\subsection{Mechanistic Studies}

The base-mediated ring-opening reactions of cyclopropanes $\mathbf{9 7}$ and $\mathbf{1 9 0}$ provide 2- $C$-branched products, rather than the ring-expanded structures typically obtained through silver-mediated reactions. Therefore, further investigation was warranted, in the hope of elucidating mechanistic information. A deuterium-labelling study was performed, in which methanol- $d_{4}$ was reacted with sodium, and cyclopropane 97 subsequently added to the resulting solution. The proton NMR spectra of the separated product anomers showed none of the peaks corresponding to the methoxy and olefinic protons in products 213 and 214. The carbon NMR spectra failed to show peaks corresponding to the methoxy carbons or the olefinic carbons, which 
might be expected due to the effect of deuterium coupling: one would expect to observe the deuterated alkenyl carbon as a triplet, and the deuterated methyl group as a complex multiplet, with both peaks of very low intensity. However, these peaks were not visible in the spectrum obtained. With the remaining peaks present in both carbon and proton spectra matching those of $\mathbf{2 1 3}$ and 214, the products were identified as tetradeutero products 219 and $\mathbf{2 2 0}$ (Scheme 2.6). Their molecular weight was corroborated by high-resolution mass spectrometric analysis of anomer 220. This significant result indicates that the H-2 proton of cyclopropane $\mathbf{9 7}$ is lost during the reaction, and that a proton (or deuteron) from methanol is incorporated into the 2-C-bromomethylene substituent. It appears that the requirement of an introduced proton (or deuteron) may only be satisfied in the presence of protic species. This observation suggests that the reason the ring-opening reaction failed to proceed in the presence of dry sodium methoxide was due to the absence of methanol (or other protic species), required to provide the necessary proton.

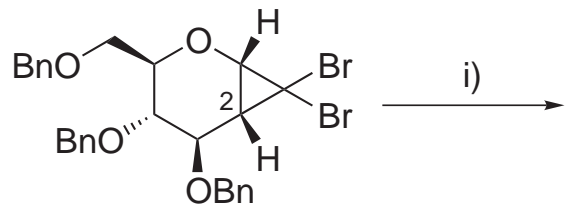

97

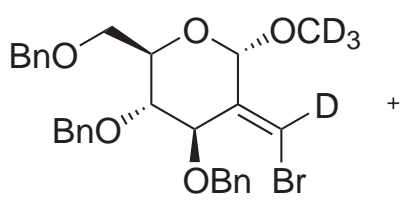

219

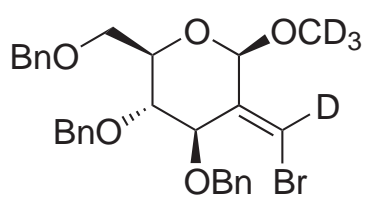

220

Reagents and Conditions: i) $\mathrm{NaOCD}_{3}, \mathrm{CD}_{3} \mathrm{OD}$, reflux, $2 \mathrm{~h}, 49 \%$.

Scheme 2.6 Synthesis of tetradeutero pyranoside sugars 219 and 220

The ring cleavage of $\mathbf{9 7}$ in this reaction could arise through several different mechanisms, as shown in Scheme 2.7. The different routes would be initiated by two possible mechanistic pathways: ring-opening of the cyclopropane to form zwitterion 221 (red and black routes), or deprotonation to form the cyclopropyl anion 222 (blue and green routes).

Zwitterion 221 forms through participation of the pyran ring oxygen in cleavage of the 1,7-exocyclic bond, as shown in the left-hand path of Scheme 2.7 (red path). This species could react further via two paths, one (shown in black) involving methanol addition to the zwitterion to provide 229 and 230 , which could undergo elimination of hydrogen bromide, forming 2- $C$-branched sugars 213 and 214. Alternatively, zwitterion $\mathbf{2 2 1}$ could be protonated at C-7 to form oxonium ion $\mathbf{2 2 3}$, then deprotonation at C-2 would lead to glycal 226. Loss of a bromide ion from $\mathbf{2 2 6}$ could be driven by participation of the pyran oxygen, to arrive at glycosyl cation 231. Finally, attack by methanol would provide 213 and 214 .

The middle path (shown in dark blue) would be dependant on the presence of base, to provide anion 222. Subsequent rearrangement to anionic glycal 224, then protonation would afford glycal 226, whereupon the previously discussed route applies. 


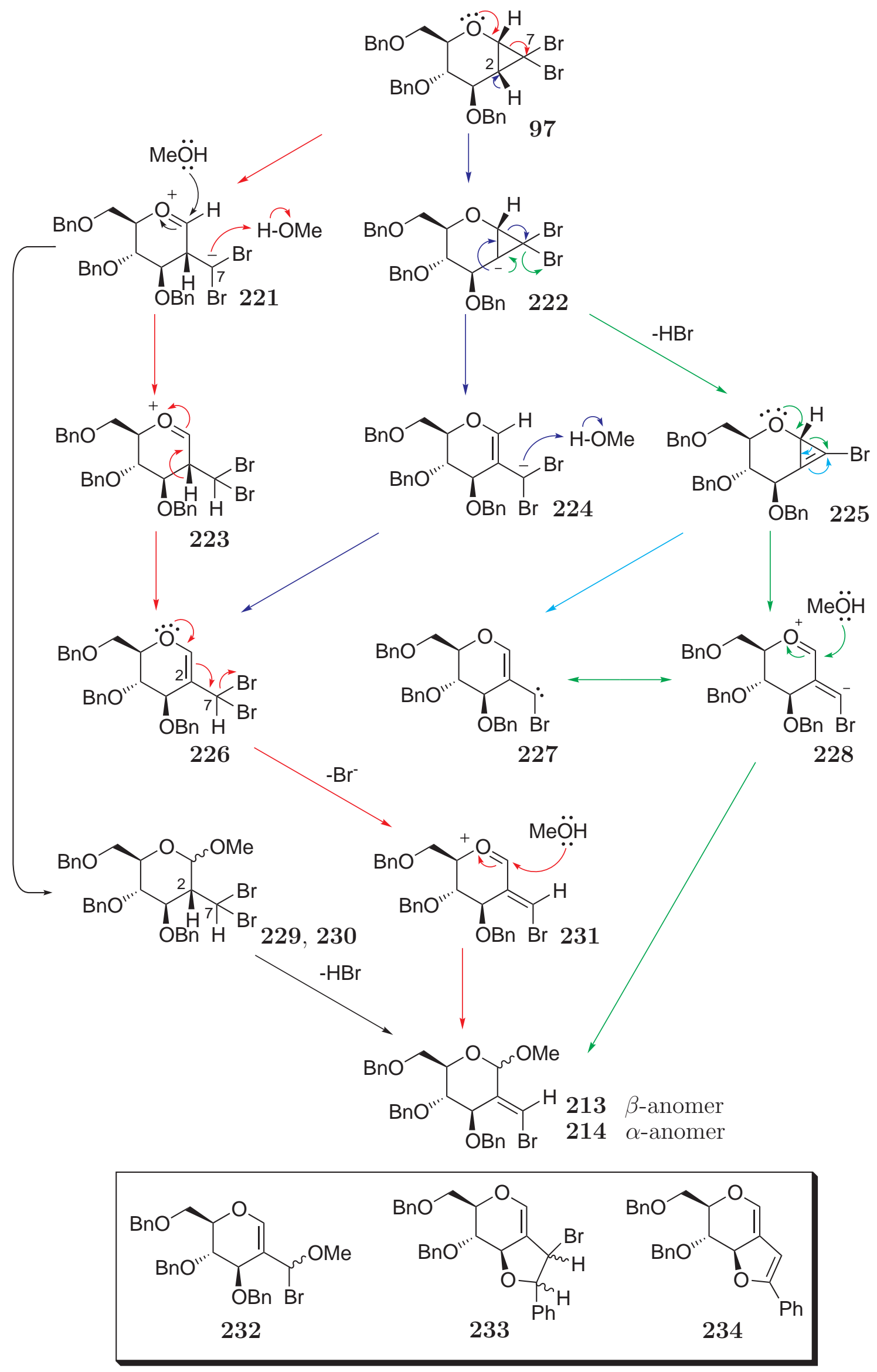

Scheme 2.7 Mechanism of base-mediated ring opening of cyclopropane 97 
Finally, the right-hand path (shown in green), involving cyclopropene 225, could also lead to the observed exocyclic bromomethylene sugars. The cyclopropene could be formed by elimination of $\mathrm{HBr}$ across the 2,7-bond (probably via anion 222), and then could undergo rearrangement to carbene $\mathbf{2 2 7}$ (path shown in light blue) or the alternative resonance form, zwitterion 228. Addition of methanol to zwitterion $\mathbf{2 2 8}$ could lead to 213 and $\mathbf{2 1 4}$.

Many of the possible mechanisms shown in Scheme 2.7 may be tentatively dismissed, given results already discussed. While all paths do explain the observed deuterium incorporation, the paths shown in black, red and dark blue involve neutral species such as 226, 229 and 230, with a dibromomethyl moiety attached to C-2. This moiety could ultimately be transformed into the key bromomethylene substituent. However, both product anomers (213 and 214) were invariably isolated as a single geometric isomer, with the double bond always in the $(E)$-configuration, as confirmed by nOe studies. None of the $(Z)$-isomer was observed in the proton NMR spectra of the crude reaction mixtures. This observation casts doubt upon these paths, as compounds 226, 229 and 230 would both seem sufficiently long-lived for free rotation of the 2,7-bond, which could lead to a mixture of $(E) /(Z)$-isomers. However, it is possible that the red route might be involved, and the formation of (Z)-isomer from 226, 229 and $\mathbf{2 3 0}$ is simply a much less favoured process, when compared to the formation of the observed $(E)$-isomer (213 and $\mathbf{2 1 4})$.

Evidence for participation of the cyclopropene (225) is more compelling. Cyclopropane rearrangements to halomethylene-branched products such as this are precedented, ${ }^{92,106,107}$ of which some are believed to form via cyclopropenes such as $\mathbf{2 2 5}{ }^{92}$ However, if the cyclopropene rearranges to carbene 227, insertion into the hydroxyl bond of methanol is likely, providing bromomethoxymethyl species 232. Alternatively, carbene insertion into one of the neighbouring benzylic $\mathrm{CH}$ bonds, would provide bicyclic pyranofuran 233, or the corresponding eliminated product $234{ }^{108}$ None of these products was observed, therefore it is unlikely carbene 227 is involved. Conversely, zwitterion $\mathbf{2 2 8}$ is likely the predominant resonance form. Due to the presence of the pyran oxygen, reaction progression via zwitterion 228 is quite plausible, which would lead to subsequent attack by methanol, providing observed 2- $C$-branched sugars 213 and 214. In addition, this mechanism would retain stereochemical integrity of the bromomethylene moiety throughout, which all other mechanisms in Scheme 2.7 fail to do.

Experiments were then performed to gain further insight into the mechanism of this reaction. In an attempt to observe the intermediacy of cyclopropene 225, cyclopropane 97 was dissolved in THF- $d_{8}$, then added to sodium methoxide in methanol. 
Proton NMR spectra were obtained at regular intervals, however only starting material and products 213 and $\mathbf{2 1 4}$ were observed, indicating that if cyclopropene $\mathbf{2 2 5}$ is present, it is an extremely short-lived species. These proton NMR spectra further demonstrated how clean this transformation is, as no byproducts were observed during the course of this reaction. Attempts to trap the cyclopropene as a Diels-Alder adduct were undertaken, by replacing the THF solvent with furan. ${ }^{92}$ Consumption of starting material was very slow when performed at room temperature, with only a trace amount of material converted into the previously observed exocyclic bromomethylene products 213 and 214. Cyclopropanes may undergo cycloadditions with a dipolarophile, presumably through zwitterions such as 228. ${ }^{109}$ To investigate this, cyclopropane $\mathbf{9 7}$ was reacted with sodium hydride in THF in the presence of methyl acrylate, which was expected to provide a cyclopentyl derivative. Unfortunately, minimal consumption of starting material was observed. Changing the solvent to DMF led to a faster reaction, generating a complex mixture, from which none of the expected products could be identified.

As mentioned earlier, this reaction proceeded well in the presence of sodium methoxide and methanol, but failed if no methanol was present. This was also observed when cyclopropene generation was attempted using sodium hydride, with which cyclopropane 97 was completely unreactive. Use of $n$-butyllithium led to debromination of 97, rather than the removal of H-2 (Scheme 2.8). Both starting material $97(26 \%)$, and bromocyclopropane 235 (26\%) were isolated from the reaction. The configuration of $\mathbf{2 3 5}$ at C-7 was determined by nOe experimentation in which irradiation of the introduced proton ( $\mathrm{H}-7,3.01 \mathrm{ppm})$, led to enhancement of a signal attributed to either $\mathrm{H}-3$ or H-5, which indicates $\mathrm{H}-7$ is in the endo-position.

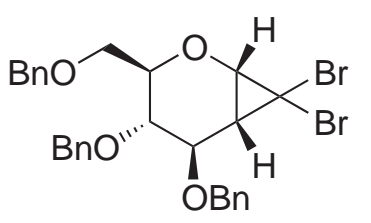

97
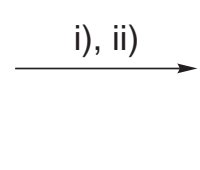

Reagents and Conditions: i) n-BuLi, THF, r.t., $10 \mathrm{~min}$; ii) $\mathrm{H}_{2} \mathrm{O}$, r.t., 5 min, $26 \%$ over 2 steps.

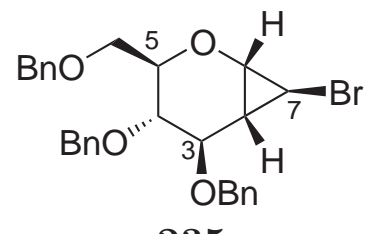

235

Scheme 2.8 Reaction of cyclopropane $\mathbf{9 7}$ with $n$-butyllithium

Unfortunately, due to the lack of success in the reactions described above, the experimental results do not provide comprehensive evidence for one mechanism over the other. However, using the limited information obtained, it is believed that the most likely mechanism for this base-mediated ring-opening reaction is via cyclopropene 225 and zwitterion 228 (green route), as shown in Scheme 2.7. 


\subsection{Base-Mediated Ring Opening with Alternative Nucleophiles}

As this reaction was able to be performed with only a moderate excess of methanol, it demonstrates that one might employ a variety of nucleophiles. The use of nucleophiles unsuitable as solvents, of significant expense, or those that would complement a larger synthetic goal, could be used to generate a range of 2 - $C$-branched carbohydrates.

With an improved method for accessing pyranoside sugars 213 and 214, other alcohols were used as nucleophiles, to determine the versatility of the above methodology. In analogy with the methoxide-based reactions, approximately 1.5 equivalents sodium alkoxide and 5 equivalents alcohol were used in these reactions. Cyclopropane 97 reacts with allyl alcohol and benzyl alcohol under these conditions (Table 2.4). For comparison, the optimised ring-opening reaction with sodium methoxide in methanol is included (entry 1). The reaction with allyl alcohol and sodium allyloxide provided glycosides 236 and 237 (entries 2 and 3); benzyl alcohol and sodium benzyloxide provided glycosides 238 and 239 (entry 4). Good yields were obtained in all cases, though longer reaction times were required, as compared to the methanol case. This methodology allows for the construction of a variety of glycosides of these unusual 2- $C$-branched sugars.

Table 2.4 Ring opening of cyclopropane $\mathbf{9 7}$ with various nucleophiles ${ }^{a b}$

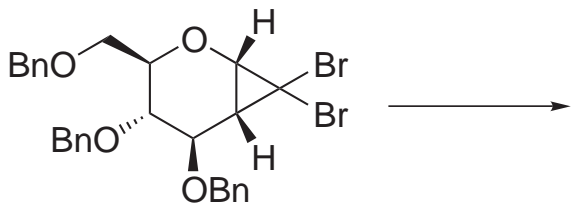

97<smiles>CCCOC1O[C@H](COc2ccccc2)[C@@H](OCc2ccccc2)[C@H](Br)/C1=C/Br</smiles>

$213 \mathrm{R}=\mathrm{Me}$

$236 \mathrm{R}=$ Allyl

$238 \mathrm{R}=\mathrm{Bn}$<smiles></smiles>

$214 \mathrm{R}=\mathrm{Me}$

$237 \mathrm{R}=$ Allyl

$239 \mathrm{R}=\mathrm{Bn}$

\begin{tabular}{|c|c|c|c|c|c|}
\hline Entry & Base & Nucleophile & Time & Yield, Products & Ratio $(\alpha: \beta)$ \\
\hline \hline 1 & 1.5 eq. $\mathrm{NaOMe}$ & 7.5 eq. $\mathrm{MeOH}$ & $1.25 \mathrm{~h}$ & $71 \%, \mathbf{2 1 3}, \mathbf{2 1 4}$ & $1: 2.2$ \\
\hline 2 & 6.6 eq. AllylONa & 5.1 eq. AllylOH & $5 \mathrm{~h}$ & $55 \%, \mathbf{2 3 6}, \mathbf{2 3 7}$ & $1: 1.9$ \\
\hline 3 & 1.7 eq. AllylONa & 5.9 eq. AllylOH & $5 \mathrm{~h}$ & $71 \%, \mathbf{2 3 6}, \mathbf{2 3 7}$ & $1: 2.2$ \\
\hline 4 & 3.2 eq. $\mathrm{NaOBn}$ & 7.1 eq. $\mathrm{BnOH}$ & $4 \mathrm{~h}$ & $63 \%, \mathbf{2 3 8}, \mathbf{2 3 9}$ & $1.1: 1$ \\
\hline
\end{tabular}

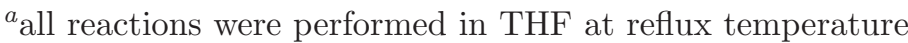

${ }^{b}$ all yields and ratios are of isolated products

The use of carbon- and nitrogen-based nucleophiles was also investigated. Treatment of tert-butyl acetylene with sodium hydride, followed by addition of cyclopropane 97 led to consumption of the cyclopropane after 20 hours in refluxing THF. The crude NMR spectrum of this mixture indicated the presence of several components, with numerous peaks observed from 5.2 to $5.8 \mathrm{ppm}$, and 6.7 to $6.9 \mathrm{ppm}$. 
Unfortunately, these products were unable to be separated and identified. The use of Meldrum's acid (2,2-dimethyl-1,3-dioxane-4,6-dione) was also studied, however this reaction was extremely sluggish. Pre-treating Meldrum's acid with sodium hydride, followed by reaction with $\mathbf{9 7}$ in refluxing THF for three days provided a mixture composed of predominantly starting material, with a minor amount of unisolated material. Presumably this lack of smooth reactivity could be due to the use of aprotic nucleophiles, which are not able to provide the required proton for the bromomethyl moiety. Alternatively, deprotonated Meldrum's acid is resonance stabilised, therefore it may not be an appropriate base or nucleophile. Diethylamine was used in an attempt to extend this methodology to nitrogen nucleophiles. This provided fairly good reactivity, with almost full conversion observed after 6 hours, providing glycosylamine derivatives 240 and 241 in approximately $45 \%$ yield (Scheme 2.9). Unfortunately these products were not very stable, and converted into mixtures of multiple products before full NMR characterisation was performed. These mixtures included aldehydic peaks in the proton NMR spectra, possibly indicating the glycosyl bonds of these sugars are quite labile.

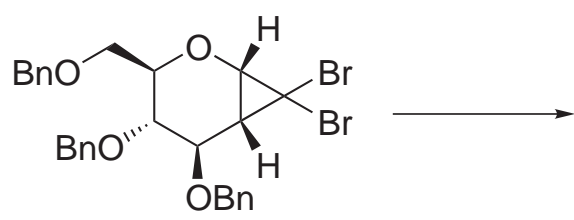

97

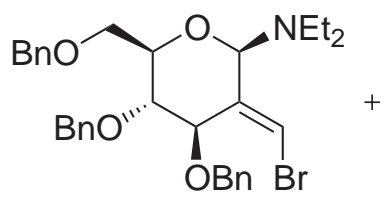

240

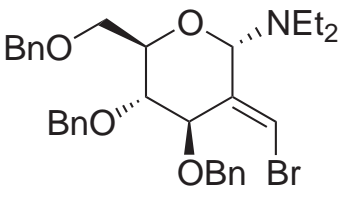

241

Reagents and Conditions: i) $\mathrm{NaNEt}_{2}, \mathrm{HNEt}_{2}$, THF, reflux, 6 h, $45 \%$.

Scheme 2.9 Synthesis of glycosylamines 240 and 241

\subsection{Palladium-Catalysed Coupling Reactions}

The exocyclic bromomethylene moiety of the 2- $C$-branched sugars represents an excellent substrate for palladium-catalysed cross-coupling reactions. Suzuki and Stille coupling reactions involving substrate $\mathbf{2 1 4}$ were both investigated. A Suzuki reaction was performed with methylboronic acid, but the reaction was quite slow, even though refluxing 1,4-dioxane was employed (Scheme 2.10). A significant portion of starting material was recovered (35\%), in addition to the previously formed exocyclic methylene $\mathbf{2 1 5}$. This presumably indicates palladium undergoes oxidative addition with substrate $\mathbf{2 1 4}$, however it appears transmetalation with the boronic acid has failed to occur.

A Stille reaction with substrate $\mathbf{2 1 4}$ was performed in the presence of allyltributylstannane and catalytic palladium-tetrakis(triphenylphosphine). Although the product was indistinguishable from the substrate by TLC, after two days the reaction 
<smiles>CO[C@H]1O[C@H](COc2ccccc2)[C@@H](OCc2ccccc2)[C@H](OBr)/C1=C\Br</smiles>

214

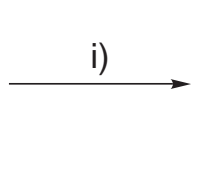

215<smiles>C=C1[C@H](OCc2ccccc2)[C@@H](Br)[C@@H](COBr)O[C@H]1OC</smiles>

Reagents and Conditions: i) $\mathrm{Pd}\left(\mathrm{PPh}_{3}\right)_{4}, \mathrm{MeB}(\mathrm{OH})_{2}, \mathrm{~K}_{2} \mathrm{CO}_{3}$, 1,4-dioxane, reflux, $2 \mathrm{~d}, 30 \%$.

Scheme 2.10 Attempted Suzuki coupling of 2- $C$-branched sugar 214

was terminated, and found to have undergone full conversion. Upon purification by column chromatography, coupled sugar $\mathbf{2 4 2}$ was isolated in 52\% yield (Scheme 2.11). This reaction demonstrates the value of the bromomethylene moiety and the potential for the rapid assembly of unnatural sugars such as 242. Further investigations into coupling reactions of these substrates are under investigation within the research group.

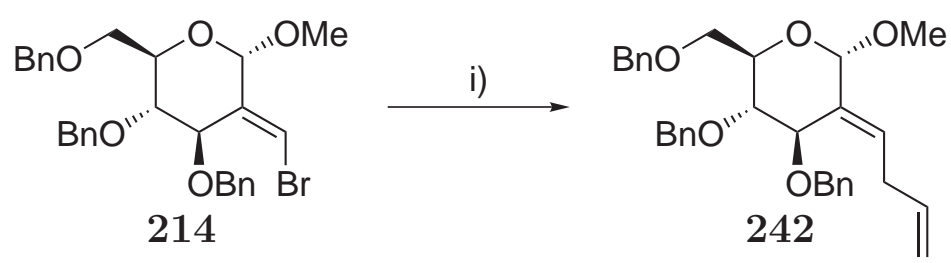

Reagents and Conditions: i) $\mathrm{Pd}\left(\mathrm{PPh}_{3}\right)_{4}, \mathrm{AllylSnBu}_{3}, \mathrm{DMF}, 100{ }^{\circ} \mathrm{C}, 2 \mathrm{~d}, 52 \%$.

Scheme 2.11 Stille coupling of 2- $C$-branched sugar 214

\subsection{Reactions of Galactose and Xylose Derivatives}

Investigations then moved on to determine if other sugars could also be used to form 2- $C$-branched sugars. Nagarajan investigated the base-mediated methanolysis of Dgalactose and D-xylose derived cyclopropanes 45 and 243 (Scheme 2.12). ${ }^{45}$ While the products obtained were reported to be oxepines, results discussed in section 2.3 suggest these products may actually be 2 - $C$-branched sugars.

Cyclopropanes derived from D-galactose (183) and D-xylose (184) were investigated, as these offer alternative stereochemical configurations, complementary to D-glucose. The glycals were prepared as described for D-glucal (185). Thus, peracetylation of 183 and 184, followed by treatment with hydrobromic acid led to $\alpha$-bromoglycosides 248 and 249 (Scheme 2.13). Reduction in the presence of zinc dust provided tri- $O$ acetyl-D-galactal $(\mathbf{1 8 0})$ and di- $O$-acetyl-D-xylal (181). The zinc reduction for the D-galactose series was performed at $0{ }^{\circ} \mathrm{C}$, then allowed to warm to room temperature overnight. This led to formation of tri- $O$-acetyl-D-galactal (180), which was used without purification. However, di-O-acetyl-D-xylal (181) was not stable to 
<smiles>Cc1ccccc1Br</smiles>

45<smiles>CC1CCCCC1Br</smiles>
243

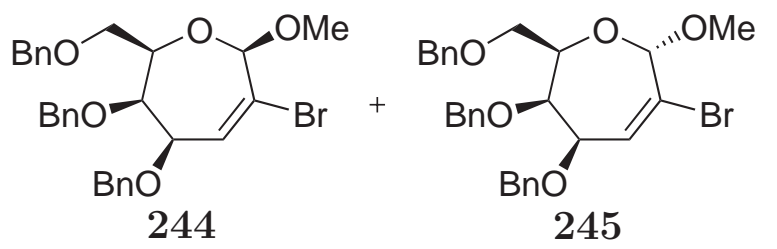

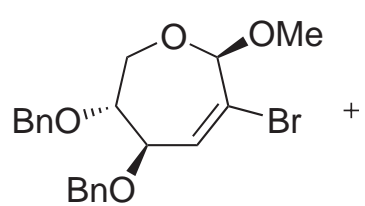

246

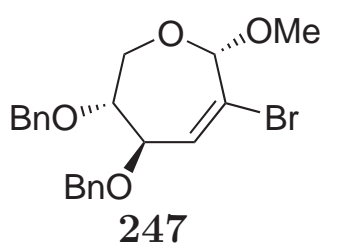

Reagents and Conditions: i) $\mathrm{K}_{2} \mathrm{CO}_{3}, \mathrm{MeOH}$, reflux.

Scheme 2.12 Nagarajan's reported synthesis of oxepines $\mathbf{2 4 4}, \mathbf{2 4 5}, \mathbf{2 4 6}$ and $\mathbf{2 4 7}{ }^{45}$

these zinc reduction conditions, which led to the isolation of $\mathbf{1 8 1}$ in very poor yield $(13 \%)$. Following literature precedence, ${ }^{110}$ the reaction was stirred for three hours at $0{ }^{\circ} \mathrm{C}$, then immediately worked up. The crude product required purification by column chromatography, providing $\mathbf{1 8 1}$ in $38 \%$ yield. Further optimisation was not investigated, though it has been recommended the reduction takes place below $0{ }^{\circ} \mathrm{C}$, or through the use of a $\mathrm{Zn} / \mathrm{Cu}$ couple, in order to maximise the yield of $\mathbf{1 8 1}$.<smiles>[R]C1O[C@H](O)[C@@H](O)[C@H](O)[C@]1([R7])[3H]</smiles>

$183 \mathrm{R}=\mathrm{CH}_{2} \mathrm{OH}, \mathrm{R}^{\prime}=\mathrm{OH}, \mathrm{R}^{\prime \prime}=\mathrm{H}$<smiles>CC#CC1CCCCC1[PH]</smiles>

248,249<smiles>[R]C1OC=C[C@@H](OC(C)=O)[C@]1(P)[P]C</smiles>

180,181
248, $180 \mathrm{R}=\mathrm{CH}_{2} \mathrm{OAc}, \mathrm{R}^{\prime}=\mathrm{OAc}, \mathrm{R}^{\prime \prime}=\mathrm{H}$ 249, $181 \mathrm{R}=\mathrm{H}, \mathrm{R}^{\prime}=\mathrm{H}, \mathrm{R}^{\prime \prime}=\mathrm{OAc}$ iv) or v)<smiles>[R]C1OC=C[C@@H](O)C1([PH])[PH]</smiles>

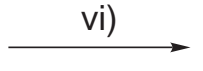

$186 \mathrm{R}=\mathrm{CH}_{2} \mathrm{OH}, \mathrm{R}^{\prime}=\mathrm{OH}, \mathrm{R}^{\prime \prime}=\mathrm{H}$ $187 \mathrm{R}=\mathrm{H}, \mathrm{R}^{\prime}=\mathrm{H}, \mathrm{R}^{\prime \prime}=\mathrm{OH}$
$43 \mathrm{R}=\mathrm{CH}_{2} \mathrm{OBn}, \mathrm{R}^{\prime}=\mathrm{OBn}, \mathrm{R}^{\prime \prime}=\mathrm{H}$ $189 \mathrm{R}=\mathrm{H}, \mathrm{R}^{\prime}=\mathrm{H}, \mathrm{R}^{\prime \prime}=\mathrm{OBn}$

Reagents and Conditions: i) $\mathrm{Ac}_{2} \mathrm{O}, \mathrm{HClO}_{4}, 40-50{ }^{\circ} \mathrm{C}, 30 \mathrm{~min}$; ii) $\mathrm{HBr}, \mathrm{AcOH}$, r.t., 90 min; iii) $\mathrm{Zn}, \mathrm{AcOH}_{(\mathrm{aq})}, 0{ }^{\circ} \mathrm{C}$ to r.t., overnight; iv) $\mathrm{NEt}_{3}, \mathrm{MeOH}_{(\mathrm{aq})}$, r.t., $90 \mathrm{~min}$; v) $\mathrm{NaOMe}, \mathrm{MeOH}$, r.t. $90 \mathrm{~min}$; vi) $\mathrm{BnBr}, \mathrm{NaH}, \mathrm{DMF}, 0{ }^{\circ} \mathrm{C}$ to r.t., overnight.

Scheme 2.13 Synthesis of galactose- and xylose-based glycals

The corresponding benzylated glycals were obtained by deprotecting the acetylated glycals, then reacting glycals 186 and $\mathbf{1 8 7}$ under benzylation conditions as described previously (section 2.2). Galactose-derived 180 was deprotected using triethylamine in aqueous methanol, then purified from a methanol/acetone/diethyl ether solvent mixture, to provide D-galactal (186) in $41 \%$ yield from D-galactose. Upon perbenzylation, tri- $O$-benzyl-D-galactal (43) was obtained in $62 \%$ yield (Scheme 2.13 ). For the xylal series, purified di- $O$-acetyl-D-xylal (181) was deprotected to provide 
D-xylal (187) using catalytic sodium methoxide in methanol, then benzylated prior to purification. Di-O-benzyl-D-xylal (189) was obtained in 66\% yield from $\mathbf{1 8 1 .}$

Using the modified Mạkosza cyclopropanation conditions discussed previously, cyclopropanation of $\mathbf{4 3}$ provided a single isomer, cyclopropane 45 (Scheme 2.14), in very good yield (71\%). The spectral data matched that reported previously. ${ }^{45}$ Due to the large degree of steric crowding upon the $\beta$-face of 43 , no $\beta$-face cyclopropanation was observed. Cyclopropanation of D-xylose-derived glycal 189 provided separable cyclopropanes 243 and 250 (76\% combined yield), in a 4.8:1 ( $\alpha: \beta)$ isolated ratio. While cyclopropanation was not as stereoselective for glycal 189 as that for tri- $O$-benzyl-D-glucal (188) or tri- $O$-benzyl-D-galactal (43), the isolated ratio indicates carbene addition to the $\alpha$-face is still favoured. This provides further evidence that the C-3 stereochemistry (and presumably, the bulk of the attached protecting group) is a very dominant factor in directing dibromocarbene addition to glycals.

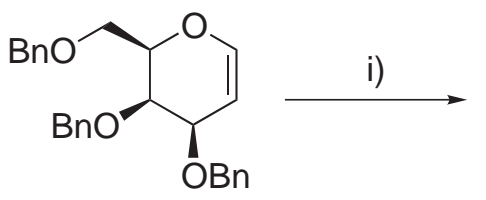

43<smiles>O[C@H]1[C@@H](Br)[C@@H](Br)O[C@H](CBr)[C@@H]1Br</smiles>

45<smiles>BrC(Br)c1ccccc1</smiles>

189

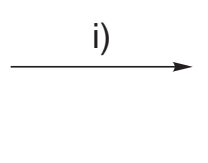

Reagents and Conditions: i)<smiles>BrC1OC[C@H](Br)[C@H](Br)C1Br</smiles>

243<smiles>BrC1[C@H]2OC[C@H](Br)[C@H](O[C@H](Br)c3ccccc3)[C@@H]12</smiles>

250

Scheme 2.14 Synthesis of galactose- and xylose-based cyclopropanes

With galactal and xylal-derived cyclopropanes 45, 243 and 250 in hand, basemediated cyclopropane ring opening was investigated. Cyclopropane $\mathbf{4 5}$ underwent ring opening in the presence of sodium methoxide and methanol to provide bromomethylene sugars 251 and 252 (1:3 ratio $\beta: \alpha$ ), in 65\% yield (Scheme 2.15). This reaction was complete after only 40 minutes in refluxing THF, however the isolated products were inseparable. Additionally, they were not as stable as the D-glucose analogues 213 and 214. This led to the NMR samples degrading significantly in chloroform- $d$ within hours; consequently, full characterisation was not possible. The NMR data set of this mixture of compounds was consistent with that obtained by Nagarajan, in the potassium carbonate mediated ring-opening reaction of 45. Through correlations of the NMR data of 251 and 252 with that of D-glucose-derived sugars 213 and 214, it is believed products 251 and 252 are also 2- $C$-bromomethylene derivatives (Scheme 2.15). 


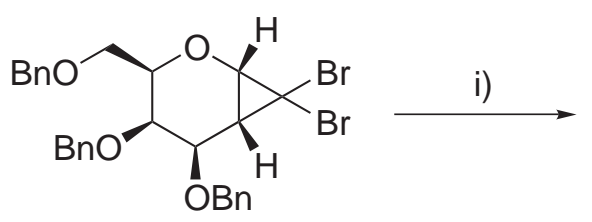

45

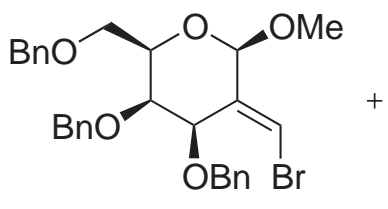

251

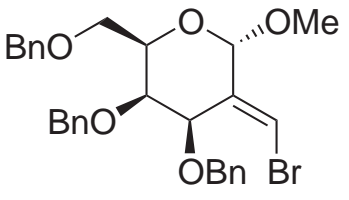

252

Reagents and Conditions: i) NaOMe, MeOH, THF, reflux, 40 min, 65\%.

Scheme 2.15 Ring opening of galactose-based cyclopropane 45

Ring opening of major D-xylal-derived cyclopropane 243 was also attempted. This reaction proceeded very efficiently, with full consumption of starting material observed after just 20 minutes at THF reflux. Upon purification by column chromatography, the corresponding bromomethylene sugars 253 and 254 (1:5 ratio $\beta: \alpha)$ were obtained in 67\% yield (Scheme 2.16). Unfortunately, the minor product was isolated with other impurities, and both isolated products degraded in the NMR tube overnight, providing complicated mixtures of aldehydic products. It may be noted that this reaction appears to provide both the fastest conversion, possibly due to the dryness of the methanol used. This reaction also provided the highest anomeric selectivity of all base-mediated reactions that have been discussed, which implies the formation of one anomer over the other is not likely influenced by the steric environment of the substrate. Comparison of the NMR data of these xylose-derived products with previously isolated 2- $C$-bromomethylene sugars, indicates products 253 and 254 are also branched pyranoside sugars (Scheme 2.16).<smiles>Br[C@H]1CO[C@H](Br)[C@H](Br)[C@H]1Br</smiles>

243

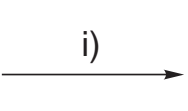<smiles>COC1OC[C@@H](Cc2ccccc2)[C@H](Br)[C@@H]1CBr</smiles>

253<smiles>CO[C@H]1COC[C@@H](OCc2ccccc2)[C@@H]1OCc1ccccc1</smiles>

254

Reagents and Conditions: i) NaOMe, $\mathrm{MeOH}$, THF, reflux, 20 min, $67 \%$.

Scheme 2.16 Ring opening of xylose-based cyclopropane $\mathbf{2 4 3}$

\subsection{Summary of Chapter 2}

Cyclopropanes derived from glucose, galactose and xylose glycals have been synthesised. The base-mediated ring-opening reaction of cyclopropane $\mathbf{9 7}$ has been thoroughly investigated, and a structure revision has been provided, indicating the products from this reaction are in fact 2- $C$-bromomethylene pyranoside sugars $\mathbf{2 1 3}$ and 214. Derivatisations of selected pyranoside sugars have provided valuable insights into the structure and mechanism of formation of these compounds, and also demonstrate the synthetic utility of these compounds. 


\section{Chapter 3}

\section{Silver-Mediated and Thermal Ring-Opening Reactions}

\subsection{Introduction}

Ring-expansion reactions of gem-dihalocyclopropanes are known to occur through the use of Lewis acids (such as halophilic silver(I) salts), or the application of heat (see subsection 1.1.2). ${ }^{9}$ Nagarajan trialled the use of several such reaction conditions for the ring expansion of cyclopropane 97, with the expectation of forming heptoseptanoside sugar derivatives (Scheme 3.1). ${ }^{45}$ All attempts using either silvermediated reaction at room temperature or thermal promotion were unsuccessful. Having determined that basic conditions produced 2- $C$-branched sugars 213 and $\mathbf{2 1 4}$, it was envisaged that careful analysis of silver-mediated reaction conditions, and/or the application of heat might provide access to oxepine structures.<smiles>BrC[C@H]1O[C@H](Br)[C@H](Br)[C@H](OBr)[C@H]1Br</smiles>

97<smiles>OCC[C@H]1OC(O)C(Br)=C[C@H](O)[C@@H]1Br</smiles>

255<smiles>O[C@H]1C=C(Br)[C@H](O)O[C@@H](COc2ccccc2)[C@@H]1Br</smiles>

256

Scheme 3.1 Ring expansion of cyclopropane $\mathbf{9 7}$

Silver-mediated cleavage of gem-dihalocyclopropanes can often provide good regiochemical control. Cleavage of the fused bond of cyclopropane $\mathbf{9 7}$ would lead to a seven-membered ring $\pi$-allyl cation intermediate, which would react with a nucle- 
ophile to introduce glycoside functionality (due to the directing effect of the pyran oxygen). Investigations into the synthesis of septanose sugars with the structures reported, but not synthesised by Nagarajan et al. were then undertaken. ${ }^{45}$

\subsection{Silver-Mediated Ring-Expansion Reactions in Acetic Acid}

Nagarajan observed that treatment of $\mathbf{9 7}$ with acetic acid and silver salts at room temperature did not lead to any reaction. ${ }^{45}$ This was confirmed after five days reaction at room temperature in the presence of silver acetate. The reaction was repeated with the addition of sodium acetate; again, no reaction was observed after four days at room temperature. The initial unsuccessful reactions at room temperature correlated with the stability of cyclopropane $\mathbf{9 7}$ when stored at room temperature. This indicates that the substrate requires harsher reaction conditions than are needed for related bicycles containing these strained three-membered rings, which has been observed in similar cases. ${ }^{111}$

Nagarajan reported that reaction of $\mathbf{9 7}$ in refluxing acetic acid or 1,4-dioxane led to decomposition of the cyclopropane. ${ }^{45}$ The reaction of cyclopropane $\mathbf{9 7}$ with silver acetate in acetic acid was trialled at various temperatures (Table 3.1), which gratifyingly provided oxepines 257 and 258 . Reaction at $70{ }^{\circ} \mathrm{C}$ provided a very modest yield (entry 2); increasing the temperature to $90{ }^{\circ} \mathrm{C}$ led to better conversion into oxepines (entry 3). Optimal reaction conditions were achieved at $100{ }^{\circ} \mathrm{C}$, providing oxepines $\mathbf{2 5 7}$ and $\mathbf{2 5 8}$ in $52 \%$ yield, after only 25 hours reaction (entry 4). However, these oxepines were almost entirely inseparable by column chromatography, with only a portion of the major oxepine (258) isolated as a single anomer. As previously reported by Nagarajan, ${ }^{45}$ reflux in acetic acid led to extensive decomposition, however by halting the reaction once all starting material had been consumed, a modest amount of oxepines $\mathbf{2 5 7}$ and $\mathbf{2 5 8}$ (15\%) was isolated (entry 5).

Acetyl septanoside products are exceedingly useful substrates, as cleavage of the acetate leads to the corresponding hemiacetals, which may be used in other areas, including oligosaccharide synthesis; subsequent applications are discussed in chapter 4 . The identity of these oxepines was determined through extensive NMR studies. In particular a ${ }^{1} \mathrm{H}-{ }^{1} \mathrm{H}$ COSY correlation was observed between the olefinic protons and an oxymethine, indicating connectivity, in contrast to the 2 - $C$-bromomethylene sugars discussed in chapter 2. 
Table 3.1 Acetic acid-mediated ring-expansions of cyclopropane $\mathbf{9 7}^{a}$

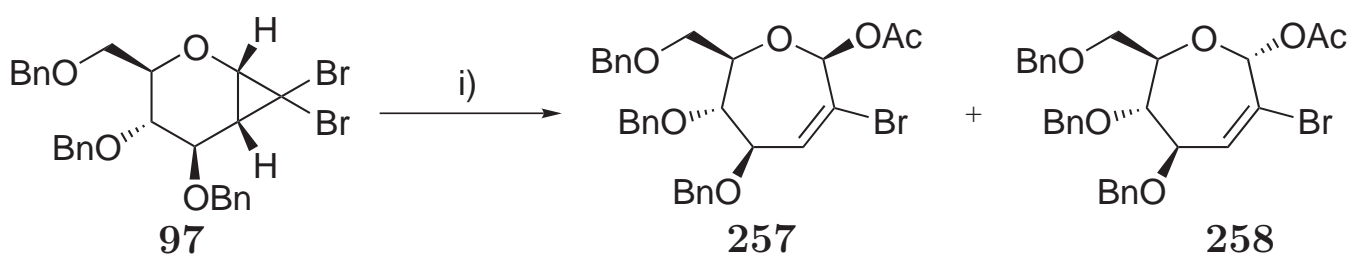

Reagents and Conditions: i) AgOAc, AcOH.

\begin{tabular}{|c|c|c|c|c|c|}
\hline Entry & Temperature & Time & Recovered 97 & Yield $(\mathbf{2 5 7}$ and 258) & Ratio $(\beta: \alpha)$ \\
\hline \hline 1 & r.t. & $5 \mathrm{~d}$ & quantitative & $0 \%$ & - \\
\hline 2 & $70{ }^{\circ} \mathrm{C}$ & $3 \mathrm{~d}$ & $10 \%$ & $27 \%$ & $1: 2.9$ \\
\hline 3 & $90{ }^{\circ} \mathrm{C}$ & $36 \mathrm{~h}$ & $4 \%$ & $37 \%$ & $1: 3.4$ \\
\hline 4 & $100{ }^{\circ} \mathrm{C}$ & $25 \mathrm{~h}$ & $0 \%$ & $52 \%$ & $1: 3.8$ \\
\hline 5 & reflux & $5 \mathrm{~h}$ & $0 \%$ & $15 \%$ & $1: 1.7$ \\
\hline
\end{tabular}

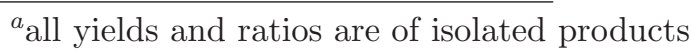

An important goal of this research was to investigate the use of only moderate excesses of various nucleophiles in ring-opening reactions of cyclopropanated sugars. This was deemed relevant for the synthesis of acetyl glycosides 257 and 258, in which milder conditions might lead to an improvement in yield. To achieve oxepine synthesis with a minimum amount of nucleophile, the use of a non-nucleophilic solvent was required. The results of the silver-mediated reactions involving acetic acid indicate that temperatures of approximately $100{ }^{\circ} \mathrm{C}$ are required, which ruled out the use of many common, low-boiling solvents (under atmospheric pressure). Toluene was chosen, due to the relatively high boiling point $\left(110-111^{\circ} \mathrm{C}\right)$, low toxicity and low cost of this solvent. Cyclopropane 97 was dissolved in toluene, treated with silver acetate (1.5 eq.) and sodium acetate (10 eq.), then the reaction was left to proceed for two days at $100{ }^{\circ} \mathrm{C}$. The reaction rate was significantly slower than acetic acid solvolysis, but a good yield was obtained (53\%) (Scheme 3.2), and a significant portion of starting material was also recovered $(22 \%)$. In addition, a small amount of acetylated glycal $\mathbf{2 5 9}$ was observed (5\% yield), due to attack of acetate at C-2 of cyclopropane $\mathbf{9 7}$, rather than C-1. This observation was not entirely unexpected, as nucleophilic attack could occur at either end of the $\pi$-allyl intermediate derived from the cyclopropane (attack at either C-1 or C-2). The high selectivity observed for $\mathrm{C}-1$ substitution is due to the participation of the ring oxygen, which promotes attack at the glycosyl cation (page 3).

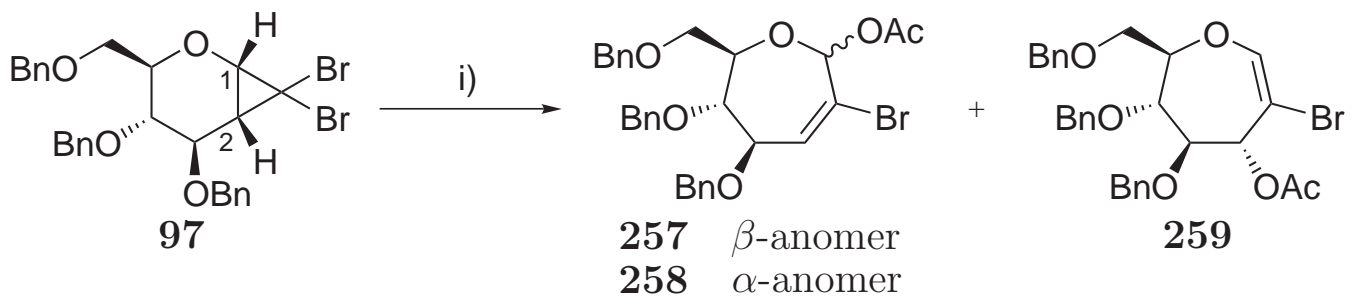

Reagents and Conditions: i) AgOAc, NaOAc, toluene, $100{ }^{\circ} \mathrm{C}, 2 \mathrm{~d}$.

Scheme 3.2 Synthesis of acetoxy oxepines 257, 258 and 259 
Repetition of this reaction using only 5 equivalents of sodium acetate at reflux led to even greater yields after only 28 hours reaction. Presumably, the elevated temperature allows for faster reactivity, without significant degradation, as observed in acetic acid. It was very pleasing to obtain oxepines $\mathbf{2 5 7}$ and $\mathbf{2 5 8}$ in $65 \%$ yield, though acetylated glycal 259 was isolated in a significantly greater quantity than in the previous reaction (14\%). Because of the reduced regioselectivity, future reactions in toluene were performed at $100{ }^{\circ} \mathrm{C}$.

\subsection{Ring-Expansions of Cyclopropane 97 with Alternative Nucleophiles}

With the development of methodology that provides oxepines in good yield, additional nucleophiles were sought to extend this chemistry. Initially the conditions outlined by Nagarajan were followed; ${ }^{45}$ treatment of cyclopropane $\mathbf{9 7}$ with silver tetrafluoroborate in methanol provided only recovered starting material, in agreement with previously reported observations. Reaction at $40{ }^{\circ} \mathrm{C}$ led to a small degree of reaction, as observed by TLC after one day. The temperature was then elevated to reflux, then stirred for an additional day. Column chromatography provided two products in a combined yield of $13 \%$. The proton NMR spectra of these two products indicated that they were different from exocyclic bromomethylene sugars $\mathbf{2 1 3}$ and 214, with the olefinic peaks shifted upfield by approximately $0.5 \mathrm{ppm}$. For each product, the olefinic peak was observed as a doublet, with a ${ }^{1} \mathrm{H}-{ }^{1} \mathrm{H}$ COSY correlation to an oxymethine. This provided further support that these silver-mediated reactions form the expected oxepines. Attempts to increase the yield through lengthening the reaction time were unsuccessful. After five days at reflux, oxepines $\mathbf{9 8}$ and 99 were isolated in only $15 \%$ combined yield (Table 3.2), with a large proportion of starting material recovered (61\%). These results correlate well with those observed using acetic acid solvolysis, implying that the temperature used is the main determinant of the rate of reaction. Attempts to increase the nucleophilicity of methanol through the addition of several equivalents of sodium methoxide provided significant consumption of starting material in only 16 hours, but as one might expect, exocyclic bromomethylene sugars 213 and 214 were observed in similar quantity (22\%) to that of oxepines 98 and 99 (17\%). Attempts to use higher temperatures, through the use of pressurised reaction vessels, is discussed in section 3.7.

This methodology was then extended to provide allyl, benzyl and phenyl glycosides incorporating an oxepine ring (Table 3.2). Solvolysis was employed in the formation of allyl septanosides, as refluxing allyl alcohol provides a convenient reaction tem- 
perature $\left(96-98^{\circ} \mathrm{C}\right)$. Benzyl alcohol and phenol were employed as stoichiometric nucleophiles. Although both could function as solvents at $100{ }^{\circ} \mathrm{C}$, it was anticipated that subsequent removal of these high-boiling alcohols would be problematic, and the use of moderate excesses of these nucleophiles would further demonstrate the efficiency of this methodology. These alcohols were employed in the synthesis of additional septanosides, as shown in Table 3.2. For completeness, key results mentioned in section 3.2 are included too (entries 2-4).

Table 3.2 Ring-expansion of cyclopropane $\mathbf{9 7}$ with various nucleophiles ${ }^{a}$<smiles>BrC[C@H]1O[C@H](Br)[C@H](Br)[C@H](OCc2ccccc2)[C@H]1Br</smiles>

97

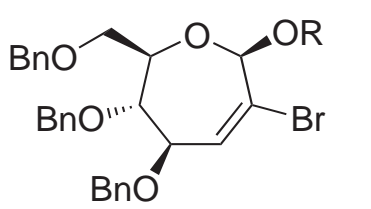

$98 \mathrm{R}=\mathrm{Me}$

$257 \mathrm{R}=\mathrm{Ac}$

$260 \mathrm{R}=$ Allyl

$262 \mathrm{R}=\mathrm{Bn}$

$264 \mathrm{R}=\mathrm{Ph}$

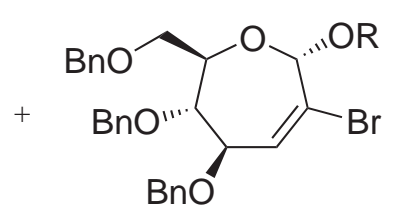

$99 \mathrm{R}=\mathrm{Me}$

$258 \mathrm{R}=\mathrm{Ac}$

$261 \mathrm{R}=$ Allyl

$263 \mathrm{R}=\mathrm{Bn}$

$265 \mathrm{R}=\mathrm{Ph}$

\begin{tabular}{|c|c|c|c|c|c|}
\hline Entry & Silver Salt $^{b}$ & Nucleophile, Solvent & Conditions & Yield, Products & Ratio $(\beta: \alpha)$ \\
\hline \hline 1 & $\mathrm{AgOAc}$ & 240 eq. $\mathrm{MeOH}$ & reflux, $5 \mathrm{~d}$ & $15 \%, \mathbf{9 8}, \mathbf{9 9}$ & $1: 2.8$ \\
\hline 2 & $\mathrm{AgOAc}$ & 170 eq. $\mathrm{AcOH}$ & $100{ }^{\circ} \mathrm{C}, 25 \mathrm{~h}$ & $52 \%, \mathbf{2 5 7}, \mathbf{2 5 8}$ & $1: 3.8$ \\
\hline 3 & $\mathrm{AgOAc}$ & $\begin{array}{c}9.9 \text { eq. } \mathrm{NaOAc} \\
\text { toluene }\end{array}$ & $100{ }^{\circ} \mathrm{C}, 2 \mathrm{~d}$ & $53 \%, \mathbf{2 5 7}, \mathbf{2 5 8}$ & $1: 3.7$ \\
\hline 4 & $\mathrm{AgOAc}$ & $\begin{array}{c}5.1 \text { eq. } \mathrm{NaOAc} \\
\text { toluene }\end{array}$ & reflux, $2 \mathrm{~d}$ & $65 \%, \mathbf{2 5 7}, \mathbf{2 5 8}$ & $1: 3.8$ \\
\hline 5 & $\mathrm{AgOAc}$ & 140 eq. AllylOH & reflux, $27 \mathrm{~h}$ & $\begin{array}{c}51 \%, \mathbf{2 6 0}, \mathbf{2 6 1} \\
4 \%, \mathbf{2 5 8}\end{array}$ & $1.1: 1$ \\
\hline 6 & $\mathrm{AgNO}_{3}$ & 140 eq. AllylOH & reflux, $29 \mathrm{~h}$ & $50 \%, \mathbf{2 6 0}, \mathbf{2 6 1}$ & $1: 1.8$ \\
\hline 7 & $\mathrm{AgOAc}$ & $\begin{array}{c}5.0 \text { eq. } \mathrm{BnOH} \\
\text { toluene }\end{array}$ & $100{ }^{\circ} \mathrm{C}, 2 \mathrm{~d}$ & $\begin{array}{c}45 \%, \mathbf{2 6 2}, \mathbf{2 6 3} \\
26 \%, \mathbf{2 5 7}, \mathbf{2 5 8}\end{array}$ & $1: 1.1$ \\
\hline 8 & $\mathrm{AgNO}_{3}$ & 5.3 eq. $\mathrm{PhOH}$ & $100{ }^{\circ} \mathrm{C}, 2 \mathrm{~d}$ & $45 \%, \mathbf{2 6 4}, \mathbf{2 6 5}$ & $6.7: 1$ \\
\hline
\end{tabular}

${ }^{a}$ all yields and ratios are of isolated products

${ }^{b}$ between 1.1 and 1.5 eq. of silver salt used in all cases

All reaction times provide good comparative indicators of the time required for complete consumption of starting material; these results indicate a two-fold difference in reaction time between solvolysis and toluene-based reactions is required. Entries 2, 5 and 6 indicate the reaction time under solvolysis conditions appears to be independent of solvent used. The relatively small amount of nucleophile employed in entries 3,7 and 8 shows the required reaction time is not drastically affected by the reduction in the amount of nucleophile present.

It is quite noteworthy that entries 5 and 7 provided mixtures of different oxepines, due to the alcohol and silver counterion both participating as nucleophiles. As one would expect, relative amounts of the different glycosides are dependant on the relative amounts of nucleophilic silver counterion and the intended nucleophile. 
Hence, the use of silver nitrate eliminated this problem, due to the non-nucleophilic nature of the nitrate counterion (entries 6 and 8).

The relative ratios of glycoside anomers proved to be exciting, with $\alpha$-glycosides predominating. Despite some anomalous results (e.g. entries 5, 6 and 7), in general the reaction exhibits good anomeric stereoselectivity. This $\alpha$-glycoside selectivity also suggests that the ring-expansion reaction might proceed via a concerted mechanism, as shown in the red path (Scheme 3.3), rather than a discrete $\pi$-allyl cation or glycosyl cation such as $\mathbf{2 6 6}$ (black arrows involved in both paths). Formation of intermediate $\mathbf{2 6 6}$ from cyclopropane $\mathbf{9 7}$ (green path) would allow anomeric mixtures to form, which does not necessarily agree with the observation that the $\alpha$-anomer (256) predominates. However, if factors such as the anomeric effect, or steric constraints are involved, the route via glycosyl cation 266 could still be the dominant pathway. It is also of interest that the bulky phenol nucleophile provided the highest anomeric selectivity, but for the $\beta$ anomer (entry 8). This disparity might indicate that reaction with phenol occurs through $\mathrm{S}_{\mathrm{N}} 2$-type stereoinversion, presumably due to the high degree of bulkiness of this alcohol.

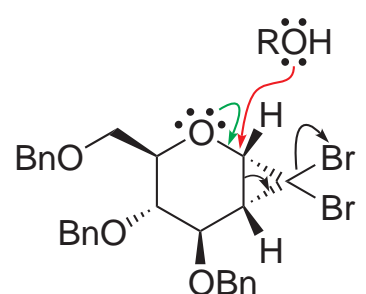

97

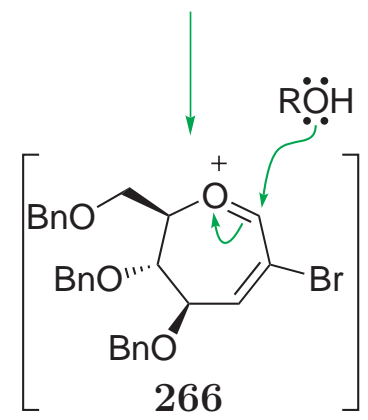

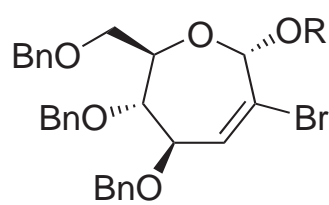

$256 \alpha$-anomer

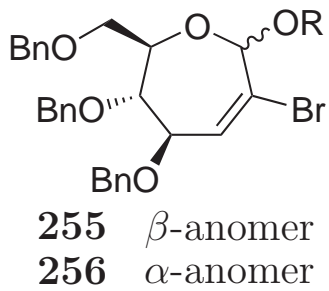

Scheme 3.3 Mechanism of the ring-expansion reaction of cyclopropane $\mathbf{9 7}$

The use of nucleophiles other than alcohols was briefly explored, with little success. Attempts to generate amino-functionalised septanosides with benzylamine and silver nitrate surprisingly led to the previously obtained benzyl $O$-glycosides 262 and 263, albeit in very low yield (15\%). This was possibly due to loss of benzyl protecting groups, and subsequent attack of the benzyl alcohol obtained. TLC evidence indicated some deprotection of the material; peaks attributed to benzyl alcohol and benzaldehyde were observed in the crude proton NMR spectrum. Use of cyclohexylamine led to very complicated mixtures, none of which were able to be characterised. The anion of Meldrum's acid was also used, in the hope that 
$C$-glycosyl sugars could be accessed. Unfortunately, this reaction provided low mass recovery. A small amount of acetate oxepines 257 and 258 (7\% combined yield) was isolated, due to interception of the silver counterion. Bicyclic acetal 267 was isolated from the same reaction in $13 \%$ yield, which arose from intramolecular nucleophilic attack of the C-6 benzyloxy group of the starting material (Scheme 3.4). ${ }^{112}$ It is likely the bulk of Meldrum's acid was too great to access the cyclopropane, which therefore allowed intramolecular cyclisation to occur. The preference for the C-6 substituent as nucleophile is supported by the fact that no other bicyclic acetals were observed. It is likely the relative distance and configurations of the other benzyl ethers attached to C-3 and C-4, precluded these substituents from participating in the reaction. It is likely, that upon silver-mediated ring opening of $\mathbf{9 7}$, the C-6 benzyloxy substituent attacks the C-1 glycosyl cation. This provides an intermediate oxonium species $(\mathbf{2 6 8})$, which could eject a benzylic cation, presumably through displacement by acetate ion. Fraser-Reid has reported similar reactivity, involving attack of a benzyl ether group upon an electrophilic centre, leading to subsequent loss of a benzylic cation, as proposed in Scheme 3.4. ${ }^{113}$

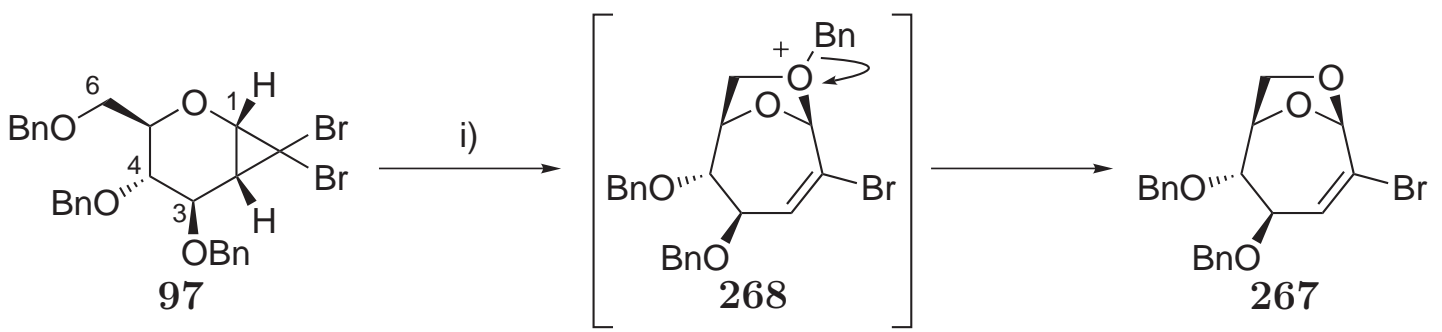

Reagents and Conditions: i) AgOAc, Meldrum's Acid, NaH, DMF, $100{ }^{\circ} \mathrm{C}, 2$ d, $13 \%$.

Scheme 3.4 Synthesis of bicyclic acetal 267

The complete lack of participation of anionic Meldrum's acid was quite disappointing, as it appears to fail to provide either 2 - $C$-bromomethylene pyranoside sugars under basic conditions (page 49), or $C$-functionalised oxepines under silver-mediated conditions. While further study of carbon nucleophiles is warranted, these observations imply that departure from oxygen nucleophiles may provide limited success.

\subsection{Scope of Oxepine Synthesis}

Alternative oxepine formation conditions were explored, in order to provide further information in regards to the ring-expansion reaction. An early attempt to synthesise allyl alcohol-based pyranoside products 236 and 237 (see section 2.5), used potassium carbonate, which led to a combination of pyranoside sugars 236 and 237 (41\%) and oxepines 260 and 261 (11\%) after 11 hours at reflux (Scheme 3.5). It is presumed that the basicity of the reaction was not sufficient to override the com- 
peting thermal process, yet the use of refluxing allyl alcohol was enough to ensure some material underwent ring expansion to the observed oxepine products. In contrast, when sodium allyloxide was employed, exclusive formation of 2- $C$-branched products was observed. However, the formation of oxepines 260 and 261 even in the absence of a silver salt implies oxepine formation from cyclopropane $\mathbf{9 7}$ may be a thermally induced process.<smiles>Cc1ccccc1Br</smiles>

97<smiles>C=CCO[C@H]1O[C@H](COBr)[C@@H](Br)[C@H](OBr)/C1=C\Br</smiles>

236, 237<smiles>C=CCO[C@H]1O[C@H](COBr)[C@@H](OBr)[C@H](Br)C=C1Br</smiles>

260,261

Reagents and Conditions: i) $\mathrm{K}_{2} \mathrm{CO}_{3}$, AllylOH, reflux, $11 \mathrm{~h}, 52 \%$.

Scheme 3.5 Synthesis of 2-C-branched sugars and oxepines

To determine if ring expansion was due to the presence of silver ion or the temperatures employed, a ring-expansion reaction of $\mathbf{9 7}$ with sodium acetate in toluene was performed. Consumption of starting material occurred within two days, as previously observed. However, after purification, mass recovery was low, with oxepines $\mathbf{2 5 7}$ and $\mathbf{2 5 8}$ obtained in only $18 \%$ yield, in addition to minor amounts of unidentified products (Table 3.3, entry 2). For comparison, the previous reaction with silver acetate and sodium acetate is detailed in entry 1.

Table 3.3 Miscellaneous ring-expansions of cyclopropane $\mathbf{9 7}$<smiles>BrC[C@H]1O[C@H](Br)[C@H](Br)[C@H](OBr)[C@H]1Br</smiles>

97

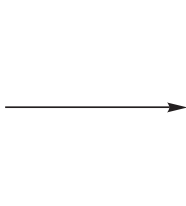

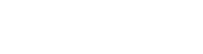<smiles>CC(=O)O[C@H]1O[C@H](COBr)[C@@H](Br)[C@H](OBr)C=C1Br</smiles>

$257 \beta$-anomer

$258 \alpha$-anomer<smiles>OC1C=C(Br)C2OCC(O2)C1Br</smiles>

267

\begin{tabular}{|c|c|c|c|c|c|}
\hline Entry & Silver Salt $^{b}$ & Nucleophile, Solvent & Conditions & Yield, Products & Ratio $(\beta: \alpha)$ \\
\hline 1 & AgOAc & $\begin{array}{c}9.9 \text { eq. NaOAc } \\
\text { toluene }\end{array}$ & $100{ }^{\circ} \mathrm{C}, 2 \mathrm{~d}$ & $53 \%, \mathbf{2 5 7}, \mathbf{2 5 8}$ & $1: 3.7$ \\
\hline 2 & - & $\begin{array}{c}5.0 \text { eq. NaOAc } \\
\text { toluene }\end{array}$ & $100{ }^{\circ} \mathrm{C}, 2 \mathrm{~d}$ & $18 \%, \mathbf{2 5 7}, \mathbf{2 5 8}$ & $1: 2.1$ \\
\hline 3 & $\mathrm{AgOAc}$ & $\begin{array}{c}5.3 \text { eq. NaOAc } \\
\text { DMF }\end{array}$ & $100{ }^{\circ} \mathrm{C}, 2 \mathrm{~d}$ & $49 \%, \mathbf{2 5 7 , 2 5 8}$ & $1.2: 1$ \\
\hline 4 & $\mathrm{AgOAc}$ & $\begin{array}{c}- \\
\text { toluene }\end{array}$ & $100{ }^{\circ} \mathrm{C}, 22 \mathrm{~h}$ & $38 \%, \mathbf{2 6 7}$ & - \\
\hline
\end{tabular}

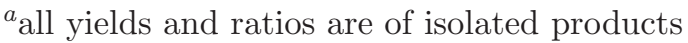

${ }^{b}$ between 1.2 and 1.5 eq. of silver salt, if used

Use of an alternative solvent was also explored. Good conversion of cyclopropane $\mathbf{9 7}$ was achieved in DMF, providing oxepines 257 and 258 (49\% yield), though two days reaction was still required (entry 3). However, the anomeric ratio had changed dramatically from approximately 3.5:1 in favour of $\alpha$-anomer 258, to only 1.2:1 in favour of $\beta$-anomer 257 (Table 3.3). These observations indicate the choice 
of solvent has minimal influence upon the rate of reaction, but may dramatically reduce the anomeric stereoselectivity previously seen.

An investigation into the behaviour of cyclopropane $\mathbf{9 7}$ in the absence of added nucleophile was explored. Thus, a solution of $\mathbf{9 7}$ and silver acetate in toluene was refluxed for 22 hours (entry 4). Upon purification of the complicated mixture obtained, bicyclic acetal 267 was obtained in $38 \%$ yield, with no acetate-derived oxepines observed (Table 3.3). Benzyl acetate was present in the proton NMR spectrum of the crude product. This result indicates that, when the intermolecular nucleophile is present in low concentration, internal attack by an appropriate benzyloxy group is preferred.

The oxepine formation reaction appears to be dependant on temperature, yet has no significant rate dependence on solvent, nucleophile or silver salt used. It appears that effective ring expansion often requires addition of a silver salt to aid cyclopropane ring cleavage, otherwise low yields are obtained. Furthermore, as the majority of oxepine syntheses were regioselective, participation of the glycosyl cation intermediate is likely. Substitution at C-2 of cyclopropane 97, which provided glycal 259, was only observed when acetate was used.

\subsection{Oxepines Derived from Galactose and Xylose}

The ring expansions of D-galactose and D-xylose-derived cyclopropanes 45 and 243 were briefly investigated. Optimised methodology from the D-glucose series (Table 3.2, entries 3 and 4) was employed, using approximately 1.5 equivalents of silver acetate and 5 equivalents of sodium acetate in toluene at $100{ }^{\circ} \mathrm{C}$.

Galactose-derived cyclopropane $\mathbf{4 5}$ was successfully ring-expanded using these conditions, providing oxepine $\mathbf{2 6 9}$ in $52 \%$ yield, with the corresponding $\beta$-anomer not observed (Scheme 3.6). The isolation of a single anomer was very pleasing, demonstrating the ability of this methodology to provide anomeric selectivity. Additionally, bicyclic acetal $\mathbf{2 7 0}$ was isolated in $7 \%$ yield, presumably formed via intramolecular nucleophilic attack of the C-4 benzyloxy substituent, providing a bicyclo[3.2.1]octane skeleton. It is likely the other two benzyloxy substituents at C-3 and C-6 of cyclopropane 45 would lie in a pseudo-equatorial conformation, which would lead to the C-4 benzyloxy substituent oriented in a pseudo-axial conformation. The C-4 oxygen would therefore be in close proximity to the cyclopropane and could provide competitive nucleophilic attack. This reactivity was observed earlier (pages 60 and 
62) with silver-mediated ring-opening of D-glucal-derived cyclopropane $\mathbf{9 7}$ in the absence an appropriate nucleophile.

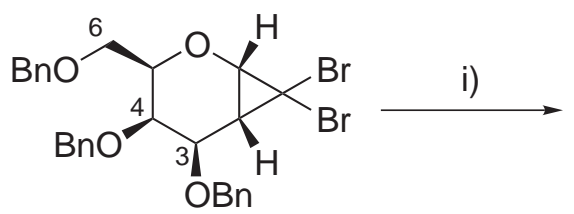

45

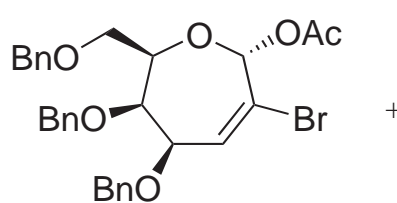

269

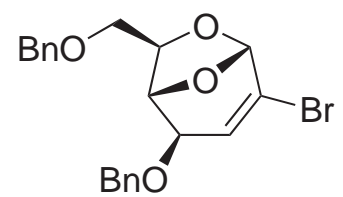

270

Reagents and Conditions: i) AgOAc, NaOAc, toluene, $100{ }^{\circ} \mathrm{C}, 2 \mathrm{~d}$.

Scheme 3.6 Formation of galactose-derived oxepine 269

Xylose-derived cyclopropane 243 was also subjected to ring-expansion reaction conditions, providing a mixture of oxepines 271 and 272 (1:1 anomeric ratio) (Scheme 3.7). While the lack of anomeric selectivity was disappointing, this might be due to a lack of significant steric bulk near C-1, the centre of nucleophilic attack.

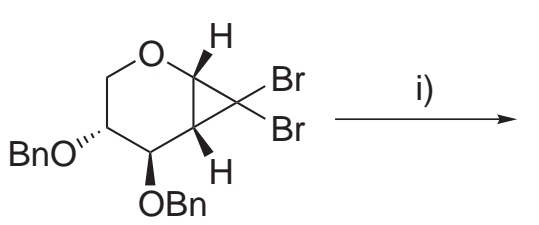

243<smiles>CC(=O)OC1OCC(Oc2ccccc2)C(Br)C=C1Br</smiles>

271<smiles>CC(=O)O[C@@H]1OC[C@@H](Br)[C@H](OBr)C=C1Br</smiles>

272

Reagents and Conditions: i) AgOAc, NaOAc, toluene, $100{ }^{\circ} \mathrm{C}, 2$ d, $45 \%$.

Scheme 3.7 Formation of xylose-derived oxepines 271 and 272

Due to the selective formation of galactose-derived oxepine 269, it appears likely that the steric environment of cyclopropane $\mathbf{4 5}$ has controlled the anomeric stereoselectivity to some extent. This was also implied in the ring expansion of xylosederived cyclopropane $\mathbf{2 4 3}$, which provided approximately equal amounts of oxepines 271 and 272 , presumably due to the lack of steric bulk.

\subsection{Oxepine Derivatisation Reactions}

Previously, 2- $C$-branched methyl glycoside pyranoside sugar 214 was subjected to lithium-halogen exchange, which upon quenching with water, provided an exocyclic methylene at C-2. To confirm unequivocally the structure of the above silvermediated oxepine products, allyl glycoside 261 was subjected to a similar reaction, as shown in Scheme 3.8. This reaction provided oxepine $\mathbf{2 7 3}$ in $34 \%$ yield as the only observed product, with the 2,3-olefin exhibiting two separate peaks in the proton NMR spectrum. These proton resonances coupled with a coupling constant of $12.0 \mathrm{~Hz}$, as one would expect for a 1,2-cis-disubstituted olefin. A ${ }^{1} \mathrm{H}^{-}{ }^{1} \mathrm{H}$ COSY correlation between $\mathrm{H}-3$ and $\mathrm{H}-4$ was observed, in addition to a two bond HMBC correlation between $\mathrm{C}-1$ and $\mathrm{H}-2$ was also seen. This provides further evidence that seven-membered rings have indeed been formed in these reactions. 


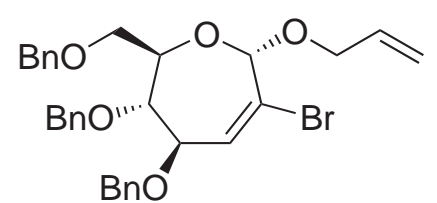

261

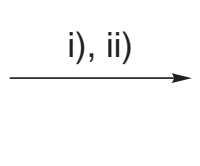

Reagents and Conditions: i) $n$-BuLi, THF, $-78{ }^{\circ} \mathrm{C}, 1 \mathrm{~h}$; ii) $\mathrm{H}_{2} \mathrm{O},-78{ }^{\circ} \mathrm{C}$ to r.t., 30 min, $34 \%$ over 2 steps.

Scheme 3.8 Lithium-halogen exchange of allyl oxepine $\mathbf{2 6 1}$

Allyl glycoside 261 underwent an intramolecular Heck reaction ${ }^{114}$ to afford bicycle $\mathbf{2 7 4}$ in $47 \%$ yield (Scheme 3.9), in addition to recovered starting material (20\%). This reaction highlights the synthetic utility of the alkenyl bromide moiety obtained through cyclopropane ring-expansion methodology. Furthermore, the synthesis of bicyclic acetal $\mathbf{2 7 4}$ demonstrates the potential of this chemistry for generating complex structures, of relevance to natural product synthesis. ${ }^{115}$ The furano $[2,3-b]$ oxepine bicycle is indeed present in several natural products, including azadirachtin, ${ }^{116}$ dysoxyhainanin $\mathrm{A},{ }^{117}$ and fabianane. ${ }^{118}$

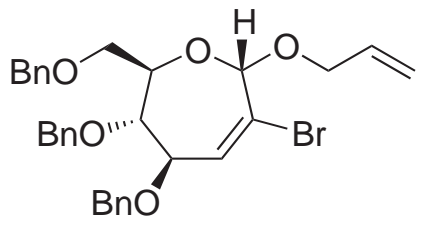

261
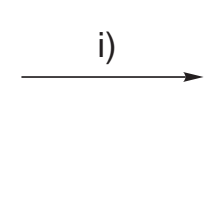

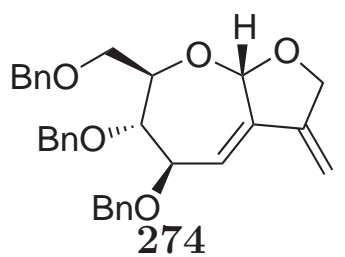

274

Reagents and Conditions: i) $\mathrm{Pd}(\mathrm{OAc})_{2}, \mathrm{PPh}_{3}, \mathrm{NEt}_{3}, \mathrm{THF}$, reflux, $1 \mathrm{~d}, 47 \%$.

Scheme 3.9 Heck cyclisation of allyl oxepine $\mathbf{2 6 1}$

\subsection{Synthesis of Furanosyl Sugars}

The silver-mediated syntheses of oxepines occasionally provided additional products, which were initially overlooked. These reactions occurred under very similar conditions to the successful oxepine syntheses, with no differences evident. Complex mixtures of products were obtained in these cases, with proton NMR analysis of the crude reaction mixtures indicating the presence of unknown products, occasionally in addition to the previously discussed oxepine derivatives. The first conclusive determination of these unusual structures occurred in a final attempt to produce methyl septanosides $\mathbf{9 8}$ and $\mathbf{9 9}$ under pressure.

As explained earlier, oxepine formation in refluxing methanol provided minimal reactivity due to the limiting reaction temperature. Later reactions with alternative nucleophiles at $100{ }^{\circ} \mathrm{C}$ provided efficient conversion of cyclopropane $\mathbf{9 7}$ into oxepines. A microwave reactor allows reactions to proceed at higher temperatures and pres- 
sures within sealed reaction vessels, and might also aid reaction due to microwave irradiation. ${ }^{119}$ The possibility of performing a ring-expansion reaction in methanol at elevated temperatures through the use of microwave heating and a sealed pressure vessel was therefore explored.

Initially, a thermal ring-expansion reaction was attempted by simply heating a solution of cyclopropane $\mathbf{9 7}$ in methanol to a temperature of $130{ }^{\circ} \mathrm{C}$. The microwave reaction vessel reached a pressure of approximately 8 bar during this reaction. The high temperature provided rapid consumption of starting material, within a total reaction time of one hour at $130{ }^{\circ} \mathrm{C}$. Upon purification by column chromatography, three sugars incorporating a furan ring were isolated in $74 \%$ combined yield (Scheme 3.10). This included isomeric dimethyl acetals 275 and 276 (61\% combined yield), in addition to methyl vinyl ether 277 (13\%), a structural isomer of the expected oxepines. The stereochemistries at C-2 and C-3 of $\mathbf{2 7 5}$ and $\mathbf{2 7 6}$, and C-3 of 277 were not determined, as implied in the structures shown.

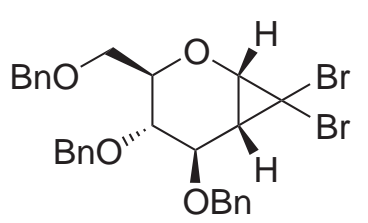

97<smiles>COC(OC)C(Br)[C@H]1O[C@H](COc2ccccc2)[C@@H](O)[C@H]1Br</smiles>
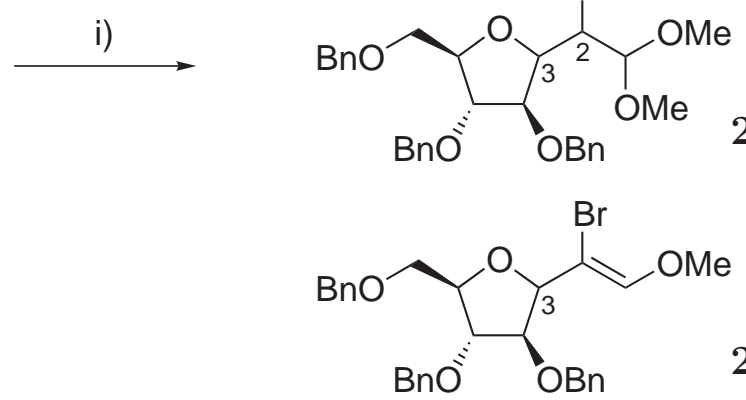

Reagents and Conditions: i) $\mathrm{MeOH}, 130{ }^{\circ} \mathrm{C}, 1 \mathrm{~h}$.

Scheme 3.10 Formation of methylated $C$-furanosyl sugars 275, 276 and 277

Microwave reaction in the presence of silver salts was then attempted. Performing the reaction at $100{ }^{\circ} \mathrm{C}$ provided significantly lower reactivity, with approximately 60\% material consumed after 110 minutes reaction. Excluding starting material, all peaks observed in the NMR spectrum of the crude reaction mixture corresponded to furanosyl products 275, 276 and $\mathbf{2 7 7}$. Repeating this reaction for a period of only 10 minutes provided a minor amount of oxepines $\mathbf{9 8}$ and $\mathbf{9 9}$ in $4 \%$ combined yield, with traces of furanosyl products 275 and 277 observed by TLC. This implied the furanosyl products were formed via oxepines $\mathbf{9 8}$ and $\mathbf{9 9}$. It was later confirmed that this chemistry was not limited to microwave-mediated reactions. One attempt at forming methanol-derived oxepines 98 and 99 via silver-mediated reaction at reflux for three days led to recovery of starting material (26\%) in addition to furanosyl acetals $\mathbf{2 7 5}$ (9\%) and $\mathbf{2 7 6}$ (22\%). Microwave reaction provides more efficient reac- 
tivity, due to the elevated temperatures one may access; consequently the reaction mixtures are often cleaner, as judged by proton NMR analysis of the crude products. These results indicate the presence of silver ion is not essential for conversion into oxepines or furanosyl products, but still might facilitate the reaction.

This unusual chemistry was not limited to methanol solvolysis. A solution of cyclopropane $\mathbf{9 7}$ in refluxing allyl alcohol led to the formation of allylated furanosyl sugars 278, 279 and $\mathbf{2 8 0}$ in 57\% combined yield (Scheme 3.11) as the only isolated products. This was also observed under silver-mediated conditions using either silver nitrate or silver trifluoroacetate in allyl alcohol.<smiles>C=CCOC(OCC=C)C(Br)C1O[C@H](COc2ccccc2)[C@@H](O)[C@H]1OCc1ccccc1</smiles><smiles>Br[C@H]1[C@H](Br)[C@H](Br)[C@@H](Br)[C@H]1Br</smiles><smiles>[C-]#C</smiles>

97<smiles>[B]OC[C@H]1OC(C(Br)C(OCC=C)OCC=C)[C@H](OCc2ccccc2)[C@@H]1Br</smiles><smiles>C=CCO/C=C(\Br)C1O[C@H](COBr)[C@@H](O)[C@H]1Br</smiles>

Reagents and Conditions: i) AllylOH, reflux, 2 d.

Scheme 3.11 Formation of allylated $C$-furanosyl sugars 278, 279 and 280

Intrigued by the presence of these methanol and allyl alcohol-derived furanosyl sugars, further analysis of this reaction and the obtained products was warranted. To confirm the identity of bromoalkene $\mathbf{2 8 0}$ treatment with $n$-butyllithium was followed by addition of water, which provided 1,2-disubstituted alkene $\mathbf{2 8 1}$ (Scheme 3.12). Starting material $\mathbf{2 8 0}$ was not completely consumed, therefore purification was not attempted. The proton NMR spectrum of the crude reaction mixture displayed a peak at $\delta_{\mathrm{H}} 6.53 \mathrm{ppm}$ corresponding to $\mathrm{H}-1$, with a coupling constant of $12.5 \mathrm{~Hz}$. This coupling constant is indicative of a trans-configuration for an enol ether moiety, ${ }^{120}$ which implies that the bromine and alkyloxy substituents are in a cis-relationship.
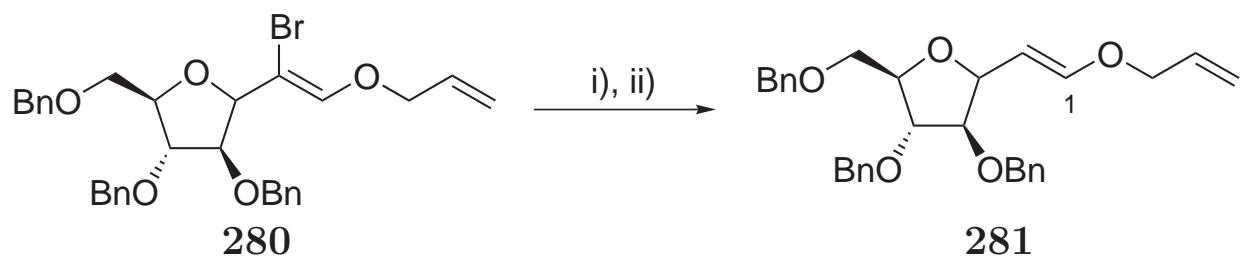

Reagents and Conditions: i) $n$-BuLi, THF, $-78{ }^{\circ} \mathrm{C}, 30 \mathrm{~min}$; ii) $\mathrm{H}_{2} \mathrm{O},-78{ }^{\circ} \mathrm{C}$ to r.t., $30 \mathrm{~min}$.

Scheme 3.12 Lithium-halogen exchange of bromoalkene $\mathbf{2 8 0}$ 
It was observed earlier that silver-mediated microwave reaction provided trace amounts of oxepine sugars after ten minutes; further reaction provided only furanosyl products. From these results it is believed the furanosyl products are obtained via oxepines 98 and 99. To confirm this, a sample of purified allyl alcohol-derived oxepine 261 was subjected to silver nitrate in allyl alcohol. After one day at reflux, a complex mixture of furanosyl products was obtained, with only a trace of starting material observed in the crude proton NMR spectrum. This indicates that the furanosyl sugars are formed from the corresponding oxepines.

While this reactivity has been observed in multiple runs of methanol and allyl alcohol solvolysis, no furanosyl sugars were observed with reactions involving sodium acetate. Furthermore, oxepine formation from cyclopropanes involves production of both silver bromide and the protonated counterion (nitric or tetrafluoroboric acid). These latter species will acidify the reaction, whereas reactions involving sodium acetate will do so to a lesser extent. The acid-sensitive acetal functionality of the target oxepines could be activated under such conditions, which might lead to furanosyl sugar formation.

Attempts to deduce the mechanism of formation of these unexpected $C$-furanosyl products from the corresponding oxepines, have led to consideration of several mechanisms as outlined in Scheme 3.13. The dark-blue path proposes that under the acidic conditions of the reaction, if wet, the acetal moiety of generic oxepines $\mathbf{2 5 5}$ and 256 might be hydrolysed to form the reducing sugar 282. This could undergo an intramolecular 1,4-addition to provide enol 283. Tautomerisation to aldehyde $\mathbf{2 8 4}$, followed by acid-catalysed acetal formation would provide acetal $\mathbf{2 8 5}$. This route however does not explain the formation of alkene 286. The green path involves acid-catalysed decomposition of the cyclic acetal to oxonium ion 287. This activated intermediate could undergo intramolecular 1,4-addition to provide furanosyl sugar 286, likely favoured due to the formation of a stable five-membered ring. Finally, the red path involves a concerted mechanism, wherein the oxepine ring oxygen could attack the alkene at C-3, forming a new 1,2-olefinic bond. This could lead to cleavage of the carbon-oxygen bond at C-1, which would provide 286 . The formation of furanosyl acetals (285) from $\mathbf{2 8 6}$ is believed to occur through acidcatalysed addition of a second equivalent of nucleophile to the enol ether moiety of bromoalkene 286 .

All paths shown involve formation of the 1,2-olefinic bond in which the alkoxy- and bromo- substituents of these carbons are likely to be in a cis-relationship, providing alkene $\mathbf{2 8 6}$ with the observed stereochemistry. Upon generation of alkene $\mathbf{2 8 6}$, acidcatalysed addition of a second equivalent would provide the corresponding acetals 


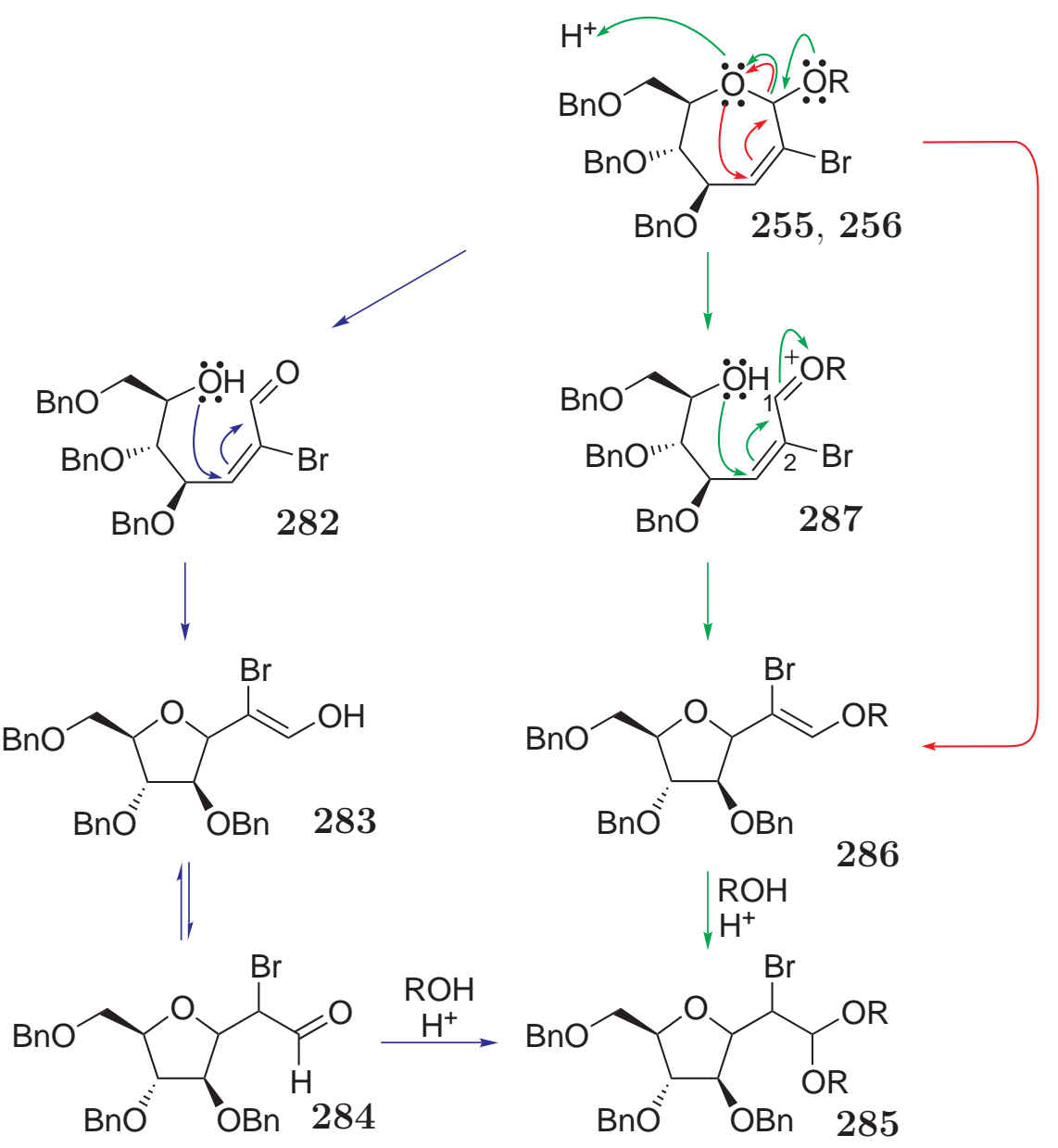

Scheme 3.13 Mechanism of conversion from oxepines into furanosyl products

shown as 285. The dark-blue path involves acid-catalysed acetal decomposition, due to the acidic reaction conditions. The formation of 282 is possible if water is present; which could be due to slightly wet solvents. Alternatively, the green path involves an intermediate one would expect in acetal decomposition, namely cation 287. Without loss of the alkyloxy group, it is possible for the conjugate cyclisation to occur, which would in turn quench the carbocation, providing alkene $\mathbf{2 8 6}$. The red path appears to be an unlikely mechanism, as the propensity for this rearrangement (either concerted or stepwise), is not very convincing. The green path is presumably the most likely; even though it is possible the 1,2-bond might rotate (to provide the $(E)$-isomer of alkene $\mathbf{2 8 6}$ ), it is likely $\mathbf{2 8 7}$ is a highly reactive intermediate. It was thought that reaction in a less acidic environment would suppress formation of these furanosyl derivatives. Unfortunately, the use of silver carbonate failed to alleviate this problem; reaction of cyclopropane $\mathbf{9 7}$ in the presence of 1.5 equivalents of silver carbonate led to a complex mixture of furanosyl sugars, with no evidence of oxepine products formed. 


\subsection{Summary of Chapter 3}

Through careful examination of methanol and acetic acid solvolysis reactions, silvermediated oxepine synthesis from cyclopropane $\mathbf{9 7}$ has been achieved. This methodology has been extended to include a number of alcohols, whereas reactions involving carbon- and nitrogen-based nucleophiles met with limited success. Analysis of the reaction through variation of the solvent, nucleophile and silver salt has also been achieved. Furthermore, unexpected furanosyl sugars have been obtained through silver(I)-mediated and thermal reactions, which are believed to occur as a result of the acid-sensitivity of the glycoside functionality in the oxepine derivatives, and the stability of the resulting five-membered rings. No distinction between reactions providing either exclusively oxepine-derived or furan-derived products was observed. 


\section{Chapter 4}

\section{Galactose and Xylose-Based Approaches to (-)-TAN-2483B}

\subsection{Introduction}

The versatile methodology described in chapters 2 and 3 for the synthesis and subsequent ring-opening of gem-dihalocyclopropanated sugars shows excellent potential for use in complex synthetic routes. The functionality obtained through this methodology could find application in the synthesis of biologically active natural products. ${ }^{13}$ Synthesis of the natural product (-)-TAN-2483B, ${ }^{82,87,88}$ isolated from a Japanese fungus, appeared amenable to such chemistry. The synthesis of this natural product would demonstrate the utility of cyclopropane chemistry, and provide the first total synthesis of this biologically relevant compound. The stereochemical configurations at positions C-2 and C-3 of (-)-TAN-2483B (154) correlate well with the stereochemistry of D-galactose (page 33). A synthetic route to (-)-TAN-2483B could involve the following sequence (Scheme 4.1).

Beginning from commercially available tri- $O$-acetyl-D-galactal (180), Ferrier rearrangement ${ }^{27}$ in the presence of thiophenol would afford the known 2,3-unsaturated sugar 289 (Scheme 4.1). ${ }^{121-123}$ Deprotection and reprotection would be required to install the $p$-methoxybenzylidene protecting group, which may be selectively deprotected at C-6. The resulting alkene (290) could subsequently undergo cyclopropanation, which would likely occur preferentially from the $\alpha$-face; upon deprotection 


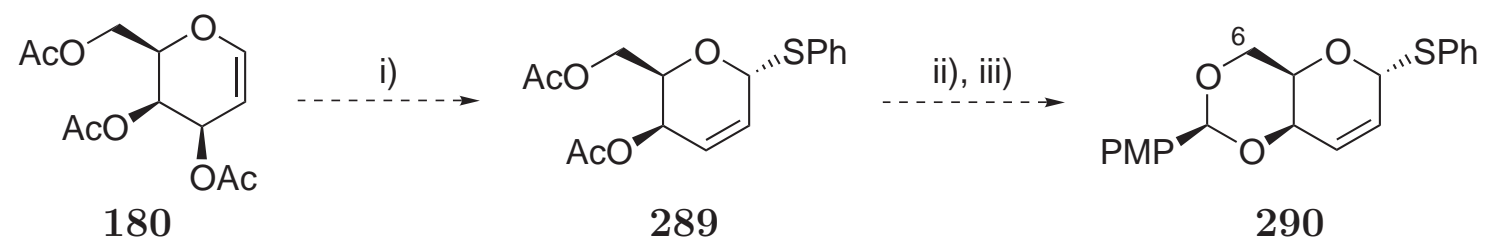

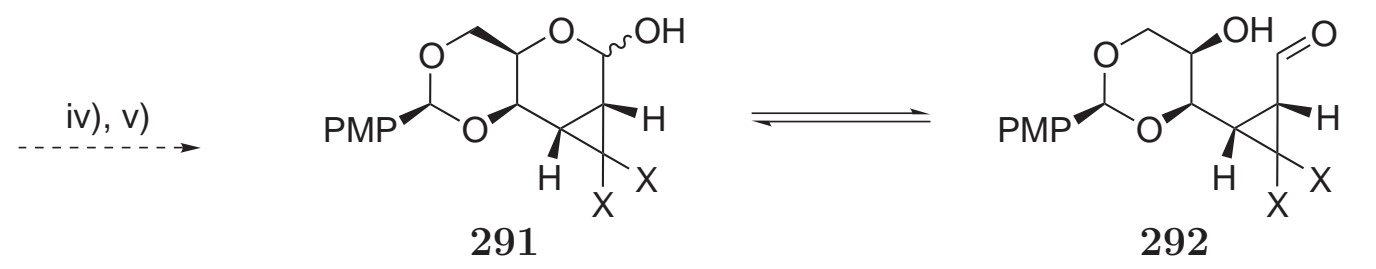

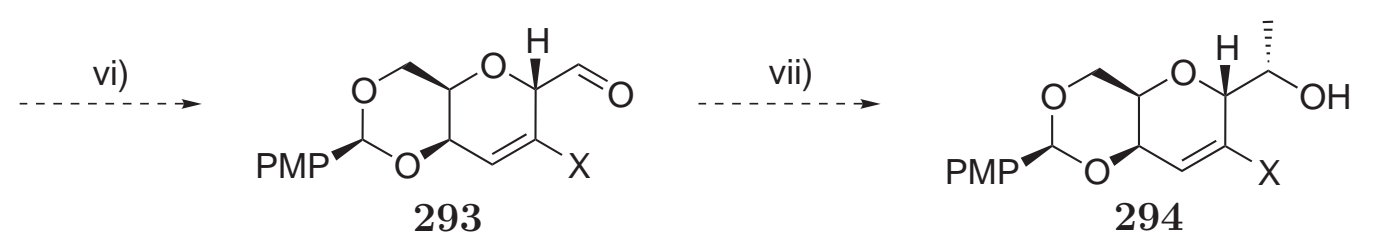

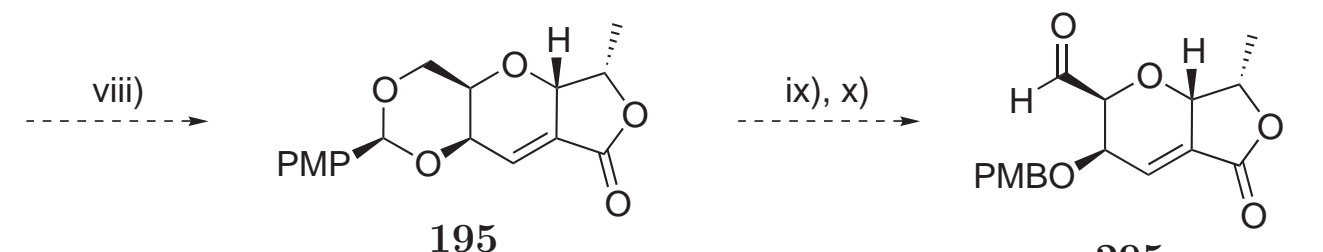
195

\section{5}<smiles>C/C=C/C1O[C@H]2C(=C[C@H]1O[R16](=O)O)C(=O)O[C@H]2C</smiles>

296

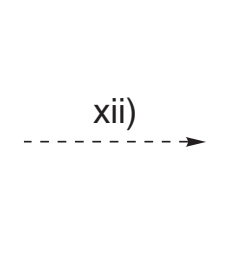


group and oxidation of the primary alcohol might be used to afford aldehyde $\mathbf{2 9 5}$. Finally, Julia-Kocienski olefination ${ }^{124,125}$ to append the sidechain (296), followed by deprotection, would provide (-)-TAN-2483B (154), completing the first total synthesis of this natural product.

\subsection{Synthesis and Cyclopropanations of 2,3-Unsaturated Sugar 290}

The $p$-methoxybenzylidene group was chosen for the protection of the C-4 and C-6 hydroxyls of galactal derivatives, as it was expected to be stable to future reactions, such as the alkaline Mạkosza cyclopropanation. This protecting group can be selectively cleaved at the C-6 hydroxyl using sodium cyanoborohydride, leaving a $p$-methoxybenzyl ether at C-4. ${ }^{90}$ Unfortunately, this acid-sensitive acetal precludes the use of an alcohol in the Ferrier rearrangement, as the resulting glycoside would require acidic deprotection. Thiophenol would generate the corresponding $S$-thioglycoside, which could be deprotected with NBS or NIS, avoiding the use of acidic media.

Formation of the required 2,3-unsaturated sugar 290 was attempted as described in Scheme 4.2. Purified tri- $O$-acetyl-D-galactal (180) was obtained in $52 \%$ yield from D-galactose. This glycal was then subjected to a Ferrier rearrangement with thiophenol, ${ }^{27}$ using a catalytic amount of iron(III) trichloride. ${ }^{122}$ Ferrier rearrangement usually provides $\alpha$-glycosides as the major product, ${ }^{123}$ which in this case provided 289 in $46 \%$ yield. Unfortunately, the low yield was due to the presence of impurities, notably the corresponding $S$ - $\beta$-glycoside $\mathbf{2 9 7}$, and what is believed to be glycal 298, which could arise from $\mathrm{S}_{\mathrm{N}} 2$ substitution of the C-3, acetoxy group by thiophenol, or most likely attack of thiophenol upon intermediate 299 at C-3 rather than C-1. Neither of these products were investigated further, though analogous compounds have been reported by Ferrier from the reaction of thiophenol with tri- $O$-acetyl-Dglucal. ${ }^{121}$

With 2,3-unsaturated carbohydrate 289 in hand, a deprotection/reprotection sequence was utilised, to provide a substrate suitable for Mąkosza cyclopropanation. Thus, diacetate $\mathbf{2 8 9}$ was hydrolysed using freshly prepared sodium methoxide in methanol to provide diol $\mathbf{3 0 0}$ in near-quantitative yield (Scheme 4.3). This diol was protected as a $p$-methoxybenzylidene acetal, through treatment with anisaldehyde dimethyl acetal and a catalytic amount of camphorsulfonic acid, to provide 2,3-unsaturated carbohydrate $\mathbf{2 9 0}$ in $\mathbf{1 6 \%}$ yield over six steps from D-galactose. 


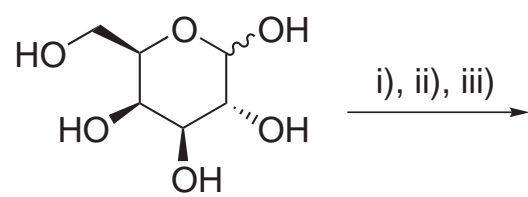

183<smiles>CC(=O)OC[C@H]1OC=C[C@@H](OC(C)=O)[C@H]1OC(C)=O</smiles>

180<smiles>CC(=O)OC[C@H]1O[C@@H](Sc2ccccc2)C=CC1OC(C)=O</smiles>

$289 \alpha$-anomer

$297 \beta$-anomer

iv)
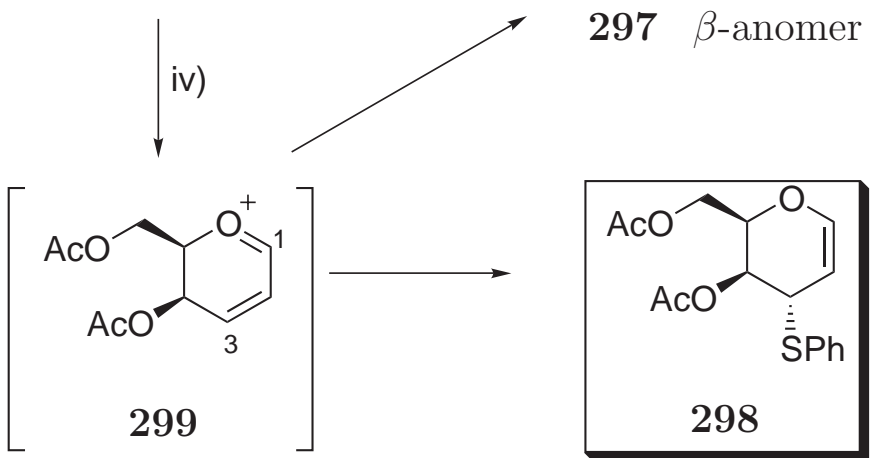

Reagents and Conditions: i) $\mathrm{Ac}_{2} \mathrm{O}, \mathrm{HClO}_{4}, 40-50{ }^{\circ} \mathrm{C}, 30 \mathrm{~min}$; ii) $\mathrm{HBr}, \mathrm{AcOH}$, r.t., 90 min; iii) Zn, $\mathrm{AcOH}_{(\mathrm{aq})}, 0{ }^{\circ} \mathrm{C}$ to r.t., overnight, $52 \%$ over 3 steps; iv) $\mathrm{PhSH}, \mathrm{FeCl}_{3}, \mathrm{CH}_{2} \mathrm{Cl}_{2}$, r.t., 25 h, $46 \%$.

Scheme 4.2 Synthesis of thioglycoside $\mathbf{2 8 9}$<smiles>CC(=O)O[C@H]1C=C[C@H](c2ccccc2)O[C@@H]1CC=C(C)C</smiles>

289

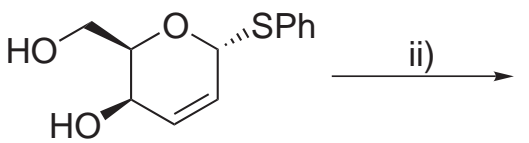

300

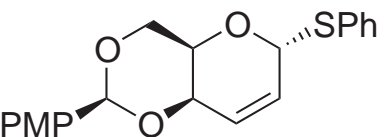

290

Reagents and Conditions: i) $\mathrm{NaOMe}, \mathrm{MeOH}$, r.t., $45 \mathrm{~min}, 99 \%$; ii) $\mathrm{PMPCH}(\mathrm{OMe})_{2}$, CSA, DMF, $50{ }^{\circ} \mathrm{C}, 22 \mathrm{~h}, 67 \%$.

Scheme 4.3 Synthesis of galactose-based substrate $\mathbf{2 9 0}$

Cyclopropanation of alkene $\mathbf{2 9 0}$ was then attempted. Unfortunately, a variety of gem-dihalocyclopropanation procedures failed to provide the desired cyclopropyl adducts. Initially alkene $\mathbf{2 9 0}$ was reacted in the presence of bromoform, the phase transfer catalyst TEBAC, and an aqueous solution of potassium fluoride and sodium hydroxide. No reaction was observed after eight days, which provided almost complete recovery of starting material. Nagarajan has reported that dichlorocarbenemediated Mạkosza cyclopropanation of glycals was successful with $50 \%$ aqueous sodium hydroxide, ${ }^{45}$ therefore this was emulated with substrate 290 . Rapid consumption of starting material was observed, though mass recovery was low. The only identifiable product appeared to be a glycal (approximate yield 15\%), probably formed through a retro-Ferrier rearrangement, as loss of the thiophenyl group was observed. Possibly this arose due to attack of chloride ion at C-3, though this compound was not identified. Finally, cyclopropanation with anhydrous potassium tert-butoxide and bromoform was attempted; ${ }^{126}$ this provided no observable reaction after several days.

The lack of reactivity under Mąkosza cyclopropanation was presumed to be a result of steric hindrance; the $p$-methoxybenzylidene acetal group blocks one face of alkene 
290, the thiophenyl group the other. Unfortunately, as the Ferrier rearrangement forms predominantly $\alpha$-glycosides, preparation of a less sterically-hindered substrate was required.

\subsection{Synthesis of Alternative 2,3-Unsaturated Carbohydrates}

Epimerisation of the anomeric thiophenyl group might aid cyclopropanation, as the lower face of the substrate would be much more accessible to carbene addition. Thus, thioglycoside $\mathbf{2 9 0}$ was treated with $n$-butyllithium and quenched with water, in the hope epimerisation would occur. Unfortunately, this provided alcohol $301(50 \%)$ and diene 302 (25\%) in addition to unreacted starting material (37\%) (Scheme 4.4). A degradation reaction had evidently taken place, presumably aided by the high conjugation of $\alpha, \beta, \gamma, \delta$-unsaturated ester 302. Diene 302 must therefore be derived from the carbon backbone of alkene 290. Mechanistically, it appears that anisaldehyde was ejected from olefin 290, upon which the anisaldehyde would have been attacked by $n$-butyllithium to provide 301 . Similar reactivity has been observed previously in the reaction of a related substrate with $n$-butyllithium, providing a stereoisomer of diene $302 .{ }^{127}$

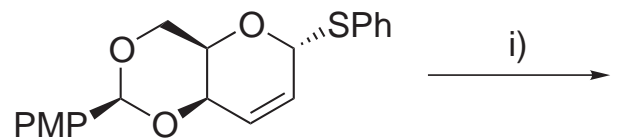

290<smiles>CCCCC([18NH2])O</smiles>

301<smiles>O=C(/C=C/C=C/CO)Sc1ccccc1</smiles>

302

Reagents and Conditions: i) $n$-BuLi, THF, $-78^{\circ} \mathrm{C}, 1 \mathrm{~h}$.

Scheme 4.4 Unsuccessful epimerisation of 290

The synthesis of other 2,3-unsaturated sugars with reduced steric bulk on the (bottom) $\alpha$-face was then pursued. Initially, a thiophenol-mediated allylic rearrangement of glycal 180 was attempted, through palladium, rather than Lewis acid catalysis. It was thought that the palladium would complex to the $\alpha$-face due to the orientation of the C-3 acetoxy group, to form a palladium- $\pi$-allyl complex. This would cause thiophenol attack at the more accessible $\beta$-face. ${ }^{128}$ Unfortunately, the use of either $\mathrm{Pd}\left(\mathrm{PPh}_{3}\right)_{4}$ or $\mathrm{PdCl}_{2}(\mathrm{dppf}) \cdot \mathrm{CH}_{2} \mathrm{Cl}_{2}$ led to no observed reactivity, possibly due to catalyst poisoning in the presence of thiophenol. Changing the nucleophile to either sodium acetate or $p$-methoxybenzyl alcohol also failed to show any reactivity, indicating this palladium-mediated allylic rearrangement was not be feasible.

It was thought this palladium-catalysed allylic rearrangement would be aided by a better leaving group at C-3. Reaction of D-galactal with anisaldehyde dimethyl 
acetal was catalysed with the mildly acidic salt PPTS (Scheme 4.5). Upon obtaining glycal 303, a carbonate group was installed at C-3 through reaction with methyl chloroformate, to provide glycal 304. No attempts were made to optimise the low yields obtained in both these reactions. A palladium-mediated Ferrier rearrangement was then attempted, using $p$-methoxybenzyl alcohol. This once again demonstrated no significant reactivity, with starting material predominating in the crude reaction mixture. No evidence of the required 2,3-unsaturated sugars was observed.

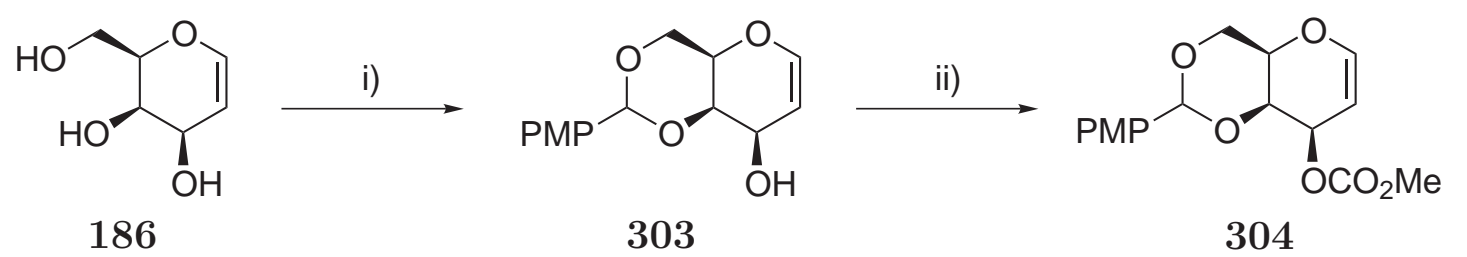

Reagents and Conditions: i) $\mathrm{PMPCH}(\mathrm{OMe})_{2}$, PPTS, $\mathrm{MeCN}$, r.t., 4 h, 31\%; ii) $\mathrm{MeOCOCl}, \mathrm{NaH}$, DMF, r.t., overnight, $19 \%$.

Scheme 4.5 Synthesis of carbonate 304

Alternative means of obtaining 2,3-unsaturated substrates were then investigated. It has been reported that the use of ammonium heptamolybdate in glycal isomerisation may provide access to 2,3-unsaturated sugars. ${ }^{129}$ This reaction was carried out in acetic anhydride, and proceeded very quickly: after 30 minutes a complicated mixture of sugars was obtained. The only separable products were the C-3 epimeric gulose derivative 305 (8\%) (Scheme 4.6), and the desired 2,3-unsaturated carbohydrate 306 in $10 \%$ yield. Allylic acetate 306 was determined to be the $\beta$ anomer through observation of nOe correlations between $\mathrm{H}-1$ and H-5. Due to the low mass recovery of this reaction, this chemistry was not pursued.

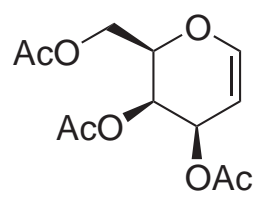

180

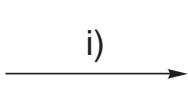

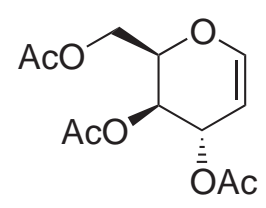

305

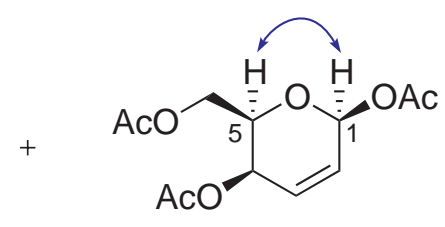

306

Reagents and Conditions: i) $\left(\mathrm{NH}_{4}\right)_{6} \mathrm{Mo}_{7} \mathrm{O}_{24} \cdot 4 \mathrm{H}_{2} \mathrm{O}, \mathrm{Ac}_{2} \mathrm{O}, 110{ }^{\circ} \mathrm{C}, 30 \mathrm{~min}$.

\section{Scheme 4.6 Isomerisation of $\mathbf{1 8 0}$}

The hydrolysis of acetylated glycals has been reported previously. ${ }^{130}$ Fraser-Reid and Tam propose that equilibration provides multiple products, including both 2,3unsaturated sugars, and ring-opened aldehydic derivatives. A 2,3-unsaturated hemiacetal could provide access to an appropriate cyclopropanation substrate, through subsequent $\beta$-selective glycosylation. Glycal 180 was refluxed in water for 100 minutes, during which most of the starting material was consumed (16\% recovered). Desired 2,3-unsaturated carbohydrate $\mathbf{3 0 7}$ was obtained in $36 \%$ yield, in addition to an unidentified mixture of aldehydic products, presumed to be $\mathbf{3 0 8}$ and corre- 
sponding isomers arising from acetate migration (Scheme 4.7). Aldehydes such as 308 are likely to arise through isomerisation of the olefin of $\mathbf{3 0 7}$ when in the ring opened form, and might explain why Fischer named D-glucal with the -al suffix. ${ }^{131}$<smiles>CC(=O)OC[C@H]1OC=C[C@@H](OC(C)=O)[C@H]1OC(C)=O</smiles>

180

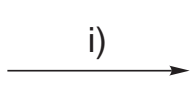

Reagents and Conditions:

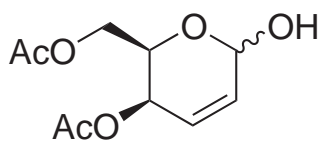

307<smiles>CC(=O)OC[C@@H](O)[C@@H](/C=C/C=O)OC(C)=O</smiles>

308

Scheme 4.7 Hydrolysis of glycal 180

Reducing sugar 307 required conversion into a $\beta$-glycoside before cyclopropanation could be attempted. Formation of the trichloroacetimidate derivative 309 was attempted, as outlined below (Scheme 4.8). A solution of sugar 307 was dissolved in dichloromethane, then treated successively with DBU and trichloroacetonitrile. Unfortunately, no reaction was observed after overnight reaction at room temperature.<smiles>CC(=O)OC[C@H]1O[C@H](O)C=C[C@H]1OC(C)=O</smiles>

307

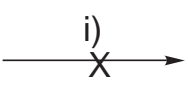

Reagents and Conditions:<smiles>CC(=O)OC[C@H]1O[C@@H](OC(=N)C(Cl)(Cl)Cl)C=CC1OC(C)=O</smiles>

309

Scheme 4.8 Formation of trichloroacetimidate 309

The above discussion has shown that the desired 2,3-unsaturated sugars are difficult to synthesise and, when obtained, are generally quite unreactive to cyclopropanation conditions. As observed in chapter 2, glycals 188, 43 and 189, which have less sterically hindered alkenes than 2,3-unsaturated glycosides (such as 290), still required long reaction times to achieve cyclopropanation. Therefore, it was decided that the synthesis of (-)-TAN-2483B would not be readily achieved using the above methodologies, hence alternative strategies were pursued.

\section{$4.4 p$-Methoxybenzyl-Protected Xylal Route to (-)-TAN-2483B}

It was thought that cyclopropanation of an appropriate glycal could be employed in the synthesis of (-)-TAN-2483B. The stereochemical configurations at C-3 and C-4 of D-xylal match those at C-2 and C-3 of (-)-TAN-2483B. A retrosynthetic scheme illustrating how a protected D-xylal-derived oxepine could provide the necessary requirements has been presented earlier (page 34). A more detailed synopsis of the proposed D-xylal-based synthesis of (-)-TAN-2483B is shown below (Scheme 4.9). 


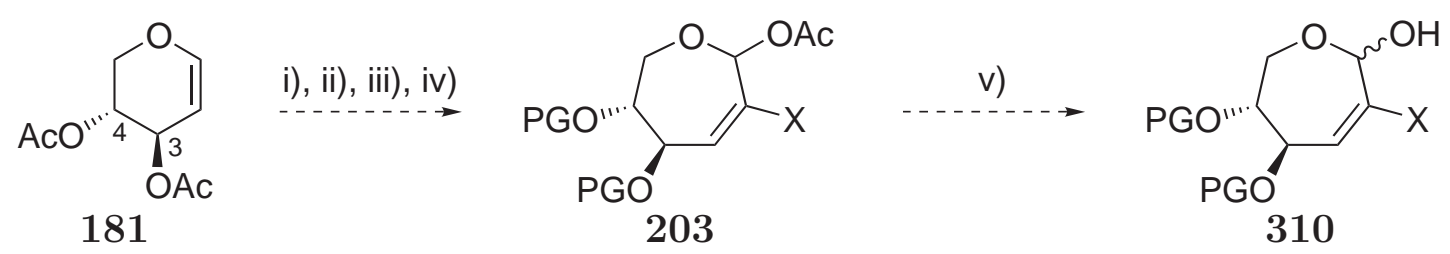

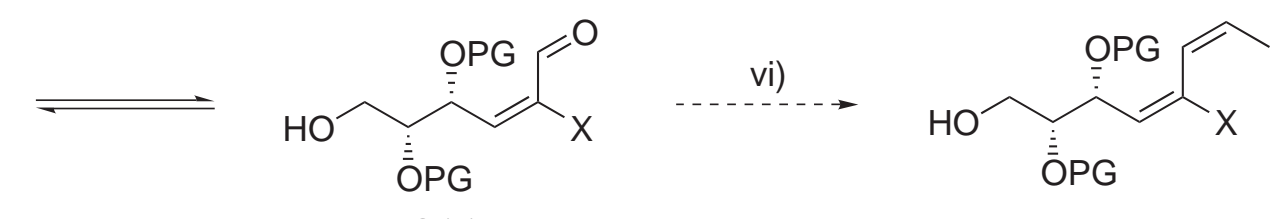

311<smiles>[X]C(=C[C@@H](O)C=CC)C1OC1C</smiles>

312

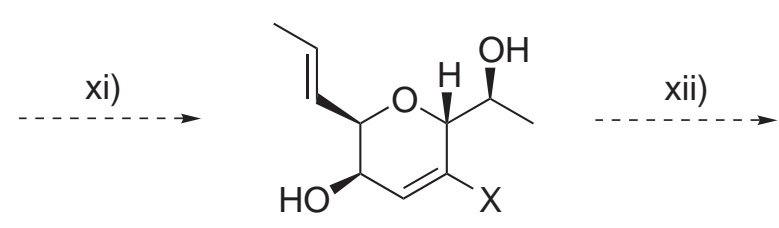

200<smiles>C/C=C/[C@H]1O[C@H]2C(=C[C@H]1O)C(=O)O[C@H]2C</smiles>

154

$(-)-\mathrm{TAN}-2483 \mathrm{~B}$

Reagents and Conditions: i) $\mathrm{NaOMe}, \mathrm{MeOH}$, r.t.; ii) $\mathrm{PGX}, \mathrm{NaH}, \mathrm{DMF}, 0{ }^{\circ} \mathrm{C}$ to r.t.; iii) $\mathrm{CHBr}_{3}$, $\mathrm{TEBAC}, \mathrm{NaOH} / \mathrm{KF}_{(\mathrm{aq})}$, r.t.; iv) $\mathrm{AgOAc}, \mathrm{NaOAc}$, toluene, $100{ }^{\circ} \mathrm{C}$; v) $\mathrm{NaOMe}, \mathrm{MeOH}$, r.t.; vi) $\mathrm{EtPPh}_{3} \mathrm{I}, n$-BuLi, $-78{ }^{\circ} \mathrm{C}$ to r.t.; vii) TEMPO, BAIB;

viii) 5-ethylsulfonyl-1-phenyl- $1 H$-tetrazole, KHMDS, DME; ix) $\mathbf{3 1 3}, \mathrm{NaOCl}_{(\mathrm{aq})}, \mathrm{CH}_{2} \mathrm{Cl}_{2}$, r.t.; x) deprotection; xi) $\mathrm{NaH}$, THF, r.t.; xii) $\mathrm{Pd}\left(\mathrm{PPh}_{3}\right)_{4}, \mathrm{CO}, \mathrm{NEt}_{3}$.

Scheme 4.9 Proposed xylose-based synthesis of (-)-TAN-2483B

Protected D-xylal-derived oxepines (203) are accessible from di- $O$-acetyl-D-xylal (181) through a four step sequence involving deprotection, reprotection, cyclopropanation and silver-mediated ring expansion (see sections 2.7 and 3.5). Deprotection of the anomeric acetate would provide sugar 310, which would be in equilibrium with the ring-opened form 311. A Wittig reaction might provide diene 202, then the sidechain of (-)-TAN-2483B could be obtained through oxidation and Julia-Kocienski olefination to provide triene 201. This triene might be selectively epoxidised through the use of $(R, R)$-Jacobsen's catalyst (313) (Figure 4.1). ${ }^{91}$ This reaction is selective for cis-alkenes, and would hopefully provide epoxidation predominantly on the top face of triene 201. Deprotection to form diol 312 could be followed by cyclisation through treatment with base. This would provide either the desired pyran derivative $\mathbf{2 0 0}$ through attack of the C-7 alcohol, or the undesired furan derivative if the C-6 alcohol attacks the epoxide. If the former cyclisation occurs, a palladium-catalysed carbonylation and lactonisation sequence would provide (-)-TAN-2483B (154). 


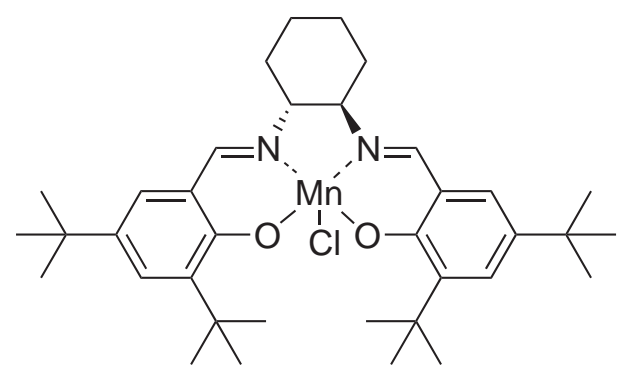

313

Figure 4.1 $(R, R)$-Jacobsen's catalyst ${ }^{91}$

The $p$-methoxybenzyl protecting group is stable to a variety conditions, and may be cleaved through reaction with DDQ, whereas standard debenzylation conditions $\left(\mathrm{Pd} / \mathrm{C}, \mathrm{H}_{2}\right)$ are likely to hydrogenate the double bonds present in the skeleton of the relevant intermediate. Di- $O$-( $p$-methoxybenzyl)-D-xylal (314) was synthesised from glycal 181 through sodium methoxide-based deprotection, then successive treatment with sodium hydride and $p$-methoxybenzyl chloride (Scheme 4.10).

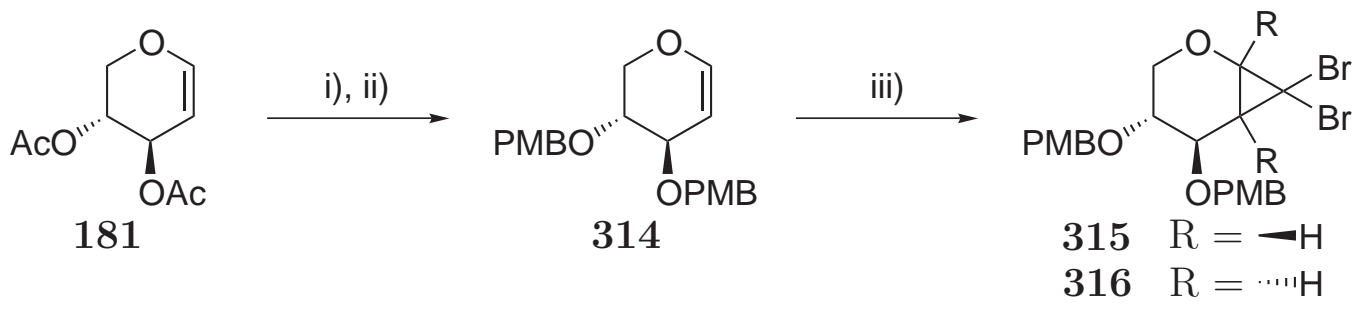

Reagents and Conditions: i) $\mathrm{NaOMe}, \mathrm{MeOH}$, r.t. 1 h; ii) $\mathrm{PMBCl}, \mathrm{NaH}, \mathrm{DMF}, 0{ }^{\circ} \mathrm{C}$ to r.t., overnight, $40 \%$ over 2 steps; iii) $\mathrm{CHBr}_{3}$, TEBAC, $\mathrm{NaOH} / \mathrm{KF}_{(\text {aq) }}$, r.t., 1 d, $30 \%$.

Scheme 4.10 Synthesis and cyclopropanation of xylose-based glycal 314

Cyclopropanation of glycal $\mathbf{3 1 4}$ was then attempted, using modified Mąkosza conditions. After one day the starting material had been consumed, providing cyclopropanes $\mathbf{3 1 5}$ and $\mathbf{3 1 6}$ in $30 \%$ yield (3.2:1 ratio as determined from the crude proton NMR spectrum). The xylal skeleton favours carbene addition from the $\alpha$-face, as seen in the cyclopropanation of the benzyl analogue 189, which provided a similar ratio of cyclopropanes $(4.8: 1)$. The low yield obtained was presumably due to degradation of material, as low-polarity spots were observed upon TLC analysis of the reaction mixture.

Major cyclopropane $\mathbf{3 1 5}$ was then subjected to silver-mediated ring expansion, using methodology developed earlier (described in section 3.2). Thus, a solution of cyclopropane $\mathbf{3 1 5}$ in acetic acid was treated with silver acetate, then allowed to react at $100{ }^{\circ} \mathrm{C}$ for two days. The major product obtained from this reaction was neither of the expected acetates 317 and 318, but bicyclic acetal 319, isolated in $40 \%$ yield (Scheme 4.11 ) 
<smiles>CC(C)C</smiles>

315

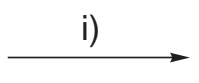

PMBO

319

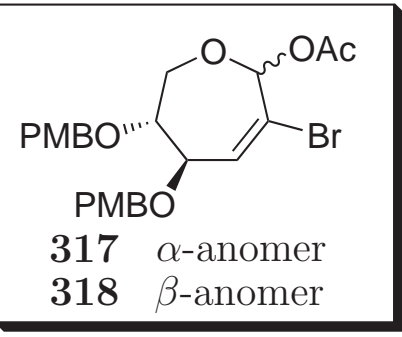

$317 \alpha$-anomer

$318 \beta$-anomer

Reagents and Conditions: i) $\mathrm{AgOAc}, \mathrm{AcOH}, 100{ }^{\circ} \mathrm{C}, 2 \mathrm{~d}, 41 \%$.

Scheme 4.11 Unsuccessful ring-expansion of cyclopropane 315

The appearance of compound 319 was not entirely unexpected, as previously discussed reactions have also exhibited similar behaviour. However, this particular reaction was noteworthy, as no acetyl glycosides were observed, even though there was a solvolytic amount of acetic acid available to participate as the nucleophile. The high propensity for intramolecular nucleophilic attack suggests that the $p$-methoxybenzyl group is too labile for this chemistry, which was further exemplified by the modest yields obtained in this series of reactions. Therefore, exploration of the benzylprotected analogues was then investigated, in the hope of testing further steps of this D-xylose-based route.

\subsection{Benzyl-Protected Xylal Route to $(-)$-TAN-2483B}

Earlier synthesis of D-xylose-derived acetyl oxepines 271 and 272 (see section 3.5) provided the opportunity to immediately test the proposed chemistry of benzylprotected D-xylal. Initial efforts focused on conversion of oxepines 271 and 272 into 320, through deacetylation and olefination (Scheme 4.12).<smiles>Br[C@H]1CO[C@H](Br)[C@H](Br)[C@H]1Br</smiles>

243<smiles>CC(=O)O[C@H]1OC[C@@H](Br)[C@@H](Br)C=C1Br</smiles>

$271 \alpha$-anomer

$272 \beta$-anomer<smiles>OC1OC[C@@H](Br)[C@H](Br)C=C1Br</smiles>

321<smiles>C/C=C\C(Br)=C/[C@H](OBr)[C@H](Br)CO</smiles>

320

Scheme 4.12 Initial benzyl xylal-based steps to (-)-TAN-2483B 
D-Xylose-derived cyclopropane $\mathbf{2 4 3}$ was subjected to the standard ring-expansion conditions outlined in chapter 3. Upon isolation of oxepines 271 and 272, the mixture was purified through a silica plug, then immediately deacetylated using catalytic sodium methoxide in methanol. However, the isolated product from this reaction was not the expected heptanose 321. NMR evidence indicated the product obtained was a mixture of isomers of aldehyde 322, (Scheme 4.13). Unfortunately the mixture of products attributed to 322 provided extremely complicated NMR spectra, which were not able to be assigned. In the proton spectrum there was a series of four aldehydic peaks from 9.5 to $9.4 \mathrm{ppm}$, which indicated aldehydes with all possible stereochemical configurations at C-2 and C-3 of $\mathbf{3 2 2}$ might have been obtained. The carbon NMR spectrum corroborated this observation, with three resolved peaks in the aldehydic region (192-190 ppm). Approximately half of the peaks corresponding to $\mathrm{sp}^{3}$-hybridised carbons were observed between 85 and $80 \mathrm{ppm}$ in the carbon NMR spectrum. Precedence within this research project shows this is indicative of $C$-furanosyl sugars; pyranose and heptanose sugars typically have the vast majority of these signals upfield of $80 \mathrm{ppm}$.

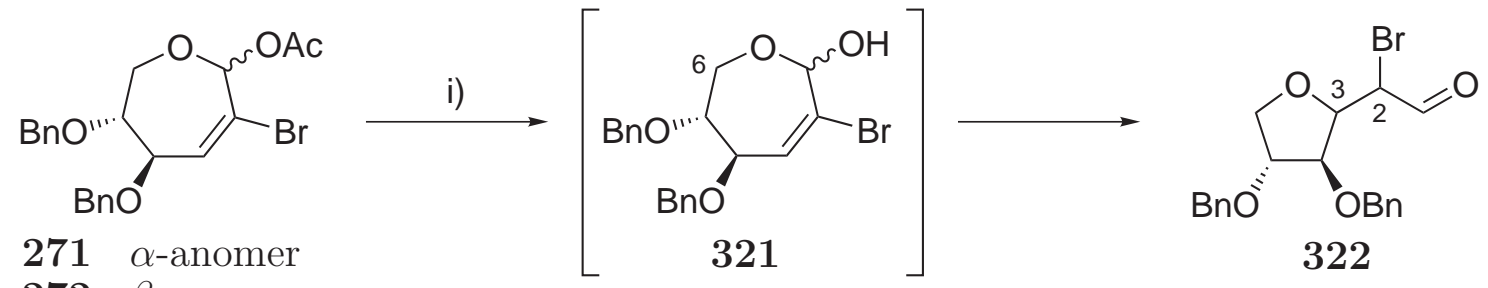

$272 \beta$-anomer

Reagents and Conditions: i) NaOMe, MeOH, r.t., 2 h.

Scheme 4.13 Formation of furanosyl aldehyde 322

The appearance of $\mathbf{3 2 2}$ is most likely due to an intramolecular 1,4-addition of the C-6 hydroxyl to the $\alpha, \beta$-unsaturated aldehyde moiety of 323, as shown in Scheme 4.14. Similar reactivity has been observed earlier (see section 3.7), although this example appears to show even greater propensity for 1,4-addition than the previously studied glycosides. As 323 has a free hydroxyl present, 1,4-conjugate addition can occur much more readily than for glycosides. Skattebøl studied the reaction of simple acetal oxepines with acid, and reported the formation of unexpected products, which were assigned as pyranosyl aldehydes. ${ }^{17}$ The proposed mechanism bore strong resemblance to that shown in Scheme 4.14, though in the previously reported case, the $\alpha$-halo aldehyde (such as $\mathbf{3 2 2}$ ) was thought to be an intermediate, not an observed product.

The formation of furanosyl product $\mathbf{3 2 2}$ might be suppressed if aldehyde $\mathbf{3 2 3}$ was immediately derivatised. Thus, a one-pot synthesis of alkene $\mathbf{3 2 0}$ was attempted, through addition of a solution of oxepines $\mathbf{2 7 1}$ and $\mathbf{2 7 2}$, in addition to potassium carbonate to the pre-formed ylide derived from treatment of ethyltriphenylphosphonium iodide (324) with KHMDS. ${ }^{132}$ However, this led to a complex mixture 
<smiles>O[C@H]1OC[C@@H](Br)[C@H](OBr)C=C1Br</smiles>

321<smiles>O=CC1=CC(Br)C(Br)C(Br)C1CO</smiles>

323

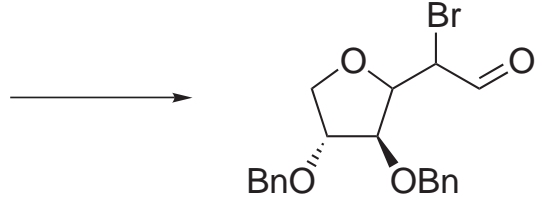

322

Scheme 4.14 Intramolecular 1,4-addition of xylose derivative 323

of products, in which $\alpha$-hydroxy acetal 325 was the only identifiable component (Scheme 4.15). Formation of the dimethyl acetal might be rationalised due to the presence of methanol within the reaction (though acid was not present); hydrolysis of the bromide moiety might have occurred upon workup.
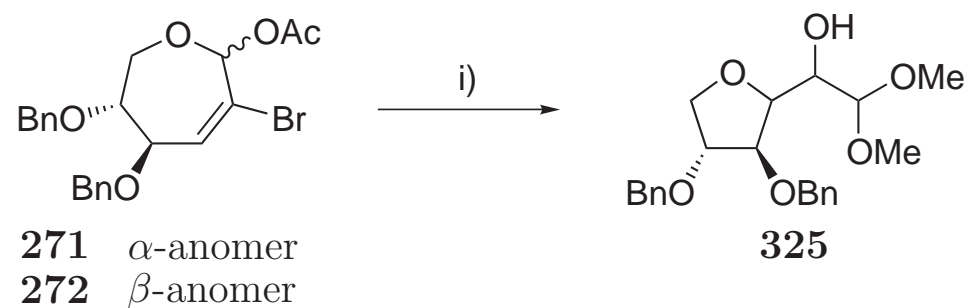

Reagents and Conditions: i) $\mathrm{K}_{2} \mathrm{CO}_{3}, \mathrm{Ph}_{3} \mathrm{P}=\mathrm{CHCH}_{3}, \mathrm{MeOH}$, THF, r.t., 2 h, $24 \%$.

Scheme 4.15 Attempted deprotection/olefination of oxepines 271 and 272

A mixture of oxepines 271 and 272 was subjected to more selective anomeric deacetylation conditions, using an established procedure ${ }^{133}$ in which 271 and 272 were treated with acetic acid and ethylene diamine. This buffered system was thought to be sufficiently mild to avoid any 1,4-addition-derived side products. Unfortunately, low consumption of starting material was observed when this reaction was performed, and the only products observed appeared to be aldehydic $C$-furanosyl sugars once again, as indicated by both proton and carbon NMR spectra of the crude reaction mixture. Therefore, this route was abandoned due to this apparently unavoidable molecular rearrangement.

\subsection{Summary of Chapter 4}

This chapter has detailed the unsuccessful attempts at synthesising (-)-TAN-2483B from galactose or xylose. Reactions with galactose show preparation of suitable 2,3-unsaturated carbohydrate precursors for cyclopropanation is often difficult, and these unsaturated sugars are not readily cyclopropanated. Studies involving xylalbased precursors has shown more success. Unfortunately, oxepines such as $\mathbf{2 7 1}$ and 272 appear to be unsuitable for deprotection at the anomeric centre, as they undergo facile intramolecular 1,4-addition to provide furanosyl sugars. An alternative route that circumvents these issues will be discussed in chapter 5 . 


\section{Chapter 5}

\section{Approaches to (-)-TAN-2483B from Mannose}

\subsection{Introduction}

The unsuccessful attempts to synthesise (-)-TAN-2483B through the use of Dgalactose-derived alkene 290 and D-xylose-derived cyclopropane 243 (Figure 5.1) were detailed in chapter 4 . Valuable insights into the chemistry involved led to the development of a new synthetic strategy through the use of D-mannose.<smiles>CC(C)[Pb]C1OC[C@H]2OC(c3ccccc3)C=C[C@@H]2O1</smiles>

290<smiles>Br[C@H](O[C@H]1OC[C@H](Br)[C@H](Br)[C@H]1Br)c1ccccc1</smiles>

243

Figure 5.1 Cyclopropanes 290 and 243

The D-galactose and D-xylose-based routes were unsuccessful for two main reasons: Firstly, cyclopropanation of 2,3-unsaturated sugars is often difficult, as exemplified by the attempted cyclopropanation of $\mathbf{2 9 0}$. Whilst xylose-derived cyclopropane $\mathbf{2 4 3}$ was synthesised in good yield, this route subsequently provided furanose sugars, requiring investigation of alternative routes. However, it appears likely that use of a lower homologue (Scheme 5.1), such as furanoglycal derivative 326, would be less prone to such a side reaction, as conjugate addition would provide a highly unfavourable four-membered oxetane ring (327). 
<smiles>COC1C(Br)C=C(C=O)C(O)C1Br</smiles>

323<smiles>[X]C(C=O)=CC(O)C([R])OBr</smiles>

326<smiles>O=CC(Br)C1OC[C@H](OCc2ccccc2)C1Br</smiles>

322<smiles>[X]C(C=O)C1OC([R])[C@H]1OBr</smiles>

327

Scheme 5.1 Intramolecular conjugate addition of 323 vs. 326

With these considerations in mind, a cyclopropanated furanoglycal precursor with general structure 328 might provide access to (-)-TAN-2483B (154) (Scheme 5.2). Cyclopropanation of a furanoglycal might proceed more readily than the unsaturated pyranoglycals used previously. gem-Dihalocyclopropanes that are part of the bicyclo[3.1.0] hexane skeleton have been used previously in natural product synthesis, ${ }^{13}$ and this provided further encouragement to explore this route.

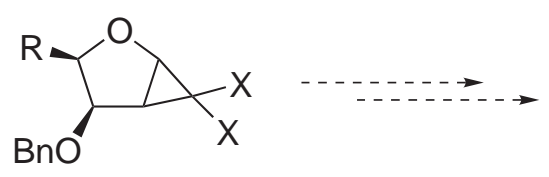

328<smiles>[X]/C(C=O)=C/[C@H](OBr)C([R])O</smiles>

326<smiles>C/C=C/[C@H]1O[C@H]2C(=C[C@H]1O)C(=O)O[C@H]2C</smiles>

154

(-)-TAN-2483B

Scheme 5.2 Proposed furanoglycal-based route to (-)-TAN-2483B

Fischer's glycal synthesis provides access to pyranoglycals, however when this methodology is applied to furanose sugars, fully unsaturated furan derivatives are obtained. ${ }^{134}$ Glycal 209 has been synthesised in four steps from D-mannose through a radical-mediated process. ${ }^{34}$ Based on the retrosynthetic scheme previously shown (page 34), the following synthesis of (-)-TAN-2483B was designed (Scheme 5.3).

Glycal 209 might undergo cyclopropanation to provide 208. Acetate-mediated ring expansion, followed by deacetylation, would afford hemiacetal 207. The ringopened form (329) could undergo a Wittig reaction with an ethylidene ylide, which could then be stereoselectively epoxidised to form intermediate 330, which contains all the required stereogenic centres of (-)-TAN-2483B. Epoxide 330 could undergo intramolecular cyclisation reaction, followed by a palladium-catalysed carbonylation/lactonisation sequence to provide 205, containing the furo[3,4- $b]$ pyran5-one skeleton. Finally, acetonide removal and diol cleavage would provide aldehyde 331. This aldehyde could then undergo Julia-Kocienski olefination to append the sidechain, then deprotection would provide (-)-TAN-2483B (154). 
<smiles>CC(C)C#CC(C)C</smiles>

209<smiles>[X]C1=CC(O)C([C@H]2COC(C)(C)O2)O[C@H]1O</smiles>

207<smiles>[X]C(=O)C1(C)CC1C(O)C(O)C(O)C1COC(C)(C)O1</smiles>

330<smiles>[X]C1C([R9])C2C([R9])C(C3COC(C)(C)O3)OC12</smiles>

208<smiles>C[C@H]1OC(=O)C2=C[C@H](O)[C@@H](C(=O)O)O[C@H]21</smiles>

331<smiles>[X]/C(C=O)=C/[C@@H](O[Ga])[C@H](O)[C@H](O)[C@H]1COC(C)(C)O1</smiles>

329

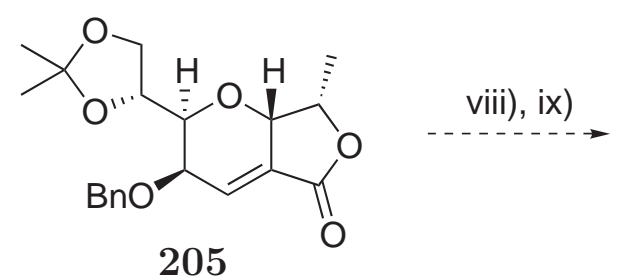<smiles>C/C=C/[C@H]1O[C@H]2C(=C[C@H]1O)C(=O)O[C@H]2C</smiles>

154

(-)-TAN-2483B

Reagents and Conditions: i) $\mathrm{CHX}_{3}, \mathrm{TEBAC}, \mathrm{NaOH} / \mathrm{KF}_{(\mathrm{aq})}$, r.t.; ii) $\mathrm{AgOAc}, \mathrm{NaOAc}$; iii) $\mathrm{NaOMe}, \mathrm{MeOH}$, r.t.; iv) $\mathrm{EtPPh}_{3} \mathrm{I}, n$-BuLi, $-78{ }^{\circ} \mathrm{C}$ to r.t.; v) $313, \mathrm{NaOCl}_{(\mathrm{aq})}, \mathrm{CH}_{2} \mathrm{Cl}_{2}$, r.t.;

vi) $\mathrm{NaH}$, THF, r.t.; vii) $\mathrm{Pd}\left(\mathrm{PPh}_{3}\right)_{4}, \mathrm{CO}, \mathrm{NEt}_{3}$; viii) $\mathrm{AcOH}_{(\text {aq })}$, r.t.; ix) $\mathrm{NaIO}_{4}$, THF (aq) , r.t.; x) 5-ethylsulfonyl-1-phenyl- $1 H$-tetrazole, KHMDS, DME; xi) deprotection.

Scheme 5.3 Proposed mannose-based synthesis of (-)-TAN-2483B

\subsection{Synthesis of Mannose-Based Glycal 209}

Synthetic ventures began with D-mannose, which was subjected to a four-step sequence previously described by Theodorakis, to afford glycal 209. ${ }^{34}$ D-Mannose (332) was reacted in acetone with catalytic iodine, to obtain the diacetonide derivative $\mathbf{3 3 3}$ in $86 \%$ yield.

Diacetonide derivative 333 was then converted into glycosyl chloride 334 , through reaction with tosyl chloride and DMAP. ${ }^{135}$ Glycosyl chloride 334 was then added to a freshly prepared solution of sodium naphthalenide in THF. Through radical initiation at the anomeric centre and loss of acetone, glycal 335 was formed. This mixture was immediately benzylated to provide fully protected glycal $\mathbf{2 0 9}$, obtained from D-mannose in $43 \%$ yield over four steps. 
<smiles>CC(C)C</smiles>

332<smiles>CC1(C)OC2C(O1)[C@@H](O)OC2[C@H]1COC(C)(C)O1</smiles><smiles>CC#CC</smiles><smiles>CC#CC1C=CC(O)C1[C@H]1COC(C)(C)O1</smiles>

335

209

Reagents and Conditions: i) $\mathrm{I}_{2}$, acetone, r.t., 3 h, $86 \%$; ii) DMAP, TsCl, $\mathrm{NEt}_{3}, \mathrm{CH}_{2} \mathrm{Cl}_{2}$, r.t., 4 h, $71 \%$; iii) $\mathrm{C}_{10} \mathrm{H}_{8}^{--} \mathrm{Na}^{+}$, THF, $0{ }^{\circ} \mathrm{C}, 10 \mathrm{~min}$; iv) $\mathrm{NaH}, \mathrm{BnBr}$, TBAI, THF, $0{ }^{\circ} \mathrm{C}$ to r.t., overnight, $71 \%$ over 2 steps.

Scheme 5.4 Synthesis of mannose-based glycal 209

\subsection{Synthesis of the Pyran Ring of (-)-TAN-2483B}

The pyran ring of (-)-TAN-2483B was constructed through a cyclopropanation/ring expansion sequence of glycal 209 (Scheme 5.5). The first of these steps would provide cyclopropane 208, which could then undergo ring expansion in the presence of an acetate nucleophile to provide glycosides 336 and $\mathbf{3 3 7}$. This could then be deprotected to provide key hemiacetal 207, suitable for homologation to the full furo $[3,4-b]$ pyran-5-one system.<smiles>CC1(C)OC[C@H](C2OC=C[C@@H]2Br)O1</smiles>

209<smiles>[X]C1=C[C@@H](O)[C@H]([C@H]2COC(C)(C)O2)O[C@H]1OC(C)=O</smiles>

336,337

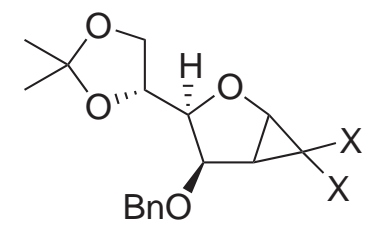

208

Scheme 5.5 Key cyclopropanation/expansion sequence 


\subsubsection{Cyclopropanation of Glycal 209}

The cyclopropanation of glycal 209 with dibromocarbene was then attempted under the modified Mąkosza conditions used previously. The reaction mixture rapidly became a very dark-brown colour, and TLC analysis indicated starting material had been consumed after only 90 minutes. This reaction provided a complicated mixture of byproducts from which ethyl glycosides 338 and 339 were isolated in a combined yield of $26 \%$ (Scheme 5.6).<smiles>CC1(C)OC[C@H](C2OC=C[C@@H]2Br)O1</smiles>

209

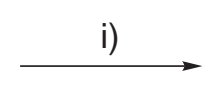

Conditions:

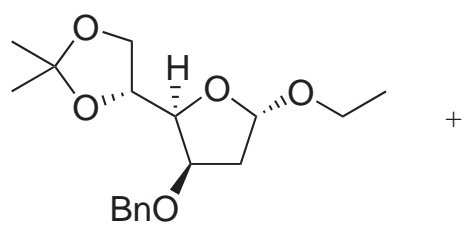

338

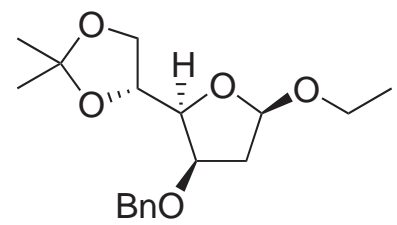

339

Reagents and Conditions: i) $\mathrm{CHBr}_{3}, \mathrm{TEBAC}, \mathrm{NaOH} / \mathrm{KF}_{(\mathrm{aq})}$, r.t., $90 \mathrm{~min}$.

Scheme 5.6 Attempted gem-dibromocyclopropanation of glycal 209

These products were presumably formed by addition of ethanol to the glycal bond. The bromoform used was stabilised with 1-3\% (v/v) ethanol, corresponding to 0.3-0.9 equivalents, relative to the amount of glycal 209. Therefore, a portion of bromoform was purified to remove the ethanol. Unfortunately, subsequent cyclopropanation using this ethanol-free bromoform led to only unidentifiable mixtures of compounds.

In contrast, cyclopropanation with dichlorocarbene, generated in the presence of $50 \%$ (w/w) sodium hydroxide, proceeded much more cleanly. These conditions provided a much cleaner reaction, with full consumption of starting material observed after just four hours. Cyclopropane $\mathbf{3 4 0}$ was obtained in 50\% isolated yield, after purification (Scheme 5.7).
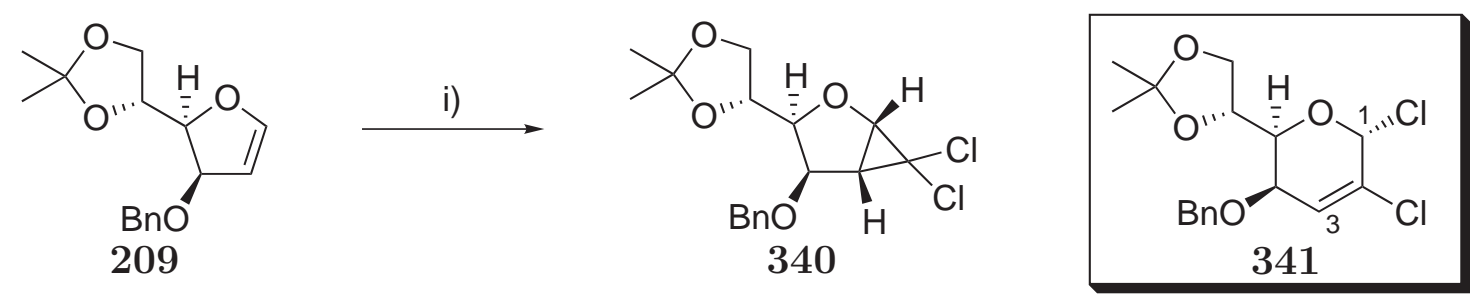

Reagents and Conditions: i) $\mathrm{CHCl}_{3}$, TEBAC, $\mathrm{NaOH}_{(\mathrm{aq})}$, r.t., 4 h, $50 \%$.

Scheme 5.7 Synthesis of mannose-based cyclopropane $\mathbf{3 4 0}$

This cyclopropanation proved to be very reproducible, and could be conducted under air, with no observable loss of yield. While the isolated yield of $50 \%$ was modest, the actual amount of cyclopropane obtained upon workup was significantly higher. A byproduct ran just below cyclopropane $\mathbf{3 4 0}$ on the TLC plate, which led to poor separation and yields of this product. The contaminant was believed to be $\mathbf{3 4 1}$, 
due to the presence of two peaks at approximately $6.2 \mathrm{ppm}$ in the proton NMR spectrum of the crude reaction mixture, which might correspond to $\mathrm{H}-1$ and $\mathrm{H}-3$ of glycosyl chloride 341. Bergman has observed a similar mixture of cyclopropane and rearranged derivative when reacting dichlorocarbene with cyclopentene. ${ }^{136}$ It was later determined that rigorous purification of this cyclopropane was not necessary for the next reaction, as byproduct 341 would likely react to provide the same ring-expanded products as cyclopropane 340. Cyclopropane 340 was obtained in $79 \%$ yield when isolated with the contaminant, presumed to be $\mathbf{3 4 1}$, the rearranged derivative of cyclopropane $\mathbf{3 4 0}$.

\subsubsection{Ring Expansion of Mannose-Derived Cyclopropane 340}

Cyclopropane 340 was ring-expanded using similar methodology to that featured in chapters 3 and 4 . It was anticipated that the use of acetate as a nucleophile in cyclopropyl ring expansion, followed by hydrolysis, would furnish the chloro- version of intermediate 207 (Scheme 5.5). Furan-fused gem-dichlorocyclopropane $\mathbf{3 4 0}$ would probably not require the high temperatures needed for pyran-based cyclopropanes discussed in chapters 3 and 4, due to greater ring strain in the bicyclic system, and the comparative stability of the six-membered ring products. Thus, the ringexpansion reaction was performed with silver acetate (1.5 eq.) and sodium acetate (5 eq.) in refluxing THF $\left(65-67^{\circ} \mathrm{C}\right)$, rather than at $100{ }^{\circ} \mathrm{C}$ in toluene. This reaction proceeded rapidly, with full consumption of starting material observed after four hours. Upon purification, an inseparable mixture of desired acetyl glycosides $\mathbf{3 4 2}$ and $\mathbf{3 4 3}$ (49\%, 3:1 ratio) was obtained (Scheme 5.8). Glycal 344, formed from acetate attack at $\mathrm{C}-2$ of $\mathbf{3 4 0}$, was also obtained (16\% yield). This result demonstrates the efficient formation and ring-expansion of these bicyclo[3.1.0]hexane-based cyclopropanes, with both reactions occurring within a few hours. In contrast, the synthesis (see chapter 2) and ring-expansion (see chapter 3) of glucal-derived cyclopropane $\mathbf{9 7}$ required two days for each reaction.

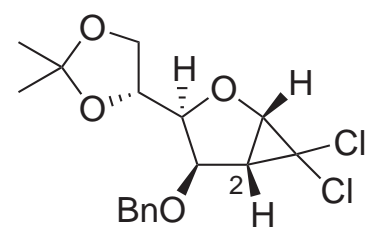

340

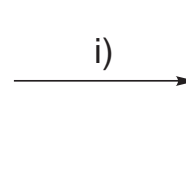

$342 \alpha$-anomer

$343 \beta$-anomer<smiles>CC(=O)OC1C(Cl)=CO[C@@H]([C@H]2COC(C)(C)O2)[C@H]1Br</smiles>

344

Reagents and Conditions: i) AgOAc, NaOAc, THF, reflux, 4 h.

Scheme 5.8 Ring-expansion of mannose-derived cyclopropane $\mathbf{3 4 0}$ 
Direct solvolysis was investigated, through substitution of sodium acetate for acetic acid. The cyclopropane was treated with silver acetate in a 4:1 mixture of THF and acetic acid, then refluxed. This led to much better regioselectivity, with a 12:1 ratio between C-1 (342 and 343) and C-3 (344) acetate isomers. However, the acetyl glycosides 342 and $\mathbf{3 4 3}$ were isolated in only $56 \%$ yield, presumably due to the presence of acetic acid causing partial deprotection of the isopropylidene protecting group. To improve the observed regioselectivity, later ring-expansion reactions were performed using glacial acetic acid as the solvent (342 and 343:344, 30:1 ratio). The absence of THF also allowed the use of higher temperatures, and a subsequent reduction in reaction time to only two hours when the reaction was performed at 80 or $90{ }^{\circ} \mathrm{C}$. In later reactions partial deprotection was still observed, presumably due to the presence of water; use of a fresh sample of glacial acetic acid solved this problem, providing glycosides $\mathbf{3 4 2}$ and $\mathbf{3 4 3}$ in $83 \%$ combined yield.

As mentioned above, mixtures of cyclopropane $\mathbf{3 4 0}$ and glycosyl chloride $\mathbf{3 4 1}$ were isolated together in high yields. It was thought that acetic acid solvolysis of both these regioisomers would provide glycosides 342 and 343, therefore the crude cyclopropanation product was cleaned by rapid elution through silica gel, which minimised degradation of $\mathbf{3 4 0}$. The mixture of $\mathbf{3 4 0}$ and $\mathbf{3 4 1}$ was immediately subjected to silver-mediated acetic acid solvolysis, which provided acetyl glycosides $\mathbf{3 4 2}$ and 343 in $77 \%$ yield over two steps (Scheme 5.9). This high yield was very pleasing, showing that conversion of glycal 209 into acetyl glycosides 342 and 343 was much more straightforward than the corresponding oxepines described in chapter 3.

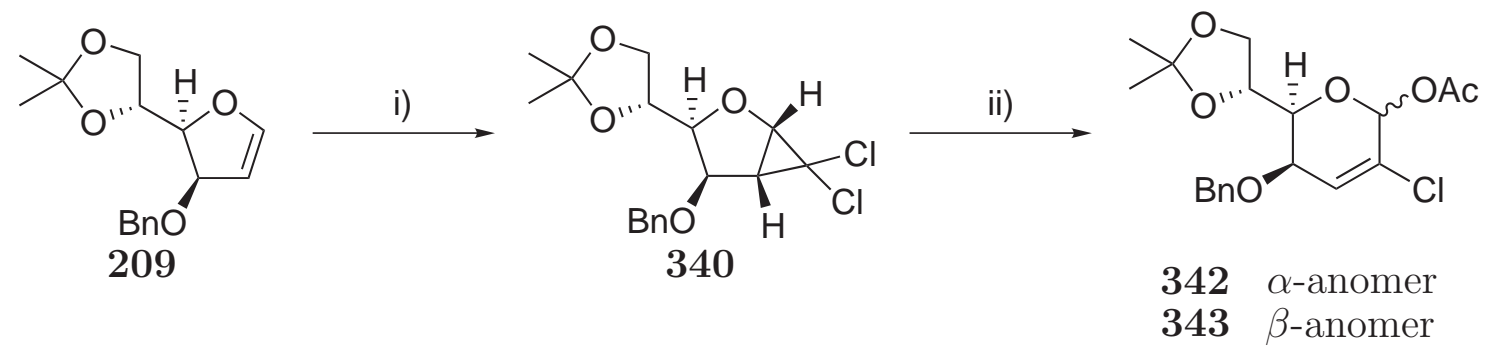

Reagents and Conditions: i) $\mathrm{CHCl}_{3}$, TEBAC, $\mathrm{NaOH}_{(\mathrm{aq})}$, r.t., 2.5 h; ii) $\mathrm{AgOAc}, \mathrm{AcOH}, 80{ }^{\circ} \mathrm{C}$, $2 \mathrm{~h}, 77 \%$ over 2 steps.

Scheme 5.9 Cyclopropanation and ring-expansion of mannose-derived glycal 209 Deprotection of 342 and 343 utilised sodium methoxide, through addition of a catalytic amount of sodium to a methanolic solution of acetates 342 and $343 .{ }^{122}$ Within one hour, full deprotection occurred, then purification by elution through a silica plug provided hemiacetal 345 in quantitative yield (Scheme 5.10). This material was deemed to be pure enough for subsequent reactions. 
<smiles>CC(=O)OC1OC([C@H]2COC(C)(C)O2)[C@H](O)C=C1Cl</smiles>

$342 \alpha$-anomer

$343 \beta$-anomer

Reagents and Conditions: i) $\mathrm{NaOMe}, \mathrm{MeOH}$, r.t., 1 h, quantitative.

Scheme 5.10 Synthesis of key hemiacetal 345

\subsection{Functionalisation of the Pyran Ring of (-)-TAN-2483B}

\subsubsection{Ethylidene Wittig-Based Route}

As outlined in Scheme 5.3, (-)-TAN-2483B was thought to be accessible through synthesis and subsequent reaction of epoxide 330, which might be formed from masked aldehyde 345 (Scheme 5.11).

Wittig reaction of hemiacetal 345 utilised ethylidenetriphenylphosphorane, generated from treatment of phosphonium salt 324 with $n$-butyllithium. After reaction in refluxing THF, an inseparable mixture of alkene isomers was obtained (2:1 ratio), in favour of the desired $(Z)$-alkene 346 ; presumably the small size of the ylide reagent substituents ( $\mathrm{H}$ vs. $\mathrm{Me}$ ) does not exclude formation of the $(E)$-alkene $\mathbf{3 4 7}$. Reaction at room temperature was sluggish, and the use of KHMDS led to greater impurities in crude product mixtures. Use of alternative solvents $\left(\mathrm{CH}_{2} \mathrm{Cl}_{2}, \mathrm{DME}\right.$, DMF) provided mixtures with $(Z):(E)$ ratios of only 1.2-1.6. When the reaction was performed in toluene, significant degradation of material was observed. The required $(Z)$-alkene might be accessed through a Corey-Fuchs reaction; subsequent methylation of the terminal alkyne and cis-selective reduction with Lindlar catalyst could provide stereoselective access to diene $\mathbf{3 4 6}$.

A cis-selective asymmetric Jacobsen epoxidation was then attempted, ${ }^{91}$ in which it was hoped epoxide $\mathbf{3 3 0}$ would be obtained. Initially, a control reaction using ( $Z$ )hex-3-en-1-ol as the epoxidation substrate provided partial conversion into epoxides. Use of $(R, R)$-Jacobsen's catalyst (313) with olefin 346 should ensure epoxidation occurs predominantly from the top face to provide the required epoxide $\mathbf{3 3 0}$. This reaction gave a multitude of products, which appeared to be due to significant degradation. A portion of starting material (25\%) was isolated, in addition to minor amounts of impure epoxides. Jacobsen epoxidation was therefore abandoned, 
<smiles>CC1(C)OC[C@H]([C@H]2OC(O)C(Cl)=C[C@H]2O)O1</smiles>

345

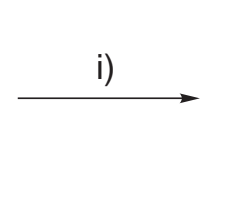

$346(2 Z, 4 E)$-isomer

$347(2 E, 4 E)$-isomer

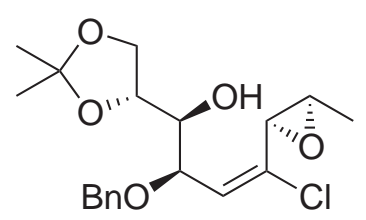

348

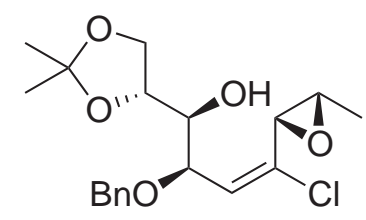

330

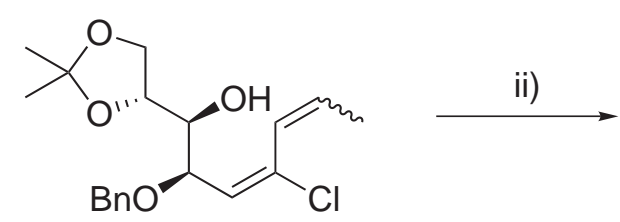

iii)<smiles>CC(O)[C@H]1O[C@@H]([C@@H]2COC(C)(C)O2)C(Cl)=C[C@H]1Br</smiles>

349

Reagents and Conditions: i) $\mathrm{EtPPh}_{3} \mathrm{I}(\mathbf{3 2 4}), n$-BuLi, THF, $-78{ }^{\circ} \mathrm{C}$ to reflux, $4.5 \mathrm{~h}, 77 \%$; ii) $\mathbf{3 1 3}$, $\mathrm{NaOCl}_{(\mathrm{aq})}, \mathrm{CH}_{2} \mathrm{Cl}_{2}$, r.t., 2 d, $3 \%$ or $m$-CPBA, $\mathrm{CH}_{2} \mathrm{Cl}_{2}$, r.t., 2 d, $56 \%$; iii) $\mathrm{NaH}$, DMF, r.t., $1 \mathrm{~d}, 11 \%$.

Scheme 5.11 Ethylidene Wittig/epoxide route

as olefin 346 appeared to be relatively unreactive to this epoxidation methodology. $m$-CPBA was then employed, though this would generate epoxides under achiral conditions. As expected, this provided a complex mixture of products, with all four possible stereoisomers observed (as indicated in the carbon NMR spectrum). No epoxidation of the chloroalkene was observed. However, the major products obtained (330 and 348, 1.5:1 ratio), arising from epoxidation of the $Z$-olefin of major alkene $\mathbf{3 4 6}$, were able to be isolated and characterised as a mixture.

Intramolecular cyclisation of epoxides 330 and 348 was expected to provide access to all C-2 and C-3 diastereomers of the cyclic alcohol 349. Reaction with sodium hydride in THF provided no consumption of starting material, while changing the solvent to DMF produced a complicated mixture of ring-opened products (349) in low yield, in addition to a mixture of starting materials 330 and $\mathbf{3 4 8}$. At this point the mixtures were deemed to be too complicated to pursue further, so alternative methodology was then investigated.

Iodoetherification of $\mathbf{3 4 6}$ was attempted in aid of providing similar functionality to pyran 349. This methodology allowed Snider to form the pyran ring of various furo $[3,4-b]$ pyran-5-one-derived natural products, through incorporation of the oxygen bond at C-2 of (-)-TAN-2483B. ${ }^{88}$ A mixture of alkenes 346 and $\mathbf{3 4 7}$ was added to a freshly prepared solution of bis(2,4,6-trimethylpyridine)iodine(I) hexafluorophosphate (350), generated in situ by treatment of the silver salt (351) with iodine. ${ }^{137}$ Unfortunately, the reaction was extremely sluggish, and was terminated after five days, by which time the electrophilic iodine reagent had decomposed. A significant amount of material had degraded, and upon purification, starting material was recovered (33\%), in addition to minor amounts of products of structure 352 (Scheme 5.12). 


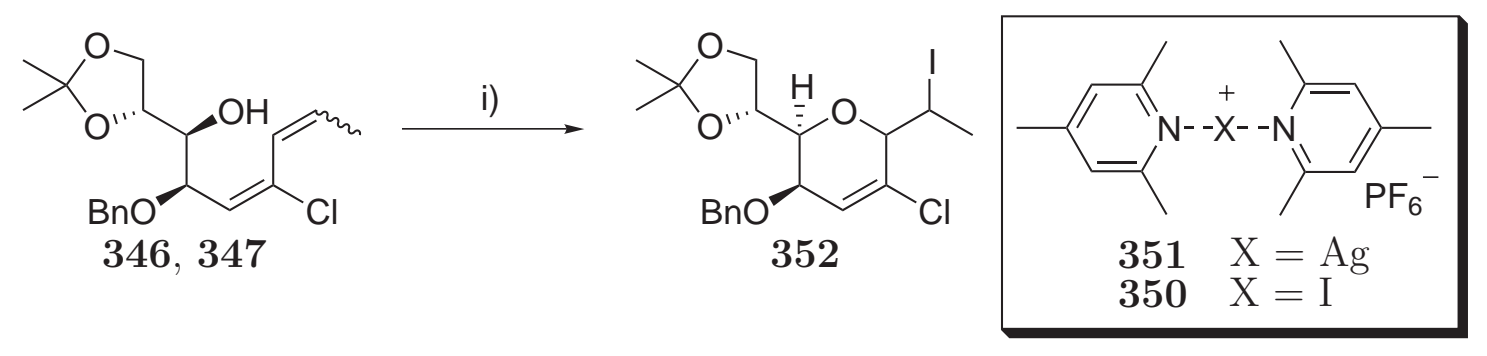

Reagents and Conditions: i) $\mathbf{3 5 0}, \mathrm{CH}_{2} \mathrm{Cl}_{2}$, r.t., $5 \mathrm{~d}, 5 \%$.

Scheme 5.12 Ethylidene Wittig/iodoetherification route

Due to the lack of success with this ethylidene Wittig-derived route, it was decided that the lower homologue, methylidene Wittig-derived diene 353 might be more useful for this epoxide-based synthetic strategy (Scheme 5.13). This would help reduce the number of stereoisomers generated in the olefination and epoxidation reactions. Diene 353 could be used to form either desmethyl (-)-TAN-2483B (354), or (-)-TAN-2483B itself (154), through late-stage installation of the methyl group.

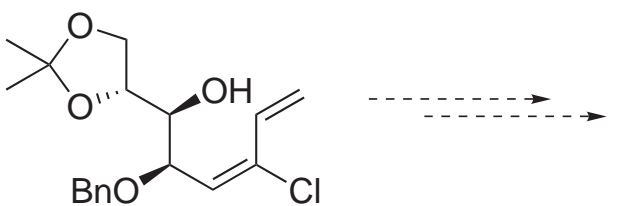

353<smiles>C/C=C/[C@H]1O[C@H]2COC(=O)C2=C[C@H]1O</smiles>

354

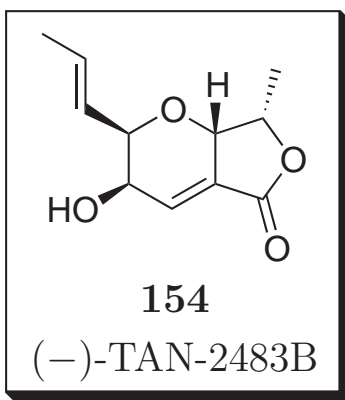

Scheme 5.13 Proposed methylidene Wittig-derived route to (-)-TAN-2483B

\subsubsection{Methylidene Wittig-Derived Route}

Synthesis of methylidene-derived diene 353 began with treatment of a solution of methyl triphenylphosphonium bromide with KHMDS, then addition of masked aldehyde $\mathbf{3 4 5}$ (Scheme 5.14). This provided diene $\mathbf{3 5 3}$ in $69 \%$ yield after 2 hours reaction at room temperature. Upon obtaining diene 353, epoxidation was attempted using $m$-CPBA. Epoxidation predominantly occurred at the terminal olefin, as required, to afford products 355 and 356 in $49 \%$ yield (1.5:1 ratio). Complicated mixtures of other products were observed, which appear to have derived from epoxidation of the alkenyl chloride moiety. Asymmetric epoxidation was also attempted using $(R, R)$-Jacobsen's catalyst $(\mathbf{3 1 3})$, though diene 353 was not very reactive to this epoxidation procedure. Epoxides 355 and 356 were obtained in low yield (18\%) and poor diastereoselectivity (1.2:1 ratio), presumably due to the lack of steric differentiation of the vinyl olefin. 
Treatment of the mixture of epoxides 355 and 356 (1.5:1 ratio) with sodium hydride in DMF initiated pyran ring closure through substitution at the epoxide, providing pyrans 357 and 358 in only $23 \%$ yield (1.5:1 ratio), primarily due to incomplete consumption of starting material (35\% recovered). Alternatively, treatment with a solution of sodium methoxide in methanol provided pyrans $\mathbf{3 5 7}$ and $\mathbf{3 5 8}$ in $42 \%$ yield (3.3:1 ratio), in addition to several minor unidentified products. Comparison of the NMR data with iodopyran 359 (Scheme 5.15) tentatively suggested the major product was the desired $\alpha$ - $C$-glycosyl alcohol 357 .<smiles>CC1(C)OC[C@H]([C@H]2O[C@H](O)C(Cl)=C[C@H]2O[As])[C@@H]1O[Mg]</smiles>

355,356<smiles>C=C/C(Cl)=C\[C@@H](O)[C@H](O)C1COC(C)(C)O1</smiles>

353

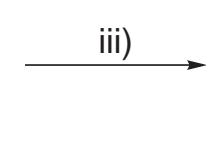<smiles>[R][C@]1(CO)O[C@H]([C@H]2COC(C)(C)O2)[C@@H](OBr)C=C1Cl</smiles>

$357 \mathrm{R}=-\mathrm{H}$

$358 \mathrm{R}=\cdots \cdot \mathrm{H}$

Reagents and Conditions: i) $\mathrm{MePPh}_{3} \mathrm{Br}$, KHMDS, THF, $-78{ }^{\circ} \mathrm{C}$ to r.t., 2 h, $69 \%$; ii) $m$-CPBA, $\mathrm{CH}_{2} \mathrm{Cl}_{2}$, r.t., 2 d, $49 \%$ or $\mathbf{3 1 3}, \mathrm{NaOCl}_{(\mathrm{aq})}, \mathrm{CH}_{2} \mathrm{Cl}_{2}$, r.t., 2 d, $18 \%$; iii) $\mathrm{NaOMe}, \mathrm{MeOH}$, r.t., 2 d, $42 \%$.

Scheme 5.14 Methylidene Wittig/epoxide route

Iodoetherification of diene $\mathbf{3 5 3}$ was also explored (Scheme 5.15). This substrate was much more reactive than the ethylidene analogue, providing iodo sugar 359 in $42 \%$ yield, as a single diastereoisomer, in addition to $38 \%$ of recovered starting material 353. The stereochemical configuration of iodopyran 359 was determined through an nOe correlation; gratifyingly this indicated the stereochemistry of the pyran ring was consistent with that of (-)-TAN-2483B.<smiles>C=C/C(Cl)=C\[C@@H](O)[C@H](O)[C@H]1COC(C)(C)O1</smiles>

353

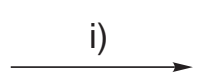

- $\mathrm{nOe}$

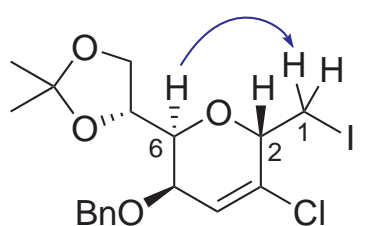

359

Reagents and Conditions: i) $\mathbf{3 5 0}, \mathrm{CH}_{2} \mathrm{Cl}_{2}$, r.t., $22 \mathrm{~h}, 42 \%$.

Scheme 5.15 Methylidene Wittig iodoetherification

Substrates 357 and $\mathbf{3 5 8}$, in addition to iodide 359 were later used for approaches to the construction of the lactone ring of (-)-TAN-2483B, as discussed below. 


\subsubsection{Stabilised Wittig-Based Route to (-)-TAN-2483B}

Given the lack of stereoselectivity observed in many of the reactions involving unstabilised Wittig-derived dienes 346, 347 and 353, reaction of a stabilised ylide with hemiacetal 345 might be exploited in a slightly modified strategy. It was thought that this route would provide intermediate 360 (Scheme 5.16), analogous to ethylidene Wittig-derived 346 (Scheme 5.11). Unfortunately, this would necessitate late-stage removal of the ester functionality, presumably through a reduction/deoxygenation sequence. Reaction of hemiacetal 345 with (ethoxycarbonylmethylene)triphenylphosphorane provided $\alpha, \beta$-unsaturated ester 360 stereoselectively. Base-mediated cyclisation of $\mathbf{3 6 0}$ provided a mixture of functionalised pyrans 361 and 362, of which the former had the same pyran ring stereochemical configuration as (-)-TAN-2483B. C-Glycosyl pyrans 361 and 362 were initially formed in a 1:1.2 ratio, isolated as an inseparable mixture. Their identities were assigned through nOe correlations; simultaneous irradiation of both $\mathrm{H}-7$ signals provided enhancement of one of the H-2 protons of $\mathbf{3 6 1}$, and H-3 of $\mathbf{3 6 2}$.<smiles>CC1(C)OC[C@H]([C@H]2OC(O)C(Cl)=C[C@H]2O)[C@@H]1Br</smiles>

345<smiles>CCCC[C@@H]1O[C@@H]([C@H]2COC(C)(C)O2)[C@H](O)C=C(Cl)[C@@H]1COCC</smiles>

361

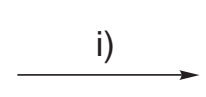

- $\mathrm{nOe}$<smiles>CCOC(=O)/C=C/C(Cl)=C/[C@H](O)[C@H](O)[C@H]1COC(C)(C)O1</smiles>

360

Reagents and Conditions: i) $\mathrm{Ph}_{3} \mathrm{P}=\mathrm{CHCO}_{2}$ Et, THF, r.t. to reflux, 2 d, $63 \%$; ii) NaH, THF, r.t., $15 \min , 94 \%$

Scheme 5.16 Ethoxycarbonylmethylene Wittig/epoxide route

Taylor et al. reported intramolecular conjugate additions of this nature proceeded with greater selectivity for the $\alpha$-glycosyl configuration when the reaction was performed at colder temperatures. ${ }^{138}$ In this case, reaction of substrate 360 at $0{ }^{\circ} \mathrm{C}$ for one hour gratifyingly provided a mixture of $C$-glycosyl pyrans 361 and 362 in a 4:1 ratio in favour of the required $\alpha$-glycosyl compound, 361 .

While the pyran ring of (-)-TAN-2483B was now successfully constructed, efforts to form the furanone ring required additional functionality. As the C-2 methylene group of 361 is at the $\alpha$-position of the ester group, a mono-deprotonation/halogenation sequence would provide an $\alpha$-halo ester such as 363 (Scheme 5.17). Inseparable substrates 361 and 362 (4:1 ratio, shown as pure 361) were added to a freshly prepared solution of LDA, followed by addition of iodine. Upon workup, starting 
materials 361 and 362 (4:1 ratio) were recovered (60\%), in addition to previously isolated unsaturated ester 360 (30\%). The re-appearance of ester $\mathbf{3 6 0}$ was probably due to rearrangement of anion 364. Conjugation within ester 360 indicates anion 364 is more likely to ring open, rather than undergo an intermolecular iodination.

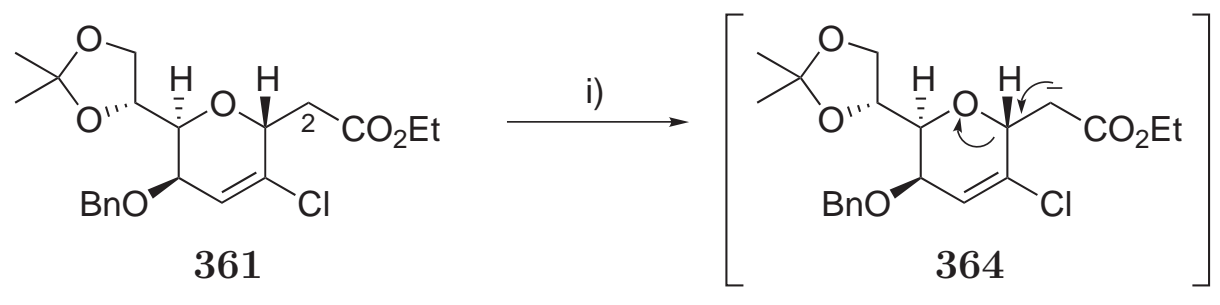<smiles>CCOC(=O)/C=C/C(Cl)=C\[C@H](O)[C@H](O)[C@H]1COC(C)(C)O1</smiles>

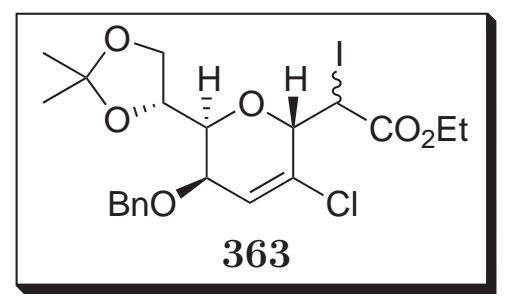

Reagents and Conditions: i) LDA, THF, $-78{ }^{\circ} \mathrm{C}$ to r.t., $50 \mathrm{~min}, 30 \%$;

Scheme 5.17 Mechanism of base-mediated ring opening of ester 361

The difficulty of functionalising the methylene group of pyran 361 is compounded by the high propensity for formation of the highly conjugated ester 360, which is likely to occur under basic conditions, regardless of the halogen source used. In addition, the requirements for late-stage reduction and deoxygenation of the ester functionality would increase the number of required steps, therefore alternative methodologies were investigated.

\subsubsection{HWE/Ramberg-Bäcklund Route to (-)-TAN-2483B}

The Ramberg-Bäcklund reaction has been widely used ${ }^{139,140}$ for converting an $\alpha$ halo-sulfone into an olefin, through base-mediated elimination of sulfur dioxide. The Meyers modification ${ }^{141}$ involves in situ halogenation of a sulfone (365) to provide the alkene (2) in a single-step, one-pot reaction (Scheme 5.18). The RambergBäcklund reaction has shown excellent utility with sulfonated carbohydrates, in which the Franck and Taylor groups have reported many interesting syntheses. ${ }^{142-144}$

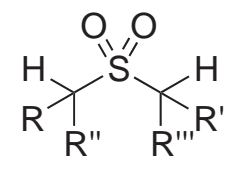

365

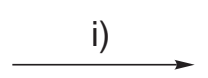<smiles>[R]C([R])=C([3H])[10B]</smiles>

2

Reagents and Conditions: i) $\mathrm{KOH}, \mathrm{CCl}_{4},{ }^{t} \mathrm{BuOH}$, r.t.

Scheme 5.18 The Ramberg-Bäcklund reaction ${ }^{139-141}$ 
It was anticipated that the following Ramberg-Bäcklund approach to (-)-TAN2483B would overcome previous difficulties (Scheme 5.19). Thus, HWE reaction of diethyl methylsulfonylmethylphosphonate (366) with hemiacetal 345 , followed by an intramolecular 1,4-conjugate addition would provide $C$-glycosyl sugar 367 , probably as a mixture with the $\beta$-anomer. Ramberg-Bäcklund reaction of this sulfone would provide vinylic sugar 368. This could be used to construct the bicyclic skeleton of (-)-TAN-2483B through lithium-halogen exchange of the alkenyl chloride, followed by treatment with dry ice, to provide carboxylate functionality. Finally, treatment with an appropriate electrophilic halogen source could lead to iodolactonisation, to provide furo[3,4- $b]$ pyran-5-one--based epimers 369 and $\mathbf{3 7 0 .}$

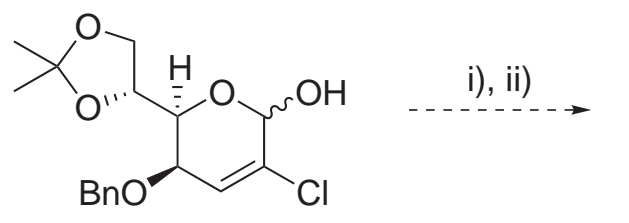

345

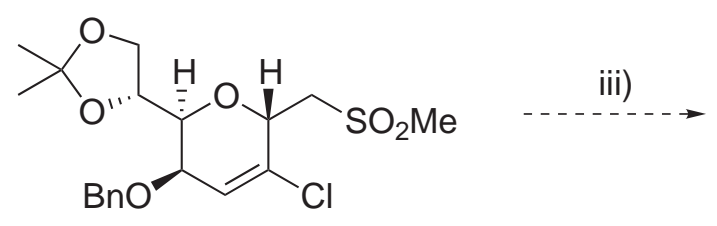

367

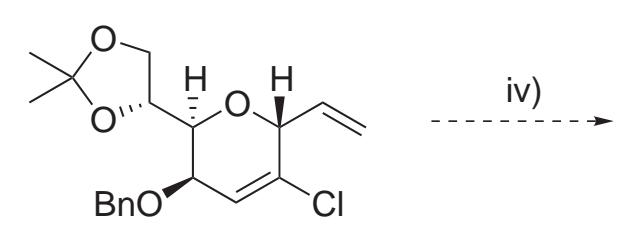

368

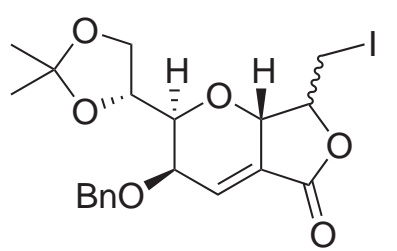

369,370

Reagents and Conditions: i) (EtO) ${ }_{2} \mathrm{P}(\mathrm{O}) \mathrm{CH}_{2} \mathrm{SO}_{2} \mathrm{Me}(\mathbf{3 6 6}), \mathrm{NaH}$; ii) base; iii) $\mathrm{KOH}, \mathrm{CCl}_{4}$, ${ }^{t} \mathrm{BuOH}$, r.t.; iv) $n$-BuLi, $\mathrm{CO}_{2}$, then $\mathbf{3 5 0}$, r.t.

Scheme 5.19 Proposed sulfone-based Ramberg-Bäcklund route

The required HWE reagent 366 was first synthesised in 96\% yield, through oxidation of commercially available diethyl methylthiomethylphosphonate with catalytic ammonium heptamolybdate and hydrogen peroxide. ${ }^{145}$ HWE reaction with hemiacetal 345 was then pursued (Scheme 5.20). After deprotonation of 366 with sodium hydride, hemiacetal $\mathbf{3 4 5}$ was added. After two hours reaction, starting material was fully consumed, and upon purification, a mixture of pyranosyl sugars 367 and 371 was obtained in $77 \%$ combined yield. The absence of $\alpha, \beta$-unsaturated sulfone $\mathbf{3 7 2}$ was presumably due to facile cyclisation to the observed products 367 and 371 . Nevertheless, it was extremely pleasing to obtain 367 and 371 in just one step, and in a surprisingly high isolated ratio of 14:1 in favour of the desired anomer 367 (identified through an nOe correlation). When the reaction was conducted at $0{ }^{\circ} \mathrm{C}$, none of minor isomer 371 was observed. Full consumption of starting material still occurred after two hours, though in some cases a small amount of diene $\mathbf{3 7 2}$ was isolated in addition to $\mathbf{3 6 7}$. Taylor has reported similar results in which in situ intramolecular cyclisation occurred during coupling of other sulfonylmethylphosphonates to carbohydrates. ${ }^{146}$ The high selectivity for isomer 367 was quite surprising, as literature precedence involving synthesis of sulfones similar to 367 and $371^{147}$ indicate colder temperatures are often required to provide high $\alpha$-glycosyl selectivity. 

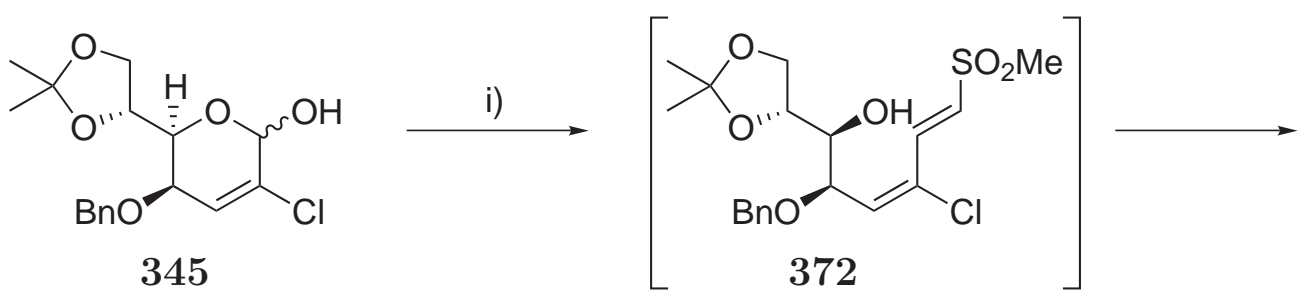

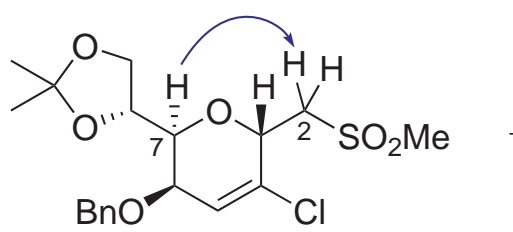

- nOe $\quad 367$<smiles>COS(=O)(=O)CC1O[C@@H]([C@H]2COC(C)(C)O2)C(Cl)=C[C@@H]1Br</smiles>

371

Reagents and Conditions: i) (EtO $)_{2} \mathrm{P}(\mathrm{O}) \mathrm{CH}_{2} \mathrm{SO}_{2} \mathrm{Me}(366), \mathrm{NaH}, \mathrm{THF}, 0{ }^{\circ} \mathrm{C}$ to r.t., 2 h, $77 \%$.

Scheme 5.20 Synthesis of sulfonated sugar 367

With sulfone 367 in hand, the key Ramberg-Bäcklund reaction was attempted, using Meyers' conditions (Scheme 5.21). ${ }^{141}$ Consumption of starting material occurred within 30 minutes, and a complex mixture of products was obtained, in which the required vinyl sugar 368 was not readily identifiable. A major component of the mixture of products was determined to be exocyclic olefin 373, due to elimination of the sulfonyl group. Presumably this occurred due to the high propensity of this system to form conjugated dienes, as observed above. It was proposed that the use of the alumina supported $\mathrm{KOH}-\mathrm{Al}_{2} \mathrm{O}_{3}$ (as used in the Chan modification of the Ramberg-Bäcklund reaction ${ }^{148}$ ), might reduce the detrimental effects observed under Meyers' conditions. However, substitution of $\mathrm{KOH}$ for $\mathrm{KOH}-\mathrm{Al}_{2} \mathrm{O}_{3}$ failed to provide $C$-glycosyl ethene $\mathbf{3 6 8}$, but afforded pure methylene-branched sugar $\mathbf{3 7 3}$ (26\%), in addition to epimerised starting material 371 (39\%). Pathak and Pal have observed similar epimerisation in their attempted synthesis of a sugar with a pendant vinyl group using the Ramberg-Bäcklund reaction. ${ }^{149}$

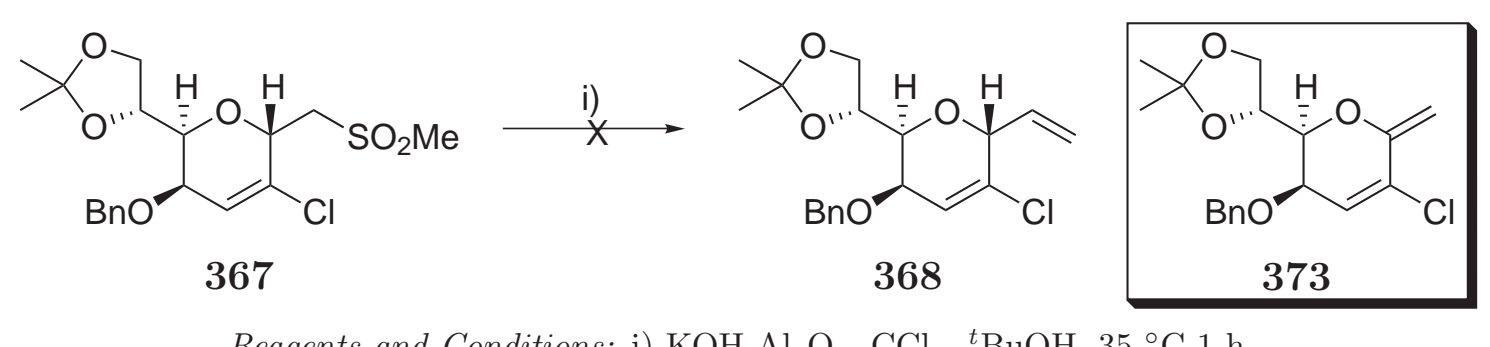

Reagents and Conditions: i) $\mathrm{KOH}-\mathrm{Al}_{2} \mathrm{O}_{3}, \mathrm{CCl}_{4},{ }^{t} \mathrm{BuOH}, 35{ }^{\circ} \mathrm{C} 1 \mathrm{~h}$.

Scheme 5.21 Attempted synthesis of alkene 368 under Meyers conditions ${ }^{141}$

A Ramberg-Bäcklund reaction was then attempted using the Chan modification, which utilises a moderate excess (4 eq.) of dibromodifluoromethane as the halogen source, in addition to $\mathrm{KOH}-\mathrm{Al}_{2} \mathrm{O}_{3} \cdot{ }^{148}$ Reaction at $5{ }^{\circ} \mathrm{C}$ was extremely sluggish, therefore the reaction was warmed successively to room temperature and dichloromethane reflux. Additional portions of dibromodifluoromethane were added approximately every hour, to compensate for the very low boiling point of this reagent 
(b.p. $23^{\circ} \mathrm{C}$ ). After several hours reaction, the mixture was worked up, providing recovered starting material 367 (57\%), in addition to a small amount of unidentified material. Maintaining the reaction at $5{ }^{\circ} \mathrm{C}$ also led to a large portion of recovered sulfone 367 (67\%) after 24 hours. A microwave reaction at $60{ }^{\circ} \mathrm{C}$ was attempted, providing greater consumption of starting material. The isolated products consisted of a small amount of diene 353 (14\%), and unidentified materials (Scheme 5.22). This observed lack of reactivity of sulfone $\mathbf{3 6 7}$ to the Ramberg-Bäcklund reaction, and in particular to the Chan modification, might be circumvented through use of an activated analogue. Replacement of the terminal methylsulfonyl group with a benzylsulfonyl group might provide a more reactive substrate. Another research group has shown that a benzylsulfone was able to undergo a Ramberg-Bäcklund reaction; yet the isopropylsulfone analogue was completely unreactive. ${ }^{150}$ However, the inability to transform the resulting Ramberg-Bäcklund reaction product into (-)-TAN-2483B meant investigation into the use of a benzylsulfone analogue was not pursued.<smiles>CO[C@H](COS(C)(=O)=O)[C@H]1O[C@H]([C@@H]2COC(C)(C)O2)[C@H](O)C=C1Cl</smiles>

367

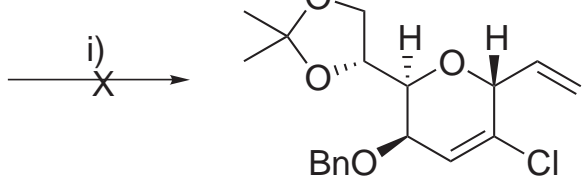

368

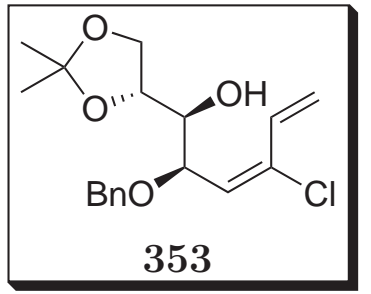

, $60{ }^{\circ} \mathrm{C}, 30 \mathrm{~min}$.

Scheme 5.22 Attempted synthesis of vinyl sugar 368 under Chan conditions ${ }^{148}$

Due to the undesired reactivity of sulfone 367 towards Ramberg-Bäcklund reaction conditions, it was thought a stepwise sequence, rather than the one-pot RambergBäcklund reaction ${ }^{141}$ might provide access to alkene 368 . Direct $\alpha$-halogenation of sulfone 367 would provide bromosulfones 374 and/or 375 , or iodosulfones 376 and/or 377. These $\alpha$-halo sulfones could then undergo a classic Ramberg-Bäcklund reaction, ${ }^{139}$ methodology which has still been employed in recent years. ${ }^{143,151}$ Efforts to achieve $\alpha$-halogenation are detailed in Table 5.1.

It was thought that repetition of the HWE reaction, followed immediately by addition of iodine, would provide iodosulfone $\mathbf{3 7 6}$ through attack of the $\mathrm{C}-1$ anion (the likely product after 1,4-addition) upon iodine (entry 1). Unfortunately, this only led to the previously isolated sulfone 367. Addition of an extra equivalent of sodium hydride concurrently with the iodine also failed to alter the outcome of the HWE reaction (entry 2). Attempts were then made to halogenate isolated sulfone $\mathbf{3 6 7}$. Entry 3 shows that deprotonation with LDA, followed by addition of NIS, failed to achieve any consumption of starting material. To ensure the halogen source was present as the deprotonated sulfone was generated, KHMDS was slowly added to a solution of sulfone $\mathbf{3 6 7}$ and NIS (entry 4). Consumption of the starting material 367 
Table 5.1 Attempts at $\alpha$-halogenating sulfone 367<smiles>CO[SH](=O)C[C@H]1O[C@H]([C@H]2COC(C)(C)O2)[C@H](O)C=C1Cl</smiles>

367<smiles>[X]C([C@H]1O[C@H]([C@@H]2COC(C)(C)O2)[C@@H](OBr)C=C1Cl)S(C)(=O)=O</smiles>

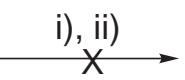

$374 \mathrm{X}=\operatorname{Br} 376 \mathrm{X}=\mathrm{I}$

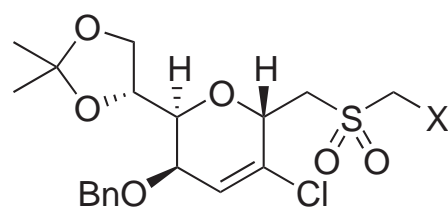

$375 \mathrm{X}=\mathrm{Br} 377 \mathrm{X}=\mathrm{I}$

Reagents and Conditions: i) Base, THF; ii) Halogen Source

\begin{tabular}{|c|c|c|c|c|}
\hline Entry & Base & Halogen Source & Conditions & Byproducts \\
\hline $1^{a}$ & - & 1.5 eq. $\mathrm{I}_{2}$ & r.t., $1 \mathrm{~h}$ & $\mathbf{3 6 7}$ \\
\hline $2^{a}$ & 1.1 eq. $\mathrm{NaH}$ & 1.5 eq. $\mathrm{I}_{2}$ & r.t., $1 \mathrm{~h}^{b}$ & $\mathbf{3 6 7}$ \\
\hline 3 & 1.2 eq. LDA & 1.8 eq. NIS & $\begin{array}{c}\text { i) } 0{ }^{\circ} \mathrm{C}, 30 \mathrm{~min} \\
\text { ii) } 0{ }^{\circ} \mathrm{C}, 1 \mathrm{~h} ; \text { r.t. } 2 \mathrm{~h}\end{array}$ & $\mathbf{3 6 7}$ \\
\hline 4 & 1.2 eq. KHMDS & 1.4 eq. $\mathrm{NIS}^{c}$ & $\begin{array}{c}\text { i) } 0{ }^{\circ} \mathrm{C}, 15 \text { min } \\
\text { ii) } 0{ }^{\circ} \mathrm{C}, 1 \mathrm{~h} ; \text { r.t. } 2 \mathrm{~h}\end{array}$ & $\mathbf{3 6 7}$ \\
\hline 5 & 1.2 eq. $n$-BuLi & 1.8 eq. NBS & $\begin{array}{l}\text { i) }-78^{\circ} \mathrm{C}, 30 \text { min } \\
\text { ii) } 0{ }^{\circ} \mathrm{C}, 1 \text { h; r.t. } 2 \text { h }\end{array}$ & $\begin{array}{c}\mathbf{3 6 7}, \mathbf{3 7 2} \\
1: 1 \text { ratio }\end{array}$ \\
\hline
\end{tabular}

${ }^{a}$ entries 1 and 2 performed on unisolated $\mathbf{3 6 7}$

${ }^{b}$ reagents added concurrently

${ }^{c}$ added prior to base

${ }^{d}$ base added dropwise over this period

was not observed. Therefore, a final attempt involved addition of $n$-butyllithium (entry 5) to a solution of sulfone 367 , followed by addition of NBS. The starting material failed to undergo halogenation, though it was observed that upon workup, a 1:1 mixture of starting material 367 and ring-opened sulfone $\mathbf{3 7 2}$ was obtained.

The initial HWE product, $\alpha, \beta$-unsaturated sulfone 372 was not readily isolated from the reaction, as the basic reaction conditions facilitated intramolecular conjugate addition, to provide pyranosyl sulfone 367. It was thought isolation of sulfone 372 and subsequent haloetherification might provide an $\alpha$-halo sulfone suitable for a Ramberg-Bäcklund reaction, although the electron-withdrawing sulfone would likely inhibit this reaction to a degree. Carrying out the HWE reaction at $-40{ }^{\circ} \mathrm{C}$ led to slightly greater proportions of diene $\mathbf{3 7 2}$ in addition to sulfone $\mathbf{3 6 7}$, though the reaction proved to be inconveniently slow. However, deprotonation of reagent 366 using LDA at $0{ }^{\circ} \mathrm{C}$ led to isolation of diene 372 in $13 \%$ yield, presumably due to lithium stabilisation of the C-6 hydroxyl of $\mathbf{3 7 2}$ (Scheme 5.23). This yield could not be increased without causing partial conversion into ring-closed sulfone 367 , which was inseparable from 372 by column chromatography. The small portion of $\mathbf{3 7 2}$ obtained was then subjected to iodoetherification using a freshly prepared solution of bis(2,4,6-trimethylpyridine)iodine(I) hexafluorophosphate (350). Unfortunately, 
diene 372 appeared to be completely unreactive after several days reaction. Upon observation of this result, the Ramberg-Bäcklund route was abandoned.

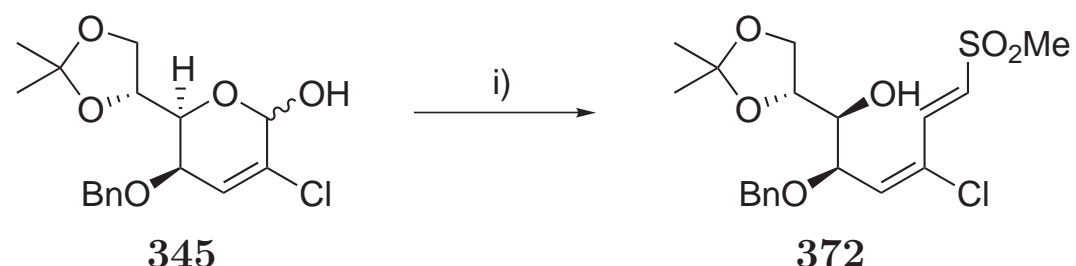

Reagents and Conditions: i) $(\mathrm{EtO}){ }_{2} \mathrm{P}(\mathrm{O}) \mathrm{CH}_{2} \mathrm{SO}_{2} \mathrm{Me}(\mathbf{3 6 6})$, LDA, THF, $0{ }^{\circ} \mathrm{C}, 3 \mathrm{~h}, 13 \%$.

Scheme 5.23 Synthesis of $\alpha, \beta$-unsaturated sulfone 372

\subsection{Approaches to the furo[3,4- $b]$ pyran-5-one skeleton of (-)-TAN-2483B}

The above section detailed various methods for elaborating the pyran ring of (-)TAN-2483B, from which methylidene Wittig-derived diene 353 seemed to provide the greatest potential for use in the construction of the furo[3,4- $b]$ pyran-5-one skeleton. Efforts to form the lactone ring required derivatisation of the alkenyl chloride moiety with carbonyl or carboxyl functionality.

A one-step synthesis of the furo[3,4- $b]$ pyran-5-one skeleton was attempted with alcohols 357 and 358, through palladium-catalysed carbonylation (Scheme 5.24). After reaction overnight, the catalyst had decomposed and only starting material was observed in the crude reaction mixture. A second attempt involved reaction with $\mathrm{PdCl}_{2}\left(\mathrm{PPh}_{3}\right)_{2}$, a catalyst which has been used for appending methyl ester functionality onto aryl iodides, ${ }^{152}$ and also chloroalkenes. ${ }^{153}$ Unfortunately, this provided no consumption of starting material. Due to this lack of reactivity, alternative means were sought.<smiles>CC1(C)OC[C@H]([C@H]2O[C@H](CO)C(Cl)=C[C@H]2O)[OH+]1</smiles>

357,358

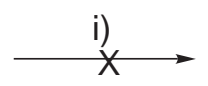

Reagents and Conditions: i) $\mathrm{CO}, \mathrm{Pd}(\mathrm{OAc})_{2}, \mathrm{PPh}_{3}, \mathrm{NEt}_{3}$, DMF, r.t., overnight, $0 \%$ or $\mathrm{CO}$, $\mathrm{PdCl}_{2}\left(\mathrm{PPh}_{3}\right)_{2}, \mathrm{NEt}_{3}, \mathrm{DMF}, 60{ }^{\circ} \mathrm{C}, 24 \mathrm{~h}, 0 \%$.

Scheme 5.24 Attempted lactone formation of alcohols 357 and 358

Carboxylation of the alkenyl chloride moiety of iodosugar 359 was attempted, through treatment with $n$-butyllithium, and carbon dioxide gas. This led to recovery of starting material 359 (63\% yield), in addition to previously isolated diene $\mathbf{3 5 3}$ (27\% yield). Presumably this reactivity is due to lithium-halogen exchange of 
the iodide, which undergoes facile ring opening to form the stable diene moiety of 353 (Scheme 5.25).

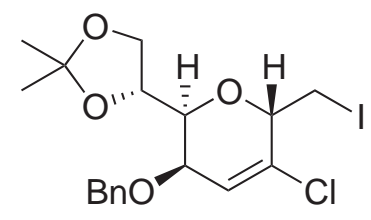

359

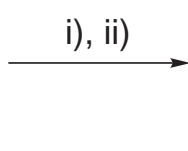

Reagents and Conditions: i) $n$-BuLi, THF, -78 to $0{ }^{\circ} \mathrm{C}, 1 \mathrm{~h}$; ii) $\mathrm{CO}_{2}$, r.t., $30 \mathrm{~min}$, $27 \%$ over 2 steps.

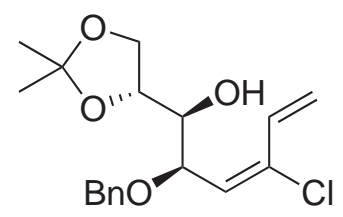

353

Scheme 5.25 Ring-opening of iodosugar 359

In order to avoid competitive reaction of iodosugar 359 with $n$-butyllithium, carboxylation of diene $\mathbf{3 5 3}$ was attempted. It was expected that the resulting acid would undergo either iodoetherification then lactonisation, or iodolactonisation followed by pyran formation, to form the furo[3,4-b]pyran-5-one skeleton. Initially, carboxylation of diene $\mathbf{3 5 3}$ was attempted, through treatment with $n$-butyllithium and subsequent addition of carbon dioxide. Unfortunately, $n$-butyllithium appeared to be unreactive to the alkenyl chloride moiety at either $-78{ }^{\circ} \mathrm{C}$ or $0{ }^{\circ} \mathrm{C}$. Use of tert-butyllithium led to consumption of starting material, but the mixture of products obtained was complicated, and did not appear to contain any carboxylate functionality at the site of the alkenyl chloride.

Palladium-catalysed carbonylation in methanol was explored with chloroalkene 353, in which methyl ester 380 was expected to form (Scheme 5.26). Thus, chloroalkene 353 was treated with the catalyst $\mathrm{PdCl}_{2}\left(\mathrm{PPh}_{3}\right)_{2}$, triphenylphosphine and triethylamine in methanol, and refluxed under an atmosphere of carbon monoxide. Gratifyingly, this provided consumption of starting material after seven hours under methanol reflux. The reaction mixture provided a black crude product, which consequently led to difficulties purifying ester 380, isolated in only $41 \%$ yield. However, it is expected that optimisation of this reaction will be possible.<smiles>C=C/C(Cl)=C\[C@@H](O)[C@H](O)[C@H]1COC(C)(C)O1</smiles>

353

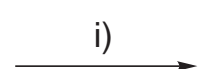

Reagents and Conditions:

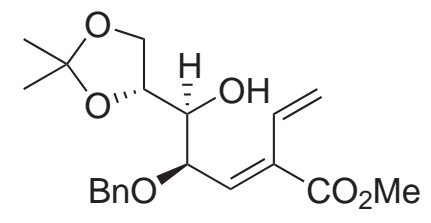

380

Scheme 5.26 Carbonylation of diene $\mathbf{3 5 3}$

With this successful functionalisation of the alkene moiety, all the carbons of the furo[3,4- $b]$ pyran-5-one skeleton were present. Construction of the pyran ring was achieved through iodoetherification (Scheme 5.27), providing a mixture of iodoesters 381 and 382 in $47 \%$ unoptimised yield (3:1 ratio). While the stereochemistry of these epimeric sugars was not proven, comparison of the NMR data with the alkenyl 
chloride analogue (359) allowed tentative assignment of the major isomer to structure 381, in which the pyran ring stereochemistry is consistent with that of (-)TAN-2483B. It may be noted these methyl esters bore similar structural similarities to the natural product waol B (page 26).<smiles>C=C/C(=C\[C@@H](O)C(C)C)[C@@H](O)[C@H]1COC(C)(C)O1</smiles>

380<smiles>COC(=O)C1=C[C@@H](O)[C@H](CI)O[C@H]1[C@H]1COC(C)(C)O1</smiles>

381<smiles>COC(=O)C1=C[C@@H](Br)[C@H]([C@H]2COC(C)(C)O2)O[C@H]1CI</smiles>

382

Reagents and Conditions: i) $350, \mathrm{CH}_{2} \mathrm{Cl}_{2}$, r.t., 1 d, $47 \%$.

Scheme $\mathbf{5 . 2 7}$ Iodoetherification of ester $\mathbf{3 8 0}$

Lactonisation of iodoesters 381 and 382 was attempted, through treatment with sodium hydroxide. It was anticipated that this could lead to hydrolysis of either the iodomethyl (green path) or ester (red path) moieties, to provide intermediates $\mathbf{3 8 3}$ and 384 or 385 and 386 (Scheme 5.28). Either pair of intermediates might undergo lactonisation to form the required bicycle $\mathbf{3 7 8}$, in addition to the $\mathrm{C}-7$ a epimer $\mathbf{3 7 9}$, which contains the bicycle of (-)-TAN-2483A. Unfortunately though, TLC and NMR evidence indicated the ester had undergone almost complete saponification. Additionally, a reduction in the signals corresponding to the iodomethyl moiety was<smiles>[R]C[C@]1(CI)O[C@H]([C@H]2COC(C)(C)O2)[C@H](O)C=C1C(C)=O</smiles>

$381 \mathrm{R}=-\mathrm{H}$

$382 \mathrm{R}=\cdots \cdot \mathrm{H}$<smiles>[R]C1(CO)OC([C@H]2COC(C)(C)O2)[C@H](O)C=C1C1(CO)CO1</smiles>

383,384

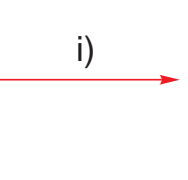

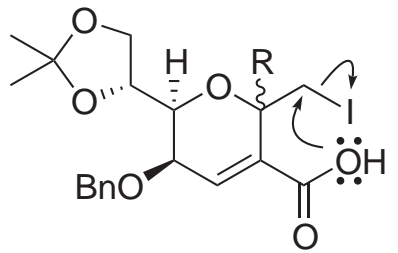

385,386

$$
\begin{array}{ll}
378 & \mathrm{R}=-\mathrm{H} \\
379 & \mathrm{R}=\cdots \cdots \mathrm{H}
\end{array}
$$

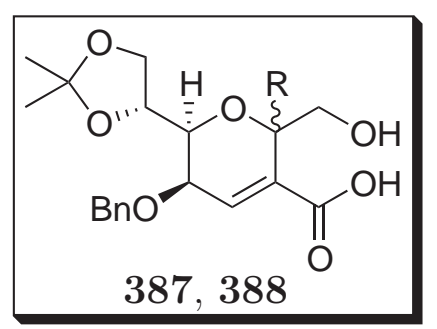

Reagents and Conditions: i) $\mathrm{NaOH}, \mathrm{THF}_{(\mathrm{aq})}$, r.t., $1 \mathrm{~d}$.

Scheme 5.28 Proposed lactonisation of iodoesters 381 and 382

observed in the proton NMR spectrum of the crude product. This indicated hydroxy acids 387 and 388 had been formed. The crude mixture of these products was subjected to DCC/DMAP-mediated lactonisation, in the hope of forming furo[3,4b]pyran-5-ones $\mathbf{3 7 8}$ and $\mathbf{3 7 9}$. TLC evidence tentatively indicated this reaction might have produced minute quantities of lactones $\mathbf{3 7 8}$ and $\mathbf{3 7 9}$. However, purification and 
characterisation were not achieved, due to the small amount of material obtained. Due to time constraints, further investigations into this promising synthetic route were not pursued.

\subsection{Summary of Chapter 5}

This chapter has reported the D-mannose-based routes to (-)-TAN-2483B, using a variety of strategies. While many olefination methodologies were deemed to be too inconvenient for further use, diene $\mathbf{3 5 3}$ provided access to the full carbon skeleton of the furo[3,4-b]pyran-5-one bicycle. Although (-)-TAN-2483B was not able to be synthesised during the timeframe of this research project, the successful synthesis of iodoesters 381 and 382 indicates the synthesis of (-)-TAN-2483B could be achievable in the near future. 


\section{Chapter 6}

\section{Future Work}

The results obtained from this project have often been unexpected and interesting, and thus prompt additional research that was not able to be completed during the timeframe of this project.

\subsection{Ring Opening Studies}

As detailed in chapters 2 and 3, cyclopropanes such as $\mathbf{9 7}$ undergo a diverse range of reactions, producing highly functionalised pyranoside (see chapter 2) or septanoside (see chapter 3) carbohydrates.

As discussed in chapter 2, the synthesis of 2- $C$-bromomethylene sugars has not been reported previously. The derivatisation of the bromomethylene moiety could lead to a wide range of new and interesting 2 - $C$-branched sugars. This research is currently underway within the research group.<smiles>O[C@@H]1[C@@H](COCc2ccccc2)O[C@@H](Br)C(=CBr)[C@H]1OCc1ccccc1</smiles>

389,390<smiles>[R]C=C1[C@@H](O)O[C@H](COc2ccccc2)[C@@H](O)[C@@H]1OCC</smiles>

391,392

Scheme 6.1 Palladium-catalysed cross-coupling of 2- $C$-bromomethylene sugars While this research showed that only $O$-glycosides were efficiently obtained through the methodologies described in chapters 2 and 3 , additional attempts to provide 
$C$-glycosyl and $N$-glycosyl derivatives would be of interest. Initial reactions mentioned within this thesis have met with limited success, yet these analogues might be accessible through optimisation of reaction conditions and through careful choice of nucleophiles.<smiles>CC#CC1O[C@H](COCc2ccccc2)[C@H](OCc2ccccc2)[C@@H](Br)C1=CBr</smiles>

393,394<smiles>BrC(Br)(Br)c1ccccc1</smiles>

97

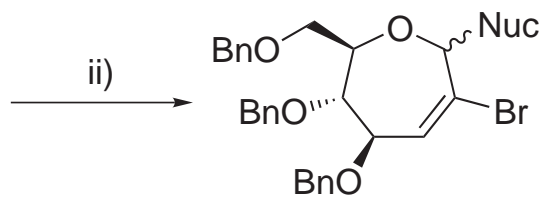

191,192

Reagents and Conditions: i) NaNuc, NucH, THF, reflux; ii) $\mathrm{Ag}^{+}$, NucH, toluene, $100{ }^{\circ} \mathrm{C}$.

Scheme 6.2 Reactions of cyclopropane $\mathbf{9 7}$ with carbon and nitrogen nucleophiles As was reported in chapter 2, monohalogenated cyclopropane 235 was obtained from treatment of dihalocyclopropane $\mathbf{9 7}$ with $n$-butyllithium. The remaining bromine atom is in the exo-configuration, which implies that this cyclopropane would fail to undergo ring expansion, due to the "cis-in trans-out" requirement discussed in subsection 1.1.2. Thus, representative ring-opening reactions of $\mathbf{2 3 5}$ under either basic or silver-mediated conditions would be of significant interest, and might provide further mechanistic insight.<smiles>CC=C(C)C</smiles>

389,390<smiles>BrC[C@H]1O[C@H](Br)[C@H](Br)[C@H](Br)[C@H]1Br</smiles>

235<smiles>CC#CC</smiles>

255,256

Reagents and Conditions: i) $\mathrm{NaOR}, \mathrm{ROH}, \mathrm{THF}$, reflux; ii) $\mathrm{Ag}^{+}$, $\mathrm{ROH}$, toluene, $100{ }^{\circ} \mathrm{C}$.

Scheme 6.3 Ring-opening reactions of cyclopropane $\mathbf{2 3 5}$

Finally, a detailed investigation into the microwave-mediated reaction of cyclopropanes such as $\mathbf{9 7}$ would be of use. Specifically, this may help define the optimal conditions for selectively generating septanosides or $C$-furanosyl sugars. Microwave reactions are generally influenced by the choice of solvent, and the corresponding ability of that solvent to absorb microwave energy. Therefore, alternative solvents (e.g. DMF, acetonitrile), may provide more efficient reactivity than solvents reported in chapter 3 .

\subsection{Natural Product Synthesis}

The synthesis of (-)-TAN-2483B through use of D-galactose or D-xylose (as discussed in chapter 4) was unsuccessful, therefore pursuing these routes is not likely to provide much headway. In contrast, work detailed in chapter 5 covered many 
similar approaches to (-)-TAN-2483B through hemiacetal 345, of which several may be elaborated further, which could provide access to (-)-TAN-2483B.

The ethoxycarbonyl-based Wittig route may hold further promise, if functionalisation of the $\alpha$-centre of ester 361 can be achieved. Possibly the 2-(phenylsulfonyl)3-phenyl-oxaziridine reagent could effect this transformation.<smiles>CCOC(=O)C[C@H]1O[C@H]([C@H]2COC(C)(C)O2)[C@H](O)C=C1Cl</smiles>

361

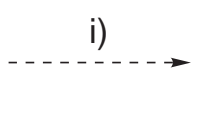

Reagents and Conditions:<smiles>CCOC(O)[C@H]1O[C@H]([C@H](O)CO)[C@@H](O)C=C1Cl</smiles>

395

Scheme 6.4 Synthesis of $\alpha$-hydroxy ester 395

The sulfone-based HWE route appears to fail from a lack of reactivity in the Ramberg-Bäcklund reaction. Incorporation of a more reactive moiety (e.g. through use of diethyl benzylsulfonylmethylphosphonate) may allow the Ramberg-Bäcklund reaction of sulfone 396 to occur. ${ }^{150}$ This could lead to phenyl-TAN-2483B (397).<smiles></smiles>

396<smiles>C/C=C/C1O[C@H]2C(=C[C@H]1O)C(=O)O[C@H]2Cc1ccccc1</smiles>

397

Scheme 6.5 Synthesis of phenyl-TAN-2483B (397)

Finally, the methylidene-derived Wittig route ultimately provided the best results, with incorporation of the full carbon skeleton of the required furo[3,4- $b]$ pyran-5one bicycle. The work described in chapter 5 has demonstrated that this route shows great promise. The required lactone ring may be constructed through optimisation of results discussed in section 5.5, with particular focus on developing an effective lactonisation strategy. Furthermore, the methyl group attached to C-7 of (-)-TAN-2483B could be installed through additional steps, or further studies upon ethylidene-based Wittig products 347 and 346 (see section 5.4) might provide the completed lactone ring. Completion of the synthesis through sidechain appendation will in all probability, finally provide access to (-)-TAN-2483B. 


\section{Chapter 7}

\section{Experimental}

\subsection{General Experimental Details}

Unless otherwise stated, the following conditions apply. All reactions were performed under argon (zero grade) in vacuum-dried or flame-dried glassware using dry solvents and standard syringe techniques. Tetrahydrofuran (THF) was freshly distilled from the sodium benzophenone ketyl radical ion. Acetonitrile (MeCN), dichloromethane $\left(\mathrm{CH}_{2} \mathrm{Cl}_{2}\right)$, triethylamine $\left(\mathrm{NEt}_{3}\right)$ and diisopropylamine $\left({ }^{i} \mathrm{Pr}_{2} \mathrm{NH}\right)$ were distilled from calcium hydride. Methanol $(\mathrm{MeOH})$ and toluene were distilled from sodium. Acetone, 1,4-dioxane and water were distilled. Acetic acid (glacial) and acetic anhydride were used as received. Allyl alcohol (AllylOH), anhydrous dimethylformamide (DMF), methanol- $d_{4}$ and THF- $d_{8}$ were purchased from Aldrich Chemical Company and were used without further purification. Chloroform and bromoform were both used as received (with a small amount of ethanol as stabiliser). Bleach was prepared as described earlier, ${ }^{91}$ from a sodium hypochlorite solution of commercial quality. Sodium hydride $(\mathrm{NaH})$ was obtained as a $60 \%$ (w/w) dispersion in paraffin oil and was used as obtained unless otherwise stated. $n$-butyllithium was obtained as a $1.6 \mathrm{molL}^{-1}$ solution in hexanes, unless otherwise stated. KHMDS was obtained as a $0.5 \mathrm{molL}^{-1}$ solution in toluene. Potassium fluoride dihydrate was crystallised from a super-saturated aqueous solution of potassium fluoride. Pyridinium $p$-toluenesulfonate (PPTS) was prepared through dissolution of $p$-toluenesulfonic acid in an excess of pyridine, then evaporation to dryness. $\mathrm{KOH}-\mathrm{Al}_{2} \mathrm{O}_{3}$ was prepared as reported by Chan. ${ }^{148} \mathrm{~m}$-CPBA was obtained as an approx. $70 \%$ dispersion 
in water and $m$-chlorobenzoic acid. All other reagents were of commercial quality and distilled prior to use if necessary.

Reaction progress was monitored using aluminium-backed thin layer chromatography (TLC) plates pre-coated with silica UV254 and visualised by either UV radiation $(254 \mathrm{~nm})$, or anisaldehyde dip. Purification of products via flash chromatography was conducted using a column filled with silica gel 60 (220-240 mesh) with solvent systems as indicated. Microwave-assisted reactions were carried out in a Milestone Microsynth reactor, monitored by a fibre optic temperature and pressure probe. ${ }^{1} \mathrm{H}$ and ${ }^{13} \mathrm{C}$ NMR spectra were recorded on a Varian Unity Inova 500 (500 $\mathrm{MHz}$ for ${ }^{1} \mathrm{H}$ and $125 \mathrm{MHz}$ for ${ }^{13} \mathrm{C}$ ) spectrometer, NOESY and ${ }^{31} \mathrm{P}$ NMR spectra were recorded on a Varian Unity Inova 300 (300 MHz for ${ }^{1} \mathrm{H}$ and $121 \mathrm{MHz}$ for $\left.{ }^{31} \mathrm{P}\right)$. All chemical shifts $(\delta)$ were referenced to solvent peaks if possible $\left(\mathrm{CDCl}_{3}:{ }^{1} \mathrm{H}\right.$ $\left.7.26 \mathrm{ppm},{ }^{13} \mathrm{C}-77.0 \mathrm{ppm}\right)$. Melting points were determined using a Gallenkamp melting point apparatus and are uncorrected. Optical rotation was measured on a Perkin-Elmer Polarimeter (sodium lamp). Infrared spectra were obtained on a Perkin-Elmer Spectrum One FT-IR spectrometer. High-resolution mass spectrometry (HRMS) was performed on a Waters Q-TOF Premier ${ }^{\text {TM }}$ Tandem Mass Spectrometer. The structure of each compound is presented with the corresponding method of preparation.

\subsection{Chapter Two Experimental}

D-Glucal (185)

Using a modification of Kozikowski's procedure, ${ }^{89}$ a magnetically stirred solution of D-glucose (126 mg, $0.7 \mathrm{mmol})$ in acetic anhydride $(75 \mathrm{~mL}, 790 \mathrm{mmol}$ ) was treated dropwise with conc. perchloric acid $(0.45 \mathrm{~mL}, 6.9 \mathrm{mmol})$. Additional D-glucose (19.9 g, $110.4 \mathrm{mmol}$ ) was slowly added over half an hour, at a rate that maintained a temperature of $40-50{ }^{\circ} \mathrm{C}$. Upon complete addition of D-glucose, the solution was allowed to cool to room temperature, then treated with a $33 \%(\mathrm{w} / \mathrm{w})$ solution of hydrobromic acid in acetic acid $(75 \mathrm{~mL}, 410 \mathrm{mmol})$. After 90 minutes, the solution was diluted with dichloromethane $(180 \mathrm{~mL})$ and washed with ice-cold water $(2 \times 50 \mathrm{~mL})$, then with cold sat. sodium bicarbonate solution $(6 \times 50 \mathrm{~mL})$. The organic phase was dried, filtered and concentrated to afford crude tetra- $O$-acetyl- $\alpha$-D-glucopyranosyl bromide (210) as a pale-yellow oil.

A mechanically stirred dispersion of zinc dust (47.9 g, $733 \mathrm{mmol})$ in water $(150 \mathrm{~mL})$ was cooled to $0{ }^{\circ} \mathrm{C}$, then diluted with acetic acid $(150 \mathrm{~mL})$, then treated dropwise with a solution of tetra- $O$-acetyl- $\alpha$-D-glucopyranosyl bromide in diethyl ether (150 $\mathrm{mL}$ ), over one hour. The reaction was allowed to warm to room temperature and left 
to proceed overnight. The solution was filtered, then diluted with dichloromethane $(200 \mathrm{~mL})$. The resulting solution was washed successively with water $(3 \times 60 \mathrm{~mL})$, sat. sodium bicarbonate solution $(4 \times 50 \mathrm{~mL})$, then brine $(60 \mathrm{~mL})$. The organic phase was dried, filtered and concentrated to provide tri- $O$-acetyl-D-glucal (179) as an off-white solid (26.6 g).

A solution of triethylamine $(110 \mathrm{~mL}, 780 \mathrm{mmol})$ in methanol $(50 \%$ of an aqueous solution, $1100 \mathrm{~mL}$ ) was treated with tri- $O$-acetyl-D-glucal (26.6 g) and stirred for 90 minutes. The mixture was concentrated to liberate D-glucal as a brown oil, contaminated with triethylamine. The crude mixture was successively treated with portions of methanol, then concentrated to remove the triethylamine. The oil was dissolved in methanol $(15 \mathrm{~mL})$, then successively diluted with acetone $(100 \mathrm{~mL})$ and diethyl ether $(200 \mathrm{~mL})$, which led to the precipitation of impurities. The solution was filtered and concentrated to afford D-glucal (185) as a brown solid (10.7 g, 66\% crude yield), which was used without further purification.

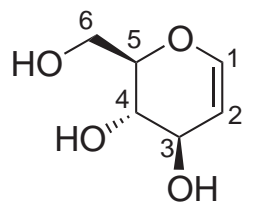

${ }^{1} \mathrm{H}-\mathrm{NMR}:\left(\mathrm{D}_{2} \mathrm{O}\right) \delta_{\mathrm{H}} 6.38(\mathrm{dd}, J=6.0,1.6 \mathrm{~Hz}, 1 \mathrm{H}, \mathrm{H}-1), 4.79$ (complex m, 3H, 3 x OH) 4.77 (dd, $J=6.1,2.4 \mathrm{~Hz}, 1 \mathrm{H}, \mathrm{H}-2), 4.19$ (dt, $J=7.1,2.1 \mathrm{~Hz}, 1 \mathrm{H}, \mathrm{H}-3), 3.92-3.82$ (complex m, 3H, H-5,6), 3.64 $(\mathrm{dd}, J=9.0,7.1 \mathrm{~Hz}, 1 \mathrm{H}, \mathrm{H}-4) ;{ }^{13} \mathrm{C}-\mathrm{NMR}:\left(\mathrm{D}_{2} \mathrm{O}\right) \delta_{\mathrm{C}} 143.8(\mathrm{CH}$, C-1), 102.7 (CH, C-2), 78.0 (CH, C-5), 68.7 (CH, C-4), $68.2(\mathrm{CH}, \mathrm{C}-3), 59.9\left(\mathrm{CH}_{2}\right.$, C-6); IR (KBr): $\nu_{\max } 3337,2923,1650,1229,1073 \mathrm{~cm}^{-1}$.

\section{Tri- $O$-benzyl-D-glucal (188)}

A solution of D-glucal $(4.99 \mathrm{~g}, 34.1 \mathrm{mmol})$ in DMF $(200 \mathrm{~mL})$ was cooled to $0{ }^{\circ} \mathrm{C}$, then treated with sodium hydride $(5.33 \mathrm{~g}, 133 \mathrm{mmol})$. The solution was stirred at $0{ }^{\circ} \mathrm{C}$ for 30 minutes, then treated with benzyl bromide $(14.0 \mathrm{~mL}, 118 \mathrm{mmol})$ and allowed to warm to room temperature. After 17 hours, the solution was quenched with water $(20 \mathrm{~mL})$ and the product extracted with diethyl ether $(3 \times 200 \mathrm{~mL})$. The organic fractions were combined, dried, filtered and concentrated to provide the crude product as a concentrated solution in DMF. Chromatography of this solution (9:1 hexanes:ethyl acetate) afforded $188(11.9 \mathrm{~g}, 84 \%)$ as a pale-yellow solid. The spectral data matched that reported previously. ${ }^{154}$

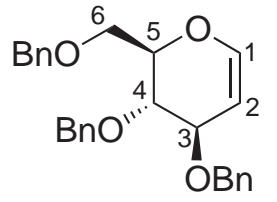

$\mathrm{R}_{f} 0.4$ (9:1 hexanes:ethyl acetate); m.p. $54-56{ }^{\circ} \mathrm{C}$ (lit. ${ }^{154}$ m.p. 53$\left.55{ }^{\circ} \mathrm{C}\right) ;{ }^{1} \mathrm{H}-\mathrm{NMR}:\left(\mathrm{CDCl}_{3}\right) \delta_{\mathrm{H}} 7.34-7.26$ (complex m, 15H, Bn), $6.42(\mathrm{dd}, J=6.1,1.2 \mathrm{~Hz}, 1 \mathrm{H}, \mathrm{H}-1), 4.88(\mathrm{dd}, J=6.1,2.7 \mathrm{~Hz}, 1 \mathrm{H}$, $\mathrm{H}-2), 4.83\left(\mathrm{~d}, J=11.2 \mathrm{~Hz}, 1 \mathrm{H}, \mathrm{PhCH}_{2}\right), 4.64$ (d, $J=11.2 \mathrm{~Hz}, 1 \mathrm{H}$, $\left.\mathrm{PhCH}_{2}\right), 4.64\left(\mathrm{~d}, J=11.7 \mathrm{~Hz}, 1 \mathrm{H}, \mathrm{PhCH}_{2}\right), 4.59-4.51$ (complex m, 3H, $\mathrm{PhCH}_{2}$ ), 4.21 (ddd, $J=6.1,2.4,1.5 \mathrm{~Hz}, 1 \mathrm{H}, \mathrm{H}-3), 4.06$ (ddd, $J=8.4,5.2,3.1 \mathrm{~Hz}, 1 \mathrm{H}, \mathrm{H}-5)$, $3.86(\mathrm{dd}, J=8.8,6.1 \mathrm{~Hz}, 1 \mathrm{H}, \mathrm{H}-4), 3.81$ (dd, $J=10.7,5.1 \mathrm{~Hz}, 1 \mathrm{H}, \mathrm{H}-6 \mathrm{a}), 3.76$ $(\mathrm{dd}, J=10.7,2.9 \mathrm{~Hz}, 1 \mathrm{H}, \mathrm{H}-6 \mathrm{~b}) ;{ }^{13} \mathrm{C}-\mathrm{NMR}:\left(\mathrm{CDCl}_{3}\right) \delta_{\mathrm{C}} 144.7$ (CH, C-1), 138.3 
(C, Bn), 138.2 (C, Bn), 138.0 (C, Bn), 128.42 (CH, Bn), 128.41 (CH, Bn), 128.38 (CH, Bn), 127.9 (CH, Bn), 127.8 (CH, Bn), 127.74 (CH, Bn), 127.65 (CH, Bn), 99.9 (CH, C-2), 76.6 (CH, C-4), 75.7 (CH, C-5), 74.4 (CH, C-3), $73.8\left(\mathrm{CH}_{2}, \mathrm{PhCH}_{2}\right)$, $73.5\left(\mathrm{CH}_{2}, \mathrm{PhCH}_{2}\right), 70.5\left(\mathrm{CH}_{2}, \mathrm{PhCH}_{2}\right), 68.5\left(\mathrm{CH}_{2}, \mathrm{C}-6\right)$; IR $(\mathrm{KBr}): \nu_{\max } 3066$, 3029, 2924, 2911, 2887, 1952, 1650, 1452, 1245, 1096, $740 \mathrm{~cm}^{-1}$.

\section{1,5-Anhydro-3,4,6-tri- $O$-benzyl-2-deoxy-1,2- $C$-(dibromomethylene)-D- glycero-D-gulo-hexitol (97) and 1,5-anhydro-3,4,6-tri- $O$-benzyl-2-deoxy- 1,2-C-(dibromomethylene)-D-glycero-D-talo-hexitol (190)}

A As reported by Nagarajan, ${ }^{45}$ a solution of tri- $O$-benzyl-D-glucal (188) (1.14 g, $2.73 \mathrm{mmol})$ and TEBAC (8 $\mathrm{mg}, 0.04 \mathrm{mmol})$ in bromoform $(4.5 \mathrm{~mL}, 51 \mathrm{mmol})$ was treated dropwise with a solution of sodium hydroxide (930 mg, $23 \mathrm{mmol}$ ) and potassium fluoride dihydrate $(6.9 \mathrm{~g})$ in water $(7.0 \mathrm{~mL})$. The biphasic mixture was stirred at room temperature for two days, then diluted with water $(20 \mathrm{~mL})$. The mixture was extracted with diethyl ether $(3 \times 40 \mathrm{~mL})$, then the organic fractions were combined and washed with brine $(20 \mathrm{~mL})$. The ethereal solution was dried, filtered and concentrated to provide a crude mixture of cyclopropanes 97 and 190 (6.5:1 ratio), which was purified by column chromatography (9:1 hexanes:ethyl acetate) to liberate cyclopropanes 97 (1.11 g, 66\%), as a light-yellow solid and 190 (170 mg, 10\%) as a yellow oil ( $76 \%$ combined yield).

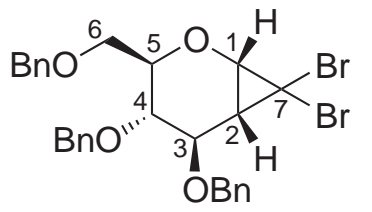

97: The spectral data matched that which was previously reported. ${ }^{45} \mathrm{R}_{f} 0.4$ (9:1 hexanes:ethyl acetate); m.p. $56-60{ }^{\circ} \mathrm{C}$ (lit. ${ }^{45}$ m.p. $\left.58-60{ }^{\circ} \mathrm{C}\right) ;[\alpha]_{\mathrm{D}}^{21}+59\left(\right.$ c $\left.1.0 \mathrm{CHCl}_{3}\right)$; ${ }^{1} \mathrm{H}-\mathrm{NMR}$ : $\left(\mathrm{CDCl}_{3}\right) \delta_{\mathrm{H}} 7.44-7.22$ (complex m, 15H, Bn), $4.90(\mathrm{~d}, J=$ $11.2 \mathrm{~Hz}, 1 \mathrm{H}, \mathrm{PhCH}_{2}$ ), 4.81 (d, $J=11.7 \mathrm{~Hz}, 1 \mathrm{H}, \mathrm{PhCH}_{2}$ ), 4.70 (d, $J=11.7 \mathrm{~Hz}, 1 \mathrm{H}$, $\mathrm{PhCH}_{2}$ ), $4.54\left(\mathrm{~d}, J=11.5 \mathrm{~Hz}, 1 \mathrm{H}, \mathrm{PhCH}_{2}\right), 4.53$ (d, $\left.J=12.2 \mathrm{~Hz}, 1 \mathrm{H}, \mathrm{PhCH}_{2}\right)$, $4.44\left(\mathrm{~d}, J=12.2 \mathrm{~Hz}, 1 \mathrm{H}, \mathrm{PhCH}_{2}\right), 3.94$ (d, $\left.J=7.8 \mathrm{~Hz}, 1 \mathrm{H}, \mathrm{H}-1\right), 3.87$ (ddd, $J=$ 7.6, 3.7, $2.8 \mathrm{~Hz}, 1 \mathrm{H}, \mathrm{H}-5), 3.79$ (dd, $J=9.5,7.6 \mathrm{~Hz}, 1 \mathrm{H}, \mathrm{H}-4$ ), 3.68 (d, $J=9.5,4.9$ $\mathrm{Hz}, 1 \mathrm{H}, \mathrm{H}-3), 3.56$ (dd, $J=10.6,2.8 \mathrm{~Hz}, 1 \mathrm{H}, \mathrm{H}-6 \mathrm{a}), 3.51$ (dd, $J=10.6,3.8 \mathrm{~Hz}, 1 \mathrm{H}$, $\mathrm{H}-6 \mathrm{~b}), 1.87$ (dd, $J=7.8,4.9 \mathrm{~Hz}, 1 \mathrm{H}, \mathrm{H}-2) ;{ }^{13} \mathrm{C}-\mathrm{NMR}:\left(\mathrm{CDCl}_{3}\right) \delta_{\mathrm{C}} 138.1$ (C, Bn), 137.9 (C, Bn), 137.7 (C, Bn), 128.5 (CH, Bn), 128.43 (CH, Bn), 128.35 (CH, Bn), 128.2 (CH, Bn), 127.87 (CH, Bn), 127.85 (CH, Bn), 127.78 (CH, Bn), $127.7(\mathrm{CH}$, Bn), 127.6 (CH, Bn), 80.2 (CH, C-5), 79.9 (CH, C-3), 75.0 (CH, C-4), $74.6\left(\mathrm{CH}_{2}\right.$, $\left.\mathrm{PhCH}_{2}\right), 73.3\left(\mathrm{CH}_{2}, \mathrm{PhCH}_{2}\right), 71.8\left(\mathrm{CH}_{2}, \mathrm{PhCH}_{2}\right), 70.1\left(\mathrm{CH}_{2}, \mathrm{C}-6\right), 59.2(\mathrm{CH}, \mathrm{C}-1)$, 35.2 (CH, C-2), 33.9 (C, C-7); IR (KBr): $\nu_{\max } 3032,2864,1949,1586,1496,1453$, $1365,1205,1129,1097,1027,735 \mathrm{~cm}^{-1}$. 


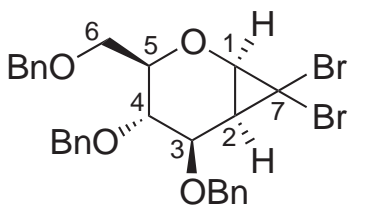

190: $\mathrm{R}_{f} 0.2$ (9:1 hexanes:ethyl acetate); $[\alpha]_{\mathrm{D}}^{21}-30$ (c 1.0 $\left.\mathrm{CHCl}_{3}\right) ;{ }^{1} \mathrm{H}-\mathrm{NMR}:\left(\mathrm{CDCl}_{3}\right) \delta_{\mathrm{H}} 7.44-7.18$ (complex m, $15 \mathrm{H}$, $\mathrm{Bn}), 4.91\left(\mathrm{~d}, J=11.7 \mathrm{~Hz}, 1 \mathrm{H}, \mathrm{PhCH}_{2}\right), 4.82(\mathrm{~d}, J=11.0$ $\left.\mathrm{Hz}, 1 \mathrm{H}, \mathrm{PhCH}_{2}\right), 4.71$ (d, $\left.J=11.7 \mathrm{~Hz}, 1 \mathrm{H}, \mathrm{PhCH}_{2}\right), 4.65$ (d, $\left.J=12.0 \mathrm{~Hz}, 1 \mathrm{H}, \mathrm{PhCH}_{2}\right), 4.56\left(\mathrm{~d}, J=12.0 \mathrm{~Hz}, 1 \mathrm{H}, \mathrm{PhCH}_{2}\right), 4.54(\mathrm{~d}, J=11.0$ $\mathrm{Hz}, 1 \mathrm{H}, \mathrm{PhCH}_{2}$ ) 4.17 (apparent t, $J=7.8 \mathrm{~Hz}, 1 \mathrm{H}, \mathrm{H}-3$ ), 4.07 (d, $J=7.8 \mathrm{~Hz}, 1 \mathrm{H}$, H-1), 3.94 (dd, $J=10.4,7.6 \mathrm{~Hz}, 1 \mathrm{H}, \mathrm{H}-4), 3.77$ (dd, $J=11.0,1.7 \mathrm{~Hz}, 1 \mathrm{H}, \mathrm{H}-6 \mathrm{a})$, 3.67 (dd, $J=11.1,5.3 \mathrm{~Hz}, 1 \mathrm{H}, \mathrm{H}-6 \mathrm{~b}), 3.50$ (ddd, $J=10.4,5.3,1.7 \mathrm{~Hz}, 1 \mathrm{H}, \mathrm{H}-5)$, 2.30 (apparent t, $J=8.1 \mathrm{~Hz}, 1 \mathrm{H}, \mathrm{H}-2)$; ${ }^{13} \mathrm{C}-\mathrm{NMR}:\left(\mathrm{CDCl}_{3}\right) \delta_{\mathrm{C}} 138.2(\mathrm{C}, \mathrm{Bn}), 138.1$ (C, Bn), 137.7 (C, Bn), 128.6 (CH, Bn), 128.3 (CH, Bn), 128.1 (CH, Bn), 127.93 (CH, Bn), 127.88 (CH, Bn), 127.69 (CH, Bn), 127.65 (CH, Bn), $127.5(\mathrm{CH}, \mathrm{Bn})$, 78.5 (CH, C-3), 78.4 (CH, C-5), $76.4(\mathrm{CH}, \mathrm{C}-4), 74.6\left(\mathrm{CH}_{2}, \mathrm{PhCH}_{2}\right), 73.6\left(\mathrm{CH}_{2}\right.$, $\left.\mathrm{PhCH}_{2}\right), 71.0\left(\mathrm{CH}_{2}, \mathrm{PhCH}_{2}\right), 68.9\left(\mathrm{CH}_{2}, \mathrm{C}-6\right), 62.7$ (CH, C-1), 33.7 (CH, C-2), 30.8 (C, C-7); IR (KBr): $\nu_{\max } 3024,2866,1496,1453,1363,1179,1093,1027,736,697$ $\mathrm{cm}^{-1}$.

B In a slight modification of Nagarajan's procedure, ${ }^{45}$ a solution of tri- $O$-benzylD-glucal (188) (495 mg, $1.19 \mathrm{mmol}$ ) and TEBAC (6 mg, $0.03 \mathrm{mmol}$ ) in bromoform $(2.0 \mathrm{~mL}, 23 \mathrm{mmol})$ was chilled to $0{ }^{\circ} \mathrm{C}$, then treated dropwise with a solution of sodium hydroxide (442 mg, $11.1 \mathrm{mmol}$ ) and potassium fluoride dihydrate (3.31 g) in water $(3.3 \mathrm{~mL})$. The biphasic mixture was then sonicated for 10 periods of 10 minutes, maintaining the temperature of the sonicator bath between approximately 20-30 ${ }^{\circ} \mathrm{C}$. The mixture was diluted with water $(20 \mathrm{~mL})$, extracted with diethyl ether $(3 \times 20 \mathrm{~mL})$, then the organic fractions were combined and washed with brine $(20 \mathrm{~mL})$. The ethereal solution was dried, filtered and concentrated, then purified by column chromatography (9:1 hexanes:ethyl acetate) to liberate recovered starting material (188) (208 mg, 42\%), in addition to cyclopropanes 97 (243 mg, 35\%) and 190 (34 mg, 5\%) (40\% combined yield).

C In a slight modification of Nagarajan's procedure, ${ }^{45}$ a solution of tri- $O$-benzylD-glucal (188) (501 mg, $1.20 \mathrm{mmol}$ ) and TEBAC (6 mg, $0.03 \mathrm{mmol}$ ) in bromoform $(2.0 \mathrm{~mL}, 23 \mathrm{mmol})$ was chilled to $0{ }^{\circ} \mathrm{C}$, then treated dropwise with a solution of sodium hydroxide (402 $\mathrm{mg}, 10.1 \mathrm{mmol}$ ) and potassium fluoride dihydrate $(2.99 \mathrm{~g}$ ) in water $(3.0 \mathrm{~mL})$. The biphasic mixture was then sonicated for eight periods of 30 minutes, maintaining the temperature of the sonicator bath between approximately 20-30 ${ }^{\circ} \mathrm{C}$. The mixture was diluted with water $(20 \mathrm{~mL})$, extracted with diethyl ether $(3 \times 20 \mathrm{~mL})$, then the organic fractions were combined and washed with brine $(20 \mathrm{~mL})$. The ethereal solution was dried, filtered and concentrated, then purified by column chromatography (9:1 hexanes:ethyl acetate) to liberate recovered starting material (188) (149 mg, 30\%), in addition to cyclopropanes 97 (340 mg, 48\%) and 
190 (43 mg, 6\%) (54\% combined yield).

D In a slight modification of Nagarajan's procedure, ${ }^{45}$ a solution of tri- $O$-benzylD-glucal (188) (533 mg, $1.28 \mathrm{mmol}$ ) and TEBAC (4 mg, $0.02 \mathrm{mmol}$ ) in bromoform $(2.0 \mathrm{~mL}, 23 \mathrm{mmol})$ was chilled to $0{ }^{\circ} \mathrm{C}$, then treated dropwise with a solution of sodium hydroxide (401 mg, $10.0 \mathrm{mmol}$ ) and potassium fluoride dihydrate (3.01 g) in water $(3.0 \mathrm{~mL})$. The biphasic mixture was then sonicated for 12 periods of 30 minutes, maintaining the temperature of the sonicator bath between approximately 20-30 ${ }^{\circ} \mathrm{C}$. The mixture was diluted with water $(20 \mathrm{~mL})$, extracted with diethyl ether $(3 \times 20 \mathrm{~mL})$, then the organic fractions were combined and washed with brine (20 mL). The ethereal solution was dried, filtered and concentrated, then purified by column chromatography (9:1 hexanes:ethyl acetate) to liberate recovered starting material (188) (132 mg, 28\%), in addition to cyclopropanes 97 (321 mg, 43\%) and 190 (56 mg, 7\%) (50\% combined yield).

E In a slight modification of Nagarajan's procedure, ${ }^{45}$ a solution of tri- $O$-benzylD-glucal (188) (1.00 g, $2.40 \mathrm{mmol})$ and TEBAC (8 mg, $0.04 \mathrm{mmol})$ in bromoform $(4.0 \mathrm{~mL}, 46 \mathrm{mmol})$ was chilled to $0{ }^{\circ} \mathrm{C}$, then treated dropwise with a solution of sodium hydroxide $(0.80 \mathrm{~g}, 20 \mathrm{mmol})$ and potassium fluoride dihydrate $(6.04 \mathrm{~g})$ in water $(6.0 \mathrm{~mL})$. The biphasic mixture was then sonicated for 12 periods of $30 \mathrm{~min}-$ utes, maintaining the temperature of the sonicator bath at approximately $20-30{ }^{\circ} \mathrm{C}$. The mixture was diluted with water $(40 \mathrm{~mL})$, extracted with diethyl ether $(3 \mathrm{x}$ $40 \mathrm{~mL})$, then the organic fractions were combined and washed with brine $(40 \mathrm{~mL})$. The ethereal solution was dried, filtered and concentrated then purified by column chromatography (9:1 hexanes:ethyl acetate) to liberate recovered starting material (188) (300 mg, 30\%), in addition to cyclopropanes 97 (678 mg, 48\%) and 190 (110 mg, 8\%) (56\% combined yield).

F In a slight modification of Nagarajan's procedure, ${ }^{45}$ a solution of tri- $O$-benzyl-Dglucal (188) (501 $\mathrm{mg}, 1.20 \mathrm{mmol}$ ) and TEBAC (9 mg, $0.04 \mathrm{mmol}$ ) in bromoform $(2.0 \mathrm{~mL}, 23 \mathrm{mmol})$ was chilled to $0{ }^{\circ} \mathrm{C}$, then treated dropwise with a solution of sodium hydroxide $(398 \mathrm{mg}, 9.95 \mathrm{mmol})$ and potassium fluoride dihydrate $(3.01 \mathrm{~g})$ in water $(3.0 \mathrm{~mL})$. The biphasic mixture was stirred at room temperature for two days. The mixture was diluted with water $(20 \mathrm{~mL})$, extracted with diethyl ether $(3 \mathrm{x}$ $20 \mathrm{~mL})$, then the organic fractions were combined and washed with brine $(20 \mathrm{~mL})$. The ethereal solution was dried, filtered and concentrated, then purified by column chromatography (9:1 hexanes:ethyl acetate) to liberate recovered starting material (188) (78 mg, 16\%), in addition to cyclopropanes 97 (448 mg, 63\%) and 190 (48 mg, $7 \%$ ) (70\% combined yield). 
Methyl 3,4,6-tri- $O$-benzyl-(2E)-2- $C$-(bromomethylene)-2-deoxy- $\beta$-Darabino-hexopyranoside (213) and methyl 3,4,6-tri- $O$-benzyl-(2E)-2- $C$ (bromomethylene)-2-deoxy- $\alpha$-D-arabino-hexopyranoside (214)

A In repetition of Nagarajan's procedure, ${ }^{45}$ a solution of cyclopropane 97 (115 $\mathrm{mg}, 0.195 \mathrm{mmol})$ in methanol $(2.1 \mathrm{~mL})$ was treated with dry potassium carbonate (136 mg, $0.984 \mathrm{mmol}$ ) and refluxed for eight hours. The solution was separated between dichloromethane $(10 \mathrm{~mL})$ and water $(10 \mathrm{~mL})$, then the aqueous phase was extracted with dichloromethane $(2 \times 10 \mathrm{~mL})$. The combined organic fractions were dried, filtered and concentrated to provide crude 2 - $C$-branched sugars 213 and 214 as a yellow oil (1:1.3 ratio). Upon purification by column chromatography (20:1 hexanes:ethyl acetate), recovered starting material 97 (4 mg, 4\%) was obtained, in addition to 2-C-branched sugars 213 (34 mg, 32\%) as a colourless oil, and 214 (37 $\mathrm{mg}, 35 \%)$ as a colourless oil (68\% combined yield). The spectral data set matched that for products previously isolated from this reaction (reported as the corresponding oxepines 98 and $\mathbf{9 9}$ ).

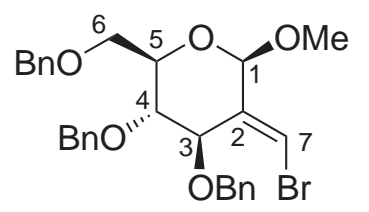

213: $\mathrm{R}_{f} 0.35$ (9:1 hexanes:ethyl acetate); $[\alpha]_{\mathrm{D}}^{17}+57$ ( c 1.0 $\left.\mathrm{CHCl}_{3}\right)$; ${ }^{1} \mathrm{H}-\mathrm{NMR}:\left(\mathrm{CDCl}_{3}\right) \delta_{\mathrm{H}} 7.41-7.18$ (complex m, 15H, $\mathrm{Bn}), 6.81(\mathrm{~d}, J=1.7 \mathrm{~Hz}, 1 \mathrm{H}, \mathrm{H}-7), 5.19$ (d, $J=1.7 \mathrm{~Hz}, 1 \mathrm{H}$, $\mathrm{H}-1), 4.73$ (d, $J=1.2 \mathrm{~Hz}, 1 \mathrm{H}, \mathrm{H}-3), 4.64$ (d, $J=12.2 \mathrm{~Hz}, 1 \mathrm{H}$, $\left.\mathrm{PhCH}_{2}\right), 4.60\left(\mathrm{~d}, J=12.2 \mathrm{~Hz}, 1 \mathrm{H}, \mathrm{PhCH}_{2}\right), 4.52\left(\mathrm{~d}, J=11.5 \mathrm{~Hz}, 1 \mathrm{H}, \mathrm{PhCH}_{2}\right)$, $4.50\left(\mathrm{~d}, J=12.2 \mathrm{~Hz}, 1 \mathrm{H}, \mathrm{PhCH}_{2}\right), 4.43$ (d, $\left.J=12.2 \mathrm{~Hz}, 1 \mathrm{H}, \mathrm{PhCH}_{2}\right), 4.29$ (d, $J=$ $11.5 \mathrm{~Hz}, 1 \mathrm{H}, \mathrm{PhCH}_{2}$ ), 3.74 (dd, $\left.J=9.0,1.5 \mathrm{~Hz}, 1 \mathrm{H}, \mathrm{H}-4\right), 3.69$ (ddd, $J=8.8,5.1$, $2.4 \mathrm{~Hz}, 1 \mathrm{H}, \mathrm{H}-5), 3.65$ (dd, $J=10.9,2.5 \mathrm{~Hz}, 1 \mathrm{H}, \mathrm{H}-6 \mathrm{a}), 3.62$ (dd, $J=10.9,5.3 \mathrm{~Hz}$, 1H, H-6b), 3.47 (s, 3H, Me); ${ }^{13} \mathrm{C}-\mathrm{NMR}:\left(\mathrm{CDCl}_{3}\right) \delta_{\mathrm{C}} 138.3$ (C, Bn), 138.1 (C, Bn), 137.7 (C, Bn), 137.5 (C, C-2), 128.32 (CH, Bn), 128.27 (CH, Bn), 128.0 (CH, Bn), $127.9(\mathrm{CH}, \mathrm{Bn}), 127.8$ (CH, Bn), $127.72(\mathrm{CH}, \mathrm{Bn}), 127.67(\mathrm{CH}, \mathrm{Bn}), 127.5(\mathrm{CH}$, Bn), 112.6 (CH, C-7), 98.9 (CH, C-1), 79.3 (CH, C-4), 76.1 (CH, C-3), $73.1\left(\mathrm{CH}_{2}\right.$, $\left.\mathrm{PhCH}_{2}\right), 71.5\left(\mathrm{CH}_{2}, \mathrm{PhCH}_{2}\right), 71.4(\mathrm{CH}, \mathrm{C}-5), 70.8\left(\mathrm{CH}_{2}, \mathrm{PhCH}_{2}\right), 69.5\left(\mathrm{CH}_{2}, \mathrm{C}-6\right)$, $55.2\left(\mathrm{CH}_{3}, \mathrm{Me}\right)$; IR (KBr): $\nu_{\max } 3054,3027,2961,2865,1629,1496,1453,1345$, 1261, 1094, 1072, 802, 735, $697 \mathrm{~cm}^{-1}$.

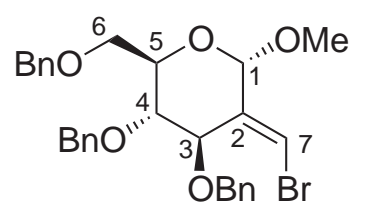

214: $\mathrm{R}_{f} 0.25$ (9:1 hexanes:ethyl acetate); $[\alpha]_{\mathrm{D}}^{17}+18$ (c 1.0 $\left.\mathrm{CHCl}_{3}\right) ;{ }^{1} \mathrm{H}-\mathrm{NMR}:\left(\mathrm{CDCl}_{3}\right) \delta_{\mathrm{H}} 7.36-7.24$ (complex m, $15 \mathrm{H}$, $\mathrm{Bn}), 6.78$ (s, 1H, H-7), 5.05 (s, 1H, H-1), 4.71 (d, $J=11.5$ $\left.\mathrm{Hz}, 1 \mathrm{H}, \mathrm{PhCH}_{2}\right), 4.69$ (d, $\left.J=3.7 \mathrm{~Hz}, 1 \mathrm{H}, \mathrm{H}-3\right), 4.62$ (d, $J=$ $\left.11.7 \mathrm{~Hz}, 1 \mathrm{H}, \mathrm{PhCH}_{2}\right), 4.52$ (s, 2H, $\mathrm{PhCH}_{2}$ ), 4.50 (d, J = 11.7 Hz, 1H, $\mathrm{PhCH}_{2}$ ), $4.48\left(\mathrm{~d}, J=11.7 \mathrm{~Hz}, 1 \mathrm{H}, \mathrm{PhCH}_{2}\right), 3.91$ (dd, $\left.J=5.0,3.8 \mathrm{~Hz}, 1 \mathrm{H}, \mathrm{H}-4\right), 3.85$ (m, $1 \mathrm{H}, \mathrm{H}-5), 3.83$ (dd, $J=10.0,5.2 \mathrm{~Hz}, 1 \mathrm{H}, \mathrm{H}-6 \mathrm{a}), 3.77$ (dd, $J=10.0,5.1 \mathrm{~Hz}, 1 \mathrm{H}$, H-6b), 3.47 (s, 3H, Me); ${ }^{13} \mathrm{C}-\mathrm{NMR}:\left(\mathrm{CDCl}_{3}\right) \delta_{\mathrm{C}} 138.4$ (C, Bn), 138.3 (C, Bn), 137.8 (C, Bn), 137.0 (C, C-2), 128.4 (CH, Bn), 128.33 (CH, Bn), 128.25 (CH, Bn), 127.9 
(CH, Bn), $127.8(\mathrm{CH}, \mathrm{Bn}), 127.7(\mathrm{CH}, \mathrm{Bn}), 127.56(\mathrm{CH}, \mathrm{Bn}), 127.55(\mathrm{CH}, \mathrm{Bn})$, 114.7 (CH, C-7), 101.1 (CH, C-1), 76.0 (CH, C-4), 74.9 (CH, C-5), 74.6 (CH, C-3), $73.3\left(\mathrm{CH}_{2}, \mathrm{PhCH}_{2}\right), 72.2\left(\mathrm{CH}_{2}, \mathrm{PhCH}_{2}\right), 70.9\left(\mathrm{CH}_{2}, \mathrm{PhCH}_{2}\right), 70.6\left(\mathrm{CH}_{2}, \mathrm{C}-6\right), 55.8$ $\left(\mathrm{CH}_{3}, \mathrm{Me}\right)$; IR $(\mathrm{KBr}): \nu_{\max } 3060,3030,2912,2865,1629,1496,1453,1363,1303$, 1205, 1097, 1072, 1027, 818, 735, $697 \mathrm{~cm}^{-1}$; HRMS: $m / z \mathrm{C}_{29} \mathrm{H}_{31} \mathrm{O}_{5}^{79} \mathrm{BrNa}^{+}[\mathrm{M}+\mathrm{Na}]^{+}$ calcd 561.1253, found 561.1245.

B A portion of methanol $(63 \mu \mathrm{L}, 1.6 \mathrm{mmol})$ was treated with metallic sodium (6.5 $\mathrm{mg}, 0.3 \mathrm{mmol}$ ), then once the sodium had reacted, the solution was diluted with THF (1.7 mL), then treated with cyclopropane 97 (100 mg, $0.17 \mathrm{mmol})$ and heated at reflux for 75 minutes. The solution was separated between dichloromethane $(10 \mathrm{~mL})$ and water $(10 \mathrm{~mL})$, then the aqueous phase was extracted with dichloromethane $(2 \times 10 \mathrm{~mL})$. The combined organic fractions were dried, filtered and concentrated to provide crude products 213 and 214 as a yellow oil. Upon separation by column chromatography (9:1 hexanes:ethyl acetate), the previously isolated 2- $C$-substituted sugars 213 (20 $\mathrm{mg}, 22 \%$ ) and $\mathbf{2 1 4}$ (45 $\mathrm{mg}, 49 \%$ ) were obtained ( $71 \%$ combined yield).

C A portion of methanol $(200 \mu \mathrm{L}, 4.9 \mathrm{mmol})$ was treated with metallic sodium (19 $\mathrm{mg}, 0.8 \mathrm{mmol}$ ), then once the sodium had reacted, the solution was diluted with THF $(1.7 \mathrm{~mL})$, then treated with cyclopropane 97 (85 mg, $0.14 \mathrm{mmol})$ and heated at reflux for three hours. The solution was separated between dichloromethane $(10 \mathrm{~mL})$ and water $(10 \mathrm{~mL})$, then the aqueous phase was extracted with dichloromethane $(2 \times 10 \mathrm{~mL})$. The combined organic fractions were dried, filtered and concentrated to provide crude products 213 and 214 as a yellow oil. Upon separation by column chromatography (9:1 hexanes:ethyl acetate), the previously isolated 2- $C$-substituted sugars 213 (30 $\mathrm{mg}, 38 \%$ ) and $\mathbf{2 1 4}$ (20 $\mathrm{mg}, 26 \%$ ) were obtained (64\% combined yield).

D A portion of methanol $(0.2 \mathrm{~mL}, 0.49 \mathrm{mmol})$ was treated with metallic sodium ( $8.5 \mathrm{mg}, 0.36 \mathrm{mmol}$ ), then once the sodium had reacted, the solution was diluted with THF (3.4 mL), then treated with cyclopropane 97 (202 mg, $0.343 \mathrm{mmol}$ ) and stirred at room temperature for 90 minutes. The solution was separated between dichloromethane $(10 \mathrm{~mL})$ and water $(10 \mathrm{~mL})$, then the aqueous phase was extracted with dichloromethane $(2 \times 10 \mathrm{~mL})$. The combined organic fractions were dried, filtered and concentrated to provide crude products 213 and 214 as a yellow oil. Upon separation by column chromatography (9:1 hexanes:ethyl acetate), the previously isolated 2- $C$-substituted sugars 213 (55 $\mathrm{mg}, 30 \%$ ) and 214 (55 $\mathrm{mg}, 30 \%$ ) were obtained (60\% combined yield). 
E A portion of methanol $(26 \mu \mathrm{L}, 0.64 \mathrm{mmol})$ was treated with metallic sodium $(1.5 \mathrm{mg}, 70 \mu \mathrm{mol})$ in a clean NMR tube, then once the sodium had reacted, was treated with a solution of cyclopropane $97(21 \mathrm{mg}, 36 \mu \mathrm{mol})$ in THF- $d_{8}(0.85 \mathrm{~mL})$. The tube was flushed with argon, sealed with a septum, then allowed to react. Proton NMR spectra were obtained after $0,7,25,49,102$ minutes reaction time. The solution was separated between dichloromethane $(10 \mathrm{~mL})$ and water $(10 \mathrm{~mL})$, then the aqueous phase was extracted with dichloromethane $(2 \times 10 \mathrm{~mL})$. The combined organic fractions were dried, filtered and concentrated to provide crude products 213 and 214.

F A portion of methanol $(82 \mu \mathrm{L}, 2.0 \mathrm{mmol})$ was treated with metallic sodium $(6 \mathrm{mg}$, $0.3 \mathrm{mmol}$ ), then once the sodium had reacted, the solution was diluted with THF $(1.7 \mathrm{~mL})$. The solution was transferred into a second flask containing minor cyclopropane 190 (92 mg, $0.16 \mathrm{mmol}$ ) and stirred at reflux for three hours. The solution was separated between dichloromethane $(10 \mathrm{~mL})$ and water $(10 \mathrm{~mL})$, then the aqueous phase was extracted with dichloromethane $(2 \times 10 \mathrm{~mL})$. The combined organic fractions were dried, filtered and concentrated to provide crude products 213 and 214 as a yellow oil. Upon separation by column chromatography (9:1 hexanes:ethyl acetate), starting material $97(15 \mathrm{mg}, 16 \%)$ and the previously isolated 2- $C$-branched sugars 213 (10 mg, 12\%) and 214 (30 mg, 36\%) were obtained (48\% combined yield).

G A portion of methanol $(50 \mu \mathrm{L}, 0.64 \mathrm{mmol})$ was treated with metallic sodium ( $3 \mathrm{mg}, 0.1 \mathrm{mmol}$ ) in a clean NMR tube, then once the sodium had reacted, was treated with a solution of cyclopropanes 97 and 190 (approximate ratio 97:190 5:4, $52 \mathrm{mg}, 88 \mu \mathrm{mol})$ in THF- $d_{8}(0.85 \mathrm{~mL})$. The tube was flushed with argon, sealed with a septum, then allowed to react. Proton NMR spectra were obtained after 0 , 15, 65, 115 minutes reaction time. The solution was separated between dichloromethane $(10 \mathrm{~mL})$ and water $(10 \mathrm{~mL})$, then the aqueous phase was extracted with dichloromethane $(2 \times 10 \mathrm{~mL})$. The combined organic fractions were dried, filtered and concentrated to provide crude products 213 and 214 .

\section{Methyl 3,4,6-tri- $O$-benzyl-2-deoxy-2- $C$-(methylene)- $\alpha$-D-arabino- hexopyranoside (215)}

A A solution of brominated sugar $214(20 \mathrm{mg}, 37 \mu \mathrm{mol})$ in THF $(0.5 \mathrm{~mL})$ was cooled to $-78{ }^{\circ} \mathrm{C}$, then treated with $n$-butyllithium $(50 \mu \mathrm{L}, 80 \mu \mathrm{mol})$ and stirred at $-78^{\circ} \mathrm{C}$ for one hour. The reaction mixture was then treated with water $(50 \mu \mathrm{L}, 2.8 \mathrm{mmol})$ and immediately allowed to warm to room temperature. After 30 minutes additional stirring, the reaction mixture was diluted with water $(10 \mathrm{~mL})$, then extracted with 
diethyl ether $(3 \times 10 \mathrm{~mL})$. The ethereal fractions were combined, dried and concentrated to provide a colourless oil. This oil was purified by flash chromatography (9:1 hexanes:ethyl acetate), to provide the major observed product, exocyclic olefin $\mathbf{2 1 5}$ as a colourless oil (5 mg, 29\%).

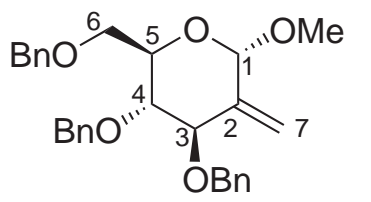

$\mathrm{R}_{f} 0.25$ (9:1 hexanes:ethyl acetate); $[\alpha]_{\mathrm{D}}^{17}+13\left(\right.$ c $\left.0.3 \mathrm{CHCl}_{3}\right)$;

${ }^{1} \mathrm{H}-\mathrm{NMR}:\left(\mathrm{CDCl}_{3}\right) \delta_{\mathrm{H}} 7.40-7.15$ (complex m, 15H, Bn), 5.30 (s, 1H, H-7a), 5.16 (s, 1H, H-7b), 5.06 (s, 1H, H-1), 4.87 (d, $\left.J=10.7 \mathrm{~Hz}, 1 \mathrm{H}, \mathrm{PhCH}_{2}\right), 4.76\left(\mathrm{~d}, J=11.2 \mathrm{~Hz}, 1 \mathrm{H}, \mathrm{PhCH}_{2}\right)$, $4.70\left(\mathrm{~d}, J=11.2 \mathrm{~Hz}, 1 \mathrm{H}, \mathrm{PhCH}_{2}\right), 4.63\left(\mathrm{~d}, J=12.0 \mathrm{~Hz}, 1 \mathrm{H}, \mathrm{PhCH}_{2}\right), 4.51$ (d, $J=$ $\left.12.2 \mathrm{~Hz}, 1 \mathrm{H}, \mathrm{PhCH}_{2}\right), 4.49$ (d, $\left.J=10.7 \mathrm{~Hz}, 1 \mathrm{H}, \mathrm{PhCH}_{2}\right), 4.42(\mathrm{dd}, J=9.0,2.0$ $\mathrm{Hz}, 1 \mathrm{H}, \mathrm{H}-3), 3.92$ (ddd, $J=9.9,3.8,1.9 \mathrm{~Hz}, 1 \mathrm{H}, \mathrm{H}-5), 3.75$ (dd, $J=10.6,3.9$ $\mathrm{Hz}, 1 \mathrm{H}, \mathrm{H}-6 \mathrm{a}$ ), 3.69 (dd, $J=10.6,1.9 \mathrm{~Hz}, 1 \mathrm{H}, \mathrm{H}-6 \mathrm{~b}$ ), 3.60 (apparent t, $J=9.4$ $\mathrm{Hz}, 1 \mathrm{H}, \mathrm{H}-4), 3.38$ (s, 3H, Me); ${ }^{13} \mathrm{C}-\mathrm{NMR}:\left(\mathrm{CDCl}_{3}\right) \delta_{\mathrm{C}} 142.3$ (C, C-2), 138.32 (C, Bn), 138.27 (C, Bn), 138.1 (C, Bn), 128.4 (CH, Bn), 128.3 (CH, Bn), 127.9 (CH, Bn), 127.8 (CH, Bn), 127.7 (CH, Bn), $127.63(\mathrm{CH}, \mathrm{Bn}), 127.61(\mathrm{CH}, \mathrm{Bn}), 110.7$ $\left(\mathrm{CH}_{2}, \mathrm{C}-7\right), 102.4(\mathrm{CH}, \mathrm{C}-1), 81.2(\mathrm{CH}, \mathrm{C}-3), 79.9(\mathrm{CH}, \mathrm{C}-4), 74.9\left(\mathrm{CH}_{2}, \mathrm{PhCH}_{2}\right)$, $73.5\left(\mathrm{CH}_{2}, \mathrm{PhCH}_{2}\right), 73.4\left(\mathrm{CH}_{2}, \mathrm{PhCH}_{2}\right), 71.5(\mathrm{CH}, \mathrm{C}-5), 68.8\left(\mathrm{CH}_{2}, \mathrm{C}-6\right), 54.5$ $\left(\mathrm{CH}_{3}, \mathrm{Me}\right)$; IR (KBr): $\nu_{\max }$ 3029, 2907, 1496, 1454, 1359, 1102, 1067, 1026, 968, 736, $697 \mathrm{~cm}^{-1}$; HRMS: $m / z \mathrm{C}_{29} \mathrm{H}_{32} \mathrm{O}_{5} \mathrm{Na}^{+}[\mathrm{M}+\mathrm{Na}]^{+}$calcd 483.2147, found 483.2145.

B Using a previously reported procedure, ${ }^{155}$ a solution of brominated sugar 214 (23 mg, $43 \mu \mathrm{mol})$ in 1,4-dioxane (1 mL) was treated successively with potassium carbonate (20 mg, $0.15 \mathrm{mmol}$ ), palladium-tetrakis(triphenylphosphine) (5 mg, $4 \mu \mathrm{mol}$ ) and methaneboronic acid $(4 \mathrm{mg}, 70 \mu \mathrm{mol})$, then refluxed for two days. The reaction mixture was then treated with water $(25 \mu \mathrm{L}, 1.4 \mathrm{mmol})$ and reacted for an additional five hours. The reaction mixture was diluted with water $(10 \mathrm{~mL})$, then extracted with dichloromethane $(2 \times 10 \mathrm{~mL})$. The organic fractions were combined, dried and concentrated to provide a green oil. This oil was purified by flash chromatography (9:1 hexanes:ethyl acetate), to provide both unreacted starting material 214 (8 $\mathrm{mg}$, $35 \%$ ) and exocyclic olefin 215 (6 mg, 30\%).

\section{Methyl- $d_{3}$ 3,4,6-tri- $O$-benzyl-( $\left.2 E\right)$-2- $C$-(bromomethylene- $\left.d_{1}\right)$-2-deoxy- $\beta$ - D-arabino-hexopyranoside (219) and methyl- $d_{3} 3,4,6$-tri- $O$-benzyl-( $\left.2 E\right)$ - 2- $C$-(bromomethylene- $\left.d_{1}\right)$-2-deoxy- $\alpha$-D-arabino-hexopyranoside (220)}

Methanol- $d_{4}(0.6 \mathrm{~mL}, 15 \mathrm{mmol})$ was treated with metallic sodium $(5 \mathrm{mg}, 0.2 \mathrm{mmol})$, then once the sodium had reacted, the solution was treated with cyclopropane $\mathbf{9 7}$ (38 $\mathrm{mg}, 0.065 \mathrm{mmol}$ ) and heated at reflux for two hours. The solution was separated between dichloromethane $(10 \mathrm{~mL})$ and water $(10 \mathrm{~mL})$, then the aqueous phase was extracted with dichloromethane $(2 \times 10 \mathrm{~mL})$. The combined organic fractions were 
dried, filtered and concentrated to provide tetra-deutero sugars 219 and 220 as a yellow oil. Upon column chromatography (9:1 hexanes:ethyl acetate), recovered starting material 97 (5 mg, 13\%) was obtained, in addition to 2-C-branched sugars 219 (7 mg, 20\%) and 220 (10 mg, 29\%), isolated as colourless oils (49\% combined yield).

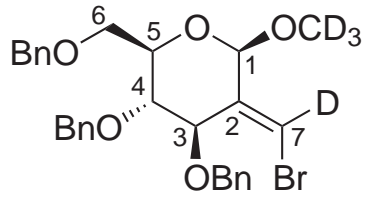

219: $\mathrm{R}_{f} 0.2$ (9:1 hexanes:ethyl acetate); ${ }^{1} \mathrm{H}-\mathrm{NMR}:\left(\mathrm{CDCl}_{3}\right)$ $\delta_{\mathrm{H}} 7.41-7.18$ (complex m, 15H, Bn), $5.19(\mathrm{~d}, J=1.7 \mathrm{~Hz}, 1 \mathrm{H}$, $\mathrm{H}-1), 4.73$ (d, $J=1.2 \mathrm{~Hz}, 1 \mathrm{H}, \mathrm{H}-3), 4.64(\mathrm{~d}, J=12.2 \mathrm{~Hz}$, $\left.1 \mathrm{H}, \mathrm{PhCH}_{2}\right), 4.60\left(\mathrm{~d}, J=12.2 \mathrm{~Hz}, 1 \mathrm{H}, \mathrm{PhCH}_{2}\right), 4.52(\mathrm{~d}, J=$ $\left.11.5 \mathrm{~Hz}, 1 \mathrm{H}, \mathrm{PhCH}_{2}\right), 4.51$ (d, $\left.J=12.2 \mathrm{~Hz}, 1 \mathrm{H}, \mathrm{PhCH}_{2}\right), 4.43$ (d, $J=12.2 \mathrm{~Hz}$, $\left.1 \mathrm{H}, \mathrm{PhCH}_{2}\right), 4.29\left(\mathrm{~d}, J=11.5 \mathrm{~Hz}, 1 \mathrm{H}, \mathrm{PhCH}_{2}\right), 3.74(\mathrm{dd}, J=9.0,1.5 \mathrm{~Hz}, 1 \mathrm{H}$, H-4), 3.69 (ddd, $J=8.7,5.5,2.7 \mathrm{~Hz}, 1 \mathrm{H}, \mathrm{H}-5$ ), 3.65-3.59 (complex m, 2H, H-6a,b); ${ }^{13} \mathrm{C}-\mathrm{NMR}:\left(\mathrm{CDCl}_{3}\right) \delta_{\mathrm{C}} 138.3$ (C, Bn), 138.1 (C, Bn), 137.6 (C, Bn), 137.4 (C, C-2), 128.32 (CH, Bn), 128.28 (CH, Bn), 128.0 (CH, Bn), $127.9(\mathrm{CH}, \mathrm{Bn}), 127.8(\mathrm{CH}$, Bn), 127.72 (CH, Bn), 127.68 (CH, Bn), 127.5 (CH, Bn), 98.9 (CH, C-1), 79.2 (CH, C-4), 76.1 (CH, C-3), $73.1\left(\mathrm{CH}_{2}, \mathrm{PhCH}_{2}\right), 71.5\left(\mathrm{CH}_{2}, \mathrm{PhCH}_{2}\right), 71.4(\mathrm{CH}, \mathrm{C}-5), 70.7$ $\left(\mathrm{CH}_{2}, \mathrm{PhCH}_{2}\right), 69.5\left(\mathrm{CH}_{2}, \mathrm{C}-6\right)$.

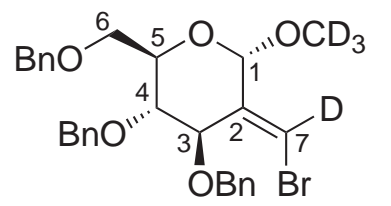

220: $\mathrm{R}_{f} 0.15$ (9:1 hexanes:ethyl acetate); ${ }^{1} \mathrm{H}-\mathrm{NMR}:\left(\mathrm{CDCl}_{3}\right)$ $\delta_{\mathrm{H}}$ 7.36-7.24 (complex m, 15H, Bn), 5.05 (s, 1H, H-1), 4.72 $\left(\mathrm{d}, J=11.5 \mathrm{~Hz}, 1 \mathrm{H}, \mathrm{PhCH}_{2}\right), 4.69(\mathrm{~d}, J=3.9 \mathrm{~Hz}, 1 \mathrm{H}, \mathrm{H}-3)$, $4.62\left(\mathrm{~d}, J=11.7 \mathrm{~Hz}, 1 \mathrm{H}, \mathrm{PhCH}_{2}\right), 4.52\left(\mathrm{~s}, 2 \mathrm{H}, \mathrm{PhCH}_{2}\right), 4.51$ $\left(\mathrm{d}, J=11.8 \mathrm{~Hz}, 1 \mathrm{H}, \mathrm{PhCH}_{2}\right), 4.49$ (d, $\left.J=11.8 \mathrm{~Hz}, 1 \mathrm{H}, \mathrm{PhCH}_{2}\right), 3.91$ (dd, $J=5.0$, $3.8 \mathrm{~Hz}, 1 \mathrm{H}, \mathrm{H}-4$ ), 3.85 (m, 1H, H-5), 3.82-3.76 (complex m, 2H, H-6a,b); ${ }^{13} \mathrm{C}-\mathrm{NMR}$ : $\left(\mathrm{CDCl}_{3}\right) \delta_{\mathrm{C}} 138.3(\mathrm{C}, \mathrm{Bn}), 138.2(\mathrm{C}, \mathrm{Bn}), 137.7$ (C, Bn), 136.8 (C, C-2), $128.4(\mathrm{CH}$, Bn), 128.3 (CH, Bn), 128.2 (CH, Bn), 127.9 (CH, Bn), 127.8 (CH, Bn), 127.7 (CH, Bn), 127.54 (CH, Bn), 127.53 (CH, Bn), 101.0 (CH, C-1), 76.0 (CH, C-4), 74.8 (CH, C-5), 74.5 (CH, C-3), $73.2\left(\mathrm{CH}_{2}, \mathrm{PhCH}_{2}\right), 72.2\left(\mathrm{CH}_{2}, \mathrm{PhCH}_{2}\right), 70.8\left(\mathrm{CH}_{2}, \mathrm{PhCH}_{2}\right)$, $70.5\left(\mathrm{CH}_{2}, \mathrm{C}-6\right)$; HRMS: $m / z \mathrm{C}_{29} \mathrm{H}_{27} \mathrm{D}_{4} \mathrm{O}_{5}^{79} \mathrm{BrNa}^{+}[\mathrm{M}+\mathrm{Na}]^{+}$calcd 565.1504, found 565.1498 .

\section{Allyl 3,4,6-tri- $O$-benzyl-(2E)-2- $C$-(bromomethylene)-2-deoxy- $\beta$-D- arabino-hexopyranoside (236) and allyl 3,4,6-tri- $O$-benzyl-(2E)-2- $C$ - (bromomethylene)-2-deoxy- $\alpha$-D-arabino-hexopyranoside (237)}

A A solution of allyl alcohol $(70 \mu \mathrm{L}, 1.0 \mathrm{mmol})$ in THF $(1.7 \mathrm{~mL})$ was treated with sodium hydride $(12 \mathrm{mg}, 0.3 \mathrm{mmol})$, then once evolution of hydrogen had ceased, the solution was treated with cyclopropane 97 (103 mg, $0.175 \mathrm{mmol}$ ) and heated at reflux for five hours. The solution was separated between dichloromethane $(10 \mathrm{~mL})$ and water $(10 \mathrm{~mL})$, then the aqueous phase was extracted with dichloromethane $(2 \times 10 \mathrm{~mL})$. The combined organic fractions were dried, filtered and concentrated 
to provide crude products 236 and 237 as a yellow oil. Upon separation by column chromatography (14:1 hexanes:ethyl acetate), allyl glycosides 236 (22 $\mathrm{mg}, 22 \%$ ) and 237 (48 mg, 49\%) were obtained as colourless oils (71\% combined yield).

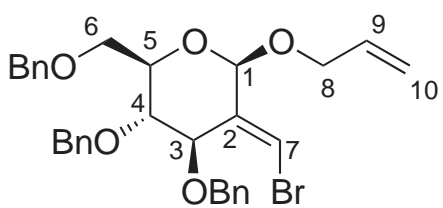

236: $\mathrm{R}_{f} 0.25$ (14:1 hexanes:ethyl acetate); $[\alpha]_{\mathrm{D}}^{20}+66(c$ $\left.1.7 \mathrm{CHCl}_{3}\right) ;{ }^{1} \mathrm{H}-\mathrm{NMR}:\left(\mathrm{CDCl}_{3}\right) \delta_{\mathrm{H}} 7.41-7.18$ (complex m, $15 \mathrm{H}, \mathrm{Bn}), 6.87$ (d, $J=1.7 \mathrm{~Hz}, 1 \mathrm{H}, \mathrm{H}-7), 5.93$ (ddd, $J=17.1,11.0,5.6 \mathrm{~Hz}, 1 \mathrm{H}, \mathrm{H}-9), 5.35(\mathrm{~s}, 1 \mathrm{H}, \mathrm{H}-1), 5.28$ (dd, $J=17.4,1.5 \mathrm{~Hz}, 1 \mathrm{H}, \mathrm{H}-10 \mathrm{a}), 5.17$ (dd, $J=10.5,1.2 \mathrm{~Hz}, 1 \mathrm{H}, \mathrm{H}-10 \mathrm{~b}), 4.75$ (s, $1 \mathrm{H}, \mathrm{H}-3), 4.65$ (d, $J=11.9 \mathrm{~Hz}, 1 \mathrm{H}, \mathrm{PhCH}_{2}$ ), 4.60 (d, $J=12.0 \mathrm{~Hz}, 1 \mathrm{H}, \mathrm{PhCH}_{2}$ ), 4.53-4.48 (complex m, 2H, $\mathrm{PhCH}_{2}$ ), 4.44 (d, $J=12.2 \mathrm{~Hz}, 1 \mathrm{H}, \mathrm{PhCH}_{2}$ ), 4.33 (dd, $J=13.1,5.1 \mathrm{~Hz}, 1 \mathrm{H}, \mathrm{H}-8 \mathrm{a}), 4.29\left(\mathrm{~d}, J=11.5 \mathrm{~Hz}, 1 \mathrm{H}, \mathrm{PhCH}_{2}\right), 4.10$ (dd, $J=$ 13.1, 6.2 Hz, 1H, H-8b), 3.77-3.74 (complex m, 2H, H-4,5), 3.65-3.60 (complex m, 2H, H-6a,b); ${ }^{13} \mathrm{C}-\mathrm{NMR}:\left(\mathrm{CDCl}_{3}\right) \delta_{\mathrm{C}} 138.3$ (C, Bn), 138.1 (C, Bn), 137.63 (C, Bn), 137.58 (C, C-2), 134.1 (CH, C-9), 128.32 (CH, Bn), 128.27 (CH, Bn), 128.0 (CH, Bn), 127.9 (CH, Bn), 127.8 (CH, Bn), $127.73(\mathrm{CH}, \mathrm{Bn}), 127.69(\mathrm{CH}, \mathrm{Bn}), 127.5$ (CH, Bn), $117.2\left(\mathrm{CH}_{2}, \mathrm{C}-10\right), 112.6$ (CH, C-7), 97.1 (CH, C-1), 79.3 (CH, C-4), 76.1 $(\mathrm{CH}, \mathrm{C}-3), 73.1\left(\mathrm{CH}_{2}, \mathrm{PhCH}_{2}\right), 71.5\left(\mathrm{CH}_{2}, \mathrm{PhCH}_{2}\right), 71.4(\mathrm{CH}, \mathrm{C}-5), 70.8\left(\mathrm{CH}_{2}\right.$, $\left.\mathrm{PhCH}_{2}\right)$, $69.5\left(\mathrm{CH}_{2}, \mathrm{C}-6\right), 68.2\left(\mathrm{CH}_{2}, \mathrm{C}-8\right)$; IR (KBr): $\nu_{\max } 3087,3063,3030,2906$ 2865, 1951, 1632, 1496, 1454, 1332, 1206, 1096, 1067, 1028, 927, 811, 736, $697 \mathrm{~cm}^{-1}$.

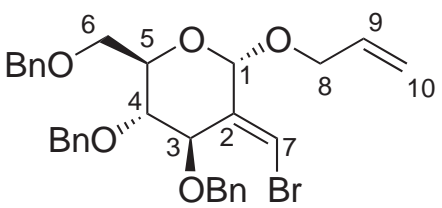

237: $\mathrm{R}_{f} 0.2$ (14:1 hexanes:ethyl acetate); $[\alpha]_{\mathrm{D}}^{20}+11(c$ $\left.4.0 \mathrm{CHCl}_{3}\right)$; ${ }^{1} \mathrm{H}-\mathrm{NMR}:\left(\mathrm{CDCl}_{3}\right) \delta_{\mathrm{H}} 7.35-7.25$ (complex m, $15 \mathrm{H}, \mathrm{Bn}), 6.79$ (s, 1H, H-7), 5.89 (ddd, $J=16.8$, $11.2,5.4 \mathrm{~Hz}, 1 \mathrm{H}, \mathrm{H}-9), 5.25$ (dd, $J=17.2,1.6 \mathrm{~Hz}, 1 \mathrm{H}$, H-10a), 5.19 (s, 1H, H-1), 5.16 (dd, $J=10.2,1.2 \mathrm{~Hz}, 1 \mathrm{H}, \mathrm{H}-10 \mathrm{~b}), 4.72$ (d, $J=$ $3.2 \mathrm{~Hz}, 1 \mathrm{H}, \mathrm{H}-3), 4.72$ (d, $\left.J=11.3 \mathrm{~Hz}, 1 \mathrm{H}, \mathrm{PhCH}_{2}\right), 4.63(\mathrm{~d}, J=11.8 \mathrm{~Hz}, 1 \mathrm{H}$, $\left.\mathrm{PhCH}_{2}\right), 4.52\left(\mathrm{~d}, J=12.2 \mathrm{~Hz}, 1 \mathrm{H}, \mathrm{PhCH}_{2}\right), 4.49\left(\mathrm{~s}, 2 \mathrm{H}, \mathrm{PhCH}_{2}\right), 4.47(\mathrm{~d}, J=$ $11.5 \mathrm{~Hz}, 1 \mathrm{H}, \mathrm{PhCH}_{2}$ ), 4.35 (dd, $\left.J=13.0,4.9 \mathrm{~Hz}, 1 \mathrm{H}, \mathrm{H}-8 \mathrm{a}\right), 4.08$ (dd, $J=13.0$, $5.8 \mathrm{~Hz}, 1 \mathrm{H}, \mathrm{H}-8 \mathrm{~b}$ ), 3.91-3.87 (complex m, 2H, H-4,5), 3.84-3.78 (complex m, 2H, H-6a,b); ${ }^{13}$ C-NMR: $\left(\mathrm{CDCl}_{3}\right) \delta_{\mathrm{C}} 138.4$ (C, Bn), 138.2 (C, Bn), 137.7 (C, Bn), 136.7 (C, C-2), 134.0 (CH, C-9), 128.4 (CH, Bn), 128.3 (CH, Bn), 128.2 (CH, Bn), 127.9 (CH, Bn), 127.8 (CH, Bn), $127.7(\mathrm{CH}, \mathrm{Bn}), 127.52(\mathrm{CH}, \mathrm{Bn}), 127.48(\mathrm{CH}, \mathrm{Bn})$, $117.1\left(\mathrm{CH}_{2}, \mathrm{C}-10\right), 114.6$ (CH, C-7), 98.8 (CH, C-1), 75.8 (CH, C-4), $74.9(\mathrm{CH}$, C-5), $74.2(\mathrm{CH}, \mathrm{C}-3), 73.2\left(\mathrm{CH}_{2}, \mathrm{PhCH}_{2}\right), 72.1\left(\mathrm{CH}_{2}, \mathrm{PhCH}_{2}\right), 70.63$ and 70.60 $\left(\mathrm{CH}_{2}, \mathrm{PhCH}_{2}\right.$ and $\left.\mathrm{CH}_{2}, \mathrm{C}-6\right), 68.7\left(\mathrm{CH}_{2}, \mathrm{C}-8\right)$; IR (KBr): $\nu_{\max } 3063,3030,2864$, 1951, 1873, 1631, 1496, 1454, 1365, 1333, 1305, 1207, 1094, 1074, 1027, 819, 735, $697 \mathrm{~cm}^{-1}$; HRMS: $m / z \mathrm{C}_{31} \mathrm{H}_{33} \mathrm{O}_{5}^{79} \mathrm{BrNa}^{+}[\mathrm{M}+\mathrm{Na}]^{+}$calcd 587.1409, found 587.1403.

B A solution of allyl alcohol $(60 \mu \mathrm{L}, 0.9 \mathrm{mmol})$ in THF $(1.7 \mathrm{~mL})$ was treated with sodium hydride (46 mg, $1.2 \mathrm{mmol}$ ), then once evolution of hydrogen had ceased, the solution was treated with cyclopropane 97 (102 mg, $0.173 \mathrm{mmol}$ ) and heated at 
reflux for five hours. The solution was separated between dichloromethane $(10 \mathrm{~mL})$ and water $(10 \mathrm{~mL})$, then the aqueous phase was extracted with dichloromethane $(2 \times 10 \mathrm{~mL})$. The combined organic fractions were dried, filtered and concentrated to provide crude products 236 and 237 as a yellow oil. Upon separation by column chromatography (14:1 hexanes:ethyl acetate), allyl glycosides 236 (19 mg, 19\%) and 237 (35 mg, 36\%) were obtained (55\% combined yield).

\section{Benzyl 3,4,6-tri- $O$-benzyl-(2E)-2- $C$-(bromomethylene)-2-deoxy- $\beta$-D- arabino-hexopyranoside (238) and benzyl 3,4,6-tri- $O$-benzyl-(2E)-2- $C$ - (bromomethylene)-2-deoxy- $\alpha$-D-arabino-hexopyranoside (239)}

Benzyl alcohol $(60 \mu \mathrm{L}, 0.58 \mathrm{mmol})$ was treated with metallic sodium $(6 \mathrm{mg}, 0.3$ mmol), then once the sodium had reacted, the solution was diluted with THF (1.7 mL). The solution was then treated with cyclopropane 97 (48 mg, $82 \mu \mathrm{mol})$ and heated at reflux for four hours. The solution was separated between dichloromethane $(10 \mathrm{~mL})$ and water $(10 \mathrm{~mL})$, then the aqueous phase was extracted with dichloromethane $(2 \times 10 \mathrm{~mL})$. The combined organic fractions were dried, filtered and concentrated to provide crude products 238 and 239 as a yellow oil. Upon separation by column chromatography (14:1 hexanes:ethyl acetate), benzyl glycosides 238 (16.5 mg, 33\%) and 239 (15 mg, 30\%) were obtained as colourless oils $(63 \%$ combined yield).

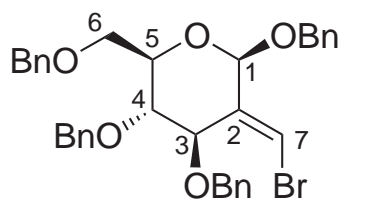

238: $\mathrm{R}_{f} 0.25$ (14:1 hexanes:ethyl acetate); $[\alpha]_{\mathrm{D}}^{20}+27(c 0.45$ $\left.\mathrm{CHCl}_{3}\right) ;{ }^{1} \mathrm{H}-\mathrm{NMR}:\left(\mathrm{CDCl}_{3}\right) \delta_{\mathrm{H}} 7.39-7.18$ (complex m, 20H, Bn), 6.87 (s, 1H, H-7), 5.40 (s, 1H, H-1), 4.87 (d, $J=11.9$ $\mathrm{Hz}, 1 \mathrm{H}, \mathrm{PhCH}_{2}$ ) 4.75 (s, 1H, H-3), 4.63 (d, $J=12.2 \mathrm{~Hz}, 1 \mathrm{H}$, $\mathrm{PhCH}_{2}$ ), 4.62-4.59 (complex m, 2H, $\mathrm{PhCH}_{2}$ ), 4.51 (d, J = 11.5 Hz, 1H, $\mathrm{PhCH}_{2}$ ), $4.50\left(\mathrm{~d}, J=12.2 \mathrm{~Hz}, 1 \mathrm{H}, \mathrm{PhCH}_{2}\right), 4.42\left(\mathrm{~d}, J=12.3 \mathrm{~Hz}, 1 \mathrm{H}, \mathrm{PhCH}_{2}\right), 4.28$ (d, $J=$ $11.4 \mathrm{~Hz}, 1 \mathrm{H}, \mathrm{PhCH}_{2}$ ) , 3.80-3.75 (complex m, 2H, H-4,5), 3.63-3.60 (complex m, 2H, H-6a,b); ${ }^{13}$ C-NMR: $\left(\mathrm{CDCl}_{3}\right) \delta_{\mathrm{C}} 138.3$ (C, Bn), 138.0 (C, Bn), 137.7 (C, Bn), 137.62 (C, Bn and C, C-2), 128.4 (CH, Bn), 128.33 (CH, Bn), 128.32 (CH, Bn), 128.28 (CH, Bn), 128.02 (CH, Bn), 127.97 (CH, Bn), $127.9(\mathrm{CH}, \mathrm{Bn}), 127.78$ (CH, Bn), 127.75 (CH, Bn), 127.70 (CH, Bn), 127.5 (CH, Bn), 112.8 (CH, C-7), 97.1 (CH, $\mathrm{C}-1), 79.3(\mathrm{CH}, \mathrm{C}-4), 76.0(\mathrm{CH}, \mathrm{C}-3), 73.1\left(\mathrm{CH}_{2}, \mathrm{PhCH}_{2}\right), 71.48$ and $71.47\left(\mathrm{CH}_{2}\right.$, $\mathrm{PhCH}_{2}$ and $\left.\mathrm{CH}, \mathrm{C}-5\right), 70.8\left(\mathrm{CH}_{2}, \mathrm{PhCH}_{2}\right), 69.5\left(\mathrm{CH}_{2}, \mathrm{C}-6\right), 69.1\left(\mathrm{CH}_{2}, \mathrm{PhCH}_{2}\right)$; IR (KBr): $\nu_{\max } 3063,3030,2865,1633,1496,1454,1335,1207,1097,1067,1027$, $807,733,694 \mathrm{~cm}^{-1}$.

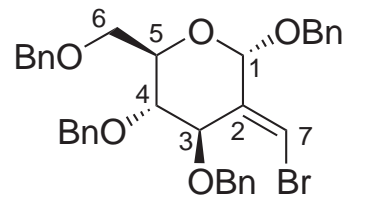

239: $\mathrm{R}_{f} 0.2$ (14:1 hexanes:ethyl acetate); $[\alpha]_{\mathrm{D}}^{20}-20(c \quad 0.3$ $\left.\mathrm{CHCl}_{3}\right) ;{ }^{1} \mathrm{H}-\mathrm{NMR}:\left(\mathrm{CDCl}_{3}\right) \delta_{\mathrm{H}} 7.40-7.24$ (complex m, 20H, Bn), 6.76 (s, 1H, H-7), 5.24 (s, 1H, H-1), 4.91 (d, $J=11.9 \mathrm{~Hz}$, $\left.1 \mathrm{H}, \mathrm{PhCH}_{2}\right), 4.75$ (d, $\left.J=2.4 \mathrm{~Hz}, 1 \mathrm{H}, \mathrm{H}-3\right), 4.72(\mathrm{~d}, J=11.5$ 
$\left.\mathrm{Hz}, 1 \mathrm{H}, \mathrm{PhCH}_{2}\right), 4.65\left(\mathrm{~d}, J=11.7 \mathrm{~Hz}, 1 \mathrm{H}, \mathrm{PhCH}_{2}\right), 4.60(\mathrm{~d}, J=11.7 \mathrm{~Hz}, 1 \mathrm{H}$, $\left.\mathrm{PhCH}_{2}\right), 4.54\left(\mathrm{~d}, J=11.5 \mathrm{~Hz}, 1 \mathrm{H}, \mathrm{PhCH}_{2}\right), 4.47$ (d, $\left.J=11.0 \mathrm{~Hz}, 1 \mathrm{H}, \mathrm{PhCH}_{2}\right)$, 4.47 (s, 2H, $\mathrm{PhCH}_{2}$ ), 3.95-3.92 (complex m, 2H, H-4,5), 3.86 (dd, J = 10.3, 6.1 $\mathrm{Hz}, 1 \mathrm{H}, \mathrm{H}-6 \mathrm{a}), 3.82$ (dd, $J=10.3,4.9 \mathrm{~Hz}, 1 \mathrm{H}, \mathrm{H}-6 \mathrm{~b}) ;{ }^{13} \mathrm{C}-\mathrm{NMR}:\left(\mathrm{CDCl}_{3}\right) \delta_{\mathrm{C}} 138.3$ (C, Bn), 138.2 (C, Bn), 137.7 (C, Bn), 137.5 (C, Bn), 136.6 (C, C-2), 128.62 (CH, Bn), 128.57 (CH, Bn), 128.4 (CH, Bn), 128.33 (CH, Bn), 128.30 (CH, Bn), 128.2 (CH, Bn), 127.9 (CH, Bn), 127.8 (CH, Bn), 127.7 (CH, Bn), 127.6 (CH, Bn), 127.5 (CH, Bn), 127.4 (CH, Bn), 114.8 (CH, C-7), 98.9 (CH, C-1), 75.8 (CH, C-4), 75.0 (CH, C-5), $74.3(\mathrm{CH}, \mathrm{C}-3), 73.2\left(\mathrm{CH}_{2}, \mathrm{PhCH}_{2}\right), 72.1\left(\mathrm{CH}_{2}, \mathrm{PhCH}_{2}\right), 70.6\left(\mathrm{CH}_{2}\right.$, C-6), $70.5\left(\mathrm{CH}_{2}, \mathrm{PhCH}_{2}\right), 69.6\left(\mathrm{CH}_{2}, \mathrm{PhCH}_{2}\right)$; IR (KBr): $\nu_{\max } 3030,2864,1629$, 1496, 1453, 1336, 1304, 1207, 1094, 1075, 1026, 819, 735, $696 \mathrm{~cm}^{-1}$; HRMS: $\mathrm{m} / z$ $\mathrm{C}_{35} \mathrm{H}_{35} \mathrm{O}_{5}^{79} \mathrm{BrNa}^{+}[\mathrm{M}+\mathrm{Na}]^{+}$calcd 637.1566 , found 637.1569 .

$N, N$-Diethyl 3,4,6-tri- $O$-benzyl-(2E)-2- $C$-(bromomethylene)-2-deoxy- $\beta$ D-arabino-hexopyranosyl amine (240) and $N, N$-diethyl 3,4,6-tri- $O$ benzyl-(2E)-2-C-(bromomethylene)-2-deoxy- $\alpha$-D-arabino-hexopyranosyl amine (241)

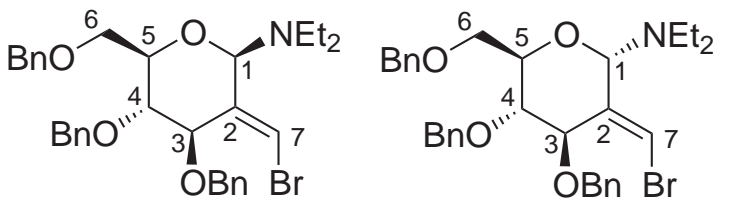

Diethylamine $(50 \mu \mathrm{L}, 0.53 \mathrm{mmol})$ was diluted with THF $(1.7 \mathrm{~mL})$, then treated with sodium hydride (18 mg, $0.45 \mathrm{mmol}$ ). Once the sodium had reacted, the solution was treated with cyclopropane 97 (109 $\mathrm{mg}, 0.185 \mathrm{mmol})$ and heated at reflux for six hours. The solution was separated between dichloromethane $(10 \mathrm{~mL})$ and water $(10 \mathrm{~mL})$, then the aqueous phase was extracted with dichloromethane $(2 \mathrm{x}$ $10 \mathrm{~mL}$ ). The combined organic fractions were dried, filtered and concentrated to provide crude products 240 and 241 as a yellow oil. Upon separation by column chromatography (20:1 hexanes:ethyl acetate), a mixture of glycosylamines $\mathbf{2 4 0}$ and $\mathbf{2 4 1}$ (48 mg, 45\%) was obtained as a pale-yellow oil, in addition to recovered starting material (15 mg, 14\%).

\section{1,5-Anhydro-3,4,6-tri- $O$-benzyl-2-deoxy-1,2- $C$-(( $S)$-bromomethylene)-D- glycero-D-gulo-hexitol (235)}

gem-Dibromocyclopropane 97 (99 $\mathrm{mg}, 0.17 \mathrm{mmol}$ ) was dissolved in THF (1.7 mL), cooled to $-78{ }^{\circ} \mathrm{C}$, then treated with $n$-butyllithium $(110 \mu \mathrm{L}, 0.176 \mathrm{mmol})$. The reaction was stirred for 10 minutes, then quenched with water $(8 \mu \mathrm{L}$ of a $50 \%$ solution in THF, $0.2 \mathrm{mmol})$. 10\% aqueous hydrochloric acid was then added (10 mL), then the mixture was extracted with dichloromethane $(3 \times 10 \mathrm{~mL})$. The organic fractions were combined, dried and concentrated to provide an orange oil. This oil was purified by flash chromatography (14:1 hexanes:ethyl acetate), to provide both unreacted 
starting material 97 (26 mg, 26\%) and bromocyclopropane 235 as a colourless oil (22 mg, 26\%).

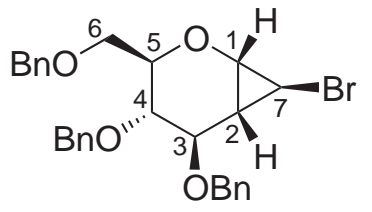

$\mathrm{R}_{f} 0.2$ (14:1 hexanes:ethyl acetate); $[\alpha]_{\mathrm{D}}^{17}+23\left(\right.$ c $\left.1.7 \mathrm{CHCl}_{3}\right)$; ${ }^{1} \mathrm{H}-\mathrm{NMR}:\left(\mathrm{CDCl}_{3}\right) \delta_{\mathrm{H}} 7.37-7.23$ (complex m, $\left.15 \mathrm{H}, \mathrm{Bn}\right), 4.74$ $\left(\mathrm{d}, J=11.7 \mathrm{~Hz}, 1 \mathrm{H}, \mathrm{PhCH}_{2}\right), 4.70(\mathrm{~d}, J=11.5 \mathrm{~Hz}, 1 \mathrm{H}$, $\mathrm{PhCH}_{2}$ ), 4.60 (d, $\left.J=11.7 \mathrm{~Hz}, 1 \mathrm{H}, \mathrm{PhCH}_{2}\right), 4.55-4.49$ (complex m, 3H, $\mathrm{PhCH}_{2}$ ), 3.81 (dd, $J=7.8,1.5 \mathrm{~Hz}, 1 \mathrm{H}, \mathrm{H}-1$ ), 3.77 (ddd, $J=6.0,5.5$, $3.7 \mathrm{~Hz}, 1 \mathrm{H}, \mathrm{H}-5$ ), 3.74 (dd, $J=6.3,2.9 \mathrm{~Hz}, 1 \mathrm{H}, \mathrm{H}-3), 3.66$ (d, $J=10.4,6.0 \mathrm{~Hz}$, 1H, H-6a), 3.56 (d, $J=3.7 \mathrm{~Hz}, 1 \mathrm{H}, \mathrm{H}-4), 3.54$ (d, $J=5.4 \mathrm{~Hz}, 1 \mathrm{H}, \mathrm{H}-6 \mathrm{~b}), 3.01$ (dd, $J=4.5,1.6 \mathrm{~Hz}, 1 \mathrm{H}, \mathrm{H}-7), 1.54$ (ddd, $J=7.8,4.7,3.0 \mathrm{~Hz}, 1 \mathrm{H}, \mathrm{H}-2) ;{ }^{13} \mathrm{C}-\mathrm{NMR}$ : $\left(\mathrm{CDCl}_{3}\right) \delta_{\mathrm{C}} 138.02(\mathrm{C}, \mathrm{Bn}), 137.99$ (C, Bn), 137.6 (C, Bn), 128.5 (CH, Bn), 128.41 (CH, Bn), 128.40 (CH, Bn), 127.91 (CH, Bn), 127.86 (CH, Bn), 127.8 (CH, Bn), 127.73 (CH, Bn), 127.70 (CH, Bn), 127.68 (CH, Bn), 75.9 (CH, C-3), 75.3 (CH, C-5), 74.8 (CH, C-4), $73.3\left(\mathrm{CH}_{2}, \mathrm{PhCH}_{2}\right), 73.1\left(\mathrm{CH}_{2}, \mathrm{PhCH}_{2}\right), 71.4\left(\mathrm{CH}_{2}, \mathrm{PhCH}_{2}\right)$, $69.3\left(\mathrm{CH}_{2}, \mathrm{C}-6\right), 56.7$ (CH, C-1), $26.3(\mathrm{CH}, \mathrm{C}-2), 20.5$ (CH, C-7); IR (KBr): $\nu_{\max }$ 3062, 3030, 2862, 1496, 1453, 1364, 1200, 1094, 1027, 736, $697 \mathrm{~cm}^{-1}$; HRMS: $\mathrm{m} / z$ $\mathrm{C}_{28} \mathrm{H}_{29} \mathrm{O}_{4}^{79} \mathrm{BrNa}^{+}[\mathrm{M}+\mathrm{Na}]^{+}$calcd 531.1147, found 531.1143.

\section{Methyl 3,4,6-tri- $O$-benzyl-(2E)-2- $C$-(but-3-enylidene)-2-deoxy- $\alpha$-D- arabino-hexopyranoside (242)}

Using a previously reported procedure, ${ }^{156}$ a solution of brominated sugar 214 (25 mg, $46 \mu \mathrm{mol})$ in DMF $(1 \mathrm{~mL})$ was treated successively with allyltributylstannane $(18 \mu \mathrm{L}$, $58 \mu \mathrm{mol}$ ) and palladium-tetrakis(triphenylphosphine) (7 mg, $6 \mu \mathrm{mol}$ ), then stirred at $100{ }^{\circ} \mathrm{C}$ for one day. The reaction mixture was treated with additional palladiumtetrakis(triphenylphosphine) $(7 \mathrm{mg}, 6 \mu \mathrm{mol})$, then stirred for an additional day. The reaction mixture was diluted with water $(10 \mathrm{~mL})$, then extracted with dichloromethane $(3 \times 10 \mathrm{~mL})$. The organic fractions were combined, dried and concentrated to provide a yellow oil. This oil was purified by flash chromatography ( $9: 1$ hexanes:ethyl acetate), to provide diene $\mathbf{2 4 2}$ (12 $\mathrm{mg}, 52 \%$ ) as a colourless oil.

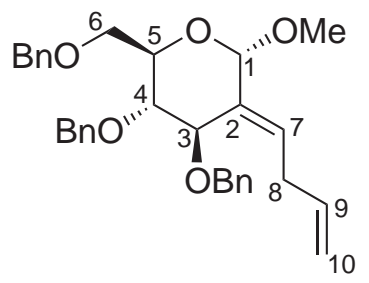

$\mathrm{R}_{f} 0.3$ (9:1 hexanes:ethyl acetate); $[\alpha]_{\mathrm{D}}^{17}-12\left(\right.$ c $\left.0.5 \mathrm{CHCl}_{3}\right)$; ${ }^{1} \mathrm{H}-\mathrm{NMR}:\left(\mathrm{CDCl}_{3}\right) \delta_{\mathrm{H}} 7.35-7.13$ (complex m, 15H, Bn), 5.87 (t, $J=7.6 \mathrm{~Hz}, 1 \mathrm{H}, \mathrm{H}-7$ ), 5.80 (ddt, $J=17.1,10.3,6.2 \mathrm{~Hz}$, 1H, H-9), 5.06 (s, 1H, H-1), 5.02 (ddd, $J=17.1,3.5,1.9 \mathrm{~Hz}$, 1H, H-10a), 4.99 (ddd, $J=10.3,3.9,1.6 \mathrm{~Hz}, 1 \mathrm{H}, \mathrm{H}-10 \mathrm{~b}), 4.68$ $\left(\mathrm{d}, J=12.0 \mathrm{~Hz}, 1 \mathrm{H}, \mathrm{PhCH}_{2}\right), 4.65\left(\mathrm{~d}, J=12.0 \mathrm{~Hz}, 1 \mathrm{H}, \mathrm{PhCH}_{2}\right), 4.63$ (d, $J=$ $\left.11.5 \mathrm{~Hz}, 1 \mathrm{H}, \mathrm{PhCH}_{2}\right), 4.52$ (d, $\left.J=12.2 \mathrm{~Hz}, 1 \mathrm{H}, \mathrm{PhCH}_{2}\right), 4.49$ (d, $J=12.0 \mathrm{~Hz}$, $1 \mathrm{H}, \mathrm{PhCH}_{2}$ ), 4.49 (m, 1H, H-3), 4.40 (d, $J=11.5 \mathrm{~Hz}, 1 \mathrm{H}, \mathrm{PhCH}_{2}$ ), 3.77 (ddd, $J=$ 9.3, 4.1, $2.9 \mathrm{~Hz}, 1 \mathrm{H}, \mathrm{H}-5$ ), 3.72 (dd, $J=9.4,4.5 \mathrm{~Hz}, 1 \mathrm{H}, \mathrm{H}-4$ ), 3.66-3.68 (complex m, 2H, H-6a,b), 3.44 (s, 3H, Me), 2.97 (apparent td, $J=7.0,1.5 \mathrm{~Hz}, 2 \mathrm{H}, \mathrm{H}-8 \mathrm{a}, \mathrm{b}$ ); 
${ }^{13} \mathrm{C}-\mathrm{NMR}:\left(\mathrm{CDCl}_{3}\right) \delta_{\mathrm{C}} 138.31$ (C, 2 x Bn), 138.0 (C, Bn), 136.5 (CH, C-9), 132.4

(C, C-2), 129.9 (CH, C-7), $128.4(\mathrm{CH}, \mathrm{Bn}), 128.30$ (CH, Bn), 128.28 (CH, Bn), 127.81 (CH, Bn), 127.79 (CH, Bn), $127.64(\mathrm{CH}, \mathrm{Bn}), 127.58(\mathrm{CH}, \mathrm{Bn}), 127.54(\mathrm{CH}$, Bn), 127.50 ( CH, Bn), $115.4\left(\mathrm{CH}_{2}, \mathrm{C}-10\right), 101.8$ (CH, C-1), 79.7 (CH, C-4), 77.3 (CH, C-3), $73.2\left(\mathrm{CH}_{2}, \mathrm{PhCH}_{2}\right), 73.0\left(\mathrm{CH}_{2}, \mathrm{PhCH}_{2}\right), 71.5\left(\mathrm{CH}_{2}, \mathrm{PhCH}_{2}\right), 71.3(\mathrm{CH}$, C-5), $69.4\left(\mathrm{CH}_{2}, \mathrm{C}-6\right), 54.8\left(\mathrm{CH}_{3}, \mathrm{Me}\right), 31.4\left(\mathrm{CH}_{2}, \mathrm{C}-8\right)$; IR ( $\left.\mathrm{KBr}\right): \nu_{\max } 3030,2920$, 1723, 1496, 1453, 1356, 1091, 1067, 1027, 750, $698 \mathrm{~cm}^{-1}$; HRMS: $m / z \mathrm{C}_{32} \mathrm{H}_{36} \mathrm{O}_{5} \mathrm{Na}^{+}$ $[\mathrm{M}+\mathrm{Na}]^{+}$calcd 523.2460, found 523.2462.

\section{D-Galactal (186)}

Using a modification of Kozikowski's procedure, ${ }^{89}$ a magnetically stirred solution of D-galactose $(125 \mathrm{mg}, 0.7 \mathrm{mmol})$ in acetic anhydride $(75 \mathrm{~mL}, 790 \mathrm{mmol})$ was treated dropwise with conc. perchloric acid $(0.45 \mathrm{~mL}, 6.9 \mathrm{mmol})$. D-galactose $(19.9 \mathrm{~g}$, $110.3 \mathrm{mmol}$ ) was slowly added over half an hour, at a rate that maintained a temperature of $40-50{ }^{\circ} \mathrm{C}$. Upon complete addition of D-galactose, the solution was allowed to cool to room temperature, then treated with a $33 \%(\mathrm{w} / \mathrm{w})$ solution of hydrobromic acid in acetic acid $(75 \mathrm{~mL}, 410 \mathrm{mmol})$. After 90 minutes, the solution was diluted with dichloromethane $(180 \mathrm{~mL})$ and washed with ice-cold water $(2 \times 50 \mathrm{~mL})$, then with cold sat. sodium bicarbonate solution $(4 \times 100 \mathrm{~mL})$. The organic phase was dried, filtered and concentrated to afford crude tetra- $O$-acetyl- $\alpha$-D-galactopyranosyl bromide (248) as a pale-yellow oil.

A mechanically stirred dispersion of zinc dust (48.0 g, $734 \mathrm{mmol})$ in water $(150 \mathrm{~mL})$ was cooled to $0{ }^{\circ} \mathrm{C}$, then diluted with acetic acid $(150 \mathrm{~mL})$, then treated dropwise with a solution of tetra- $O$-acetyl- $\alpha$-D-galactopyranosyl bromide in diethyl ether $(150 \mathrm{~mL})$, over one hour. The reaction was allowed to warm to room temperature and left to proceed overnight. The solution was filtered, then diluted with dichloromethane $(200 \mathrm{~mL})$. The solution was then washed successively with water $(3 \mathrm{x}$ $60 \mathrm{~mL})$, sat. sodium bicarbonate solution $(3 \times 50 \mathrm{~mL})$, then brine $(60 \mathrm{~mL})$. The organic phase was dried, filtered and concentrated to provide tri- $O$-acetyl-D-galactal (180) (27.7 g) as a pale-yellow oil.

A solution of triethylamine $(110 \mathrm{~mL}, 780 \mathrm{mmol})$ in methanol $(50 \%$ of an aqueous solution, $1100 \mathrm{~mL}$ ) was treated with tri- $O$-acetyl-D-galactal $(27.7 \mathrm{~g})$ and stirred for 90 minutes. The mixture was concentrated to liberate crude D-galactal, contaminated with triethylamine. The crude mixture was successively treated with portions of methanol, then concentrated to remove the triethylamine. The oil was dissolved in methanol $(15 \mathrm{~mL})$, then diluted successively with acetone $(100 \mathrm{~mL})$ and diethyl ether $(200 \mathrm{~mL})$, which led to the precipitation of impurities. The solution was filtered and concentrated to afford D-galactal (186) as a pale-brown solid (6.63 g, 41\% crude yield), which was used without further purification. 


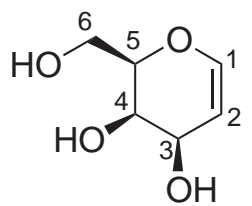

${ }^{1} \mathrm{H}-\mathrm{NMR}:\left(\mathrm{D}_{2} \mathrm{O}\right) \delta_{\mathrm{H}} 6.25(\mathrm{dd}, J=6.3,1.2 \mathrm{~Hz}, 1 \mathrm{H}, \mathrm{H}-1), 4.77$ (dd, $J=6.1,2.4 \mathrm{~Hz}, 1 \mathrm{H}, \mathrm{H}-2), 4.66$ (complex m, 3H, 3 x OH) 4.19 (dt, $J=7.1,2.1 \mathrm{~Hz}, 1 \mathrm{H}, \mathrm{H}-3), 3.84$ (complex m, 3H, H-5,6), 3.64 (dd, $J=9.0,7.1 \mathrm{~Hz}, 1 \mathrm{H}, \mathrm{H}-4) ;{ }^{13} \mathrm{C}-\mathrm{NMR}:\left(\mathrm{D}_{2} \mathrm{O}\right) \delta_{\mathrm{H}} 143.8(\mathrm{CH}, \mathrm{C}-1)$, 102.7 (CH, C-2), 78.0 (CH, C-5), 68.7 (CH, C-4), 68.2 (CH, C-3), $59.9\left(\mathrm{CH}_{2}, \mathrm{C}-6\right)$; IR $(\mathrm{KBr}): \nu_{\max } 3383,3060,2952,2892,1643,1219,1020 \mathrm{~cm}^{-1}$.

\section{Tri- $O$-benzyl-D-galactal (43)}

A solution of D-galactal (186) (2.00 g, $13.7 \mathrm{mmol})$ in DMF (80 mL) was cooled to $0{ }^{\circ} \mathrm{C}$, then treated with sodium hydride $(2.13 \mathrm{~g}, 53.2 \mathrm{mmol})$. The solution was stirred at $0{ }^{\circ} \mathrm{C}$ for 30 minutes, then treated with benzyl bromide $(5.6 \mathrm{~mL}, 47 \mathrm{mmol})$ and allowed to warm to room temperature. After 19 hours, the solution was diluted with water $(40 \mathrm{~mL})$ and the product extracted with diethyl ether $(3 \times 100 \mathrm{~mL})$. The organic fractions were combined, dried, filtered and concentrated to provide the crude product as a concentrated solution in DMF. Purification by column chromatography (9:1 hexanes:ethyl acetate) afforded 43 (3.53 g, 62\%) as a white solid. The spectral data matched that reported previously. ${ }^{154}$

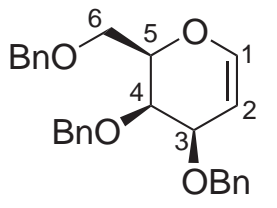

$\mathrm{R}_{f} 0.3$ (9:1 hexanes:ethyl acetate); m.p. $47-51{ }^{\circ} \mathrm{C}$ (lit. ${ }^{154}$ m.p. $52-$ $54{ }^{\circ} \mathrm{C}$ ); ${ }^{1} \mathrm{H}-\mathrm{NMR}:\left(\mathrm{CDCl}_{3}\right) \delta_{\mathrm{H}} 7.35-7.26$ (complex m, $15 \mathrm{H}, \mathrm{Bn}$ ), $6.38(\mathrm{dd}, J=6.3,1.2 \mathrm{~Hz}, 1 \mathrm{H}, \mathrm{H}-1), 4.89(\mathrm{~d}, J=12.0 \mathrm{~Hz}, 1 \mathrm{H}$, $\mathrm{PhCH}_{2}$ ), 4.86 (m, 1H, H-2), 4.69-4.61 (complex m, 3H, $\mathrm{PhCH}_{2}$ ), $4.51\left(\mathrm{~d}, J=12.0 \mathrm{~Hz}, 1 \mathrm{H}, \mathrm{PhCH}_{2}\right), 4.43\left(\mathrm{~d}, J=12.0 \mathrm{~Hz}, 1 \mathrm{H}, \mathrm{PhCH}_{2}\right), 4.20-4.19$ (complex m, 1H, H-3,5), 3.96 (m, 1H, H-4), 3.79 (dd, $J=10.3,7.3 \mathrm{~Hz}, 1 \mathrm{H}, \mathrm{H}-6 \mathrm{a})$, $3.65(\mathrm{dd}, J=10.3,5.1 \mathrm{~Hz}, 1 \mathrm{H}, \mathrm{H}-6 \mathrm{~b}) ;{ }^{13} \mathrm{C}-\mathrm{NMR}:\left(\mathrm{CDCl}_{3}\right) \delta_{\mathrm{C}} 144.2(\mathrm{CH}, \mathrm{C}-1), 138.5$ (C, Bn), 138.3 (C, Bn), 138.0 (C, Bn), 128.4 (CH, Bn), 128.3 (CH, Bn), 128.2 (CH, $\mathrm{Bn}), 127.9$ (CH, Bn), 127.7 (CH, Bn), 127.6 (CH, Bn), 127.4 (CH, Bn), 100.0 (CH, C-2), 75.7 (CH, C-5), $73.4\left(\mathrm{CH}_{2}, \mathrm{PhCH}_{2}\right), 73.3\left(\mathrm{CH}_{2}, \mathrm{PhCH}_{2}\right), 71.2(\mathrm{CH}, \mathrm{C}-4), 70.9$ $\left(\mathrm{CH}_{2}, \mathrm{PhCH}_{2}\right), 70.7(\mathrm{CH}, \mathrm{C}-3), 68.4\left(\mathrm{CH}_{2}, \mathrm{C}-6\right)$; IR $(\mathrm{KBr}): \nu_{\max } 3087,3064,3025$, $2874,1951,1809,1642,1453,1159,1098,747 \mathrm{~cm}^{-1}$.

\section{Di- $O$-acetyl-D-xylal (181)}

Using a modification of Kozikowski's procedure, ${ }^{89}$ a magnetically stirred solution of D-xylose (30 mg, $0.2 \mathrm{mmol})$ in acetic anhydride $(15 \mathrm{~mL}, 160 \mathrm{mmol})$ was treated dropwise with conc. perchloric acid $(0.09 \mathrm{~mL}, 1.4 \mathrm{mmol})$. Additional D-xylose $(3.90 \mathrm{~g}$, $26.0 \mathrm{mmol}$ ) was slowly added over half an hour, at a rate that maintained a temperature of $40-50{ }^{\circ} \mathrm{C}$. Upon complete addition of D-xylose, the solution was allowed to cool to room temperature, then treated with a $33 \%(\mathrm{w} / \mathrm{w})$ solution of hydrobromic acid in acetic acid $(15 \mathrm{~mL}, 83 \mathrm{mmol})$. After 90 minutes, the solution was diluted 
with dichloromethane $(40 \mathrm{~mL})$ and washed with ice-cold water $(3 \times 20 \mathrm{~mL})$, then with cold sat. sodium bicarbonate solution $(2 \times 20 \mathrm{~mL})$. The organic phase was dried, filtered and concentrated to afford crude tetra- $O$-acetyl- $\alpha$-D-xylopyranosyl bromide (249) as an orange oil.

A mechanically stirred dispersion of zinc dust $(9.9 \mathrm{~g}, 150 \mathrm{mmol})$ in water $(30 \mathrm{~mL})$ was cooled to $0{ }^{\circ} \mathrm{C}$, then diluted with acetic acid $(30 \mathrm{~mL})$, then treated dropwise with a solution of tetra- $O$-acetyl- $\alpha$-D-xylopyranosyl bromide in diethyl ether $(40 \mathrm{~mL})$, over half an hour, then allowed to react further at $0{ }^{\circ} \mathrm{C}$ for three hours. The solution was filtered, then diluted with dichloromethane $(40 \mathrm{~mL})$. The resulting solution was washed successively with water $(3 \mathrm{x} 20 \mathrm{~mL})$, sat. sodium bicarbonate solution $(3 \mathrm{x}$ $20 \mathrm{~mL})$, then brine $(20 \mathrm{~mL})$. The organic phase was dried, filtered and concentrated to provide crude di- $O$-acetyl-D-xylal as a colourless oil $(5.18 \mathrm{~g})$, which was purified by column chromatography (3:1 hexanes:ethyl acetate) to liberate glycal 181 (2.00 g, $38 \%)$ as an off-white solid. The spectral data matched that reported previously. ${ }^{157}$<smiles>CC(=O)O[C@H]1COC=C[C@H]1OC(C)=O</smiles>

$\mathrm{R}_{f} 0.4$ (3:1 hexanes:ethyl acetate); m.p. $54-56{ }^{\circ} \mathrm{C}$; (lit. ${ }^{110}$ m.p. $\left.51^{\circ} \mathrm{C}\right)$; ${ }^{1} \mathrm{H}-\mathrm{NMR}:\left(\mathrm{CDCl}_{3}\right) \delta_{\mathrm{H}} 6.60(\mathrm{~d}, J=5.6 \mathrm{~Hz}, 1 \mathrm{H}, \mathrm{H}-1), 4.99-4.94(\mathrm{com}-$ plex m, 3H, H-2,3,4), 4.18 (ddd, $J=12.2,3.1,1.5 \mathrm{~Hz}, 1 \mathrm{H}, \mathrm{H}-5 \mathrm{a}$ ), 3.97 (dd, $J=12.2,1.2 \mathrm{~Hz}, 1 \mathrm{H}, \mathrm{H}-5 \mathrm{~b}), 2.10$ (s, 3H, Ac), 2.07 (s, 3H, Ac); ${ }^{13} \mathrm{C}-\mathrm{NMR}:\left(\mathrm{CDCl}_{3}\right) \delta_{\mathrm{C}} 169.9\left(\mathrm{C}, \mathrm{CH}_{3} \mathrm{CO}\right), 169.8$ (C, $\left.\mathrm{CH}_{3} \mathrm{CO}\right), 148.0$ (CH, C-1), 97.4 (CH, C-2), $67.2(\mathrm{CH}, \mathrm{C}-4), 63.6\left(\mathrm{CH}_{2}, \mathrm{C}-5\right), 63.4(\mathrm{CH}, \mathrm{C}-3), 21.1\left(\mathrm{CH}_{3}, \mathrm{Ac}\right), 21.0$ $\left(\mathrm{CH}_{3}, \mathrm{Ac}\right)$.

\section{Di- $O$-benzyl-D-xylal (189)}

A solution of di- $O$-acetyl-D-xylal (181) $(672 \mathrm{mg}, 3.35 \mathrm{mmol})$ in methanol $(35 \mathrm{~mL})$ was treated with metallic sodium (4 mg, $0.17 \mathrm{mmol}$ ) and stirred for 90 minutes at room temperature. The mixture was treated with water $(5 \mathrm{~mL})$ and a piece of dry ice $(5 \mathrm{~g})$, then concentrated to liberate crude D-xylal as a colourless oil (413 $\mathrm{mg}$ ). This oil was dissolved in DMF $(35 \mathrm{~mL})$, cooled to $0{ }^{\circ} \mathrm{C}$, then treated with tetrabutylammonium iodide $(71 \mathrm{mg}, 0.19 \mathrm{mmol})$, followed by sodium hydride (364 mg, $9.10 \mathrm{mmol})$. The solution was stirred at $0{ }^{\circ} \mathrm{C}$ for 30 minutes, then treated with benzyl bromide $(0.92 \mathrm{~mL}, 7.7 \mathrm{mmol})$ and allowed to warm to room temperature. The mixture was stirred overnight, then quenched with water $(30 \mathrm{~mL})$ and sat. copper sulfate solution $(30 \mathrm{~mL})$, then the product was extracted with diethyl ether $(3 \mathrm{x}$ $50 \mathrm{~mL})$. The organic fractions were combined, washed with brine $(20 \mathrm{~mL})$, dried, filtered and concentrated to provide crude di- $O$-benzyl-D-xylal (189). Purification by column chromatography (9:1 hexanes:ethyl acetate) afforded 189 (665 mg, 66\%) as a white solid. The spectral data matched that reported previously. ${ }^{154}$ 
<smiles>Oc1ccccc1Br</smiles>

$\mathrm{R}_{f} 0.35$ (9:1 hexanes:ethyl acetate); m.p. $28-30{ }^{\circ} \mathrm{C}$ (lit. ${ }^{154}$ m.p. 30 $32{ }^{\circ} \mathrm{C}$ ); ${ }^{1} \mathrm{H}-\mathrm{NMR}:\left(\mathrm{CDCl}_{3}\right) \delta_{\mathrm{H}} 7.37-7.28$ (complex m, 10H, Bn), 6.55 $(\mathrm{d}, J=6.1 \mathrm{~Hz}, 1 \mathrm{H}, \mathrm{H}-1), 4.94(\mathrm{ddd}, J=6.1,4.6,1.5 \mathrm{~Hz}, 1 \mathrm{H}, \mathrm{H}-$ 2), 4.65-4.64 (complex m, 2H, $\left.\mathrm{PhCH}_{2}\right), 4.60(\mathrm{~d}, J=11.7 \mathrm{~Hz}, 1 \mathrm{H}$, $\mathrm{PhCH}_{2}$ ), $4.53\left(\mathrm{~d}, J=12.0 \mathrm{~Hz}, 1 \mathrm{H}, \mathrm{PhCH}_{2}\right.$ ), 4.11 (ddd, $J=11.7,3.9,1.5 \mathrm{~Hz}, 1 \mathrm{H}$, H-5a), 3.96 (dd, $J=11.6,1.6 \mathrm{~Hz}, 1 \mathrm{H}, \mathrm{H}-5 \mathrm{~b}), 3.84$ (m, 1H, H-3), 3.67 (m, 1H, H-4); ${ }^{13} \mathrm{C}-\mathrm{NMR}:\left(\mathrm{CDCl}_{3}\right) \delta_{\mathrm{C}} 146.7(\mathrm{CH}, \mathrm{C}-1), 138.3(\mathrm{C}, \mathrm{Bn}), 137.9(\mathrm{C}, \mathrm{Bn}), 128.5(\mathrm{CH}$, Bn), 128.4 (CH, Bn), 127.84 (CH, Bn), 127.76 (CH, Bn), 127.67 (CH, Bn), 127.65 (CH, Bn), 98.9 (CH, C-2), $72.6(\mathrm{CH}, \mathrm{C}-4), 71.2\left(\mathrm{CH}_{2}, \mathrm{PhCH}_{2}\right), 70.0\left(\mathrm{CH}_{2}, \mathrm{PhCH}_{2}\right)$, $69.0(\mathrm{CH}, \mathrm{C}-3), 63.9\left(\mathrm{CH}_{2}, \mathrm{C}-5\right)$.

\section{1,5-anhydro-3,4,6-tri- $O$-benzyl-2-deoxy-1,2- $C$-(dibromomethylene)-D- glycero-L-manno-hexitol (45)}

As reported by Nagarajan, ${ }^{45}$ a solution of tri- $O$-benzyl-D-galactal (43) (375 mg, $0.90 \mathrm{mmol})$ and TEBAC (4 mg, $0.02 \mathrm{mmol})$ in bromoform $(2.0 \mathrm{~mL}, 23 \mathrm{mmol})$ was treated dropwise with a solution of sodium hydroxide (382 $\mathrm{mg}, 9.5 \mathrm{mmol}$ ) and anhydrous potassium fluoride $(2.95 \mathrm{~g})$ in water $(2.9 \mathrm{~mL})$. The biphasic mixture was stirred at room temperature for two days, then diluted with water $(20 \mathrm{~mL})$. The mixture was extracted with diethyl ether $(3 \times 30 \mathrm{~mL})$, then the organic fractions were combined and washed with brine $(20 \mathrm{~mL})$. The ethereal solution was dried, filtered and concentrated to provide a crude mixture of cyclopropane $\mathbf{4 5}$, which was purified by column chromatography (9:1 hexanes:ethyl acetate) to liberate cyclopropane 45 (375 mg, 71\%), as a yellow oil. The spectral data matched that which was previously reported. ${ }^{45}$

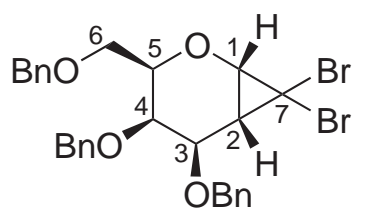

$\mathrm{R}_{f} 0.5$ (5:1 hexanes:ethyl acetate); $[\alpha]_{\mathrm{D}}^{18}+13\left(\right.$ c $\left.2.2 \mathrm{CHCl}_{3}\right)$; ${ }^{1} \mathrm{H}-\mathrm{NMR}:\left(\mathrm{CDCl}_{3}\right) \delta_{\mathrm{H}} 7.41-7.26$ (complex m, $\left.15 \mathrm{H}, \mathrm{Bn}\right), 4.93$ $\left(\mathrm{d}, J=11.5 \mathrm{~Hz}, 1 \mathrm{H}, \mathrm{PhCH}_{2}\right), 4.80(\mathrm{~d}, J=12.2 \mathrm{~Hz}, 1 \mathrm{H}$, $\left.\mathrm{PhCH}_{2}\right), 4.64\left(\mathrm{~d}, J=12.0 \mathrm{~Hz}, 1 \mathrm{H}, \mathrm{PhCH}_{2}\right), 4.59$ (d, $J=11.5$

$\left.\mathrm{Hz}, 1 \mathrm{H}, \mathrm{PhCH}_{2}\right), 4.48\left(\mathrm{~d}, J=11.7 \mathrm{~Hz}, 1 \mathrm{H}, \mathrm{PhCH}_{2}\right), 4.41(\mathrm{~d}, J=12.0 \mathrm{~Hz}, 1 \mathrm{H}$, $\mathrm{PhCH}_{2}$ ), 3.96 (dd, $\left.J=8.8,1.5 \mathrm{~Hz}, 1 \mathrm{H}, \mathrm{H}-1\right), 3.87$ (d, $\left.J=2.0 \mathrm{~Hz}, 1 \mathrm{H}, \mathrm{H}-4\right), 3.85$ $(\mathrm{dd}, J=7.1,6.1 \mathrm{~Hz}, 1 \mathrm{H}, \mathrm{H}-5), 3.59(\mathrm{dd}, J=9.3,7.1 \mathrm{~Hz}, 1 \mathrm{H}, \mathrm{H}-6 \mathrm{a}), 3.54$ (dd, $J=9.4,5.8 \mathrm{~Hz}, 1 \mathrm{H}, \mathrm{H}-6 \mathrm{~b}), 3.51(\mathrm{dd}, J=4.3,1.9 \mathrm{~Hz}, 1 \mathrm{H}, \mathrm{H}-3), 2.06(\mathrm{dd}, J=$ 8.9, $4.3 \mathrm{~Hz}, 1 \mathrm{H}, \mathrm{H}-2) ;{ }^{13} \mathrm{C}-\mathrm{NMR}:\left(\mathrm{CDCl}_{3}\right) \delta_{\mathrm{C}} 138.5$ (C, Bn), 137.8 (C, Bn), 137.5 (C, Bn), 128.5 (CH, Bn), 128.4 (CH, Bn), 128.3 (CH, Bn), 127.88 (CH, Bn), 127.86 (CH, Bn), $127.8(\mathrm{CH}, \mathrm{Bn}), 127.6(\mathrm{CH}, \mathrm{Bn}), 78.4(\mathrm{CH}, \mathrm{C}-5), 77.7(\mathrm{CH}, \mathrm{C}-3), 74.5$ $\left(\mathrm{CH}_{2}, \mathrm{PhCH}_{2}\right), 73.4\left(\mathrm{CH}_{2}, \mathrm{PhCH}_{2}\right), 71.7(\mathrm{CH}, \mathrm{C}-4), 71.0\left(\mathrm{CH}_{2}, \mathrm{PhCH}_{2}\right), 69.0\left(\mathrm{CH}_{2}\right.$, C-6), 59.0 (CH, C-1), 35.0 (C, C-7), 32.0 (CH, C-2); IR (KBr): $\nu_{\max } 3088,3063$, 3030, 2917, 2867, 1951, 1876, 1728, 1605, 1586, 1496, 1454, 1363, 1219, 1173, 1126, 1090, 1064, 1028, 750, $699 \mathrm{~cm}^{-1}$; HRMS: $m / z \mathrm{C}_{28} \mathrm{H}_{28} \mathrm{O}_{4}^{79} \mathrm{Br}_{2} \mathrm{Na}^{+}[\mathrm{M}+\mathrm{Na}]^{+}$calcd 
609.0252, found 609.0251.

\section{1,5-Anhydro-3,4-di- $O$-benzyl-2-deoxy-1,2- $C$-(dibromomethylene)-D- gulo-pentitol (243) and 1,5-anhydro-3,4-di- $O$-benzyl-2-deoxy-1,2- $C$ - (dibromomethylene)-D-talo-pentitol (250)}

As reported by Nagarajan, ${ }^{45}$ a solution of di- $O$-benzyl-D-xylal (189) (287 mg, $0.97 \mathrm{mmol})$ and TEBAC (6 mg, $0.03 \mathrm{mmol})$ in bromoform $(2.0 \mathrm{~mL}, 23 \mathrm{mmol})$ was treated dropwise with a solution of sodium hydroxide (426 mg, $11 \mathrm{mmol}$ ) and anhydrous potassium fluoride $(3.2 \mathrm{~g})$ in water $(3.2 \mathrm{~mL})$. The biphasic mixture was stirred at room temperature for two days, then diluted with water $(20 \mathrm{~mL})$. The mixture was extracted with diethyl ether $(3 \times 20 \mathrm{~mL})$, then the organic fractions were combined and washed with brine $(20 \mathrm{~mL})$. The ethereal solution was dried, filtered and concentrated to provide a crude mixture of cyclopropanes $\mathbf{2 4 3}$ and $\mathbf{2 5 0}$ (3.8:1 ratio), which was purified by column chromatography (9:1 hexanes:ethyl acetate) to liberate cyclopropanes 243 (271 mg, 63\%) as a light-yellow oil and 250 (57 $\mathrm{mg}, 13 \%)$ as a yellow oil $(76 \%$ combined yield).

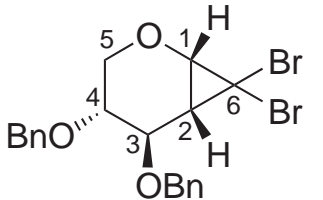

243: The spectral data matched that which was previously reported. ${ }^{45} \mathrm{R}_{f} 0.5$ (5:1 hexanes:ethyl acetate); $[\alpha]_{\mathrm{D}}^{19}-48$ (c 1.2 $\left.\mathrm{CHCl}_{3}\right) ;{ }^{1} \mathrm{H}-\mathrm{NMR}:\left(\mathrm{CDCl}_{3}\right) \delta_{\mathrm{H}} 7.49-7.27$ (complex m, 10H, Bn), $4.79\left(\mathrm{~d}, J=11.7 \mathrm{~Hz}, 1 \mathrm{H}, \mathrm{PhCH}_{2}\right), 4.76(\mathrm{~d}, J=12.0 \mathrm{~Hz}, 1 \mathrm{H}$, $\left.\mathrm{PhCH}_{2}\right), 4.70\left(\mathrm{~d}, J=11.7 \mathrm{~Hz}, 1 \mathrm{H}, \mathrm{PhCH}_{2}\right), 4.61$ (d, $J=12.0 \mathrm{~Hz}, 1 \mathrm{H}, \mathrm{PhCH}_{2}$ ), $3.92(\mathrm{dd}, J=11.5,5.6 \mathrm{~Hz}, 1 \mathrm{H}, \mathrm{H}-5 \mathrm{a}), 3.84$ (d, $J=7.8 \mathrm{~Hz}, 1 \mathrm{H}, \mathrm{H}-1), 3.78$ (dd, $J=$ 11.3, $6.0 \mathrm{~Hz}, 1 \mathrm{H}, \mathrm{H}-5 \mathrm{~b}), 3.71$ (m, 1H, H-4), 3.66 (dd, $J=7.6,3.9$ Hz, 1H, H-3), 1.86 $(\mathrm{dd}, J=7.8,3.7 \mathrm{~Hz}, 1 \mathrm{H}, \mathrm{H}-2) ;{ }^{13} \mathrm{C}-\mathrm{NMR}:\left(\mathrm{CDCl}_{3}\right) \delta_{\mathrm{C}} 138.1(\mathrm{C}, \mathrm{Bn}), 137.6(\mathrm{C}, \mathrm{Bn})$, 128.5 (CH, Bn), 128.4 (CH, Bn), 128.0 (CH, Bn), 127.9 (CH, Bn), 127.74 (CH, Bn), 127.72 (CH, Bn), 78.6 (CH, C-3), $75.2(\mathrm{CH}, \mathrm{C}-4), 72.8\left(\mathrm{CH}_{2}, \mathrm{PhCH}_{2}\right), 71.9\left(\mathrm{CH}_{2}\right.$, $\left.\mathrm{PhCH}_{2}\right), 69.4\left(\mathrm{CH}_{2}, \mathrm{C}-5\right), 60.3(\mathrm{CH}, \mathrm{C}-1), 33.9(\mathrm{CH}, \mathrm{C}-2), 32.6(\mathrm{C}, \mathrm{C}-6)$; IR $(\mathrm{KBr})$ : $\nu_{\max } 3064,3028,2981,2872,1720,1496,1454,1365,1270,1205,1097,1026,736,697$ $\mathrm{cm}^{-1}$; HRMS: $m / z \mathrm{C}_{20} \mathrm{H}_{20} \mathrm{O}_{3}^{79} \mathrm{Br}_{2} \mathrm{Na}^{+}[\mathrm{M}+\mathrm{Na}]^{+}$calcd 488.9677, found 488.9682.

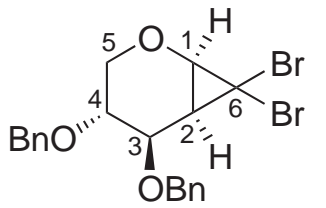

250: $\mathrm{R}_{f} 0.45$ (5:1 hexanes:ethyl acetate); $[\alpha]_{\mathrm{D}}^{19}+3\left(\right.$ c $\left.1.0 \mathrm{CHCl}_{3}\right)$; ${ }^{1} \mathrm{H}-\mathrm{NMR}:\left(\mathrm{CDCl}_{3}\right) \delta_{\mathrm{H}}$ 7.49-7.28 (complex m, 10H, Bn), $4.91(\mathrm{~d}$, $\left.J=12.0 \mathrm{~Hz}, 1 \mathrm{H}, \mathrm{PhCH}_{2}\right), 4.76\left(\mathrm{~d}, J=11.5 \mathrm{~Hz}, 1 \mathrm{H}, \mathrm{PhCH}_{2}\right)$, $4.74\left(\mathrm{~d}, J=11.5 \mathrm{~Hz}, 1 \mathrm{H}, \mathrm{PhCH}_{2}\right), 4.60(\mathrm{~d}, J=11.5 \mathrm{~Hz}, 1 \mathrm{H}$, $\mathrm{PhCH}_{2}$ ), 4.09 (apparent t, $\left.J=7.9 \mathrm{~Hz}, 1 \mathrm{H}, \mathrm{H}-3\right), 4.01$ (dd, $J=7.8,1.2 \mathrm{~Hz}, 1 \mathrm{H}, \mathrm{H}-1$ ), $3.98(\mathrm{~m}, 1 \mathrm{H}, \mathrm{H}-4), 3.84$ (dd, $J=10.9,4.8 \mathrm{~Hz}, 1 \mathrm{H}, \mathrm{H}-5 \mathrm{a}), 3.17$ (apparent t, $J=11.0$ $\mathrm{Hz}, 1 \mathrm{H}, \mathrm{H}-5 \mathrm{~b}$ ), 2.28 (apparent t, $J=8.0 \mathrm{~Hz}, 1 \mathrm{H}, \mathrm{H}-2) ;{ }^{13} \mathrm{C}-\mathrm{NMR}$ : $\left(\mathrm{CDCl}_{3}\right) \delta_{\mathrm{C}} 138.2$ (C, Bn), 137.9 (C, Bn), 128.5 (CH, Bn), 128.4 (CH, Bn), 127.94 (CH, Bn), 127.86 (CH, Bn), 127.74 (CH, Bn), 127.65 (CH, Bn), 77.2 (CH, C-3), 75.9 (CH, C-4), 73.3 $\left(\mathrm{CH}_{2}, \mathrm{PhCH}_{2}\right), 71.1\left(\mathrm{CH}_{2}, \mathrm{PhCH}_{2}\right), 67.2\left(\mathrm{CH}_{2}, \mathrm{C}-5\right), 63.4(\mathrm{CH}, \mathrm{C}-1), 33.8(\mathrm{CH}$, 
C-2), 30.8 (C, C-6); IR (KBr): $\nu_{\max }$ 3087, 3062, 3031, 2979, 2929, 2873, 1721, 1495, 1454, 1374, 1189, 1094, 1076, 1028, 738, $697 \mathrm{~cm}^{-1}$; HRMS: $m / z \mathrm{C}_{20} \mathrm{H}_{20} \mathrm{O}_{3}^{79} \mathrm{Br}_{2} \mathrm{Na}^{+}$ $[\mathrm{M}+\mathrm{Na}]^{+}$calcd 488.9677 , found 488.9673 .

\section{Methyl 3,4,6-tri- $O$-benzyl-(2E)-2- $C$-(bromomethylene)-2-deoxy- $\beta$-D- lyxo-hexopyranoside (251) and methyl 3,4,6-tri- $O$-benzyl-(2E)-2- $C$ - (bromomethylene)-2-deoxy- $\alpha$-D-lyxo-hexopyranoside (252)}

A portion of methanol $(100 \mu \mathrm{L}, 2.5 \mathrm{mmol})$ was treated with metallic sodium $(7 \mathrm{mg}$, $0.3 \mathrm{mmol}$ ), then once the sodium had reacted, the solution was diluted with THF $(1.7 \mathrm{~mL})$, then treated with cyclopropane 45 (101 $\mathrm{mg}, 0.172 \mathrm{mmol})$ and heated at reflux for 40 minutes. The solution was diluted with water $(10 \mathrm{~mL})$, then extracted with dichloromethane $(3 \times 10 \mathrm{~mL})$. The combined organic fractions were dried, filtered and concentrated to provide crude products 251 and 252 as a yellow oil. Upon separation by column chromatography (14:1 hexanes:ethyl acetate), 2- $C$ substituted sugars $\mathbf{2 5 1}$ and $\mathbf{2 5 2}$ were obtained as an inseparable colourless oil (60 mg, $65 \%$ combined yield, 1:3 ratio).

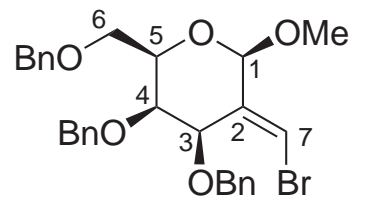

251: The following data set was obtained by subtracting the NMR signals for $\mathbf{2 5 2}$ from that of the mixture obtained.

$\mathrm{R}_{f} 0.10$ (14:1 hexanes:ethyl acetate); ${ }^{1} \mathrm{H}-\mathrm{NMR}:\left(\mathrm{CDCl}_{3}\right) \delta_{\mathrm{H}}$ 7.39-7.27 (complex m, 15H, Bn), 6.65 (s, 1H, H-7), 4.93 (s, 1H, H-1), 4.82 (m, 1H, H-3), 4.73 (d, $J=12.0 \mathrm{~Hz}, 1 \mathrm{H}, \mathrm{PhCH}_{2}$ ), 4.68 (d, $J=12.7$ $\left.\mathrm{Hz}, 1 \mathrm{H}, \mathrm{PhCH}_{2}\right), 4.59$ (d, $\left.J=12.0 \mathrm{~Hz}, 1 \mathrm{H}, \mathrm{PhCH}_{2}\right), 4.48(\mathrm{~d}, J=12.8 \mathrm{~Hz}, 1 \mathrm{H}$, $\mathrm{PhCH}_{2}$ ), 4.47 (m, 1H, H-6a), 4.43 (d, $J=11.0 \mathrm{~Hz}, 1 \mathrm{H}, \mathrm{PhCH}_{2}$ ), 4.41 (d, $J=11.7$ $\mathrm{Hz}, 1 \mathrm{H}, \mathrm{PhCH}_{2}$ ), 4.24 (m, 1H, H-5), 3.84 (dd, $J=10.7,2.2 \mathrm{~Hz}, 1 \mathrm{H}, \mathrm{H}-6 \mathrm{~b}$ ), 3.72 (dd, $J=5.9,3.2 \mathrm{~Hz}, 1 \mathrm{H}, \mathrm{H}-4), 3.45$ (s, 3H, Me); ${ }^{13} \mathrm{C}-\mathrm{NMR}:\left(\mathrm{CDCl}_{3}\right) \delta_{\mathrm{C}} 138.6-137.7(\mathrm{C}$, Bn), 136.5 (C, C-2), 128.5-127.5 (CH, Bn), 112.8 (CH, C-7), 101.4 (CH, C-1), 75.7 $(\mathrm{CH}, \mathrm{C}-4), 74.5(\mathrm{CH}, \mathrm{C}-5), 73.2(\mathrm{CH}, \mathrm{C}-3), 73.5-71.2\left(\mathrm{CH}_{2}, \mathrm{PhCH}_{2}\right), 70.5\left(\mathrm{CH}_{2}\right.$, C-6), $55.6\left(\mathrm{CH}_{3}, \mathrm{Me}\right)$.

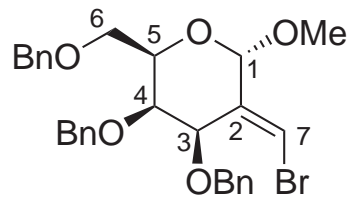

252: $\mathrm{R}_{f} 0.10$ (14:1 hexanes:ethyl acetate); ${ }^{1} \mathrm{H}-\mathrm{NMR}:\left(\mathrm{CDCl}_{3}\right)$ $\delta_{\mathrm{H}} 7.39-7.27$ (complex m, 15H, Bn), $6.64(\mathrm{~s}, 1 \mathrm{H}, \mathrm{H}-7), 5.05(\mathrm{~s}$, $1 \mathrm{H}, \mathrm{H}-1), 4.85$ (d, $J=11.7 \mathrm{~Hz}, 1 \mathrm{H}, \mathrm{PhCH}_{2}$ ), 4.80 (d, $J=12.2$ $\left.\mathrm{Hz}, 1 \mathrm{H}, \mathrm{PhCH}_{2}\right), 4.70$ (d, $\left.J=3.2 \mathrm{~Hz}, 1 \mathrm{H}, \mathrm{H}-3\right), 4.61$ (d, $J=$ $\left.11.7 \mathrm{~Hz}, 1 \mathrm{H}, \mathrm{PhCH}_{2}\right), 4.53\left(\mathrm{~d}, J=11.7 \mathrm{~Hz}, 1 \mathrm{H}, \mathrm{PhCH}_{2}\right), 4.52\left(\mathrm{~m}, 1 \mathrm{H}, \mathrm{PhCH}_{2}\right)$, $4.46\left(\mathrm{~d}, J=12.2 \mathrm{~Hz}, 1 \mathrm{H}, \mathrm{PhCH}_{2}\right), 4.17$ (m, 1H, H-5), 3.88 (apparent t, $J=3.2$ $\mathrm{Hz}, 1 \mathrm{H}, \mathrm{H}-4), 3.80$ (dd, $J=10.4,7.2 \mathrm{~Hz}, 1 \mathrm{H}, \mathrm{H}-6 \mathrm{a}), 3.68$ (dd, $J=10.3,5.1 \mathrm{~Hz}$, 1H, H-6b), 3.42 (s, 3H, Me); ${ }^{13}$ C-NMR: $\left(\mathrm{CDCl}_{3}\right) \delta_{\mathrm{C}} 138.2$ (C, Bn), 138.12 (C, Bn), 138.06 (C, Bn), 136.9 (C, C-2), 128.33 (CH, Bn), 128.25 (CH, Bn), 128.2 (CH, Bn), 127.7 (CH, Bn), 127.64 (CH, Bn), 127.59 (CH, Bn), 127.52 (CH, Bn), 127.50 (CH, Bn), 107.5 (CH, C-7), 100.3 (CH, C-1), 77.1 (CH, C-3), $76.4(\mathrm{CH}, \mathrm{C}-4), 73.3\left(\mathrm{CH}_{2}\right.$, 
$\left.\mathrm{PhCH}_{2}\right), 73.0\left(\mathrm{CH}_{2}, \mathrm{PhCH}_{2}\right), 72.5(\mathrm{CH}, \mathrm{C}-5), 72.1\left(\mathrm{CH}_{2}, \mathrm{PhCH}_{2}\right), 68.3\left(\mathrm{CH}_{2}, \mathrm{C}-6\right)$, $55.5\left(\mathrm{CH}_{3}, \mathrm{Me}\right)$; HRMS: $m / z \mathrm{C}_{29} \mathrm{H}_{31} \mathrm{O}_{5}^{79} \mathrm{BrNa}^{+}[\mathrm{M}+\mathrm{Na}]^{+}$calcd 561.1253, found 561.1250 .

\title{
Methyl 3,4-di- $O$-benzyl-(2E)-2- $C$-(bromomethylene)-2-deoxy- $\beta$-D- threo-pentopyranoside (253) and methyl 3,4-di- $O$-benzyl-(2E)-2- $C$ - (bromomethylene)-2-deoxy- $\alpha$-D-threo-pentopyranoside (254)
}

A portion of methanol $(100 \mu \mathrm{L}, 2.5 \mathrm{mmol})$ was treated with metallic sodium $(7 \mathrm{mg}$, $0.3 \mathrm{mmol}$ ), then once the sodium had reacted, the solution was diluted with THF $(1.7 \mathrm{~mL})$, then treated with cyclopropane $243(84 \mathrm{mg}, 0.18 \mathrm{mmol})$ and heated at reflux for 20 minutes. The solution was diluted with water $(10 \mathrm{~mL})$, then extracted with dichloromethane $(3 \times 10 \mathrm{~mL})$. The combined organic fractions were dried, filtered and concentrated to provide crude products 253 and 254 as a yellow oil (1:5 ratio). Upon separation by column chromatography (14:1 hexanes:ethyl acetate), the major 2- $C$-substituted sugar was isolated as a colourless oil (38 $\mathrm{mg}, 50 \%$ ), in addition to an impure mixture of $\mathbf{2 5 3}$ and $\mathbf{2 5 4}$ (20 $\mathrm{mg}, \mathbf{1 7 \%}$, 1:2 ratio), isolated as a colourless oil (67\% combined yield).<smiles>COC1OC[C@@H](OCc2ccccc2)[C@H](O)[C@@H]1CBr</smiles>

The following data set was obtained for the major isomer, of which the anomeric configuration was not determined. $\mathrm{R}_{f} 0.3$ (14:1 hexanes:ethyl acetate); ${ }^{1} \mathrm{H}-\mathrm{NMR}:\left(\mathrm{CDCl}_{3}\right) \delta_{\mathrm{H}} 7.43-7.27$ (complex m, 10H, Bn), 6.76 (d, $=1.9 \mathrm{~Hz}, 1 \mathrm{H}, \mathrm{H}-6), 5.00$ (s, 1H, H-1), 4.76 (m, 1H, H-3), 4.68 (d, J = $111.2 \mathrm{~Hz}$, $1 \mathrm{H}, \mathrm{PhCH}_{2}$ ), 4.65 (d, $J=12.3 \mathrm{~Hz}, 1 \mathrm{H}, \mathrm{PhCH}_{2}$ ), 4.56 (d, $J=12.2 \mathrm{~Hz}, 1 \mathrm{H}, \mathrm{PhCH}_{2}$ ), $4.43\left(\mathrm{~d}, J=11.3 \mathrm{~Hz}, 1 \mathrm{H}, \mathrm{PhCH}_{2}\right), 4.28$ (d, $\left.J=12.2 \mathrm{~Hz}, 1 \mathrm{H}, \mathrm{H}-5 \mathrm{a}\right), 3.64$ (d, $J=$ $12.4 \mathrm{~Hz}, 1 \mathrm{H}, \mathrm{H}-5 \mathrm{~b}), 3.57$ (m, 1H, H-4), 3.43 (s, 3H, Me).

\subsection{Chapter Three Experimental}

\author{
Acetyl 4,5,7-tri- $O$-benzyl-2-bromo-2,3-dideoxy- $\beta$-D-arabino-hept-2- \\ enoseptanoside (257) and acetyl 4,5,7-tri- $O$-benzyl-2-bromo-2,3-dideoxy- \\ $\alpha$-D-arabino-hept-2-enoseptanoside (258)
}

A A solution of cyclopropane $97(101 \mathrm{mg}, 0.172 \mathrm{mmol})$ in acetic acid $(1.7 \mathrm{~mL})$ was treated with silver acetate $(39 \mathrm{mg}, 0.23 \mathrm{mmol})$, then stirred at $100{ }^{\circ} \mathrm{C}$ for 25 hours. The solution was diluted with dichloromethane $(20 \mathrm{~mL})$, filtered through a pad of Celite ${ }^{\circledR}$, then washed with water $(10 \mathrm{~mL})$. The aqueous phase was extracted further with dichloromethane $(2 \times 10 \mathrm{~mL})$, then the organic fractions were combined, dried, filtered and concentrated to provide a mixture of oxepines 257 and 258 (1:3.5 
ratio) as a light-yellow oil. Upon purification by column chromatography (14:1 hexanes:ethyl acetate), major oxepine 258 (14 mg, 14\%) and mixtures of 257 and 258 (37 mg, 38\%) were obtained as colourless oils (1:3.8 ratio, 52\% combined yield).

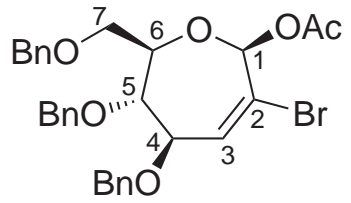

257: The following data set was obtained by subtracting the NMR signals for 258 from that of the purified mixture.

$\mathrm{R}_{f} 0.15$ (14:1 hexanes:ethyl acetate); ${ }^{1} \mathrm{H}-\mathrm{NMR}:\left(\mathrm{CDCl}_{3}\right) \delta_{\mathrm{H}}$ 7.36-7.14 (complex m, 15H, Bn), 6.45 (m, 1H, H-3), 6.33 (s, 1H, H-1), 4.69-4.37 (complex m, 6H, $\left.\mathrm{PhCH}_{2}\right), 4.15$ (m, 1H, H-4), $3.92(\mathrm{dd}, J=$ 11.0, 5.9 Hz, 1H, H-6), 3.80 (dd, $J=6.3,4.4 \mathrm{~Hz}, 1 \mathrm{H}, \mathrm{H}-5$ ), 3.67-3.52 (complex m, 2H, H-7a,b), 2.15 (s, 3H, Ac); ${ }^{13} \mathrm{C}-\mathrm{NMR}:\left(\mathrm{CDCl}_{3}\right) \delta_{\mathrm{C}} 169.0\left(\mathrm{C}, \mathrm{CH}_{3} \mathrm{CO}\right), 138.1(\mathrm{C}$, Bn), 137.8 (C, Bn), 137.5 (C, Bn), 133.4 (CH, C-3), 128.5-127.6 (CH, Bn), 125.6 (C, C-2), 94.4 (CH, C-1), 79.2 (CH, C-5), 78.6 (CH, C-6), $75.4(\mathrm{CH}, \mathrm{C}-4), 73.3\left(\mathrm{CH}_{2}\right.$, $\left.\mathrm{PhCH}_{2}\right), 72.7\left(\mathrm{CH}_{2}, \mathrm{PhCH}_{2}\right), 71.6\left(\mathrm{CH}_{2}, \mathrm{PhCH}_{2}\right), 70.4\left(\mathrm{CH}_{2}, \mathrm{C}-7\right), 20.9\left(\mathrm{CH}_{3}, \mathrm{Ac}\right)$.

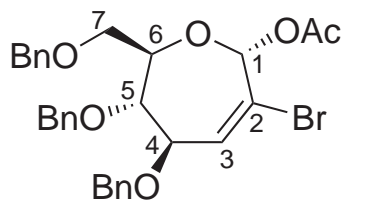

258: $\mathrm{R}_{f} 0.15$ (14:1 hexanes:ethyl acetate); $[\alpha]_{\mathrm{D}}^{19}-23$ ( $c 0.7$ $\left.\mathrm{CHCl}_{3}\right)$; ${ }^{1} \mathrm{H}-\mathrm{NMR}:\left(\mathrm{CDCl}_{3}\right) \delta_{\mathrm{H}} 7.36-7.16$ (complex m, $15 \mathrm{H}$, Bn), 6.57 (s, 1H, H-1), 6.46 (dd, $J=6.3,1.2 \mathrm{~Hz}, 1 \mathrm{H}, \mathrm{H}-3)$, $4.68\left(\mathrm{~d}, J=12.0 \mathrm{~Hz}, 1 \mathrm{H}, \mathrm{PhCH}_{2}\right), 4.57$ (d, $J=11.2 \mathrm{~Hz}, 1 \mathrm{H}$, $\left.\mathrm{PhCH}_{2}\right), 4.56\left(\mathrm{~d}, J=12.0 \mathrm{~Hz}, 1 \mathrm{H}, \mathrm{PhCH}_{2}\right), 4.51$ (d, $\left.J=11.7 \mathrm{~Hz}, 1 \mathrm{H}, \mathrm{PhCH}_{2}\right)$, $4.45\left(\mathrm{~d}, J=11.7 \mathrm{~Hz}, 1 \mathrm{H}, \mathrm{PhCH}_{2}\right), 4.38\left(\mathrm{~d}, J=11.2 \mathrm{~Hz}, 1 \mathrm{H}, \mathrm{PhCH}_{2}\right), 4.17-4.14$ (complex m, 2H, H-4,6), 3.74 (dd, $J=9.1,4.2 \mathrm{~Hz}, 1 \mathrm{H}, \mathrm{H}-5$ ), 3.61 (dd, $J=10.5,2.4$ $\mathrm{Hz}, 1 \mathrm{H}, \mathrm{H}-7 \mathrm{a}$ ), 3.54 (dd, $J=10.5,7.1 \mathrm{~Hz}, 1 \mathrm{H}, \mathrm{H}-7 \mathrm{~b}), 1.93$ (s, 3H, Ac); ${ }^{13} \mathrm{C}-\mathrm{NMR}$ : $\left(\mathrm{CDCl}_{3}\right) \delta_{\mathrm{C}} 169.3\left(\mathrm{C}, \mathrm{CH}_{3} \mathrm{CO}\right), 138.0$ (C, Bn), 137.8 (C, Bn), 137.5 (C, Bn), 133.5 (CH, C-3), 128.5 (CH, Bn), 128.4 (CH, Bn), 128.3 (CH, Bn), 128.0 (CH, Bn), 127.95 (CH, Bn), 127.93 (CH, Bn), 127.88 (CH, Bn), 127.8 (CH, Bn), 127.6 (CH, Bn), 123.9 (C, C-2), 93.4 (CH, C-1), 81.1 (CH, C-5), 77.2 (CH, C-4), $74.4(\mathrm{CH}, \mathrm{C}-6), 73.4\left(\mathrm{CH}_{2}\right.$, $\left.\mathrm{PhCH}_{2}\right), 73.2\left(\mathrm{CH}_{2}, \mathrm{PhCH}_{2}\right), 71.7\left(\mathrm{CH}_{2}, \mathrm{PhCH}_{2}\right), 70.3\left(\mathrm{CH}_{2}, \mathrm{C}-7\right), 20.7\left(\mathrm{CH}_{3}, \mathrm{Ac}\right)$; IR (KBr): $\nu_{\max }$ 3086, 3058, 3030, 2863, 1753, 1639, 1496, 1454, 1368, 1221, 1071 , 1005, 952, 737, $698 \mathrm{~cm}^{-1}$; HRMS: $m / z \mathrm{C}_{30} \mathrm{H}_{31} \mathrm{O}_{6}^{79} \mathrm{BrNa}^{+}[\mathrm{M}+\mathrm{Na}]^{+}$calcd 589.1202, found 589.1202 .

B A solution of cyclopropane 97 (100 $\mathrm{mg}, 0.170 \mathrm{mmol})$ in acetic acid (1.7 mL) was treated with silver acetate $(56 \mathrm{mg}, 0.34 \mathrm{mmol})$ then stirred at room temperature for five days. No reaction was observed by TLC, therefore this reaction was abandoned.

C A solution of cyclopropane 97 (98 $\mathrm{mg}, 0.17 \mathrm{mmol})$ in acetic acid $(1.7 \mathrm{~mL})$ was treated with silver acetate $(54 \mathrm{mg}, 0.32 \mathrm{mmol})$ and sodium acetate $(70 \mathrm{mg}$, $0.85 \mathrm{mmol}$ ), then stirred at room temperature for four days. The solution was diluted with diethyl ether $(20 \mathrm{~mL})$, filtered through a pad of Celite ${ }^{\circledR}$, then washed with sat. sodium bicarbonate solution $(20 \mathrm{~mL})$, then washed with brine $(20 \mathrm{~mL})$. The ethereal solution was dried, filtered and concentrated to provide a colourless 
oil. Proton NMR analysis of this material showed only starting material was present.

D A solution of cyclopropane 97 (97 $\mathrm{mg}, 0.17 \mathrm{mmol}$ ) in acetic acid $(1.7 \mathrm{~mL})$ was treated with silver acetate $(60 \mathrm{mg}, 0.36 \mathrm{mmol})$, then stirred at $70{ }^{\circ} \mathrm{C}$ for three days. The solution was treated with a further portion of silver acetate $(61 \mathrm{mg}, 0.37 \mathrm{mmol})$, then stirred at $70{ }^{\circ} \mathrm{C}$ for an additional two days. The solution was diluted with water $(10 \mathrm{~mL})$, then extracted with dichloromethane $(3 \times 10 \mathrm{~mL})$, then the organic fractions were combined, dried, filtered and concentrated to provide a mixture of oxepines 257 and 258 as a light-yellow oil. Upon purification by column chromatography (9:1 hexanes:ethyl acetate), starting material (10 mg, 10\%) was obtained, in addition to a mixture of oxepines 257 and 258 (1:3.4 ratio, $25 \mathrm{mg}, 27 \%$ ).

E A solution of cyclopropane $97(98 \mathrm{mg}, 0.17 \mathrm{mmol})$ in acetic acid $(1.7 \mathrm{~mL})$ was treated with silver acetate $(54 \mathrm{mg}, 0.32 \mathrm{mmol})$, then stirred at $90{ }^{\circ} \mathrm{C}$ for 36 hours. The solution was diluted with water $(10 \mathrm{~mL})$, then extracted with dichloromethane $(3 \times 10 \mathrm{~mL})$, then the organic fractions were combined, dried, filtered and concentrated to provide a mixture of oxepines 257 and 258 as a light-yellow oil. Upon purification by column chromatography (20:1 hexanes:ethyl acetate), mixtures of oxepines 257 and 258 (1:2.9 ratio, $35 \mathrm{mg}, 37 \%$ ) were obtained.

F A solution of cyclopropane $97(103 \mathrm{mg}, 0.175 \mathrm{mmol})$ in acetic acid $(1.7 \mathrm{~mL})$ was treated with silver acetate $(56 \mathrm{mg}, 0.34 \mathrm{mmol})$, then stirred at reflux for five hours. The solution was diluted with water $(10 \mathrm{~mL})$, then extracted with dichloromethane $(3 \times 10 \mathrm{~mL})$, then the organic fractions were combined, dried, filtered and concentrated to provide a light-yellow oil. Upon purification by column chromatography (8:1 hexanes:diethyl ether), mixtures of oxepines 257 and 258 (1:1.7 ratio, $15 \mathrm{mg}$, $15 \%$ ) were obtained, in addition to a mixture of two unidentified aldehydic products (18 mg).

G A solution of cyclopropane $97(100 \mathrm{mg}, 0.170 \mathrm{mmol})$ in toluene $(1.7 \mathrm{~mL})$ was treated with sodium acetate $(70 \mathrm{mg}, 0.85 \mathrm{mmol})$, then stirred at $100{ }^{\circ} \mathrm{C}$ for two days. The solution was diluted with water $(10 \mathrm{~mL})$ and extracted with dichloromethane $(3 \times 10 \mathrm{~mL})$, then the organic fractions were combined, dried, filtered and concentrated to provide a mixture of oxepines 257 and 258 as a pale-yellow oil. Upon purification by column chromatography (14:1 hexanes:ethyl acetate), a mixture of oxepines 257 and 258 (1:2.1 ratio, $17 \mathrm{mg}, \mathbf{1 8 \%}$ ) was obtained.

H A solution of cyclopropane 97 (102 mg, $0.173 \mathrm{mmol})$ in DMF (1.7 mL) was treated with silver acetate (42 mg, $0.25 \mathrm{mmol}$ ) and sodium acetate $(75 \mathrm{mg}, 0.91 \mathrm{mmol}$ ), then stirred at $100{ }^{\circ} \mathrm{C}$ for two days. The solution was diluted with water $(10 \mathrm{~mL})$ and sat. 
copper sulfate solution $(10 \mathrm{~mL})$, then extracted with dichloromethane $(3 \times 10 \mathrm{~mL})$. The organic fractions were combined, dried, filtered and concentrated to provide a mixture of oxepines 257 and 258 as a brown oil. Upon purification by column chromatography (9:1 hexanes:ethyl acetate), a mixture of oxepines $\mathbf{2 5 7}$ and $\mathbf{2 5 8}$ (1.2:1 ratio, $48 \mathrm{mg}, 49 \%)$.

\section{3- $O$-Acetyl-1,6-anhydro-4,5,7-tri- $O$-benzyl-2-bromo-2-deoxy-D-arabino- hept-1-enitol (259)}

A A solution of cyclopropane $97(100 \mathrm{mg}, 0.170 \mathrm{mmol})$ in toluene $(3.4 \mathrm{~mL})$ was treated with silver acetate $(44 \mathrm{mg}, 0.26 \mathrm{mmol})$ and sodium acetate $(138 \mathrm{mg}, 1.68$ mmol), then stirred at $100{ }^{\circ} \mathrm{C}$ for two days. The solution was diluted with dichloromethane $(10 \mathrm{~mL})$, then washed with water $(10 \mathrm{~mL})$. The aqueous phase was extracted further with dichloromethane $(2 \times 10 \mathrm{~mL})$, then the organic fractions were combined, filtered through a pad of Celite ${ }^{\circledR}$, dried, filtered and concentrated to provide a mixture of oxepines 257 and 258 (1:3.5 ratio) as a light-yellow oil, in addition to 3 -acetate oxepine 259. Upon purification by column chromatography (9:1 hexanes:ethyl acetate), starting material 97 (22 $\mathrm{mg}, 22 \%)$ was recovered, in addition to 3-acetate oxepine 259 ( $5 \mathrm{mg}, 5 \%$ ), obtained as a colourless oil and a mixture of oxepines $\mathbf{2 5 7}$ and $\mathbf{2 5 8}$ (1:3.7 ratio, $51 \mathrm{mg}, 53 \%$ ).

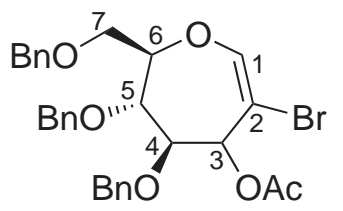

259: $\mathrm{R}_{f} 0.4$ (5:1 hexanes:ethyl acetate); $[\alpha]_{\mathrm{D}}^{18}+78\left(c 0.5 \mathrm{CHCl}_{3}\right)$; ${ }^{1} \mathrm{H}-\mathrm{NMR}:\left(\mathrm{CDCl}_{3}\right) \delta_{\mathrm{H}} 7.43-7.06$ (complex m, 15H, Bn), $6.94(\mathrm{~s}$, $1 \mathrm{H}, \mathrm{H}-1), 5.63$ (d, $J=5.9 \mathrm{~Hz}, 1 \mathrm{H}, \mathrm{H}-3), 4.69$ (d, $J=12.2 \mathrm{~Hz}$, $\left.1 \mathrm{H}, \mathrm{PhCH}_{2}\right), 4.62\left(\mathrm{~d}, J=12.5 \mathrm{~Hz}, 1 \mathrm{H}, \mathrm{PhCH}_{2}\right), 4.58(\mathrm{~d}, J=$ $12.2 \mathrm{~Hz}, 1 \mathrm{H}, \mathrm{PhCH}_{2}$ ), 4.50 (d, $J=12.2 \mathrm{~Hz}, 1 \mathrm{H}, \mathrm{PhCH}_{2}$ ), 4.45 (dd, $J=10.5,4.6$ $\mathrm{Hz}, 1 \mathrm{H}, \mathrm{H}-6), 4.16$ (d, $J=10.7 \mathrm{~Hz}, 1 \mathrm{H}, \mathrm{PhCH}_{2}$ ), 4.14 (d, $\left.J=5.9 \mathrm{~Hz}, 1 \mathrm{H}, \mathrm{H}-4\right), 4.07$ $\left(\mathrm{d}, J=10.7 \mathrm{~Hz}, 1 \mathrm{H}, \mathrm{PhCH}_{2}\right.$ ), 3.73 (d, $\left.J=10.2 \mathrm{~Hz}, 1 \mathrm{H}, \mathrm{H}-5\right), 3.66-3.60$ (complex m, 2H, H-7a,b), 1.89 (s, 3H, Ac); ${ }^{13} \mathrm{C}-\mathrm{NMR}:\left(\mathrm{CDCl}_{3}\right) \delta_{\mathrm{C}} 170.6$ (C, $\left.\mathrm{CH}_{3} \mathrm{CO}\right), 151.8$ (CH, C-1), 137.9 (C, Bn), 137.5 (C, Bn), 137.0 (C, Bn), 128.5 (CH, Bn), 128.4 (CH, Bn), 128.3 (CH, Bn), 128.21 (CH, Bn), 128.20 (CH, Bn), 128.1 (CH, Bn), 128.0 (CH, $\mathrm{Bn}), 127.8$ (CH, Bn), 127.7 (CH, Bn), 99.4 (C, C-2), 84.1 (CH, C-6), 78.8 (CH, C-5), 75.6 (CH, C-3), $75.3(\mathrm{CH}, \mathrm{C}-4), 73.4\left(\mathrm{CH}_{2}, \mathrm{PhCH}_{2}\right), 72.5\left(\mathrm{CH}_{2}, \mathrm{PhCH}_{2}\right), 72.2\left(\mathrm{CH}_{2}\right.$, $\left.\mathrm{PhCH}_{2}\right), 69.8\left(\mathrm{CH}_{2}, \mathrm{C}-7\right), 20.9\left(\mathrm{CH}_{3}, \mathrm{Ac}\right)$; IR (KBr): $\nu_{\max } 3065,3027,2928,2871$, 1739, 1454, 1367, 1223, 1072, 1025, 750, $698 \mathrm{~cm}^{-1}$; HRMS: $m / z \mathrm{C}_{30} \mathrm{H}_{31} \mathrm{O}_{6}^{79} \mathrm{BrNa}^{+}$ $[\mathrm{M}+\mathrm{Na}]^{+}$calcd 589.1202, found 589.1198.

B A solution of cyclopropane $97(102 \mathrm{mg}, 0.173 \mathrm{mmol})$ in toluene $(1.7 \mathrm{~mL})$ was treated with silver acetate $(41 \mathrm{mg}, 0.24 \mathrm{mmol})$ and sodium acetate $(72 \mathrm{mg}, 0.88$ mmol), then stirred at reflux for 28 hours. The solution was filtered through a pad of silica, eluting further with dichloromethane $(40 \mathrm{~mL})$, then the organic fractions 
were combined, dried, filtered and concentrated to provide a mixture of 3-acetate oxepine 259 and oxepines 257 and 258 as a colourless oil. Upon purification by column chromatography (14:1 hexanes:ethyl acetate), 3-acetate oxepine 259 (14 mg, $14 \%$ ) and a mixture of oxepines $\mathbf{2 5 7}$ and $\mathbf{2 5 8}$ (1:3.8 ratio, $64 \mathrm{mg}, 65 \%$ ).

\section{Methyl 4,5,7-tri- $O$-benzyl-2-bromo-2,3-dideoxy- $\beta$-D-arabino-hept-2- enoseptanoside (98) and methyl 4,5,7-tri- $O$-benzyl-2-bromo-2,3-dideoxy- $\alpha$-D-arabino-hept-2-enoseptanoside (99)}

A A solution of cyclopropane $97(91 \mathrm{mg}, 0.15 \mathrm{mmol})$ in methanol $(1.7 \mathrm{~mL})$ was treated with silver tetrafluoroborate $\left(88 \mathrm{mg}, 0.45 \mathrm{mmol}\right.$ ), then stirred at $40{ }^{\circ} \mathrm{C}$ for one day, followed by heating at reflux for one further day. The solution was diluted with diethyl ether $(20 \mathrm{~mL})$, filtered through a pad of Celite ${ }^{\circledR}$, then washed with sat. sodium bicarbonate solution $(20 \mathrm{~mL})$, followed by brine $(20 \mathrm{~mL})$. The ethereal solution was dried, filtered and concentrated to provide a light-yellow oil, comprised predominantly of starting material 97. Upon separation by column chromatography (9:1 hexanes:ethyl acetate), oxepines 98 (5 mg, 6\%) and 99 (6 mg, 7\%) were obtained, isolated as colourless oils (13\% combined yield).

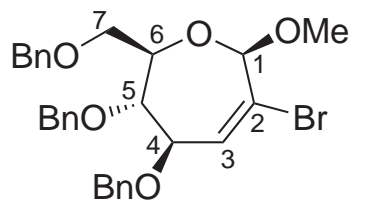

98: $\mathrm{R}_{f} 0.3$ (9:1 hexanes:ethyl acetate); ${ }^{1} \mathrm{H}-\mathrm{NMR}:\left(\mathrm{CDCl}_{3}\right) \delta_{\mathrm{H}}$ 7.36-7.15 (complex m, 15H, Bn), 6.33 (d, $J=5.1 \mathrm{~Hz}, 1 \mathrm{H}$, H-3), 5.14 (s, 1H, H-1), 4.68 (d, $J=12.0 \mathrm{~Hz}, 1 \mathrm{H}, \mathrm{PhCH}_{2}$ ), $4.65\left(\mathrm{~d}, J=11.2 \mathrm{~Hz}, 1 \mathrm{H}, \mathrm{PhCH}_{2}\right), 4.59(\mathrm{~d}, J=11.7 \mathrm{~Hz}, 1 \mathrm{H}$, $\mathrm{PhCH}_{2}$ ), 4.59 (d, $J=12.2 \mathrm{~Hz}, 1 \mathrm{H}, \mathrm{PhCH}_{2}$ ), 4.51 (d, $J=12.0 \mathrm{~Hz}, 1 \mathrm{H}, \mathrm{PhCH}_{2}$ ), 4.45 $\left(\mathrm{d}, J=11.2 \mathrm{~Hz}, 1 \mathrm{H}, \mathrm{PhCH}_{2}\right.$ ), 4.17-4.14 (complex m, 2H, H-4,6), 3.77 (dd, $J=8.9$, $5.7 \mathrm{~Hz}, 1 \mathrm{H}, \mathrm{H}-5), 3.70$ (dd, $J=10.5,5.6 \mathrm{~Hz}, 1 \mathrm{H}, \mathrm{H}-7 \mathrm{a}), 3.61$ (dd, $J=10.5,2.7 \mathrm{~Hz}$, 1H, H-7b), 3.46 (s, 3H, Me); ${ }^{13} \mathrm{C}-\mathrm{NMR}:\left(\mathrm{CDCl}_{3}\right) \delta_{\mathrm{C}} 138.2$ (C, Bn), 138.1 (C, Bn), 137.9 (C, Bn), 132.1 (CH, C-3), 128.5 (CH, Bn), 128.4 (CH, Bn), 128.3 (CH, Bn), 127.90 (CH, Bn), 127.87 (CH, Bn), 127.85 (CH, Bn), 127.8 (CH, Bn), 127.7 (CH, Bn), 127.6 (CH, Bn), 124.7 (C, C-2), 102.1 (CH, C-1), 80.5 (CH, C-5), 78.9 (CH, C-4), $73.7\left(\mathrm{CH}_{2}, \mathrm{PhCH}_{2}\right), 73.2\left(\mathrm{CH}_{2}, \mathrm{PhCH}_{2}\right), 72.1\left(\mathrm{CH}_{2}, \mathrm{PhCH}_{2}\right), 71.5(\mathrm{CH}, \mathrm{C}-6)$, $70.4\left(\mathrm{CH}_{2}, \mathrm{C}-7\right), 56.5\left(\mathrm{CH}_{3}, \mathrm{Me}\right)$.

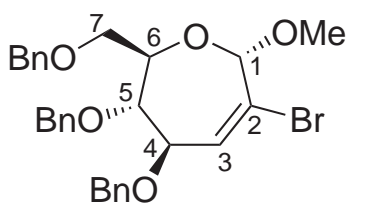

99: $\mathrm{R}_{f} 0.25$ (9:1 hexanes:ethyl acetate); ${ }^{1} \mathrm{H}-\mathrm{NMR}:\left(\mathrm{CDCl}_{3}\right) \delta_{\mathrm{H}}$ 7.37-7.14 (complex m, 15H, Bn), 6.44 (dd, $J=7.1,1.0 \mathrm{~Hz}$, $1 \mathrm{H}, \mathrm{H}-3), 5.05$ (s, 1H, H-1), 4.68 (d, $J=12.2 \mathrm{~Hz}, 1 \mathrm{H}, \mathrm{PhCH}_{2}$ ), 4.57-4.56 (complex m, 2H, $\mathrm{PhCH}_{2}$ ), 4.51 (d, $J=12.4 \mathrm{~Hz}, 1 \mathrm{H}$, $\mathrm{PhCH}_{2}$ ), 4.49 (d, $\left.J=12.2 \mathrm{~Hz}, 1 \mathrm{H}, \mathrm{PhCH}_{2}\right), 4.33$ (d, $J=11.5 \mathrm{~Hz}, 1 \mathrm{H}, \mathrm{PhCH}_{2}$ ), 4.09 (dd, $J=7.1,1.2 \mathrm{~Hz}, 1 \mathrm{H}, \mathrm{H}-4$ ), 3.70-3.69 (complex m, 2H, H-5,6), 3.65-3.63 (complex m, 2H, H-7a,b), 3.47 (s, 3H, Me); ${ }^{13} \mathrm{C}-\mathrm{NMR}:\left(\mathrm{CDCl}_{3}\right) \delta_{\mathrm{C}} 138.2(\mathrm{C}, \mathrm{Bn})$, 137.9 (C, Bn), 137.4 (C, Bn), 132.2 (CH, C-3), 128.5 (CH, Bn), 128.42 (CH, Bn), 128.37 (CH, Bn), 128.0 (CH, Bn), 127.93 (CH, Bn), 127.85 (CH, Bn), 127.7 (CH, 
Bn), 127.6 (CH, Bn), 123.5 (C, C-2), 104.2 (CH, C-1), 80.0 (CH, C-5), $77.4(\mathrm{CH}$, C-6), $75.3(\mathrm{CH}, \mathrm{C}-4), 73.3\left(\mathrm{CH}_{2}, \mathrm{PhCH}_{2}\right), 72.7\left(\mathrm{CH}_{2}, \mathrm{PhCH}_{2}\right), 71.3\left(\mathrm{CH}_{2}, \mathrm{PhCH}_{2}\right)$, $71.0\left(\mathrm{CH}_{2}, \mathrm{C}-7\right), 55.8\left(\mathrm{CH}_{3}, \mathrm{Me}\right)$; HRMS: $m / z \mathrm{C}_{29} \mathrm{H}_{31} \mathrm{O}_{5}^{79} \mathrm{BrNa}^{+}[\mathrm{M}+\mathrm{Na}]^{+}$calcd 561.1253 , found 561.1256 .

B A solution of cyclopropane 97 (49 $\mathrm{mg}, 0.083 \mathrm{mmol}$ ) in methanol $(1.7 \mathrm{~mL})$ was treated with silver tetrafluoroborate $(22 \mathrm{mg}, 0.11 \mathrm{mmol})$ and $3 \AA$ molecular sieves, then stirred in the dark at room temperature for two days. The solution was diluted with diethyl ether $(20 \mathrm{~mL})$, filtered through a pad of Celite ${ }^{\circledR}$, washed with sat. sodium bicarbonate solution $(20 \mathrm{~mL})$, then washed with brine $(20 \mathrm{~mL})$. The ethereal solution was dried, filtered and concentrated to provide a light-yellow oil. Proton NMR analysis of this material indicated only starting material was present.

C A solution of cyclopropane $97(104 \mathrm{mg}, 0.177 \mathrm{mmol})$ in methanol $(1.7 \mathrm{~mL})$ was treated with silver acetate $(37 \mathrm{mg}, 0.22 \mathrm{mmol})$, then stirred at reflux for five days. The solution was diluted with diethyl ether $(10 \mathrm{~mL})$, filtered through a pad of Celite ${ }^{\circledR}$, then washed with water $(10 \mathrm{~mL})$. The aqueous wash was extracted further with ether $(2 \times 10 \mathrm{~mL})$, then the ethereal solutions were combined, dried, filtered and concentrated to provide a light-yellow oil. Upon separation by column chromatography (9:1 hexanes:ethyl acetate), recovered starting material 97 (63 $\mathrm{mg}, 61 \%$ ) was obtained, in addition to oxepines 98 (4 mg, 4\%) and 99 (11 mg, 11\%) (15\% combined yield).

D A solution of cyclopropane 97 (101 mg, $0.172 \mathrm{mmol})$ in methanol (1.7 mL) was treated with silver tetrafluoroborate $(67 \mathrm{mg}, 0.34 \mathrm{mmol})$ and potassium carbonate (44 mg, $0.32 \mathrm{mmol}$ ), then stirred at reflux for 16 hours. The solution was diluted with diethyl ether $(20 \mathrm{~mL})$, filtered through a pad of Celite ${ }^{\circledR}$, then washed with sat. sodium bicarbonate solution $(20 \mathrm{~mL})$, then washed with brine $(20 \mathrm{~mL})$. The ethereal solution was dried, filtered and concentrated to provide a light-yellow oil. Proton NMR analysis of this crude mixture indicated the presence of primarily starting material 97, in addition to oxepines 98 and 99 (approx. 17\% combined yield) and previously isolated 2- $C$-branched sugars 213 and 214 (approx. 22\% combined yield).

\section{Allyl 4,5,7-tri- $O$-benzyl-2-bromo-2,3-dideoxy- $\beta$-D-arabino-hept-2- enoseptanoside (260) and allyl 4,5,7-tri- $O$-benzyl-2-bromo-2,3-dideoxy- $\alpha$ - D-arabino-hept-2-enoseptanoside (261)}

A A solution of cyclopropane 97 (102 $\mathrm{mg}, 0.173 \mathrm{mmol})$ in allyl alcohol (1.7 mL) was treated with silver nitrate $(39 \mathrm{mg}, 0.23 \mathrm{mmol})$, then stirred at reflux for 29 hours. 
The solution was diluted with dichloromethane $(10 \mathrm{~mL})$, filtered through a pad of Celite ${ }^{\circledR}$, then washed with water $(10 \mathrm{~mL})$. The aqueous phase was extracted further with dichloromethane $(2 \times 10 \mathrm{~mL})$, then the organic fractions were combined, dried, filtered and concentrated to provide a light-yellow oil. Upon separation by column chromatography (14:1 hexanes:ethyl acetate), recovered starting material 97 (16 mg, 16\%), plus oxepines 260 (18 mg, 18\%) and 261 (31 mg, 32\%) were obtained as white solids (50\% combined yield).

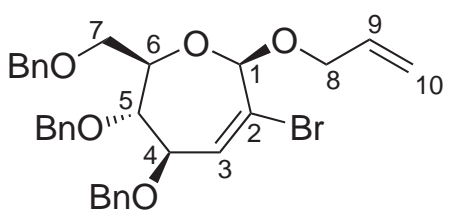

260: $\mathrm{R}_{f} 0.25$ (14:1 hexanes:ethyl acetate); m.p. $48-51^{\circ} \mathrm{C}$; $[\alpha]_{\mathrm{D}}^{19}-4.3\left(c 1.0 \mathrm{CHCl}_{3}\right) ;{ }^{1} \mathrm{H}-\mathrm{NMR}:\left(\mathrm{CDCl}_{3}\right) \delta_{\mathrm{H}} 7.37-$ 7.15 (complex m, 15H, Bn), $6.32(\mathrm{~d}, J=5.1 \mathrm{~Hz}, 1 \mathrm{H}$, H-3), 5.89 (ddt, $J=17.1,10.5,6.4 \mathrm{~Hz}, 1 \mathrm{H}, \mathrm{H}-9$ ), 5.29 (ddd, $J=17.3,3.2,1.7 \mathrm{~Hz}, 1 \mathrm{H}, \mathrm{H}-10 \mathrm{a}), 5.27$ (s, 1H, H-1), 5.18 (ddd, $J=10.5,2.9$, $1.2 \mathrm{~Hz}, 1 \mathrm{H}, \mathrm{H}-10 \mathrm{~b}), 4.68$ (d, $J=12.0 \mathrm{~Hz}, 1 \mathrm{H}, \mathrm{PhCH}_{2}$ ), 4.64 (d, $J=11.2 \mathrm{~Hz}, 1 \mathrm{H}$, $\left.\mathrm{PhCH}_{2}\right), 4.58\left(\mathrm{~d}, J=12.0 \mathrm{~Hz}, 1 \mathrm{H}, \mathrm{PhCH}_{2}\right), 4.56\left(\mathrm{~d}, J=12.2 \mathrm{~Hz}, 1 \mathrm{H}, \mathrm{PhCH}_{2}\right)$, $4.50\left(\mathrm{~d}, J=12.0 \mathrm{~Hz}, 1 \mathrm{H}, \mathrm{PhCH}_{2}\right), 4.44\left(\mathrm{~d}, J=11.2 \mathrm{~Hz}, 1 \mathrm{H}, \mathrm{PhCH}_{2}\right.$ ), 4.28 (ddt, $J=12.9,5.1,1.5 \mathrm{~Hz}, 1 \mathrm{H}, \mathrm{H}-8 \mathrm{a}), 4.19$ (ddd, $J=9.0,6.1,2.4 \mathrm{~Hz}, 1 \mathrm{H}, \mathrm{H}-6), 4.16$ (apparent t, $J=5.4 \mathrm{~Hz}, 1 \mathrm{H}, \mathrm{H}-4$ ), 4.06 (ddd, $J=12.9,6.3,1.2 \mathrm{~Hz}, 1 \mathrm{H}, \mathrm{H}-8 \mathrm{~b}$ ), 3.75 (dd, $J=9.0,5.6 \mathrm{~Hz}, 1 \mathrm{H}, \mathrm{H}-5), 3.68$ (dd, $J=10.5,5.9 \mathrm{~Hz}, 1 \mathrm{H}, \mathrm{H}-7 \mathrm{a}), 3.59$ (dd, $J=10.5,2.4 \mathrm{~Hz}, 1 \mathrm{H}, \mathrm{H}-7 \mathrm{~b}) ;{ }^{13} \mathrm{C}-\mathrm{NMR}:\left(\mathrm{CDCl}_{3}\right) \delta_{\mathrm{C}} 138.0$ (C, Bn), 137.91 (C, Bn), 137.86 (C, Bn), 133.5 (CH, C-9), 132.1 (CH, C-3), 128.5 (CH, Bn), 128.4 (CH, Bn), 128.3 (CH, Bn), 127.9 (CH, Bn), 127.84 (CH, Bn), 128.76 (CH, Bn), 127.7 (CH, Bn), 127.6 (CH, Bn), 124.8 (C, C-2), $117.9\left(\mathrm{CH}_{2}, \mathrm{C}-10\right), 99.7$ (CH, C-1), 80.5 (CH, C-5), $78.9(\mathrm{CH}, \mathrm{C}-4), 73.7\left(\mathrm{CH}_{2}, \mathrm{PhCH}_{2}\right), 73.2\left(\mathrm{CH}_{2}, \mathrm{PhCH}_{2}\right), 72.0\left(\mathrm{CH}_{2}, \mathrm{PhCH}_{2}\right)$, $71.4(\mathrm{CH}, \mathrm{C}-6), 70.4\left(\mathrm{CH}_{2}, \mathrm{C}-7\right), 69.2\left(\mathrm{CH}_{2}, \mathrm{C}-8\right)$; IR (KBr): $\nu_{\max } 3060,3029,2925$, 2871, 1723, 1647, 1496, 1453, 1364, 1111, 1072, 1049, 735, $697 \mathrm{~cm}^{-1}$; HRMS: $\mathrm{m} / z$ $\mathrm{C}_{31} \mathrm{H}_{33} \mathrm{O}_{5}^{79} \mathrm{BrNa}^{+}[\mathrm{M}+\mathrm{Na}]^{+}$calcd 587.1409, found 587.1406.

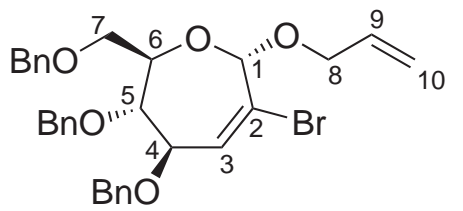

261: $\mathrm{R}_{f} 0.2$ (14:1 hexanes:ethyl acetate); m.p. $28-30{ }^{\circ} \mathrm{C}$; $[\alpha]_{\mathrm{D}}^{19}-37\left(\right.$ c $\left.2.2 \mathrm{CHCl}_{3}\right) ;{ }^{1} \mathrm{H}-\mathrm{NMR}:\left(\mathrm{CDCl}_{3}\right) \delta_{\mathrm{H}} 7.35-7.14$ (complex m, 15H, Bn), 6.43 (d, $J=7.1 \mathrm{~Hz}, 1 \mathrm{H}, \mathrm{H}-3)$, 5.95 (ddt, $J=17.0,10.8,5.7 \mathrm{~Hz}, 1 \mathrm{H}, \mathrm{H}-9), 5.31$ (d, $J=$

$17.3 \mathrm{~Hz}, 1 \mathrm{H}, \mathrm{H}-10 \mathrm{a}), 5.20$ (d, $J=10.3 \mathrm{~Hz}, 1 \mathrm{H}, \mathrm{H}-10 \mathrm{~b}), 5.11$ (s, 1H, H-1), 4.69 (d, $\left.J=12.2 \mathrm{~Hz}, 1 \mathrm{H}, \mathrm{PhCH}_{2}\right), 4.55\left(\mathrm{~s}, 2 \mathrm{H}, \mathrm{PhCH}_{2}\right), 4.52$ (d, $\left.J=12.2 \mathrm{~Hz}, 1 \mathrm{H}, \mathrm{PhCH}_{2}\right)$, $4.50\left(\mathrm{~d}, J=11.2 \mathrm{~Hz}, 1 \mathrm{H}, \mathrm{PhCH}_{2}\right), 4.34\left(\mathrm{~d}, J=11.2 \mathrm{~Hz}, 1 \mathrm{H}, \mathrm{PhCH}_{2}\right), 4.33(\mathrm{~m}$, 1H, H-8a), 4.21 (dd, $J=12.8,6.4 \mathrm{~Hz}, 1 \mathrm{H}, \mathrm{H}-8 \mathrm{~b}), 4.10$ (d, $J=6.8 \mathrm{~Hz}, 1 \mathrm{H}, \mathrm{H}-4)$, 3.69-3.66 (complex m, 2H, H-5,6), 3.64-3.60 (complex m, 2H, H-7a,b); ${ }^{13} \mathrm{C}-\mathrm{NMR}$ : $\left(\mathrm{CDCl}_{3}\right) \delta_{\mathrm{C}} 138.2(\mathrm{C}, \mathrm{Bn}), 137.9$ (C, Bn), 137.4 (C, Bn), 133.8 (CH, C-9), 132.2 (CH, C-3), 128.42 (CH, Bn), 128.40 (CH, Bn), 128.35 (CH, Bn), 128.1 (C, C-2), 127.98 (CH, Bn), 127.96 (CH, Bn), 127.9 (CH, Bn), 127.8 (CH, Bn), 127.7 (CH, Bn), 127.6 (CH, Bn), $118.0\left(\mathrm{CH}_{2}, \mathrm{C}-10\right), 102.1$ (CH, C-1), 80.0 (CH, C-5), 77.3 (CH, C-6), $75.5(\mathrm{CH}, \mathrm{C}-4), 73.3\left(\mathrm{CH}_{2}, \mathrm{PhCH}_{2}\right), 72.8\left(\mathrm{CH}_{2}, \mathrm{PhCH}_{2}\right), 71.3\left(\mathrm{CH}_{2}\right.$, 
$\left.\mathrm{PhCH}_{2}\right)$, $71.0\left(\mathrm{CH}_{2}, \mathrm{C}-7\right), 69.2\left(\mathrm{CH}_{2}, \mathrm{C}-8\right)$; IR (KBr): $\nu_{\max } 3087,3063,3029,2863$, 1637, 1496, 1453, 1360, 1264, 1075, 1027, 736, $697 \mathrm{~cm}^{-1}$.

B A solution of cyclopropane 97 (103 mg, $0.175 \mathrm{mmol})$ in allyl alcohol (1.7 mL) was treated with silver acetate $(32 \mathrm{mg}, 0.19 \mathrm{mmol})$, then stirred at reflux for 27 hours. The solution was diluted with dichloromethane $(10 \mathrm{~mL})$, filtered through a pad of Celite ${ }^{\circledR}$, then washed with water $(10 \mathrm{~mL})$. The aqueous phase was extracted further with dichloromethane $(2 \times 10 \mathrm{~mL})$, then the organic fractions were combined, dried, filtered and concentrated to provide a colourless oil. Upon separation by column chromatography (14:1 hexanes:ethyl acetate), recovered starting material (13 mg, 13\%), plus oxepines 260 (27 mg, 27\%) and 261 (24 mg, 24\%) were obtained (51\% combined yield) in addition to major acetate oxepine 258 (4 mg, 4\%).

C A solution of cyclopropane 97 (100 mg, $0.170 \mathrm{mmol})$ in allyl alcohol $(1.7 \mathrm{~mL})$ was treated with potassium carbonate $(139 \mathrm{mg}, 1.00 \mathrm{mmol})$, then stirred at reflux for 11 hours. The solution was diluted with water $(10 \mathrm{~mL})$, extracted with dichloromethane $(3 \times 10 \mathrm{~mL})$, then the organic fractions were combined, dried, filtered and concentrated to provide a colourless oil. Upon separation by column chromatography (9:1 hexanes:ethyl acetate), recovered starting material (6 mg, 6\%), plus a mixture of oxepine $\mathbf{2 6 0}$ (9\%) and 2-C-branched sugar 236 (20\%) (28 mg combined yield) and a mixture of oxepine $\mathbf{2 6 1}$ (2\%) and 2-C-branched sugar $\mathbf{2 3 7}$ (21\%) (22 mg combined yield), were obtained (percentages determined by NMR analysis).

\section{Benzyl 4,5,7-tri- $O$-benzyl-2-bromo-2,3-dideoxy- $\beta$-D-arabino-hept-2- enoseptanoside (262) and benzyl 4,5,7-tri- $O$-benzyl-2-bromo-2,3-dideoxy- $\alpha$-D-arabino-hept-2-enoseptanoside (263)}

A A solution of cyclopropane $97(100 \mathrm{mg}, 0.170 \mathrm{mmol})$ in toluene $(1.7 \mathrm{~mL})$ was treated with silver acetate $(39 \mathrm{mg}, 0.23 \mathrm{mmol})$, followed by benzyl alcohol $(90 \mu \mathrm{L}$, $0.85 \mathrm{mmol}$ ), then stirred at $100{ }^{\circ} \mathrm{C}$ for two days. The solution was diluted with dichloromethane $(10 \mathrm{~mL})$, filtered through a pad of Celite ${ }^{\circledR}$, then washed with water $(10 \mathrm{~mL})$. The aqueous phase was extracted further with dichloromethane $(2 \mathrm{x}$ $10 \mathrm{~mL}$ ), then the organic fractions were combined, dried, filtered and concentrated to provide a yellow oil. Upon separation by column chromatography (14:1 hexanes:ethyl acetate), oxepine $\mathbf{2 6 2}$ (23 $\mathrm{mg}, 22 \%$ ) was obtained as a white solid and oxepine 263 (24 mg, 23\%) as a colourless oil (45\% combined yield), plus a mixture of acetate oxepines $\mathbf{2 5 7}$ and $\mathbf{2 5 8}$ (26 $\mathrm{mg}, \mathbf{2 6 \%}$, 1:4.6 ratio). 


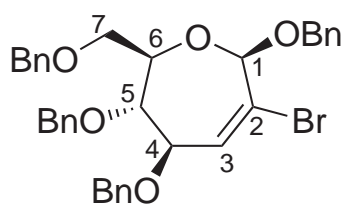

262: $\mathrm{R}_{f} 0.25$ (14:1 hexanes:ethyl acetate); m.p. $67-71^{\circ} \mathrm{C} ;[\alpha]_{\mathrm{D}}^{19}$ $+3.2\left(\right.$ c $\left.1.8 \mathrm{CHCl}_{3}\right) ;{ }^{1} \mathrm{H}-\mathrm{NMR}:\left(\mathrm{CDCl}_{3}\right) \delta_{\mathrm{H}} 7.40-7.16$ (complex m, 20H, Bn), 6.34 (d, $J=5.4 \mathrm{~Hz}, 1 \mathrm{H}, \mathrm{H}-3), 5.32$ (s, 1H, H-1), $4.85\left(\mathrm{~d}, J=12.0 \mathrm{~Hz}, 1 \mathrm{H}, \mathrm{PhCH}_{2}\right), 4.68$ (d, $J=11.7 \mathrm{~Hz}, 1 \mathrm{H}$, $\left.\mathrm{PhCH}_{2}\right), 4.65\left(\mathrm{~d}, J=11.2 \mathrm{~Hz}, 1 \mathrm{H}, \mathrm{PhCH}_{2}\right), 4.59\left(\mathrm{~d}, J=11.7 \mathrm{~Hz}, 1 \mathrm{H}, \mathrm{PhCH}_{2}\right)$, $4.58\left(\mathrm{~d}, J=12.0 \mathrm{~Hz}, 1 \mathrm{H}, \mathrm{PhCH}_{2}\right), 4.57$ (d, $\left.J=12.0 \mathrm{~Hz}, 1 \mathrm{H}, \mathrm{PhCH}_{2}\right), 4.51$ (d, $J=$ $12.0 \mathrm{~Hz}, 1 \mathrm{H}, \mathrm{PhCH}_{2}$ ), 4.45 (d, $J=11.2 \mathrm{~Hz}, 1 \mathrm{H}, \mathrm{PhCH}_{2}$ ), 4.19 (ddd, $J=8.3,6.2$, $2.0 \mathrm{~Hz}, 1 \mathrm{H}, \mathrm{H}-6$ ), 4.16 (apparent t, $J=5.4 \mathrm{~Hz}, 1 \mathrm{H}, \mathrm{H}-4), 3.76$ (dd, $J=8.9,5.5 \mathrm{~Hz}$, 1H, H-5), 3.69 (dd, $J=10.5,5.9 \mathrm{~Hz}, 1 \mathrm{H}, \mathrm{H}-7 \mathrm{a}), 3.59$ (dd, $J=10.5,2.0 \mathrm{~Hz}, 1 \mathrm{H}$, H-7b); ${ }^{13} \mathrm{C}-\mathrm{NMR}:\left(\mathrm{CDCl}_{3}\right) \delta_{\mathrm{C}} 138.0$ (C, Bn), 137.9 (C, Bn), 137.8 (C, Bn), 137.1 (C, Bn), 132.0 (CH, C-3), 128.5 (CH, Bn), 128.36 (CH, Bn), 128.35 (CH, Bn), 128.3 (CH, Bn), 128.1 (CH, Bn), 127.89 (CH, Bn), 127.86 (CH, Bn), 127.83 (CH, Bn), 127.76 (CH, Bn), 127.6 (CH, Bn), 124.9 (C, C-2), 100.0 (CH, C-1), 80.7 (CH, C-5), 78.7 (CH, C-4), $73.7\left(\mathrm{CH}_{2}, \mathrm{PhCH}_{2}\right), 73.2\left(\mathrm{CH}_{2}, \mathrm{PhCH}_{2}\right), 72.0\left(\mathrm{CH}_{2}, \mathrm{PhCH}_{2}\right), 71.6$ (CH, C-6), $70.5\left(\mathrm{CH}_{2}, \mathrm{C}-7\right), 70.4\left(\mathrm{CH}_{2}, \mathrm{PhCH}_{2}\right)$; IR (KBr): $\nu_{\max } 3087,3062,3030$, 2864, 1736, 1639, 1496, 1453, 1363, 1110, 1070, 1027, 736, $697 \mathrm{~cm}^{-1}$; HRMS: m/z $\mathrm{C}_{35} \mathrm{H}_{35} \mathrm{O}_{5}^{79} \mathrm{BrNa}^{+}[\mathrm{M}+\mathrm{Na}]^{+}$calcd 637.1566, found 637.1568.

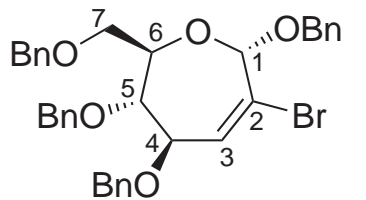

263: $\quad \mathrm{R}_{f} 0.2$ (14:1 hexanes:ethyl acetate); $[\alpha]_{\mathrm{D}}^{19}-30$ (c 0.7 $\left.\mathrm{CHCl}_{3}\right)$; ${ }^{1} \mathrm{H}-\mathrm{NMR}:\left(\mathrm{CDCl}_{3}\right) \delta_{\mathrm{H}} 7.41-7.13$ (complex m, 20H, $\mathrm{Bn}), 6.44$ (d, $J=7.1 \mathrm{~Hz}, 1 \mathrm{H}, \mathrm{H}-3), 5.11$ (s, 1H, H-1), 4.86 (d, $\left.J=11.7 \mathrm{~Hz}, 1 \mathrm{H}, \mathrm{PhCH}_{2}\right), 4.73\left(\mathrm{~d}, J=12.0 \mathrm{~Hz}, 1 \mathrm{H}, \mathrm{PhCH}_{2}\right)$, 4.69 (d, $\left.J=12.0 \mathrm{~Hz}, 1 \mathrm{H}, \mathrm{PhCH}_{2}\right), 4.56\left(\mathrm{~s}, 2 \mathrm{H}, \mathrm{PhCH}_{2}\right), 4.51$ (d, $J=12.2 \mathrm{~Hz}, 1 \mathrm{H}$, $\mathrm{PhCH}_{2}$ ), 4.49 (d, $J=11.5 \mathrm{~Hz}, 1 \mathrm{H}, \mathrm{PhCH}_{2}$ ), 4.33 (d, $J=11.5 \mathrm{~Hz}, 1 \mathrm{H}, \mathrm{PhCH}_{2}$ ), 4.09 (dd, $J=6.9,3.1 \mathrm{~Hz}, 1 \mathrm{H}, \mathrm{H}-4$ ), 3.71-3.61 (complex m, 4H, H-5,6,7a,b); ${ }^{13} \mathrm{C}-\mathrm{NMR}$ : $\left(\mathrm{CDCl}_{3}\right) \delta_{\mathrm{C}} 138.2(\mathrm{C}, \mathrm{Bn}), 137.9(\mathrm{C}, \mathrm{Bn}), 137.4$ (C, Bn), 137.1 (C, Bn), $132.2(\mathrm{CH}$, C-3), 128.5 (CH, Bn), 128.43 (CH, Bn), 128.41 (CH, Bn), 128.37 (CH, Bn), 128.31 (CH, Bn), 128.26 (CH, Bn), 128.2 (C, C-2), 127.99 (CH, Bn), 127.96 (CH, Bn), 127.9 (CH, Bn), 127.8 (CH, Bn), 127.7 (CH, Bn), 127.6 (CH, Bn), 102.0 (CH, C-1), 80.0 (CH, C-5), 77.3 (CH, C-6), $75.5(\mathrm{CH}, \mathrm{C}-4), 73.3\left(\mathrm{CH}_{2}, \mathrm{PhCH}_{2}\right), 72.8\left(\mathrm{CH}_{2}\right.$, $\left.\mathrm{PhCH}_{2}\right), 71.2\left(\mathrm{CH}_{2}, \mathrm{PhCH}_{2}\right), 71.0\left(\mathrm{CH}_{2}, \mathrm{C}-7\right), 69.7\left(\mathrm{CH}_{2}, \mathrm{PhCH}_{2}\right)$; IR $(\mathrm{KBr}): \nu_{\max }$ 3062, 3030, 2864, 1737, 1637, 1496, 1453, 1363, 1210, 1072, 1027, 736, $697 \mathrm{~cm}^{-1}$.

B A solution of cyclopropane $97(100 \mathrm{mg}, 0.170 \mathrm{mmol})$ in toluene $(1.7 \mathrm{~mL})$ was treated with silver nitrate $(48 \mathrm{mg}, 0.28 \mathrm{mmol})$, followed by benzylamine $(90 \mu \mathrm{L}$, $0.85 \mathrm{mmol}$ ), then stirred at $100{ }^{\circ} \mathrm{C}$ for two days. The solution was treated with additional silver nitrate (42 $\mathrm{mg}, 0.25 \mathrm{mmol}$ ) and after refluxing for one further day, the mixture was diluted with dichloromethane $(10 \mathrm{~mL})$, filtered through a plug of silica, then washed with water $(10 \mathrm{~mL})$. The aqueous phase was extracted further with dichloromethane $(2 \times 10 \mathrm{~mL})$, then the organic fractions were combined, dried, filtered and concentrated to provide a yellow oil. Upon separation by column chro- 
matography (14:1 hexanes:ethyl acetate), oxepines $\mathbf{2 6 2}$ and $\mathbf{2 6 3}$ were obtained (4:1 ratio, $16 \mathrm{mg}, 15 \%$ combined yield).

\section{Phenyl 4,5,7-tri- $O$-benzyl-2-bromo-2,3-dideoxy- $\beta$-D-arabino-hept-2-} enoseptanoside (264) and phenyl 4,5,7-tri- $O$-benzyl-2-bromo-2,3dideoxy- $\alpha$-D-arabino-hept-2-enoseptanoside (265)

A solution of cyclopropane $97(101 \mathrm{mg}, 0.172 \mathrm{mmol})$ in toluene $(1.7 \mathrm{~mL})$ was treated with silver nitrate $(53 \mathrm{mg}, 0.31 \mathrm{mmol})$ and phenol $(86 \mathrm{mg}, 0.91 \mathrm{mmol})$, then stirred at $100{ }^{\circ} \mathrm{C}$ for two days. The solution was diluted with water $(10 \mathrm{~mL})$, then extracted with dichloromethane $(3 \times 10 \mathrm{~mL})$, then the organic fractions were combined, dried, filtered and concentrated to provide a yellow oil. Upon separation by column chromatography (14:1 hexanes:ethyl acetate), an inseparable mixture of oxepines $\mathbf{2 6 4}$ and 265 (6.7:1 ratio) was obtained as a colourless oil (46 mg, 45\%).
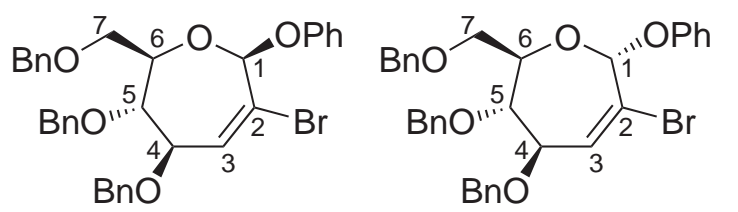

The given NMR data set is of major $\beta$-oxepine 264, unless otherwise stated. $\mathrm{R}_{f} 0.55$ (5:1 hexanes:ethyl acetate); ${ }^{1} \mathrm{H}-\mathrm{NMR}:\left(\mathrm{CDCl}_{3}\right) \delta_{\mathrm{H}} 7.36-7.12$ (complex m, 19H, Bn, Ph), 7.03 (t, J = 7.3 Hz, 1H, Ph), 6.51 (d, J=7.1 Hz, 1H, H-3 (265)), $6.45(\mathrm{~d}, J=5.4 \mathrm{~Hz}, 1 \mathrm{H}, \mathrm{H}-3), 5.91$ (s, 1H, H-1), 5.64 (s, 1H, H-1 (265)), 4.72 (d, $\left.J=12.0 \mathrm{~Hz}, 1 \mathrm{H}, \mathrm{PhCH}_{2}\right)$, 4.67-4.62 (complex m, 2H, $\mathrm{PhCH}_{2}$ ), 4.51-4.46 (complex m, $\left.2 \mathrm{H}, \mathrm{PhCH}_{2}\right), 4.40$ (d, $J=11.9 \mathrm{~Hz}, 1 \mathrm{H}, \mathrm{PhCH}_{2}$ ), 4.29 (ddd, $J=8.6,4.6,2.9 \mathrm{~Hz}$, $1 \mathrm{H}, \mathrm{H}-6), 4.25$ (t, $J=5.5 \mathrm{~Hz}, 1 \mathrm{H}, \mathrm{H}-4), 3.92(\mathrm{dd}, J=8.6,5.5 \mathrm{~Hz}, 1 \mathrm{H}, \mathrm{H}-5), 3.68$ (dd, $J=10.7,4.6 \mathrm{~Hz}, 1 \mathrm{H}, \mathrm{H}-7 \mathrm{a}), 3.45$ (dd, $J=10.6,2.6 \mathrm{~Hz}, 1 \mathrm{H}, \mathrm{H}-7 \mathrm{~b}$ ); ${ }^{13} \mathrm{C}-\mathrm{NMR}$ : $\left(\mathrm{CDCl}_{3}\right) \delta_{\mathrm{C}} 157.2(\mathrm{C}, \mathrm{Ph}), 138.0(\mathrm{C}, \mathrm{Bn}), 137.9$ (C, Bn), 137.8 (C, Bn), 133.1 (CH, C-3), 129.5 (CH, Ph), 128.5 (CH, Bn), 128.4 (CH, Bn), 128.3 (CH, Bn), 127.92 (CH, Bn), 127.90 (CH, Bn), 127.8 (CH, Bn), 127.7 (CH, Bn), 127.5 (CH, Bn), 123.8 (C, C-2), 122.6 (CH, Ph), 116.8 (CH, Ph), 99.8 (CH, C-1), $80.2(\mathrm{CH}, \mathrm{C}-5), 78.7$ $(\mathrm{CH}, \mathrm{C}-4), 73.7\left(\mathrm{CH}_{2}, \mathrm{PhCH}_{2}\right), 73.2\left(\mathrm{CH}_{2}, \mathrm{PhCH}_{2}\right), 72.4(\mathrm{CH}, \mathrm{C}-6), 72.1\left(\mathrm{CH}_{2}\right.$, $\left.\mathrm{PhCH}_{2}\right), 70.0\left(\mathrm{CH}_{2}, \mathrm{C}-7\right)$; IR (KBr): $\nu_{\max } 3077,3060,3030,2912,2862,1590,1495$, 1454, 1363, 1222, 1102, 1070, 1028, 736, $697 \mathrm{~cm}^{-1}$; HRMS: $m / z \mathrm{C}_{34} \mathrm{H}_{33} \mathrm{O}_{5}^{79} \mathrm{BrNa}^{+}$ $[\mathrm{M}+\mathrm{Na}]^{+}$calcd 623.1409 , found 623.1405 .

\section{1,7-Anhydro-4,5-di- $O$-benzyl-2-bromo-2,3-dideoxy- $\beta$-D-arabino-hept-2- enoseptanose (267)}

A A solution of cyclopropane $97(106 \mathrm{mg}, 0.180 \mathrm{mmol})$ in toluene $(1.7 \mathrm{~mL})$ was treated with silver acetate $(35 \mathrm{mg}, 0.21 \mathrm{mmol})$, then stirred at $100{ }^{\circ} \mathrm{C}$ for 22 hours. The solution was diluted with water $(10 \mathrm{~mL})$, extracted with dichloromethane $(3 \mathrm{x}$ $10 \mathrm{~mL}$ ), then the organic fractions were combined, dried, filtered and concentrated 
to provide a yellow oil. Upon purification by column chromatography (9:1 hexanes:ethyl acetate), bicyclic sugar 267 (30 mg, 38\%) was obtained as a colourless oil.

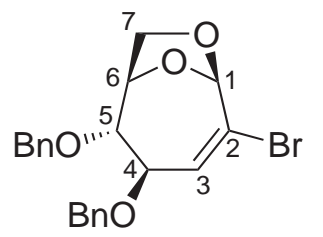

267: $\mathrm{R}_{f} 0.35$ (9:1 hexanes:ethyl acetate); $[\alpha]_{\mathrm{D}}^{20}-22\left(c 0.4 \mathrm{CHCl}_{3}\right)$; ${ }^{1} \mathrm{H}-\mathrm{NMR}:\left(\mathrm{CDCl}_{3}\right) \delta_{\mathrm{H}} 7.40-7.27$ (complex m, 10H, Bn), 6.20 (dd, $J=2.2,1.2 \mathrm{~Hz}, 1 \mathrm{H}, \mathrm{H}-3), 5.66(\mathrm{~s}, 1 \mathrm{H}, \mathrm{H}-1), 4.79$ (d, $J=11.7$ $\mathrm{Hz}, 1 \mathrm{H}, \mathrm{PhCH}_{2}$ ), 4.73 (d, $J=11.5 \mathrm{~Hz}, 1 \mathrm{H}, \mathrm{PhCH}_{2}$ ), 4.68 (d, $\left.J=11.5 \mathrm{~Hz}, 1 \mathrm{H}, \mathrm{PhCH}_{2}\right), 4.61\left(\mathrm{~d}, J=11.7 \mathrm{~Hz}, 1 \mathrm{H}, \mathrm{PhCH}_{2}\right), 4.60$ (m, 1H, H-4), 4.48 (dd, $J=8.0,5.5 \mathrm{~Hz}, 1 \mathrm{H}, \mathrm{H}-6$ ), 4.13 (apparent t, $J=8.1 \mathrm{~Hz}, 1 \mathrm{H}, \mathrm{H}-7 \mathrm{a}$ ), 3.63 $(\mathrm{d}, J=8.5 \mathrm{~Hz}, 1 \mathrm{H}, \mathrm{H}-5), 3.55(\mathrm{dd}, J=8.4,5.5 \mathrm{~Hz}, 1 \mathrm{H}, \mathrm{H}-7 \mathrm{~b}) ;{ }^{13} \mathrm{C}-\mathrm{NMR}:\left(\mathrm{CDCl}_{3}\right)$ $\delta_{\mathrm{C}} 137.9(\mathrm{C}, \mathrm{Bn}), 137.7$ (C, Bn), $\left.134.1 \mathrm{CH}, \mathrm{C}-3\right), 128.49$ (CH, Bn), 128.47 (CH, Bn), 127.9 (CH, Bn), 127.84 (CH, Bn), 127.82 (CH, Bn), 118.8 (C, C-2), 104.2 (CH, C-1), 88.7 (CH, C-5), 78.6 (CH, C-4), 77.8 (CH, C-6), $73.5\left(\mathrm{CH}_{2}, \mathrm{PhCH}_{2}\right)$, $73.3\left(\mathrm{CH}_{2}, \mathrm{PhCH}_{2}\right), 66.4\left(\mathrm{CH}_{2}, \mathrm{C}-7\right)$; IR (KBr): $\nu_{\max } 3063,3031,2928,2862,1725$, 1639, 1601, 1539, 1496, 1453, 1349, 1272, 1210, 1091, 1027, 933, 865, 737, $698 \mathrm{~cm}^{-1}$; HRMS: $m / z \mathrm{C}_{21} \mathrm{H}_{21} \mathrm{O}_{4}^{79} \mathrm{BrNa}^{+}[\mathrm{M}+\mathrm{Na}]^{+}$calcd 439.0521, found 439.0515.

B A solution of Meldrum's acid (41 mg, $0.28 \mathrm{mmol})$ in DMF (1.7 mL) was treated with sodium hydride ( $7 \mathrm{mg}, 0.2 \mathrm{mmol}$ ) and allowed to react at room temperature. Upon completion of hydrogen evolution, the solution was treated with silver acetate (53 mg, $0.32 \mathrm{mmol}$ ), then cyclopropane 97 (108 mg, $0.184 \mathrm{mmol}$ ), then stirred at $100{ }^{\circ} \mathrm{C}$ for two days. The solution was diluted with dichloromethane $(20 \mathrm{~mL})$, filtered through a silica plug, then washed with water $(10 \mathrm{~mL})$ and extracted further with dichloromethane $(2 \times 10 \mathrm{~mL})$. The organic fractions were combined, dried, filtered and concentrated to provide a brown oil. Upon purification by column chromatography (9:1 hexanes:ethyl acetate), bicyclic sugar 267 (10 mg, 13\%) was obtained, in addition to a mixture of acetate oxepines 257 and 258 (7 $\mathrm{mg}, 7 \%$ ).

\section{1,6-Anhydro-4,7-di- $O$-benzyl-2-bromo-2,3-dideoxy- $\beta$-D-lyxo-hept-2- enopyranose (270) and acetyl 4,5,7-tri- $O$-benzyl-2-bromo-2,3-dideoxy- $\alpha$ - D-lyxo-hept-2-enoseptanoside (269)}

A solution of cyclopropane 45 (103 $\mathrm{mg}, 0.175 \mathrm{mmol})$ in toluene $(1.7 \mathrm{~mL})$ was treated with silver acetate $(42 \mathrm{mg}, 0.25 \mathrm{mmol})$ and sodium acetate $(71 \mathrm{mg}, 0.86 \mathrm{mmol})$, then stirred at $100{ }^{\circ} \mathrm{C}$ for two days. The solution was diluted with dichloromethane $(10 \mathrm{~mL})$, filtered through a pad of silica, then eluted further with dichloromethane $(30 \mathrm{~mL})$. The organic fractions were combined, dried, filtered and concentrated to provide a mixture of bicyclic compound 270 and oxepine 269 as a yellow oil. Upon purification by column chromatography (14:1 hexanes:ethyl acetate), bicyclic sugar $\mathbf{2 7 0}(7 \mathrm{mg}, 7 \%)$ and oxepine 269 (51 mg, 52\%) were obtained as colourless oils. 


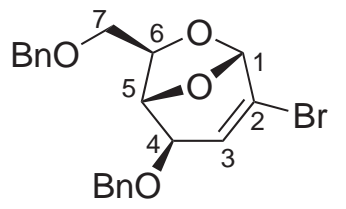

270: $\mathrm{R}_{f} 0.5$ (5:1 hexanes:ethyl acetate); $[\alpha]_{\mathrm{D}}^{18}-69\left(c 0.3 \mathrm{CHCl}_{3}\right)$; ${ }^{1} \mathrm{H}-\mathrm{NMR}:\left(\mathrm{CDCl}_{3}\right) \delta_{\mathrm{H}} 7.40-7.24$ (complex m, 10H, Bn), $6.10(\mathrm{~d}$, $J=3.5 \mathrm{~Hz}, 1 \mathrm{H}, \mathrm{H}-3), 5.51(\mathrm{~s}, 1 \mathrm{H}, \mathrm{H}-1), 4.64\left(\mathrm{~s}, 2 \mathrm{H}, \mathrm{PhCH}_{2}\right)$, 4.61 ( $\mathrm{m}, 1 \mathrm{H}, \mathrm{H}-6), 4.56$ (s, 2H, $\mathrm{PhCH}_{2}$ ), 3.79 ( $\left.\mathrm{m}, 1 \mathrm{H}, \mathrm{H}-5\right), 3.63$ $(\mathrm{d}, J=4.6 \mathrm{~Hz}, 1 \mathrm{H}, \mathrm{H}-4), 3.58$ (dd, $J=9.6,5.2 \mathrm{~Hz}, 1 \mathrm{H}, \mathrm{H}-7 \mathrm{a}), 3.43$ (dd, $J=9.4$, $7.5 \mathrm{~Hz}, 1 \mathrm{H}, \mathrm{H}-7 \mathrm{~b}) ;{ }^{13} \mathrm{C}-\mathrm{NMR}:\left(\mathrm{CDCl}_{3}\right) \delta_{\mathrm{C}} 137.6$ (C, Bn), 137.5 (C, Bn), $128.6(\mathrm{CH}$, Bn), 128.5 (CH, Bn), 128.0 (CH, Bn), 127.9 (CH, Bn), 127.8 (CH, Bn), 127.6 (CH, Bn), 126.3 (C, C-2), 123.9 (CH, C-3), 101.4 (CH, C-1), 76.5 (CH, C-6), 74.0 (CH, C-4), $73.8(\mathrm{CH}, \mathrm{C}-5), 73.6\left(\mathrm{CH}_{2}, \mathrm{PhCH}_{2}\right), 70.8\left(\mathrm{CH}_{2}, \mathrm{PhCH}_{2}\right), 70.2\left(\mathrm{CH}_{2}, \mathrm{C}-7\right)$; IR (KBr): $\nu_{\max }$ 3029, 2917, 2866, 1756, 1630, 1496, 1454, 1261, 1217, 1105, 1064, 1026, 920, 737, $698 \mathrm{~cm}^{-1}$; HRMS: $m / z \mathrm{C}_{21} \mathrm{H}_{21} \mathrm{O}_{4}^{79} \mathrm{BrNa}^{+}[\mathrm{M}+\mathrm{Na}]^{+}$calcd 439.0521, found 439.0516 .

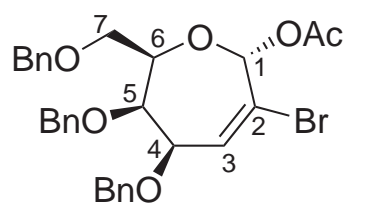

269: $\mathrm{R}_{f} 0.4$ (5:1 hexanes:ethyl acetate); $[\alpha]_{\mathrm{D}}^{18}-25$ (c 2.0

$\left.\mathrm{CHCl}_{3}\right) ;{ }^{1} \mathrm{H}-\mathrm{NMR}:\left(\mathrm{CDCl}_{3}\right) \delta_{\mathrm{H}} 7.34-7.24$ (complex m, $15 \mathrm{H}$,

Bn), 6.44 (s, 1H, H-1), 6.38 (s, 1H, H-3), 4.80 (d, $J=12.0$ $\mathrm{Hz}, 1 \mathrm{H}, \mathrm{PhCH}_{2}$ ), 4.64 (d, $J=12.0 \mathrm{~Hz}, 1 \mathrm{H}, \mathrm{PhCH}_{2}$ ), 4.54 (d, $\left.J=11.2 \mathrm{~Hz}, 1 \mathrm{H}, \mathrm{PhCH}_{2}\right), 4.49\left(\mathrm{~d}, J=11.7 \mathrm{~Hz}, 1 \mathrm{H}, \mathrm{PhCH}_{2}\right), 4.43(\mathrm{~m}, 1 \mathrm{H}, \mathrm{H}-4)$, $4.38\left(\mathrm{~d}, J=11.7 \mathrm{~Hz}, 1 \mathrm{H}, \mathrm{PhCH}_{2}\right), 4.38\left(\mathrm{~d}, J=11.7 \mathrm{~Hz}, 1 \mathrm{H}, \mathrm{PhCH}_{2}\right), 4.11$ (m, 1H, H-6), 4.09 (m, 1H, H-5), 3.68 (apparent d, $J=6.3 \mathrm{~Hz}, 2 \mathrm{H}, \mathrm{H}-7 \mathrm{a}, \mathrm{b}), 2.08$ (s, 3H, Ac); ${ }^{13} \mathrm{C}-\mathrm{NMR}:\left(\mathrm{CDCl}_{3}\right) \delta_{\mathrm{C}} 168.8$ (C, $\left.\mathrm{CH}_{3} \mathrm{CO}\right), 138.2$ (C, Bn), 138.0 (C, Bn), 137.4 (C, Bn), 134.4 (CH, C-3), 128.6 (CH, Bn), 128.4 (CH, Bn), 128.3 (CH, Bn), 128.00 (CH, Bn), 127.95 (CH, Bn), 127.9 (CH, Bn), 127.7 (CH, Bn), $127.62(\mathrm{CH}$, Bn), 127.61 (CH, Bn), 119.9 (C, C-2), 91.6 (CH, C-1), 80.4 (CH, C-4), 77.0 (CH, C-5), $75.4(\mathrm{CH}, \mathrm{C}-6), 73.6\left(\mathrm{CH}_{2}, \mathrm{PhCH}_{2}\right), 73.2\left(\mathrm{CH}_{2}, \mathrm{PhCH}_{2}\right), 71.9\left(\mathrm{CH}_{2}, \mathrm{PhCH}_{2}\right)$, $67.5\left(\mathrm{CH}_{2}, \mathrm{C}-7\right), 20.9\left(\mathrm{CH}_{3}, \mathrm{Ac}\right)$; IR $(\mathrm{KBr}): \nu_{\max } 3063,3030,2870,1775,1604$, 1496, 1454, 1370, 1219, 1061, 1027, 752, $698 \mathrm{~cm}^{-1}$; HRMS: $m / z \mathrm{C}_{30} \mathrm{H}_{31} \mathrm{O}_{6}^{79} \mathrm{BrNa}^{+}$ $[\mathrm{M}+\mathrm{Na}]^{+}$calcd 589.1202, found 589.1199.

\section{Acetyl 4,5-di- $O$-benzyl-2-bromo-2,3-dideoxy- $\beta$-D-threo-hex-2- enoseptanoside (271) and acetyl 4,5-di- $O$-benzyl-2-bromo-2,3-dideoxy- $\alpha$ - D-threo-hex-2-enoseptanoside (272)}

A Cyclopropane 243 (95 mg, $0.20 \mathrm{mmol})$ in toluene $(2.1 \mathrm{~mL})$ was treated with silver acetate $(53 \mathrm{mg}, 0.32 \mathrm{mmol})$ and sodium acetate $(89 \mathrm{mg}, 1.1 \mathrm{mmol})$, then stirred at $100{ }^{\circ} \mathrm{C}$ for two days. The solution was filtered through a pad of silica, then eluted further with diethyl ether $(100 \mathrm{~mL})$. The organic fractions were combined, dried, filtered and concentrated to provide a mixture of oxepines 271 and 272 as a yellow oil. Upon purification by column chromatography (14:1 hexanes:ethyl acetate), oxepines 271 and 272 (41 $\mathrm{mg}, \mathbf{4 5 \%}$ ) were obtained as an inseparable colourless oil (1:1 ratio). 

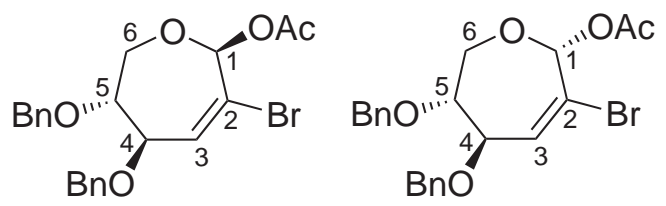

271 and 272: $\mathrm{R}_{f} 0.5$ (5:1 hexanes:ethyl acetate); ${ }^{1} \mathrm{H}-\mathrm{NMR}:\left(\mathrm{CDCl}_{3}\right) \delta_{\mathrm{H}} 7.35-7.28$ (complex m, 10H, Bn), 6.55 (apparent t, $J=$ $1.7 \mathrm{~Hz}, 1 \mathrm{H}, \mathrm{H}-1), 6.40$ (dd, $J=3.0,1.5 \mathrm{~Hz}$, 1H, H-3), 6.38 (d, J = 3.4 Hz, 1H, H-3), 6.18 (d, $J=1.7 \mathrm{~Hz}, 1 \mathrm{H}, \mathrm{H}-1), 4.72-4.60$ (complex m, 5H, $\mathrm{PhCH}_{2}$ and $\mathrm{H}-4$ ), 4.53 (ddd, $J=7.6,3.4,1.5 \mathrm{~Hz}, 1 \mathrm{H}, \mathrm{H}-4$ ), 4.25 $(\mathrm{dd}, J=13.8,5.0 \mathrm{~Hz}, 1 \mathrm{H}, \mathrm{H}-6 \mathrm{a}), 4.03$ (m, 1H, H-6a), 3.88 (td, $J=4.7,1.5 \mathrm{~Hz}$, 1H, H-5), 3.85 (d, $J=13.9$ Hz, 1H, H-6b), 3.83-3.78 (complex m, 2H, H-5,6b), 2.15 (s, 3H, Ac), 2.13 (s, 3H, Ac); ${ }^{13} \mathrm{C}-\mathrm{NMR}:\left(\mathrm{CDCl}_{3}\right) \delta_{\mathrm{C}} 169.1$ (C, $\left.\mathrm{CH}_{3} \mathrm{CO}\right), 138.1$ (C, Bn), 137.9 (C, Bn), 137.7 (C, Bn), 137.6 (C, Bn), 137.5 (CH, C-3), 136.5 (CH, C-3), 128.5 (CH, Bn), 128.41 (CH, Bn), 128.38 (CH, Bn), 127.9 (CH, Bn), 127.79 (CH, Bn), 127.78 (CH, Bn), 127.68 (CH, Bn), 127.67 (CH, Bn), 119.2 (C, C-2), 118.5 (C, C-2), 95.6 (CH, C-1), 94.3 (CH, C-1), 82.7 (CH, C-5), 80.3 (CH, C-5), 80.0 (CH, C-4), 79.2 (CH, C-4), $73.1\left(\mathrm{CH}_{2}, \mathrm{PhCH}_{2}\right), 73.0\left(\mathrm{CH}_{2}, \mathrm{PhCH}_{2}\right), 72.8\left(\mathrm{CH}_{2}, \mathrm{PhCH}_{2}\right)$, $72.4\left(\mathrm{CH}_{2}, \mathrm{PhCH}_{2}\right), 66.0\left(\mathrm{CH}_{2}, \mathrm{C}-6\right), 65.3\left(\mathrm{CH}_{2}, \mathrm{C}-6\right), 20.8\left(\mathrm{CH}_{3}, \mathrm{Ac}\right)$; IR $(\mathrm{KBr})$ : $\nu_{\max }$ 3061, 3030, 2939, 2880, 1755, 1640, 1496, 1454, 1370, 1273, 1218, 1092, 997, 946, 738, $698 \mathrm{~cm}^{-1}$; HRMS: $m / z \mathrm{C}_{22} \mathrm{H}_{23} \mathrm{O}_{5}^{79} \mathrm{BrNa}^{+}[\mathrm{M}+\mathrm{Na}]^{+}$calcd 469.0627, found 469.0630 .

B A solution of cyclopropane $243(820 \mathrm{mg}, 1.75 \mathrm{mmol})$ in toluene $(17.5 \mathrm{~mL})$ was treated with silver acetate $(437 \mathrm{mg}, 2.62 \mathrm{mmol})$ and sodium acetate $(724 \mathrm{mg}$, $8.82 \mathrm{mmol}$ ), then stirred at $95^{\circ} \mathrm{C}$ for three days. The solution was filtered through a pad of silica, then eluted further with diethyl ether $(100 \mathrm{~mL})$. The organic fractions were combined, dried, filtered and concentrated to provide a mixture of oxepines 271 and 272 as a yellow oil. Upon purification by column chromatography (14:1 hexanes:ethyl acetate), oxepines 271 and 272 (313 $\mathrm{mg}, 40 \%$ ) were obtained as an inseparable mixture (1:1 ratio).

C A solution of cyclopropane $243(89 \mathrm{mg}, 0.19 \mathrm{mmol})$ in toluene $(2.1 \mathrm{~mL})$ was treated with sodium acetate $(82 \mathrm{mg}, 1.0 \mathrm{mmol})$, then stirred at $100{ }^{\circ} \mathrm{C}$ for two days. The solution was diluted with water $(10 \mathrm{~mL})$, then extracted with dichloromethane $(3 \times 10 \mathrm{~mL})$. The organic fractions were combined, dried, filtered and concentrated to provide a mixture of oxepines 271 and 272 as a pale-yellow oil. Upon purification by column chromatography (14:1 hexanes:ethyl acetate), oxepines 271 and 272 (20 mg, 24\%) were obtained as an inseparable mixture (1.5:1 ratio).

\section{Allyl 4,5,7-tri- $O$-benzyl-2,3-dideoxy- $\alpha$-D-arabino-hept-2-enoseptanoside (273)}

A solution of oxepine $261(7 \mathrm{mg}, 12 \mu \mathrm{mol})$ in THF $(0.5 \mathrm{~mL})$ was cooled to $-78{ }^{\circ} \mathrm{C}$, 
then treated with $n$-butyllithium $(20 \mu \mathrm{L}, 32 \mu \mathrm{mol})$ and stirred for one hour. The solution was then treated with water $(20 \mu \mathrm{L}, 1.1 \mathrm{mmol})$ and immediately allowed to warm to room temperature. After an additional 30 minutes reaction, the solution was diluted with water $(10 \mathrm{~mL})$, extracted with dichloromethane $(3 \times 10 \mathrm{~mL})$, then the organic fractions were combined, dried, filtered and concentrated to provide a yellow oil. Upon purification by column chromatography (14:1 hexanes:ethyl acetate), diene $\mathbf{2 7 3}$ (2 mg, 34\%) was obtained as a colourless oil.

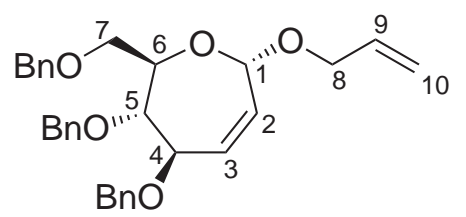

$\mathrm{R}_{f} 0.2$ (14:1 hexanes:ethyl acetate); ${ }^{1} \mathrm{H}-\mathrm{NMR}:\left(\mathrm{CDCl}_{3}\right)$ $\delta_{\mathrm{H}}$ 7.47-7.15 (complex m, 15H, Bn), 5.89 (ddd, $J=12.0$, $5.4,1.5 \mathrm{~Hz}, 1 \mathrm{H}, \mathrm{H}-3), 5.86(\mathrm{~m}, 1 \mathrm{H}, \mathrm{H}-9), 5.74$ (dd, $J=$ 12.1, $1.8 \mathrm{~Hz}, 1 \mathrm{H}, \mathrm{H}-2), 5.38$ (s, 1H, H-1), 5.21 (dd, $J=$ 17.2, $1.1 \mathrm{~Hz}, 1 \mathrm{H}, \mathrm{H}-10 \mathrm{a}), 5.12$ (dd, $J=10.4,0.6 \mathrm{~Hz}, 1 \mathrm{H}, \mathrm{H}-10 \mathrm{~b}), 4.68$ (d, $J=$ $12.2 \mathrm{~Hz}, 1 \mathrm{H}, \mathrm{PhCH}_{2}$ ), 4.58 (d, $J=12.0 \mathrm{~Hz}, 1 \mathrm{H}, \mathrm{PhCH}_{2}$ ), 4.57 (d, $J=11.2 \mathrm{~Hz}, 1 \mathrm{H}$, $\mathrm{PhCH}_{2}$ ), 4.55 (d, $J=12.0 \mathrm{~Hz}, 1 \mathrm{H}, \mathrm{PhCH}_{2}$ ), 4.48 (d, $J=12.2 \mathrm{~Hz}, 1 \mathrm{H}, \mathrm{PhCH}_{2}$ ), 4.36 $\left(\mathrm{d}, J=11.2 \mathrm{~Hz}, 1 \mathrm{H}, \mathrm{PhCH}_{2}\right), 4.32(\mathrm{dd}, J=12.6,5.0 \mathrm{~Hz}, 1 \mathrm{H}, \mathrm{H}-8 \mathrm{a}), 4.14$ (apparent t, $J=4.8 \mathrm{~Hz}, 1 \mathrm{H}, \mathrm{H}-4), 4.10(\mathrm{ddd}, J=8.8,6.2,2.4 \mathrm{~Hz}, 1 \mathrm{H}, \mathrm{H}-6), 4.00$ (dd, $J=$ $12.7,5.9 \mathrm{~Hz}, 1 \mathrm{H}, \mathrm{H}-8 \mathrm{~b}), 3.75$ (dd, $J=8.9,4.0 \mathrm{~Hz}, 1 \mathrm{H}, \mathrm{H}-5), 3.68$ (dd, $J=10.5,5.9$ $\mathrm{Hz}, 1 \mathrm{H}, \mathrm{H}-7 \mathrm{a}), 3.59$ (dd, $J=10.5,2.2 \mathrm{~Hz}, 1 \mathrm{H}, \mathrm{H}-7 \mathrm{~b}) ;{ }^{13} \mathrm{C}-\mathrm{NMR}:\left(\mathrm{CDCl}_{3}\right) \delta_{\mathrm{C}} 138.3$ (C, Bn), 138.2 (C, Bn), 137.9 (C, Bn), 134.4 (CH, C-9), 132.3 (CH, C-2), 129.3 (CH, C-3), 128.4 (CH, Bn), 128.32 (CH, Bn), 128.27 (CH, Bn), 127.9 (CH, Bn), 127.8 (CH, Bn), 127.70 (CH, Bn), 127.67 (CH, Bn), 127.5 (CH, Bn), $117.1\left(\mathrm{CH}_{2}, \mathrm{C}-10\right)$, 97.9 (CH, C-1), 81.4 (CH, C-5), $76.2(\mathrm{CH}, \mathrm{C}-4), 73.1\left(\mathrm{CH}_{2}, \mathrm{PhCH}_{2}\right), 72.9\left(\mathrm{CH}_{2}\right.$, $\left.\mathrm{PhCH}_{2}\right), 71.6(\mathrm{CH}, \mathrm{C}-6), 71.3\left(\mathrm{CH}_{2}, \mathrm{PhCH}_{2}\right), 70.7\left(\mathrm{CH}_{2}, \mathrm{C}-7\right), 68.3\left(\mathrm{CH}_{2}, \mathrm{C}-8\right)$; HRMS: $m / z \mathrm{C}_{31} \mathrm{H}_{34} \mathrm{O}_{5} \mathrm{Na}^{+}[\mathrm{M}+\mathrm{Na}]^{+}$calcd 509.2304, found 509.2300.

\section{4-Methylene-2-(4,5,7-tri- $O$-benzyl-1,2,3-dideoxy- $\alpha$-D-arabino-hept-2- enoseptanoso) $[1,2-b]$ oxolane $(274)$}

A solution of allyl oxepine $261(15 \mathrm{mg}, 27 \mu \mathrm{mol})$ in THF $(1 \mathrm{~mL})$ was treated successively with triphenylphosphine $(2 \mathrm{mg}, 8 \mu \mathrm{mol})$, triethylamine $(8 \mu \mathrm{L}, 60 \mu \mathrm{mol})$ and palladium(II)diacetate $(1 \mathrm{mg}, 5 \mu \mathrm{mol})$, then stirred at reflux for one day. The reaction was then charged with additional triphenylphosphine $(10 \mathrm{mg} 38 \mu \mathrm{mol})$ and palladium(II)diacetate ( $4 \mathrm{mg}, 18 \mu \mathrm{mol}$ ), then reacted for an additional three hours. The solution was diluted with water $(10 \mathrm{~mL})$, extracted with dichloromethane $(3 \mathrm{x}$ $10 \mathrm{~mL}$ ), then the organic fractions were combined, dried, filtered and concentrated to provide a yellow solid. Upon separation by column chromatography (5:1 hexanes:ethyl acetate), starting material 261 was recovered (3 $\mathrm{mg}, 20 \%)$, in addition to diene $\mathbf{2 7 4}$ (6 $\mathrm{mg}, \mathbf{4 7 \%}$ ), obtained as a yellow solid. 


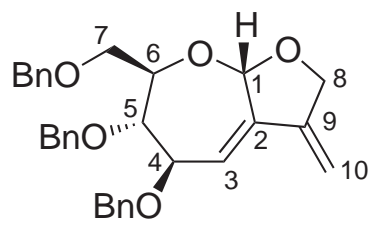

$\mathrm{R}_{f} 0.3$ (5:1 hexanes:ethyl acetate); m.p. $78-81{ }^{\circ} \mathrm{C} ;[\alpha]_{\mathrm{D}}^{19}-2$ (c $\left.0.4 \mathrm{CHCl}_{3}\right) ;{ }^{1} \mathrm{H}-\mathrm{NMR}:\left(\mathrm{CDCl}_{3}\right) \delta_{\mathrm{H}} 7.73-7.69$ (complex m, 2H, Bn), 7.43-7.12 (complex m, 13H, Bn), 6.19 (dd, $J=3.5$, $1.6 \mathrm{~Hz}, 1 \mathrm{H}, \mathrm{H}-3), 5.50$ (d, $J=1.7 \mathrm{~Hz}, 1 \mathrm{H}, \mathrm{H}-1$ ), 5.29 (apparent t, $J=2.2 \mathrm{~Hz}, 1 \mathrm{H}, \mathrm{H}-10 \mathrm{a}), 4.94$ (s, 1H, H-10b), 4.91 (d, $J=10.5 \mathrm{~Hz}, 1 \mathrm{H}, \mathrm{H}-8 \mathrm{a})$, $4.78\left(\mathrm{dt}, J=12.7,2.4 \mathrm{~Hz}, 1 \mathrm{H}, \mathrm{PhCH}_{2}\right), 4.73\left(\mathrm{~d}, J=12.0 \mathrm{~Hz}, 1 \mathrm{H}, \mathrm{PhCH}_{2}\right), 4.62$ (d, $J=12.2 \mathrm{~Hz}, 1 \mathrm{H}, \mathrm{PhCH}_{2}$ ), 4.54-4.50 (complex m, 4H, $\left.\mathrm{PhCH}_{2}, \mathrm{H}-8 \mathrm{~b}\right), 4.41$ (dd, $J=$ 9.1, $3.5 \mathrm{~Hz}, 1 \mathrm{H}, \mathrm{H}-4), 3.94$ (dd, $J=10.0,3.9 \mathrm{~Hz}, 1 \mathrm{H}, \mathrm{H}-7 \mathrm{a}), 3.90$ (ddd, $J=8.7$, 3.9, $2.1 \mathrm{~Hz}, 1 \mathrm{H}, \mathrm{H}-6$ ), 3.77 (dd, $J=10.0,2.0 \mathrm{~Hz}, 1 \mathrm{H}, \mathrm{H}-7 \mathrm{~b}), 3.63$ (apparent t, $J=$ $9.0 \mathrm{~Hz}, 1 \mathrm{H}, \mathrm{H}-5) ;{ }^{13} \mathrm{C}-\mathrm{NMR}:\left(\mathrm{CDCl}_{3}\right) \delta_{\mathrm{C}} 141.4$ (C, C-9), 141.2 (C, C-2), 138.3 (C, Bn), 138.2 (C, Bn), 138.1 (C, Bn), 135.2 (CH, Bn), 128.4 (CH, Bn), 128.3 (CH, Bn), 127.99 (CH, Bn), 127.97 (CH, Bn), 127.9 (CH, Bn), 127.7 (CH, Bn), 127.60 (CH, Bn), 127.56 (CH, Bn), 125.0 (CH, C-3), 105.2 (CH, C-1), $103.3\left(\mathrm{CH}_{2}, \mathrm{C}-10\right), 82.5$ (CH, C-4), 80.7 (CH, C-6), 76.1 (CH, C-5), $75.3\left(\mathrm{CH}_{2}, \mathrm{C}-8\right), 73.6\left(\mathrm{CH}_{2}, \mathrm{PhCH}_{2}\right)$, $73.2\left(\mathrm{CH}_{2}, \mathrm{PhCH}_{2}\right), 72.7\left(\mathrm{CH}_{2}, \mathrm{PhCH}_{2}\right), 70.7\left(\mathrm{CH}_{2}, \mathrm{C}-7\right)$; IR $(\mathrm{KBr}): \nu_{\max } 3063$, 3030, 2918, 2866, 1723, 1604, 1496, 1453, 1359, 1091, 1072, 1027, 750, $697 \mathrm{~cm}^{-1}$; HRMS: $m / z \mathrm{C}_{31} \mathrm{H}_{32} \mathrm{O}_{5} \mathrm{Na}^{+}[\mathrm{M}+\mathrm{Na}]^{+}$calcd 507.2147, found 507.2148.

\section{3,6-Anhydro-4,5,7-tri- $O$-benzyl-2-bromo-2-deoxy-D-arabino-septanose} dimethyl acetal (275) and 3,6-anhydro-4,5,7-tri- $O$-benzyl-2-bromo-2deoxy-D-arabino-septanose dimethyl acetal (276) and (Z)-3,6-anhydro4,5,7-tri- $O$-benzyl-2-bromo-2-deoxy-1- $O$-methyl-D-arabino-hept-1-enitol (277)

A A solution of cyclopropane $97(96 \mathrm{mg}, 0.16 \mathrm{mmol})$ in methanol $(1.7 \mathrm{~mL})$ was subjected to microwave reaction at $130{ }^{\circ} \mathrm{C}$ (pressure approximately eight bar) for periods of 10, 30 and 20 minutes (total one hour). The mixture was transferred from the reaction vessel by dilution with methanol $(10 \mathrm{~mL})$ and dichloromethane $(20 \mathrm{~mL})$, then concentrated to provide a yellow oil. This oil was purified by column chromatography (14:1 hexanes:ethyl acetate) to provide a trace of starting material 97 (4 mg, 4\%), in addition to dimethyl acetals 275 (14 mg, 15\%) and 276 (43 mg, 46\%) and olefin 277 (11 mg, 13\%), isolated as colourless oils.

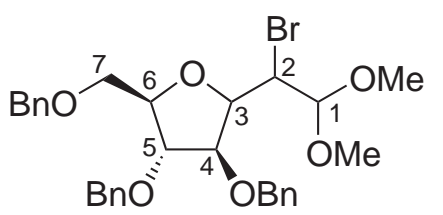

275: $\mathrm{R}_{f} 0.4$ (5:1 hexanes:ethyl acetate); $[\alpha]_{\mathrm{D}}^{20}+29$ ( c 1.2 $\left.\mathrm{CHCl}_{3}\right) ;{ }^{1} \mathrm{H}-\mathrm{NMR}:\left(\mathrm{CDCl}_{3}\right) \delta_{\mathrm{H}} 7.36-7.26$ (complex m, $15 \mathrm{H}, \mathrm{Bn}), 4.61$ (d, $\left.J=11.7 \mathrm{~Hz}, 1 \mathrm{H}, \mathrm{PhCH}_{2}\right), 4.58-4.55$ (complex m, 6H, $\mathrm{PhCH}_{2}, \mathrm{H}-1$ ), 4.33 (dd, $J=6.6,4.7 \mathrm{~Hz}$, 1H, H-4), 4.25 (apparent q, $J=5.0 \mathrm{~Hz}, 1 \mathrm{H}, \mathrm{H}-6), 4.22$ (dd, $J=6.6,3.2 \mathrm{~Hz}, 1 \mathrm{H}, \mathrm{H}-$ 3), 4.19 (apparent t, $J=5.0 \mathrm{~Hz}, 1 \mathrm{H}, \mathrm{H}-5$ ), 4.04 (dd, $J=7.3,2.9 \mathrm{~Hz}, 1 \mathrm{H}, \mathrm{H}-2$ ), 3.59 (apparent t, $J=5.0 \mathrm{~Hz}, 2 \mathrm{H}, \mathrm{H}-7 \mathrm{a}, \mathrm{b}), 3.40$ (s, 3H, Me), 3.38 (s, 3H, Me); ${ }^{13} \mathrm{C}-\mathrm{NMR}$ : $\left(\mathrm{CDCl}_{3}\right) \delta_{\mathrm{C}} 138.1(\mathrm{C}, \mathrm{Bn}), 137.87$ (C, 2 x Bn), 128.5 (CH, Bn), 128.41 (CH, Bn), 
128.38 (CH, Bn), 127.9 (CH, Bn), 127.84 (CH, Bn), 127.81 (CH, Bn), 127.79 (CH, Bn), 127.7 (CH, Bn), 127.6 (CH, Bn), 104.4 (CH, C-1), 85.9 (CH, C-4), 84.3 (CH, C-5), 81.8 (CH, C-6), $79.9(\mathrm{CH}, \mathrm{C}-3), 73.4\left(\mathrm{CH}_{2}, \mathrm{PhCH}_{2}\right), 72.4\left(\mathrm{CH}_{2}, \mathrm{PhCH}_{2}\right), 72.2$ $\left(\mathrm{CH}_{2}, \mathrm{PhCH}_{2}\right), 70.1\left(\mathrm{CH}_{2}, \mathrm{C}-7\right), 55.7\left(\mathrm{CH}_{3}, \mathrm{Me}\right), 55.0(\mathrm{CH}, \mathrm{C}-2), 54.4\left(\mathrm{CH}_{3}, \mathrm{Me}\right)$; IR (KBr): $\nu_{\max } 3088,3063,3030,2930,2862,1723,1496,1454,1362,1108,1072$, $1027,737,698 \mathrm{~cm}^{-1}$.

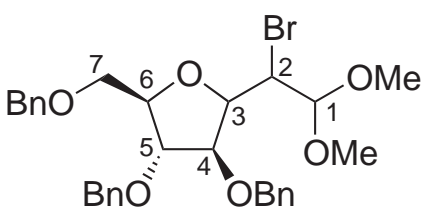

276: $\quad \mathrm{R}_{f} 0.3$ (5:1 hexanes:ethyl acetate); $[\alpha]_{\mathrm{D}}^{20}-5.0(c$ $\left.3.3 \mathrm{CHCl}_{3}\right) ;{ }^{1} \mathrm{H}-\mathrm{NMR}:\left(\mathrm{CDCl}_{3}\right) \delta_{\mathrm{H}} 7.37-7.19$ (complex m, $15 \mathrm{H}, \mathrm{Bn}), 4.64$ (d, $\left.J=12.2 \mathrm{~Hz}, 1 \mathrm{H}, \mathrm{PhCH}_{2}\right), 4.59$ (d, $J=$ $\left.12.0 \mathrm{~Hz}, 1 \mathrm{H}, \mathrm{PhCH}_{2}\right), 4.53\left(\mathrm{~d}, J=12.2 \mathrm{~Hz}, 1 \mathrm{H}, \mathrm{PhCH}_{2}\right)$, $4.52\left(\mathrm{~d}, J=12.0 \mathrm{~Hz}, 1 \mathrm{H}, \mathrm{PhCH}_{2}\right), 4.46\left(\mathrm{~d}, J=11.7 \mathrm{~Hz}, 1 \mathrm{H}, \mathrm{PhCH}_{2}\right), 4.28$ (dd, $J=9.0,3.2 \mathrm{~Hz}, 1 \mathrm{H}, \mathrm{H}-2), 4.24\left(\mathrm{~d}, J=11.2 \mathrm{~Hz}, 1 \mathrm{H}, \mathrm{PhCH}_{2}\right), 4.24$ (dd, $J=9.0$, $3.4 \mathrm{~Hz}, 1 \mathrm{H}, \mathrm{H}-3), 4.17$ (ddd, $J=7.7,5.1,2.5 \mathrm{~Hz}, 1 \mathrm{H}, \mathrm{H}-6), 4.12$ (d, J = 3.2 Hz, $1 \mathrm{H}, \mathrm{H}-1), 4.11$ (d, J = 2.2 Hz, 1H, H-5), 3.90 (d, $J=3.7 \mathrm{~Hz}, 1 \mathrm{H}, \mathrm{H}-4), 3.71$ (dd, $J=9.8,5.1 \mathrm{~Hz}, 1 \mathrm{H}, \mathrm{H}-7 \mathrm{a}), 3.51$ (td, $J=9.8,2.0 \mathrm{~Hz}, 1 \mathrm{H}, \mathrm{H}-7 \mathrm{~b}), 3.33$ (s, 3H, Me), 3.28 (s, 3H, Me); ${ }^{13} \mathrm{C}-\mathrm{NMR}:\left(\mathrm{CDCl}_{3}\right) \delta_{\mathrm{C}} 138.2$ (C, Bn), 137.8 (C, Bn), 137.1 (C, $\mathrm{Bn}), 128.52$ (CH, Bn), 128.50 (CH, Bn), 128.4 (CH, Bn), $128.2(\mathrm{CH}, \mathrm{Bn}), 128.1$ (CH, Bn), 127.9 (CH, Bn), 127.84 (CH, Bn), 127.81 (CH, Bn), 127.7 (CH, Bn), 103.7 (CH, C-1), 83.2 (CH, C-5), 82.3 (CH, C-4), 82.0 (CH, C-6), 81.1 (CH, C-3), $73.3\left(\mathrm{CH}_{2}, \mathrm{PhCH}_{2}\right), 71.3\left(\mathrm{CH}_{2}, \mathrm{PhCH}_{2}\right), 70.9\left(\mathrm{CH}_{2}, \mathrm{PhCH}_{2}\right), 70.1\left(\mathrm{CH}_{2}, \mathrm{C}-7\right), 56.2$ $\left(\mathrm{CH}_{3}, \mathrm{Me}\right), 55.5\left(\mathrm{CH}_{3}, \mathrm{Me}\right), 51.5(\mathrm{CH}, \mathrm{C}-2)$; IR (KBr): $\nu_{\max } 3087,3062,3030,2926$, 2865, 1726, 1604, 1496, 1454, 1362, 1210, 1090, 1072, 738, $698 \mathrm{~cm}^{-1}$; HRMS: $\mathrm{m} / z$ $\mathrm{C}_{30} \mathrm{H}_{35} \mathrm{O}_{6}^{79} \mathrm{BrNa}^{+}[\mathrm{M}+\mathrm{Na}]^{+}$calcd 593.1515, found 593.1506.

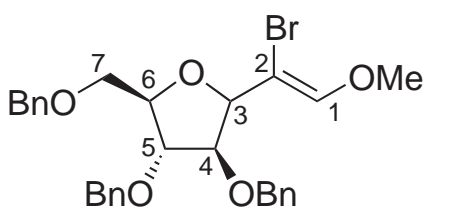

277: $\mathrm{R}_{f} 0.25$ (5:1 hexanes:ethyl acetate); $[\alpha]_{\mathrm{D}}^{20}+8$ (c 0.6 $\left.\mathrm{CHCl}_{3}\right) ;{ }^{1} \mathrm{H}-\mathrm{NMR}:\left(\mathrm{CDCl}_{3}\right) \delta_{\mathrm{H}} 7.35-7.25$ (complex m, $15 \mathrm{H}, \mathrm{Bn}), 6.60$ (s, 1H, H-1), 4.62-4.51 (complex m, 6H, $\mathrm{PhCH}_{2}$ ), 4.38-4.34 (complex m, 2H, H-3,4), 4.23-4.19

(complex m, 2H, H-5,6), 3.74 (s, 3H, Me), 3.60 (dd, $J=10.4,3.5 \mathrm{~Hz}, 1 \mathrm{H}, \mathrm{H}-7 \mathrm{a}$ ), 3.55 $(\mathrm{dd}, J=10.0,3.9 \mathrm{~Hz}, 1 \mathrm{H}, \mathrm{H}-7 \mathrm{~b}) ;{ }^{13} \mathrm{C}-\mathrm{NMR}:\left(\mathrm{CDCl}_{3}\right) \delta_{\mathrm{C}} 147.9$ (CH, C-1), 138.1 (C, Bn), 138.0 (C, Bn), 137.9 (C, Bn), 128.41 (CH, Bn), 128.39 (CH, Bn), 128.37 (CH, $\mathrm{Bn}), 127.82$ (CH, Bn), 127.79 (CH, Bn), 127.76 (CH, Bn), 127.7 (CH, Bn), 127.6 (CH, Bn), 101.2 (C, C-2), 85.9 (CH, C-4), 83.6 (CH, C-5), 82.4 (CH, C-3), 81.1 (CH, C-6), $73.4\left(\mathrm{CH}_{2}, \mathrm{PhCH}_{2}\right), 72.5\left(\mathrm{CH}_{2}, \mathrm{PhCH}_{2}\right), 72.2\left(\mathrm{CH}_{2}, \mathrm{PhCH}_{2}\right), 70.2\left(\mathrm{CH}_{2}, \mathrm{C}-7\right)$, $60.7\left(\mathrm{CH}_{3}, \mathrm{Me}\right)$; IR $(\mathrm{KBr}): \nu_{\max } 3054,2986,2917,2848,1666,1496,1454,1421$, 1363, 1265, 1098, 1027, 895, 738, $703 \mathrm{~cm}^{-1}$; HRMS: $m / z \mathrm{C}_{29} \mathrm{H}_{31} \mathrm{O}_{5}^{79} \mathrm{BrNa}^{+}[\mathrm{M}+\mathrm{Na}]^{+}$ calcd 561.1253, found 561.1246 .

B A solution of cyclopropane $97(60 \mathrm{mg}, 0.10 \mathrm{mmol})$ in methanol $(3.4 \mathrm{~mL})$ was treated with silver acetate $(25 \mathrm{mg}, 0.15 \mathrm{mmol})$, then subjected to microwave reaction at $100{ }^{\circ} \mathrm{C}$ (pressure approximately three bar) for a period of 10 minutes. The 
mixture was transferred with methanol $(20 \mathrm{~mL})$, then concentrated to provide a yellow oil. This oil was purified by column chromatography (14:1 hexanes:ethyl acetate) to provide a large proportion of starting material $\mathbf{9 7}$, in addition to oxepines $98(0.6 \mathrm{mg}, 1 \%)$ and $99(1.5 \mathrm{mg}, 3 \%)$. Traces of the higher moving dimethyl acetal 275 and olefin 277 were detected by TLC.

C A solution of cyclopropane $97(100 \mathrm{mg}, 0.17 \mathrm{mmol})$ in methanol (3.4 mL) was treated with silver nitrate $(38 \mathrm{mg}, 0.21 \mathrm{mmol})$, then subjected to microwave reaction at $100{ }^{\circ} \mathrm{C}$ (pressure approximately three bar) for periods of 10,50 and 50 minutes (total 110 minutes). The mixture was transferred with methanol $(20 \mathrm{~mL})$, then concentrated to provide a yellow oil. Proton NMR analysis of this crude oil showed the presence of starting material 97 and $C$-furanosyl sugars 275, 276 and olefin $\mathbf{2 7 7}$, indicating conversion of approximately $60 \%$.

D A solution of cyclopropane $97(100 \mathrm{mg}, 0.17 \mathrm{mmol})$ in methanol $(1.7 \mathrm{~mL})$ was treated with silver tetrafluoroborate $(29 \mathrm{mg}, 0.15 \mathrm{mmol})$, then refluxed for three days. The solution was diluted with diethyl ether $(10 \mathrm{~mL})$, filtered through a pad of Celite ${ }^{\circledR}$, then washed with water $(10 \mathrm{~mL})$. The aqueous wash was extracted further with ether $(2 \times 10 \mathrm{~mL})$, then the ethereal solutions were combined, dried, filtered and concentrated to provide a yellow oil. Upon separation by column chromatography (9:1 hexanes:ethyl acetate), starting material 97 (26 mg, 26\%) was recovered, in addition to $C$-furanosyl sugars 275 (9 $\mathrm{mg}, 9 \%)$ and 276 (21 mg, 22\%).

\section{3,6-Anhydro-4,5,7-tri- $O$-benzyl-2-bromo-2-deoxy-D-arabino-septanose} diallyl acetal (278) and 3,6-anhydro-4,5,7-tri- $O$-benzyl-2-bromo-2-deoxyD-arabino-septanose diallyl acetal (279) and (Z)-1- $O$-allyl-3,6-anhydro4,5,7-tri- $O$-benzyl-2-bromo-2-deoxy-D-arabino-hept-1-enitol (280)

A A solution of cyclopropane $97(100 \mathrm{mg}, 0.170 \mathrm{mmol})$ in allyl alcohol $(1.7 \mathrm{~mL})$ was heated under reflux for two days, then filtered through a plug of silica, which was eluted further with 5:1 hexanes:ethyl acetate $(30 \mathrm{~mL})$. The eluents were concentrated to provide a colourless oil, which was purified by column chromatography (14:1 hexanes:ethyl acetate). A complex mixture of unidentified products was isolated (30 $\mathrm{mg}, \mathrm{R}_{f} 0.2$ ), in addition to diallyl acetals 278 (18 $\left.\mathrm{mg}, 17 \%\right)$ and 279 (30 $\mathrm{mg}$, $28 \%$ ) and olefin 280 (11 mg, 12\%), all isolated as colourless oils.

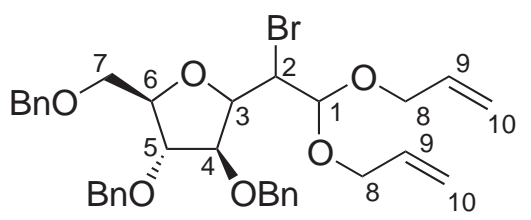

278: $\mathrm{R}_{f} 0.15$ (14:1 hexanes:ethyl acetate); $[\alpha]_{\mathrm{D}}^{21}-3(c$ $\left.0.3 \mathrm{CHCl}_{3}\right) ;{ }^{1} \mathrm{H}-\mathrm{NMR}:\left(\mathrm{CDCl}_{3}\right) \delta_{\mathrm{H}} 7.40-7.26$ (complex m, 15H, Bn), 5.95-5.82 (complex m, 2H, H-9), $5.30(\mathrm{~d}, J=17.1 \mathrm{~Hz}, 1 \mathrm{H}, \mathrm{H}-10 \mathrm{a}), 5.26$ (d, $J=17.1$ Hz, 1H, H-10a), 5.18 (d, $J=10.5$ Hz, 1H, H-10b), 5.14 (d, $J=10.5$ Hz, 1H, H-10b), 
$4.80(\mathrm{dd}, J=7.2,2.1 \mathrm{~Hz}, 1 \mathrm{H}, \mathrm{H}-1), 4.60-4.48$ (complex m, 6H, $\mathrm{PhCH}_{2}$ ), 4.38 (dd, $J=6.6,4.6 \mathrm{~Hz}, 1 \mathrm{H}, \mathrm{H}-4$ ), 4.31 (dd, $J=5.7,2.3 \mathrm{~Hz}, 1 \mathrm{H}, \mathrm{H}-6$ ), 4.28 (dd, $J=6.3$, $3.2 \mathrm{~Hz}, 1 \mathrm{H}, \mathrm{H}-3$ ), 4.25 (apparent t, $J=4.9 \mathrm{~Hz}, 1 \mathrm{H}, \mathrm{H}-5$ ) , 4.22-4.17 (complex m, 2H, H-8a,b), 4.14-4.07 (complex m, 3H, H-2,8), 3.58 (apparent td, $J=5.0,2.0 \mathrm{~Hz}, 2 \mathrm{H}$, H-7a,b); ${ }^{13}$ C-NMR: $\left(\mathrm{CDCl}_{3}\right) \delta_{\mathrm{C}} 138.1$ (C, Bn), 137.8 (C, Bn), 137.7 (C, Bn), 134.1 and 133.9 (C, C-9), $128.4(\mathrm{CH}, \mathrm{Bn}), 128.3$ (CH, Bn), $127.82(\mathrm{CH}, \mathrm{Bn}), 127.78(\mathrm{CH}$, Bn), 127.75 (CH, Bn), $127.71(\mathrm{CH}, \mathrm{Bn}), 127.65(\mathrm{CH}, \mathrm{Bn}), 127.6(\mathrm{CH}, \mathrm{Bn}), 117.5$ and $117.3\left(\mathrm{CH}_{2}, \mathrm{C}-10\right), 101.9(\mathrm{CH}, \mathrm{C}-1), 85.8$ (CH, C-4), $84.3(\mathrm{CH}, \mathrm{C}-5), 81.7(\mathrm{CH}$, C-3), $79.9(\mathrm{CH}, \mathrm{C}-6), 73.4\left(\mathrm{CH}_{2}, \mathrm{PhCH}_{2}\right), 72.4\left(\mathrm{CH}_{2}, \mathrm{PhCH}_{2}\right), 72.1\left(\mathrm{CH}_{2}, \mathrm{PhCH}_{2}\right)$, $70.2\left(\mathrm{CH}_{2}, \mathrm{C}-7\right), 69.2$ and $68.3\left(\mathrm{CH}_{2}, \mathrm{C}-8\right), 55.7$ (CH, C-2); IR (KBr): $\nu_{\max } 3060$, 3027, 2912, 2862, 1723, 1454, 1361, 1219, 1095, 925, 770, $698 \mathrm{~cm}^{-1}$.

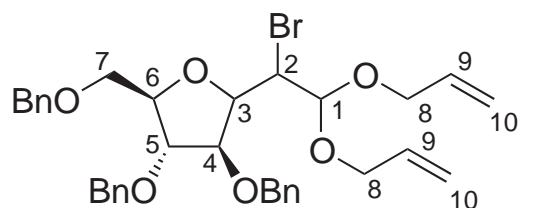

279: $\mathrm{R}_{f} 0.1$ (14:1 hexanes:ethyl acetate); $[\alpha]_{\mathrm{D}}^{21}-8(c$ $\left.0.7 \mathrm{CHCl}_{3}\right) ;{ }^{1} \mathrm{H}-\mathrm{NMR}:\left(\mathrm{CDCl}_{3}\right) \delta_{\mathrm{H}} 7.33-7.14$ (complex m, 15H, Bn), 5.85-5.77 (complex m, 2H, H-9), $5.20(\mathrm{dd}, J=17.1,1.5 \mathrm{~Hz}, 1 \mathrm{H}, \mathrm{H}-10 \mathrm{a}), 5.17$ (dd, $J=17.1,1.5 \mathrm{~Hz}, 1 \mathrm{H}, \mathrm{H}-10 \mathrm{a}), 5.14$ (d, $J=10.5 \mathrm{~Hz}, 1 \mathrm{H}, \mathrm{H}-10 \mathrm{~b}), 5.10$ (d, $J=$ $10.4 \mathrm{~Hz}, 1 \mathrm{H}, \mathrm{H}-10 \mathrm{~b}), 4.58$ (d, $J=12.0 \mathrm{~Hz}, 1 \mathrm{H}, \mathrm{PhCH}_{2}$ ), 4.55 (d, $J=12.0 \mathrm{~Hz}, 1 \mathrm{H}$, $\mathrm{PhCH}_{2}$ ), 4.49 (d, $J=12.2 \mathrm{~Hz}, 1 \mathrm{H}, \mathrm{PhCH}_{2}$ ), 4.48 (d, $J=12.2 \mathrm{~Hz}, 1 \mathrm{H}, \mathrm{PhCH}_{2}$ ), 4.44 (apparent t, $J=2.2 \mathrm{~Hz}, 1 \mathrm{H}, \mathrm{H}-1), 4.41$ (d, $J=11.5 \mathrm{~Hz}, 1 \mathrm{H}, \mathrm{PhCH}_{2}$ ), 4.29-4.25 (complex m, 2H, H-2,3), 4.22 (d, $J=11.5 \mathrm{~Hz}, 1 \mathrm{H}, \mathrm{PhCH}_{2}$ ), 4.13 (m, 1H, H-6), 4.10 (m, 1H, H-8), 4.06 (m, 1H, H-5), 4.00 (ddd, $J=12.8,5.4,1.2 \mathrm{~Hz}, 1 \mathrm{H}, \mathrm{H}-8$ ), 3.94 (ddd, $J=12.8,5.4,1.2 \mathrm{~Hz}, 1 \mathrm{H}, \mathrm{H}-8), 3.92$ (m, 1H, H-4), 3.88 (ddd, $J=13.0$, 6.3, $1.2 \mathrm{~Hz}, 1 \mathrm{H}, \mathrm{H}-8), 3.67$ (ddd, $J=9.8,5.2,2.0 \mathrm{~Hz}, 1 \mathrm{H}, \mathrm{H}-7 \mathrm{a}), 3.48$ (ddd, $J=$ 9.8, 7.9, $2.0 \mathrm{~Hz}, 1 \mathrm{H}, \mathrm{H}-7 \mathrm{~b}) ;{ }^{13} \mathrm{C}-\mathrm{NMR}:\left(\mathrm{CDCl}_{3}\right) \delta_{\mathrm{C}} 138.2(\mathrm{C}, \mathrm{Bn}), 137.8$ (C, Bn), $137.3(\mathrm{C}, \mathrm{Bn}), 133.89$ and 133.85 (CH, C-9), $128.44(\mathrm{CH}, \mathrm{Bn}), 128.41$ (CH, Bn), 128.3 (CH, Bn), 127.9 (CH, Bn), 127.81 (CH, Bn), 127.80 (CH, Bn), 127.79 (CH, $\mathrm{Bn}), 127.7(\mathrm{CH}, \mathrm{Bn}), 127.6(\mathrm{CH}, \mathrm{Bn}), 117.8$ and $117.4\left(\mathrm{CH}_{2}, \mathrm{C}-10\right), 100.2(\mathrm{CH}$, C-1), 83.1 (CH, C-5), 82.4 (CH, C-4), 82.0 (CH, C-6), $81.0(\mathrm{CH}, \mathrm{C}-3), 73.3\left(\mathrm{CH}_{2}\right.$, $\left.\mathrm{PhCH}_{2}\right), 71.3\left(\mathrm{CH}_{2}, \mathrm{PhCH}_{2}\right), 70.9\left(\mathrm{CH}_{2}, \mathrm{PhCH}_{2}\right), 70.1\left(\mathrm{CH}_{2}, \mathrm{C}-7\right), 69.4$ and 68.4 $\left(\mathrm{CH}_{2}, \mathrm{C}-8\right), 52.3$ (CH, C-2); IR (KBr): $\nu_{\max } 3064,3031,2918,2867,1723,1647$, 1603, 1496, 1455, 1363, 1340, 1270, 1208, 1106, 1055, 1028, 928, 738, $699 \mathrm{~cm}^{-1}$; HRMS: $m / z \mathrm{C}_{34} \mathrm{H}_{39} \mathrm{O}_{6}^{79} \mathrm{BrNa}^{+}[\mathrm{M}+\mathrm{Na}]^{+}$calcd 645.1828 , found 645.1823

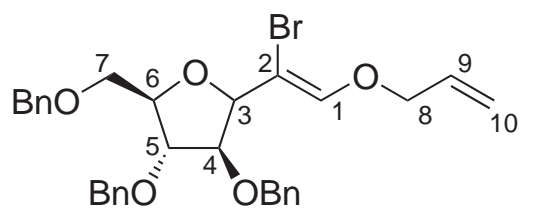

280: $\mathrm{R}_{f} 0.05$ (14:1 hexanes:ethyl acetate); ${ }^{1} \mathrm{H}-\mathrm{NMR}$ : $\left(\mathrm{CDCl}_{3}\right) \delta_{\mathrm{H}} 7.35-7.22$ (complex m, 15H, Bn), $6.69(\mathrm{~d}$, $J=2.0 \mathrm{~Hz}, 1 \mathrm{H}, \mathrm{H}-1), 5.90$ (dddd, $J=17.1,10.4$, $5.0,2.0 \mathrm{~Hz}, 1 \mathrm{H}, \mathrm{H}-9), 5.33(\mathrm{dt}, J=17.1,1.5 \mathrm{~Hz}, 1 \mathrm{H}$, H-10a), 5.26 (dt, $J=10.5,1.3 \mathrm{~Hz}, 1 \mathrm{H}, \mathrm{H}-10 \mathrm{~b}$ ), 4.63-4.51 (complex m, 6H, $\mathrm{PhCH}_{2}$ ), 4.40 (d, $J=5.4 \mathrm{~Hz}, 2 \mathrm{H}, \mathrm{H}-8 \mathrm{a}, \mathrm{b}), 4.37$ (complex m, 2H, H-3,4), 4.21-4.20 (complex m, 2H, H-5,6), 3.60 (dd, $J=10.5,3.0 \mathrm{~Hz}, 1 \mathrm{H}, \mathrm{H}-7 \mathrm{a}$ ), 3.55 (dd, $J=10.5,1.5 \mathrm{~Hz}$, 1H, H-7b); ${ }^{13} \mathrm{C}-\mathrm{NMR}:\left(\mathrm{CDCl}_{3}\right) \delta_{\mathrm{C}} 146.2$ (CH, C-1), 138.1 (C, Bn), 137.91 (C, Bn), 
137.90 (C, Bn), 132.8 (CH, C-9), 128.44 (CH, Bn), 128.38 (CH, Bn), 128.35 (CH, Bn), 128.3 (CH, Bn), 127.9 (CH, Bn), 127.79 (CH, Bn), 127.77 (CH, Bn), 127.7 (CH, $\mathrm{Bn}), 127.6$ (CH, Bn), 118.7 ( $\left.\mathrm{CH}_{2}, \mathrm{C}-10\right), 101.6$ (CH, C-2), 85.8 (CH, C-4), 83.4 (CH, C-5), 82.4 (CH, C-3), 81.0 (CH, C-6), $73.7\left(\mathrm{CH}_{2}, \mathrm{C}-8\right), 73.4\left(\mathrm{CH}_{2}, \mathrm{PhCH}_{2}\right), 72.5$ $\left(\mathrm{CH}_{2}, \mathrm{PhCH}_{2}\right), 72.2\left(\mathrm{CH}_{2}, \mathrm{PhCH}_{2}\right), 70.1\left(\mathrm{CH}_{2}, \mathrm{C}-7\right)$.

B A solution of cyclopropane 97 (104 mg, $0.177 \mathrm{mmol})$ in allyl alcohol $(1.7 \mathrm{~mL})$ was treated with silver nitrate $(31 \mathrm{mg}, 0.19 \mathrm{mmol})$, then heated under reflux for three days. The solution was separated between water $(10 \mathrm{~mL})$ and dichloromethane $(10 \mathrm{~mL})$, then the aqueous phase was extracted further with dichloromethane $(2 \times 10 \mathrm{~mL})$. The organic fractions were combined, filtered through a pad of silica, then dried, filtered and concentrated to provide a yellow oil, which was purified by column chromatography (14:1 hexanes:ethyl acetate). A complex mixture of aldehydes and oxepines 260 and 261 (9 mg) was obtained, in addition to previously isolated $C$-furanosyl sugars 278 (12 mg, 11\%), 279 (28 mg, 25\%) and olefin 280 (16 mg, 15\%).

C A solution of cyclopropane $97(100 \mathrm{mg}, 0.170 \mathrm{mmol})$ in allyl alcohol $(1.7 \mathrm{~mL})$ was treated with silver trifluoroacetate $(54 \mathrm{mg}, 0.24 \mathrm{mmol})$, then heated under reflux for three days. The solution was separated between water $(10 \mathrm{~mL})$ and dichloromethane $(10 \mathrm{~mL})$, then the aqueous phase was extracted further with dichloromethane $(2 \mathrm{x}$ $10 \mathrm{~mL}$ ). The organic fractions were combined, filtered through a pad of Celite ${ }^{\circledR}$, then dried, filtered and concentrated to provide a yellow oil, which was purified by column chromatography (14:1 hexanes:ethyl acetate). Starting material 97 (5 mg, 5\%), oxepines 260 (8 $\mathrm{mg}, 8 \%)$ and 261 (13 mg, 14\%), diallyl acetal 279 (8 $\mathrm{mg}, 8 \%$ ) and olefin $\mathbf{2 8 0}(23 \mathrm{mg}, 22 \%)$ were isolated.

\subsection{Chapter Four Experimental}

\section{Tri- $O$-acetyl-D-galactal (180)}

Using a modification of Kozikowski's procedure, ${ }^{89}$ a magnetically stirred solution of D-galactose (124 mg, $0.7 \mathrm{mmol})$ in acetic anhydride $(75 \mathrm{~mL}, 790 \mathrm{mmol})$ was treated dropwise with conc. perchloric acid $(0.45 \mathrm{~mL}, 6.9 \mathrm{mmol})$. D-Galactose (20.00 g, $111.0 \mathrm{mmol}$ ) was slowly added over half an hour, at a rate that maintained a temperature of $40-50{ }^{\circ} \mathrm{C}$. Upon complete addition of D-galactose, the solution was allowed to cool to room temperature, then treated with a $33 \%$ (w/w) solution of hydrobromic acid in acetic acid $(75 \mathrm{~mL}, 410 \mathrm{mmol})$. After 90 minutes, the solution was diluted with dichloromethane $(180 \mathrm{~mL})$ and washed with ice-cold water $(2 \times 50 \mathrm{~mL})$, 
then with cold sat. sodium bicarbonate solution $(4 \times 100 \mathrm{~mL})$. The organic phase was dried, filtered and concentrated to afford crude tetra- $O$-acetyl- $\alpha$-D-galactopyranosyl bromide (248) as a pale-yellow oil.

A mechanically stirred dispersion of zinc dust (48.1 g, $736 \mathrm{mmol})$ in water $(150 \mathrm{~mL})$ was cooled to $0{ }^{\circ} \mathrm{C}$, then diluted with acetic acid $(150 \mathrm{~mL})$, then treated dropwise with a solution of tetra- $O$-acetyl- $\alpha$-D-galactopyranosyl bromide in diethyl ether $(150 \mathrm{~mL})$, over 20 minutes. The reaction was allowed to warm to room temperature and left to proceed overnight. The solution was filtered, then diluted with dichloromethane $(200 \mathrm{~mL})$. The solution was then washed with water $(3 \mathrm{x} 60 \mathrm{~mL})$, sat. sodium bicarbonate solution $(2 \times 50 \mathrm{~mL})$, then brine $(60 \mathrm{~mL})$. The organic phase was dried, filtered and concentrated to provide tri- $O$-acetyl-D-galactal $(\mathbf{1 8 0})$ as a pale-yellow oil. Purification by flash chromatography (3:1 hexanes:ethyl acetate), provided glycal 180 as a pale-yellow oil (15.9 g, 52\%). The spectral data matched that reported previously. ${ }^{157}$

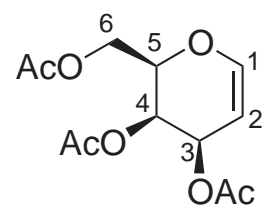

$\mathrm{R}_{f} 0.3$ (3:1 hexanes:ethyl acetate); ${ }^{1} \mathrm{H}-\mathrm{NMR}:\left(\mathrm{CDCl}_{3}\right) \delta_{\mathrm{H}} 6.46$ (dd, $J=6.1,1.8 \mathrm{~Hz}, 1 \mathrm{H}, \mathrm{H}-1), 5.54(\mathrm{~m}, 1 \mathrm{H}, \mathrm{H}-3), 5.42(\mathrm{dt}, J=4.5,1.5$ $\mathrm{Hz}, 1 \mathrm{H}, \mathrm{H}-4), 4.72$ (ddd, $J=6.3,2.6,1.5 \mathrm{~Hz}, 1 \mathrm{H}, \mathrm{H}-2), 4.31$ (m, $1 \mathrm{H}, \mathrm{H}-5), 4.26$ (dd, $J=11.5,7.3 \mathrm{~Hz}, 1 \mathrm{H}, \mathrm{H}-6 \mathrm{a}), 4.21$ (dd, $J=11.5$,

$5.1 \mathrm{~Hz}, 1 \mathrm{H}, \mathrm{H}-6 \mathrm{~b}), 2.12$ (s, 3H, Ac), 2.08 (s, 3H, Ac), 2.03 (s, 3H, Ac); ${ }^{13} \mathrm{C}-\mathrm{NMR}$ : $\left(\mathrm{CDCl}_{3}\right) \delta_{\mathrm{C}} 170.6\left(\mathrm{C}, \mathrm{CH}_{3} \mathrm{CO}\right), 170.3\left(\mathrm{C}, \mathrm{CH}_{3} \mathrm{CO}\right), 170.2\left(\mathrm{C}, \mathrm{CH}_{3} \mathrm{CO}\right), 145.4(\mathrm{CH}$, C-1), 98.8 (CH, C-2), 72.8 (CH, C-5), $63.9(\mathrm{CH}, \mathrm{C}-3), 63.7(\mathrm{CH}, \mathrm{C}-4), 61.9\left(\mathrm{CH}_{2}\right.$, C-6), $20.83\left(\mathrm{CH}_{3}, \mathrm{Ac}\right), 20.78\left(\mathrm{CH}_{3}, \mathrm{Ac}\right), 20.7\left(\mathrm{CH}_{3}, \mathrm{Ac}\right)$.

\section{Phenyl 4,6-di- $O$-acetyl-2,3-dideoxy-1-thio- $\alpha$-D-threo-hex-2-enopyranoside (289)}

Using a known procedure, ${ }^{122}$ a solution of tri- $O$-acetyl-D-galactal (180) (4.06 g, $14.9 \mathrm{mmol})$ in dichloromethane $(70 \mathrm{~mL})$ was treated with thiophenol $(1.79 \mathrm{~g}, 16.2$ $\mathrm{mmol})$, then iron trichloride $\left(2.5 \mathrm{~mL}\right.$ of a $0.01 \mathrm{molL}^{-1}$ solution in dichloromethane, $0.025 \mathrm{mmol}$ ) and stirred under argon for 20 hours. Further iron trichloride was added ( $3 \mathrm{~mL}$ of a $0.01 \mathrm{molL}^{-1}$ solution in dichloromethane, $0.03 \mathrm{mmol}$ ), then the reaction allowed to proceed for 5 hours. The mixture was then diluted with sat. sodium bicarbonate $(75 \mathrm{~mL})$ and the phases separated. The aqueous fraction was extracted further with dichloromethane $(2 \times 75 \mathrm{~mL})$, then the organic fractions were combined, dried, filtered and concentrated. The oil obtained was then subjected to flash chromatography (5:1 hexanes:ethyl acetate), to provide 2,3-unsaturated sugar 289 (2.23 g, 46\% yield) as a white crystalline solid. The spectral data matched that reported previously. ${ }^{123}$ 


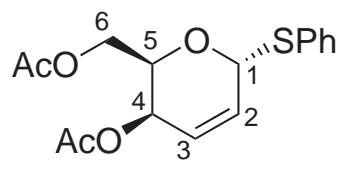

$\mathrm{R}_{f} 0.35$ (5:1 hexanes:ethyl acetate); m.p. $87-90{ }^{\circ} \mathrm{C}$ (lit. ${ }^{123} \mathrm{~m} . \mathrm{p}$. 92-93 ${ }^{\circ} \mathrm{C}$ ); ${ }^{1} \mathrm{H}-\mathrm{NMR}:\left(\mathrm{CDCl}_{3}\right) \delta_{\mathrm{H}} 7.59-7.55$ (complex m, 2H, $\mathrm{Ph}$ ), 7.34-7.28 (complex m, 3H, Ph), 6.23 (dd, $J=10.0,3.4$ $\mathrm{Hz}, 1 \mathrm{H}, \mathrm{H}-2), 6.11$ (ddd, $J=9.9,5.5,1.7 \mathrm{~Hz}, 1 \mathrm{H}, \mathrm{H}-3), 5.85$ (dd, $J=3.1,1.6 \mathrm{~Hz}$, 1H, H-1), 5.13 (dd, $J=5.4,2.4 \mathrm{~Hz}, 1 \mathrm{H}, \mathrm{H}-4), 4.71$ (td, $J=6.0,2.4 \mathrm{~Hz}, 1 \mathrm{H}, \mathrm{H}-5)$, 4.28-4.26 (complex m, 2H, H-6a,b), 2.02 (s, 3H, Ac), 1.97 (s, 3H, Ac); ${ }^{13} \mathrm{C}-\mathrm{NMR}$ : $\left(\mathrm{CDCl}_{3}\right) \delta_{\mathrm{C}} 170.6\left(\mathrm{C}, \mathrm{CH}_{3} \mathrm{CO}\right), 170.3\left(\mathrm{C}, \mathrm{CH}_{3} \mathrm{CO}\right), 134.6(\mathrm{C}, \mathrm{Ph}), 131.8(\mathrm{CH}, \mathrm{Ph})$, 131.3 (CH, C-2), 128.9 (CH, Ph), 127.6 (CH, Ph), 124.5 (CH, C-3), 83.4 (CH, C-1), $67.2(\mathrm{CH}, \mathrm{C}-5), 63.3$ (CH, C-4), $62.7\left(\mathrm{CH}_{2}, \mathrm{C}-6\right), 20.8\left(\mathrm{CH}_{3}, \mathrm{Ac}\right), 20.7\left(\mathrm{CH}_{3}, \mathrm{Ac}\right)$; IR (KBr): $\nu_{\max }$ 3061, 3004, 2964, 2934, 2902, 1739, 1370, 1265, 1241, 1080, 1053, $1020,817,789,690 \mathrm{~cm}^{-1}$.

\section{Phenyl 2,3-dideoxy-1-thio- $\alpha$-D-threo-hex-2-enopyranoside (300)}

Diacetate 289 (331 mg, $1.03 \mathrm{mmol}$ ) was dissolved in methanol (20 mL) and treated with a small piece of sodium metal $(4 \mathrm{mg}, 0.2 \mathrm{mmol})$. The mixture was stirred for 45 minutes, then neutralised with Amberlite ${ }^{\circledR}$ IR 120 resin $\left(\mathrm{H}^{+}\right.$form $)(0.7 \mathrm{~g})$. The solution was concentrated to afford diol $\mathbf{3 0 0}$ (242 mg, 99\% yield) as a white solid, which was used without further purification.

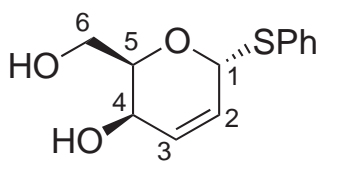

$\mathrm{R}_{f} 0.2$ (2:1 hexanes:ethyl acetate); m.p. $112-114^{\circ} \mathrm{C}$ (lit. ${ }^{158} \mathrm{~m} . \mathrm{p}$. $\left.108-110{ }^{\circ} \mathrm{C}\right) ;[\alpha]_{\mathrm{D}}^{20}+138\left(\right.$ c $\left.0.7 \mathrm{CH}_{2} \mathrm{Cl}_{2}\right) ;{ }^{1} \mathrm{H}-\mathrm{NMR}:\left(\mathrm{CDCl}_{3}\right) \delta_{\mathrm{H}}$ 7.56-7.54 (complex m, 2H, Ph), 7.35-7.29 (complex m, 3H, Ph), 6.16-6.14 (complex m, 2H, H-2,3), 5.85 (d, $J=2.2 \mathrm{~Hz}, 1 \mathrm{H}, \mathrm{H}-1$ ), 4.41 (ddd, $J=$ 6.4, 4.5, $2.2 \mathrm{~Hz}, 1 \mathrm{H}, \mathrm{H}-5$ ), 4.03 (ddd, $J=9.0,4.6,2.4 \mathrm{~Hz}, 1 \mathrm{H}, \mathrm{H}-4), 3.99$ (m, 1H, H-6a), 3.91 (ddd, $J=12.0,8.0,4.7 \mathrm{~Hz}, 1 \mathrm{H}, \mathrm{H}-6 \mathrm{~b}$ ), 2.00 (dd, $J=8.0,4.9 \mathrm{~Hz}, 1 \mathrm{H}$, $\mathrm{OH}), 1.90(\mathrm{~d}, J=9.3 \mathrm{~Hz}, 1 \mathrm{H}, \mathrm{OH}) ;{ }^{13} \mathrm{C}-\mathrm{NMR}:\left(\mathrm{CDCl}_{3}\right) \delta_{\mathrm{C}} 134.6(\mathrm{C}, \mathrm{Ph}), 132.0$ (CH, Ph), 129.5 (CH, C-2), 129.1 (CH, Ph), 128.5 (CH, C-3), 127.7 (CH, Ph), 83.9 (CH, C-1), 70.9 (CH, C-5), 63.1 (CH, C-4), $62.8\left(\mathrm{CH}_{2}, \mathrm{C}-6\right)$; IR (KBr): $\nu_{\max } 3366$, 2969, 2931, 2893, 1582, 1479, 1437, 1331, 1078, 1037, 972, 865, 792, 736, $688 \mathrm{~cm}^{-1}$.

\section{Phenyl 2,3-dideoxy-4,6- $O$-((S)-p-methoxyphenylmethylene)-1-thio- $\alpha$-D- threo-hex-2-enopyranoside (290)}

A magnetically stirred solution of diol $\mathbf{3 0 0}(97 \mathrm{mg}, 0.41 \mathrm{mmol})$ in DMF $(3.8 \mathrm{~mL})$ was treated with anisaldehyde dimethyl acetal (86 $\mathrm{mg}, 0.47 \mathrm{mmol}$ ) and $(1 S)-(+)$ 10 -camphorsulfonic acid $\left(0.4 \mathrm{~mL}\right.$ of a $0.01 \mathrm{molL}^{-1}$ solution in DMF, $\left.4 \mu \mathrm{mol}\right)$. The solution was heated to $50{ }^{\circ} \mathrm{C}$ and allowed to proceed for 22 hours. The reaction was quenched with triethylamine (1 drop) and concentrated under reduced pressure. The mixture was then separated between water $(50 \mathrm{~mL})$ and dichloromethane $(50 \mathrm{~mL})$. The aqueous layer was extracted further with dichloromethane $(2 \times 50 \mathrm{~mL})$ and the 
organic fractions were combined and washed with brine $(50 \mathrm{~mL})$. The organic phase was dried, filtered and concentrated to afford the crude product as a white solid. Recrystallisation from ethanol provided 290 (98 $\mathrm{mg}, 67 \%$ yield) as white crystalline needles.

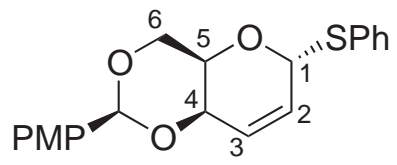

$\mathrm{R}_{f} 0.4$ (3:1 hexanes:ethyl acetate); m.p. $174-176{ }^{\circ} \mathrm{C} ;[\alpha]_{\mathrm{D}}^{20}$ $-260\left(\right.$ c $\left.0.3 \mathrm{CH}_{2} \mathrm{Cl}_{2}\right) ;{ }^{1} \mathrm{H}-\mathrm{NMR}:\left(\mathrm{CDCl}_{3}\right) \delta_{\mathrm{H}} 7.52(\mathrm{~d}, J=$ $8.1 \mathrm{~Hz}, 2 \mathrm{H}, \mathrm{Ph}), 7.42$ (d, J = 8.5 Hz, 2H, PMP), 7.28 (apparent t, $J=7.4 \mathrm{~Hz}, 2 \mathrm{H}, \mathrm{Ph}), 7.22(\mathrm{~m}, 1 \mathrm{H}, \mathrm{Ph}), 6.84$ (d, $J=8.8 \mathrm{~Hz}, 2 \mathrm{H}, \mathrm{PMP})$, $6.20(\mathrm{dd}, J=10.0,3.4 \mathrm{~Hz}, 1 \mathrm{H}, \mathrm{H}-2), 6.07$ (dd, $J=9.8,5.4 \mathrm{~Hz}, 1 \mathrm{H}, \mathrm{H}-3), 6.00$ (s, 1H, H-1), 5.55 (s, 1H, PMPCH), 4.34 (d, $J=12.9 \mathrm{~Hz}, 1 \mathrm{H}, \mathrm{H}-6 \mathrm{a}), 4.26$ (dd, $J=$ 12.9, $1.8 \mathrm{~Hz}, 1 \mathrm{H}, \mathrm{H}-6 \mathrm{~b}), 4.24$ (m, 1H, H-4), 4.16 (m, 1H, H-5), 3.76 (s, 3H, Me); ${ }^{13} \mathrm{C}-\mathrm{NMR}:\left(\mathrm{CDCl}_{3}\right) \delta_{\mathrm{C}} 160.0$ (C, PMP), 135.4 (C, Ph), 130.9 (C-2), 130.7 (CH, Ph), 130.3 (C, PMP), 129.0 (CH, Ph), 127.5 (CH, PMP), 127.1 (CH, Ph), 125.2 (C-3), 113.5 (CH, PMP), 100.7 (CH, PMPC), 84.1 (CH, C-1), $69.9\left(\mathrm{CH}_{2}, \mathrm{C}-6\right), 67.8$ (CH, C-4), 62.5 (CH, C-5), $55.3\left(\mathrm{CH}_{3}, \mathrm{Me}\right)$; IR (KBr): $\nu_{\max } 3045,3001,2981,2859,2840$, 1614, 1517, 1312, 1250, 1134, 1073, 1060, 1031, 983, 827, 789, $689 \mathrm{~cm}^{-1}$; HRMS: $m / z$ $\mathrm{C}_{20} \mathrm{H}_{20} \mathrm{O}_{4} \mathrm{SNa}^{+}[\mathrm{M}+\mathrm{Na}]^{+}$calcd 379.0980, found 379.0984.

\section{1-( $p$-Methoxyphenyl)-pentan-1-ol (301) and phenyl $(2 E, 4 E)$-6-hydroxy- thiohexadienoate $(302)$}

A solution of thioglycoside $290(201 \mathrm{mg}, 0.564 \mathrm{mmol})$ in THF $(5.6 \mathrm{~mL})$ was cooled to $-78{ }^{\circ} \mathrm{C}$, then treated with $n$-butyllithium $(0.5 \mathrm{~mL}, 0.8 \mathrm{mmol})$ and stirred for one hour.The reaction was quenched through addition of water $(1 \mathrm{~mL})$, then was diluted further with water $(20 \mathrm{~mL})$ and extracted with dichloromethane $(3 \times 20 \mathrm{~mL})$. The organic fractions were dried, filtered and concentrated to provide a white solid. Purification by flash chromatography (9:1 to 3:1 hexanes:ethyl acetate) provided starting material 290 (73 mg, 36\%), in addition to alcohol 301 (55 mg, 50\%) and diene 302 (31 mg, 25\%), both isolated as yellow oils.

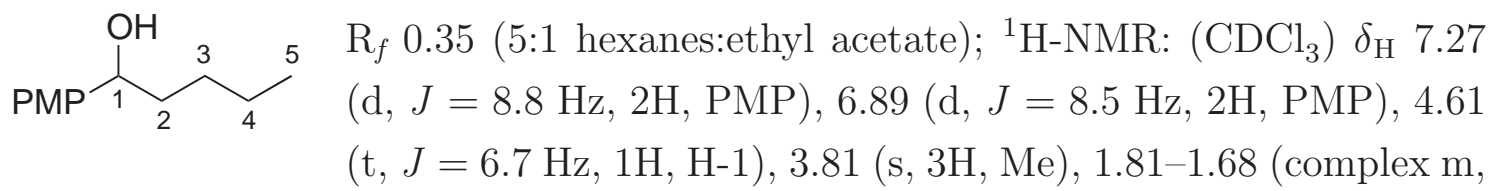
$3 \mathrm{H}), 1.37-1.23$ (complex m, 4H), 0.89 (t, $J=7.1 \mathrm{~Hz}, 3 \mathrm{H}, \mathrm{H}-5) ;{ }^{13} \mathrm{C}-\mathrm{NMR}:\left(\mathrm{CDCl}_{3}\right)$ $\delta_{\mathrm{C}} 128.2(\mathrm{CH}, \mathrm{PMP}), 127.2$ (CH, PMP), 113.6 (CH, PMP), 77.6 (CH, C-1), 55.2 $\left(\mathrm{CH}_{3}, \mathrm{Me}\right), 38.2\left(\mathrm{CH}_{2}\right), 28.0\left(\mathrm{CH}_{2}\right), 22.6\left(\mathrm{CH}_{2}\right), 14.0\left(\mathrm{CH}_{3}, \mathrm{C}-5\right)$.

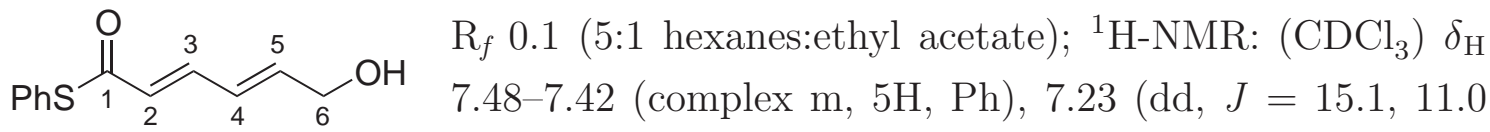
$\mathrm{Hz}, 1 \mathrm{H}, \mathrm{H}-3), 6.44$ (dd, $J=15.3,10.9 \mathrm{~Hz}, 1 \mathrm{H}, \mathrm{H}-4), 6.34$ (dt, $J=15.3,4.6 \mathrm{~Hz}, 1 \mathrm{H}, \mathrm{H}-5), 6.26$ (d, $J=15.1 \mathrm{~Hz}, 1 \mathrm{H}, \mathrm{H}-2), 4.34$ (d, $J=4.6 \mathrm{~Hz}$, 2H, H-6a,b); ${ }^{13} \mathrm{C}-\mathrm{NMR}:\left(\mathrm{CDCl}_{3}\right) \delta_{\mathrm{C}} 188.1$ (C, C-1), 143.1 (CH, C-5), $140.6(\mathrm{CH}$, 
C-3), 134.6 (CH, Ph), 129.4 (CH, Ph), 129.2 (CH, Ph), 127.6 (C, Ph), 127.4 (CH, C-4), 127.3 ( $\mathrm{CH}, \mathrm{C}-2), 62.7\left(\mathrm{CH}_{2}, \mathrm{C}-6\right)$.

\section{4,6-O-(( $S)$-p-methoxyphenylmethylene)-D-galactal (303)}

A solution of D-galactal (186) (298 $\mathrm{mg}, 2.04 \mathrm{mmol})$ in acetonitrile (10 mL) was treated with anisaldehyde dimethyl acetal $(500 \mu \mathrm{L}, 2.9 \mathrm{mmol})$ and PPTS $(27 \mathrm{mg}$, $0.11 \mathrm{mmol}$ ), then the reaction was allowed to proceed at room temperature for four hours. The mixture was then diluted with water $(20 \mathrm{~mL})$, extracted with dichloromethane $(3 \times 40 \mathrm{~mL})$, then the organic fractions were combined, dried, filtered and concentrated. The oil obtained was then subjected to flash chromatography (5:1 hexanes:ethyl acetate) to provide glycal 303 (169 mg, 31\% yield) as a colourless oil.

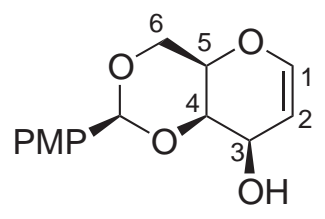

$\mathrm{R}_{f} 0.3$ (2:1 hexanes:ethyl acetate); $[\alpha]_{\mathrm{D}}^{18}-22\left(\right.$ c $\left.0.05 \mathrm{CHCl}_{3}\right)$; ${ }^{1} \mathrm{H}-\mathrm{NMR}:\left(\mathrm{CDCl}_{3}\right) \delta_{\mathrm{H}} 7.44(\mathrm{~d}, J=8.5 \mathrm{~Hz}, 2 \mathrm{H}, \mathrm{PMP}), 6.90(\mathrm{~d}$, $J=8.8 \mathrm{~Hz}, 2 \mathrm{H}, \mathrm{PMP}), 6.42(\mathrm{dd}, J=6.3,2.0 \mathrm{~Hz}, 1 \mathrm{H}, \mathrm{H}-1)$,

$5.63(\mathrm{~s}, 1 \mathrm{H}, \mathrm{PMPH}), 4.74(\mathrm{dt}, J=6.3,1.7 \mathrm{~Hz}, 1 \mathrm{H}, \mathrm{H}-2), 4.49$ (dddd, $J=11.7,5.1,2.6,0.9 \mathrm{~Hz}, 1 \mathrm{H}, \mathrm{H}-3), 4.35$ (dd, $J=12.5,1.8 \mathrm{~Hz}, 1 \mathrm{H}, \mathrm{H}-6 \mathrm{a})$, 4.21 (d, $J=5.2 \mathrm{~Hz}, 1 \mathrm{H}, \mathrm{H}-4), 4.03$ (dd, $J=12.7,5.2 \mathrm{~Hz}, 1 \mathrm{H}, \mathrm{H}-6 \mathrm{~b}), 3.91$ (m, 1H, H-5), 3.81 (s, 3H, Me), 2.38 (d, $J=11.7 \mathrm{~Hz}, 1 \mathrm{H}, \mathrm{OH}) ;{ }^{13} \mathrm{C}-\mathrm{NMR}:\left(\mathrm{CDCl}_{3}\right) \delta_{\mathrm{C}} 160.2$ (C, PMP), 143.8 (CH, C-1), 129.8 (C, PMP), 127.6 (CH, PMP), 113.6 (CH, PMP), 102.1 (CH, C-2), 101.3 (CH, PMPC), 72.3 (CH, C-4), $69.4\left(\mathrm{CH}_{2}, \mathrm{C}-6\right), 68.0(\mathrm{CH}$, C-5), 62.9 (CH, C-3), $55.3\left(\mathrm{CH}_{3}, \mathrm{Me}\right)$; IR (KBr): $\nu_{\max } 3417,2951,2912,2835,1709$, $1605,1518,1251,1169,1029,830 \mathrm{~cm}^{-1}$.

\section{3-O-methoxycarbonyl-4,6-O-((S)-p-methoxyphenylmethylene)-D- galactal (304)}

A A solution of glycal 303 (35 mg, $0.13 \mathrm{mmol})$ in DMF (2 mL) was treated with sodium hydride (9 mg, $0.23 \mathrm{mmol}$ ) at room temperature, then stirred for $30 \mathrm{~min}$ utes. The reaction mixture was then treated with methyl chloroformate $(25 \mu \mathrm{L}$, $0.32 \mathrm{mmol}$ ) and reacted at room temperature overnight. The mixture was then diluted with water $(20 \mathrm{~mL})$, extracted with dichloromethane $(3 \times 20 \mathrm{~mL})$, then the organic fractions were combined, dried, filtered and concentrated. The liquid obtained was then subjected to flash chromatography (2:1 hexanes:ethyl acetate) to provide carbonate 304 ( $8 \mathrm{mg}, 19 \%$ yield) as a colourless oil.

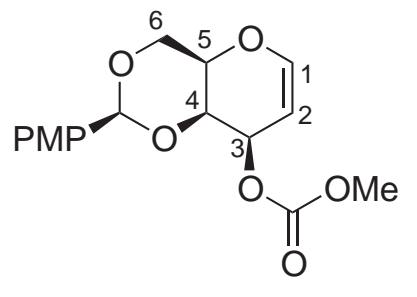

$\mathrm{R}_{f} 0.5$ (1:1 hexanes:ethyl acetate); $[\alpha]_{\mathrm{D}}^{18}+72\left(c 0.1 \mathrm{CHCl}_{3}\right)$; ${ }^{1} \mathrm{H}-\mathrm{NMR}:\left(\mathrm{CDCl}_{3}\right) \delta_{\mathrm{H}} 7.42(\mathrm{~d}, J=8.4 \mathrm{~Hz}, 2 \mathrm{H}, \mathrm{PMP}), 6.87$ (d, $J=8.5 \mathrm{~Hz}, 2 \mathrm{H}, \mathrm{PMP}), 6.52(\mathrm{~d}, J=6.1 \mathrm{~Hz}, 1 \mathrm{H}, \mathrm{H}-1)$, $5.59(\mathrm{~s}, 1 \mathrm{H}, \mathrm{PMPH}), 5.38(\mathrm{~m}, 1 \mathrm{H}, \mathrm{H}-3), 4.75(\mathrm{~d}, J=6.3$ $\mathrm{Hz}, 1 \mathrm{H}, \mathrm{H}-2), 4.53(\mathrm{~d}, J=4.9 \mathrm{~Hz}, 1 \mathrm{H}, \mathrm{H}-4), 4.36$ (d, $J=$ 
$12.0 \mathrm{~Hz}, 1 \mathrm{H}, \mathrm{H}-6 \mathrm{a}), 4.05$ (d, $J=12.4 \mathrm{~Hz}, 1 \mathrm{H}, \mathrm{H}-6 \mathrm{~b}), 3.95$ (m, 1H, H-5), 3.79 (s, 6H, $2 \times \mathrm{Me}) ;{ }^{13} \mathrm{C}-\mathrm{NMR}:\left(\mathrm{CDCl}_{3}\right) \delta_{\mathrm{C}} 160.0$ (C, PMP), 155.3 (C, $\left.\mathrm{CH}_{3} \mathrm{OCO}\right), 145.5(\mathrm{CH}$, C-1), 130.0 (C, PMP), 127.5 (CH, PMP), 113.5 (CH, PMP), 100.9 (CH, PMPC), 96.9 (CH, C-2), 69.5 (CH, C-4), 69.1 ( $\left.\mathrm{CH}_{2}, \mathrm{C}-6\right), 69.0$ (CH, C-3), 67.8 (CH, C-5), 55.3 and $54.9\left(\mathrm{CH}_{3}, \mathrm{Me}\right)$; IR (KBr): $\nu_{\max } 2995,2956,2912,2846,1745,1616,1519$, $1443,1369,1268,1235,1172,1099,998,830,794 \mathrm{~cm}^{-1}$.

B A solution of protected glycal $304(5 \mathrm{mg}, 16 \mu \mathrm{mol})$ in dichloromethane (1 $\mathrm{mL}$ ) was treated with $p$-methoxybenzyl alcohol (14 $\mathrm{mg}, 0.1 \mathrm{mmol}$ ) and palladiumtetrakis(triphenylphosphine) $(2 \mathrm{mg}, 2 \mu \mathrm{mol})$, then stirred at room temperature for one day, followed by heating at reflux for one further day. The reaction mixture was diluted with water $(10 \mathrm{~mL})$, then extracted with dichloromethane $(2 \times 10 \mathrm{~mL})$. The organic fractions were combined, dried and concentrated to provide a pale-yellow oil. Proton NMR analysis of this crude mixture showed the presence of a significant amount of starting material; no peaks corresponding to 2,3-unsaturated sugars were observed.

\section{Tri- $O$-acetyl-D-gulal (305) and acetyl 4,6-di- $O$-acetyl-2,3-dideoxy- $\beta$-D- threo-hex-2-enopyranoside (306)}

Tri- $O$-acetyl-D-galactal (180) $(231 \mathrm{mg}, 0.85 \mathrm{mmol})$ in acetic anhydride $(3 \mathrm{~mL})$ was treated with ammonium heptamolybdate tetrahydrate (209 $\mathrm{mg}, 0.17 \mathrm{mmol})$, then stirred at $110{ }^{\circ} \mathrm{C}$ for 30 minutes. The reaction mixture was diluted with water $(10 \mathrm{~mL})$, extracted twice with diethyl ether $(2 \times 10 \mathrm{~mL})$, then concentrated to provide a yellow oil. Chromatographic purification of this material (5:1 hexanes:ethyl acetate), provided glycal 305 (18 mg, 8\%) and 2,3-unsaturated sugar 306 (22 mg, $10 \%$ ) as colourless oils, in addition to mixtures of more polar sugars.

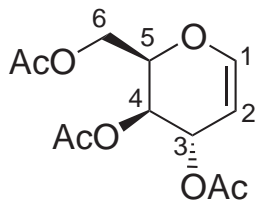

305: The spectral data matched that which was previously reported. ${ }^{157} \mathrm{R}_{f} 0.2$ (5:1 hexanes:ethyl acetate); ${ }^{1} \mathrm{H}-\mathrm{NMR}:\left(\mathrm{CDCl}_{3}\right) \delta_{\mathrm{H}}$ $6.63(\mathrm{~d}, J=6.1 \mathrm{~Hz}, 1 \mathrm{H}, \mathrm{H}-1), 5.04-5.02$ (complex m, 2H, H-2,4), 4.91 (dd, $J=4.8,2.4 \mathrm{~Hz}, 1 \mathrm{H}, \mathrm{H}-3$ ), 4.28-4.21 (complex m, 3H, H-5,6a,b), 2.11 (s, 3H, Ac), 2.10 (s, 3H, Ac), 2.07 (s, 3H, Ac); ${ }^{13} \mathrm{C}-\mathrm{NMR}:\left(\mathrm{CDCl}_{3}\right)$ $\delta_{\mathrm{C}} 170.6\left(\mathrm{C}, \mathrm{CH}_{3} \mathrm{CO}\right), 169.7\left(\mathrm{C}, \mathrm{CH}_{3} \mathrm{CO}\right), 169.6\left(\mathrm{C}, \mathrm{CH}_{3} \mathrm{CO}\right), 147.6(\mathrm{CH}, \mathrm{C}-1), 97.5$ (CH, C-2), 70.6 (CH, C-5), $66.3(\mathrm{CH}, \mathrm{C}-4), 63.4(\mathrm{CH}, \mathrm{C}-3), 62.5\left(\mathrm{CH}_{2}, \mathrm{C}-6\right), 21.1$ $\left(\mathrm{CH}_{3}, \mathrm{Ac}\right), 20.8\left(\mathrm{CH}_{3}, \mathrm{Ac}\right), 20.7\left(\mathrm{CH}_{3}, \mathrm{Ac}\right)$.

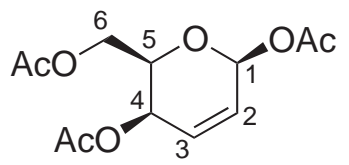

306: $\mathrm{R}_{f} 0.15$ (5:1 hexanes:ethyl acetate); ${ }^{1} \mathrm{H}-\mathrm{NMR}:\left(\mathrm{CDCl}_{3}\right)$ $\delta_{\mathrm{H}} 6.36(\mathrm{~d}, J=3.0 \mathrm{~Hz}, 1 \mathrm{H}, \mathrm{H}-1), 6.24(\mathrm{dd}, J=10.0,5.3 \mathrm{~Hz}$, $1 \mathrm{H}, \mathrm{H}-3), 6.04$ (dd, $J=10.0,3.2 \mathrm{~Hz}, 1 \mathrm{H}, \mathrm{H}-2), 5.06$ (dd, $J=$ 5.6, 3.4 Hz, 1 H, H-4), 4.34 (m, 1H, H-5), 4.26-4.18 (complex m, 2H, H-6a,b), 2.08 (s, 3H, Ac), 2.07 (s, 3H, Ac), $2.06(\mathrm{~s}, 3 \mathrm{H}, \mathrm{Ac}) ;{ }^{13} \mathrm{C}-\mathrm{NMR}:\left(\mathrm{CDCl}_{3}\right) \delta_{\mathrm{C}} 170.6(\mathrm{C}$, 
$\mathrm{CH}_{3} \mathrm{CO}$ ), 170.3 (C, $\left.\mathrm{CH}_{3} \mathrm{CO}\right), 169.7$ (C, $\left.\mathrm{CH}_{3} \mathrm{CO}\right), 128.7$ (CH, C-2), 126.3 (CH, C-3), 87.9 (CH, C-1), 68.8 (CH, C-5), $62.3\left(\mathrm{CH}_{2}, \mathrm{C}-6\right), 62.0$ (CH, C-4), $21.1\left(\mathrm{CH}_{3}, \mathrm{Ac}\right)$, $20.75\left(\mathrm{CH}_{3}, \mathrm{Ac}\right), 20.73\left(\mathrm{CH}_{3}, \mathrm{Ac}\right)$.

\section{4,6-Di- $O$-acetyl-2,3-dideoxy-D-threo-hex-2-enopyranoside (307)}

A solution of tri- $O$-acetyl-D-galactal $(\mathbf{1 8 0})(116 \mathrm{mg}, 0.426 \mathrm{mmol})$ in water $(2.7 \mathrm{~mL})$ was stirred at reflux for 100 minutes. The reaction mixture was concentrated, then co-evaporated with methanol $(2 \times 5 \mathrm{~mL})$ to provide an orange oil. Chromatographic purification of this material (3:1 hexanes:ethyl acetate), provided starting material 180 (16 mg, 16\%) and 2,3-unsaturated sugar 307 (35 $\mathrm{mg}, 36 \%$ ), isolated as a colourless oil, in addition to mixtures of unidentified aldehydes.

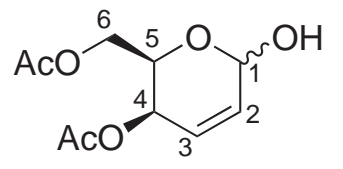

$\mathrm{R}_{f} 0.1$ (3:1 hexanes:ethyl acetate); ${ }^{1} \mathrm{H}-\mathrm{NMR}:\left(\mathrm{CDCl}_{3}\right) \delta_{\mathrm{H}} 6.15$ $(\mathrm{dd}, J=10.0,5.4 \mathrm{~Hz}, 1 \mathrm{H}, \mathrm{H}-3), 6.08$ (dd, $J=10.0,2.9 \mathrm{~Hz}, 1 \mathrm{H}$, H-2), 5.51 (apparent t, $J=3.7 \mathrm{~Hz}, 1 \mathrm{H}, \mathrm{H}-1$ ), 5.03 (dd, $J=5.4$, $2.7 \mathrm{~Hz}, 1 \mathrm{H}, \mathrm{H}-4), 4.46$ (ddd, $J=7.5,5.2,2.3 \mathrm{~Hz}, 1 \mathrm{H}, \mathrm{H}-5), 4.27$ (dd, $J=11.5,5.1$ Hz, 1H, H-6a), 4.19 (dd, $J=11.5,7.3 \mathrm{~Hz}, 1 \mathrm{H}, \mathrm{H}-6 \mathrm{~b}$ ), 2.99 (d, $J=4.6 \mathrm{~Hz}, \mathrm{OH}), 2.08$ (s, 6H, Ac); ${ }^{13} \mathrm{C}-\mathrm{NMR}:\left(\mathrm{CDCl}_{3}\right) \delta_{\mathrm{C}} 170.8$ (C, $\left.\mathrm{CH}_{3} \mathrm{CO}\right), 170.4$ (C, $\left.\mathrm{CH}_{3} \mathrm{CO}\right), 131.1$ (CH, C-2), 125.2 (CH, C-3), 88.6 (CH, C-1), 66.6 (CH, C-5), $62.9\left(\mathrm{CH}_{2}, \mathrm{C}-6\right), 62.2$ $(\mathrm{CH}, \mathrm{C}-4), 21.0\left(\mathrm{CH}_{3}, \mathrm{Ac}\right), 20.7\left(\mathrm{CH}_{3}, \mathrm{Ac}\right)$.

\section{Di- $O$ - $p$-methoxybenzyl-D-xylal (314)}

A solution of di- $O$-acetyl-D-xylal (181) $(505 \mathrm{mg}, 2.52 \mathrm{mmol})$ in methanol $(10 \mathrm{~mL})$ was treated with metallic sodium (4 mg, $0.17 \mathrm{mmol}$ ) and stirred for one hour at room temperature. The mixture was treated with a piece of dry ice (ca. $5 \mathrm{~g}$ ) and stirred for 15 minutes, then concentrated to liberate crude D-xylal as a colourless oil (298 $\mathrm{mg})$. The oil was dissolved in DMF $(25 \mathrm{~mL})$, cooled to $0{ }^{\circ} \mathrm{C}$, then treated with sodium hydride $(266 \mathrm{mg}, 6.65 \mathrm{mmol})$. The solution was stirred at $0{ }^{\circ} \mathrm{C}$ for 30 minutes, then treated with $p$-methoxybenzyl chloride $(0.75 \mathrm{~mL}, 5.5 \mathrm{mmol})$ and allowed to warm to room temperature. The mixture was stirred overnight, then quenched with water $(20 \mathrm{~mL})$ and sat. copper sulfate solution $(20 \mathrm{~mL})$, then the product was extracted with diethyl ether $(3 \times 50 \mathrm{~mL})$. The organic fractions were combined, washed with brine $(50 \mathrm{~mL})$, dried, filtered and concentrated to provide crude di$O$-p-methoxybenzyl-D-xylal (314). Purification by column chromatography (3:1 hexanes:ethyl acetate) afforded $\mathbf{3 1 4}(360 \mathrm{mg}, 40 \%)$ as a pale-yellow oil.

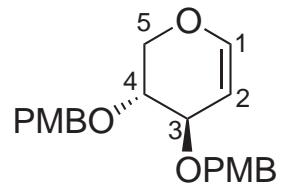

$\mathrm{R}_{f} 0.4$ (3:1 hexanes:ethyl acetate); $[\alpha]_{\mathrm{D}}^{18}-101\left(\right.$ c $\left.0.5 \mathrm{CHCl}_{3}\right)$;

${ }^{1} \mathrm{H}-\mathrm{NMR}:\left(\mathrm{CDCl}_{3}\right) \delta_{\mathrm{H}} 7.26(\mathrm{~d}, J=8.5 \mathrm{~Hz}, 2 \mathrm{H}, \mathrm{PMB}), 7.23(\mathrm{~d}$, $J=8.5 \mathrm{~Hz}, 2 \mathrm{H}, \mathrm{PMB}), 6.88(\mathrm{~d}, J=8.4 \mathrm{~Hz}, 2 \mathrm{H}, \mathrm{PMB}), 6.87$ (d, $J=8.8 \mathrm{~Hz}, 2 \mathrm{H}, \mathrm{PMB}), 6.54$ (d, $J=6.1 \mathrm{~Hz}, 1 \mathrm{H}, \mathrm{H}-1), 4.91$ (ddd, 
$J=6.1,4.6,1.2 \mathrm{~Hz}, 1 \mathrm{H}, \mathrm{H}-2), 4.56\left(\mathrm{~s}, 2 \mathrm{H}, \mathrm{PMPCH}_{2}\right), 4.52(\mathrm{~d}, J=11.5 \mathrm{~Hz}, 1 \mathrm{H}$, $\left.\mathrm{PMPCH}_{2}\right), 4.45\left(\mathrm{~d}, J=11.5 \mathrm{~Hz}, 1 \mathrm{H}, \mathrm{PMPCH}_{2}\right), 4.07$ (ddd, $J=11.6,4.0,1.4 \mathrm{~Hz}$, 1H, H-5a), 3.93 (dd, $J=11.7,2.0 \mathrm{~Hz}, 1 \mathrm{H}, \mathrm{H}-5 \mathrm{~b}$ ), 3.81-3.79 (complex m, 7H, 2 x Me, H-3), 3.63 (m, 1H, H-4); ${ }^{13} \mathrm{C}-\mathrm{NMR}:\left(\mathrm{CDCl}_{3}\right) \delta_{\mathrm{C}} 159.3$ (C, PMP), 159.2 (C, PMP), 146.6 (CH, C-1), 130.5 (C, PMP), 130.0 (C, PMP), 129.4 (CH, PMP), 129.3 (CH, PMP), 113.9 (CH, PMP), 113.8 (CH, PMP), 99.1 (CH, C-2), 72.3 (CH, C-4), $70.9\left(\mathrm{CH}_{2}, \mathrm{PMPCH}_{2}\right), 69.7\left(\mathrm{CH}_{2}, \mathrm{PMPCH}_{2}\right), 68.7(\mathrm{CH}, \mathrm{C}-3), 64.0\left(\mathrm{CH}_{2}, \mathrm{C}-5\right), 55.3$ $\left(\mathrm{CH}_{3}, 2 \times \mathrm{xe}\right)$; IR (KBr): $\nu_{\max } 3082,3005,2929,2851,1713,1612,1513,1463$, 1245, 1172, 1091, 1033, 820, $668 \mathrm{~cm}^{-1}$; HRMS: $m / z \mathrm{C}_{21} \mathrm{H}_{24} \mathrm{O}_{5} \mathrm{Na}^{+}[\mathrm{M}+\mathrm{Na}]^{+}$calcd 379.1521 , found 379.1516 .

\section{1,5-Anhydro-3,4-di- $O$ - $p$-methoxybenzyl-2-deoxy-1,2- $C$ -}

(dibromomethylene)-D-gulo-pentitol (315) and 1,5-anhydro-3,4-di- $O$ - $p$ methoxybenzyl-2-deoxy-1,2-C-(dibromomethylene)-D-talo-pentitol (316)

A solution of di- $O$ - $p$-methoxybenzyl-D-xylal (314) $(90 \mathrm{mg}, 0.25 \mathrm{mmol})$ and TEBAC $(10 \mathrm{mg}, 0.05 \mathrm{mmol})$ in bromoform $(1.0 \mathrm{~mL}, 12 \mathrm{mmol})$ was treated dropwise with a solution of sodium hydroxide $(200 \mathrm{mg}, 5.0 \mathrm{mmol})$ and anhydrous potassium fluoride $(1.5 \mathrm{~g})$ in water $(1.5 \mathrm{~mL})$. The biphasic mixture was stirred at room temperature for one day, then diluted with water $(20 \mathrm{~mL})$. The mixture was extracted with diethyl ether ( $3 \times 40 \mathrm{~mL})$, the organic fractions were then combined, dried, filtered and concentrated to provide a crude mixture of cyclopropanes 315 and 316 (3.2:1 ratio), which was purified by column chromatography (5:1 hexanes:ethyl acetate) to liberate cyclopropane $\mathbf{3 1 5}$ (27 mg, 20\%) as a light-yellow oil and a mixture of cyclopropanes 315 and 316 (1:1 ratio, 13 mg, 10\%) as a yellow oil (30\% combined yield).

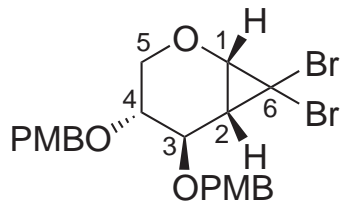

315: $\mathrm{R}_{f} 0.3$ (5:1 hexanes:ethyl acetate); ${ }^{1} \mathrm{H}-\mathrm{NMR}:\left(\mathrm{CDCl}_{3}\right) \delta_{\mathrm{H}}$ $7.35(\mathrm{~d}, J=8.3 \mathrm{~Hz}, 2 \mathrm{H}, \mathrm{PMB}), 7.22(\mathrm{~d}, J=8.1 \mathrm{~Hz}, 2 \mathrm{H}$, PMB), 6.92 (d, $J=8.5 \mathrm{~Hz}, 2 \mathrm{H}, \mathrm{PMB}), 6.86(\mathrm{~d}, J=8.3 \mathrm{~Hz}$, 2H, PMB), 4.71 (d, $\left.J=11.2 \mathrm{~Hz}, 1 \mathrm{H}, \mathrm{PMPCH}_{2}\right), 4.67$ (d, $J=$ $\left.11.5 \mathrm{~Hz}, 1 \mathrm{H}, \mathrm{PMPCH}_{2}\right), 4.63$ (d, $\left.J=11.2 \mathrm{~Hz}, 1 \mathrm{H}, \mathrm{PMPCH}_{2}\right), 4.52(\mathrm{~d}, J=11.5$ $\mathrm{Hz}, 1 \mathrm{H}, \mathrm{PMPCH}_{2}$ ), 3.88 (dd, $J=11.5,6.0 \mathrm{~Hz}, 1 \mathrm{H}, \mathrm{H}-5 \mathrm{a}$ ), 3.83-3.81 (complex m, 4H, Me, H-1), 3.80 (s, 3H, Me), 3.73 (dd, $J=11.7,5.8$ Hz, 1H, H-5b), 3.66 (m, 1H, $\mathrm{H}-4), 3.62$ (m, 1H, H-3), 1.83 (dd, $J=7.8,1.8 \mathrm{~Hz}, 1 \mathrm{H}, \mathrm{H}-2) ;{ }^{13} \mathrm{C}-\mathrm{NMR}:\left(\mathrm{CDCl}_{3}\right)$ $\delta_{\mathrm{C}} 159.4$ (C, PMP), 159.2 (C, PMP), 130.2 (C, PMP), 129.7 (CH, PMP), 129.6 (C, PMP), 129.4 (CH, PMP), 113.9 (CH, PMP), 113.8 (CH, PMP), 78.2 (CH, C-3), $74.8(\mathrm{CH}, \mathrm{C}-4), 72.5\left(\mathrm{CH}_{2}, \mathrm{PMPCH}_{2}\right), 71.6\left(\mathrm{CH}_{2}, \mathrm{PMPCH}_{2}\right), 69.3\left(\mathrm{CH}_{2}, \mathrm{C}-5\right), 60.2$ (CH, C-1), $55.29\left(\mathrm{CH}_{3}, \mathrm{Me}\right), 55.26\left(\mathrm{CH}_{3}, \mathrm{Me}\right), 34.0$ (CH, C-2), 32.7 (C, C-6). 
PMBO

316: The following data set was obtained from a mixture of $\mathbf{3 1 5}$ and $\mathbf{3 1 6}$ (2:3 ratio). $\mathrm{R}_{f} 0.25$ (5:1 hexanes:ethyl acetate); ${ }^{1} \mathrm{H}-\mathrm{NMR}:\left(\mathrm{CDCl}_{3}\right) \delta_{\mathrm{H}} 7.40(\mathrm{~d}, J=8.5 \mathrm{~Hz}, 2 \mathrm{H}, \mathrm{PMB}), 7.21$ $(\mathrm{d}, J=8.3 \mathrm{~Hz}, 2 \mathrm{H}, \mathrm{PMB}), 6.92(\mathrm{~d}, J=8.3 \mathrm{~Hz}, 2 \mathrm{H}, \mathrm{PMB})$, $6.85(\mathrm{~d}, J=8.6 \mathrm{~Hz}, 2 \mathrm{H}, \mathrm{PMB}), 4.82\left(\mathrm{~d}, J=11.5 \mathrm{~Hz}, 1 \mathrm{H}, \mathrm{PMPCH}_{2}\right), 4.69-4.66$ (complex m, 2H, $\mathrm{PMPCH}_{2}$ ), 4.51 (d, $J=10.9 \mathrm{~Hz}, 1 \mathrm{H}, \mathrm{PMPCH}_{2}$ ), 4.03 (apparent t, $J=8.0 \mathrm{~Hz}, 1 \mathrm{H}, \mathrm{H}-4), 3.99$ (d, $J=7.8 \mathrm{~Hz}, 1 \mathrm{H}, \mathrm{H}-1), 3.82$ (s, 3H, Me), 3.80-3.78 (complex m, 4H, Me,H-5a), 3.93 (m, 1H, H-3), 3.13 (apparent t, $J=11.1 \mathrm{~Hz}, 1 \mathrm{H}$, H-5b), 2.24 (apparent t, $J=8.1 \mathrm{~Hz}, 1 \mathrm{H}, \mathrm{H}-2) ;{ }^{13} \mathrm{C}-\mathrm{NMR}:\left(\mathrm{CDCl}_{3}\right) \delta_{\mathrm{C}} 159.3(\mathrm{C}$, PMP), 159.2 (C, PMP), 129.9 (C, PMP), 129.6 (CH, PMP), 129.4 (CH, PMP), 129.3 (C, PMP), 113.9 (CH, PMP), 113.8 (CH, PMP), 76.9 (CH, C-4), 75.5 (CH, C-3), $73.0\left(\mathrm{CH}_{2}, \mathrm{PMPCH}_{2}\right), 70.8\left(\mathrm{CH}_{2}, \mathrm{PMPCH}_{2}\right), 67.2\left(\mathrm{CH}_{2}, \mathrm{C}-5\right), 63.4(\mathrm{CH}$, C-1), $55.28\left(\mathrm{CH}_{3}, \mathrm{Me}\right), 55.25\left(\mathrm{CH}_{3}, \mathrm{Me}\right), 33.9$ (CH, C-2), 30.9 (C, C-6); IR (KBr): $\nu_{\max }$ 2994, 2932, 2835, 1716, 1611, 1513, 1463, 1302, 1248, 1173, 1096, 1033, 820, $769 \mathrm{~cm}^{-1}$; HRMS: $m / z \mathrm{C}_{22} \mathrm{H}_{24} \mathrm{O}_{5}^{79} \mathrm{Br}_{2} \mathrm{Na}^{+}[\mathrm{M}+\mathrm{Na}]^{+}$calcd 548.9888, found 548.9885 .

\section{1,6-Anhydro-4- $O$ - $p$-methoxybenzyl-2-bromo-2,3-dideoxy- $\alpha$-D-threo-hex- 2-enopyranose (319)}

A solution of cyclopropane (315) $(31 \mathrm{mg}, 59 \mu \mathrm{mol})$ in acetic acid $(0.6 \mathrm{~mL})$ was treated with silver acetate $(14 \mathrm{mg}, 84 \mu \mathrm{mol})$ and stirred in the dark at $100{ }^{\circ} \mathrm{C}$ for two days. Further silver acetate was added $(16 \mathrm{mg}, 96 \mu \mathrm{mol})$ and the reaction was allowed to proceed for an additional six hours. The reaction mixture was diluted with water $(20 \mathrm{~mL})$, then extracted with diethyl ether $(2 \times 20 \mathrm{~mL})$. The organic fractions were combined, washed with sat. sodium bicarbonate $(10 \mathrm{~mL})$, then washed with brine $(10 \mathrm{~mL})$, dried, filtered and concentrated to provide a yellow oil. Purification by column chromatography (5:1 hexanes:ethyl acetate) provided bicyclic acetal $\mathbf{3 1 9}$ (8 $\mathrm{mg}, 41 \%)$ as a colourless oil, in addition to $p$-methoxybenzylacetate.

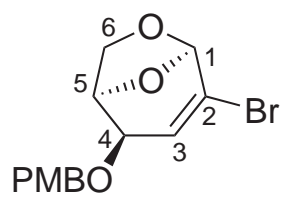

319: $\mathrm{R}_{f} 0.45$ (5:1 hexanes:ethyl acetate); ${ }^{1} \mathrm{H}-\mathrm{NMR}:\left(\mathrm{CDCl}_{3}\right) \delta_{\mathrm{H}}$ $7.23(\mathrm{~d}, J=7.8 \mathrm{~Hz}, 2 \mathrm{H}, \mathrm{PMB}), 6.89$ (d, $J=8.8 \mathrm{~Hz}, 2 \mathrm{H}, \mathrm{PMB})$, 6.06 (d, $J=1.2 \mathrm{~Hz}, 1 \mathrm{H}, \mathrm{H}-3), 5.44$ (s, 1H, H-1), 4.60 (d, $J=11.5$ $\mathrm{Hz}, 1 \mathrm{H}, \mathrm{PMPCH}_{2}$ ), 4.54 (m, 1H, H-5), 4.51 (m, 1H, H-4), 4.46 (d, $\left.J=11.5 \mathrm{~Hz}, 1 \mathrm{H}, \mathrm{PMPCH}_{2}\right), 4.26(\mathrm{~d}, J=8.1 \mathrm{~Hz}, 1 \mathrm{H}, \mathrm{H}-6 \mathrm{a}), 3.88$ (apparent $\mathrm{t}, J=$ $7.0 \mathrm{~Hz}, 1 \mathrm{H}, \mathrm{H}-6 \mathrm{~b}), 3.82$ (s, 3H, Me); ${ }^{13} \mathrm{C}-\mathrm{NMR}:\left(\mathrm{CDCl}_{3}\right) \delta_{\mathrm{C}} 159.5$ (C, PMP), 129.44 (C, PMP), 129.42 (CH, PMP), 126.8 (CH, C-3), 122.0 (C, C-2), 114.0 (CH, PMP), 101.0 (CH, C-1), 75.4 (CH, C-4), $73.0(\mathrm{CH}, \mathrm{C}-5), 71.2\left(\mathrm{CH}_{2}, \mathrm{PMPCH}_{2}\right), 63.3\left(\mathrm{CH}_{2}\right.$, C-6), $55.3\left(\mathrm{CH}_{3}, \mathrm{Me}\right)$; IR (KBr): $\nu_{\max } 2932,2857,1722,1613,1511,1463,1302$, $1249,1174,1121,1101,1033,913,896,820,727 \mathrm{~cm}^{-1}$. 


\section{Ethyltriphenylphosphonium iodide (324)}

As reported previously, ${ }^{132}$ a solution of ethyl iodide $(5.87 \mathrm{~g}, 37.6 \mathrm{mmol})$ in toluene $(50 \mathrm{~mL})$ was treated with triphenylphosphine $(10.7 \mathrm{~g}, 40.9 \mathrm{mmol})$, then stirred at $80{ }^{\circ} \mathrm{C}$ for 18 hours. The mixture was filtered, then the precipitate was washed with cold toluene $(150 \mathrm{~mL})$, then dried under vacuum to provide phosphine salt $\mathbf{3 2 4}$ as a white solid (12.2 g, $77 \%$ ).

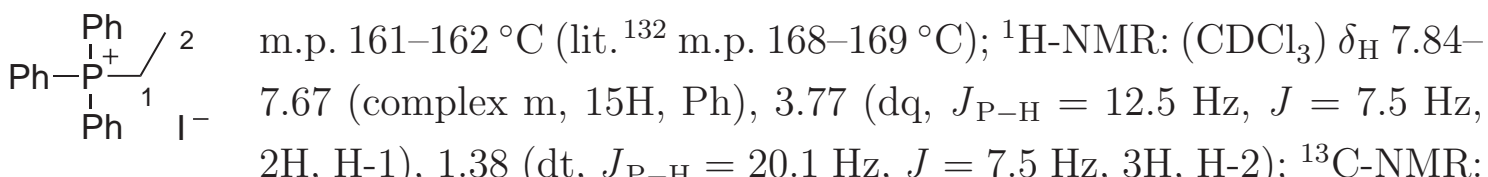
$\left(\mathrm{CDCl}_{3}\right) \delta_{\mathrm{C}} 135.1(\mathrm{CH}, \mathrm{Ph}), 133.7\left(\mathrm{~d}, J_{\mathrm{C}-\mathrm{P}}=10.0 \mathrm{~Hz}, \mathrm{CH}, \mathrm{Ph}\right), 130.6\left(\mathrm{~d}, J_{\mathrm{C}-\mathrm{P}}\right.$ $=12.5 \mathrm{~Hz}, \mathrm{CH}, \mathrm{Ph}), 117.8\left(\mathrm{~d}, J_{\mathrm{C}-\mathrm{P}}=88.3 \mathrm{~Hz}, \mathrm{C}, \mathrm{Ph}\right), 17.6\left(\mathrm{~d}, J_{\mathrm{C}-\mathrm{P}}=51.4 \mathrm{~Hz}\right.$, $\left.\mathrm{CH}_{2}, \mathrm{C}-1\right), 6.9\left(\mathrm{~d}, J_{\mathrm{C}-\mathrm{P}}=5.2 \mathrm{~Hz}, \mathrm{CH}_{3}, \mathrm{C}-2\right) ;{ }^{31} \mathrm{P}-\mathrm{NMR}:\left(\mathrm{CDCl}_{3}\right) \delta_{\mathrm{P}} 26.3(\mathrm{~s}, 1 \mathrm{P})$.

\section{3,6-Anhydro-4,5-di- $O$-benzyl-D-threo-hexanose dimethyl acetal (325)}

A solution of ethyltriphenylphosphonium iodide (186 $\mathrm{mg}, 0.445 \mathrm{mmol}$ ) in THF $(2 \mathrm{~mL})$ was cooled to $-78{ }^{\circ} \mathrm{C}$, then treated with KHMDS $(0.9 \mathrm{~mL}, 0.45 \mathrm{mmol})$ and stirred for 30 minutes. To this was added a solution of oxepines $\mathbf{2 7 1}$ and $\mathbf{2 7 2}$ (102 $\mathrm{mg}, 0.228 \mathrm{mmol}$ ) and potassium carbonate $(64 \mathrm{mg}, 0.46 \mathrm{mmol})$ in methanol $(1 \mathrm{~mL})$. The reaction mixture was immediately warmed to room temperature, then allowed to react for five hours. The mixture was then diluted with sat. ammonium chloride solution and extracted with dichloromethane $(3 \times 10 \mathrm{~mL})$. The organic fractions were combined, dried, filtered and concentrated to provide a yellow oil. Upon purification by column chromatography (2:1 hexanes:ethyl acetate), acetal $\mathbf{3 2 5}$ was obtained as a colourless oil (21 mg, 24\%).

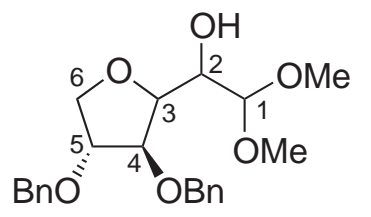

$\mathrm{R}_{f} 0.45$ (1:1 hexanes:ethyl acetate); $[\alpha]_{\mathrm{D}}^{18}+13\left(\right.$ c $\left.1.9 \mathrm{CHCl}_{3}\right)$; ${ }^{1} \mathrm{H}-\mathrm{NMR}:\left(\mathrm{CDCl}_{3}\right) \delta_{\mathrm{H}} 7.34-7.19$ (complex m, 10H, Bn), 4.544.40 (complex m, 4H, $\mathrm{PhCH}_{2}$ ), 4.31 (dd, $J=7.1,2.0 \mathrm{~Hz}, 1 \mathrm{H}$, $\mathrm{H}-1), 4.14$ (dd, $J=2.3,1.1 \mathrm{~Hz}, 1 \mathrm{H}, \mathrm{H}-4), 4.04$ (m, 1H, H-3), 4.00 (m, 1H, H-6a), 3.96 (m, 1H, H-5), 3.83 (ddd, J = 9.9, 3.7, 2.0 Hz, 1H, H-6b), $3.62(\mathrm{~m}, 1 \mathrm{H}, \mathrm{H}-2), 3.37$ (s, 6H, Me), 2.76 (d, $J=5.6 \mathrm{~Hz}, 1 \mathrm{H}, \mathrm{OH}) ;{ }^{13} \mathrm{C}-\mathrm{NMR}$ : $\left(\mathrm{CDCl}_{3}\right) \delta_{\mathrm{C}} 137.6(\mathrm{C}, \mathrm{Bn}), 137.2(\mathrm{C}, \mathrm{Bn}), 128.51$ (CH, Bn), $128.49(\mathrm{CH}, \mathrm{Bn}), 127.94$ (CH, Bn), 127.90 (CH, Bn), 127.85 (CH, Bn), 127.7 (CH, Bn), 104.7 (CH, C-1), 84.0 (CH, C-4), $83.3(\mathrm{CH}, \mathrm{C}-3), 82.3(\mathrm{CH}, \mathrm{C}-5), 72.0$ and $71.8\left(\mathrm{CH}_{2}, \mathrm{PhCH}_{2}\right.$ and $\mathrm{CH}_{2}$, C-6), 71.1 and $71.0\left(\mathrm{CH}_{2}, \mathrm{PhCH}_{2}\right.$ and $\left.\mathrm{CH}, \mathrm{C}-2\right), 55.8\left(\mathrm{CH}_{3}, \mathrm{Me}\right), 54.0\left(\mathrm{CH}_{3}, \mathrm{Me}\right)$; IR (KBr): $\nu_{\max } 3514,3027,2925,2868,1731,1496,1455,1368,1260,1206,1075$, 800, 749, $698 \mathrm{~cm}^{-1}$; HRMS: $m / z \mathrm{C}_{22} \mathrm{H}_{28} \mathrm{O}_{6} \mathrm{Na}^{+}[\mathrm{M}+\mathrm{Na}]^{+}$calcd 411.1784, found 411.1778. 


\subsection{Chapter Five Experimental}

\section{2,3:5,6-Di- $O$-isopropylidene-D-mannofuranose (333)}

A solution of D-mannose $(27.0 \mathrm{~g}, 150 \mathrm{mmol})$ in acetone $(1.35 \mathrm{~L})$ was treated with iodine $(7.70 \mathrm{~g}, 30.3 \mathrm{mmol})$, then stirred at room temperature for three hours. The solution was diluted with sat. sodium thiosulfate solution $(400 \mathrm{~mL})$ and sat. sodium bicarbonate solution ( $400 \mathrm{~mL}$ ), extracted with chloroform (3 x $400 \mathrm{~mL})$, then dried, filtered and concentrated to provide a pale-yellow solid. This crude product was dissolved in acetone $(300 \mathrm{~mL})$, diluted with hexanes $(600 \mathrm{~mL})$, then concentrated by rotary evaporation at $50{ }^{\circ} \mathrm{C}$, until crystallisation occurred. The solution was cooled and filtered to provide $\mathbf{3 3 3}$ as pale off-white crystals $(23.8 \mathrm{~g}, 61 \%)$. The mother liquor was concentrated further, until crystallisation reoccurred. The mixture was left overnight in the freezer, then filtered to provide a second crop of off-white crystals $(9.8 \mathrm{~g}, 25 \%)$. The spectral data matched that reported previously. ${ }^{34}$

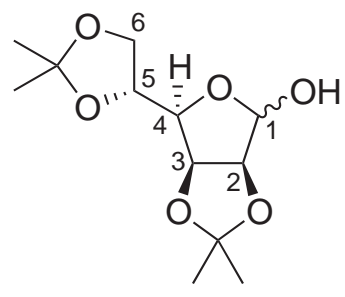

m.p. $117-119{ }^{\circ} \mathrm{C}$ (lit. ${ }^{159}$ m.p. $\left.121-122{ }^{\circ} \mathrm{C}\right) ;{ }^{1} \mathrm{H}-\mathrm{NMR}:\left(\mathrm{CDCl}_{3}\right)$ $\delta_{\mathrm{H}} 5.38(\mathrm{~d}, J=3.5 \mathrm{~Hz}, 1 \mathrm{H}, \mathrm{H}-1), 4.82(\mathrm{dd}, J=5.8,3.6 \mathrm{~Hz}$, $1 \mathrm{H}, \mathrm{H}-3), 4.62(\mathrm{~d}, J=5.8 \mathrm{~Hz}, 1 \mathrm{H}, \mathrm{H}-2), 4.40$ (dd, $J=11.3$, $6.7 \mathrm{~Hz}, 1 \mathrm{H}, \mathrm{H}-5), 4.19$ (dd, $J=7.1,3.6 \mathrm{~Hz}, 1 \mathrm{H}, \mathrm{H}-4), 4.09$ $(\mathrm{dd}, J=8.7,6.2 \mathrm{~Hz}, 1 \mathrm{H}, \mathrm{H}-6 \mathrm{a}), 4.04(\mathrm{dd}, J=8.7,4.8 \mathrm{~Hz}, 1 \mathrm{H}$, H-6b), 2.60 (s, 1H, OH), $1.464\left(\mathrm{~s}, 3 \mathrm{H},\left(\mathrm{CH}_{3}\right)_{2} \mathrm{C}\right), 1.457$ (s, 3H, $\left.\left(\mathrm{CH}_{3}\right)_{2} \mathrm{C}\right), 1.38$ (s, $\left.3 \mathrm{H},\left(\mathrm{CH}_{3}\right)_{2} \mathrm{C}\right), 1.33\left(\mathrm{~s}, 3 \mathrm{H},\left(\mathrm{CH}_{3}\right)_{2} \mathrm{C}\right) ;{ }^{13} \mathrm{C}-\mathrm{NMR}:\left(\mathrm{CDCl}_{3}\right) \delta_{\mathrm{C}} 112.6\left(\mathrm{C},\left(\mathrm{CH}_{3}\right)_{2} \mathrm{C}\right)$, $109.1\left(\mathrm{C},\left(\mathrm{CH}_{3}\right)_{2} \mathrm{C}\right), 101.3(\mathrm{CH}, \mathrm{C}-1), 85.4(\mathrm{CH}, \mathrm{C}-2), 80.3(\mathrm{CH}, \mathrm{C}-4), 79.6(\mathrm{CH}$, $\mathrm{C}-3), 73.2(\mathrm{CH}, \mathrm{C}-5), 66.6\left(\mathrm{CH}_{2}, \mathrm{C}-6\right), 26.8\left(\mathrm{CH}_{3},\left(\mathrm{CH}_{3}\right)_{2} \mathrm{C}\right), 25.8\left(\mathrm{CH}_{3},\left(\mathrm{CH}_{3}\right)_{2} \mathrm{C}\right)$, $25.1\left(\mathrm{CH}_{3},\left(\mathrm{CH}_{3}\right)_{2} \mathrm{C}\right), 24.4\left(\mathrm{CH}_{3},\left(\mathrm{CH}_{3}\right)_{2} \mathrm{C}\right)$.

\section{2,3:5,6-Di- $O$-isopropylidene- $\alpha$-D-mannofuranosyl chloride (334)}

2,3:5,6-Di- $O$-isopropylidene-D-mannofuranose (333) (10.2 g, $39.0 \mathrm{mmol}$ ) was dissolved in dichloromethane $(190 \mathrm{~mL})$, then treated sequentially with DMAP (2.86 g, $23.4 \mathrm{mmol})$, tosyl chloride $(8.81 \mathrm{~g}, 46.2 \mathrm{mmol})$ and triethylamine $(5.4 \mathrm{~mL}, 39 \mathrm{mmol})$. The mixture was stirred at room temperature for four hours, then washed sequentially with sat. copper sulfate solution $(200 \mathrm{~mL})$, sat. sodium bicarbonate solution $(200 \mathrm{~mL})$ and brine $(100 \mathrm{~mL})$. The solution obtained was dried, filtered and concentrated to provide a viscous yellow-orange oil. This material was purified by column chromatography (9:1 hexanes:ethyl acetate) to provide mannosyl chloride $\mathbf{3 3 4}$ as a colourless oil (7.76 g, 71\%). The spectral data matched that reported previously. ${ }^{34}$ 


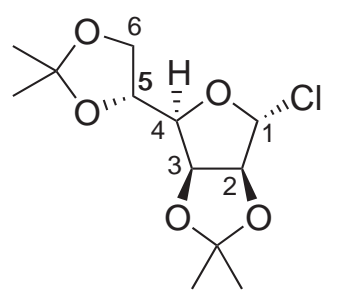

$\mathrm{R}_{f} 0.35$ (9:1 hexanes:ethyl acetate); ${ }^{1} \mathrm{H}-\mathrm{NMR}:\left(\mathrm{CDCl}_{3}\right) \delta_{\mathrm{H}} 6.07$ $(\mathrm{d}, J=1.9 \mathrm{~Hz}, 1 \mathrm{H}, \mathrm{H}-1), 4.96$ (dd, $J=5.6,2.7 \mathrm{~Hz}, 1 \mathrm{H}, \mathrm{H}-2)$, 4.89 (m, 1H, H-3), 4.44 (m, 1H, H-5), 4.21 (dd, $J=7.8,3.0 \mathrm{~Hz}$, $1 \mathrm{H}, \mathrm{H}-4), 4.10$ (ddd, $J=8.9,6.3,2.7 \mathrm{~Hz}, 1 \mathrm{H}, \mathrm{H}-6 \mathrm{a}), 4.02$ (ddd, $J=8.8,4.4,2.7 \mathrm{~Hz}, 1 \mathrm{H}, \mathrm{H}-6 \mathrm{~b}), 1.47\left(\mathrm{~s}, 6 \mathrm{H},\left(\mathrm{CH}_{3}\right)_{2} \mathrm{C}\right), 1.39(\mathrm{~s}$, $\left.3 \mathrm{H},\left(\mathrm{CH}_{3}\right)_{2} \mathrm{C}\right), 1.33\left(\mathrm{~s}, 3 \mathrm{H},\left(\mathrm{CH}_{3}\right)_{2} \mathrm{C}\right) ;{ }^{13} \mathrm{C}-\mathrm{NMR}:\left(\mathrm{CDCl}_{3}\right) \delta_{\mathrm{C}} 113.3\left(\mathrm{C},\left(\mathrm{CH}_{3}\right)_{2} \mathrm{C}\right)$, $109.5\left(\mathrm{C},\left(\mathrm{CH}_{3}\right)_{2} \mathrm{C}\right), 97.6(\mathrm{CH}, \mathrm{C}-1), 89.1(\mathrm{CH}, \mathrm{C}-2), 82.3(\mathrm{CH}, \mathrm{C}-4), 78.5(\mathrm{CH}$, $\mathrm{C}-3), 72.3(\mathrm{CH}, \mathrm{C}-5), 66.7\left(\mathrm{CH}_{2}, \mathrm{C}-6\right), 26.9\left(\mathrm{CH}_{3},\left(\mathrm{CH}_{3}\right)_{2} \mathrm{C}\right), 25.8\left(\mathrm{CH}_{3},\left(\mathrm{CH}_{3}\right)_{2} \mathrm{C}\right)$, $25.1\left(\mathrm{CH}_{3},\left(\mathrm{CH}_{3}\right)_{2} \mathrm{C}\right), 24.6\left(\mathrm{CH}_{3},\left(\mathrm{CH}_{3}\right)_{2} \mathrm{C}\right)$.

\section{1,4-Anhydro-3- $O$-benzyl-2-deoxy-5,6- $O$-isopropylidene-D-arabino-hex-1- enitol (209)}

A solution of naphthalene $(38.4 \mathrm{~g}, 299 \mathrm{mmol})$ in THF $(100 \mathrm{~mL})$ was treated with freshly cut sodium $(2.71 \mathrm{~g}, 118 \mathrm{mmol})$ and stirred at room temperature for two hours to generate the deep-green coloured sodium naphthalenide radical. The mixture was cooled to $0{ }^{\circ} \mathrm{C}$, then treated with mannosyl chloride $334(7.54 \mathrm{~g}, 27.1 \mathrm{mmol})$ as a solution in THF $(60 \mathrm{~mL})$. The reaction mixture was allowed to stir for ten minutes, then quenched with sat. ammonium chloride solution $(100 \mathrm{~mL})$, followed by dilution with water $(100 \mathrm{~mL})$. The phases were separated, then the aqueous layer was extracted with diethyl ether $(2 \times 100 \mathrm{~mL})$. The combined organic fractions were washed with brine $(100 \mathrm{~mL})$, then dried, filtered and concentrated to provide a mixture of naphthalene and furanoglycal 335. This mixture was immediately dissolved in a minimum volume of THF $(100 \mathrm{~mL})$, cooled to $0{ }^{\circ} \mathrm{C}$, then treated successively with tetrabutylammonium iodide (506 mg, $1.37 \mathrm{mmol})$, sodium hydride $(1.43 \mathrm{~g}, 35.8$ $\mathrm{mmol}$ ) and benzyl bromide $(4.0 \mathrm{~mL}, 33 \mathrm{mmol})$. The mixture was allowed to warm to room temperature, then stirred overnight. The reaction was quenched with brine $(100 \mathrm{~mL})$ and water $(100 \mathrm{~mL})$, then the mixture was extracted with diethyl ether $(3$ x $200 \mathrm{~mL})$. The combined organic fractions were washed with brine $(100 \mathrm{~mL})$, then dried, filtered and concentrated to provide a mixture of naphthalene and glycal 209. This crude product was purified by column chromatography (20:1 hexanes:ethyl acetate) to provide glycal 209 as a pale-yellow oil (5.30 g, 71\%). The spectral data matched that reported previously. ${ }^{34}$

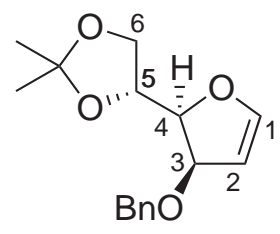

$\mathrm{R}_{f} 0.4$ (9:1 hexanes:ethyl acetate); ${ }^{1} \mathrm{H}-\mathrm{NMR}:\left(\mathrm{CDCl}_{3}\right) \delta_{\mathrm{H}} 7.36-7.27$ (complex m, 5H, Bn), 6.62 (d, $J=2.7 \mathrm{~Hz}, 1 \mathrm{H}, \mathrm{H}-1), 5.29$ (apparent t, $J=2.5 \mathrm{~Hz}, 1 \mathrm{H}, \mathrm{H}-2), 4.65$ (dd, $J=7.1,2.4 \mathrm{~Hz}, 1 \mathrm{H}, \mathrm{H}-3)$, $4.59(\mathrm{dd}, J=11.6,6.5 \mathrm{~Hz}, 1 \mathrm{H}, \mathrm{H}-5), 4.57$ (d, $J=11.4 \mathrm{~Hz}, 1 \mathrm{H}$, $\left.\mathrm{PhCH}_{2}\right), 4.51\left(\mathrm{~d}, J=11.7 \mathrm{~Hz}, 1 \mathrm{H}, \mathrm{PhCH}_{2}\right), 4.44(\mathrm{dd}, J=6.8,5.4 \mathrm{~Hz}, 1 \mathrm{H}, \mathrm{H}-4), 4.11$ (dd, $J=8.6,6.8 \mathrm{~Hz}, 1 \mathrm{H}, \mathrm{H}-6 \mathrm{a}), 3.99$ (dd, $J=8.5,6.4 \mathrm{~Hz}, 1 \mathrm{H}, \mathrm{H}-6 \mathrm{~b}), 1.48$ (s, 3H, $\left.\left(\mathrm{CH}_{3}\right)_{2} \mathrm{C}\right), 1.40\left(\mathrm{~s}, 3 \mathrm{H},\left(\mathrm{CH}_{3}\right)_{2} \mathrm{C}\right) ;{ }^{13} \mathrm{C}-\mathrm{NMR}:\left(\mathrm{CDCl}_{3}\right) \delta_{\mathrm{C}} 150.6(\mathrm{CH}, \mathrm{C}-1), 138.4(\mathrm{C}$, 
Bn), 128.4 (CH, Bn), 127.6 (CH, Bn), 127.5 (CH, Bn), $108.7\left(\mathrm{C},\left(\mathrm{CH}_{3}\right)_{2} \mathrm{C}\right), 101.9$ (CH, C-2), 84.1 (CH, C-4), $79.2(\mathrm{CH}, \mathrm{C}-3), 73.1$ (CH, C-5), $71.0\left(\mathrm{CH}_{2}, \mathrm{PhCH}_{2}\right)$, $65.9\left(\mathrm{CH}_{2}, \mathrm{C}-6\right), 26.5\left(\mathrm{CH}_{3},\left(\mathrm{CH}_{3}\right)_{2} \mathrm{C}\right), 25.2\left(\mathrm{CH}_{3},\left(\mathrm{CH}_{3}\right)_{2} \mathrm{C}\right)$.

\section{Ethyl 3- $O$-benzyl-2-deoxy-5,6- $O$-isopropylidene- $\alpha$-D-arabino- hexofuranoside (338) and ethyl 3-O-benzyl-2-deoxy-5,6- $O$ - isopropylidene- $\beta$-D-arabino-hexofuranoside (339)}

A solution of glycal 209 (171 mg, $0.62 \mathrm{mmol}$ ) in bromoform $(1 \mathrm{~mL})$ was treated with TEBAC (4 mg, $0.02 \mathrm{mmol}$ ), then a solution of sodium hydroxide (212 $\mathrm{mg}$, $5.3 \mathrm{mmol})$ and potassium fluoride $(1.56 \mathrm{~g})$ in water $(1.6 \mathrm{~mL})$ and stirred rapidly for 90 minutes. The mixture was diluted with water $(10 \mathrm{~mL})$, then extracted with diethyl ether ( $3 \times 10 \mathrm{~mL})$. The combined ethereal fractions were dried, filtered and concentrated to provide a dark-brown liquid. This crude product was purified by column chromatography (9:1 hexanes:ethyl acetate) to afford complicated mixtures of unidentified material, plus ethyl glycosides 338 (38 mg, 12\%) and 339 (46 mg, $14 \%)$, isolated as yellow oils (26\% combined yield).

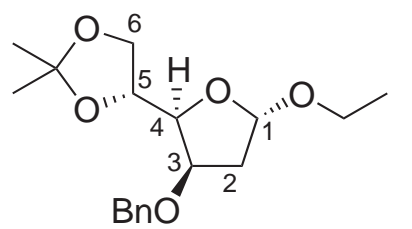

338: $\mathrm{R}_{f} 0.5$ (5:1 hexanes:ethyl acetate); $[\alpha]_{\mathrm{D}}^{22}+27$ ( c 0.9 $\left.\mathrm{CHCl}_{3}\right) ;{ }^{1} \mathrm{H}-\mathrm{NMR}:\left(\mathrm{CDCl}_{3}\right) \delta_{\mathrm{H}} 7.36-7.26$ (complex m, 5H, $\mathrm{Bn}$ ), 5.26 (dd, $J=5.4,3.4 \mathrm{~Hz}, 1 \mathrm{H}, \mathrm{H}-1), 4.57$ (d, $J=12.0$ $\left.\mathrm{Hz}, 1 \mathrm{H}, \mathrm{PhCH}_{2}\right), 4.52\left(\mathrm{~d}, J=12.0 \mathrm{~Hz}, 1 \mathrm{H}, \mathrm{PhCH}_{2}\right), 4.43$ $(\mathrm{q}, J=6.5 \mathrm{~Hz}, 1 \mathrm{H}, \mathrm{H}-4), 4.21(\mathrm{~m}, 1 \mathrm{H}, \mathrm{H}-3), 4.11$ (dd, $J=7.8,6.6 \mathrm{~Hz}, 1 \mathrm{H}, \mathrm{H}-6 \mathrm{a})$, $4.04(\mathrm{dd}, J=7.2,4.2 \mathrm{~Hz}, 1 \mathrm{H}, \mathrm{H}-5), 4.00$ (dd, $J=8.3,6.0 \mathrm{~Hz}, 1 \mathrm{H}, \mathrm{H}-6 \mathrm{~b}), 3.74$ (dq, $J=9.8,7.1 \mathrm{~Hz}, 1 \mathrm{H}, \mathrm{Et}), 3.45(\mathrm{dq}, J=9.8,7.0 \mathrm{~Hz}, 1 \mathrm{H}, \mathrm{Et}), 2.26$ (ddd, $J=$ 14.4, 5.7, 2.0 Hz, 1H, H-2a), 2.06 (ddd, $J=14.3,6.0,3.3 \mathrm{~Hz}, 1 \mathrm{H}, \mathrm{H}-2 \mathrm{~b}$ ), 1.44 (s, $\left.3 \mathrm{H},\left(\mathrm{CH}_{3}\right)_{2} \mathrm{C}\right), 1.39\left(\mathrm{~s}, 3 \mathrm{H},\left(\mathrm{CH}_{3}\right)_{2} \mathrm{C}\right), 1.18(\mathrm{td}, J=7.1,0.7 \mathrm{~Hz}, 3 \mathrm{H}, \mathrm{Et}) ;{ }^{13} \mathrm{C}-\mathrm{NMR}$ : $\left(\mathrm{CDCl}_{3}\right) \delta_{\mathrm{C}} 138.3(\mathrm{C}, \mathrm{Bn}), 128.3(\mathrm{CH}, \mathrm{Bn}), 127.5(\mathrm{CH}, \mathrm{Bn}), 127.4(\mathrm{CH}, \mathrm{Bn}), 108.8$ $\left(\mathrm{C},\left(\mathrm{CH}_{3}\right)_{2} \mathrm{C}\right), 103.2(\mathrm{CH}, \mathrm{C}-1), 80.9(\mathrm{CH}, \mathrm{C}-5), 78.3(\mathrm{CH}, \mathrm{C}-3), 73.4(\mathrm{CH}, \mathrm{C}-4)$, $71.5\left(\mathrm{CH}_{2}, \mathrm{PhCH}_{2}\right), 67.1\left(\mathrm{CH}_{2}, \mathrm{C}-6\right), 63.4\left(\mathrm{CH}_{2}, \mathrm{Et}\right), 40.0\left(\mathrm{CH}_{2}, \mathrm{C}-2\right), 26.7\left(\mathrm{CH}_{3}\right.$, $\left.\left(\mathrm{CH}_{3}\right)_{2} \mathrm{C}\right), 25.5\left(\mathrm{CH}_{3},\left(\mathrm{CH}_{3}\right)_{2} \mathrm{C}\right), 15.2\left(\mathrm{CH}_{3}, \mathrm{Et}\right)$; IR $(\mathrm{KBr}): \nu_{\max } 3030,2979,2938$, 2907, 2360, 1727, 1455, 1371, 1255, 1212, 1067, 1048, 955, 849, 737, $698 \mathrm{~cm}^{-1}$; HRMS: $m / z \mathrm{C}_{18} \mathrm{H}_{26} \mathrm{O}_{5} \mathrm{Na}^{+}[\mathrm{M}+\mathrm{Na}]^{+}$calcd 345.1678, found 345.1682.

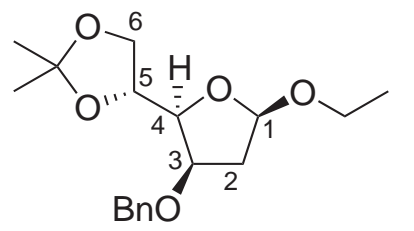

339: $\mathrm{R}_{f} 0.35$ (5:1 hexanes:ethyl acetate); $[\alpha]_{\mathrm{D}}^{22}-58$ ( c 1.5 $\left.\mathrm{CHCl}_{3}\right) ;{ }^{1} \mathrm{H}-\mathrm{NMR}:\left(\mathrm{CDCl}_{3}\right) \delta_{\mathrm{H}} 7.38-7.28$ (complex m, 5H, $\mathrm{Bn}), 5.15(\mathrm{~d}, J=5.9 \mathrm{~Hz}, 1 \mathrm{H}, \mathrm{H}-1), 4.64(\mathrm{~d}, J=12.2 \mathrm{~Hz}$, $1 \mathrm{H}, \mathrm{PhCH}_{2}$ ), 4.49 (d, $J=12.0 \mathrm{~Hz}, 1 \mathrm{H}, \mathrm{PhCH}_{2}$ ), 4.45 (q, $J=6.6 \mathrm{~Hz}, 1 \mathrm{H}, \mathrm{H}-4), 4.17$ (td, $J=5.1,0.9 \mathrm{~Hz}, 1 \mathrm{H}, \mathrm{H}-5), 4.13$ (m, 1H, H-3), 4.08 (d, $J=6.6 \mathrm{~Hz}, 2 \mathrm{H}, \mathrm{H}-6 \mathrm{a}, \mathrm{b}), 3.80$ (dq, $J=8.3,7.1 \mathrm{~Hz}, 1 \mathrm{H}, \mathrm{Et}), 3.48$ (dq, $J=$ 8.3, 7.1 Hz, 1H, Et), 2.20 (dd, $J=14.3,1.5 \mathrm{~Hz}, 1 \mathrm{H}, \mathrm{H}-2 \mathrm{a}), 2.14$ (ddd, $J=14.3$, 6.0, $1.2 \mathrm{~Hz}, 1 \mathrm{H}, \mathrm{H}-2 \mathrm{~b}), 1.45\left(\mathrm{~s}, 3 \mathrm{H},\left(\mathrm{CH}_{3}\right)_{2} \mathrm{C}\right), 1.38\left(\mathrm{~s}, 3 \mathrm{H},\left(\mathrm{CH}_{3}\right)_{2} \mathrm{C}\right), 1.23(\mathrm{td}, J=$ 
7.1, $1.2 \mathrm{~Hz}, 3 \mathrm{H}, \mathrm{Et}) ;{ }^{13} \mathrm{C}-\mathrm{NMR}:\left(\mathrm{CDCl}_{3}\right) \delta_{\mathrm{C}} 138.4$ (C, Bn), 128.5 (CH, Bn), 128.2 (CH, Bn), $127.5(\mathrm{CH}, \mathrm{Bn}), 108.4\left(\mathrm{C},\left(\mathrm{CH}_{3}\right)_{2} \mathrm{C}\right), 103.4(\mathrm{CH}, \mathrm{C}-1), 82.9$ (CH, C-5), 77.1 (CH, C-3), 74.5 (CH, C-4), $71.2\left(\mathrm{CH}_{2}, \mathrm{PhCH}_{2}\right), 66.7\left(\mathrm{CH}_{2}, \mathrm{C}-6\right), 63.5\left(\mathrm{CH}_{2}\right.$, Et), $37.9\left(\mathrm{CH}_{2}, \mathrm{C}-2\right), 26.6\left(\mathrm{CH}_{3},\left(\mathrm{CH}_{3}\right)_{2} \mathrm{C}\right), 25.4\left(\mathrm{CH}_{3},\left(\mathrm{CH}_{3}\right)_{2} \mathrm{C}\right), 15.1\left(\mathrm{CH}_{3}, \mathrm{Et}\right)$; IR (KBr): $\nu_{\max } 3027,2983,2891,1752,1454,1370,1253,1210,1113,1067,1048$, $973,852,737,698 \mathrm{~cm}^{-1}$.

\section{1,4-Anhydro-3- $O$-benzyl-2-deoxy-1,2- $C$-(dichloromethylene)-5,6- $O$ - isopropylidene-D-glycero-D-gulo-hexitol (340)}

A Glycal 209 (161 mg, $0.582 \mathrm{mmol}$ ) was dissolved in chloroform (2 mL), treated with TEBAC (7 mg, $0.03 \mathrm{mmol})$, then a solution of sodium hydroxide $(1.42 \mathrm{~g}$, $35.5 \mathrm{mmol})$ in water $(1.5 \mathrm{~mL})$ and allowed to stir at room temperature for four hours. The reaction mixture was dissolved in water $(20 \mathrm{~mL})$, then extracted with diethyl ether $(3 \times 20 \mathrm{~mL})$. The ethereal fractions were combined, dried, filtered and concentrated to afford a brown oil. This oil was purified by column chromatography (9:1 hexanes:ethyl acetate) to afford $\mathrm{gem}$-dichlorocyclopropane $\mathbf{3 4 0}$ as a pale-yellow oil (104 mg, 50\%).

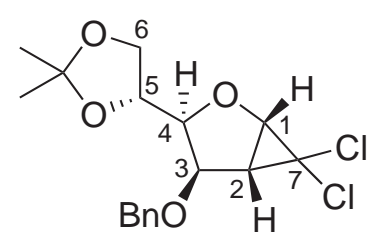

$\mathrm{R}_{f} 0.45$ (9:1 hexanes:ethyl acetate); $[\alpha]_{\mathrm{D}}^{22}+16\left(\right.$ c $\left.1.2 \mathrm{CHCl}_{3}\right)$; ${ }^{1} \mathrm{H}-\mathrm{NMR}:\left(\mathrm{CDCl}_{3}\right) \delta_{\mathrm{H}}$ 7.39-7.31 (complex m, 5H, Bn), 4.67 $\left(\mathrm{d}, J=11.8 \mathrm{~Hz}, 1 \mathrm{H}, \mathrm{PhCH}_{2}\right), 4.61(\mathrm{~d}, J=11.5 \mathrm{~Hz}, 1 \mathrm{H}$, $\left.\mathrm{PhCH}_{2}\right), 4.48$ (apparent t, $\left.J=5.9 \mathrm{~Hz}, 1 \mathrm{H}, \mathrm{H}-5\right), 4.33$ (d, $J=6.4 \mathrm{~Hz}, 1 \mathrm{H}, \mathrm{H}-3), 4.31$ (d, $J=5.9 \mathrm{~Hz}, 1 \mathrm{H}, \mathrm{H}-4), 4.27$ (dd, $J=5.9,0.8 \mathrm{~Hz}$, $1 \mathrm{H}, \mathrm{H}-1), 4.05$ (dd, $J=8.3,6.9 \mathrm{~Hz}, 1 \mathrm{H}, \mathrm{H}-6 \mathrm{a}), 3.93(\mathrm{dd}, J=7.9,6.5 \mathrm{~Hz}, 1 \mathrm{H}$, $\mathrm{H}-6 \mathrm{~b}), 2.47$ (d, $J=5.9 \mathrm{~Hz}, 1 \mathrm{H}, \mathrm{H}-2), 1.44\left(\mathrm{~s}, 3 \mathrm{H},\left(\mathrm{CH}_{3}\right)_{2} \mathrm{C}\right), 1.36\left(\mathrm{~s}, 3 \mathrm{H},\left(\mathrm{CH}_{3}\right)_{2} \mathrm{C}\right)$; ${ }^{13} \mathrm{C}-\mathrm{NMR}:\left(\mathrm{CDCl}_{3}\right) \delta_{\mathrm{C}} 137.2(\mathrm{C}, \mathrm{Bn}), 128.5(\mathrm{CH}, \mathrm{Bn}), 128.0(\mathrm{CH}, \mathrm{Bn}), 127.6(\mathrm{CH}$, $\mathrm{Bn}), 108.8\left(\mathrm{C},\left(\mathrm{CH}_{3}\right)_{2} \mathrm{C}\right), 87.6(\mathrm{CH}, \mathrm{C}-5), 80.0(\mathrm{CH}, \mathrm{C}-3), 73.5(\mathrm{CH}, \mathrm{C}-4), 73.2$ $\left(\mathrm{CH}_{2}, \mathrm{PhCH}_{2}\right), 67.9(\mathrm{CH}, \mathrm{C}-1), 66.2\left(\mathrm{CH}_{2}, \mathrm{C}-6\right), 61.2(\mathrm{C}, \mathrm{C}-7), 39.3(\mathrm{CH}, \mathrm{C}-2)$, $26.5\left(\mathrm{CH}_{3},\left(\mathrm{CH}_{3}\right)_{2} \mathrm{C}\right), 25.3\left(\mathrm{CH}_{3},\left(\mathrm{CH}_{3}\right)_{2} \mathrm{C}\right)$; IR $(\mathrm{KBr}): \nu_{\max } 2986,2937,2885,1497$, 1455, 1370, 1255, 1211, 1157, 1129, 1108, 1069, 1052, 1029, 909, 853, 737, $698 \mathrm{~cm}^{-1}$; HRMS: $m / z \mathrm{C}_{18} \mathrm{H}_{23} \mathrm{O}_{5}^{35} \mathrm{ClNa}^{+}$(product of methanol-mediated ring opening reaction during HRMS acquisition) $[\mathrm{M}+\mathrm{Na}]^{+}$calcd 377.1132 , found 377.1132.

B Glycal 209 (508 mg, $1.84 \mathrm{mmol}$ ) was dissolved in chloroform (3 mL), treated with TEBAC (9 mg, $0.04 \mathrm{mmol})$, then a solution of sodium hydroxide (2.97 g, 74.3 $\mathrm{mmol})$ in water $(3.0 \mathrm{~mL})$ and allowed to stir at room temperature for three hours. The reaction mixture was dissolved in water $(20 \mathrm{~mL})$, then extracted with diethyl ether $(3 \times 20 \mathrm{~mL})$. The ethereal fractions were combined, dried, filtered and concentrated to afford a brown oil. This oil was purified by column chromatography (14:1 hexanes:ethyl acetate) to afford gem-dichlorocyclopropane $\mathbf{3 4 0}$ as a pale-yellow oil 
(519 mg, 79\%), contaminated with a minor amount of an impurity.

3-O-Acetyl-1,5-anhydro-4- $O$-benzyl-2-chloro-2-deoxy-6,7- $O$ isopropylidene-D-gluco-hept-1-enitol (344), acetyl 4-O-benzyl-2-chloro2,3-dideoxy-6,7-O-isopropylidene- $\alpha$-D-arabino-hept-2-enopyranoside (342) and acetyl 4- $O$-benzyl-2-chloro-2,3-dideoxy-6,7- $O$-isopropylidene$\beta$-D-arabino-hept-2-enopyranoside (343)

A A solution of cyclopropane 340 (151 $\mathrm{mg}, 0.420 \mathrm{mmol})$ in THF $(4.2 \mathrm{~mL})$ was treated with silver acetate $(104 \mathrm{mg}, 0.623 \mathrm{mmol})$ and sodium acetate (180 mg, $2.19 \mathrm{mmol}$ ), then refluxed for four hours. The solution was filtered through a silica plug and eluted with diethyl ether $(50 \mathrm{~mL})$. The solution was concentrated to provide a mixture of ring-opened products as a yellow oil. This mixture was purified by column chromatography (5:1 hexanes:ethyl acetate), providing acetylated glycal $\mathbf{3 4 4}$ (26 mg, 16\%), in addition to acetyl pyranosides 342 and 343 (3:1 ratio, 79 mg, 49\%), as an inseparable yellow oil.

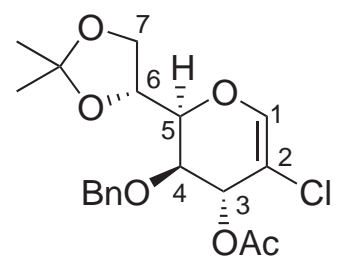

344: $\mathrm{R}_{f} 0.5$ (5:1 hexanes:ethyl acetate); ${ }^{1} \mathrm{H}-\mathrm{NMR}:\left(\mathrm{CDCl}_{3}\right) \delta_{\mathrm{H}}$ 7.37-7.30 (complex m, 5H, Bn), 6.79 (d, $J=1.6 \mathrm{~Hz}, 1 \mathrm{H}, \mathrm{H}-1$ ), $5.36(\mathrm{~d}, J=2.0 \mathrm{~Hz}, 1 \mathrm{H}, \mathrm{H}-3), 4.83(\mathrm{~d}, J=11.7 \mathrm{~Hz}, 1 \mathrm{H}$, $\left.\mathrm{PhCH}_{2}\right), 4.66\left(\mathrm{~d}, J=11.7 \mathrm{~Hz}, 1 \mathrm{H}, \mathrm{PhCH}_{2}\right), 4.28(\mathrm{~m}, 1 \mathrm{H}, \mathrm{H}-5)$, 4.08 (ddd, $J=8.6,6.0,1.8 \mathrm{~Hz}, 1 \mathrm{H}, \mathrm{H}-7 \mathrm{a}$ ), 4.01 (ddd, $J=8.7$, 4.9, $1.7 \mathrm{~Hz}, 1 \mathrm{H}, \mathrm{H}-7 \mathrm{~b}), 3.81$ (d, $J=1.5 \mathrm{~Hz}, 1 \mathrm{H}, \mathrm{H}-4), 3.68$ (d, $J=8.3 \mathrm{~Hz}, 1 \mathrm{H}, \mathrm{H}-6$ ), $2.13(\mathrm{~s}, 3 \mathrm{H}, \mathrm{Ac}), 1.37\left(\mathrm{~s}, 3 \mathrm{H},\left(\mathrm{CH}_{3}\right)_{2} \mathrm{C}\right), 1.32\left(\mathrm{~s}, 3 \mathrm{H},\left(\mathrm{CH}_{3}\right)_{2} \mathrm{C}\right) ;{ }^{13} \mathrm{C}-\mathrm{NMR}:\left(\mathrm{CDCl}_{3}\right)$ $\delta_{\mathrm{C}} 170.0\left(\mathrm{C}, \mathrm{CH}_{3} \mathrm{CO}\right), 145.0(\mathrm{CH}, \mathrm{C}-1), 137.2(\mathrm{C}, \mathrm{Bn}), 128.4(\mathrm{CH}, \mathrm{Bn}), 128.1(\mathrm{CH}$, $\mathrm{Bn}), 128.0$ (CH, Bn), $109.4\left(\mathrm{C},\left(\mathrm{CH}_{3}\right)_{2} \mathrm{C}\right), 107.3$ (C, C-2), 74.5 (CH, C-6), 72.8 (CH, C-4), $72.5\left(\mathrm{CH}_{2}, \mathrm{PhCH}_{2}\right), 72.4(\mathrm{CH}, \mathrm{C}-5), 67.2(\mathrm{CH}, \mathrm{C}-3), 66.8\left(\mathrm{CH}_{2}, \mathrm{C}-7\right), 26.8$ $\left(\mathrm{CH}_{3},\left(\mathrm{CH}_{3}\right)_{2} \mathrm{C}\right), 25.2\left(\mathrm{CH}_{3},\left(\mathrm{CH}_{3}\right)_{2} \mathrm{C}\right), 21.0\left(\mathrm{CH}_{3}, \mathrm{Ac}\right)$.

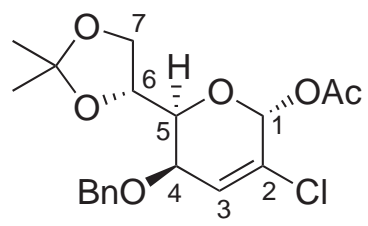

342: $\mathrm{R}_{f} 0.4$ (5:1 hexanes:ethyl acetate); ${ }^{1} \mathrm{H}-\mathrm{NMR}:\left(\mathrm{CDCl}_{3}\right)$ $\delta_{\mathrm{H}}$ 7.42-7.30 (complex m, 5H, Bn), 6.28-6.24 (complex m, $2 \mathrm{H}, \mathrm{H}-1,3), 4.70$ (s, 2H, $\left.\mathrm{PhCH}_{2}\right), 4.39$ (m, 1H, H-6), 4.12 (dd, $J=8.8,6.3 \mathrm{~Hz}, 1 \mathrm{H}, \mathrm{H}-7 \mathrm{a}), 3.98(\mathrm{dd}, J=5.9,2.5 \mathrm{~Hz}, 1 \mathrm{H}$, H-4), 3.88 (dd, $J=8.8,4.7 \mathrm{~Hz}, 1 \mathrm{H}, \mathrm{H}-7 \mathrm{~b}), 3.84$ (dd, $J=8.5,2.4 \mathrm{~Hz}, 1 \mathrm{H}, \mathrm{H}-5)$, $2.13(\mathrm{~s}, 3 \mathrm{H}, \mathrm{Ac}), 1.41\left(\mathrm{~s}, 3 \mathrm{H},\left(\mathrm{CH}_{3}\right)_{2} \mathrm{C}\right), 1.38\left(\mathrm{~s}, 3 \mathrm{H},\left(\mathrm{CH}_{3}\right)_{2} \mathrm{C}\right) ;{ }^{13} \mathrm{C}-\mathrm{NMR}:\left(\mathrm{CDCl}_{3}\right)$ $\delta_{\mathrm{C}} 169.5\left(\mathrm{C}, \mathrm{CH}_{3} \mathrm{CO}\right), 137.8(\mathrm{C}, \mathrm{Bn}), 132.4$ (C, C-2), $128.42(\mathrm{CH}, \mathrm{Bn}), 127.96(\mathrm{CH}$, $\mathrm{Bn}), 127.93$ (CH, Bn), 125.9 (CH, C-3), $109.4\left(\mathrm{C},\left(\mathrm{CH}_{3}\right)_{2} \mathrm{C}\right), 89.0(\mathrm{CH}, \mathrm{C}-1), 73.1$ (CH, C-5), 72.9 (CH, C-6), $72.0\left(\mathrm{CH}_{2}, \mathrm{PhCH}_{2}\right), 68.3(\mathrm{CH}, \mathrm{C}-4), 67.1\left(\mathrm{CH}_{2}, \mathrm{C}-7\right)$, $26.9\left(\mathrm{CH}_{3},\left(\mathrm{CH}_{3}\right)_{2} \mathrm{C}\right), 25.2\left(\mathrm{CH}_{3},\left(\mathrm{CH}_{3}\right)_{2} \mathrm{C}\right), 20.90\left(\mathrm{CH}_{3}, \mathrm{Ac}\right)$; IR $(\mathrm{KBr}): \nu_{\max } 2986$, 2935, 2874, 1754, 1655, 1455, 1372, 1213, 1133, 1074, 1004, 930, 849, $698 \mathrm{~cm}^{-1}$; HRMS: $m / z \mathrm{C}_{19} \mathrm{H}_{23} \mathrm{O}_{6} \mathrm{Na}^{+}[\mathrm{M}+\mathrm{Na}]^{+}$calcd 405.1081, found 405.1078. 


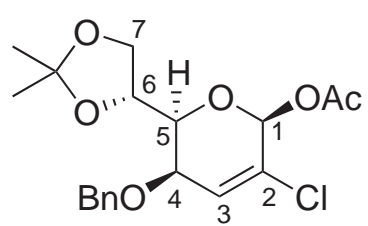

343: $\mathrm{R}_{f} 0.4$ (5:1 hexanes:ethyl acetate); ${ }^{1} \mathrm{H}-\mathrm{NMR}:\left(\mathrm{CDCl}_{3}\right)$ $\delta_{\mathrm{H}}$ 7.42-7.30 (complex m, 5H, Bn), 6.28-6.24 (complex m, $2 \mathrm{H}, \mathrm{H}-1,3), 4.68$ (s, 2H, $\mathrm{PhCH}_{2}$ ), 4.38 (m, 1H, H-6), 4.10 (m, 1H, H-7a), 4.04 (dd, J = 8.8, 4.7 Hz, 1H, H-7b), 4.00 (m, 1H, $\mathrm{H}-4), 3.67$ (dd, $J=8.6,1.9 \mathrm{~Hz}, 1 \mathrm{H}, \mathrm{H}-5), 2.16$ (s, 3H, Ac), 1.40 (s, 3H, $\left(\mathrm{CH}_{3}\right)_{2} \mathrm{C}$ ), $1.37\left(\mathrm{~s}, 3 \mathrm{H},\left(\mathrm{CH}_{3}\right)_{2} \mathrm{C}\right) ;{ }^{13} \mathrm{C}-\mathrm{NMR}:\left(\mathrm{CDCl}_{3}\right) \delta_{\mathrm{C}} 169.2\left(\mathrm{C}, \mathrm{CH}_{3} \mathrm{CO}\right), 138.0(\mathrm{C}, \mathrm{Bn})$, 133.7 (C, C-2), 128.35 (CH, Bn), 128.02 (CH, Bn), 127.85 (CH, Bn), $127.2(\mathrm{CH}$, C-3), $109.4\left(\mathrm{C},\left(\mathrm{CH}_{3}\right)_{2} \mathrm{C}\right), 90.0(\mathrm{CH}, \mathrm{C}-1), 76.8$ (CH, C-5), 73.0 (CH, C-6), 71.9 $\left(\mathrm{CH}_{2}, \mathrm{PhCH}_{2}\right), 68.5(\mathrm{CH}, \mathrm{C}-4), 67.0\left(\mathrm{CH}_{2}, \mathrm{C}-7\right), 27.0\left(\mathrm{CH}_{3},\left(\mathrm{CH}_{3}\right)_{2} \mathrm{C}\right), 25.3\left(\mathrm{CH}_{3}\right.$, $\left.\left(\mathrm{CH}_{3}\right)_{2} \mathrm{C}\right), 20.86\left(\mathrm{CH}_{3}, \mathrm{Ac}\right)$.

B A solution of cyclopropane 340 (175 mg, $0.487 \mathrm{mmol}$ ) in THF (4 mL) was treated with acetic acid $(1 \mathrm{~mL})$ and silver acetate $(124 \mathrm{mg}, 0.743 \mathrm{mmol})$, then refluxed for four hours. The solution was filtered through a silica plug and eluted with diethyl ether $(100 \mathrm{~mL})$. The solution was concentrated to provide a mixture of acetylated glycal 344 and acetyl pyranosides 342 and 343 (344:342 and 343, 1:12 ratio), as a yellow oil. This mixture was purified by column chromatography (5:1 hexanes:ethyl acetate), providing acetyl pyranosides 342 and 343 (3:1 ratio, $105 \mathrm{mg}, 56 \%$ ), as an inseparable mixture.

C A solution of cyclopropane 340 (499 mg, $1.39 \mathrm{mmol}$ ) in acetic acid (14 mL) was treated with silver acetate $(353 \mathrm{mg}, 2.11 \mathrm{mmol})$, then stirred at $90{ }^{\circ} \mathrm{C}$ for two hours. The solution was filtered through Celite ${ }^{\circledR}$ and eluted with diethyl ether $(50 \mathrm{~mL})$. The solution was concentrated to provide a mixture of acetylated glycal 344 and acetyl pyranosides 342 and 343 (344:342 and 343, 1:21 ratio), as a yellow oil. This mixture was purified by column chromatography (9:1 hexanes:ethyl acetate), providing acetyl pyranosides $\mathbf{3 4 2}$ and $\mathbf{3 4 3}$ (3:1 ratio, $442 \mathrm{mg}, 83 \%$ ), as an inseparable mixture.

D Glycal 209 (462 $\mathrm{mg}, 1.67 \mathrm{mmol})$ was dissolved in chloroform (3 mL), treated with TEBAC ( $8 \mathrm{mg}, 0.04 \mathrm{mmol})$, then a solution of sodium hydroxide (3.01 g, 75.3 $\mathrm{mmol})$ in water $(3.0 \mathrm{~mL})$ and allowed to stir under air at room temperature for 2.5 hours. The reaction mixture was dissolved in water $(20 \mathrm{~mL})$, then extracted with diethyl ether $(3 \times 20 \mathrm{~mL})$. The ethereal fractions were combined, dried, filtered and concentrated to afford a brown oil. This oil was purified by column chromatography (5:1 hexanes:ethyl acetate) to afford impure cyclopropane $\mathbf{3 4 0}$ as a yellow oil. This sample was dissolved in acetic acid $(17 \mathrm{~mL})$, treated with silver acetate (435 $\mathrm{mg}$, $2.61 \mathrm{mmol}$ ), then stirred at $80^{\circ} \mathrm{C}$ for two hours. The solution was filtered through Celite ${ }^{\circledR}$ and eluted with diethyl ether $(20 \mathrm{~mL})$. The solution was concentrated to provide a mixture of acetylated glycal 344 and acetyl pyranosides 342 and 343 (344:342 and 343, 1:30 ratio), as a yellow oil. This mixture was purified by column 
chromatography (5:1 hexanes:ethyl acetate), providing acetyl pyranosides 342 and 343 (3:1 ratio, $495 \mathrm{mg}, 77 \%$ ), as an inseparable mixture.

\section{4- $O$-Benzyl-2-chloro-2,3-dideoxy-6,7- $O$-isopropylidene-D- arabino-hept-2- enopyranose (345)}

Using a previously reported procedure, ${ }^{122}$ a solution of acetates 342 and 343 (574 $\mathrm{mg}, 1.50 \mathrm{mmol})$ in methanol $(15 \mathrm{~mL})$ was treated with a small piece of sodium (3 $\mathrm{mg}$, $0.1 \mathrm{mmol}$ ), then stirred at room temperature for one hour. The mixture was concentrated, then passed through a silica plug, which was eluted with dichloromethane (10 mL). The organic solution was concentrated to provide hemiacetal 345 (517 mg, quantitative) as a viscous yellow oil, which was used without further purification.

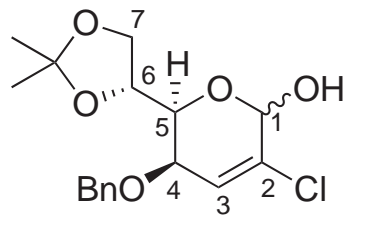

$\mathrm{R}_{f} 0.2$ (5:1 hexanes:ethyl acetate); $[\alpha]_{\mathrm{D}}^{23}-79\left(\right.$ c $\left.1.1 \mathrm{CHCl}_{3}\right)$;

${ }^{1} \mathrm{H}-\mathrm{NMR}:\left(\mathrm{CDCl}_{3}\right) \delta_{\mathrm{H}} 7.36-7.30$ (complex m, 5H, Bn), 6.17 $(\mathrm{d}, J=5.7 \mathrm{~Hz}, 1 \mathrm{H}, \mathrm{H}-3), 5.34(\mathrm{~d}, J=4.6 \mathrm{~Hz}, 1 \mathrm{H}, \mathrm{H}-1), 4.68$

$\left(\mathrm{s}, 2 \mathrm{H}, \mathrm{PhCH}_{2}\right), 4.40(\mathrm{~m}, 1 \mathrm{H}, \mathrm{H}-6), 4.14(\mathrm{dd}, J=8.4,6.6$ $\mathrm{Hz}, 1 \mathrm{H}, \mathrm{H}-7 \mathrm{a}), 4.02$ (m, 1H, H-5), 3.99 (m, 1H, H-7b), 3.92 (d, J = 5.9 Hz, 1H, H-4), 2.82 (brs, 1H, OH), 1.42 (s, 3H, $\left.\left(\mathrm{CH}_{3}\right)_{2} \mathrm{C}\right), 1.40$ (s, 3H, $\left.\left(\mathrm{CH}_{3}\right)_{2} \mathrm{C}\right) ;{ }^{13} \mathrm{C}-\mathrm{NMR}$ : $\left(\mathrm{CDCl}_{3}\right) \delta_{\mathrm{C}} 137.9(\mathrm{C}, \mathrm{Bn}), 134.6(\mathrm{C}, \mathrm{C}-2), 128.4(\mathrm{CH}, \mathrm{Bn}), 128.0(\mathrm{CH}, \mathrm{Bn}), 127.9$ (CH, Bn), 124.6 (CH, C-3), $109.3\left(\mathrm{C},\left(\mathrm{CH}_{3}\right)_{2} \mathrm{C}\right), 90.9(\mathrm{CH}, \mathrm{C}-1), 73.2$ (CH, C-6), $72.1\left(\mathrm{CH}_{2}, \mathrm{PhCH}_{2}\right), 71.2(\mathrm{CH}, \mathrm{C}-5), 68.8(\mathrm{CH}, \mathrm{C}-4), 67.2\left(\mathrm{CH}_{2}, \mathrm{C}-7\right), 26.9\left(\mathrm{CH}_{3}\right.$, $\left.\left(\mathrm{CH}_{3}\right)_{2} \mathrm{C}\right), 25.4\left(\mathrm{CH}_{3},\left(\mathrm{CH}_{3}\right)_{2} \mathrm{C}\right)$; IR $(\mathrm{KBr}): \nu_{\max } 3400,2986,2933,2874,1654,1455$, 1372, 1254, 1213, 1073, 1043, 913, 850, 738, $698 \mathrm{~cm}^{-1}$; HRMS: $m / z \mathrm{C}_{17} \mathrm{H}_{21} \mathrm{O}_{5} \mathrm{Na}^{+}$ $[\mathrm{M}+\mathrm{Na}]^{+}$calcd 363.0975, found 363.0971 .

(2Z,4E)-6- $O$-Benzyl-4-chloro-1,2,3,4,5-pentadeoxy-8,9- $O$-isopropylideneD-arabino-nona-2,4-dienitol (346) and ( $2 E, 4 E)$-6- $O$-benzyl-4-chloro1,2,3,4,5-pentadeoxy-8,9- $O$-isopropylidene-D-arabino-nona-2,4-dienitol (347)

A solution of ethyl triphenylphosphonium iodide (860 mg, $2.05 \mathrm{mmol})$ in THF (6 mL) was cooled to $-78{ }^{\circ} \mathrm{C}$, then treated with $n$-butyllithium $(1.25 \mathrm{~mL}, 2.00 \mathrm{mmol})$ and stirred at $-78{ }^{\circ} \mathrm{C}$ for one hour. The mixture was then treated with a solution of hemiacetal 345 (304 mg, $0.892 \mathrm{mmol}$ ) in THF (4 mL), then heated at reflux for 4.5 hours. The reaction mixture was diluted with sat. ammonium chloride solution $(20 \mathrm{ml})$ and extracted with dichloromethane $(3 \times 20 \mathrm{~mL})$. The organic fractions were combined, dried, filtered and concentrated to provide a crude brown oil. Upon purification by column chromatography (5:1 hexanes:ethyl acetate), an inseparable mixture of dienes 346 and 347 (2.0:1 ratio, $245 \mathrm{mg}, 77 \%$ ) was obtained as a colourless oil. 


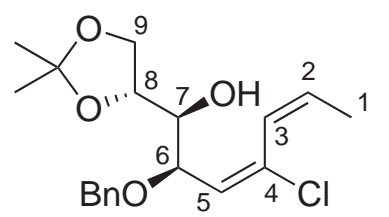

346: $\mathrm{R}_{f} 0.45$ (5:1 hexanes:ethyl acetate); ${ }^{1} \mathrm{H}-\mathrm{NMR}:\left(\mathrm{CDCl}_{3}\right)$ $\delta_{\mathrm{H}} 7.36-7.28$ (complex m, 5H, Bn), $5.99(\mathrm{~d}, J=10.3 \mathrm{~Hz}, 1 \mathrm{H}$, $\mathrm{H}-5), 5.95(\mathrm{dd}, J=11.3,1.5 \mathrm{~Hz}, 1 \mathrm{H}, \mathrm{H}-3), 5.79(\mathrm{dq}, J=$ $11.3,7.1 \mathrm{~Hz}, 1 \mathrm{H}, \mathrm{H}-2), 4.59\left(\mathrm{~d}, J=11.5 \mathrm{~Hz}, 1 \mathrm{H}, \mathrm{PhCH}_{2}\right)$, $4.36\left(\mathrm{~d}, J=11.3 \mathrm{~Hz}, 1 \mathrm{H}, \mathrm{PhCH}_{2}\right.$ ), 4.19 (dd, $\left.J=10.0,2.2 \mathrm{~Hz}, 1 \mathrm{H}, \mathrm{H}-6\right), 4.11-4.05$ (complex m, 2H, H-8,9a), 3.98 (dd, $J=8.3,5.4 \mathrm{~Hz}, 1 \mathrm{H}, \mathrm{H}-9 \mathrm{~b}$ ), 3.45 (m, 1H, H-7), $2.37(\mathrm{~d}, J=9.1 \mathrm{~Hz}, 1 \mathrm{H}, \mathrm{OH}), 1.77(\mathrm{dd}, J=7.1,1.6 \mathrm{~Hz}, 3 \mathrm{H}, \mathrm{H}-1), 1.37(\mathrm{~s}, 3 \mathrm{H}$, $\left.\left(\mathrm{CH}_{3}\right)_{2} \mathrm{C}\right), 1.33\left(\mathrm{~s}, 3 \mathrm{H},\left(\mathrm{CH}_{3}\right)_{2} \mathrm{C}\right) ;{ }^{13} \mathrm{C}-\mathrm{NMR}:\left(\mathrm{CDCl}_{3}\right) \delta_{\mathrm{C}} 137.7(\mathrm{C}, \mathrm{Bn}), 132.3(\mathrm{CH}$, C-2), 128.37 (C, C-4), 128.34 (CH, Bn), 127.82 (CH, Bn), 127.80 (CH, Bn), 127.4 $(\mathrm{CH}, \mathrm{C}-5), 124.3(\mathrm{CH}, \mathrm{C}-3), 109.2\left(\mathrm{C},\left(\mathrm{CH}_{3}\right)_{2} \mathrm{C}\right), 75.2(\mathrm{CH}, \mathrm{C}-8), 74.9(\mathrm{CH}, \mathrm{C}-7)$, 74.7 (CH, C-6), $70.5\left(\mathrm{CH}_{2}, \mathrm{PhCH}_{2}\right), 67.0\left(\mathrm{CH}_{2}, \mathrm{C}-9\right), 26.7\left(\mathrm{CH}_{3},\left(\mathrm{CH}_{3}\right)_{2} \mathrm{C}\right), 25.2$ $\left(\mathrm{CH}_{3},\left(\mathrm{CH}_{3}\right)_{2} \mathrm{C}\right), 15.1\left(\mathrm{CH}_{3}, \mathrm{C}-1\right)$; HRMS: $m / z \mathrm{C}_{19} \mathrm{H}_{25} \mathrm{O}_{4}^{35} \mathrm{ClNa}^{+}[\mathrm{M}+\mathrm{Na}]^{+}$calcd 375.1339 , found 375.1344 .

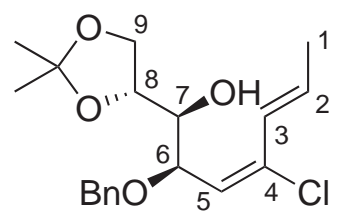

347: The following data set was obtained by subtracting the NMR signals for $\mathbf{3 4 6}$ from that of the purified mixture.

${ }^{1} \mathrm{H}-\mathrm{NMR}:\left(\mathrm{CDCl}_{3}\right) \delta_{\mathrm{H}} 7.37-7.28$ (complex m, 5H, Bn), $6.31(\mathrm{~m}$, $1 \mathrm{H}, \mathrm{H}-2), 6.25$ (d, $J=14.7 \mathrm{~Hz}, 1 \mathrm{H}, \mathrm{H}-3), 5.82(\mathrm{~d}, J=10.0 \mathrm{~Hz}$, $1 \mathrm{H}, \mathrm{H}-5), 4.64\left(\mathrm{~d}, J=11.7 \mathrm{~Hz}, 1 \mathrm{H}, \mathrm{PhCH}_{2}\right), 4.45(\mathrm{dd}, J=9.8,3.0 \mathrm{~Hz}, 1 \mathrm{H}, \mathrm{H}-6)$, $4.41\left(\mathrm{~d}, J=11.7 \mathrm{~Hz}, 1 \mathrm{H}, \mathrm{PhCH}_{2}\right.$ ), 4.14-3.96 (complex m, 3H, H-8,9a,b), 3.52 (td, $J=7.6,2.7 \mathrm{~Hz}, 1 \mathrm{H}, \mathrm{H}-7), 2.45(\mathrm{~d}, J=8.0 \mathrm{~Hz}, 1 \mathrm{H}, \mathrm{OH}), 1.84(\mathrm{~d}, J=6.3 \mathrm{~Hz}, 3 \mathrm{H}$, $\mathrm{H}-1), 1.40\left(\mathrm{~s}, 3 \mathrm{H},\left(\mathrm{CH}_{3}\right)_{2} \mathrm{C}\right), 1.35\left(\mathrm{~s}, 3 \mathrm{H},\left(\mathrm{CH}_{3}\right)_{2} \mathrm{C}\right) ;{ }^{13} \mathrm{C}-\mathrm{NMR}:\left(\mathrm{CDCl}_{3}\right) \delta_{\mathrm{C}} 137.5$ (C, Bn), 134.5 (CH, C-4), 133.4 (CH, C-2), 128.3 (CH, Bn), 127.9 (CH, Bn), 127.84 (CH, Bn), $125.1(\mathrm{CH}, \mathrm{C}-5), 123.3(\mathrm{CH}, \mathrm{C}-3), 109.1\left(\mathrm{C},\left(\mathrm{CH}_{3}\right)_{2} \mathrm{C}\right), 75.5(\mathrm{CH}, \mathrm{C}-8)$, 74.8 (CH, C-7), 73.3 (CH, C-6), $70.4\left(\mathrm{CH}_{2}, \mathrm{PhCH}_{2}\right), 66.5\left(\mathrm{CH}_{2}, \mathrm{C}-9\right), 26.8\left(\mathrm{CH}_{3}\right.$, $\left.\left(\mathrm{CH}_{3}\right)_{2} \mathrm{C}\right), 25.3\left(\mathrm{CH}_{3},\left(\mathrm{CH}_{3}\right)_{2} \mathrm{C}\right), 18.1\left(\mathrm{CH}_{3}, \mathrm{C}-1\right)$.

\section{(E)-2,3-Anhydro-6- $O$-benzyl-4-chloro-1,4,5-trideoxy-8,9- $O$ -} isopropylidene-D-glycero-D-talo-nona-4-enopyranitol (330) and $(E)-2,3-$ anhydro-6- $O$-benzyl-4-chloro-1,4,5-trideoxy-8,9- $O$-isopropylidene-Dglycero-D-gulo-nona-4-enopyranitol (348)

A A solution of dienes $\mathbf{3 4 6}$ and $\mathbf{3 4 7}$ (2.0:1 ratio, $90 \mathrm{mg}, 0.26 \mathrm{mmol}$ ) in dichloromethane $(4 \mathrm{~mL})$ was treated with $m$-CPBA $(129 \mathrm{mg}, 0.52 \mathrm{mmol})$. The mixture was stirred at room temperature for two days, then diluted with sat. sodium bicarbonate solution $(20 \mathrm{~mL})$ and dichloromethane $(10 \mathrm{~mL})$. The biphasic mixture was stirred rapidly for ten minutes, then separated. The organic fraction was dried, filtered and concentrated to provide a colourless oil. Upon purification by column chromatography (4:1 hexanes:ethyl acetate, $1 \%$ triethylamine), a mixture of epoxides 330 and 348 (1.3:1 ratio), in addition to a minor amount of a mixture of all four stereoisomeric epoxides, all obtained as colourless oils (53 mg, $56 \%$ combined yield). 


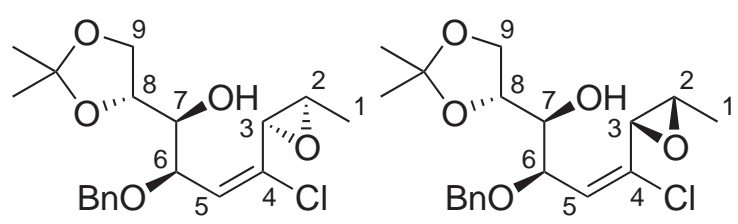

330 and 348: $\mathrm{R}_{f} 0.35$ (3:1 hexanes:ethyl acetate); ${ }^{1} \mathrm{H}-\mathrm{NMR}:\left(\mathrm{CDCl}_{3}\right) \delta_{\mathrm{H}}$

7.37-7.28 (complex m, 5H, Bn), 6.09 (d, $J=9.5 \mathrm{~Hz}, 1 \mathrm{H}, \mathrm{H}-5), 6.05(\mathrm{dd}, J=$ $10.2 \mathrm{~Hz}, 1 \mathrm{H}, \mathrm{H}-5$ ), 4.81 (apparent dt, $J=9.7,3.2 \mathrm{~Hz}, 1 \mathrm{H}, \mathrm{H}-6$ ), 4.65 (d, $J=11.2$ $\left.\mathrm{Hz}, 1 \mathrm{H}, \mathrm{PhCH}_{2}\right), 4.59\left(\mathrm{~d}, J=11.5 \mathrm{~Hz}, 1 \mathrm{H}, \mathrm{PhCH}_{2}\right.$ ), 4.50-4.46 (complex m, 2H, $\mathrm{PhCH}_{2}$ ), 4.15-3.99 (complex m, 3H, H-8,9a,b), 3.60 (m, 1H, H-3), 3.55 (dd, $J=$ 7.0, $3.2 \mathrm{~Hz}, 1 \mathrm{H}, \mathrm{H}-7), 3.50$ (m, 1H, H-7), 3.24 (dq, $J=5.4,0.7 \mathrm{~Hz}, 1 \mathrm{H}, \mathrm{H}-2), 3.16$ $(\mathrm{dq}, J=5.4,1.7 \mathrm{~Hz}, 1 \mathrm{H}, \mathrm{H}-2), 2.28(\mathrm{brs}, 1 \mathrm{H}, \mathrm{OH}), 1.42\left(\mathrm{~s}, 3 \mathrm{H},\left(\mathrm{CH}_{3}\right)_{2} \mathrm{C}\right), 1.36-1.34$ (complex m, 6H, H-1, $\left(\mathrm{CH}_{3}\right)_{2} \mathrm{C}$ ), 1.28 (dd, $J=5.4,1.7 \mathrm{~Hz}, 3 \mathrm{H}, \mathrm{H}-1$ ); ${ }^{13} \mathrm{C}-\mathrm{NMR}$ : $\left(\mathrm{CDCl}_{3}\right) \delta_{\mathrm{C}} 137.8(\mathrm{C}, \mathrm{Bn}), 137.7$ (C, Bn), 131.1 (C, C-5), 129.9 (C, C-5), $128.4(\mathrm{CH}$, $\mathrm{Bn}), 127.9(\mathrm{CH}, \mathrm{Bn}), 127.8(\mathrm{CH}, \mathrm{Bn}), 109.2\left(\mathrm{C},\left(\mathrm{CH}_{3}\right)_{2} \mathrm{C}\right), 109.1\left(\mathrm{C},\left(\mathrm{CH}_{3}\right)_{2} \mathrm{C}\right), 75.8$ (CH, C-8), 75.7 (CH, C-8), 75.0 (CH, C-7), 74.5 (CH, C-7), 74.0 (CH, C-6), 73.8 $(\mathrm{CH}, \mathrm{C}-6), 71.4\left(\mathrm{CH}_{2}, \mathrm{PhCH}_{2}\right), 71.0\left(\mathrm{CH}_{2}, \mathrm{PhCH}_{2}\right), 66.6\left(\mathrm{CH}_{2}, \mathrm{C}-9\right), 66.4\left(\mathrm{CH}_{2}\right.$, C-9), 56.7 (CH, C-3), $56.2(\mathrm{CH}, \mathrm{C}-3), 54.5$ (CH, C-2), $52.9(\mathrm{CH}, \mathrm{C}-2), 26.73\left(\mathrm{CH}_{3}\right.$, $\left.\left(\mathrm{CH}_{3}\right)_{2} \mathrm{C}\right), 26.71\left(\mathrm{CH}_{3},\left(\mathrm{CH}_{3}\right)_{2} \mathrm{C}\right), 25.34\left(\mathrm{CH}_{3},\left(\mathrm{CH}_{3}\right)_{2} \mathrm{C}\right), 25.32\left(\mathrm{CH}_{3},\left(\mathrm{CH}_{3}\right)_{2} \mathrm{C}\right)$, $13.5\left(\mathrm{CH}_{3}, \mathrm{C}-1\right), 13.2\left(\mathrm{CH}_{3}, \mathrm{C}-1\right)$; HRMS: $m / z \mathrm{C}_{19} \mathrm{H}_{25} \mathrm{O}_{5}^{35} \mathrm{ClNa}^{+}[\mathrm{M}+\mathrm{Na}]^{+}$calcd 391.1288, found 391.1289.

B Using a known procedure, ${ }^{91}$ a solution of dienes 346 and 347 (2.0:1 ratio, 69 mg, $0.20 \mathrm{mmol})$ in dichloromethane $(2 \mathrm{~mL})$ was treated with $(R, R)$-Jacobsen's catalyst 313 (4 mg, $6 \mu \mathrm{mol})$, then bleach $\left(0.55 \mathrm{molL}^{-1}\right.$, pH 11.3, $\left.2 \mathrm{~mL}\right) .{ }^{91}$ The mixture was stirred at room temperature for two days, then diluted with water $(20 \mathrm{~mL})$ and extracted with diethyl ether $(2 \times 20 \mathrm{~mL})$. The ethereal fractions were combined, dried, filtered and concentrated to provide a brown oil. Upon purification by column chromatography (3:1 hexanes:ethyl acetate), starting material $\mathbf{3 4 6}$ was obtained (17 mg, $25 \%$ ), in addition to a minor amount of impure epoxides 330 and 348 (1.5:1 ratio, $2 \mathrm{mg}, 3 \%$ ), which was not able to be characterised.

\section{3,7-Anhydro-6- $O$-benzyl-4-chloro-1,4,5-trideoxy-8,9- $O$-isopropylidene-D- arabino-nona-4-enopyranitol (349)}

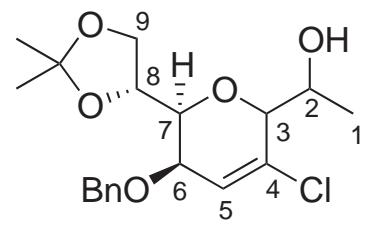

A solution of epoxides 330 and 348 (2:1 ratio, $76 \mathrm{mg}, 0.21$ $\mathrm{mmol})$ in DMF $(2 \mathrm{~mL})$ was treated with sodium hydride (13 mg, $0.33 \mathrm{mmol}$ ). The mixture was stirred at room temperature for 24 hours, then diluted with sat. copper sulfate solution $(20 \mathrm{~mL})$ and diethyl ether $(20 \mathrm{~mL})$. The phases were separated, then the ethereal fraction was dried, filtered and concentrated to provide a yellow oil. Upon purification by column chromatography (5:1 hexanes:ethyl acetate), a mixture of starting materials $\mathbf{3 3 0}$ and $\mathbf{3 4 8}$ was obtained (19 $\mathrm{mg}, \mathbf{2 5 \%}$ ), in addition to com- 
plicated mixtures of epoxide-opened products (349) as colourless oils (11 mg, 14\% combined yield), which were not sufficiently pure for characterisation.

\section{bis (2,4,6-Trimethylpyridine)silver(I) hexafluorophosphate (351)}

As reported previously, ${ }^{137}$ a solution of silver nitrate (4.99 g, $\left.29.4 \mathrm{mmol}\right)$ and potassium hexafluorophosphate $(5.49 \mathrm{~g}, 29.8 \mathrm{mmol})$ in water $(100 \mathrm{~mL})$ was treated with 2,4,6-trimethylpyridine $(11 \mathrm{~mL}, 81 \mathrm{mmol})$, then stirred at room temperature for one hour. The mixture was filtered and the precipitate washed with water $(50 \mathrm{~mL})$, then dried to provide silver salt $\mathbf{3 5 1}$ as a white solid (12.8 g, 88\%).

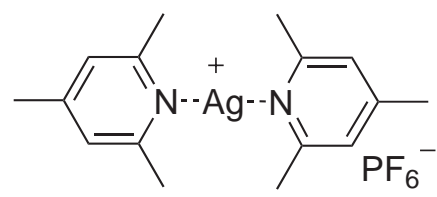

m.p. $213-216{ }^{\circ} \mathrm{C}$ (lit. ${ }^{137}$ m.p. $210-253{ }^{\circ} \mathrm{C}$ ).

\section{3,7-Anhydro-6- $O$-benzyl-4-chloro-1,2,4,5-tetradeoxy-2-iodo-8,9- $O$ - isopropylidene-D-arabino-nona-4-enopyranitol (352)}

A solution of silver salt 351 (418 $\mathrm{mg}, 0.844 \mathrm{mmol})$ in dichloromethane $(5 \mathrm{~mL})$ was treated with iodine $(154 \mathrm{mg}, 0.606 \mathrm{mmol})$ and stirred at room temperature for ten minutes, in which the iodine was consumed. A solution of dienes 346 and 347 (2.0:1 ratio, $135 \mathrm{mg}, 0.383 \mathrm{mmol})$ in dichloromethane $(3 \mathrm{~mL})$ was added, then the solution was stirred at room temperature for five days, in which the solution turned dark brown due to decomposition of the iodine complex. The mixture was filtered through Celite ${ }^{\circledR}$ and eluted with dichloromethane $(20 \mathrm{~mL})$. The eluents were washed successively with sat. sodium thiosulfate solution $(20 \mathrm{~mL}), 10 \%$ aqueous hydrochloric acid $(20 \mathrm{~mL})$, then sat. sodium bicarbonate solution $(20 \mathrm{~mL})$. The organic fraction was dried, filtered and concentrated to provide an orange oil. Upon purification by column chromatography (9:1 hexanes:ethyl acetate), iodopyranose sugars were obtained (352), of which two were obtained as an inseparable yellow oil $\left(\mathrm{R}_{f} 0.55\right.$ (5:1 hexanes:ethyl acetate), $6 \mathrm{mg}, 3 \%)$; a third iodopyranose sugar $\left(\mathrm{R}_{f} 0.45\right.$ (5:1 hexanes:ethyl acetate), $4 \mathrm{mg}, 2 \%$ ) was obtained as a yellow oil (5\% combined yield), in addition to unreacted starting materials 346 and 347 (1.1:1 ratio, $44 \mathrm{mg}, 33 \%$ ).

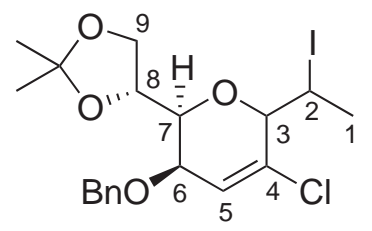

$\mathrm{R}_{f} 0.55$ (5:1 hexanes:ethyl acetate); ${ }^{1} \mathrm{H}-\mathrm{NMR}:\left(\mathrm{CDCl}_{3}\right) \delta_{\mathrm{H}}$ 7.37-7.28 (complex m, 5H, Bn), $6.31(\mathrm{~d}, J=6.1 \mathrm{~Hz}, 1 \mathrm{H}$, $\mathrm{H}-5), 6.24(\mathrm{~d}, J=5.8 \mathrm{~Hz}, 1 \mathrm{H}, \mathrm{H}-5), 4.71$ (qd, $J=7.3,2.7$ $\mathrm{Hz}, 1 \mathrm{H}, \mathrm{H}-2)$, 4.68-4.67 (complex m, 2H, $\mathrm{PhCH}_{2}$ ), 4.64 (dd, $J=8.0,1.7 \mathrm{~Hz}, 1 \mathrm{H}, \mathrm{H}-3), 4.51$ (qd, $J=7.1,2.7 \mathrm{~Hz}, 1 \mathrm{H}, \mathrm{H}-2), 4.44$ (m, 1H, H-3), $4.41-4.35$ (m, 1H, H-8), 4.30 (dd, $J=8.8,4.9$ Hz, 1H, H-9a), 4.22 (m, 1H, H-9a), 4.19 (m, 1H, H-9b), 4.17 (m, 1H, H-9b), 4.02 (dd, $J=6.2,2.1$ Hz, 1H, H-6), 3.99 (dd, $J=5.9,2.2 \mathrm{~Hz}, 1 \mathrm{H}, \mathrm{H}-6), 3.93$ (m, 1H, H-7), 3.89 (m, 1H, H-7), 2.02 (d, $J=$ 
$7.0 \mathrm{~Hz}, 3 \mathrm{H}, \mathrm{H}-1), 1.87$ (d, J = 7.4 Hz, 3H, H-1), $1.42\left(\mathrm{~s}, 3 \mathrm{H},\left(\mathrm{CH}_{3}\right)_{2} \mathrm{C}\right), 1.41(\mathrm{~s}, 3 \mathrm{H}$, $\left.\left(\mathrm{CH}_{3}\right)_{2} \mathrm{C}\right), 1.39\left(\mathrm{~s}, 3 \mathrm{H},\left(\mathrm{CH}_{3}\right)_{2} \mathrm{C}\right) ;{ }^{13} \mathrm{C}-\mathrm{NMR}:\left(\mathrm{CDCl}_{3}\right) \delta_{\mathrm{C}} 138.0(\mathrm{C}, \mathrm{Bn}), 136.4(\mathrm{C}$, C-4), 133.8 (C, C-4), 128.4 (CH, Bn), 128.0 (CH, Bn), 127.9 (CH, Bn), 127.8 (CH, $\mathrm{Bn}), 125.0(\mathrm{CH}, \mathrm{C}-5), 124.3(\mathrm{CH}, \mathrm{C}-5), 109.3\left(\mathrm{C},\left(\mathrm{CH}_{3}\right)_{2} \mathrm{C}\right), 109.2\left(\mathrm{C},\left(\mathrm{CH}_{3}\right)_{2} \mathrm{C}\right)$, 79.4 (CH, C-3), 78.1 (CH, C-7), 75.7 (CH, C-7), 74.2 (CH, C-3), 73.7 (CH, C-8), $73.6(\mathrm{CH}, \mathrm{C}-8), 71.79\left(\mathrm{CH}_{2}, \mathrm{PhCH}_{2}\right), 71.76\left(\mathrm{CH}_{2}, \mathrm{PhCH}_{2}\right), 69.5(\mathrm{CH}, \mathrm{C}-6), 69.4$ (CH, C-6), $67.42\left(\mathrm{CH}_{2}, \mathrm{C}-9\right), 67.37\left(\mathrm{CH}_{2}, \mathrm{C}-9\right), 27.1\left(\mathrm{CH}_{3},\left(\mathrm{CH}_{3}\right)_{2} \mathrm{C}\right), 26.7\left(\mathrm{CH}_{2}\right.$, $\mathrm{C}-2), 26.5\left(\mathrm{CH}_{2}, \mathrm{C}-2\right), 25.6\left(\mathrm{CH}_{3}, \mathrm{C}-1\right) 25.5\left(\mathrm{CH}_{3},\left(\mathrm{CH}_{3}\right)_{2} \mathrm{C}\right), 25.4\left(\mathrm{CH}_{3},\left(\mathrm{CH}_{3}\right)_{2} \mathrm{C}\right)$, $25.1\left(\mathrm{CH}_{3},\left(\mathrm{CH}_{3}\right)_{2} \mathrm{C}\right), 24.0\left(\mathrm{CH}_{3}, \mathrm{C}-1\right)$; IR $(\mathrm{KBr}): \nu_{\max } 2985,2932,2868,1728,1650$, 1455, 1380, 1260, 1214, 1139, 1069, 1046, 1024, 912, 847, 737, $698 \mathrm{~cm}^{-1}$; HRMS: $\mathrm{m} / z$ $\mathrm{C}_{19} \mathrm{H}_{24} \mathrm{O}_{4}^{35} \mathrm{ClINa}^{+}[\mathrm{M}+\mathrm{Na}]^{+}$calcd 501.0306, found 501.0301.

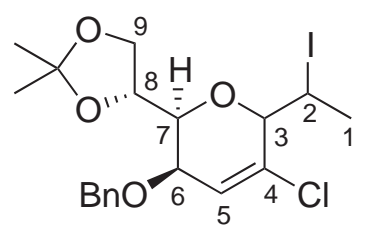

$\mathrm{R}_{f} 0.45$ (5:1 hexanes:ethyl acetate); $[\alpha]_{\mathrm{D}}^{22}-60\left(c 0.35 \mathrm{CHCl}_{3}\right)$; ${ }^{1} \mathrm{H}-\mathrm{NMR}:\left(\mathrm{CDCl}_{3}\right) \delta_{\mathrm{H}} 7.42-7.27$ (complex m, 5H, Bn), 6.27 $(\mathrm{dd}, J=6.1,1.7 \mathrm{~Hz}, 1 \mathrm{H}, \mathrm{H}-5), 4.77$ (d, $J=11.9 \mathrm{~Hz}, 1 \mathrm{H}$, $\left.\mathrm{PhCH}_{2}\right), 4.64$ (m, 1H, H-2), 4.63 (d, $J=12.0 \mathrm{~Hz}, 1 \mathrm{H}, \mathrm{PhCH}_{2}$ ), 4.41 (dd, $J=12.2,6.2 \mathrm{~Hz}, 1 \mathrm{H}, \mathrm{H}-8$ ), 4.11 (apparent d, $J=5.9 \mathrm{~Hz}, 2 \mathrm{H}, \mathrm{H}-9 \mathrm{a}, \mathrm{b}), 4.00$ (apparent dt, $J=6.1,2.0 \mathrm{~Hz}, 1 \mathrm{H}, \mathrm{H}-6), 3.68$ (dd, $J=6.5,2.1 \mathrm{~Hz}, 1 \mathrm{H}, \mathrm{H}-7), 3.44$ (d, $J=1.7 \mathrm{~Hz}, 1 \mathrm{H}, \mathrm{H}-3), 1.97$ (d, $J=7.0 \mathrm{~Hz}, 3 \mathrm{H}, \mathrm{H}-1), 1.41$ (s, 3H, $\left.\left(\mathrm{CH}_{3}\right)_{2} \mathrm{C}\right), 1.37$ (s, $\left.3 \mathrm{H},\left(\mathrm{CH}_{3}\right)_{2} \mathrm{C}\right) ;{ }^{13} \mathrm{C}-\mathrm{NMR}:\left(\mathrm{CDCl}_{3}\right) \delta_{\mathrm{C}} 138.54$ and 138.50 (C, Bn and C, C-4), 128.3 (CH, Bn), $127.6(\mathrm{CH}, \mathrm{Bn}), 127.5(\mathrm{CH}, \mathrm{Bn}), 124.5(\mathrm{CH}, \mathrm{C}-5), 108.9\left(\mathrm{C},\left(\mathrm{CH}_{3}\right)_{2} \mathrm{C}\right)$, 80.5 (CH, C-3), $77.4(\mathrm{CH}, \mathrm{C}-7), 74.2(\mathrm{CH}, \mathrm{C}-8), 70.6\left(\mathrm{CH}_{2}, \mathrm{PhCH}_{2}\right), 69.2(\mathrm{CH}, \mathrm{C}-6)$, $66.4\left(\mathrm{CH}_{2}, \mathrm{C}-9\right), 26.8\left(\mathrm{CH}_{3},\left(\mathrm{CH}_{3}\right)_{2} \mathrm{C}\right), 26.6\left(\mathrm{CH}_{2}, \mathrm{C}-2\right), 25.4\left(\mathrm{CH}_{3},\left(\mathrm{CH}_{3}\right)_{2} \mathrm{C}\right), 25.0$ $\left(\mathrm{CH}_{3}, \mathrm{C}-1\right)$; IR (KBr): $\nu_{\max } 2985,2924,2874,1645,1454,1371,1250,1212,1144$, 1071, 1046, 1027, 850, 737, $698 \mathrm{~cm}^{-1}$; HRMS: $m / z \mathrm{C}_{19} \mathrm{H}_{24} \mathrm{O}_{4}^{35} \mathrm{ClINa}^{+}[\mathrm{M}+\mathrm{Na}]^{+}$ calcd 501.0306, found 501.0304.

\section{(3E)-5- $O$-Benzyl-3-chloro-1,2,3,4-tetradeoxy-7,8- $O$-isopropylidene-D- arabino-octa-1,3-dienitol (353)}

A solution of methyl triphenylphosphonium bromide (1.06 g, $2.97 \mathrm{mmol})$ in THF $(10 \mathrm{~mL})$ was cooled to $-78{ }^{\circ} \mathrm{C}$, then treated with KHMDS $(6.0 \mathrm{~mL}, 3.0 \mathrm{mmol})$ and stirred at $-78{ }^{\circ} \mathrm{C}$ for one hour. The mixture was treated with a solution of hemiacetal 345 (478 $\mathrm{mg}, 1.40 \mathrm{mmol})$ in THF $(10 \mathrm{~mL})$, then allowed to warm to room temperature. The reaction was stirred at room temperature for two hours, then diluted with sat. ammonium chloride solution $(50 \mathrm{ml})$ and extracted with diethyl ether $(2 \times 50 \mathrm{~mL})$. The organic fractions were combined, dried, filtered and concentrated to provide a crude brown oil. Upon purification by column chromatography (5:1 hexanes:ethyl acetate), diene 353 (326 mg, 69\%) was obtained as a colourless oil. 


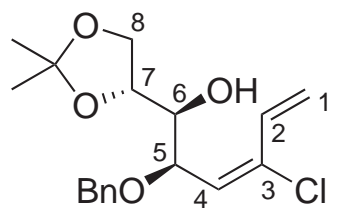

$\mathrm{R}_{f} 0.3$ (5:1 hexanes:ethyl acetate); $[\alpha]_{\mathrm{D}}^{19}-34$ ( c $1.4 \mathrm{CHCl}_{3}$ ); ${ }^{1} \mathrm{H}-\mathrm{NMR}:\left(\mathrm{CDCl}_{3}\right) \delta_{\mathrm{H}} 7.37-7.30$ (complex m, 5H, Bn), 6.61 $(\mathrm{dd}, J=16.3,10.7 \mathrm{~Hz}, 1 \mathrm{H}, \mathrm{H}-2), 5.99(\mathrm{dd}, J=9.8,0.8 \mathrm{~Hz}$, $1 \mathrm{H}, \mathrm{H}-4), 5.82$ (d, $J=16.1 \mathrm{~Hz}, 1 \mathrm{H}, \mathrm{H}-1 \mathrm{a}), 5.40$ (dd, $J=10.8$, $1.0 \mathrm{~Hz}, 1 \mathrm{H}, \mathrm{H}-1 \mathrm{~b}), 4.64$ (d, $J=11.5 \mathrm{~Hz}, 1 \mathrm{H}, \mathrm{PhCH}_{2}$ ), 4.49 (dd, $J=9.8,3.0 \mathrm{~Hz}, \mathrm{H}-$ 5), 4.41 (d, $J=11.8 \mathrm{~Hz}, 1 \mathrm{H}, \mathrm{PhCH}_{2}$ ), 4.11 (dt, $J=7.5,5.9 \mathrm{~Hz}, 1 \mathrm{H}, \mathrm{H}-7$ ), 4.04 (dd, $J=8.4,6.2 \mathrm{~Hz}, 1 \mathrm{H}, \mathrm{H}-8 \mathrm{a}), 3.98$ (dd, $J=8.4,5.5 \mathrm{~Hz}, 1 \mathrm{H}, \mathrm{H}-8 \mathrm{~b}), 3.51$ (dd, $J=7.5$, $2.7 \mathrm{~Hz}, 1 \mathrm{H}, \mathrm{H}-6), 2.40$ (brs, $1 \mathrm{H}, \mathrm{OH}), 1.38\left(\mathrm{~s}, 3 \mathrm{H},\left(\mathrm{CH}_{3}\right)_{2} \mathrm{C}\right), 1.34\left(\mathrm{~s}, 3 \mathrm{H},\left(\mathrm{CH}_{3}\right)_{2} \mathrm{C}\right)$; ${ }^{13} \mathrm{C}-\mathrm{NMR}:\left(\mathrm{CDCl}_{3}\right) \delta_{\mathrm{C}} 137.4(\mathrm{C}, \mathrm{Bn}), 134.9$ (C, C-3), $128.5(\mathrm{CH}, \mathrm{Bn}), 128.4(\mathrm{CH}$, C-2), 128.2 (CH, C-4), 127.9 (CH, Bn), $120.7\left(\mathrm{CH}_{2}, \mathrm{C}-1\right), 109.2\left(\mathrm{C},\left(\mathrm{CH}_{3}\right)_{2} \mathrm{C}\right), 75.4$ (CH, C-7), 75.0 (CH, C-6), $73.3(\mathrm{CH}, \mathrm{C}-5), 70.6\left(\mathrm{CH}_{2}, \mathrm{PhCH}_{2}\right), 66.7\left(\mathrm{CH}_{2}, \mathrm{C}-8\right)$, $26.8\left(\mathrm{CH}_{3},\left(\mathrm{CH}_{3}\right)_{2} \mathrm{C}\right), 25.3\left(\mathrm{CH}_{3},\left(\mathrm{CH}_{3}\right)_{2} \mathrm{C}\right)$; IR $(\mathrm{KBr}): \nu_{\max } 3454,3065,3031,2987$, 2934, 2879, 1587, 1455, 1381, 1371, 1253, 1216, 1152, 1059, 847, 736, $698 \mathrm{~cm}^{-1}$; HRMS: $m / z \mathrm{C}_{18} \mathrm{H}_{23} \mathrm{O}_{4}^{35} \mathrm{ClNa}^{+}[\mathrm{M}+\mathrm{Na}]^{+}$calcd 361.1183, found 361.1180.

\section{( $E$ )-1,2-Anhydro-5- $O$-benzyl-3-chloro-3,4-dideoxy-7,8- $O$-isopropylidene- D-gluco-octa-3-enopyranitol (355) and $(E)$-1,2-anhydro-5- $O$-benzyl-3- chloro-3,4-dideoxy-7,8-O-isopropylidene-D-manno-octa-3-enopyranitol $(356)$}

A A solution of diene $353(112 \mathrm{mg}, 0.33 \mathrm{mmol})$ in dichloromethane $(5 \mathrm{~mL})$ was treated with $m$-CPBA (163 mg, $0.66 \mathrm{mmol})$. The mixture was stirred at room temperature for two days, then diluted with sat. sodium bicarbonate solution $(20 \mathrm{~mL})$ and water $(10 \mathrm{~mL})$, then diethyl ether $(30 \mathrm{~mL})$. The biphasic mixture was stirred rapidly for ten minutes, then the phases were separated. The ethereal fraction was washed with brine $(10 \mathrm{~mL})$, dried, filtered and concentrated to provide a colourless oil. Upon purification by column chromatography (3:1 hexanes:ethyl acetate), a mixture of epimeric epoxides $\mathbf{3 5 5}$ and $\mathbf{3 5 6}$ were obtained as a colourless oil (1.5:1 ratio, $57 \mathrm{mg}, 49 \%$ ), in addition to complicated mixtures of unidentified products.
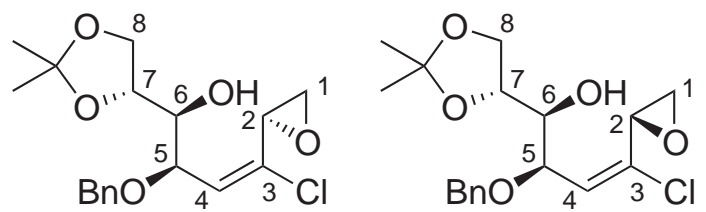

355 and 356: $\mathrm{R}_{f} 0.3$ (3:1 hexanes:ethyl acetate); ${ }^{1} \mathrm{H}-\mathrm{NMR}:\left(\mathrm{CDCl}_{3}\right) \delta_{\mathrm{H}} 7.41-7.29$ (complex m, 5H, Bn), 6.17 (d, $J=9.8 \mathrm{~Hz}$, $1 \mathrm{H}, \mathrm{H}-4), 6.14(\mathrm{~d}, J=9.8 \mathrm{~Hz}, 1 \mathrm{H}, \mathrm{H}-4)$, $4.68\left(\mathrm{~d}, J=11.4 \mathrm{~Hz}, 1 \mathrm{H}, \mathrm{PhCH}_{2}\right), 4.59(\mathrm{dd}, J=9.8,2.7 \mathrm{~Hz}, 1 \mathrm{H}, \mathrm{H}-5), 4.48$ (d, $J=$ $11.5 \mathrm{~Hz}, 1 \mathrm{H}, \mathrm{PhCH}_{2}$ ), 4.14-3.97 (complex m, 3H, H-7,8a,b), 3.72 (m, 1H, H-2), 3.66 (m, 1H, H-2), 3.53-3.48 (complex m, 1H, H-6), 3.05 (dd, J = 5.5, $2.3 \mathrm{~Hz}, 1 \mathrm{H}, \mathrm{H}-1 \mathrm{a})$, $2.99(\mathrm{dd}, J=5.5,2.4 \mathrm{~Hz}, 1 \mathrm{H}, \mathrm{H}-1 \mathrm{a}), 2.92$ (dd, $J=5.4,4.2 \mathrm{~Hz}, 1 \mathrm{H}, \mathrm{H}-1 \mathrm{~b}), 2.86$ (dd, $J=5.4,4.1 \mathrm{~Hz}, 1 \mathrm{H}, \mathrm{H}-1 \mathrm{~b}), 2.46$ (brs, 1H, OH), 1.39 (s, 3H, $\left.\left(\mathrm{CH}_{3}\right)_{2} \mathrm{C}\right), 1.37$ (s, $\left.3 \mathrm{H},\left(\mathrm{CH}_{3}\right)_{2} \mathrm{C}\right), 1.35\left(\mathrm{~s}, 3 \mathrm{H},\left(\mathrm{CH}_{3}\right)_{2} \mathrm{C}\right), 1.33\left(\mathrm{~s}, 3 \mathrm{H},\left(\mathrm{CH}_{3}\right)_{2} \mathrm{C}\right) ;{ }^{13} \mathrm{C}-\mathrm{NMR}:\left(\mathrm{CDCl}_{3}\right) \delta_{\mathrm{C}}$ 137.2 (C, 2 x Bn), 133.7 (C, C-3), 133.5 (C, C-3), 132.0 (CH, C-4), 131.3 (CH, C-4), 
128.5 (CH, Bn), 128.03 (CH, Bn), 128.00 (CH, Bn), $127.96(\mathrm{CH}, \mathrm{Bn}), 127.92(\mathrm{CH}$, $\mathrm{Bn}), 109.3\left(\mathrm{C},\left(\mathrm{CH}_{3}\right)_{2} \mathrm{C}\right), 109.2\left(\mathrm{C},\left(\mathrm{CH}_{3}\right)_{2} \mathrm{C}\right), 75.5(\mathrm{CH}, 2 \times \mathrm{C}-7), 75.2(\mathrm{CH}, \mathrm{C}-6)$, 75.0 (CH, C-6), 73.3 (CH, C-5), 73.1 (CH, C-5), $70.9\left(\mathrm{CH}_{2}, 2 \times \mathrm{PhCH}_{2}\right), 66.8\left(\mathrm{CH}_{2}\right.$, C-8), $66.7\left(\mathrm{CH}_{2}, \mathrm{C}-8\right), 49.4(\mathrm{CH}, \mathrm{C}-2), 48.7(\mathrm{CH}, \mathrm{C}-2), 46.6\left(\mathrm{CH}_{2}, \mathrm{C}-1\right), 46.5\left(\mathrm{CH}_{2}\right.$, $\mathrm{C}-1), 26.8\left(\mathrm{CH}_{3},\left(\mathrm{CH}_{3}\right)_{2} \mathrm{C}\right), 26.7\left(\mathrm{CH}_{3},\left(\mathrm{CH}_{3}\right)_{2} \mathrm{C}\right), 25.3\left(\mathrm{CH}_{3},\left(\mathrm{CH}_{3}\right)_{2} \mathrm{C}\right), 25.2\left(\mathrm{CH}_{3}\right.$, $\left.\left(\mathrm{CH}_{3}\right)_{2} \mathrm{C}\right)$; HRMS: $m / z \mathrm{C}_{18} \mathrm{H}_{23} \mathrm{O}_{5}^{35} \mathrm{ClNa}^{+}[\mathrm{M}+\mathrm{Na}]^{+}$calcd 377.1132 , found 377.1128.

B Using a known procedure, ${ }^{91}$ a solution of diene $\mathbf{3 5 3}(27 \mathrm{mg}, 80 \mu \mathrm{mol})$ in dichloromethane $(1 \mathrm{~mL})$ was treated with $(R, R)$-Jacobsen's catalyst 313 (2 mg, $3 \mu \mathrm{mol})$, then bleach $\left(0.55 \mathrm{molL}^{-1}, \mathrm{pH} 11.3,1 \mathrm{~mL}\right) .{ }^{91}$ The mixture was stirred at room temperature for two days, then separated between water $(20 \mathrm{~mL})$ and diethyl ether $(20 \mathrm{~mL})$. The ethereal fraction was washed with brine $(10 \mathrm{~mL})$, dried, filtered and concentrated to provide a brown oil. Upon purification by column chromatography (3:1 hexanes:ethyl acetate), a mixture of epimeric epoxides 355 and 356 were obtained (1.2:1 ratio, $5 \mathrm{mg}, 18 \%$ ), in addition to complex mixtures of unidentified material.

\section{$(E)$-2,6-Anhydro-5- $O$-benzyl-3-chloro-3,4-dideoxy-7,8- $O$-isopropylidene- D-gluco-octa-3-enopyranitol (357) and $(E)$-2,6-anhydro-5- $O$-benzyl-3- chloro-3,4-dideoxy-7,8-O-isopropylidene-D-manno-octa-3-enopyranitol (358)}

A A solution of epoxides 355 and 356 (69 $\mathrm{mg}, 0.19 \mathrm{mmol}$ ) in DMF (2 mL) was treated with sodium hydride $(11 \mathrm{mg}, 0.28 \mathrm{mmol})$. The mixture was stirred at room temperature for one day, then diluted with sat. copper sulfate solution $(10 \mathrm{~mL})$ and water $(10 \mathrm{~mL})$, then extracted with dichloromethane $(2 \times 20 \mathrm{~mL})$. The organic fractions were combined, dried, filtered and concentrated to provide a yellow oil. Upon purification by column chromatography (3:1 hexanes:ethyl acetate), a mixture of epimeric alcohols $\mathbf{3 5 7}$ and $\mathbf{3 5 8}$ were obtained as an inseparable pale-yellow oil (1.5:1 ratio, $16 \mathrm{mg}, 23 \%$ ), in addition to recovered starting material (24 $\mathrm{mg}, 35 \%$ ).

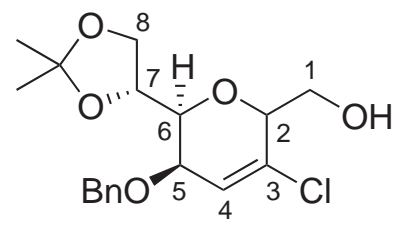

357 and 358: $\mathrm{R}_{f} 0.3$ (2:1 hexanes:ethyl acetate); ${ }^{1} \mathrm{H}-\mathrm{NMR}$ : $\left(\mathrm{CDCl}_{3}\right) \delta_{\mathrm{H}} 7.35-7.29$ (complex m, 5H, Bn), 6.29 (d, $J=6.3$ $\mathrm{Hz}, 1 \mathrm{H}, \mathrm{H}-4), 6.19$ (d, $J=5.9 \mathrm{~Hz}, 1 \mathrm{H}, \mathrm{H}-4), 4.694$ (d, $J=$ $11.7 \mathrm{~Hz}, 1 \mathrm{H}, \mathrm{PhCH}_{2}$ ), 4.688 (d, $J=11.7 \mathrm{~Hz}, 1 \mathrm{H} \mathrm{PhCH}_{2}$ ), $4.61\left(\mathrm{~d}, J=11.7 \mathrm{~Hz}, 2 \mathrm{H}, \mathrm{PhCH}_{2}\right.$ ), 4.38-4.33 (complex m, 3H, H-2,6), 4.25 (m, 1H, H-2), 4.16-4.10 (complex m, 2H, H-8a), 4.06-3.98 (complex m, 3H, H-5,8b), 3.91 (m, 1H, H-5), 3.89-3.86 (complex m, 5H, H-1a,b,7), 3.54 (d, J = 8.0 Hz, 1H, H-7), 1.89 (brs, 1H, OH), $1.41\left(\mathrm{~s}, 3 \mathrm{H},\left(\mathrm{CH}_{3}\right)_{2} \mathrm{C}\right), 1.40\left(\mathrm{~s}, 3 \mathrm{H},\left(\mathrm{CH}_{3}\right)_{2} \mathrm{C}\right), 1.39\left(\mathrm{~s}, 3 \mathrm{H},\left(\mathrm{CH}_{3}\right)_{2} \mathrm{C}\right)$, $1.38\left(\mathrm{~s}, 3 \mathrm{H},\left(\mathrm{CH}_{3}\right)_{2} \mathrm{C}\right) ;{ }^{13} \mathrm{C}-\mathrm{NMR}:\left(\mathrm{CDCl}_{3}\right) \delta_{\mathrm{C}} 138.0$ (C, Bn), 137.9 (C, Bn), 136.0 (C, C-3), 134.8 (C, C-3), 128.42 (CH, Bn), 128.37 (CH, Bn), 128.0 (CH, Bn), 127.9 
(CH, Bn), 127.8 (CH, Bn), 125.0 (CH, C-4), $123.6(\mathrm{CH}, \mathrm{C}-4), 109.2\left(\mathrm{C},\left(\mathrm{CH}_{3}\right)_{2} \mathrm{C}\right)$, $108.9\left(\mathrm{C},\left(\mathrm{CH}_{3}\right)_{2} \mathrm{C}\right), 78.1(\mathrm{CH}, \mathrm{C}-2), 77.6(\mathrm{CH}, \mathrm{C}-7), 77.0$ (CH, C-2), $74.2(\mathrm{CH}, \mathrm{C}-6)$, $73.5(\mathrm{CH}, \mathrm{C}-6), 72.4(\mathrm{CH}, \mathrm{C}-7), 71.6\left(\mathrm{CH}_{2}, \mathrm{PhCH}_{2}\right), 71.4\left(\mathrm{CH}_{2}, \mathrm{PhCH}_{2}\right), 69.29(\mathrm{CH}$, C-5), 69.26 (CH, C-5), $67.0\left(\mathrm{CH}_{2}, \mathrm{C}-8\right), 66.7\left(\mathrm{CH}_{2}, \mathrm{C}-8\right), 62.6\left(\mathrm{CH}_{2}, \mathrm{C}-1\right), 60.6\left(\mathrm{CH}_{2}\right.$, $\mathrm{C}-1), 26.9\left(\mathrm{CH}_{3},\left(\mathrm{CH}_{3}\right)_{2} \mathrm{C}\right), 26.6\left(\mathrm{CH}_{3},\left(\mathrm{CH}_{3}\right)_{2} \mathrm{C}\right), 25.4\left(\mathrm{CH}_{3},\left(\mathrm{CH}_{3}\right)_{2} \mathrm{C}\right), 25.3\left(\mathrm{CH}_{3}\right.$, $\left.\left(\mathrm{CH}_{3}\right)_{2} \mathrm{C}\right)$; IR $(\mathrm{KBr}): \nu_{\max } 3463,2988,2933,2874,1651,1455,1372,1255,1219$, 1121, 1072, 914, 850, 771, $698 \mathrm{~cm}^{-1}$; HRMS: $m / z \mathrm{C}_{18} \mathrm{H}_{23} \mathrm{O}_{5}^{35} \mathrm{ClNa}^{+}[\mathrm{M}+\mathrm{Na}]^{+}$calcd 377.1132 , found 377.1137 .

B Using a previously reported procedure, ${ }^{160}$ a solution of epoxides 355 and 356 (73 mg, $0.20 \mathrm{mmol})$ in methanol $(2 \mathrm{~mL})$ was treated with sodium (12 mg, $0.52 \mathrm{mmol})$, then stirred at room temperature for two days. The mixture was concentrated, then passed through a silica plug, which was eluted with dichloromethane $(10 \mathrm{~mL})$. The solution was dried and concentrated to provide a yellow oil. Upon purification by column chromatography (3:1 hexanes:ethyl acetate), a mixture of epimeric alcohols 357 and 358 were obtained as an inseparable pale-yellow oil (3.3:1 ratio, $31 \mathrm{mg}$, $42 \%$ ), in addition to minor amounts of unidentified products (13 mg).

\section{2,6-Anhydro-5- $O$-benzyl-3-chloro-1,3,4-trideoxy-1-iodo-7,8- $O$ - isopropylidene-D-manno-octa-1,3-dienopyranitol (359)}

A A solution of silver salt 351 (113 $\mathrm{mg}, 0.228 \mathrm{mmol})$ in dichloromethane $(2 \mathrm{~mL})$ was treated with iodine $(53 \mathrm{mg}, 0.21 \mathrm{mmol})$ and stirred at room temperature for five minutes, in which the iodine was consumed. A solution of diene 353 (50 mg, $0.15 \mathrm{mmol})$ in dichloromethane $(1 \mathrm{~mL})$ was added, then the solution was stirred at room temperature for 22 hours. The mixture was filtered through Celite ${ }^{\circledR}$ and eluted with dichloromethane $(20 \mathrm{~mL})$. The eluents were washed successively with sat. sodium thiosulfate solution $(20 \mathrm{~mL}), 10 \%$ aqueous hydrochloric acid $(20 \mathrm{~mL})$, then sat. sodium bicarbonate solution $(20 \mathrm{~mL})$. The organic fraction was dried, filtered and concentrated to provide a yellow oil. Upon purification by column chromatography (9:1 hexanes:ethyl acetate), iodopyranose sugar 359 (29 mg, 42\%) was obtained as a yellow oil, in addition to unreacted starting material 353 (19 mg, $38 \%)$.

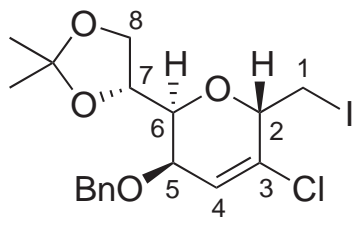

$\mathrm{R}_{f} 0.4$ (9:1 hexanes:ethyl acetate); $[\alpha]_{\mathrm{D}}^{22}-87$ ( $\left.c 0.45 \mathrm{CHCl}_{3}\right) ;$ ${ }^{1} \mathrm{H}-\mathrm{NMR}:\left(\mathrm{CDCl}_{3}\right) \delta_{\mathrm{H}} 7.38-7.29$ (complex m, 5H, Bn), 6.13 $(\mathrm{dd}, J=5.9,1.5 \mathrm{~Hz}, 1 \mathrm{H}, \mathrm{H}-4), 4.69\left(\mathrm{~s}, 2 \mathrm{H}, \mathrm{PhCH}_{2}\right), 4.41(\mathrm{~m}$, $1 \mathrm{H}, \mathrm{H}-2), 4.37$ (m, 1H, H-6), 4.25 (dd, $J=8.8,4.9 \mathrm{~Hz}, 1 \mathrm{H}$, H-8a), 4.18 (dd, $J=8.8,6.1 \mathrm{~Hz}, 1 \mathrm{H}, \mathrm{H}-8 \mathrm{~b}), 3.89$ (dd, $J=5.9,2.2 \mathrm{~Hz}, 1 \mathrm{H}, \mathrm{H}-5), 3.61$ $(\mathrm{dd}, J=8.6,2.2 \mathrm{~Hz}, 1 \mathrm{H}, \mathrm{H}-7), 3.58$ (dd, $J=11.5,2.7 \mathrm{~Hz}, 1 \mathrm{H}, \mathrm{H}-1 \mathrm{a}), 3.32$ (apparent t, $J=11.1 \mathrm{~Hz}, 1 \mathrm{H}, \mathrm{H}-1 \mathrm{~b}), 1.43\left(\mathrm{~s}, 3 \mathrm{H},\left(\mathrm{CH}_{3}\right)_{2} \mathrm{C}\right), 1.40\left(\mathrm{~s}, 3 \mathrm{H},\left(\mathrm{CH}_{3}\right)_{2} \mathrm{C}\right) ;{ }^{13} \mathrm{C}-\mathrm{NMR}$ : 
$\left(\mathrm{CDCl}_{3}\right) \delta_{\mathrm{C}} 138.0(\mathrm{C}, \mathrm{Bn}), 135.4(\mathrm{C}, \mathrm{C}-3), 128.4(\mathrm{CH}, \mathrm{Bn}), 128.0(\mathrm{CH}, \mathrm{Bn}), 127.8$ $(\mathrm{CH}, \mathrm{Bn}), 123.6(\mathrm{CH}, \mathrm{C}-4), 109.3\left(\mathrm{C},\left(\mathrm{CH}_{3}\right)_{2} \mathrm{C}\right), 76.1$ (CH, C-6), 73.2 (CH, C-2), $72.6(\mathrm{CH}, \mathrm{C}-7), 72.0\left(\mathrm{CH}_{2}, \mathrm{PhCH}_{2}\right), 69.3(\mathrm{CH}, \mathrm{C}-5), 67.8\left(\mathrm{CH}_{2}, \mathrm{C}-8\right), 27.0\left(\mathrm{CH}_{3}\right.$, $\left.\left(\mathrm{CH}_{3}\right)_{2} \mathrm{C}\right), 25.3\left(\mathrm{CH}_{3},\left(\mathrm{CH}_{3}\right)_{2} \mathrm{C}\right), 2.3\left(\mathrm{CH}_{2}, \mathrm{C}-1\right)$; IR $(\mathrm{KBr}): \nu_{\max } 2952,2930,2874$, 1723, 1652, 1454, 1422, 1335, 1185, 1131, 1083, 1048, 1013, 994, 917, 885, 860, 748, $694 \mathrm{~cm}^{-1}$; HRMS: $m / z \mathrm{C}_{18} \mathrm{H}_{22} \mathrm{O}_{4}^{35} \mathrm{ClINa}^{+}[\mathrm{M}+\mathrm{Na}]^{+}$calcd 487.0149 , found 487.0149 .

B A solution of iodide $359(60 \mathrm{mg}, 0.13 \mathrm{mmol})$ in THF $(2 \mathrm{~mL})$ was cooled to $-78{ }^{\circ} \mathrm{C}$, then treated with $n$-butyllithium $\left(80 \mu \mathrm{L}\right.$ of a $2.0 \mathrm{molL}^{-1}$ solution in cyclohexane, $0.16 \mathrm{mmol}$ ). The mixture was stirred for 30 minutes, then warmed to $0{ }^{\circ} \mathrm{C}$ and stirred for an additional 30 minutes. Carbon dioxide was bubbled through the solution for 30 minutes, then the solution was quenched with sat. ammonium chloride solution $(10 \mathrm{ml})$, then extracted with dichloromethane $(3 \times 10 \mathrm{~mL})$. The organic fractions were combined, dried, filtered and concentrated to provide a yellow oil. Upon purification by column chromatography (9:1 hexanes:ethyl acetate), starting material 359 (38 mg, 63\%) was obtained, in addition to diene $\mathbf{3 5 3}$ (12 mg, 27\%).

\section{Ethyl $(2 E, 4 E)$-6- $O$-benzyl-4-chloro-2,3,4,5-tetradeoxy-8,9- $O$ - isopropylidene-D-arabino-nona-2,4-dienoate (360)}

A solution of hemiacetal 345 (36 mg, $0.11 \mathrm{mmol})$ in THF $(1 \mathrm{~mL})$ was treated with (ethoxycarbonylmethylene)triphenylphosphorane (112 mg, $0.321 \mathrm{mmol}$ ) and stirred at room temperature overnight, then heated at reflux for 24 hours. The reaction mixture was diluted with sat. ammonium chloride solution $(10 \mathrm{ml})$ and extracted with dichloromethane $(3 \times 10 \mathrm{~mL})$. The organic fractions were combined, dried, filtered and concentrated to provide a crude yellow oil. Upon purification by column chromatography (3:1 hexanes:ethyl acetate), diene $\mathbf{3 6 0}$ (27 mg, 63\%) was obtained as a colourless oil.

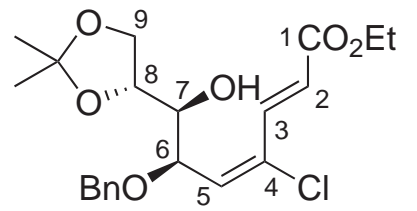

$\mathrm{R}_{f} 0.3$ (5:1 hexanes:ethyl acetate); $[\alpha]_{\mathrm{D}}^{23}-74\left(\right.$ c $\left.0.3 \mathrm{CHCl}_{3}\right)$; ${ }^{1} \mathrm{H}-\mathrm{NMR}:\left(\mathrm{CDCl}_{3}\right) \delta_{\mathrm{H}} 7.55(\mathrm{~d}, J=14.7 \mathrm{~Hz}, 1 \mathrm{H}, \mathrm{H}-3), 7.37-$ 7.29 (complex m, 5H, Bn), 6.41 (d, $J=14.7 \mathrm{~Hz}, 1 \mathrm{H}, \mathrm{H}-2)$, $6.27(\mathrm{dd}, J=9.7,0.5 \mathrm{~Hz}, 1 \mathrm{H}, \mathrm{H}-5), 4.61(\mathrm{~d}, J=11.5 \mathrm{~Hz}$, $1 \mathrm{H}, \mathrm{PhCH}_{2}$ ), 4.58 (d, $\left.J=2.7 \mathrm{~Hz}, 1 \mathrm{H}, \mathrm{H}-6\right), 4.44$ (d, $J=11.5 \mathrm{~Hz}, 1 \mathrm{H}, \mathrm{PhCH}_{2}$ ), 4.24 (qd, $J=7.1,1.1 \mathrm{~Hz}, 2 \mathrm{H}, \mathrm{Et}), 4.12-4.04$ (complex m, 2H, H-8,9a), 3.98 (dd, $J=$ $8.4,5.0 \mathrm{~Hz}, 1 \mathrm{H}, \mathrm{H}-9 \mathrm{~b}), 3.47$ (td, $J=8.0,2.6 \mathrm{~Hz}, 1 \mathrm{H}, \mathrm{H}-7), 2.43$ (d, $J=8.5 \mathrm{~Hz}, 1 \mathrm{H}$, $\mathrm{OH}), 1.39\left(\mathrm{~s}, 3 \mathrm{H},\left(\mathrm{CH}_{3}\right)_{2} \mathrm{C}\right), 1.34\left(\mathrm{~s}, 3 \mathrm{H},\left(\mathrm{CH}_{3}\right)_{2} \mathrm{C}\right), 1.31$ (t, $\left.J=7.1 \mathrm{~Hz}, 3 \mathrm{H}, \mathrm{Et}\right)$; ${ }^{13} \mathrm{C}-\mathrm{NMR}:\left(\mathrm{CDCl}_{3}\right) \delta_{\mathrm{C}} 166.0$ (C, C-1), 137.0 (C, Bn), 135.4 (CH, C-5), 134.9 (CH, C-3), 132.1 (C, C-4), 128.5 (CH, Bn), 128.1 (CH, Bn), 128.0 (CH, Bn), 124.7 (CH, C-2), 109.4 (C, $\left.\left(\mathrm{CH}_{3}\right)_{2} \mathrm{C}\right), 75.3$ (CH, C-8), 75.1 (CH, C-7), 73.8 (CH, C-6), 71.2 $\left(\mathrm{CH}_{2}, \mathrm{PhCH}_{2}\right), 66.8\left(\mathrm{CH}_{2}, \mathrm{C}-9\right), 60.9\left(\mathrm{CH}_{2}, \mathrm{Et}\right), 26.8\left(\mathrm{CH}_{3},\left(\mathrm{CH}_{3}\right)_{2} \mathrm{C}\right), 25.3\left(\mathrm{CH}_{3}\right.$, $\left.\left(\mathrm{CH}_{3}\right)_{2} \mathrm{C}\right), 14.2\left(\mathrm{CH}_{3}, \mathrm{Et}\right)$; IR $(\mathrm{KBr}): \nu_{\max } 3443,2985,2933,1716,1635,1455,1371$, 
1303, 1266, 1182, 1070, 964, 848, 740, $699 \mathrm{~cm}^{-1}$; HRMS: $m / z \quad \mathrm{C}_{21} \mathrm{H}_{27} \mathrm{O}_{6}^{35} \mathrm{ClNa}^{+}$ $[\mathrm{M}+\mathrm{Na}]^{+}$calcd 433.1394, found 433.1391.

\section{Ethyl 3,7-anhydro-6- $O$-benzyl-4-chloro-2,4,5-trideoxy-8,9- $O$ -} isopropylidene-D-manno-non-4-enoate (361) and ethyl 3,7-anhydro-6- $O$ benzyl-4-chloro-2,4,5-trideoxy-8,9- $O$-isopropylidene-D-gluco-non-4enoate (362)

A A solution of $\alpha, \beta$ unsaturated ester 360 (47 mg, $0.11 \mathrm{mmol})$ in THF $(2 \mathrm{~mL})$ was treated with sodium hydride $(10 \mathrm{mg}, 0.25 \mathrm{mmol})$, then stirred at room temperature for 15 minutes. The reaction mixture was diluted with sat. ammonium chloride solution $(10 \mathrm{ml})$ and extracted with dichloromethane $(2 \times 10 \mathrm{~mL})$. The organic fractions were combined, dried, filtered and concentrated to provide a crude yellow oil. Upon purification by column chromatography (9:1 hexanes:ethyl acetate), ringclosed products 361 and $\mathbf{3 6 2}$ were obtained as an inseparable colourless oil (1:1.2 ratio, $44 \mathrm{mg}, 94 \%)$.

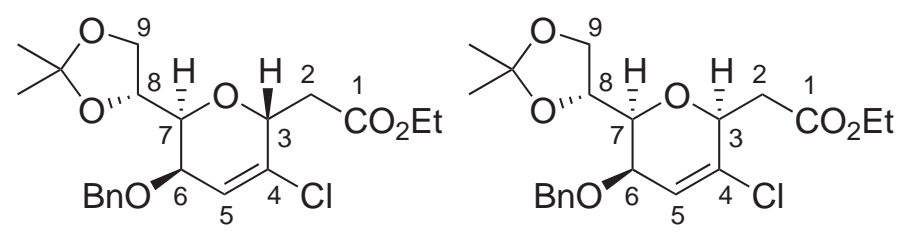

361 and $362: \mathrm{R}_{f} 0.3(5: 1$ hexanes:ethyl acetate); ${ }^{1} \mathrm{H}-\mathrm{NMR}$ : $\left(\mathrm{CDCl}_{3}\right) \delta_{\mathrm{H}} 7.42-7.27$ (complex $\mathrm{m}, 5 \mathrm{H}, \mathrm{Bn}), 6.11(\mathrm{~m}, 1 \mathrm{H}, \mathrm{H}-5)$, 4.76 (ddd, $J=9.3,3.1,1.5 \mathrm{~Hz}, 1 \mathrm{H}, \mathrm{H}-3$ (361)), 4.69-4.64 (complex m, 2H, $\mathrm{PhCH}_{2}$ ), 4.59 (ddd, $J=7.6,3.2,1.6$ Hz, 1H, H-3 (362)), 4.34 (m, 1H, H-8), 4.22-4.13 (complex m, 2H, Et), 4.08 (d, $J=8.6 \mathrm{~Hz}, 1 \mathrm{H}, \mathrm{H}-9 \mathrm{a}), 4.06$ (d, $J=8.5 \mathrm{~Hz}, 1 \mathrm{H}, \mathrm{H}-9 \mathrm{a})$, 3.99-3.95 (complex m, 2H, H-6,9b), 3.91 (dd, $J=8.5,5.4$ Hz, 1H, H-9b), 3.58 (dd, $J=8.3,2.2 \mathrm{~Hz}, 1 \mathrm{H}, \mathrm{H}-7$ (361)), 3.51 (dd, $J=8.1,2.0 \mathrm{~Hz}, 1 \mathrm{H}, \mathrm{H}-7$ (362)), 2.98 (dd, $J=15.6,3.5 \mathrm{~Hz}, 1 \mathrm{H}, \mathrm{H}-2 \mathrm{a}(\mathbf{3 6 2})), 2.79(\mathrm{dd}, J=14.9,3.0 \mathrm{~Hz}, 1 \mathrm{H}, \mathrm{H}-2 \mathrm{a}(\mathbf{3 6 1}))$, $2.67(\mathrm{dd}, J=14.9,10.8 \mathrm{~Hz}, 1 \mathrm{H}, \mathrm{H}-2 \mathrm{~b}(\mathbf{3 6 1})), 2.58(\mathrm{dd}, J=15.6,9.3 \mathrm{~Hz}, 1 \mathrm{H}$, $\mathrm{H}-2 \mathrm{~b}(\mathbf{3 6 2})), 1.40\left(\mathrm{~s}, 3 \mathrm{H},\left(\mathrm{CH}_{3}\right)_{2} \mathrm{C}\right), 1.39\left(\mathrm{~s}, 3 \mathrm{H},\left(\mathrm{CH}_{3}\right)_{2} \mathrm{C}\right), 1.38\left(\mathrm{~s}, 3 \mathrm{H},\left(\mathrm{CH}_{3}\right)_{2} \mathrm{C}\right)$, $1.37\left(\mathrm{~s}, 3 \mathrm{H},\left(\mathrm{CH}_{3}\right)_{2} \mathrm{C}\right), 1.28$ (t, $\left.J=7.1 \mathrm{~Hz}, 3 \mathrm{H}, \mathrm{Et}\right), 1.25$ (t, $\left.J=7.1 \mathrm{~Hz}, 3 \mathrm{H}, \mathrm{Et}\right)$; ${ }^{13} \mathrm{C}-\mathrm{NMR}:\left(\mathrm{CDCl}_{3}\right) \delta_{\mathrm{C}} 170.3$ (C, C-1), 170.1 (C, C-1), 138.2 (C, Bn), 138.1 (C, Bn), 137.4 (C, C-4), 136.6 (C, C-4), 128.3 (CH, Bn), 128.1 (CH, Bn), 128.0 (CH, Bn), 127.9 (CH, Bn), 127.8 (CH, Bn), 123.8 (CH, C-5), 123.1 (CH, C-5), 109.2 (C, $\left.\left(\mathrm{CH}_{3}\right)_{2} \mathrm{C}\right), 109.1\left(\mathrm{C},\left(\mathrm{CH}_{3}\right)_{2} \mathrm{C}\right), 78.4(\mathrm{CH}, \mathrm{C}-7(\mathbf{3 6 2})), 74.8(\mathrm{CH}, \mathrm{C}-3(\mathbf{3 6 2})), 73.6$ (CH, C-3 (361)), 73.4 (CH, C-8), 73.2 (CH, C-8), 72.6 (CH, C-7 (361)), $71.8\left(\mathrm{CH}_{2}\right.$, $\left.\mathrm{PhCH}_{2}\right), 71.5\left(\mathrm{CH}_{2}, \mathrm{PhCH}_{2}\right), 69.5(\mathrm{CH}, \mathrm{C}-6), 69.2(\mathrm{CH}, \mathrm{C}-6), 67.2\left(\mathrm{CH}_{2}, \mathrm{C}-9\right), 66.9$ $\left(\mathrm{CH}_{2}, \mathrm{C}-9\right), 61.0\left(\mathrm{CH}_{2}, \mathrm{Et}\right), 60.7\left(\mathrm{CH}_{2}, \mathrm{Et}\right), 38.4\left(\mathrm{CH}_{2}, \mathrm{C}-2(\mathbf{3 6 2})\right), 35.4\left(\mathrm{CH}_{2}, \mathrm{C}-2\right.$ (361)), $26.86\left(\mathrm{CH}_{3},\left(\mathrm{CH}_{3}\right)_{2} \mathrm{C}\right), 26.84\left(\mathrm{CH}_{3},\left(\mathrm{CH}_{3}\right)_{2} \mathrm{C}\right), 25.4\left(\mathrm{CH}_{3},\left(\mathrm{CH}_{3}\right)_{2} \mathrm{C}\right), 25.3$ $\left(\mathrm{CH}_{3},\left(\mathrm{CH}_{3}\right)_{2} \mathrm{C}\right), 24.8\left(\mathrm{CH}_{3}, \mathrm{Et}\right), 14.2\left(\mathrm{CH}_{3}, \mathrm{Et}\right)$; IR $(\mathrm{KBr}): \nu_{\max } 2986,2934,2885$, 1734, 1652, 1455, 1372, 1272, 1181, 1095, 1072, 847, 737, $698 \mathrm{~cm}^{-1}$; HRMS: $\mathrm{m} / z$ $\mathrm{C}_{21} \mathrm{H}_{27} \mathrm{O}_{6}^{35} \mathrm{ClNa}^{+}[\mathrm{M}+\mathrm{Na}]^{+}$calcd 433.1394, found 433.1386 . 
B A solution of $\alpha, \beta$ unsaturated ester 360 (21 $\mathrm{mg}, 0.05 \mathrm{mmol})$ in THF $(2 \mathrm{~mL})$ was cooled to $0{ }^{\circ} \mathrm{C}$, then treated with sodium hydride $(5 \mathrm{mg}, 0.1 \mathrm{mmol})$. The reaction was stirred for one hour at $0{ }^{\circ} \mathrm{C}$, then quenched with sat. ammonium chloride solution $(10 \mathrm{ml})$ and extracted with dichloromethane $(2 \times 10 \mathrm{~mL})$. The organic fractions were combined, dried, filtered and concentrated to provide a crude yellow oil. Upon purification by column chromatography (9:1 hexanes:ethyl acetate), ringclosed products 361 and 362 (4:1 ratio) were obtained (11 mg, 55\%).

C A solution of diisopropyl amine $(20 \mu \mathrm{L}, 0.14 \mathrm{mmol})$ in THF (1 mL) was cooled to $-78{ }^{\circ} \mathrm{C}$, then treated with $n$-butyllithium $\left(70 \mu \mathrm{L}\right.$ of a $2.0 \mathrm{molL}^{-1}$ solution in cyclohexane, $0.14 \mathrm{mmol}$ ) and stirred for 30 minutes to generate a solution of LDA. This solution was then treated with a solution of ring-closed pyranosyl sugars 361 and 362 (4:1 ratio, $30 \mathrm{mg}, 73 \mu \mathrm{mol})$ in THF $(1 \mathrm{~mL})$ and allowed to react for 20 minutes. The mixture was treated with iodine $(39 \mathrm{mg}, 0.15 \mathrm{mmol})$, then the solution was stirred at $-78{ }^{\circ} \mathrm{C}$ for 30 minutes. The mixture was warmed to room temperature and stirred for 30 minutes, then the reaction mixture was diluted with dichloromethane $(20 \mathrm{~mL})$, then washed sequentially with sat. sodium thiosulfate solution $(20 \mathrm{ml}), 10 \%$ aqueous hydrochloric acid $(20 \mathrm{~mL})$ and sat. sodium bicarbonate solution $(20 \mathrm{~mL})$. The organic fraction was dried, filtered and concentrated to provide a crude yellow oil. Upon purification by column chromatography (5:1 hexanes:ethyl acetate), ring-closed products 361 and 362 (4:1 ratio) were obtained (18 mg, 60\%), in addition to previously isolated diene $\mathbf{3 6 0}$ (9 $\mathrm{mg}, 30 \%)$.

\section{Diethyl methylsulfonylmethylphosphonate (366)}

A solution of diethyl methylthiomethylphosphonate $(1.99 \mathrm{~g}, 10.0 \mathrm{mmol})$ in ethanol $(50 \mathrm{~mL})$ was treated successively with a $30 \%$ aqueous solution of hydrogen peroxide $(10 \mathrm{~mL}, 100 \mathrm{mmol})$ and ammonium heptamolybdate tetrahydrate $(1.24 \mathrm{~g}$, $1.00 \mathrm{mmol}$ ), then stirred at room temperature for one hour. The mixture was then diluted with water $(200 \mathrm{~mL})$ and extracted with dichloromethane $(3 \times 100 \mathrm{~mL})$, to provide diethyl methylsulfonylmethylphosphonate (366) as a white solid $(2.22 \mathrm{~g}$, $96 \%)$.

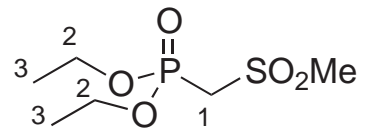

m.p. 89-91 ${ }^{\circ} \mathrm{C}$ (lit. ${ }^{161}$ m.p. $\left.96{ }^{\circ} \mathrm{C}\right) ;{ }^{1} \mathrm{H}-\mathrm{NMR}:\left(\mathrm{CDCl}_{3}\right) \delta_{\mathrm{H}} 4.24$ (apparent quintet, $J=7.1 \mathrm{~Hz}, 4 \mathrm{H}, \mathrm{H}-2), 3.59\left(\mathrm{~d}, J_{\mathrm{P}-\mathrm{H}}=16.3\right.$ $\mathrm{Hz}, 2 \mathrm{H}, \mathrm{H}-1$ ), 3.21 (s, 3H, Me), 1.37 (t, $J=7.1 \mathrm{~Hz}, 6 \mathrm{H}, \mathrm{H}-3$ );

${ }^{13} \mathrm{C}-\mathrm{NMR}:\left(\mathrm{CDCl}_{3}\right) \delta_{\mathrm{C}} 63.8\left(\mathrm{~d}, J_{\mathrm{C}-\mathrm{P}}=6.7 \mathrm{~Hz}, \mathrm{CH}_{2}, \mathrm{C}-2\right), 52.3\left(\mathrm{~d}, J_{\mathrm{C}-\mathrm{P}}=6.7 \mathrm{~Hz}\right.$, $\left.\mathrm{CH}_{2}, \mathrm{C}-1\right), 42.6\left(\mathrm{CH}_{3}, \mathrm{Me}\right), 16.3\left(\mathrm{~d}, J_{\mathrm{C}-\mathrm{P}}=6.2 \mathrm{~Hz}, \mathrm{CH}_{3}, \mathrm{C}-3\right) ;{ }^{31} \mathrm{P}-\mathrm{NMR}:\left(\mathrm{CDCl}_{3}\right)$ $\delta_{\mathrm{P}} 11.6(\mathrm{~s}, 1 \mathrm{P})$. 
2,6-Anhydro-5- $O$-benzyl-3-chloro-1,3,4-trideoxy-7,8- $O$-isopropylidene-1[(methyl)sulfonyl]-D-gluco-oct-3-enopyranitol (371) and 2,6-anhydro-5- $O$ benzyl-3-chloro-1,3,4-trideoxy-7,8- $O$-isopropylidene-1-[(methyl)sulfonyl]D-manno-oct-3-enopyranitol (367)

A A solution of sulfone $366(274 \mathrm{mg}, 1.19 \mathrm{mmol})$ in THF $(6 \mathrm{~mL})$ was treated with sodium hydride $(46 \mathrm{mg}, 1.2 \mathrm{mmol})$, then stirred at $0{ }^{\circ} \mathrm{C}$ for 15 minutes. To this was added hemiacetal 345 (249 $\mathrm{mg}, 0.73 \mathrm{mmol})$ in THF $(3 \mathrm{~mL})$, then the mixture was allowed to warm to room temperature and reacted for two hours. The reaction mixture was diluted with sat. ammonium chloride solution $(20 \mathrm{ml})$, then extracted with dichloromethane $(3 \times 20 \mathrm{~mL})$. The organic fractions were combined, dried, filtered and concentrated to provide a crude yellow oil. Upon purification by column chromatography (1:1 hexanes:ethyl acetate), pyranosyl sugar 371 (15 mg, $5 \%$ ) was obtained as a colourless oil and pyranosyl sugar 367 (220 mg, 72\%) was obtained as a white crystalline solid (77\% combined yield).

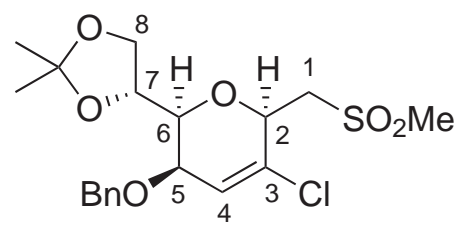

371: $\mathrm{R}_{f} 0.4$ (1:1 hexanes:ethyl acetate); $[\alpha]_{\mathrm{D}}^{21}-70$ ( $c 0.7$ $\left.\mathrm{CHCl}_{3}\right) ;{ }^{1} \mathrm{H}-\mathrm{NMR}:\left(\mathrm{CDCl}_{3}\right) \delta_{\mathrm{H}} 7.40-7.30$ (complex m, $5 \mathrm{H}, \mathrm{Bn}), 6.23$ (dd, $J=6.3,1.6 \mathrm{~Hz}, 1 \mathrm{H}, \mathrm{H}-4), 4.72-4.67$ (complex m, 2H, $\mathrm{PhCH}_{2}, \mathrm{H}-2$ ), 4.61 (d, $J=11.7 \mathrm{~Hz}, 1 \mathrm{H}$, $\mathrm{PhCH}_{2}$ ), 4.34 (dd, $J=11.7,6.3 \mathrm{~Hz}, 1 \mathrm{H}, \mathrm{H}-7$ ), 4.09-4.02 (complex m, 2H, H-8a,b), 3.96 (apparent dt, $J=6.1,2.0 \mathrm{~Hz}, 1 \mathrm{H}, \mathrm{H}-5$ ), 3.65-3.59 (complex m, 2H, H-1a,6), $3.34(\mathrm{dd}, J=15.1,10.0 \mathrm{~Hz}, 1 \mathrm{H}, \mathrm{H}-1 \mathrm{~b}), 3.02$ (s, 3H, Me), 1.41 (s, 3H, $\left(\mathrm{CH}_{3}\right)_{2} \mathrm{C}$ ), $1.36\left(\mathrm{~s}, 3 \mathrm{H},\left(\mathrm{CH}_{3}\right)_{2} \mathrm{C}\right) ;{ }^{13} \mathrm{C}-\mathrm{NMR}:\left(\mathrm{CDCl}_{3}\right) \delta_{\mathrm{C}} 137.7$ (C, Bn), $134.9(\mathrm{CH}, \mathrm{C}-3), 128.5$ (CH, Bn), 128.05 (CH, Bn), 127.98 (CH, Bn), $124.9(\mathrm{CH}, \mathrm{C}-4), 109.2\left(\mathrm{C},\left(\mathrm{CH}_{3}\right)_{2} \mathrm{C}\right)$, 78.4 (CH, C-6), 73.8 (CH, C-2), $73.5(\mathrm{CH}, \mathrm{C}-7), 71.8\left(\mathrm{CH}_{2}, \mathrm{PhCH}_{2}\right), 69.2(\mathrm{CH}$, C-5), $66.4\left(\mathrm{CH}_{2}, \mathrm{C}-8\right), 57.3\left(\mathrm{CH}_{2}, \mathrm{C}-1\right), 43.5\left(\mathrm{CH}_{3}, \mathrm{Me}\right), 26.7\left(\mathrm{CH}_{3},\left(\mathrm{CH}_{3}\right)_{2} \mathrm{C}\right), 24.8$ $\left(\mathrm{CH}_{3},\left(\mathrm{CH}_{3}\right)_{2} \mathrm{C}\right)$; IR $(\mathrm{KBr}): \nu_{\max } 3027,2987,2933,2868,1646,1455,1372,1305$, 1133, 1071, 1044, 963, 861, 771, 753, $699 \mathrm{~cm}^{-1}$.

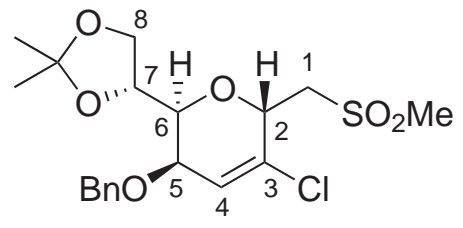

367: $\mathrm{R}_{f} 0.3$ (1:1 hexanes:ethyl acetate);

m.p. $109-112{ }^{\circ} \mathrm{C} ;[\alpha]_{\mathrm{D}}^{19}-69\left(\right.$ c $\left.0.3 \mathrm{CHCl}_{3}\right) ;{ }^{1} \mathrm{H}-\mathrm{NMR}$ : $\left(\mathrm{CDCl}_{3}\right) \delta_{\mathrm{H}} 7.38-7.31$ (complex m, 5H, Bn), 6.22 (dd, $J=5.8,1.6 \mathrm{~Hz}, 1 \mathrm{H}, \mathrm{H}-4), 4.95(\mathrm{dt}, J=8.6,1.5 \mathrm{~Hz}, 1 \mathrm{H}$, $\mathrm{H}-2), 4.68\left(\mathrm{~d}, J=11.4 \mathrm{~Hz}, 1 \mathrm{H}, \mathrm{PhCH}_{2}\right), 4.59$ (d, $\left.J=11.5 \mathrm{~Hz}, 1 \mathrm{H}, \mathrm{PhCH}_{2}\right), 4.32$ (dd, $J=12.0,6.4 \mathrm{~Hz}, 1 \mathrm{H}, \mathrm{H}-7$ ), 4.07-4.05 (complex m, 2H, H-8a,b), 3.93 (dd, $J=5.7$, $2.5 \mathrm{~Hz}, 1 \mathrm{H}, \mathrm{H}-5), 3.80$ (dd, $J=5.5,2.3 \mathrm{~Hz}, 1 \mathrm{H}, \mathrm{H}-6), 3.42(\mathrm{dd}, J=15.2,10.0 \mathrm{~Hz}$, $1 \mathrm{H}, \mathrm{H}-1 \mathrm{a}), 3.31$ (d, J = 15.1 Hz, 1H, H-1b), 3.04 (s, 3H, Me), 1.37 (s, 3H, $\left(\mathrm{CH}_{3}\right)_{2} \mathrm{C}$ ), 1.35 (s, 3H, $\left.\left(\mathrm{CH}_{3}\right)_{2} \mathrm{C}\right) ;{ }^{13} \mathrm{C}-\mathrm{NMR}:\left(\mathrm{CDCl}_{3}\right) \delta_{\mathrm{C}} 137.5$ (C, Bn), $134.5(\mathrm{CH}, \mathrm{C}-3), 128.5$ (CH, Bn), 128.1 (CH, Bn), 128.0 (CH, Bn), 124.0 (CH, C-4), 108.5 (C, $\left.\left(\mathrm{CH}_{3}\right)_{2} \mathrm{C}\right)$, 74.3 (CH, C-7), 72.1 (CH, C-2), 71.8 (CH, C-6), $71.7\left(\mathrm{CH}_{2}, \mathrm{PhCH}_{2}\right), 68.9(\mathrm{CH}, \mathrm{C}-5)$, $66.0\left(\mathrm{CH}_{2}, \mathrm{C}-8\right), 53.2\left(\mathrm{CH}_{2}, \mathrm{C}-1\right), 42.5\left(\mathrm{CH}_{3}, \mathrm{Me}\right), 26.7\left(\mathrm{CH}_{3},\left(\mathrm{CH}_{3}\right)_{2} \mathrm{C}\right), 25.1\left(\mathrm{CH}_{3}\right.$, 
$\left.\left(\mathrm{CH}_{3}\right)_{2} \mathrm{C}\right)$; IR (KBr): $\nu_{\max } 3027,2987,2933,2896,1655,1456,1372,1338,1306$, 1133, 1091, 1070, 1044, 926, 860, 755, $699 \mathrm{~cm}^{-1}$; HRMS: $m / z \mathrm{C}_{19} \mathrm{H}_{25} \mathrm{O}_{6} \mathrm{~S}^{35} \mathrm{ClNa}^{+}$ $[\mathrm{M}+\mathrm{Na}]^{+}$calcd 439.0958, found 439.0953.

B A solution of sulfone 366 (87 mg, $0.38 \mathrm{mmol})$ in THF $(2 \mathrm{~mL})$ was treated with sodium hydride $(15 \mathrm{mg}, 0.38 \mathrm{mmol})$, then stirred at $0{ }^{\circ} \mathrm{C}$ for 30 minutes. To this was added hemiacetal 345 (93 $\mathrm{mg}, 0.27 \mathrm{mmol})$ in THF $(2 \mathrm{~mL})$, then the mixture was stirred at $0{ }^{\circ} \mathrm{C}$ for two hours. Upon completion of the HWE reaction (as determined by TLC), iodine (149 $\mathrm{mg}, 0.587 \mathrm{mmol}$ ) was added, then the reaction was stirred at room temperature for one hour. The reaction mixture was diluted with sat. sodium thiosulfate solution $(10 \mathrm{ml})$ and water $(10 \mathrm{~mL})$, then extracted with dichloromethane $(2 \times 20 \mathrm{~mL})$. The organic fractions were combined, dried, filtered and concentrated to provide a crude sample of pyranosyl sugar 367 .

C A solution of sulfone 366 (116 $\mathrm{mg}, 0.504 \mathrm{mmol})$ in THF $(5 \mathrm{~mL})$ was treated with sodium hydride $(22 \mathrm{mg}, 0.55 \mathrm{mmol})$, then stirred at $0{ }^{\circ} \mathrm{C}$ for 30 minutes. To this was added hemiacetal 345 (129 $\mathrm{mg}, 0.378 \mathrm{mmol})$ in THF (2 mL), then the mixture was stirred at $0{ }^{\circ} \mathrm{C}$ for three hours. Upon completion of the HWE reaction (as determined by TLC), iodine (149 $\mathrm{mg}, 0.587 \mathrm{mmol}$ ) was added, followed by a further portion of sodium hydride $(16 \mathrm{mg}, 0.4 \mathrm{mmol})$, then the reaction was stirred at room temperature for one hour. The reaction mixture was diluted with sat. sodium thiosulfate solution $(10 \mathrm{ml})$ and water $(10 \mathrm{~mL})$, then extracted with dichloromethane $(2 \times 20 \mathrm{~mL})$. The organic fractions were combined, dried, filtered and concentrated to provide a crude yellow oil. Upon purification by column chromatography (1:1 hexanes:ethyl acetate), pyranosyl sugar 367 (100 mg, 70\%) was obtained.

D A solution of sugar sulfone $\mathbf{3 6 7}(29 \mathrm{mg}, 70 \mu \mathrm{mol})$ in THF $(1 \mathrm{~mL})$ was cooled to $0{ }^{\circ} \mathrm{C}$, then treated with NIS (23 mg, $0.10 \mathrm{mmol}$ ). The solution was slowly treated with KHMDS $(170 \mu \mathrm{L}, 85 \mu \mathrm{mol})$ over a period of 15 minutes. The mixture was stirred at $0{ }^{\circ} \mathrm{C}$ for an hour, then stirred at room temperature for two hours. The reaction mixture was diluted with sat. sodium thiosulfate solution (10 ml) and water $(10 \mathrm{~mL})$, then extracted with dichloromethane $(2 \times 20 \mathrm{~mL})$. The organic fractions were combined, dried, filtered and concentrated to provide a crude mixture of NIS and starting material sugar 367 .

E A solution of diisopropyl amine $(20 \mu \mathrm{L}, 0.14 \mathrm{mmol})$ in THF $(1 \mathrm{~mL})$ was cooled to $0{ }^{\circ} \mathrm{C}$, then treated with $n$-butyllithium $\left(60 \mu \mathrm{L}\right.$ of a $2.0 \mathrm{molL}^{-1}$ solution in cyclohexane, $0.12 \mathrm{mmol}$ ) and stirred for 15 minutes to generate a solution of LDA. This solution was then treated with a solution of sugar sulfone $\mathbf{3 6 7}$ (42 $\mathrm{mg}, 0.10 \mathrm{mmol}$ ) in THF $(1 \mathrm{~mL})$. The solution was allowed to react for 15 minutes, then treated with 
NBS (32 mg, $0.18 \mathrm{mmol}$ ). The mixture was stirred at $0{ }^{\circ} \mathrm{C}$ for an additional hour, then stirred at room temperature for two hours. The reaction mixture was diluted with sat. sodium thiosulfate solution $(10 \mathrm{ml})$ and water $(10 \mathrm{~mL})$, then extracted with dichloromethane $(2 \times 20 \mathrm{~mL})$. The organic fractions were combined, dried, filtered and concentrated to provide a crude mixture of NBS and starting material sugar 367.

F A solution of sulfone 367 (47 mg, $0.11 \mathrm{mmol})$ in tert-butanol $(0.5 \mathrm{~mL})$ and dichloromethane $(1 \mathrm{~mL})$ was treated with $\mathrm{KOH}-\mathrm{Al}_{2} \mathrm{O}_{3}(212 \mathrm{mg}, 0.9 \mathrm{mmol})$ and dibromodifluoromethane $(50 \mu \mathrm{L}, 0.5 \mathrm{mmol})$, then stirred at $5{ }^{\circ} \mathrm{C}$ for one hour. The mixture was warmed to room temperature, then stirred for one hour. Additional dibromodifluoromethane $(50 \mu \mathrm{L}, 0.5 \mathrm{mmol})$ was introduced, then the mixture was stirred for one hour. The reaction was heated to reflux, then a further portion of dibromodifluoromethane $(50 \mu \mathrm{L}, 0.5 \mathrm{mmol})$ was flashed onto the reaction mixture. After 30 minutes additional stirring, the reaction mixture was diluted with water $(20 \mathrm{ml})$ and extracted with diethyl ether $(2 \times 20 \mathrm{~mL})$. The organic fractions were combined, washed with brine $(10 \mathrm{~mL})$, then dried, filtered and concentrated to provide a crude yellow oil. Upon purification by column chromatography (3:1 hexanes:ethyl acetate), a complex mixture of products was obtained ( $5 \mathrm{mg}$ ), in addition to pyranosyl sugar 367 (27 mg, 57\%).

G A solution of sulfone 367 (57 mg, $0.14 \mathrm{mmol})$ in tert-butanol $(0.5 \mathrm{~mL})$ and dichloromethane $(1 \mathrm{~mL})$ was treated with $\mathrm{KOH}-\mathrm{Al}_{2} \mathrm{O}_{3}(286 \mathrm{mg}, 1.2 \mathrm{mmol})$ and dibromodifluoromethane $(50 \mu \mathrm{L}, 0.5 \mathrm{mmol})$, then stored in the fridge for eight hours. Additional dibromodifluoromethane $(50 \mu \mathrm{L}, 0.5 \mathrm{mmol})$ was introduced, then the mixture was stored in the fridge for 16 hours. The reaction mixture was filtered through Celite ${ }^{\circledR}$, eluting with dichloromethane $(20 \mathrm{~mL})$. The organic fraction was dried, filtered and concentrated to provide a crude yellow oil. Upon purification by column chromatography (3:1 hexanes:ethyl acetate), a complex mixture of products was obtained (8 mg), in addition to pyranosyl sugar 367 (38 mg, 67\%).

H A solution of sulfone 367 (27 mg, $0.07 \mathrm{mmol}$ ) in tert-butanol (1 mL) and dichloromethane $(2 \mathrm{~mL})$ was treated with $\mathrm{KOH}-\mathrm{Al}_{2} \mathrm{O}_{3}(154 \mathrm{mg}, 0.7 \mathrm{mmol})$ and dibromodifluoromethane $(50 \mu \mathrm{L}, 0.5 \mathrm{mmol})$, then subjected to microwave reaction at $60{ }^{\circ} \mathrm{C}$ (dibromodifluoromethane pressure approximately four bar) for a period of 30 minutes. The reaction mixture was filtered through Celite ${ }^{\circledR}$, eluting with dichloromethane $(20 \mathrm{~mL})$. The organic fraction was dried, filtered and concentrated to provide a crude yellow oil. Upon purification by column chromatography $(3: 1$ hexanes:ethyl acetate), complex mixtures of products and starting material were obtained (9 mg), in addition to diene 353 (3 mg, 14\%). 
(2E,4E)-5- $O$-Benzyl-3-chloro-1,2,3,4-tetradeoxy-7,8- $O$-isopropylidene-1[(methyl)sulfonyl]-D-arabino-octa-1,3-dienopyranitol (372)

A A solution of diisopropyl amine $(50 \mu \mathrm{L}, 0.36 \mathrm{mmol})$ in THF $(4 \mathrm{~mL})$ was cooled to $-78{ }^{\circ} \mathrm{C}$, then treated with $n$-butyllithium $\left(0.18 \mathrm{~mL}\right.$ of a $2.0 \mathrm{molL}^{-1}$ solution in cyclohexane, $0.36 \mathrm{mmol}$ ) and stirred for 30 minutes to generate a solution of LDA. This solution was warmed to $0{ }^{\circ} \mathrm{C}$ and treated with sulfone 366 (86 mg, $0.37 \mathrm{mmol}$ ), then stirred at $0{ }^{\circ} \mathrm{C}$ for one hour. To this was added hemiacetal $\mathbf{3 4 5}$ (108 $\mathrm{mg}, 0.317 \mathrm{mmol}$ ) in THF (2 mL), then the mixture was stirred for three hours at $0{ }^{\circ} \mathrm{C}$. Upon completion of the HWE reaction (as determined by TLC), iodine (126 mg, $0.496 \mathrm{mmol}$ ) was added, then the reaction was stirred at room temperature for 17 hours. The reaction mixture was diluted with sat. sodium thiosulfate solution $(10 \mathrm{ml})$ and water $(10 \mathrm{~mL})$, then extracted with dichloromethane $(2 \times 20 \mathrm{~mL})$. The organic fractions were combined, dried, filtered and concentrated to provide a crude yellow oil. Upon purification by column chromatography (1:1 hexanes:ethyl acetate), starting material 345 (32 mg, 30\%) was obtained, in addition to $\alpha, \beta$-unsaturated sulfone 372 and a minor quantity of pyranosyl sugar 367 (17 mg, 13\%), obtained as a colourless oil.

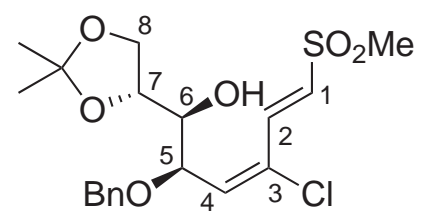

372: $\mathrm{R}_{f} 0.3$ (1:1 hexanes:ethyl acetate); ${ }^{1} \mathrm{H}-\mathrm{NMR}:\left(\mathrm{CDCl}_{3}\right)$ $\delta_{\mathrm{H}} 7.54(\mathrm{~d}, J=14.2 \mathrm{~Hz}, 1 \mathrm{H}, \mathrm{H}-2), 7.38-7.29$ (complex m, $5 \mathrm{H}, \mathrm{Bn}), 6.91$ (d, $J=14.5 \mathrm{~Hz}, 1 \mathrm{H}, \mathrm{H}-1), 6.40$ (d, $J=9.5$ $\mathrm{Hz}, 1 \mathrm{H}, \mathrm{H}-4$ ), 4.59-4.55 (complex m, 2H, $\mathrm{PhCH}_{2}, \mathrm{H}-5$ ), $4.46\left(\mathrm{~d}, J=11.2 \mathrm{~Hz}, 1 \mathrm{H}, \mathrm{PhCH}_{2}\right.$ ), 4.11-4.04 (complex m, 2H, H-7,8a), 3.98 (dd, $J=7.8,4.1 \mathrm{~Hz}, 1 \mathrm{H}, \mathrm{H}-8 \mathrm{~b}), 3.45$ (m, 1H, H-6), 2.98 (s, 3H, Me), 2.50 (d, $J=8.0$ $\mathrm{Hz}, 1 \mathrm{H}, \mathrm{OH}), 1.39\left(\mathrm{~s}, 3 \mathrm{H},\left(\mathrm{CH}_{3}\right)_{2} \mathrm{C}\right), 1.33\left(\mathrm{~s}, 3 \mathrm{H},\left(\mathrm{CH}_{3}\right)_{2} \mathrm{C}\right) ;{ }^{13} \mathrm{C}-\mathrm{NMR}:\left(\mathrm{CDCl}_{3}\right) \delta_{\mathrm{C}}$ 138.8 (CH, C-4), 136.7 (C, Bn), 134.7 (CH, C-2), 132.4 (CH, C-1), 129.5 (CH, C-3), $128.6(\mathrm{CH}, \mathrm{Bn}), 128.2(\mathrm{CH}, \mathrm{Bn}), 128.1(\mathrm{CH}, \mathrm{Bn}), 109.5\left(\mathrm{C},\left(\mathrm{CH}_{3}\right)_{2} \mathrm{C}\right), 75.2(\mathrm{CH}$, C-7), 75.1 (CH, C-6), 74.1 ( $\mathrm{CH}, \mathrm{C}-5), 71.6\left(\mathrm{CH}_{2}, \mathrm{PhCH}_{2}\right), 66.9\left(\mathrm{CH}_{2}, \mathrm{C}-8\right), 42.9$ $\left(\mathrm{CH}_{3}, \mathrm{Me}\right), 26.8\left(\mathrm{CH}_{3},\left(\mathrm{CH}_{3}\right)_{2} \mathrm{C}\right), 25.2\left(\mathrm{CH}_{3},\left(\mathrm{CH}_{3}\right)_{2} \mathrm{C}\right)$.

B A solution of diisopropyl amine $(100 \mu \mathrm{L}, 0.71 \mathrm{mmol})$ in THF $(4 \mathrm{~mL})$ was cooled to $-78^{\circ} \mathrm{C}$, then treated with $n$-butyllithium $\left(0.36 \mathrm{~mL}\right.$ of a $2.0 \mathrm{molL}^{-1}$ solution in cyclohexane, $0.72 \mathrm{mmol}$ ) and stirred for 30 minutes to generate a solution of LDA. This solution was treated with sulfone 366 (83 $\mathrm{mg}, 0.36 \mathrm{mmol})$, then allowed to warm to $0{ }^{\circ} \mathrm{C}$ over the course of 90 minutes. To this was added hemiacetal 345 (87 mg, $0.26 \mathrm{mmol})$ in THF $(1 \mathrm{~mL})$, then the mixture was stirred at $0{ }^{\circ} \mathrm{C}$ for 2.5 hours at $0{ }^{\circ} \mathrm{C}$. The reaction mixture was diluted with sat. ammonium chloride solution $(10 \mathrm{ml})$ and water $(10 \mathrm{~mL})$, then extracted with dichloromethane $(2 \times 20 \mathrm{~mL})$. The organic fractions were combined, dried, filtered and concentrated to provide a crude yellow oil containing starting material 345, diene sulfone 372 and cyclised sulfone 367 (1:1:2.5 ratio as judged by the proton NMR spectrum). Upon purifica- 
tion by column chromatography (1:1 hexanes:ethyl acetate), an inseparable mixture of diene sulfone $\mathbf{3 7 2}$ and cyclised sulfone $\mathbf{3 6 7}$ (66 mg, 62\%, 1:2.6 ratio) was obtained.

C A solution of sugar sulfone $\mathbf{3 6 7}(28 \mathrm{mg}, 67 \mu \mathrm{mol})$ in THF $(1 \mathrm{~mL})$ was cooled to $-78^{\circ} \mathrm{C}$, then treated with $n$-butyllithium $\left(40 \mu \mathrm{L}\right.$ of a $2.0 \mathrm{molL}^{-1}$ solution in cyclohexane, $80 \mu \mathrm{mol})$. The solution was allowed to react for 30 minutes, then treated with NBS (22 mg, $0.12 \mathrm{mmol}$ ). The mixture was stirred at $-78^{\circ} \mathrm{C}$ for an additional hour, then diluted with sat. ammonium chloride solution $(20 \mathrm{ml})$ and extracted with dichloromethane $(2 \times 20 \mathrm{~mL})$. The organic fractions were combined, dried, filtered and concentrated to provide a crude mixture of NBS and sugar sulfones 367 and 372 (1:1 ratio as judged by the proton NMR spectrum).

D A solution of silver salt $351(39 \mathrm{mg}, 79 \mu \mathrm{mol})$ in dichloromethane $(1 \mathrm{~mL})$ was treated with iodine $(16 \mathrm{mg}, 63 \mu \mathrm{mol})$ and stirred at room temperature for five minutes, in which the iodine was consumed. A solution of diene 372 (12 mg, $29 \mu \mathrm{mol}$ ) in dichloromethane $(1 \mathrm{~mL})$ was added, then the solution was stirred at room temperature for three days. The mixture was diluted with dichloromethane $(10 \mathrm{~mL})$, then washed successively with sat. sodium thiosulfate solution $(10 \mathrm{~mL}), 10 \%$ aqueous hydrochloric acid $(10 \mathrm{~mL})$ and sat. sodium bicarbonate solution $(10 \mathrm{~mL})$. The organic fraction was dried, filtered and concentrated to provide a crude sample of starting material 372 (11 mg, 92\%).

\section{2,6-Anhydro-5- $O$-benzyl-3-chloro-1,3,4-trideoxy-7,8- $O$-isopropylidene- D-gluco-octa-1,3-dienopyranitol (398)}

A A solution of sulfone 367 (57 $\mathrm{mg}, 0.14 \mathrm{mmol})$ in tert-butanol (1.25 mL) was treated with $\mathrm{KOH}-\mathrm{Al}_{2} \mathrm{O}_{3}(230 \mathrm{mg}, 1.0 \mathrm{mmol})$ and carbon tetrachloride $(2 \mathrm{~mL})$, then stirred at $35{ }^{\circ} \mathrm{C}$ for one hour. The reaction mixture was diluted with water $(20 \mathrm{ml})$ and extracted with diethyl ether $(2 \times 20 \mathrm{~mL})$. The organic fractions were combined, washed with brine $(10 \mathrm{~mL})$, then dried, filtered and concentrated to provide a crude yellow oil. Upon purification by column chromatography (2:1 hexanes:ethyl acetate), diene 373 (12 mg, 26\%) was obtained as a colourless oil, in addition to pyranosyl sugar 371 (22 mg, 39\%).

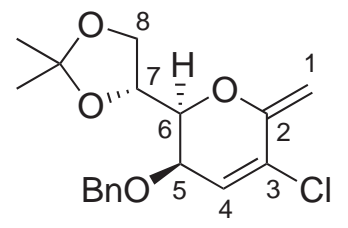

373: $\quad \mathrm{R}_{f} 0.7$ (2:1 hexanes:ethyl acetate); $[\alpha]_{\mathrm{D}}^{21}-99$ (c 0.25 $\left.\mathrm{CHCl}_{3}\right) ;{ }^{1} \mathrm{H}-\mathrm{NMR}:\left(\mathrm{CDCl}_{3}\right) \delta_{\mathrm{H}} 7.37-7.29$ (complex m, 5H, Bn), $6.22(\mathrm{~d}, J=6.4 \mathrm{~Hz}, 1 \mathrm{H}, \mathrm{H}-4), 4.94$ (s, 1H, H-1a), 4.74 (s, 1H, H-1b), 4.70 (d, $J=11.7 \mathrm{~Hz}, 1 \mathrm{H}, \mathrm{PhCH}_{2}$ ), 4.65 (d, $J=11.7$ $\left.\mathrm{Hz}, 1 \mathrm{H}, \mathrm{PhCH}_{2}\right), 4.44(\mathrm{dt}, J=8.0,5.7 \mathrm{~Hz}, 1 \mathrm{H}, \mathrm{H}-7), 4.18(\mathrm{dd}, J=8.5,6.3 \mathrm{~Hz}$, 1H, H-8a), 4.11-4.07 (complex m, 2H, H-5,8b), 3.76 (dd, J = 8.3, $1.9 \mathrm{~Hz}, 1 \mathrm{H}, \mathrm{H}-6$ ), $1.41\left(\mathrm{~s}, 3 \mathrm{H},\left(\mathrm{CH}_{3}\right)_{2} \mathrm{C}\right), 1.39\left(\mathrm{~s}, 3 \mathrm{H},\left(\mathrm{CH}_{3}\right)_{2} \mathrm{C}\right) ;{ }^{13} \mathrm{C}-\mathrm{NMR}:\left(\mathrm{CDCl}_{3}\right) \delta_{\mathrm{C}} 151.7(\mathrm{C}, \mathrm{C}-2)$, 
138.0 (C, Bn), 130.9 (CH, C-3), 128.4 (CH, Bn), 127.9 (CH, Bn), 127.8 (CH, Bn), $123.7(\mathrm{CH}, \mathrm{C}-4), 109.3\left(\mathrm{C},\left(\mathrm{CH}_{3}\right)_{2} \mathrm{C}\right), 95.9\left(\mathrm{CH}_{2}, \mathrm{C}-1\right), 78.5(\mathrm{CH}, \mathrm{C}-6), 73.2(\mathrm{CH}, \mathrm{C}-$ 7), $71.4\left(\mathrm{CH}_{2}, \mathrm{PhCH}_{2}\right), 69.0(\mathrm{CH}, \mathrm{C}-5), 66.9\left(\mathrm{CH}_{2}, \mathrm{C}-8\right), 26.9\left(\mathrm{CH}_{3},\left(\mathrm{CH}_{3}\right)_{2} \mathrm{C}\right), 25.4$ $\left(\mathrm{CH}_{3},\left(\mathrm{CH}_{3}\right)_{2} \mathrm{C}\right)$; IR $(\mathrm{KBr}): \nu_{\max } 2988,2931,2885,1635,1598,1455,1381,1372$, 1254, 1224, 1128, 1081, 1028, 845, 738, $698 \mathrm{~cm}^{-1}$; HRMS: $m / z \mathrm{C}_{18} \mathrm{H}_{21} \mathrm{O}_{4}^{35} \mathrm{ClNa}^{+}$ $[\mathrm{M}+\mathrm{Na}]^{+}$calcd 359.1024, found 359.1026.

B A solution of sulfone 367 (105 $\mathrm{mg}, 0.25 \mathrm{mmol})$ in tert-butanol $(1.25 \mathrm{~mL})$ was treated with powdered $\mathrm{KOH}(297 \mathrm{mg}, 5.29 \mathrm{mmol})$ and carbon tetrachloride $(1.25$ $\mathrm{mL}$ ), then stirred at $35{ }^{\circ} \mathrm{C}$ for 30 minutes. The reaction mixture was diluted with sat. ammonium chloride solution $(10 \mathrm{ml})$ and extracted with dichloromethane $(3 \mathrm{x}$ $10 \mathrm{~mL}$ ). The organic fractions were combined, dried, filtered and concentrated to provide a crude yellow oil. Upon purification by column chromatography (5:1 hexanes:ethyl acetate), a mixture of several inseparable products including diene $\mathbf{3 7 3}$ (31 mg) was obtained.

\section{(3E)-5- $O$-Benzyl-3- $O$-methoxycarbonyl-1,2,3,4-tetradeoxy-7,8- $O$ - isopropylidene-D-arabino-octa-1,3-dienitol (380)}

A solution of diene 353 (50 mg, $0.15 \mathrm{mmol}$ ) in methanol (2 mL) was treated with bis(triphenylphosphine)palladium(II) chloride (21 mg, $0.03 \mathrm{mmol}$ ) and triethylamine (30 $\mu \mathrm{L}, 0.2 \mathrm{mmol}$ ), then purged with carbon monoxide three times. The mixture was stirred at reflux for seven hours, then diluted with ethyl acetate $(20 \mathrm{ml})$, washed with water $(20 \mathrm{~mL})$, then brine $(20 \mathrm{~mL})$. The organic fraction was combined, dried, filtered and concentrated to provide a crude brown oil. Upon purification by column chromatography (5:1 hexanes:ethyl acetate), ester 380 (22 mg, 41\%) was obtained as a light-brown oil.

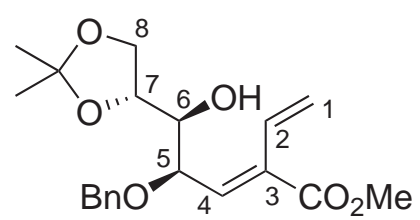

$\mathrm{R}_{f} 0.3$ (3:1 hexanes:ethyl acetate); $[\alpha]_{\mathrm{D}}^{19}-42\left(\right.$ c $\left.1.0 \mathrm{CHCl}_{3}\right)$; ${ }^{1} \mathrm{H}-\mathrm{NMR}:\left(\mathrm{CDCl}_{3}\right) \delta_{\mathrm{H}} 7.36-7.29$ (complex m, 5H, Bn), 6.77 $(\mathrm{d}, J=9.5 \mathrm{~Hz}, 1 \mathrm{H}, \mathrm{H}-4), 6.44(\mathrm{dd}, J=17.7,11.7 \mathrm{~Hz}, 1 \mathrm{H}$, H-2), 5.60 (dd, $J=17.8,1.7 \mathrm{~Hz}, 1 \mathrm{H}, \mathrm{H}-1 \mathrm{a}), 5.43$ (dt, $J=$ 11.5, $1.2 \mathrm{~Hz}, 1 \mathrm{H}, \mathrm{H}-1 \mathrm{~b}), 4.65$ (dd, $J=9.4,2.4 \mathrm{~Hz}, \mathrm{H}-5), 4.59$ (d, $J=11.4 \mathrm{~Hz}, 1 \mathrm{H}$, $\mathrm{PhCH}_{2}$ ), 4.40 (d, $\left.J=11.4 \mathrm{~Hz}, 1 \mathrm{H}, \mathrm{PhCH}_{2}\right), 4.14(\mathrm{~m}, 1 \mathrm{H}, \mathrm{H}-7), 4.07$ (dd, $J=8.4$, $6.2 \mathrm{~Hz}, 1 \mathrm{H}, \mathrm{H}-8 \mathrm{a}), 4.00$ (dd, $J=8.6,5.1 \mathrm{~Hz}, 1 \mathrm{H}, \mathrm{H}-8 \mathrm{~b}), 3.80$ (s, 3H, Me), 3.51 (td, $J=7.9,2.2 \mathrm{~Hz}, 1 \mathrm{H}, \mathrm{H}-6), 2.47(\mathrm{~d}, J=8.6 \mathrm{~Hz}, 1 \mathrm{H}, \mathrm{OH}), 1.39\left(\mathrm{~s}, 3 \mathrm{H},\left(\mathrm{CH}_{3}\right)_{2} \mathrm{C}\right)$, $1.33\left(\mathrm{~s}, 3 \mathrm{H},\left(\mathrm{CH}_{3}\right)_{2} \mathrm{C}\right) ;{ }^{13} \mathrm{C}-\mathrm{NMR}:\left(\mathrm{CDCl}_{3}\right) \delta_{\mathrm{C}} 166.7$ (C, $\left.\mathrm{CH}_{3} \mathrm{OCO}\right), 139.6(\mathrm{CH}, \mathrm{C}-4)$, 137.5 (C, Bn), 133.5 (C, C-3), 128.41 (CH, C-2), 128.40 (CH, Bn), 127.95 (CH, Bn), $127.93(\mathrm{CH}, \mathrm{Bn}), 121.6\left(\mathrm{CH}_{2}, \mathrm{C}-1\right), 109.2\left(\mathrm{C},\left(\mathrm{CH}_{3}\right)_{2} \mathrm{C}\right), 75.5(\mathrm{CH}, \mathrm{C}-7), 75.2(\mathrm{CH}$, C-6), $73.6(\mathrm{CH}, \mathrm{C}-5), 71.2\left(\mathrm{CH}_{2}, \mathrm{PhCH}_{2}\right), 66.8\left(\mathrm{CH}_{2}, \mathrm{C}-8\right), 52.1\left(\mathrm{CH}_{3}, \mathrm{Me}\right), 26.8$ $\left(\mathrm{CH}_{3},\left(\mathrm{CH}_{3}\right)_{2} \mathrm{C}\right), 25.2\left(\mathrm{CH}_{3},\left(\mathrm{CH}_{3}\right)_{2} \mathrm{C}\right)$; IR $(\mathrm{KBr}): \nu_{\max } 3456,2988,2934,1724$, 
1455, 1372, 1248, 1219, 1153, 1069, 848, 771, $698 \mathrm{~cm}^{-1}$; HRMS: $m / z \mathrm{C}_{20} \mathrm{H}_{26} \mathrm{O}_{6} \mathrm{Na}^{+}$ $[\mathrm{M}+\mathrm{Na}]^{+}$calcd 385.1627, found 385.1631.

\section{2,6-Anhydro-5- $O$-benzyl-3- $O$-methoxycarbonyl-1,3,4-trideoxy-1-iodo- 7,8- $O$-isopropylidene-D-manno-octa-1,3-dienopyranitol (381) and 2,6-anhydro-5- $O$-benzyl-3- $O$-methoxycarbonyl-1,3,4-trideoxy-1-iodo- 7,8-O-isopropylidene-D-gluco-octa-1,3-dienopyranitol (382)}

A solution of silver salt 351 (86 mg, $0.17 \mathrm{mmol}$ ) in dichloromethane $(1 \mathrm{~mL})$ was treated with iodine $(32 \mathrm{mg}, 0.13 \mathrm{mmol})$ and stirred at room temperature for five minutes, in which the iodine was consumed. A solution of diene 380 (24 mg, $66 \mu \mathrm{mol}$ ) in dichloromethane $(1 \mathrm{~mL})$ was added, then the solution was stirred at room temperature for one day. The mixture was diluted with dichloromethane (20 mL), then washed successively with sat. sodium thiosulfate solution $(20 \mathrm{~mL}), 10 \%$ aqueous hydrochloric acid $(20 \mathrm{~mL})$ and sat. sodium bicarbonate solution $(20 \mathrm{~mL})$. The organic fraction was dried, filtered and concentrated to provide a yellow oil. Upon purification by column chromatography (3:1 hexanes:ethyl acetate), iodopyranose sugars $\mathbf{3 8 1}$ and $\mathbf{3 8 2}$ (15 mg, 47\%) was obtained as a off-white solid.

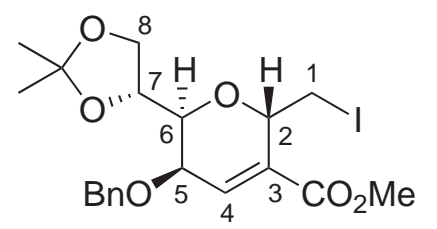

$\mathrm{R}_{f} 0.4$ (3:1 hexanes:ethyl acetate); ${ }^{1} \mathrm{H}-\mathrm{NMR}:\left(\mathrm{CDCl}_{3}\right) \delta_{\mathrm{H}}$ 7.40-7.28 (complex m, 5H, Bn), 7.07 (dd, $J=5.2,1.3$ $\mathrm{Hz}, 1 \mathrm{H}, \mathrm{H}-4)$, 4.78-4.71 (complex m, 2H, $\mathrm{PhCH}_{2}$ ), 4.69 $(\mathrm{d}, J=9.3 \mathrm{~Hz}, 1 \mathrm{H}, \mathrm{H}-2), 4.44(\mathrm{dt}, J=8.5,5.6 \mathrm{~Hz}, 1 \mathrm{H}$, H-6), 4.32 (dd, $J=8.8,5.1 \mathrm{~Hz}, 1 \mathrm{H}, \mathrm{H}-8 \mathrm{a}), 4.20$ (dd, $J=8.8,6.3 \mathrm{~Hz}, 1 \mathrm{H}, \mathrm{H}-8 \mathrm{~b})$, $3.90(\mathrm{dd}, J=5.3,2.5 \mathrm{~Hz}, 1 \mathrm{H}, \mathrm{H}-5), 3.78$ (s, 3H, Me), 3.61-3.56 (complex m, 2H, H-1a,7), 3.26 (apparent t, $J=11.1 \mathrm{~Hz}, 1 \mathrm{H}, \mathrm{H}-1 \mathrm{~b}), 1.44$ (s, 3H, $\left.\left(\mathrm{CH}_{3}\right)_{2} \mathrm{C}\right), 1.40$ (s, $\left.3 \mathrm{H},\left(\mathrm{CH}_{3}\right)_{2} \mathrm{C}\right) ;{ }^{13} \mathrm{C}-\mathrm{NMR}:\left(\mathrm{CDCl}_{3}\right) \delta_{\mathrm{C}} 164.9$ (C, $\left.\mathrm{CH}_{3} \mathrm{OCO}\right), 138.0$ (C, Bn), 135.8 (C, C-4), 133.1 (CH, C-3), 128.4 (CH, Bn), 128.1 (CH, Bn), 127.9 (CH, Bn), 109.2 (C, $\left.\left(\mathrm{CH}_{3}\right)_{2} \mathrm{C}\right), 73.2(\mathrm{CH}, \mathrm{C}-6), 72.7\left(\mathrm{CH}_{2}, \mathrm{PhCH}_{2}\right), 72.18$ and $72.17(\mathrm{CH}, \mathrm{C}-2$ and $\mathrm{CH}$, C-7), $68.0\left(\mathrm{CH}_{2}, \mathrm{C}-8\right), 67.4(\mathrm{CH}, \mathrm{C}-5), 52.3\left(\mathrm{CH}_{3}, \mathrm{Me}\right), 27.1\left(\mathrm{CH}_{3},\left(\mathrm{CH}_{3}\right)_{2} \mathrm{C}\right), 25.3$ $\left(\mathrm{CH}_{3},\left(\mathrm{CH}_{3}\right)_{2} \mathrm{C}\right), 3.8\left(\mathrm{CH}_{2}, \mathrm{C}-1\right)$; IR $(\mathrm{KBr}): \nu_{\max } 2988,2929,2868,1718,1454,1438$, 1371, 1273, 1249, 1144, 1076, 913, 851, 741, $698 \mathrm{~cm}^{-1}$; HRMS: $m / z \mathrm{C}_{20} \mathrm{H}_{25} \mathrm{O}_{6} \mathrm{INa}^{+}$ $[\mathrm{M}+\mathrm{Na}]^{+}$calcd 511.0594, found 511.0589.

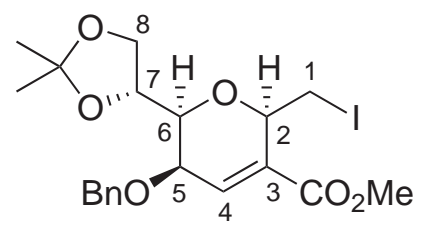

$\mathrm{R}_{f} 0.4$ (3:1 hexanes:ethyl acetate); ${ }^{1} \mathrm{H}-\mathrm{NMR}:\left(\mathrm{CDCl}_{3}\right) \delta_{\mathrm{H}}$ 7.40-7.28 (complex m, 5H, Bn), 7.14 (dd, $J=5.8,1.7 \mathrm{~Hz}$, $1 \mathrm{H}, \mathrm{H}-4)$, 4.78-4.71 (complex m, 2H, $\mathrm{PhCH}_{2}$ ), 4.38 (m, $1 \mathrm{H}, \mathrm{H}-6), 4.15$ (dd, $J=8.7,6.3 \mathrm{~Hz}, 1 \mathrm{H}, \mathrm{H}-8 \mathrm{a}), 4.08$ (dd, $J=8.8,5.2 \mathrm{~Hz}, 1 \mathrm{H}, \mathrm{H}-8 \mathrm{~b}), 4.03$ (dt, $J=5.9,1.8 \mathrm{~Hz}, 1 \mathrm{H}, \mathrm{H}-5), 3.78$ (s, 3H, Me), 3.64 (dd, $J=10.4,2.5 \mathrm{~Hz}, 1 \mathrm{H}, \mathrm{H}-2$ ), 3.61-3.56 (complex m, 2H, H-1a,b), 3.44 (dd, $J=7.8,2.0 \mathrm{~Hz}, 1 \mathrm{H}, \mathrm{H}-7), 1.40\left(\mathrm{~s}, 3 \mathrm{H},\left(\mathrm{CH}_{3}\right)_{2} \mathrm{C}\right), 1.37\left(\mathrm{~s}, 3 \mathrm{H},\left(\mathrm{CH}_{3}\right)_{2} \mathrm{C}\right) ;{ }^{13} \mathrm{C}-\mathrm{NMR}$ : $\left(\mathrm{CDCl}_{3}\right) \delta_{\mathrm{C}} 165.1\left(\mathrm{C}, \mathrm{CH}_{3} \mathrm{OCO}\right), 138.4(\mathrm{C}, \mathrm{Bn}), 136.4$ (C, C-4), 135.5 (CH, C-3), 
$128.3(\mathrm{CH}, \mathrm{Bn}), 127.8$ (CH, Bn), $127.7(\mathrm{CH}, \mathrm{Bn}), 109.1\left(\mathrm{C},\left(\mathrm{CH}_{3}\right)_{2} \mathrm{C}\right), 77.4(\mathrm{CH}$, C-7), 73.7 (CH, C-2), 73.5 (CH, C-6), $71.7\left(\mathrm{CH}_{2}, \mathrm{PhCH}_{2}\right), 67.2(\mathrm{CH}, \mathrm{C}-5), 67.0$ $\left(\mathrm{CH}_{2}, \mathrm{C}-8\right), 52.1\left(\mathrm{CH}_{3}, \mathrm{Me}\right), 26.9\left(\mathrm{CH}_{3},\left(\mathrm{CH}_{3}\right)_{2} \mathrm{C}\right), 25.4\left(\mathrm{CH}_{3},\left(\mathrm{CH}_{3}\right)_{2} \mathrm{C}\right), 8.3\left(\mathrm{CH}_{2}\right.$, C-1); IR (KBr): $\nu_{\max }$ 2988, 2929, 2868, 1718, 1454, 1438, 1371, 1273, 1249, 1144, $1076,913,851,741,698 \mathrm{~cm}^{-1}$.

\section{2,6-Anhydro-5- $O$-benzyl-3- $O$-methoxycarbonyl-1,3,4-trideoxy-1-iodo- 7,8- $O$-isopropylidene-D-manno-octa-1,3-dienopyranitol (381) and 2,6-anhydro-5- $O$-benzyl-3- $O$-methoxycarbonyl-1,3,4-trideoxy-1-iodo- 7,8-O-isopropylidene-D-gluco-octa-1,3-dienopyranitol (382)}

A solution of iodopyranose sugars 381 and $382(8 \mathrm{mg}, 16 \mu \mathrm{mol})$ in $50 \%(\mathrm{v} / \mathrm{v})$ aqueous THF $(1 \mathrm{~mL})$ was treated with sodium hydroxide $\left(12 \mu \mathrm{L}\right.$ of a $2.0 \mathrm{molL}^{-1}$ aqueous solution, $24 \mu \mathrm{mol}$ ) and stirred at room temperature for one day. The mixture was diluted with diethyl ether $(20 \mathrm{~mL})$, then washed successively with $10 \%$ aqueous hydrochloric acid $(20 \mathrm{~mL})$, water $(20 \mathrm{~mL})$ and brine $(20 \mathrm{~mL})$. The organic fraction was dried, filtered and concentrated to provide a yellow oil, in which TLC and NMR evidence indicated this material was composed of hydroxy acids 387 and 388. This crude product was diluted with dichloromethane $(1 \mathrm{~mL})$, then treated with DCC (5 mg, $0.02 \mathrm{~mol}$ ) and DMAP (2 mg, $0.02 \mathrm{mmol}$ ), then stirred at room temperature for 16 hours. The reaction mixture was diluted with sat. ammonium chloride solution $(10 \mathrm{ml})$, then extracted with dichloromethane $(3 \times 10 \mathrm{~mL})$. The organic fractions were combined, dried, filtered and concentrated to provide a crude yellow oil. Upon purification by column chromatography (9:1 hexanes:ethyl acetate), a mixure of iodopyranose sugars $\mathbf{3 8 1}$ and $\mathbf{3 8 2}(2 \mathrm{mg}, 25 \%)$ was obtained, in addition to impure mixtures of products $\left(3 \mathrm{mg}, \mathrm{R}_{f}\right.$ 0.5, 0.6 (1:1 hexanes:ethyl acetate)), tentatively assigned as lactones $\mathbf{3 7 8}$ and $\mathbf{3 7 9}$. 


\section{Spectra}

Selected spectra of key compounds

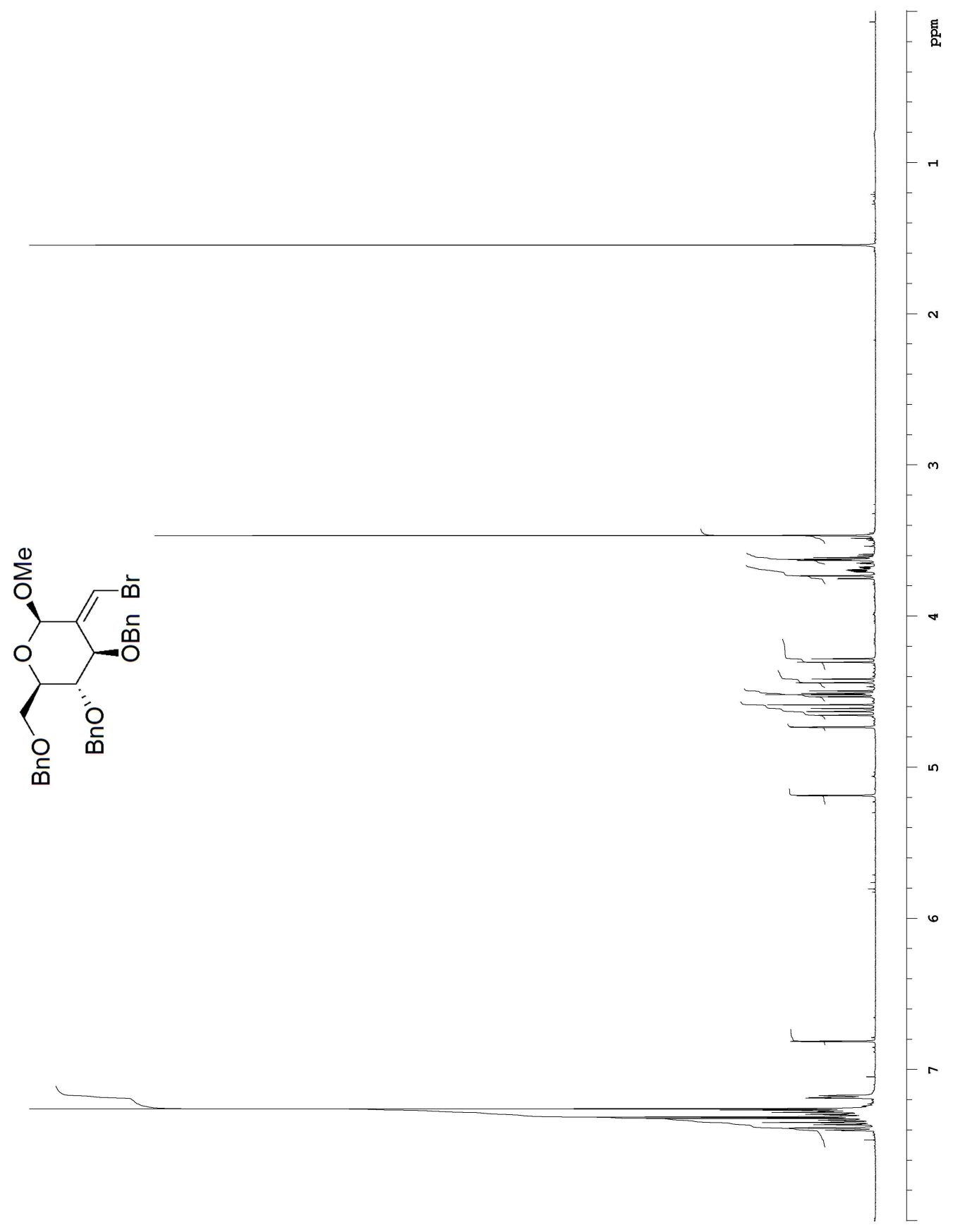

${ }^{1} \mathrm{H}-\mathrm{NMR}$ spectrum of 2-C-branched sugar $213\left(500 \mathrm{MHz}, \mathrm{CDCl}_{3}\right)$ 


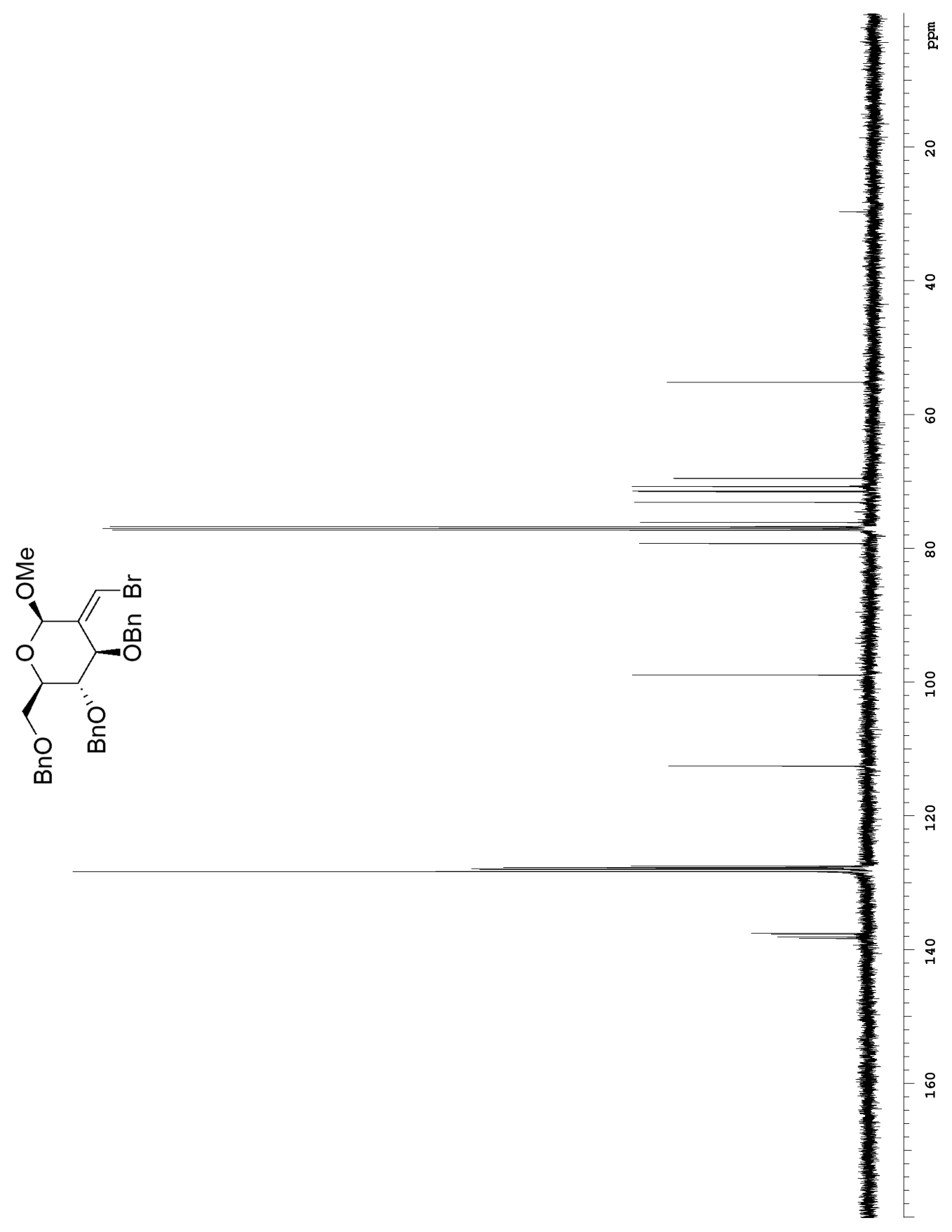

${ }^{13} \mathrm{C}-\mathrm{NMR}$ spectrum of 2-C-branched sugar $213\left(125 \mathrm{MHz}, \mathrm{CDCl}_{3}\right)$ 


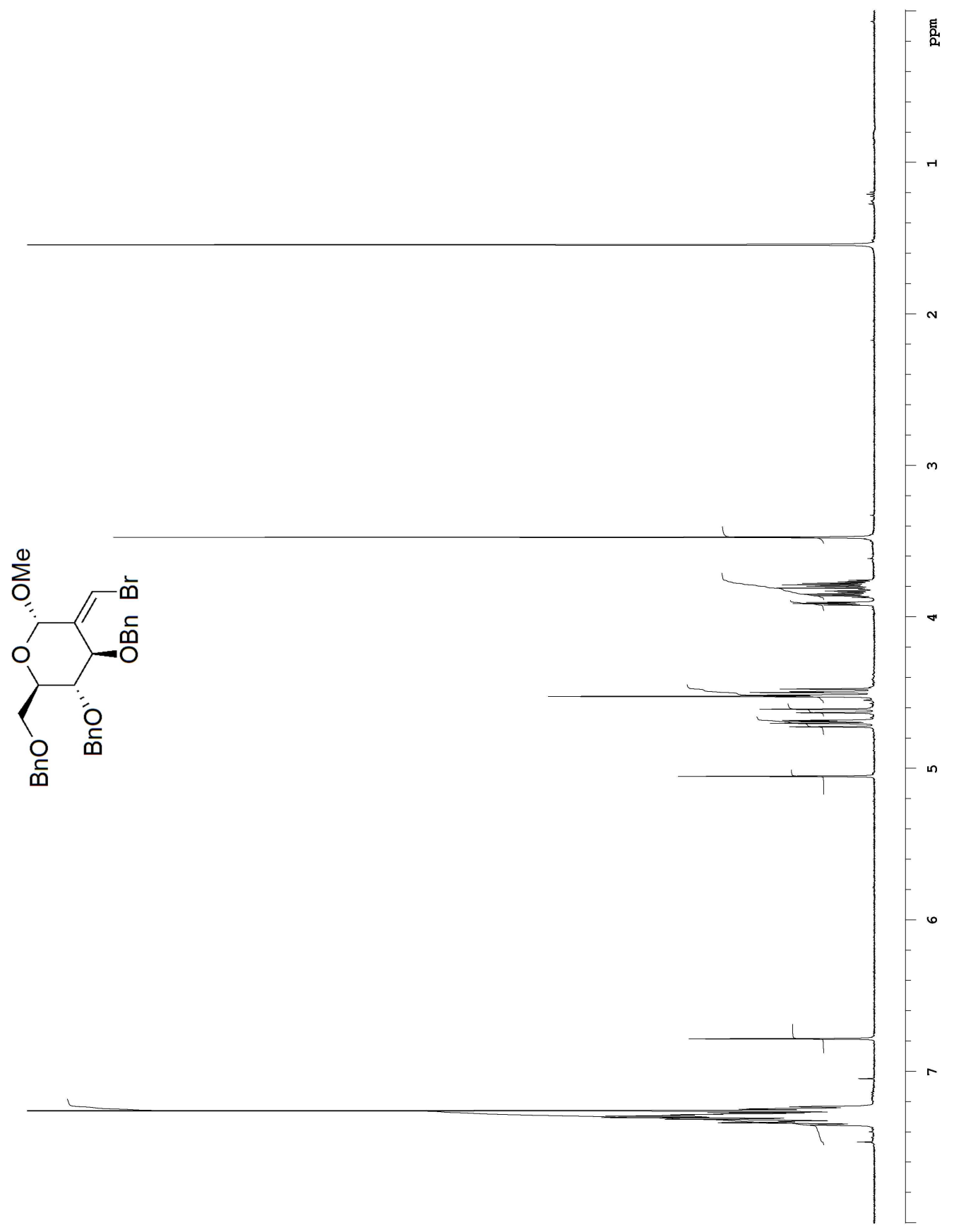

${ }^{1} \mathrm{H}-\mathrm{NMR}$ spectrum of 2-C-branched sugar $214\left(500 \mathrm{MHz}, \mathrm{CDCl}_{3}\right)$ 


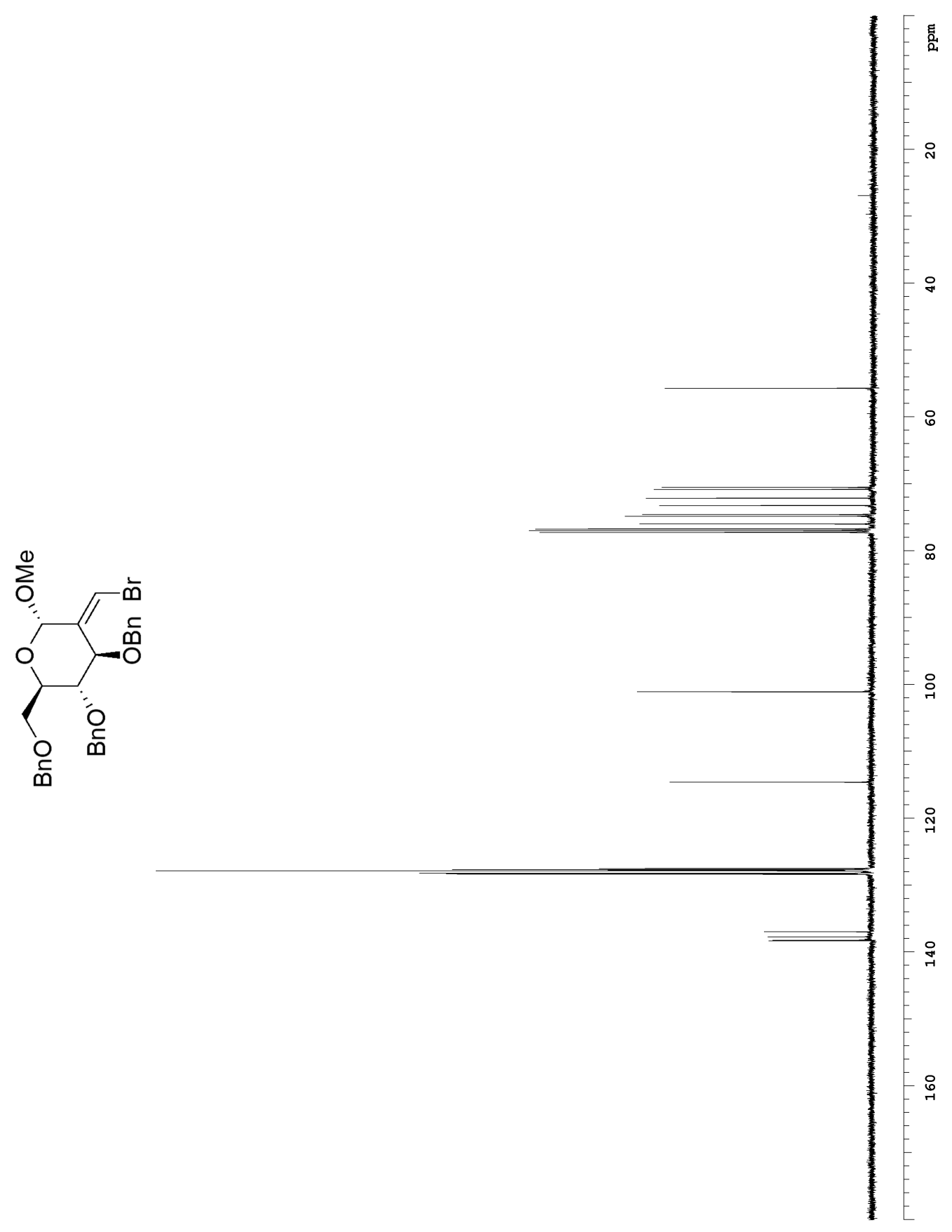

${ }^{13} \mathrm{C}$-NMR spectrum of 2-C-branched sugar $214\left(125 \mathrm{MHz}, \mathrm{CDCl}_{3}\right)$ 


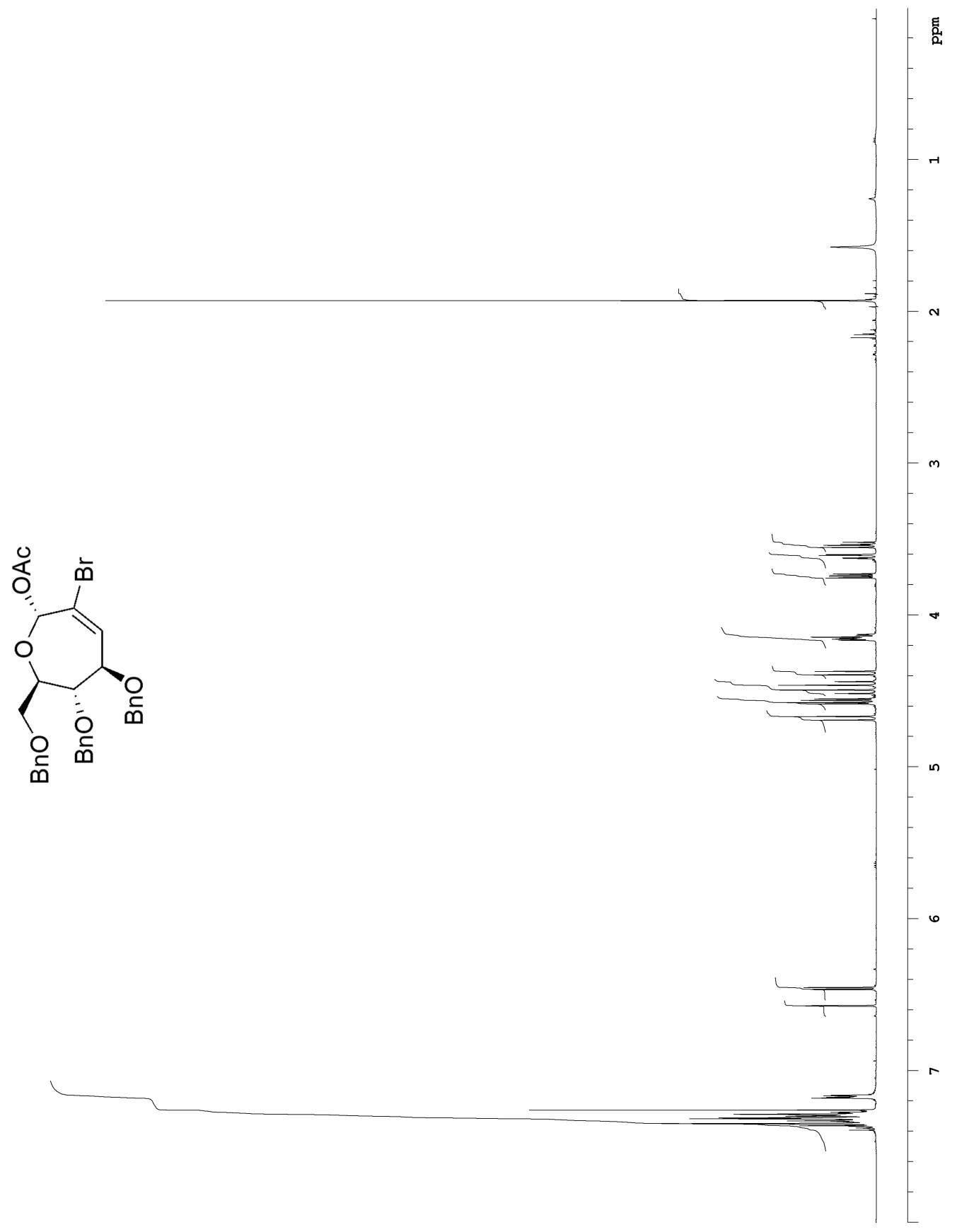

${ }^{1} \mathrm{H}-\mathrm{NMR}$ spectrum of oxepine sugar $258\left(500 \mathrm{MHz}, \mathrm{CDCl}_{3}\right)$ 


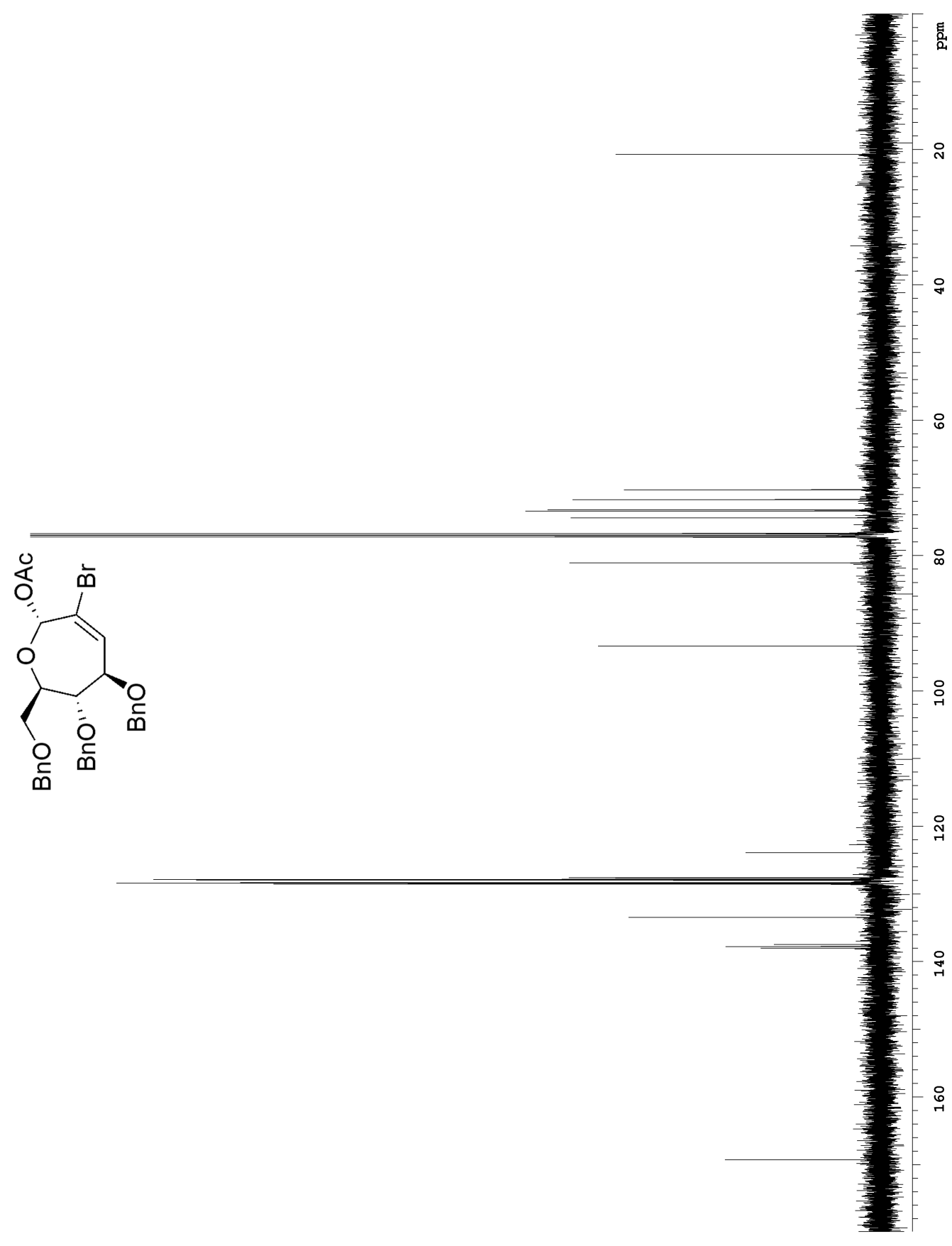

${ }^{13} \mathrm{C}-\mathrm{NMR}$ spectrum of oxepine sugar $258\left(125 \mathrm{MHz}, \mathrm{CDCl}_{3}\right)$ 


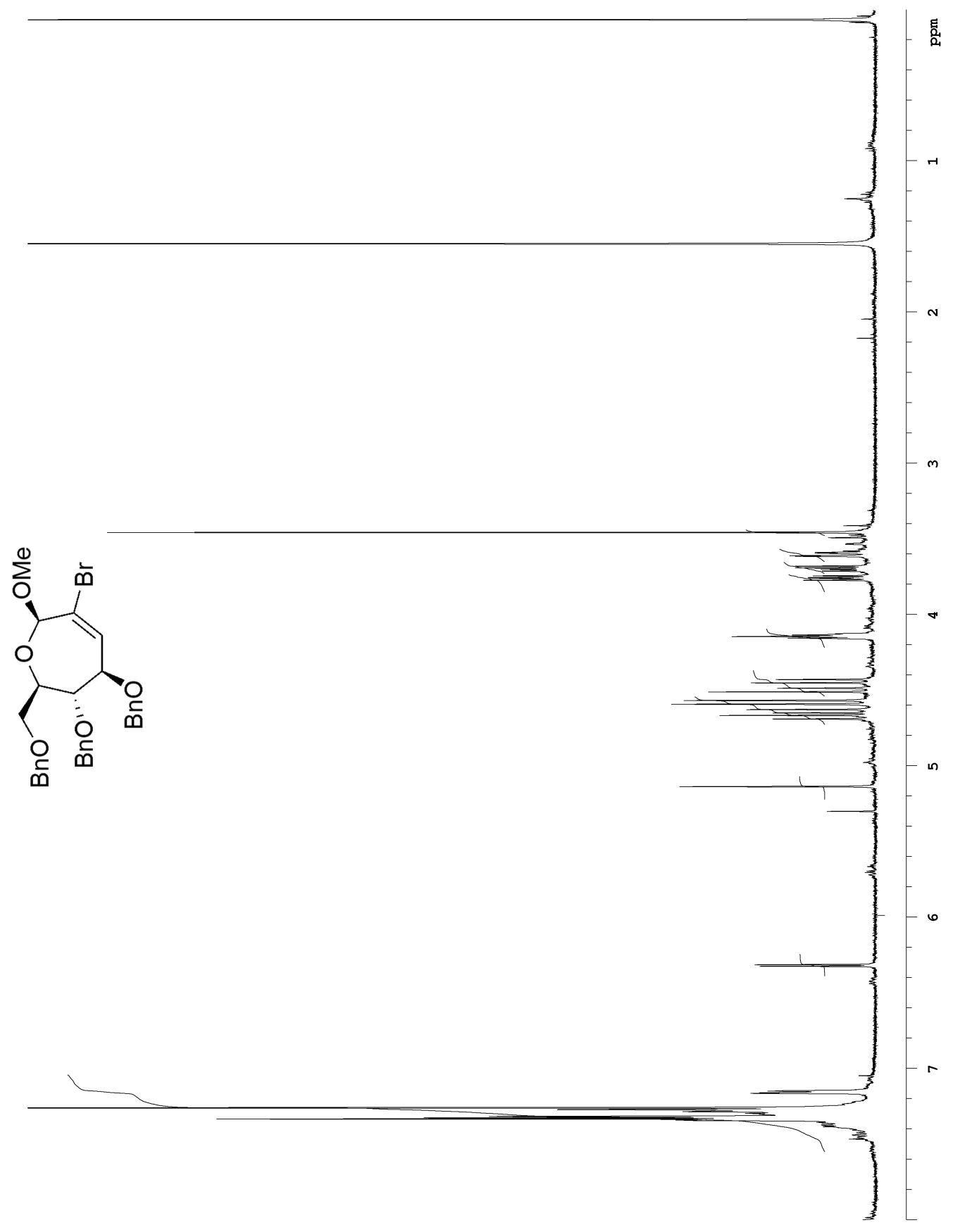

${ }^{1} \mathrm{H}-\mathrm{NMR}$ spectrum of oxepine sugar $98\left(500 \mathrm{MHz}, \mathrm{CDCl}_{3}\right)$ 


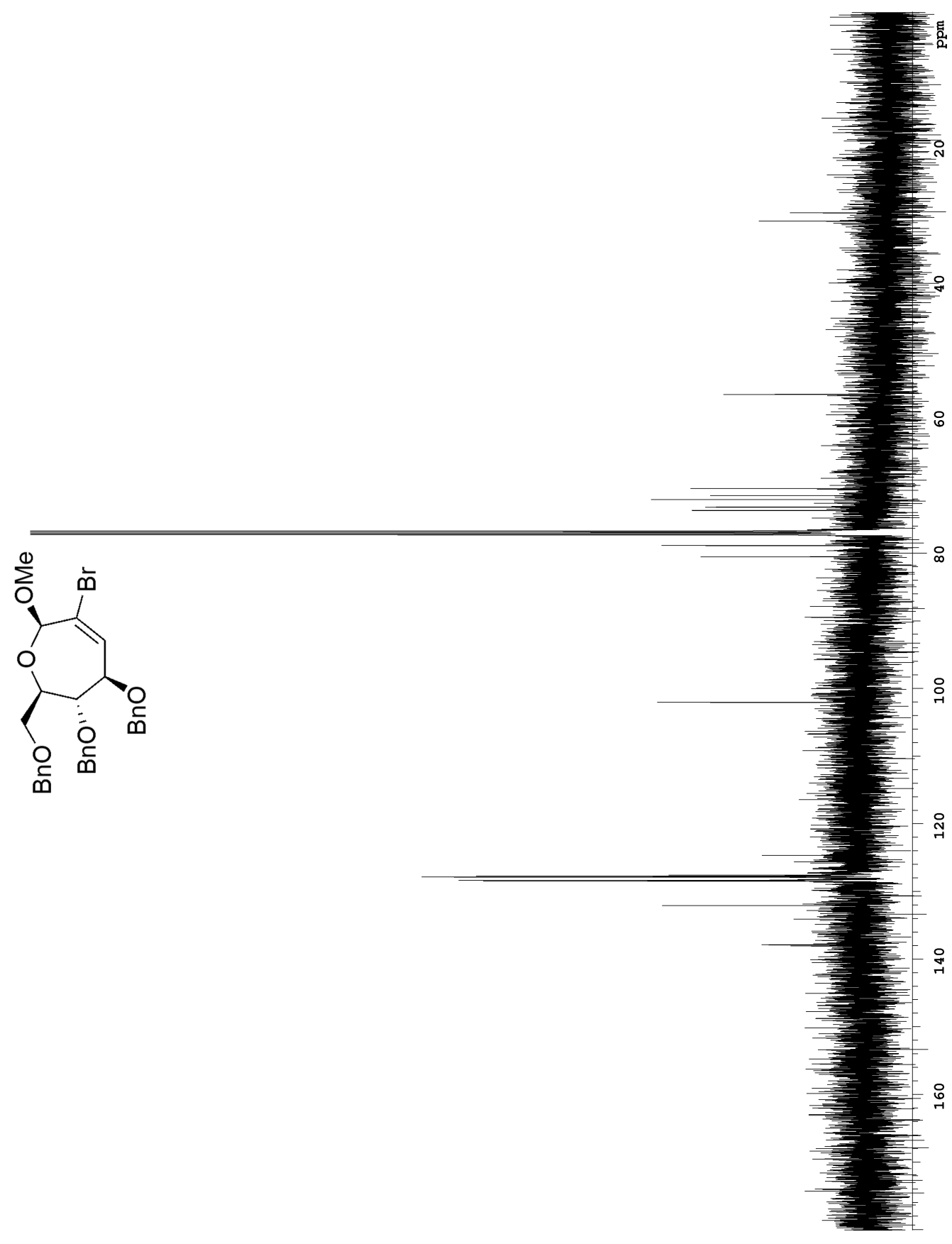

${ }^{13} \mathrm{C}-\mathrm{NMR}$ spectrum of oxepine sugar $98\left(125 \mathrm{MHz}, \mathrm{CDCl}_{3}\right)$ 


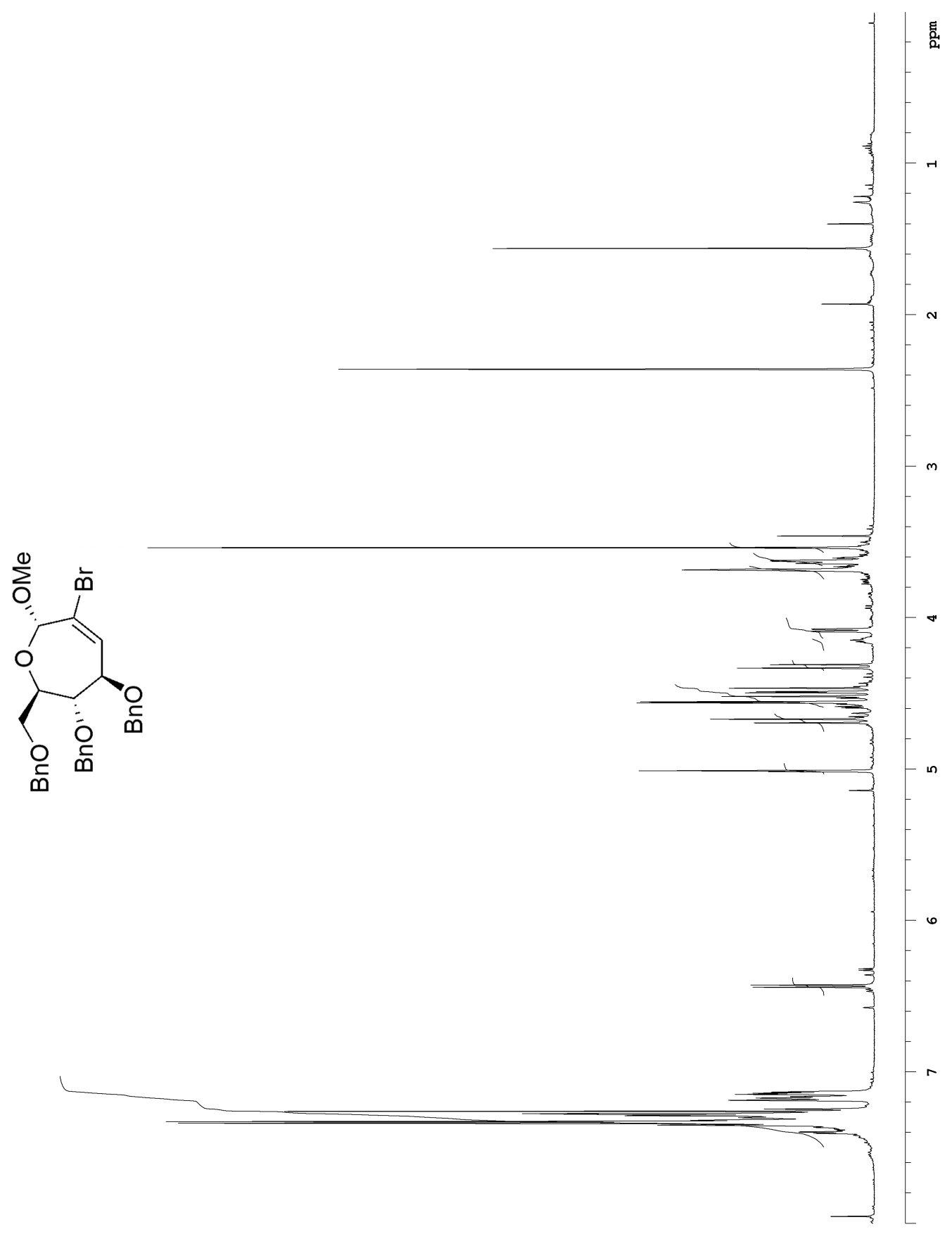

${ }^{1} \mathrm{H}-\mathrm{NMR}$ spectrum of oxepine sugar $99\left(500 \mathrm{MHz}, \mathrm{CDCl}_{3}\right)$ 


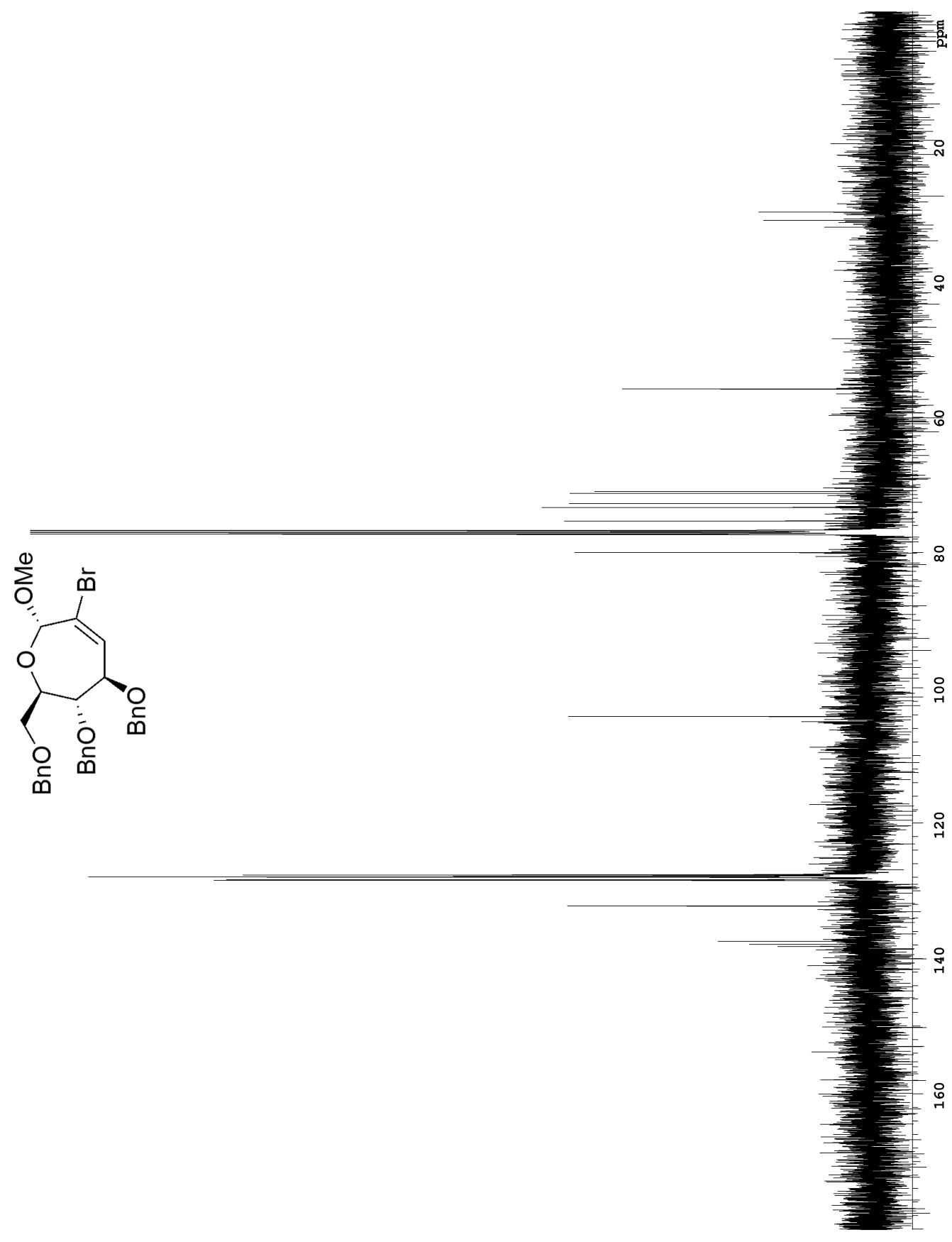

${ }^{13} \mathrm{C}-\mathrm{NMR}$ spectrum of oxepine sugar $99\left(125 \mathrm{MHz}, \mathrm{CDCl}_{3}\right)$ 


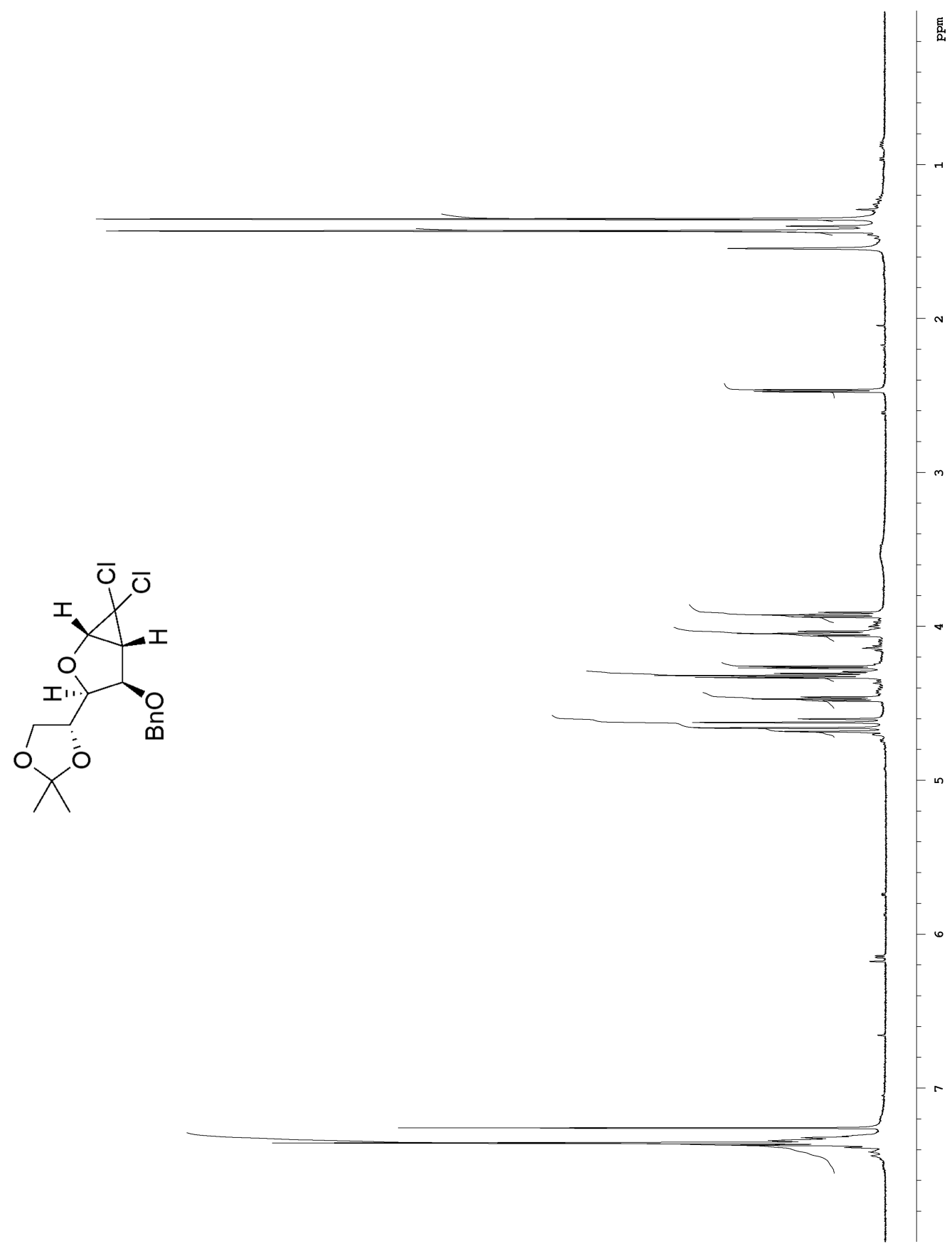

${ }^{1} \mathrm{H}-\mathrm{NMR}$ spectrum of cyclopropane $340\left(500 \mathrm{MHz}, \mathrm{CDCl}_{3}\right.$ ) 


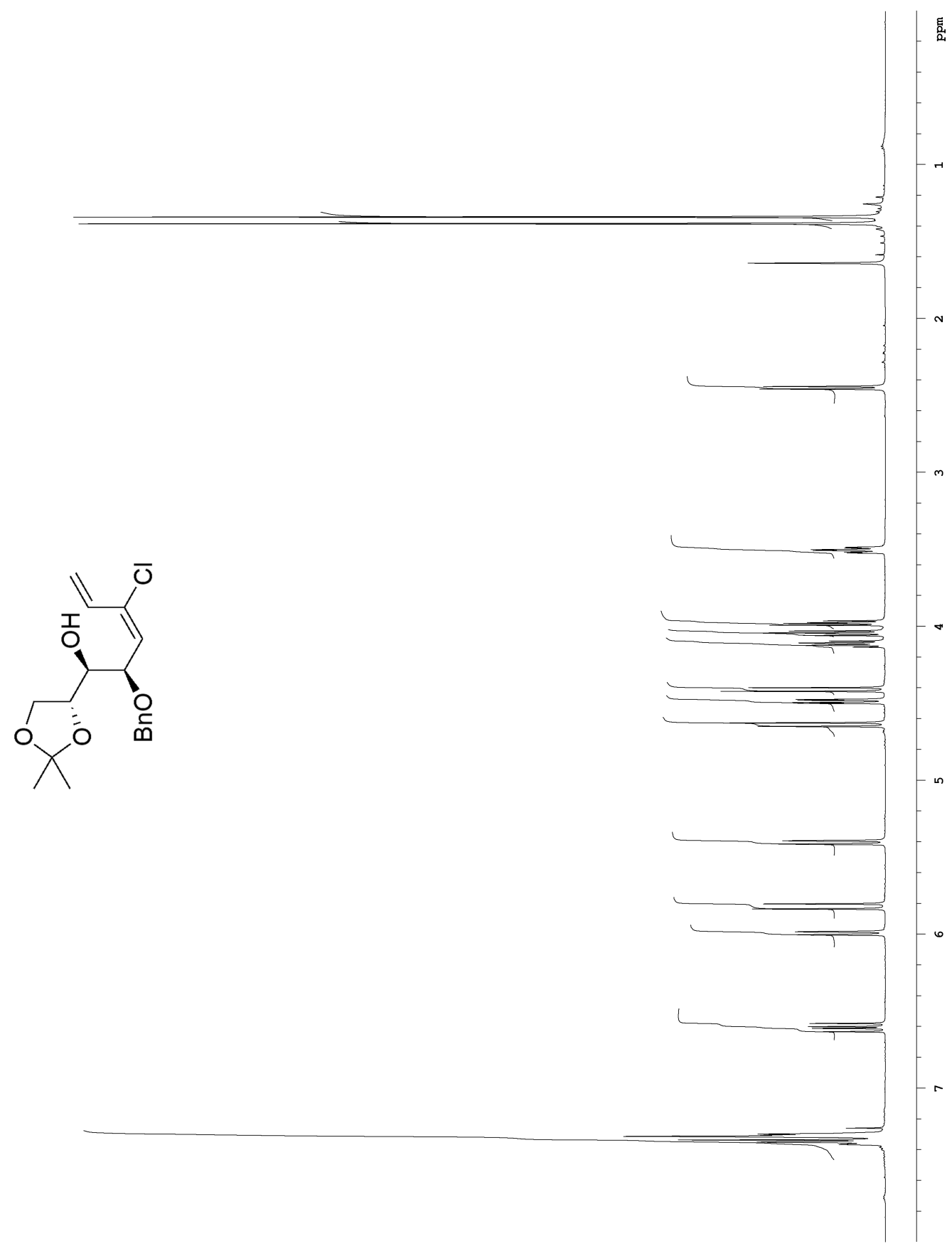

${ }^{1} \mathrm{H}-\mathrm{NMR}$ spectrum of diene $353\left(500 \mathrm{MHz}, \mathrm{CDCl}_{3}\right)$ 


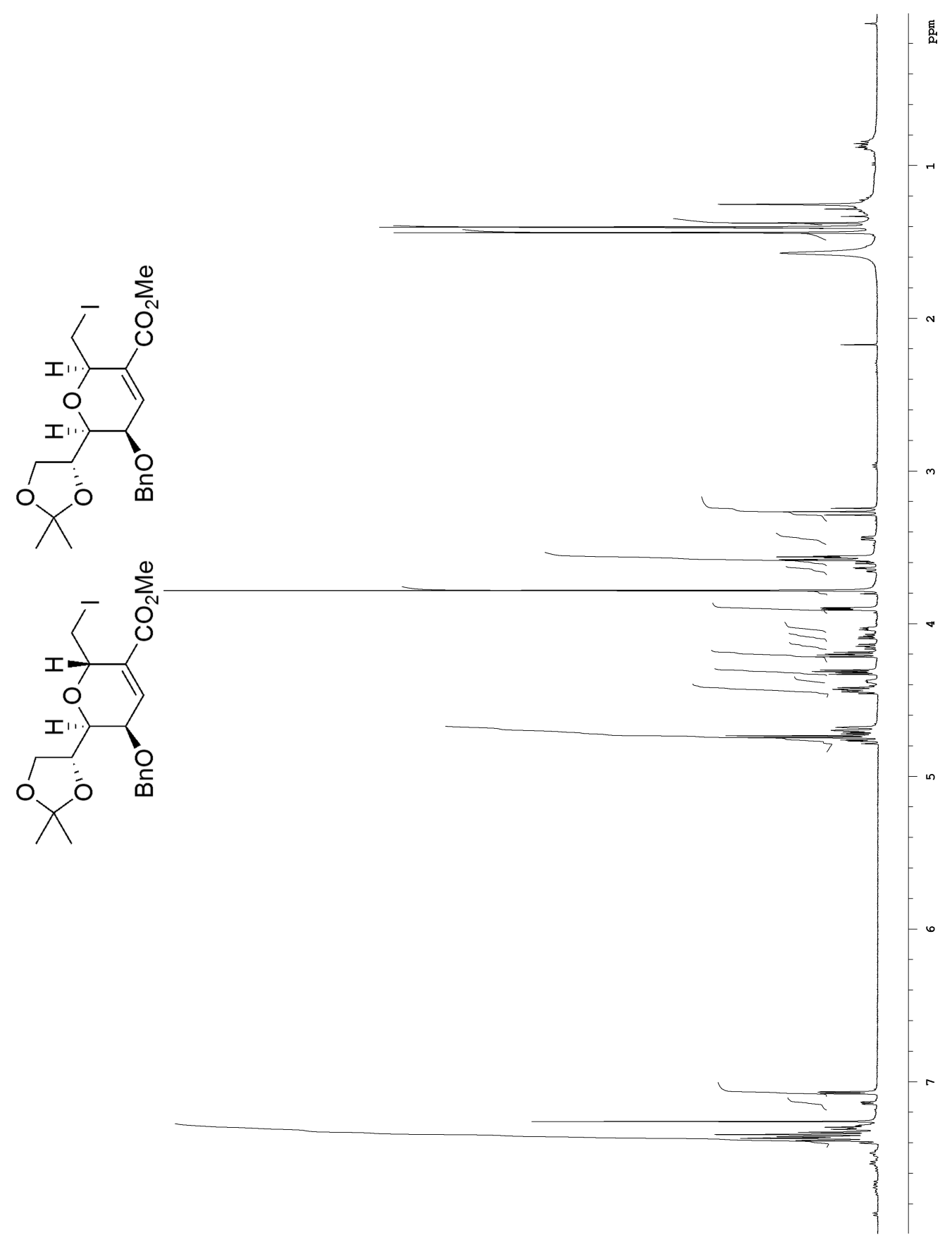

${ }^{1} \mathrm{H}-\mathrm{NMR}$ spectrum of iodoesters 381 and $382\left(500 \mathrm{MHz}, \mathrm{CDCl}_{3}\right.$ ) 


\section{References}

1. McNaught, A. D. Pure Appl. Chem. 1996, 68, 1919-2008.

2. Special issue on cyclopropanes and related rings: Chem. Rev. 2003, 103(4).

3. Perkin, J., W. H. Ber. Dtsch. Chem. Ges. 1884, 54-59.

4. Perkin, J., W. H. Ber. Dtsch. Chem. Ges. 1884, 323-325.

5. Rappoport, Z. Chemistry of the Cyclopropyl Group, Volume 1; John Wiley and Sons, Inc. New York, 1987.

6. Rappoport, Z. Chemistry of the Cyclopropyl Group, Volume 2; John Wiley and Sons, Inc. New York, 1995.

7. Barlet, R.; Vo-Quang, Y. Bull. Soc. Chim. Fr. 1969, 3729-3760.

8. Banwell, M. G.; Reum, M. E. In Advances in Strain in Organic Chemistry; Halton, B., Ed.; JAI Press Ltd: London, 1991; Vol. 1, pp 19-64.

9. Fedoryński, M. Chem. Rev. 2003, 103, 1099-1132.

10. Halton, B.; Harvey, J. Synlett 2006, 1975-2000.

11. Simmons, H. E.; Smith, R. D. J. Am. Chem. Soc. 1959, 81, 4256-4264.

12. Mąkosza, M.; Wawrzyniewicz, M. Tetrahedron Lett. 1969, 4659-4662.

13. Banwell, M. G. Pure Appl. Chem. 2008, 80, 669-679.

14. Stanislawski, P. C.; Willis, A. C.; Banwell, M. G. Chem.-Asian. J. 2007, 2, 1127-1136.

15. Bissember, A. C.; Phillis, A. T.; Banwell, M. G.; Willis, A. C. Org. Lett. 2007, 9, 5421-5424.

16. Sandler, S. R. J. Org. Chem. 1967, 32, 3876-3881.

17. Skattebøl, L. J. Org. Chem. 1970, 35, 3200-3201.

18. Woodward, R. B.; Hoffmann, R. J. Am. Chem. Soc. 1965, 87, 395-397.

19. DePuy, C. H.; Schack, L. G.; Hausser, J. W.; Wiedemann, W. J. Am. Chem. Soc. 1965, 87, 4006.

20. Cristol, S. J.; Sequeira, R. M.; DePuy, C. H. J. Am. Chem. Soc. 1965, 87, 4007-4008. 
21. Davis, B. G.; Fairbanks, A. J. Carbohydrate Chemistry; Oxford University Press Oxford, 2002.

22. Cousins, G. S.; Hoberg, J. O. Chem. Soc. Rev. 2000, 29, 165-174.

23. Meldgaard, M.; Wengel, J. J. Chem. Soc., Perkin Trans. 1 2000, 3539-3554.

24. Shekhani, M. S.; Latif, F.; Fatima, A.; Malik, A.; Voelter, W. J. Chem. Soc., Chem. Commun. 1988, 1419-1420.

25. Testero, S. A.; Spanevello, R. A. Carbohydr. Res. 2006, 341, 1057-1060.

26. Okabe, M.; Sun, R. C. Tetrahedron Lett. 1989, 30, 2203-2206.

27. Ferrier, R. J.; Prasad, N. J. Chem. Soc. C 1969, 570-575.

28. Albano, E. L.; Horton, D.; Lauterbach, J. H. Carbohydr. Res. 1969, 9, 149-161.

29. Furukawa, J.; Kawabata, N.; Nishimura, J. Tetrahedron Lett. 1966, 3353-3354.

30. Furukawa, J.; Kawabata, N.; Nishimura, J. Tetrahedron 1968, 24, 53-58.

31. Gagneron, J.; Gosselin, G.; Mathé, C. J. Org. Chem. 2005, 70, 6891-6897.

32. Storkey, C. M.; Win, A. L.; Hoberg, J. O. Carbohydr. Res. 2004, 339, 897-899.

33. Sridhar, P. R.; Kumar, P. V.; Seshadri, K.; Satyavathi, R. Chem.-Eur. J. 2009, $15,7526-7529$.

34. Kim, C.; Hoang, R.; Theodorakis, E. A. Org. Lett. 1999, 1, 1295-1297.

35. Timmers, C. M.; Leeuwenburgh, M. A.; Verheijen, J. C.; van der Marel, G. A.; van Boom, J. H. Tetrahedron: Asymmetry 1996, 7, 49-52.

36. Samano, V.; Robins, M. J. Tetrahedron Lett. 1994, 35, 3445-3448.

37. Waldraff, C.; Bernet, B.; Vasella, A. Helv. Chim. Acta 1997, 80, 1882-1900.

38. Vasella, A.; Uhlmann, P.; Waldraff, C. A. A.; Diederich, F.; Thilgen, C. Angew. Chem., Int. Ed. 1992, 31, 1388-1390.

39. Meyer zu Reckendorf, W.; Kamprath-Scholtz, U. Angew. Chem., Int. Ed. 1968, 7, 142-143.

40. Sakakibara, T.; Takamoto, T.; Sudoh, R.; Nakagawa, T. Chem. Lett. 1972, $1219-1222$.

41. Brimacombe, J. S.; Evans, M. E.; Forbes, E. J.; Foster, A. B.; Webber, J. M. Carbohydr. Res. 1967, 4, 239-243.

42. Parham, W. E.; Schweizer, E. E. J. Org. Chem. 1959, 24, 1733-1735.

43. Petter, R. C.; Powers, D. G. Tetrahedron Lett. 1989, 30, 659-662.

44. Murali, R.; Ramana, C. V.; Nagarajan, M. J. Chem. Soc., Chem. Commun. 1995, 217-218.

45. Ramana, C. V.; Murali, R.; Nagarajan, M. J. Org. Chem. 1997, 62, 7694-7703. 
46. Corsaro, A.; Chiacchio, U.; Adamo, R.; Pistarà, V.; Rescifina, A. et al. Tetrahedron 2004, 60, 3787-3795.

47. Gurjar, M. K.; Ravindranadh, S. V.; Kumar, P. Chem. Commun. 2001, 917918.

48. Gurjar, M. K.; Ravindranadh, S. V.; Karmakar, S. Chem. Commun. 2001, $241-242$.

49. Gurjar, M. K.; Ravindranadh, S. V.; Sankar, K.; Karmakar, S.; Cherian, J. et al. Org. Biomol. Chem. 2003, 1, 1366-1373.

50. Kar, P.; Nagaiah, K.; Gurjar, M. K. J. Indian Chem. Soc. 2005, 82, 428-432.

51. Meng, D.; Bertinato, P.; Balog, A.; Su, D.-S.; Kamenecka, T. et al. J. Am. Chem. Soc. 1997, 119, 10073-10092.

52. Fernandez-Megia, E.; Gourlaouen, N.; Ley, S. V.; Rowlands, G. J. Synlett 1998, 991-994.

53. Raman, C. V.; Nagarajan, M. Carbohydr. Lett. 1998, 3, 117-120.

54. Beyer, J.; Madsen, R. J. Am. Chem. Soc. 1998, 120, 12137-12138.

55. Beyer, J.; Skaanderup, P. R.; Madsen, R. J. Am. Chem. Soc. 2000, 122, 95759583.

56. Brady, T. P.; Kim, S. H.; Wen, K.; Kim, C.; Theodorakis, E. A. Chem.-Eur. J. 2005, 11, 7175-7190.

57. Kim, C.; Brady, T.; Kim, S. H.; Theodorakis, E. A. Synth. Commun. 2004, 34, 1951-1965.

58. Gharpure, S. J.; Shukla, M. K.; Vijayasree, U. Org. Lett. 2009, 11, 5466-5469.

59. Boyer, F. D.; Lallemand, J. Y. Tetrahedron 1994, 50, 10443-10458.

60. Hoberg, J. O. J. Org. Chem. 1997, 62, 6615-6618.

61. Batchelor, R.; Hoberg, J. O. Tetrahedron Lett. 2003, 44, 9043-9045.

62. Duchaussoy, P.; Di Cesare, P.; Gross, B. Synthesis 1979, 198-200.

63. Al-Harrasi, A.; Reissig, H.-U. Synlett 2005, 2376-2378.

64. Ganesh, N. V.; Jayaraman, N. J. Org. Chem. 2007, 72, 5500-5504.

65. Ganesh, N. V.; Jayaraman, N. J. Org. Chem. 2009, 74, 739-746.

66. Ganesh, N. V.; Raghothama, S.; Sonti, R.; Jayaraman, N. J. Org. Chem. 2010, $75,215-218$.

67. Hoberg, J. O. Tetrahedron 1998, 54, 12631-12670.

68. Snyder, N. L.; Haines, H. M.; Peczuh, M. W. Tetrahedron 2006, 62, 9301-9320.

69. Pakulski, Z. Pol. J. Chem. 2006, 80, 1293-1326. 
70. Sabatino, D.; Damha, M. J. J. Am. Chem. Soc. 2007, 129, 8259-8270.

71. Peczuh, M. W.; Snyder, N. L. Tetrahedron Lett. 2003, 44, 4057-4061.

72. Peczuh, M. W.; Snyder, N. L.; Fyvie, W. S. Carbohydr. Res. 2004, 339, 11631171.

73. Nowakowski, M.; Hoffmann, H. M. R. Tetrahedron Lett. 1997, 38, 1001-1004.

74. Shaabani, A.; Soleimani, E.; Sarvary, A.; Rezayan, A. H. Bioorg. Med. Chem. Lett. 2008, 18, 3968-3970.

75. Witt, U. G.; Schultz, J. E.; Dölker, M.; Eger, K. Bioorg. Med. Chem. 2000, 8, 807-813.

76. Paquette, L. A.; Sivik, M. R. Synth. Commun. 1991, 21, 467-479.

77. Krohn, K.; Biele, C.; Drogies, K.-H.; Steingröver, K.; Aust, H.-J. et al. Eur. J. Org. Chem. 2002, 2331-2336.

78. Qin, S.; Krohn, K.; Flörke, U.; Schulz, B.; Draeger, S. et al. Eur. J. Org. Chem. 2009, 3279-3284.

79. Oh, H.; Swenson, D. C.; Gloer, J. B.; Shearer, C. A. Tetrahedron Lett. 2001, 42, 975-977.

80. Kock, I.; Krohn, K.; Egold, H.; Draeger, S.; Schulz, B. et al. Eur. J. Org. Chem. 2007, 2186-2190.

81. Krohn, K.; Zia, U.; Hussain, H.; Flörke, U.; Schulz, B. et al. Chirality 2007, 19, 464-470.

82. Hayashi, K.; Takizawa, M.; Noguchi, K. Jpn. Patent 10287679 1998, 17 pp.

83. Nozawa, O.; Okazaki, T.; Sakai, N.; Komurasaki, T.; Hanada, K. et al. J. Antibiot. 1995, 48, 113-118.

84. Nozawa, O.; Okazaki, T.; Morimoto, S.; Chen, Z.-X.; He, B.-M. et al. J. Antibiot. 2000, 53, 1296-1300.

85. Wang, J.; Hsung, R. P.; Ghosh, S. K. Org. Lett. 2004, 6, 1939-1942.

86. Suzuki, E.; Takao, K.-I.; Tadano, K.-I. Heterocycles 2000, 52, 519-523.

87. Gao, X.; Nakadai, M.; Snider, B. B. Org. Lett. 2003, 5, 451-454.

88. Gao, X.; Snider, B. B. J. Org. Chem. 2004, 69, 5517-5527.

89. Kozikowski, A. P.; Lee, J. J. Org. Chem. 1990, 55, 863-870.

90. Greene, T. W.; Wuts, P. G. M. Protective Groups in Organic Synthesis, 3rd ed.; John Wiley and Sons, Inc. New York, 1999.

91. Jacobsen, E. N.; Zhang, W.; Muci, A. R.; Ecker, J. R.; Deng, L. J. Am. Chem. Soc. 1991, 113, 7063-7064.

92. Banwell, M. G.; Corbett, M.; Gulbis, J.; Mackay, M. F.; Reum, M. E. J. Chem. Soc., Perkin Trans. 1 1993, 945-963. 
93. Fischer, E.; Zach, C. Sitzb. kgl. preuss. Akad. 1913, 311-317.

94. Fischer, E. Ber. Dtsch. Chem. Ges. 1914, 47, 196-210.

95. Fischer, E.; Curme, J., George O. Ber. Dtsch. Chem. Ges. 1914, 47, $2047-$ 2057.

96. Roth, W.; Pigman, W. In Methods in Carbohydrate Chemistry; Whistler, R. L., Wolfrom, M. L., Eds.; Academic Press: New York, 1963; Vol. II, pp 405-408.

97. Fedoryński, M.; Ma̧kosza, M. J. Organomet. Chem. 1973, 51, 89-91.

98. Regen, S. L.; Singh, A. J. Org. Chem. 1982, 47, 1587-1588.

99. Booma, C.; Balasubramanian, K. K. J. Chem. Soc., Chem. Commun. 1993, 1394-1395.

100. Gupta, A.; Vankar, Y. D. Tetrahedron 2000, 56, 8525-8531.

101. Kashyap, S.; Vidadala, S. R.; Hotha, S. Tetrahedron Lett. 2007, 48, 8960-8962.

102. Agelis, G.; Tzioumaki, N.; Botić, T.; Cencič, A.; Komiotis, D. Bioorg. Med. Chem. 2007, 15, 5448-5456.

103. Sato, K.-i.; Sekiguchi, T.; Hozumi, T.; Yamazaki, T.; Akai, S. Tetrahedron Lett. 2002, 43, 3087-3090.

104. Chen, J.; Fu, X.-G.; Zhou, L.; Zhang, J.-T.; Qi, X.-L. et al. J. Org. Chem. 2009, 74, 4149-4157.

105. Shao, J.; Zhou, B.; Chu, B.; Yen, Y. Curr. Cancer Drug Targets 2006, 6, 409-431.

106. Banwell, M. G.; Gable, R. W.; Halton, B.; Phyland, J. R. Aust. J. Chem. 1994, 47, 1879-1884.

107. Banwell, M. G.; Phillis, A. T.; Willis, A. C. Org. Lett. 2006, 8, 5341-5344.

108. Baird, M. S. Chem. Rev. 2003, 103, 1271-1294.

109. Feldman, K. S.; Schildknegt, K. J. Org. Chem. 1994, 59, 1129-1134.

110. Weygand, F. In Methods in Carbohydrate Chemistry; Whistler, R. L., Wolfrom, M. L., Eds.; Academic Press: New York, 1962; Vol. I, pp 182-185.

111. Vorogushin, A. V.; Wulff, W. D.; Hansen, H.-J. Org. Lett. 2001, 3, 2641-2644.

112. Hoberg, J. O.; Bozell, J. J. Tetrahedron Lett. 1995, 36, 6831-6834.

113. Mootoo, D. R.; Fraser-Reid, B. J. Chem. Soc., Chem. Commun. 1986, 15701571.

114. Schoenberg, A.; Bartoletti, I.; Heck, R. F. J. Org. Chem. 1974, 39, 3318-3326.

115. Danishefsky, S. J.; Masters, J. J.; Young, W. B.; Link, J. T.; Snyder, L. B. et al. J. Am. Chem. Soc. 1996, 118, 2843-2859. 
116. Veitch, G. E.; Pinto, A.; Boyer, A.; Beckmann, E.; Anderson, J. C.; Ley, S. V. Org. Lett. 2008, 10, 569-572.

117. He, X.-F.; Wang, X.-N.; Gan, L.-S.; Dong, L.; Yue, J.-M. Org. Lett. 2008, 10, 4327-4330.

118. Brown, G. J. Nat. Prod. 1994, 57, 328-330.

119. de la Hoz, A.; Díaz-Ortiz, A.; Moreno, A. Chem. Soc. Rev. 2005, 34, 164-178.

120. Takayama, H.; Koike, T.; Aimi, N.; Sakai, S. J. Org. Chem. 1992, 57, 21732176.

121. De Raadt, A.; Ferrier, R. J. Carbohydr. Res. 1991, 216, 93-107.

122. Krohn, K.; Flörke, U.; Gehle, D. J. Carbohydr. Chem. 2002, 21, 431-443.

123. Halcomb, R. L.; Boyer, S. H.; Wittman, M. D.; Olson, S. H.; Denhart, D. J. et al. J. Am. Chem. Soc. 1995, 117, 5720-5749.

124. Blakemore, P. R.; Cole, W. J.; Kocieński, P. J.; Morley, A. Synlett 1998, 26-28.

125. Aïssa, C. Eur. J. Org. Chem. 2009, 1831-1844.

126. Doering, W. v. E.; Hoffmann, A. K. J. Am. Chem. Soc. 1954, 76, 6162-6165.

127. Valverde, S.; García-Ochoa, S.; Martín-Lomas, M. J. Chem. Soc., Chem. Commun. 1987, 383-384.

128. Kim, H.; Men, H.; Lee, C. J. Am. Chem. Soc. 2004, 126, 1336-1337.

129. Klaić, B.; Raza, Z.; Sanković, M.; Šunjić, V. Helv. Chim. Acta 1987, 70, 59-62.

130. Tam, S. Y. K.; Fraser-Reid, B. Carbohydr. Res. 1975, 45, 29-43.

131. Fraser-Reid, B.; Radatus, B. J. Am. Chem. Soc. 1970, 92, 5288-5290.

132. Ruffin, B.; Grelier, S.; Nourmamode, A.; Castellan, A. Can. J. Chem. 2002, $80,1223-1231$.

133. Excoffier, G.; Gagnare, D.; Utille, J. P. Carbohydr. Res. 1975, 39, 368-373.

134. Forbes, C. L.; Franck, R. W. J. Org. Chem. 1999, 64, 1424-1425.

135. Hwang, C. K.; Li, W. S.; Nicolaou, K. C. Tetrahedron Lett. 1984, 25, 22952296.

136. Bergman, E. J. Org. Chem. 1963, 28, 2210-2215.

137. Homsi, F.; Robin, S.; Rousseau, G. Org. Synth. 2000, 77, 206-211.

138. Harvey, J. E.; Raw, S. A.; Taylor, R. J. K. Org. Lett. 2004, 6, 2611-2614.

139. Ramberg, L.; Backlund, B. Ark. Kemi, Mineral. Geol. 1940, 13A, 1-50.

140. Taylor, R. J. K.; Casy, G. Org. React. 2003, 62, 357-475.

141. Meyers, C. Y.; Malte, A. M.; Matthews, W. S. J. Am. Chem. Soc. 1969, 91, $7510-7512$. 
142. Griffin, F. K.; Murphy, P. V.; Paterson, D. E.; Taylor, R. J. K. Tetrahedron Lett. 1998, 39, 8179-8182.

143. McAllister, G. D.; Taylor, R. J. K. Tetrahedron Lett. 2001, 42, 1197-1200.

144. Taylor, R. J. K.; McAllister, G. D.; Franck, R. W. Carbohydr. Res. 2006, 341, $1298-1311$.

145. Fuchs, E.; Keller, M.; Breit, B. Chem.-Eur. J. 2006, 12, 6930-6939.

146. McAllister, G. D.; Paterson, D. E.; Taylor, R. J. K. Angew. Chem., Int. Ed. 2003, 42, 1387-1391.

147. Aucagne, V.; Tatibouët, A.; Rollin, P. Tetrahedron Lett. 2008, 49, 4750-4753.

148. Chan, T.-L.; Fong, S.; Li, Y.; Man, T.-O.; Poon, C.-D. J. Chem. Soc., Chem. Commun. 1994, 1771-1772.

149. Pal, T. K.; Pathak, T. Carbohydr. Res. 2008, 343, 2826-2829.

150. Pal, T. K.; Pathak, T. Synlett 2008, 2263-2266.

151. Boeckman, J., Robert K.; Yoon, S. K.; Heckendorn, D. K. J. Am. Chem. Soc. 1991, 113, 9682-9684.

152. Carpentier, J.-F.; Castanet, Y.; Brocard, J.; Mortreux, A.; Petit, F. Tetrahedron Lett. 1991, 32, 4705-4708.

153. Pellegrini, S.; Castanet, Y.; Brocard, J.; Mortreux, A. J. Mol. Catal. A: Chem. 2007, 277, 21-26.

154. Chmielewski, M.; Fokt, I.; Grodner, J.; Grynkiewicz, G.; Szeja, W. J. Carbohydr. Chem. 1989, 8, 735-744.

155. Rebstock, A.-S.; Mongin, F.; Trécourt, F.; Quéguiner, G. Tetrahedron 2004, 60, 2181-2186.

156. Stachel, S. J.; Coburn, C. A.; Sankaranarayanan, S.; Price, E. A.; Pietrak, B. L. et al. J. Med. Chem. 2006, 49, 6147-6150.

157. Rico, M.; Santoro, J. Organic Magnetic Resonance 1976, 8, 49-55.

158. Valverde, S.; García-Ochoa, S. J. Carbohydr. Chem. 1989, 8, 553-563.

159. Kartha, K. P. R. Tetrahedron Lett. 1986, 27, 3415-3416.

160. Tony, K. A.; Denton, R. W.; Dilhas, A.; Jiménez-Barbero J.; Mootoo, D. R. Org. Lett. 2007, 9, 1441-1444.

161. Posner, G. H.; Brunelle, D. J. J. Org. Chem. 1972, 37, 3547-3549. 